\title{
MODELLING OF COOLING TOWER SPLASH PACK
}

\author{
André Alexis Dreyer \\ Dissertation presented for the degree Doctor of Philosophy \\ (Mechanical Engineering) at the University of Stellenbosch
}

Thesis supervisor : Prof. P.J. Erens

\section{Department of Mechanical Engineering \\ University of Stellenbosch \\ Stellenbosch, South Africa}

January 1994 


\section{DECLARATION}

I, André Alexis Dreyer, the undersigned, hereby declare that the work contained in this thesis is my own original work and has not previously, in its entirety or in part, been submitted for a degree.

ISt day of February............... 1994. 


\section{ABSTRACT}

A mathematical model and a computer simulation program have been developed for the modelling of counterflow cooling tower splash pack thermal performance. The onedimensional model uses basic aerodynamic, hydrodynamic and heat/mass transfer information to predict the performance of the splash pack without depending on cooling tower test data.

Some basic experimental work has been done to clarify aspects such as the volume of water splashing from the grids, the size distribution of splash drops and the size distribution of the drops dripping from the lower edges of the slats. Tests were also performed on twelve different splash packs to generate reference data against which the simulation program could be verified.

The predicted transfer characteristics and pressure drop data obtained with the simulation program are compared to experimental data. It has been found that the model predicts the correct trends for both the transfer characteristics and the pressure drop across the packing. Quantitatively the simulation program was found to over-predict the transfer characteristics and the pressure drop across the packing. Possible reasons for the differences are discussed.

The program was used to obtain rough guidelines for optimum splash pack layout. The program was also employed to study the effect of reduced surface tension (resulting in smaller drops) on the thermal performance of splash pack.

A number of recommendations for further research are made. 


\section{OPSOMMING}

'n Wiskundige model en 'n rekenaar simulasie program is ontwikkel om die vertoning van teenvloei koeltoring spat-pakking ("splash pack") te voorspel. Die een-dimensionele model gebruik basiese lugdinamiese, hidrodinamiese en warmte/massa-oordrag data om die vertoning te voorspel sonder om van gemete koeltoring toetsdata gebruik te maak.

Sekere basiese eksperimentele werk is gedoen om die volgende aspekte meer duidelik te maak: die massa water wat spat vanaf die nat latte, die grootte-verdeling van die spat druppels en die grootte-verdeling van die druppels wat afdrup vanaf die latte. Toetse is ook gedoen op twaalf verskillende pakkings om verwysingsdata te genereer waarteen die akkuraatheid van die simulasie-program getoets kon word.

Die gemete oordrags-karakteristieke en drukval data is vergelyk met die voorspelde data wat met die program gegenereer is. Daar is gevind data die model die korrekte tendense in drukval en oordrags-karakteristieke voorspel. Die program het die oordrags-karakteristieke en die drukval oor die pakking oor-voorspel en moontlike redes hiervoor word bespreek.

Die simulasie program is gebruik om ontwerpsriglyne te genereer vir die optimum pakkingsmateriaal uitleg. Met behulp van die program was dit ook moontlik om die effek van verlaagde oppervlakspanning (en gevolglik kleiner druppels) op die termiese vertoning van pakkingsmateriaal te ondersoek.

Verskeie voorstelle vir verdere werk word gemaak. 
iv

\section{ACKNOWLEDGEMENTS}

The work described in this dissertation forms part of an on-going research program on wet and dry cooling in the Department of Mechanical Engineering at the University of Stellenbosch.

I would like to acknowledge the valued contributions and support of the following persons and organisations:

Prof. P.J. Erens for his guidance and support throughout the project;

Prof. D.G. Kröger for his suggestions and interest in this project;

The other lecturers in the Mechanical Engineering Department and my fellow-students for many interesting discussions;

Mr. B. Vincent and the Centre for Mechanical Services for the construction of the experimental equipment and assistance with the experimental work;

Monika and my family for their moral support;

The Energy Branch of the Department of Mineral and Energy Affairs of South Africa for their financial support. 


\section{TABLE OF CONTENTS}

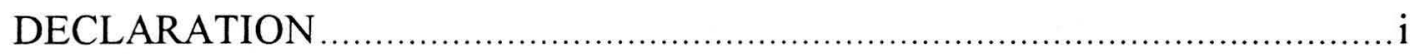

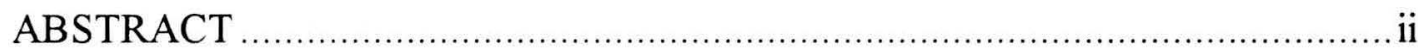

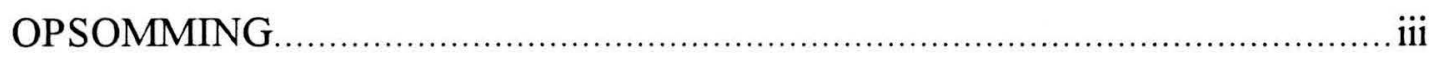

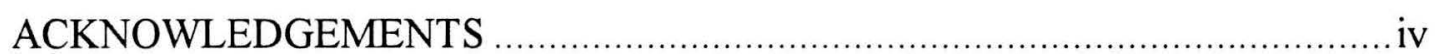

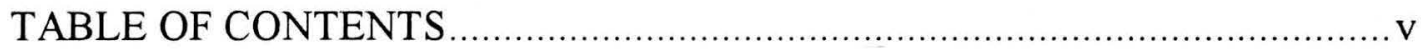

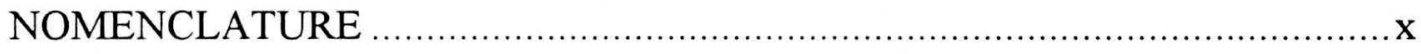

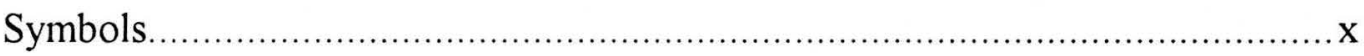

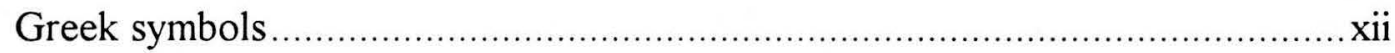

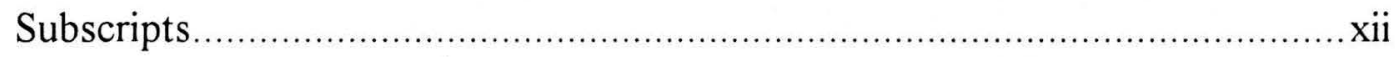

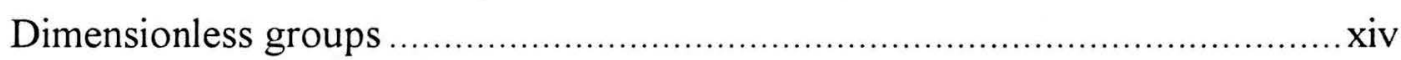

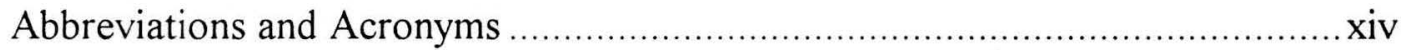

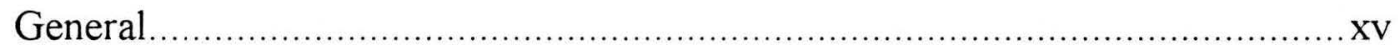

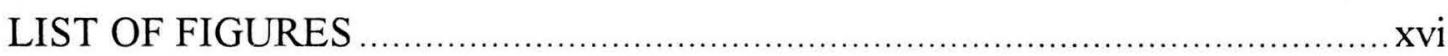

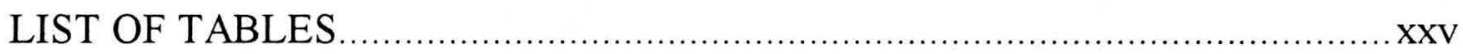

CHAPTER 1 INTRODUCTION ........................................................... 1

CHAPTER 2 LITERATURE SURVEY .................................................. 4

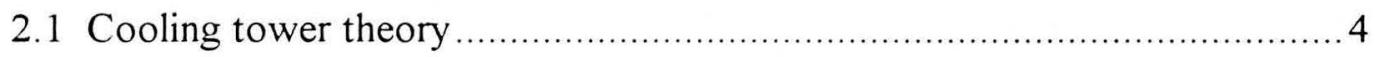

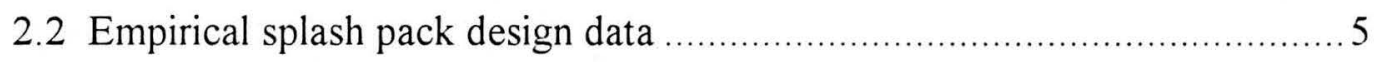

2.3 Mathematical modelling from basic principles................................... 8

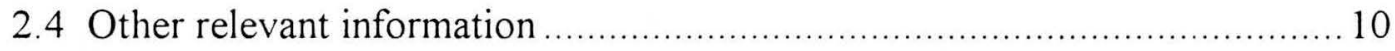

2.4.1 Drag on spheres and drops ............................................ 10

2.4.2 Heat and mass transfer from spheroids and drops...................... 17

2.4.3 Aerodynamic break-up ................................................. 21 


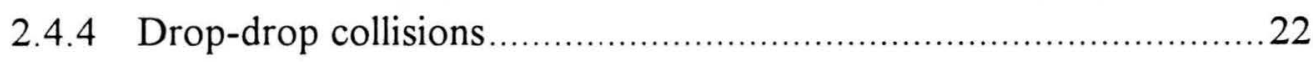

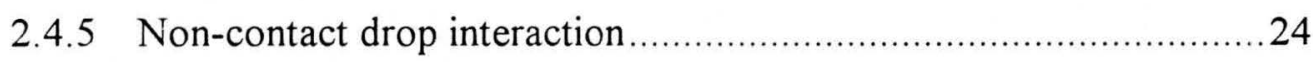

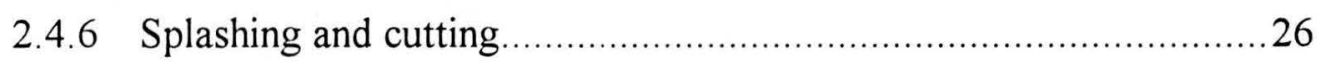

2.4.7 Dripping below horizontal surfaces..........................................29

2.4.8 Flow through and heat/mass transfer from grids .......................... 30

2.4 .9 Free-stream turbulence ....................................................... 32

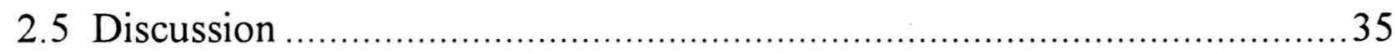

CHAPTER 3 MODELLING APPROACH................................................. 36

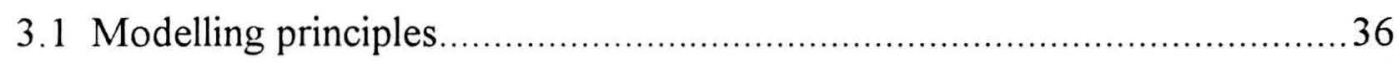

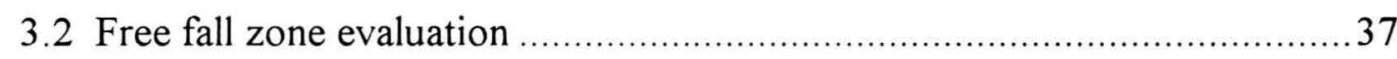

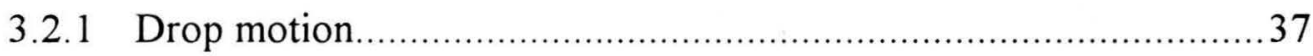

3.2.2 Heat and mass transfer from drops.................................... 40

3.2.3 Aerodynamic drop break-up ............................................ 42

3.2.4 Drop-drop collisions........................................................... 42

3.2.5 Pressure drop .............................................................. 43

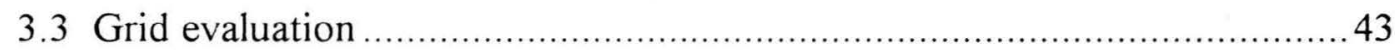

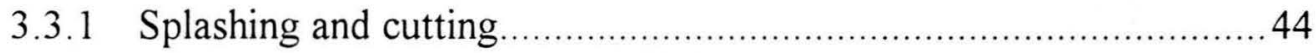

3.3.2 Interference between adjacent impacts ................................... 51

3.3.3 Cooling of upward moving splash drops ................................ 52

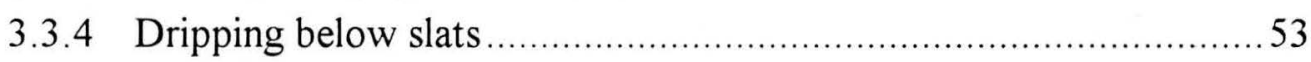

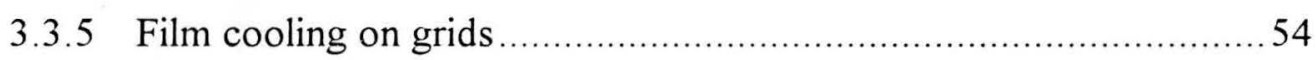

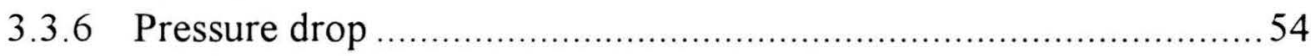

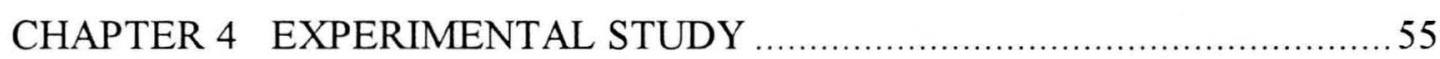

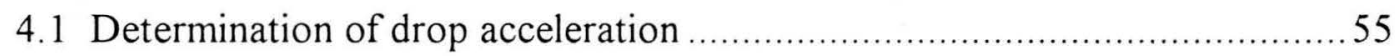

4.2 Film thickness measurements on slats in a cooling tower ......................61

4.3 Experimental investigation of the splashing and cutting processes.............65 65

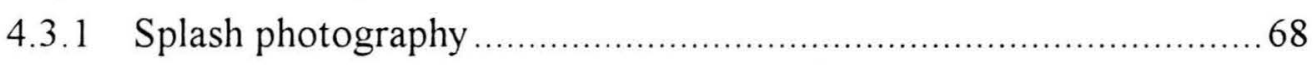

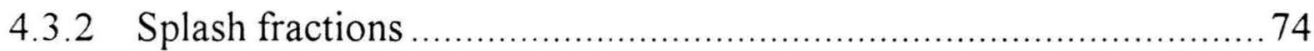

4.3.3 Splash and cutting drop size distributions............................. 82

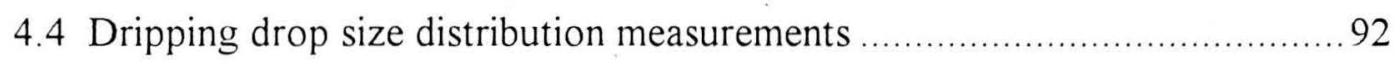

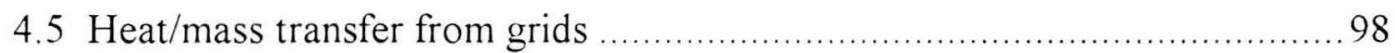


vii

4.6 Cooling tower packing performance tests...................................... 101

4.6.1 Initial drop size distribution ................................................. 104

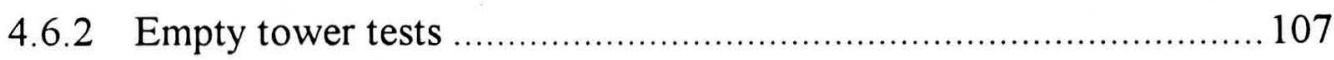

4.6.3 Splash pack performance tests .................................................. 109

4.6.4 Drop size measurements in a cooling tower ............................. 112

4.6.5 Pressure drop across the splash grids in dry operation.................. 113

CHAPTER 5 COMPUTER SIMULATION .............................................. 115

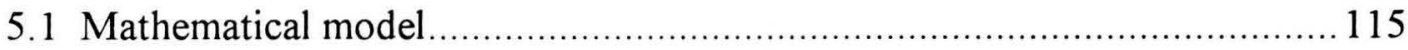

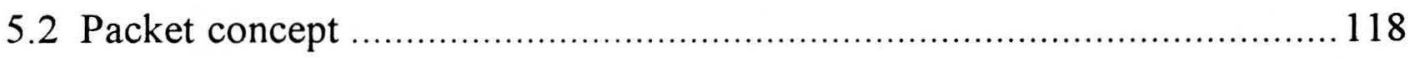

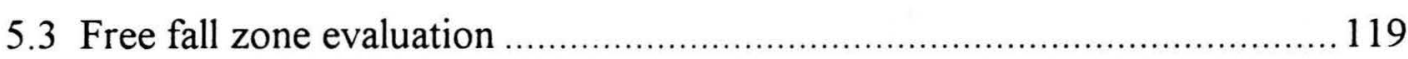

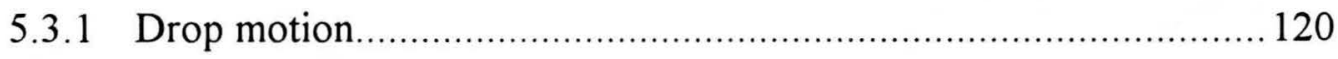

5.3.2 Heat and mass transfer from drops........................................ 121

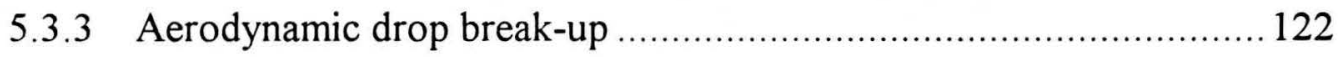

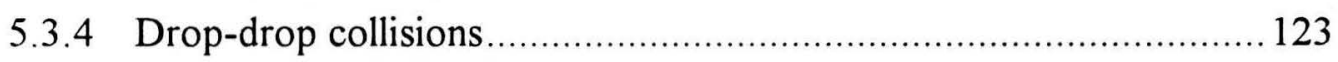

5.3.5 Handling of upward flowing drops...................................... 125

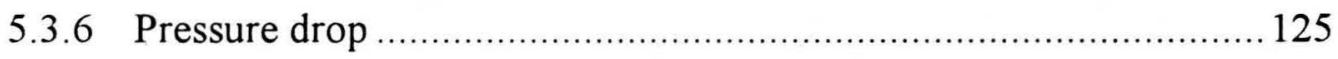

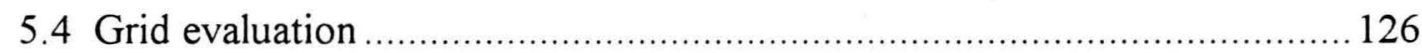

5.4.1 Splashing and cutting ........................................................ 127

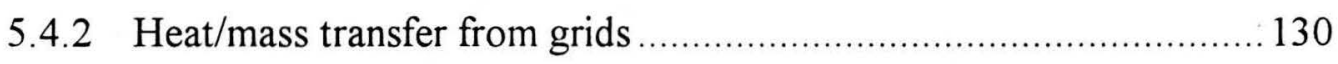

5.4 .3 Dripping below grids ......................................................... 130

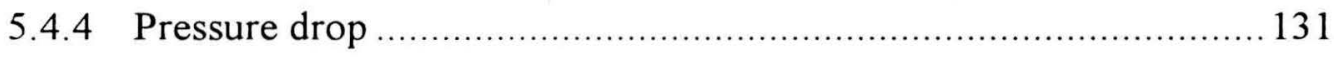

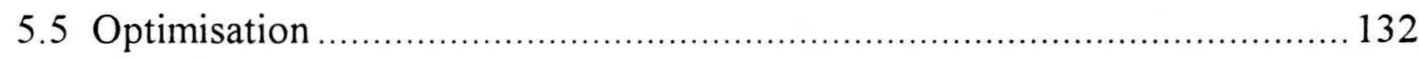

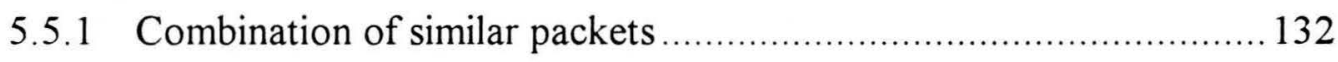

5.5.2 Distribution of splashing/cutting/dripping drops ...................... 133

CHAPTER 6 RESULTS AND DISCUSSION .......................................... 134

6.1 Typical simulation results ............................................................. 134

6.1.1 Empty tower with mono-disperse drop distribution..................... 134

6.1.2 Empty tower with poly-disperse drop distribution..................... 138

6.1.3 Splash pack simulation sensitivity analysis............................. 141

6.2 Comparison with measured splash pack performance .......................... 153

6.2.1 Thermal performance....................................................... 153

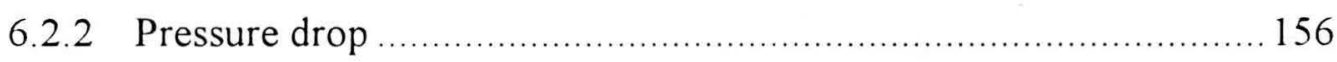




\section{viii}

6.2.3 Drop size

6.3 Design trends for splash pack optimisation 158

CHAPTER 7 CONCLUSIONS AND RECOMMENDATIONS 164

REFERENCES

APPENDIX A Re-correlation of the data by Yao [74YA1] for heat/mass transfer from free-falling, accelerating water drops.

APPENDIX B Description of the digital image processing technique used for the measurement of drop sizes from photographs.

APPENDIX C Description of apparatus for measuring water film thicknesses.

APPENDIX D Derivation of simplified analytic equations to describe the motion of splash drop above the impact plane

APPENDIX E Drop deflection around.slats

APPENDIX F Low flow rate drop formation from cylindrical orifices (and surface tension measurement).

APPENDIX G Properties of air, water and air-water mixtures 203

APPENDIX $\mathrm{H}$ Tchebycheff numerical integration method 209

APPENDIX I Numerical sample calculation

APPENDIX J Measured drop acceleration data

APPENDIX K Experimentally determined water film thicknesses on the splash grids in a cooling tower

APPENDIX L Summary of data obtained during experiments to measure the mass of water splashing from narrow slats

APPENDIX M Summary of drop size distribution data obtained from drop splashing and cutting experiments

APPENDIX N Summary of drop size distribution data for water dripping below different slats 
APPENDIX O Summary of data obtained from experiments to determine the heat transfer coefficients on the outside of tubes with different profiles.

APPENDIX P Summary of drop size distribution data obtained for the water distribution system

APPENDIX Q Drop size distribution data from measurements in a cooling tower fitted with splash pack

APPENDIX R Summary of measured pressure drop across experimental splash grids during dry operation.

APPENDIX S Program for the simulation of the thermal performance of counterflow cooling tower splash pack 


\section{NOMENCLATURE}

\section{Symbols}

a

$a^{\prime}$

A

$\mathrm{A}_{\mathrm{m}}$

b

$c_{1}, c_{2} \ldots$

$c_{p}$

$\mathrm{c}_{\mathrm{pm}}$

$\mathrm{C}$

$\mathrm{C}_{\mathrm{D}}$

d

$d_{a}$

$d_{b}$

$d_{e}$

$d_{m}$

$d_{R R}$

$\mathrm{d}_{30}$

$\mathrm{d}_{32}$

$\mathrm{d}_{50}$

D

E

$f_{c}$
Pack density, surface area per unit volume, $\mathrm{m}^{2} / \mathrm{m}^{3}$

Pack density, surface area per unit air flow volume, $\mathrm{m}^{2} / \mathrm{m}^{3}$

Area, $m^{2}$

Amplitude of drop oscillation, $\left(\mathrm{d}(\mathrm{t})-\mathrm{d}_{\mathrm{e}}\right) / 2, m$

Dimension defined by Figure 3.7 and Equation (3.18), $m$

Constants, dimensionless

Specific heat (heat capacity), $J /(\mathrm{kgK})$

Specific heat of moist air, $J /(\mathrm{kgK})$

Dimensionless drop size, $\mathrm{d}\left(\mathrm{g}\left(\rho_{\mathrm{w}}-\rho_{\mathrm{a}}\right) / \sigma\right)^{0.5}$, dimensionless

Drag coefficient, dimensionless

Diameter (characteristic length), $m$

Minor axis of spheroid (see Figure 2.4), $m$

Major axis of spheroid (see Figure 2.4), $\mathrm{d}_{\mathrm{b}}=\mathrm{d}_{\mathrm{e}} \mathrm{E}^{-1 / 3}, m$

Equivalent drop diameter, $(6 \mathrm{~V} / \pi)^{1 / 3}, m$

Maximum stable drop diameter, $m$

Scale parameter for Rosin-Rammler distribution, $m$

Mass mean diameter, defined in Equation (2.11), $m$

Sauter mean diameter, defined in Equation (2.10), $m$

Mass median diameter, drop size above (or below) which $50 \%$ of the total mass of a given distribution lies, $m$

Diffusivity, $\mathrm{m}^{2} / \mathrm{s}$

Drop deformation, ratio of minor to major axes, $\mathrm{d}_{\mathrm{a}} / \mathrm{d}_{\mathrm{b}}$, dimensionless

Cutting fraction, ratio of the mass of drops formed by cutting to that of the incoming drop, dimensionless

Interference factor, ratio of the splash fraction for a splash in the proximity of other splashes to that for an undisturbed splash, dimensionless

Frequency of oscillation in mode $\mathrm{n}, \mathrm{Hz}$

Splash fraction, ratio of the mass of splash drops to that of the incoming drop, dimensionless

Force, $N$

Gravitational acceleration, $\mathrm{m} / \mathrm{s}^{2}$

Correction factor defined by Yao [74YA1], dimensionless

Heat transfer coefficient, $W /\left(m^{2} K\right)$

Convection heat transfer coefficient, $W /\left(\mathrm{m}^{2} \mathrm{~K}\right)$

Convection mass transfer coefficient, $\mathrm{m} / \mathrm{s}$

Height (thickness of slat), $m$ 


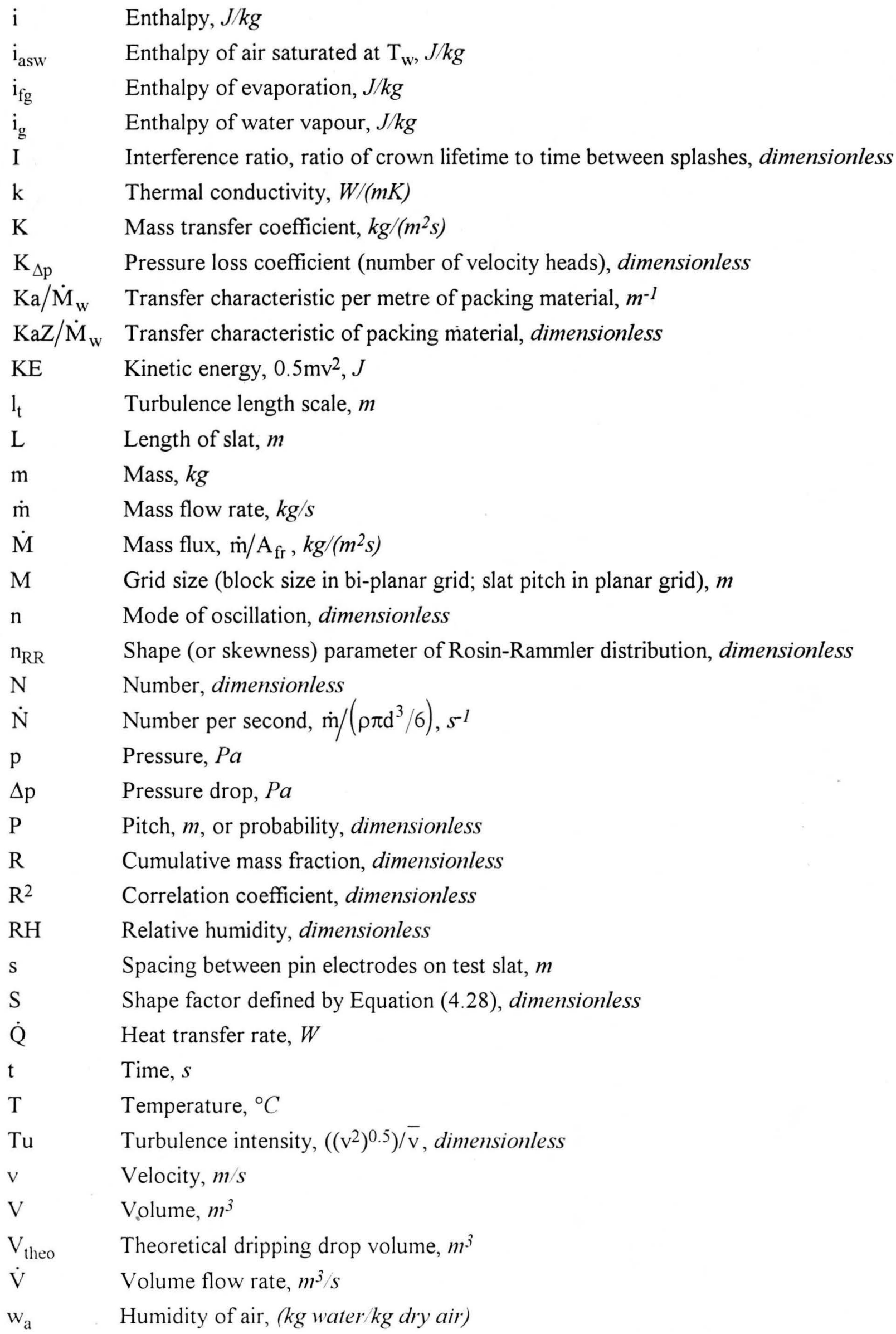


xii

$\begin{array}{ll}\mathrm{w}_{\text {asw }} & \text { Humidity of air saturated at } \mathrm{T}_{\mathrm{w}},(\mathrm{kg} \text { water } / \mathrm{kg} \text { dry air) } \\ \mathrm{W} & \text { Width of slat or crown width, } m \\ \mathrm{x} & \text { Horizontal distance from centre of a slat, } m \\ \mathrm{y} & \text { Horizontal distance along the length of a slat, } m \\ \mathrm{z} & \text { Vertical height or fall distance, } m \\ \mathrm{Z} & \text { Packing thickness, } m\end{array}$

\section{Greek symbols}

$\alpha$

Thermal diffusivity, $\mathrm{k} /\left(\rho \mathrm{c}_{\mathrm{p}}\right), \mathrm{m}^{2} / \mathrm{s}$

$\beta$

Porosity, ratio of open area to total frontal area of a grid, dimensionless

$\beta_{\text {ex }}$

Volume coefficient of expansion, for ideal gas $\beta_{\mathrm{ex}}=1 /(\mathrm{T}+273.15), K^{-1}$

$\chi$

Dimensionless drop impact point on slat, $\mathrm{x} /\left(\left(\mathrm{W}+\mathrm{d}_{\mathrm{i}}\right) / 2\right)$, dimensionless

$\delta$

Depth of water film at the centreline of a slat, $m$

$\varepsilon$

Emissivity, dimensionless

$\phi$

Mixing ratio defined by Equation (3.26), dimensionless

$\Phi$

"Thermal function" defined by Nottage and Boelter [40NO1], $s^{-1}$

$\Gamma$

Mass flow rate per unit length, $\mathrm{kg} /(\mathrm{ms})$

$\eta_{1}$

Efficiency of packing material, ratio of transfer characteristic per metre to pressure drop per metre, $\mathrm{Pa}^{-1}$

$\eta_{2} \quad$ Efficiency of packing material, ratio of transfer characteristic per metre to pressure loss coefficient per metre, dimensionless

$\eta_{\text {coll }} \quad$ Collection efficiency, fraction of in-line drops colliding with an object, dimensionless

$\eta_{\text {coal }} \quad$ Coalescence efficiency, fraction of colliding drops that coalesce on impact, dimensionless

$\varphi \quad$ Harkins-Brown correction factor, defined in Appendix F, dimensionless

$\mu \quad$ Dynamic viscosity, $\mathrm{kg} /\left(\mathrm{m}^{2} \mathrm{~s}\right)$

$\checkmark \quad$ Kinematic viscosity, $m^{2} / s$

$\theta \quad$ Splash angle, relative to the horizontal plane (xy), degrees

$\rho \quad$ Density, $\mathrm{kg} / \mathrm{m}^{3}$

$\sigma \quad$ Surface tension, $N / m$

$\sigma_{\text {rad }} \quad$ Stefan-Boltzmann constant, $5.669 \times 10^{-8} \mathrm{~W} /\left(\mathrm{m}^{2} \mathrm{~K}^{4}\right)$

$\Psi \quad$ "Dynamic function" defined by Nottage and Boelter [40NO1], $\mathrm{m} / \mathrm{s}$

\section{Subscripts}

a

as

asi
Air (including vapour)

Saturated air

Air saturated at the water interface temperature, $\mathrm{T}_{\mathrm{i}}$ 
xiii

\begin{tabular}{|c|c|}
\hline asw & Air saturated at the water temperature, $T_{w}$ \\
\hline atm & Atmospheric \\
\hline c & Cutting or condensate \\
\hline coll & Colliding \\
\hline crit & Critical \\
\hline cyl & Cylinder \\
\hline $\mathrm{d}$ & Dripping \\
\hline $\mathrm{da}$ & Dry air \\
\hline $\exp$ & Experimental \\
\hline $\mathrm{f}$ & Film or fluid \\
\hline $\mathrm{fc}$ & Forced convection \\
\hline $\mathrm{fr}$ & Frontal \\
\hline g & Gas \\
\hline $\mathrm{h}$ & Hydraulic or horizontal \\
\hline $\mathrm{HG}$ & Mercury \\
\hline $\mathrm{i}$ & Inlet, interface or incoming \\
\hline IP & Inflection point \\
\hline 1 & Liquid or laminar \\
\hline $\mathrm{L}$ & Large \\
\hline $\mathrm{m}$ & Mean (or average) \\
\hline $\max$ & Maximum \\
\hline $\min$ & Minimum \\
\hline nc & Natural convection \\
\hline $\mathrm{N}$ & Nozzle \\
\hline o & Outlet \\
\hline $\mathrm{p}$ & Primary or particle \\
\hline $\mathrm{rad}$ & Radiation \\
\hline ref & Reference \\
\hline rel & Relative \\
\hline res & Resident \\
\hline s & Splashing, satellite (secondary) or steam \\
\hline $\mathrm{S}$ & Small \\
\hline sat & Saturation \\
\hline $\mathrm{t}$ & Turbulent \\
\hline $\mathrm{T}$ & Terminal \\
\hline $\mathrm{v}$ & Vertical or vapour \\
\hline w & Water \\
\hline$\infty$ & Free stream \\
\hline
\end{tabular}


xiv

* Impact un-effected by the proximity of an edge

\section{Dimensionless groups}

Eo

Eotvos number, $\quad \frac{g\left(\rho_{\mathrm{p}}-\rho_{\mathrm{f}}\right) \mathrm{d}^{2}}{\sigma}$

Gr Grashof number,

$$
\frac{g \beta_{e x}\left(T-T_{\infty}\right) d^{3}}{v^{2}}
$$

Le Lewis number, $\quad \frac{\alpha}{\mathrm{D}}$ or $\frac{\mathrm{Sc}}{\mathrm{Pr}}$

$\mathrm{Le}_{\mathrm{f}} \quad$ Lewis factor, $\quad \cdot \quad \frac{\mathrm{h}_{\mathrm{c}}}{\mathrm{Kc}_{\mathrm{pm}}}$

$M_{\ddot{z}} \quad$ Modulus of acceleration, $\frac{(\partial v / \partial t) d}{v^{2}}$

$\mathrm{Nu} \quad$ Nusselt number, $\quad \frac{h_{c} d}{k}=f(R e, P r)$

Pr Prandtl number, $\quad \frac{v}{\alpha}$ or $\frac{\mathrm{c}_{\mathrm{p}} \mu}{\mathrm{k}}$

Re Reynolds number, $\quad \frac{\rho v d}{\mu}$

Sc Schmidt number,

$$
\frac{v}{D} \text { or } \frac{\mu}{\rho D}
$$

Sh Sherwood number, $\quad \frac{h_{D} d}{D}=f(R e, S c)$

We Weber number, $\quad \frac{\rho v^{2} d}{\sigma}$

\section{Abbreviations and Acronyms}

A-D Analogue to digital

AERE Atomic Energy Research Establishment 
XV

AIChE American Institute of Chemical Engineers

ASAE American Society of Agricultural Engineers

ASHRAE American Society of Heating, Refrigeration and Air-Conditioning Engineers

ASHVE American Society of Heating and Ventilating Engineers

ASME American Society of Mechanical Engineers

ATPCD American Tower Plastics Cool Drop

BHRA British Hydromechanics Research Association

BS British Standards

CTI Cooling Tower Institute

EPRI Electric Power Research Institute

IAHR International Association for Hydraulic Research

IChemE Institution of Chemical Engineers

IMechE Institution of Mechanical Engineers

I/O Input/output

IBM International Business Machines

LDA Laser Doppler anemometer

LOCA Loss-of-coolant accident

PVA Polyvinyl acrylic

PVC Polyvinyl chloride

PWR Pressurised water reactor

SHASE Society of Heating, Air-Conditioning and Sanitary Engineers

STP Standard temperature and pressure, $20^{\circ} \mathrm{C}$ and $101325 \mathrm{~Pa}$

TTL Transistor transistor logic

VDI Verein Deutscher Ingenieure (Society of German Engineers)

\section{General}

$\underline{\partial}$

Differential

Averaged or mean 
xvi

\section{LIST OF FIGURES}

Figure 1.1 Mechanical draft counterflow cooling tower.................................... 1

Figure 1.2 Natural draft counterflow cooling tower....................................... 2

Figure 2.1 Variation of transfer characteristic with hydraulic diameter (based on Table 2.1). 6

Figure 2.2 Comparison between the transfer characteristic correlation by Tezuka et al. ([73TE1],[75TE1],[86FU1]) and the data listed in Table 2.1 8

Figure 2.3 Comparison between the drop terminal velocity measured by Gunn and Kinzer and the predicted drop terminal velocity data obtained from the solid sphere correlation of Turton and Levenspiel [86TU1]. 12

Figure 2.4 Approximate geometry of a deformed drop. 14

Figure 2.5 Loss coefficients for flow through sharp-edged grids. 31

Figure 2.6 Turbulence decay behind a bi-planar grid. 34

Figure 3.1 Layout of the imaginary integration elements along the packing height. 36

Figure 3.2 Drop deformation at terminal velocity versus Eotvos number. 38

Figure 3.3 Variation of drop drag coefficient with drop deformation for drops travelling at terminal velocity.

Figure 3.4 Comparison between the different models for predicting the heat/mass transfer from an accelerating drop and the data of Yao [74YA1]

Figure 3.5 Schematic description of the splashing and cutting processes. 43

Figure 3.6 Variation of total splash drop mass with drop impact velocity determined by Stedman [79ST1].

Figure 3.7 Schematic diagram to describe the cutting fraction.

Figure 3.8 Average cutting fraction for various combinations of slat width and drop diameter as predicted by Equation (3.20). 46

Figure 3.9 Variation of local splash fraction with drop impact position on a slat. 
xvii

Figure 3.10 Fraction of the original drop mass contained in the splash drops.

Figure 3.11 Diagram to describe the size of drops formed by cutting. 49

Figure 3.12 Variation of interference ratio with impacting drop size.

Figure 4.1 Layout of the opto-electronic drop detector used in strobe/flash timing, drop velocity measurement (in which two similar detectors were used) and drop position detection.

Figure 4.2 Layout of the drop detectors used in velocity measurements.

Note that the one drop detector was mounted approximately 50 $\mathrm{mm}$ above the other.

Figure 4.3 Comparison between the drop deformation obtained from the experimental data and the drop deformation predicted by Equation (4.8).

Figure 4.4 Measured versus predicted drop deformation near drop terminal velocity.

Figure 4.5 Comparison between measured drop velocity and the predicted drop velocity, assuming that drop drag can be calculated by the drag curve for a solid sphere

Figure 4.6 Comparison between measured drop velocity and the predicted drop velocity, calculated with the deforming drop drag model described above.

Figure 4.7 Comparison between measured drop velocity and the predicted drop velocity, when assuming that the drop drag can be calculated by the model of Beard [77BE1]

Figure 4.8 Variation of the water film thickness on different slats placed below 10 layers of splash grids in an operational cooling tower.

Figure 4.9 Predicted variation of the average water film thickness with slat width at a constant air velocity.

Figure 4.10 Predicted variation of the average water film thickness with slat width at a constant water mass flux.

Figure 4.11 Layout of the experimental set-up used to study the splashing and cutting of single drops on narrow slats.

Figure 4.12 Layout of a typical test slat showing the feed water supply and the film thickness measuring probe. 
xviii

Figure 4.13 Layout of the drop catcher used to intercept "skew" drops. 68

Figure 4.14 Layout of the set-up used for the photography of the splashing and cutting phenomena.

Figure 4.15 Sequence of photographs showing the crown and the splash drops at various delay times after the impact of a $5.6 \mathrm{~mm}$ water drop striking the centre of a $25 \mathrm{~mm}$ wide slat covered by a 1.0 $\mathrm{mm}$ thick water layer.

Figure 4.16 Sequence of photographs showing the crown and the splash drops at various delay times after the impact of a $5.6 \mathrm{~mm}$ water drop striking the centre of a $5 \mathrm{~mm}$ wide slat covered by a 0.7 $\mathrm{mm}$ thick water layer. 72

Figure 4.17 Summary of splash angle data. 74

Figure 4.18 Graphical description of the cutting/splashing phenomena for drop impacts (a) near an edge, (b) impacts on an edge at high Weber numbers and (c) impacts on an edge at low Weber numbers.

Figure 4.19 Typical instantaneous water film thicknesses on a slat before, during and after being struck by a falling drop.

Figure 4.20(a) Accuracy of the correlations for the average splash fraction on narrow slats .78

Figure 4.20(b) Accuracy of the correlations for the average splash fraction on wide slats.

Figure 4.21 Average splash fractions on a $2 \mathrm{~mm}$ wide slat covered by a 0.5 $\mathrm{mm}$ thick water film for various combinations of drop velocity and drop size.

Figure 4.22 Average splash fractions on a $5 \mathrm{~mm}$ wide slat covered by a 0.5 $\mathrm{mm}$ thick water film for various combinations of drop velocity and drop size. 80

Figure 4.23 Average splash fractions on a $10 \mathrm{~mm}$ wide slat covered by a 0.5 $\mathrm{mm}$ thick water film for various combinations of drop velocity and drop size. 80

Figure 4.24 Average splash fractions on a $25 \mathrm{~mm}$ wide slat covered by a 0.5 $\mathrm{mm}$ thick water film for various combinations of drop velocity and drop size. 
Figure 4.25 Average splash fractions on a $25 \mathrm{~mm}$ wide slat covered by a 1 $\mathrm{mm}$ thick water film for various combinations of drop velocity and drop size.

Figure 4.26 Layout of the light box used to photograph the water drops caught in the oil filled Petri dishes.

Figure 4.27 Drop size distributions formed after drop impact on narrow slats, $\mathrm{di} / \mathrm{W}>3$.

Figure 4.28 Correlation accuracy for the size of the drops formed by cutting.

Figure 4.29 Comparison between the experimental data and correlations for the Sauter mean drop size after impact on a narrow slat with increasing Weber number.

Figure 4.30 Typical splash drop size distribution data and the corresponding Rosin-Rammler correlations for different drop fall heights.

Figure 4.31 Accuracy of the correlations for Rosin-Rammler shape parameter and mass median drop diameter describing the distribution of drops formed by splashing.....

Figure 4.32 Comparison between the experimentally determined mean initial splash drop velocity and the correlation based on the work by Ghadiri and Payne [78GH1]. 90

Figure 4.33 Comparison between measured and predicted splash and cutting drop size distribution (small incoming drop).

Figure 4.34 Comparison between measured and predicted splash and cutting drop size distributions (large incoming drop)...

Figure 4.35 Cross sectional profiles of the slats used in the experiments to measure dripping drop sizes. Dimensions in $\mathrm{mm}$.

Figure 4.36 Water distributor used to supply water to the slats

Figure 4.37 Layout of the photographic system used to photograph the drops dripping from below the test slats.

Figure 4.38 Typical photograph of water drops dripping below slat $\mathrm{A}$ at a low water flow rate $(\Gamma \approx 16 \mathrm{~g} / \mathrm{ms})$.

Figure 4.39 Typical photograph of water drops dripping below slat $\mathrm{A}$ at a high water flow rate $(\Gamma \approx 64 \mathrm{~g} / \mathrm{ms})$. 
Figure 4.40 Relative mass fraction distribution for dripping below slat A...............95

Figure 4.41 Relative mass fraction distribution for dripping below slat E. ...............95

Figure 4.42 Explanatory diagram to define the shape factor.............................. 97

Figure 4.43 Data and correlation for non-dimensional primary drop size ................97

Figure 4.44 Experimental apparatus used to determine the heat transfer from different tubes. ............................................................. 99

Figure 4.45 Comparison between the experimental data and the correlation of Gnielinski............................................................................. 101

Figure 4.46 Counterflow cooling tower packing material test facility...................... 102

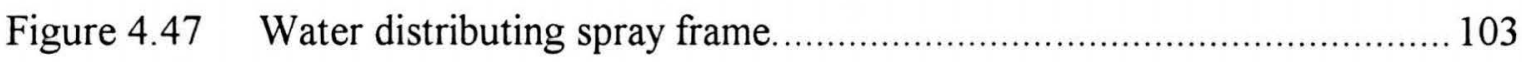

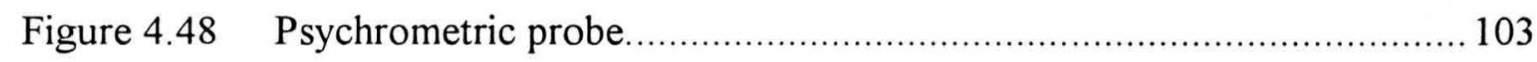

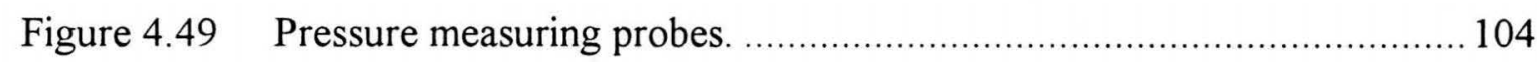

Figure 4.50 Model of the water distributing spray frame ..................................... 105

Figure 4.51 Measured cumulative mass distribution for the spray frame

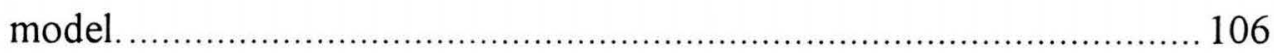

Figure 4.52 Rosin-Rammler distribution parameters describing the spray frame drop distribution. ......................................................... 107

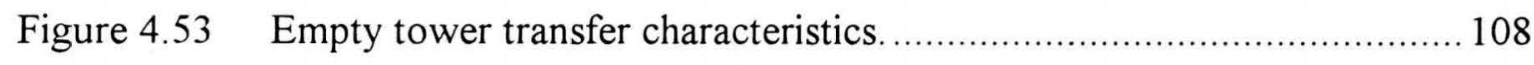

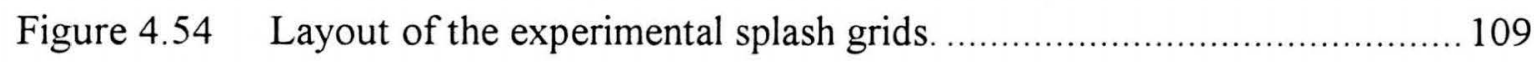

Figure 4.55 Comparison between experimental data and the general correlation by Tezuka et al. [73TE1],[75TE1],[86FU1] ................... 112

Figure 4.56 Layout of the shutter used to catch a sample of drops in a cooling tower......................................................................... 112

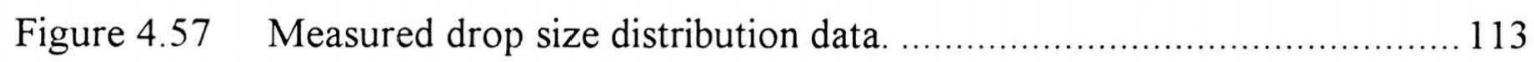

Figure 4.58 Experimentally determined pressure loss coefficients for flow through sharp-edged grids.

Figure 5.1 Layout of the imaginary integration elements along the packing height.

Figure 5.2 Flow diagram showing the main calculation steps in the splash pack simulation program. 
$\mathrm{xxi}$

Figure 5.3 Flow diagram showing the calculation steps required to evaluate a typical free fall zone between grids.

Figure 5.4 Calculation procedure to find the drop velocity change in an integration element.

Figure 5.5 Calculation procedure to find the drop temperature change in an integration element.

Figure 5.6 Flow diagram showing the calculation steps required to evaluate the influence of a grid on the drops falling through the packing.

Figure 5.7 Schematic layout of the control volume used to model the cooling of the water film flowing over the slats.

Figure 6.1 Pack density for mono-disperse spray systems (with no packing).

Figure 6.2 Transfer characteristics for mono-disperse spray systems (with no packing).

Figure 6.3 Pressure drop per metre for mono-disperse spray systems (with no packing).

Figure 6.4 Spray efficiencies for mono-disperse spray systems with no packing, according to Equation (6.2).

Figure 6.5 Spray efficiencies for mono-disperse spray systems with no packing, according to Equation (6.3).

Figure 6.6 Comparison between the transfer characteristics based on the model of Nottage and Boelter [40NO1] and the predicted values obtained with SPSIM.

Figure 6.7 Modified Rosin-Rammler distributions used in poly-disperse spray calculations.

Figure 6.8 Predicted transfer characteristics for poly-disperse sprays in an empty tower.

Figure 6.9 Predicted pressure drop for poly-disperse sprays in an empty tower.

Figure 6.10 Predicted efficiency of poly-disperse sprays in an empty tower........ 140

Figure 6.11 Predicted transfer characteristics with different correlations for heat/mass transfer from the drops. 
xxii

Figure 6.12 Predicted transfer characteristics with different drop drag models.

Figure 6.13 Predicted pressure drop with different drop drag models. 143

Figure 6.14 Predicted transfer characteristics with different film thicknesses. 144

Figure 6.15 Predicted pressure drop with different film thicknesses. 144

Figure 6.16 Predicted transfer characteristics with different rain densities. 145

Figure 6.17 Predicted pressure drop with different rain densities. 145

Figure 6.18 Predicted transfer characteristics with different splash mass models.

Figure 6.19 Predicted pressure drop with different splash mass models.

Figure 6.20 Predicted transfer characteristics when ignoring upward splash drop motion and when using a logarithmic drop size zone distribution.

Figure 6.21 Predicted pressure drop when ignoring upward splash drop motion and when using a logarithmic drop size zone distribution.

Figure 6.22 Predicted radial displacement of splash drops from the point of impact.

Figure 6.23 Predicted re-entry angles of the splash drops at $z=0$

Figure 6.24 Predicted transfer characteristics showing the influence of the cooling of the water film covering the slats and the crown splash angle.

Figure 6.25 Predicted transfer characteristics with different initial drop size distributions.

Figure 6.26 Predicted pressure drop with different initial drop size distributions.

Figure 6.27 Comparison between predicted and measured transfer characteristic for experimental splash pack I.

Figure 6.28 Correlation between predicted and uncorrected experimentally determined transfer characteristics.

Figure 6.29 Correlation between predicted and corrected experimentally determined transfer characteristics. 
xxiii

Figure 6.30 Comparison between predicted and measured pressure drop across experimental splash pack I.

Figure 6.31 Correlation between predicted and experimentally determined pressure drop.

Figure 6.32 Comparison between measured and predicted drop size distributions below experimental splash pack I.

Figure 6.33 Predicted transfer characteristic variation with slat width when cooling of the films covering the slats is taken into account.

Figure 6.34 Predicted transfer characteristic variation with slat width when cooling of the films covering the slats is NOT taken into account.

Figure 6.35 Predicted pressure drop variation with slat width.

Figure 6.36 Predicted packing efficiency variation with slat width when cooling of the films covering the slats is taken into account......

Figure 6.37 Predicted packing efficiency variation with slat width when cooling of the films covering the slats is NOT taken into account.

Figure 6.38 Predicted splash pack efficiency with and without surface tension reducing additives.

Figure 6.39 Contribution of pressure drop across the grids to the total predicted pressure drop across splash pack I.

Figure A.1 Comparison between the recalculated Yao correction factor data and the correlation by Yao [74YA1].

Figure C.1 Flush mounted pin electrodes used in water film thickness measurements.

Figure C.2 Block diagram of the electronic device used to measure liquid film thicknesses.

Figure D.1 Schematic description of splash drop trajectory from impact point.

Figure D.2 Comparison between the drop temperatures predicted by the accurate numerical integration procedure and by the simplified model.

Figure E.1 Drop collection on the rear surface of a cylinder. 
xxiv

Figure E.2 Drop collection efficiency on the rear surface of a cylinder. 200

Figure F.1 Drop hanging below a square-edged cylindrical nozzle. 201 
$\mathrm{XXV}$

\section{LIST OF TABLES}

Table 2.1 Summary of experimentally determined splash pack performance data................................................................. 7

Table 2.2 Dimensionless drop size constant. 29

Table 2.3 Typical values for the constants describing turbulence decay behind a grid.

Table 4.1 Slats and probes used in the film thickness measurements on splash grids.

Table 4.2 Ranges of variables covered by the photographic study..... 70

Table 4.3 Splash angle and crown growth time data obtained from the splash photographs. 73

Table 4.4 Ranges of variable parameters covered in splash fraction measurements. 75

Table 4.5 Constants in the correlations for the average splash fraction. 78

Table 4.6 Summary of Sauter mean drop sizes of drop distributions formed by cutting. 85

Table 4.7 Rosin-Rammler distribution data describing the mass distribution of splash drops. 88

Table 4.8 Summary of drop distribution data at lowest liquid flow rate $(\Gamma \approx 18 \mathrm{~g} / \mathrm{ms})$ 96

Table 4.9 Tube profiles used in the experiments to measure the external heat transfer coefficient. 99

Table 4.10 Rosin-Rammler parameters describing the spray frame drop size distribution. 106

Table 4.11 Splash grid layouts used in splash pack tests. 110

Table 4.12 Correlation coefficients for the experimental splash pack.

Table J.1 Measured drop velocities. 223

Table K.1 Average film thicknesses measured on slats in a cooling tower. 224

Table L. 1 Measured splash fraction data for $5 \mathrm{~mm}$ wide slat. 225

Table L.2 Measured splash fraction data for $10 \mathrm{~mm}$ wide slat.... 227

Table L.3 Measured splash fraction data for $25 \mathrm{~mm}$ wide slat. 229 
xxvi

Table M.1 Measured splash drop size distribution.

Table M.2 Measured size distribution of drops formed by cutting (W=0.3 $\mathrm{mm}$ ).

Table M.3 Measured drop size distribution of drops formed by cutting $(\mathrm{W}=2 \mathrm{~mm})$.

Table N.1 Measured dripping drop size distribution for slat A. 236

Table N.2 Measured dripping drop size distribution for slat B. 237

Table N.3 Measured dripping drop size distribution for slat C. 238

Table N.4 Measured dripping drop size distribution for slat D 239

Table N.5 Measured dripping drop size distribution for slat E. 240

Table O.1 Experimental data for heat transfer from tubes of different profiles.

Table P.1 Measured drop size distribution for the water distribution system of the cooling tower packing material test facility.

Table Q.1 Drop size distribution in a cooling tower fitted with splash pack.

Table R.1 Measured single phase pressure drop across the experimental splash grids. 244 


\section{CHAPTER 1 INTRODUCTION}

In many processes the ambient air is used as a heat sink for low-quality waste heat. The most commonly employed methods of rejecting heat to the surroundings are dry surface heat exchangers and direct contact (or wet) cooling towers.

Direct contact cooling towers have the advantages of being cheaper to construct and operate than dry surface heat exchangers. Since direct contact cooling employs both heat and mass transfer to exchange energy, it is possible to cool process fluid to below the ambient dry-bulb temperature. This gives the direct contact cooling tower a definite advantage over the dry surface heat exchangers in achieving high plant efficiencies. The advantages of direct contact cooling towers are often offset by the fact that due to the mass transfer process there is an evaporation loss associated with direct contact cooling. Also, their use is restricted to water temperatures below about $80^{\circ} \mathrm{C}$.

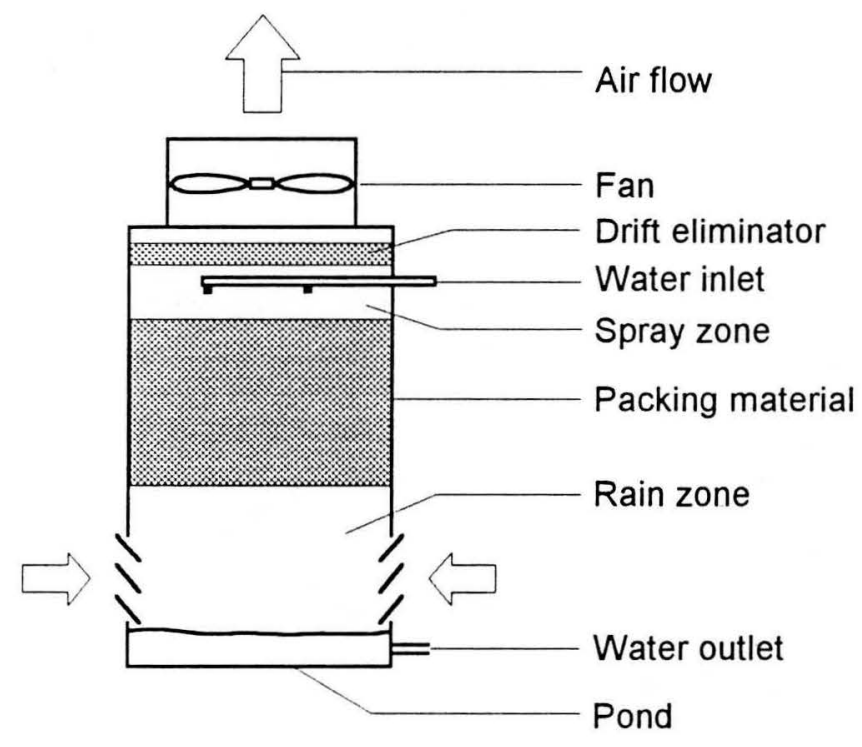

Figure 1.1 Mechanical draft counterflow cooling tower.

Direct contact cooling towers are produced in all shapes and sizes. The two main groups are distinguished by the air and water flow directions. The first group, referred to as counterflow cooling towers (see Figure 1.1), employs an upward flowing, vertical air stream cooling a gravity driven water stream (which obviously flows downwards). The other main group, known as crossflow cooling towers, has the air stream flowing horizontally across a gravity driven water flow. In this study, only counterflow cooling towers are considered but the principles are also applicable to crossflow cooling towers. 
If the airflow is produced by a fan, the cooling tower is referred to as a mechanical draft cooling tower. A natural draft cooling tower uses the rising hot air leaving the water/air contact zone to draw in fresh cold air. A natural draft cooling tower is easily identified by the large shell required to ensure sufficient draft (see Figure 1.2).

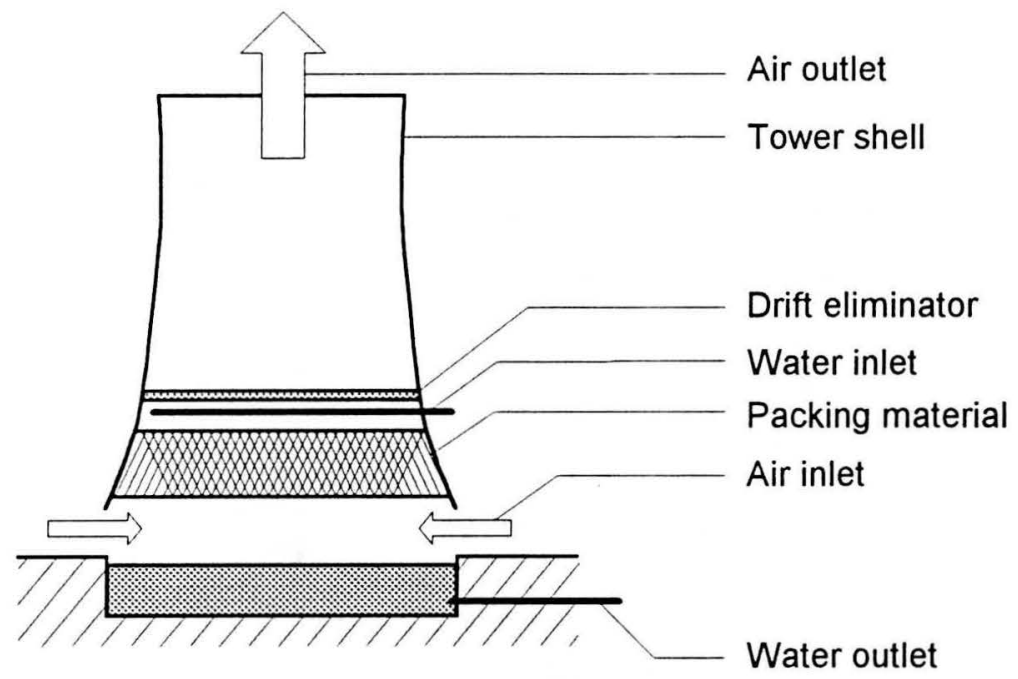

Figure 1.2 Natural draft counterflow cooling tower.

In a counterflow cooling tower, the hot water from the process which is to be cooled, is sprayed into an upward flowing air stream. Due to heat and mass transfer, the water temperature is reduced while the air enthalpy is increased. In order to increase the interface area between the air and the water, packing material is placed inside the cooling tower as shown in Figures 1.1 and 1.2. Three types of packing material are commonly used : (i) splash pack, (ii) film pack and (iii) film-grid pack. Splash packing material consists of horizontal wooden, plastic or metal slats and grids which break up the drops falling through the tower, thereby reducing the average velocity and size of the drops. The smaller drop size and lower drop velocities result in increased interface area and increased drop residence time in the tower. Film packing material consists of large, thin sheets of asbestos, plastic or metal which spread the water into thin films flowing down the sides of these sheets. Film packing is often used in smaller mechanical draft cooling towers. Film-grid packings fall between the splash and film packings discussed above. As in the case of splash packing, the grids tend to break up the falling water drops while the relatively large grid surface area (in comparison with splash packs) is covered with a thin water film which contributes significantly to the total interface area.

In order to obtain thermal and pressure drop design data on a given packing material, it is normally necessary to obtain the required information experimentally. To minimise wall effects, it is necessary to conduct these tests using large test sections, which requires large amounts of energy for the heating of the inlet water. It is often difficult to compare results obtained for the 
same packing material due to differences in the water distribution systems and sizes of the spray and rain zones above and below the packing. Development of new types of packing requires many prototypes to be built and evaluated, resulting in very high development costs.

This study is aimed at developing a mathematical model to describe the performance of splash packing material using basic aerodynamic, hydrodynamic and heat/mass transfer information without depending on empirically determined data from cooling tower testing. A mathematical model that predicts the performance of splash packing material accurately, could be used to optimise the layout of splash packing material. Such a model would also make it possible to study the effects of different types of water distribution systems on the performance of a given packing. A model that correctly predicts the drop size and velocity distributions through the packing zone would also allow accurate prediction of the performance of the rain zone below the packing. 


\section{CHAPTER 2 LITERATURE SURVEY}

In this chapter the literature which is relevant to the modelling of splash pack from first principles is reviewed. A brief review of cooling tower theory is included as well as examples of empirically determined transfer coefficients. Since the modelling of spray cooling ponds and the rain zones in large natural draft cooling towers is based on the same principles as those governing the operation of splash pack, literature on these subjects is also reviewed briefly. The main part of this literature review is concerned with literature on drop drag, mass/heat transfer, drop-drop collisions, aerodynamic break-up, splashing and other mechanisms which influence the thermal performance of splash pack. Shortcomings in the available literature are highlighted.

\subsection{Cooling tower theory}

The total heat transfer rate between a water layer with an interface temperature of $T_{i}$ and air at $\mathrm{T}_{\mathrm{a}}$ and a vapour content of $\mathrm{w}_{\mathrm{a}}$ can be expressed as

$$
\partial \dot{Q}=h_{c}\left(T_{i}-T_{a}\right) \partial A+K\left(w_{a s i}-w_{a}\right) i_{g} \partial A
$$

Merkel [26ME1] assumed (i) a Lewis factor of unity $\left(\mathrm{Le}_{\mathrm{f}}=\left(\mathrm{h}_{\mathrm{c}} / \mathrm{c}_{\mathrm{pm}} \mathrm{K}\right) \approx 1\right)$ and (ii) negligible water mass loss (due to evaporation) to simplify this relation to

$$
\partial \dot{\mathrm{Q}} \approx \mathrm{K}\left(\mathrm{i}_{\mathrm{asi}}-\mathrm{i}_{\mathrm{a}}\right) \partial \mathrm{A}
$$

Noting that $\dot{\mathrm{m}}_{\mathrm{da}} \partial \mathrm{i}_{\mathrm{a}}=\dot{\mathrm{m}}_{\mathrm{w}} \mathrm{c}_{\mathrm{pw}} \partial \mathrm{T}_{\mathrm{w}}$ and assuming that the water interface temperature is equal to the bulk water temperature (i.e. $\mathrm{T}_{\mathrm{i}} \approx \mathrm{T}_{\mathrm{w}}$ ), integration of Equation (2.2) between the water inlet and outlet positions of a cooling tower yields the following integral

$$
\frac{\mathrm{KaZ}}{\dot{\mathrm{M}}_{\mathrm{w}}}=\int_{\mathrm{Z}} \frac{\mathrm{c}_{\mathrm{pw}} \partial \mathrm{T}_{\mathrm{w}}}{\left(\mathrm{i}_{\mathrm{asw}}-\mathrm{i}_{\mathrm{a}}\right)}
$$

Since the relation between air saturation enthalpy and temperature is not a simple linear function of temperature, numerical integration is usually required to solve this integral. The Tchebycheff integration method (see Cale [82CA1]) allows approximate evaluation of this relation. The effect of the assumption that the water interface temperature is equal to the bulk water temperature has been investigated by Baker and Shryock [61BA1], Webb [88WE1] and Marseille et al. [91MA1].

The non-dimensional term, $\mathrm{KaZ} / \dot{\mathrm{M}}_{\mathrm{w}}$, is known as the Merkel number or the transfer characteristic of the packing. If the packing transfer characteristic of a given cooling tower is 
known, it is possible to use the above theory to calculate the performance of the cooling tower under various air and water inlet conditions. Similarly, if the tower performance is known for specific inlet conditions (from measurements), it is possible to calculate the transfer characteristic by solution of Equation (2.3).

In determining the operating point of natural draft cooling towers, the condition of the air leaving the packing zone is required to facilitate the calculation of the draft potential. When modelling the operation of a cooling tower with the Merkel theory, only the water temperature and the air enthalpy are known at every position in the cooling tower and it is usual to assume that the outlet air is saturated. This assumption allows the calculation of the temperature and density of the air leaving the packing.

In his 1972 thesis, Poppe (see Poppe and Rögener [91PO1]) rewrote Equation (2.1) without resorting to the Merkel assumptions for heat and mass transfer between the water and nonsaturated air in an element as

$$
\partial \dot{\mathrm{Q}}=\mathrm{K}\left(\mathrm{Le}_{\mathrm{f}}\left(\mathrm{i}_{\mathrm{asi}}-\mathrm{i}_{\mathrm{a}}\right)+\left(1-\mathrm{Le}_{\mathrm{f}}\right) \mathrm{i}_{\mathrm{g}}\left(\mathrm{w}_{\mathrm{asi}}-\mathrm{w}_{\mathrm{a}}\right)\right) \partial \mathrm{A}
$$

The complete Poppe model includes differential equations describing the air humidity, water temperature and water mass flow rate changes in an imaginary element of cooling tower packing. Poppe also derived separate equations describing the heat and mass transfer between a water surface and saturated or supersaturated air, since the driving potential for mass transfer to saturated air, ( $\mathrm{w}_{\mathrm{asi}}-\mathrm{w}_{\mathrm{as}}$ ), differs from the driving potential for mass transfer to nonsaturated air, $\left(\mathrm{w}_{\mathrm{asi}}-\mathrm{w}_{\mathrm{a}}\right)$. Various other models based on a similar approach to that of Poppe have been proposed for the mathematical modelling of cooling towers, e.g., Bourillot [83BO1], Sutherland [83SU1], Webb [88WE1] and Feltzin and Benton [91FE1]. When using one of the accurate models for cooling tower design purposes, it is important to use transfer characteristics which have been calculated from experimental data using the same model. If the Merkel model was used to calculate the transfer characteristics from experimental data, the Merkel model should be employed when using this data to predict the performance of a cooling tower. The Merkel model employing transfer characteristic data determined with the Merkel model can be expected to yield better results than a more accurate model using the same transfer characteristic data (based on the Merkel model).

\subsection{Empirical splash pack design data}

The experimentally determined values for the transfer characteristic of a given type of cooling tower packing can usually be correlated by relations of the form 


$$
\frac{\mathrm{Ka}}{\dot{\mathrm{M}}_{\mathrm{w}}}=\mathrm{c}_{1}\left(\frac{\dot{\mathrm{M}}_{\mathrm{w}}}{\dot{\mathrm{M}}_{\mathrm{a}}}\right)^{\mathrm{c}_{2}}
$$

or

$$
\frac{\mathrm{Ka}}{\dot{\mathrm{M}}_{\mathrm{w}}}=\mathrm{c}_{3} \dot{\mathrm{M}}_{\mathrm{w}}^{\mathrm{c}_{4}} \dot{\mathrm{M}}_{\mathrm{a}}^{\mathrm{c}_{5}}
$$

The pressure loss coefficient for a given pack is usually expressed as

$$
\mathrm{K}_{\Delta \mathrm{p}, \mathrm{fill}}=\frac{\Delta \mathrm{p} / \mathrm{Z}_{\mathrm{fill}}}{0.5 \rho_{\mathrm{a}} \mathrm{v}_{\mathrm{a}}^{2}}=\mathrm{c}_{6} \dot{\mathrm{M}}_{\mathrm{w}}^{\mathrm{c}_{7}} \dot{\mathrm{M}}_{\mathrm{a}}^{\mathrm{c}_{8}}
$$

Various researchers measured and correlated experimental transfer characteristic and pressure loss data for different types of splash pack. See Lowe and Christie [62LO1], Cale [82CA1] and Johnson [89JO1]. A summary of the data available in open literature is given in Table 2.1. No indication of the initial drop size distribution was given for any of the studies listed in Table 2.1. For many of the cases, the sizes of the spray zone and the rain zone above and below the packing material was not known. The variation of the transfer characteristic with hydraulic diameter $\left(d_{h}=4 / a^{\prime}\right)$ is shown in Figure 2.1 .

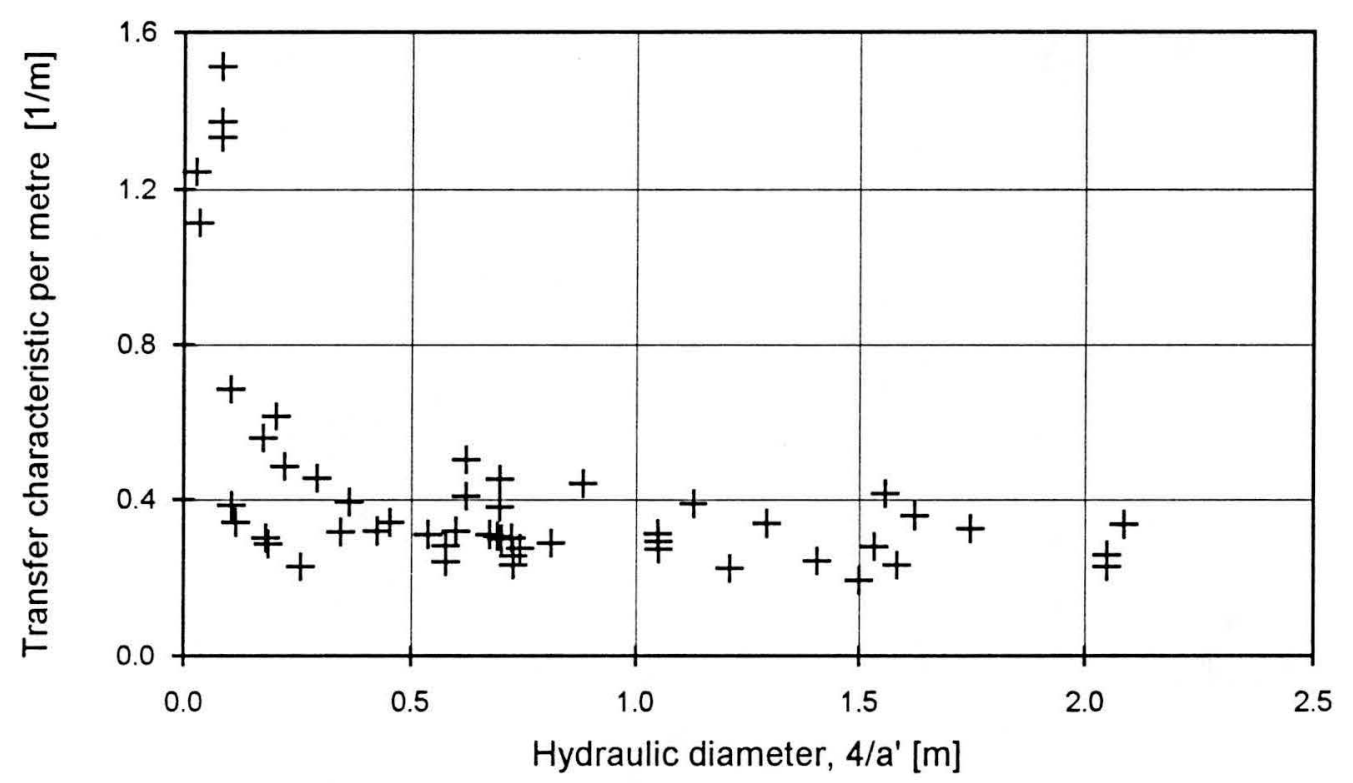

Figure 2.1 Variation of transfer characteristic with hydraulic diameter (based on Table 2.1).

Based on a similar table of packing data, Tezuka et al. ([73TE1],[75TE1],[86FU1]) derived the following general correlation describing the thermal transfer characteristics of splash pack

$$
\frac{\mathrm{Ka}}{\dot{\mathrm{M}}_{\mathrm{w}}} \approx 0.6 \mathrm{Z}^{-0.74} \mathrm{~d}_{\mathrm{h}}^{-0.21} \dot{\mathrm{M}}_{\mathrm{w}}^{-0.55} \dot{\mathrm{M}}_{\mathrm{a}}^{0.46}
$$


Table 2.1 Summary of experimentally determined splash pack performance data.

\begin{tabular}{|c|c|c|c|c|c|c|c|c|c|c|}
\hline Reference & $\begin{array}{c}\mathbf{Z}_{\text {pack }} \\
m\end{array}$ & $\begin{array}{l}\mathbf{A}_{\mathbf{f r}} \\
m^{2}\end{array}$ & Slat & $\begin{array}{c}\mathbf{W} \\
m m\end{array}$ & $\begin{array}{l}\mathbf{P}_{\mathbf{v}} \\
m m\end{array}$ & $\begin{array}{l}\beta \\
\%\end{array}$ & $\begin{array}{c}\text { a } \\
m^{2} / m^{3}\end{array}$ & $\begin{array}{c}\mathbf{a}^{\prime} \\
m^{2} / m^{3}\end{array}$ & $\begin{array}{c}\mathbf{c}_{1} \\
m^{-1}\end{array}$ & $\begin{array}{c}c_{2} \\
-\end{array}$ \\
\hline in [43LIl] & 81 & 3.34 & Rectangular & 9.5 & 381 & 67 & 1.10 & 11.61 & 0.319 & -0.62 \\
\hline Kelly\&Swenson [56KE1] & 6.10 & 3.03 & Flat bar & 22.0 & 610 & 78 & & & .328 & -0.51 \\
\hline Kelly\&Swenson [56KE1] & 6.10 & 3.03 & Rectangular & 22.0 & 610 & 71 & 3.02 & 3.09 & 0.341 & -0.57 \\
\hline Kelly\&Swenson [56KE1] & 6.10 & 3.03 & Rotated square & 31.1 & 610 & 45 & 2.53 & 2.57 & 0.417 & -0.47 \\
\hline Kelly\&Swenson [56KEl] & 6.10 & 3.03 & Flat bar & 9.0 & 381 & 69 & 5.61 & 5.71 & 0.302 & -0.60 \\
\hline Kelly\&Swenson [56KE1] & 6.10 & 3.03 & Flat bar & 9.0 & 610 & 69 & 3.50 & 3.54 & 0.390 & -0.58 \\
\hline Kelly\&Swenson [56KE1] & 6.10 & 3.03 & Trapezium & 48.6 & 610 & 49 & 2.42 & 2.46 & 0.361 & -0.46 \\
\hline Kelly\&Swenson [56KE1] & 6.10 & 3.03 & Square & 22.0 & 610 & 71 & 1.90 & 1.92 & 0.338 & -0.54 \\
\hline Kelly\&Swenson [56KE1] & 6.10 & 3.03 & Rectangular & 13.0 & 610 & 54 & 4.45 & 4.54 & 443 & -0.57 \\
\hline Kelly\&Swenson [56KE1] & 6.10 & 3.03 & Flat bar & 51.0 & 305 & 75 & 1.94 & 1.95 & 0.230 & -0.62 \\
\hline Kelly\&Swenson [56KE1] & 6.10 & 3.03 & Flat bar & 51.0 & 228 & 75 & 2.64 & 2.67 & 0.197 & -0.62 \\
\hline Smith [56SM1] & 2.44 & 0.37 & Rectangular & 9.1 & 76 & 65 & 34.27 & 38.72 & 0.684 & -0.50 \\
\hline Smith [56SM1] & 2.44 & 0.37 & Rectangular & 9.1 & 152 & 65 & 17.13 & 18.18 & 0.484 & -0.50 \\
\hline Smith [56SM1] & 2.44 & 0.37 & Rectangular & 9.1 & 305 & 65 & 8.57 & 8.82 & 0.342 & -0.50 \\
\hline Smith [56SM1] & 2.44 & 0.37 & Rectangular & 15.0 & 76 & 65 & 21.21 & 22.84 & 0.558 & -0.50 \\
\hline Smith [ & 2.44 & 0.37 & Rectangular & 15.0 & 152 & 65 & 10.61 & 11.00 & 0.394 & -0.50 \\
\hline Smith [ & 2.44 & 0.37 & Rect & 15.0 & 305 & 64 & 5.30 & 5.40 & 279 & -0.50 \\
\hline Smith [ & 2.44 & 0.37 & $\operatorname{Rec}$ & 25.4 & 76 & 63 & 13.05 & 13.65 & .454 & -0.50 \\
\hline Smith [56SM1] & 2.44 & 0.37 & Rect & 25.4 & 152 & 63 & 6.53 & 6.67 & 0.321 & -0.50 \\
\hline Smith [56SM1] & 2.44 & 0.37 & $\operatorname{Rec}$ & 25.4 & 305 & 63 & 3.26 & 3.30 & 0.227 & -0.50 \\
\hline Lowe \& Christie [62LO1] & 2.44 & 1.49 & Triar & 53.7 & 152 & 65 & 5.62 & 5.80 & 0.308 & -0.50 \\
\hline$e[62 \mathrm{LO} 1]$ & 2.44 & 1.49 & triangle & 26.1 & 190 & 79 & 5.75 & 5.93 & 0.312 & -0.49 \\
\hline e [62LOl] & 2.44 & 1.49 & Inve & 53.7 & 229 & 65 & 3.73 & 3.81 & 0.276 & -0.49 \\
\hline Christie [62LOI] & 2.44 & 1.49 & Tria & 53.7 & 229 & 65 & 3.74 & 3.81 & 0.315 & -0.45 \\
\hline Lowe \& Christie [62LO1] & 2.44 & 1.49 & triangle & 26.1 & 203 & 74 & 6.70 & 6.95 & 0.285 & -0.55 \\
\hline Lowe \& Christie [62LOl] & 2.44 & 1.49 & Tria & 53.7 & 457 & 53 & 2.49 & 2.53 & 0.236 & -0.47 \\
\hline Christie [62LO1] & 2.44 & 1.49 & Skewed triangle & 26.1 & 254 & 74 & 5.35 & 5.51 & .236 & -0.54 \\
\hline Christie [62LOl] & 2.44 & 1.49 & Flat & 51.0 & 305 & 75 & 1.94 & 1.95 & .262 & -0.53 \\
\hline Lowe \& Christie [62LOl] & 2.44 & 1.49 & tria & 26.1 & 152 & 74 & 8.94 & 9.39 & 322 & -0.54 \\
\hline Lowe \& Christie [62LO1] & 2.44 & 1.49 & Tri & 53.7 & 305 & 65 & 2.80 & 2.84 & .246 & -0.42 \\
\hline Christie [62LOI] & 2.44 & 1.49 & Ske & 26.1 & 152 & 49 & 17.89 & 19.77 & 0.614 & -0.65 \\
\hline Christie [62LO1] & 2.44 & 1.49 & Fla & 51.0 & 229 & 75 & 2.58 & 2.61 & 0.282 & -0.52 \\
\hline e [62LOl] & 2.44 & 1.49 & & 26.1 & 254 & 74 & 5.35 & 5.51 & 0.259 & -0.58 \\
\hline Christie [62LO1] & 2.44 & 1.49 & angle & 26.1 & 190 & 83 & 4.80 & 4.93 & 92 & -0.47 \\
\hline \& Christie [62LOl] & 2.44 & 1.49 & le & 26.1 & 203 & 79 & 5.38 & 5.54 & .305 & -0.46 \\
\hline Lowe \& Christie [62LOl] & 2.44 & 1.49 & iangle & 26.1 & 190 & 74 & 7.16 & 7.44 & 312 & -0.53 \\
\hline Lowe \& Christie [62LOl] & 2.44 & 1.49 & triangle & 26.1 & 203 & 74 & 6.70 & 6.95 & 43 & -0.52 \\
\hline Lowe \& Christie [62LOl] & 2.44 & 1.49 & & 53.7 & 229 & 65 & 3.73 & 3.81 & 0.295 & -0.50 \\
\hline Ohta \& Hojoh [73OH1] & 1.30 & & & 5.0 & 100 & 80 & 44.00 & 48.89 & - & - \\
\hline Ohta \& Hojoh [73OHl] & 0.90 & & & 5.0 & 100 & 80 & 44.00 & 48.89 & - & - \\
\hline Hojoh [73OHl] & 1.50 & & $\operatorname{Re}$ & 5.0 & 100 & 80 & 44.00 & 48.89 & - & - \\
\hline 1. [75BU1] & 2.70 & & & 10.0 & 260 & 62 & 32.54 & 38.19 & 0.385 & -0.50 \\
\hline Bulanina et al. [75BU1] & 2.70 & & & 10.0 & 260 & 83 & 14.59 & 15.63 & 0.230 & -0.50 \\
\hline Bulanina et al. [75BU1] & 2.70 & & $\operatorname{Rec}$ & 10.0 & 260 & 76 & 20.15 & 22.18 & 0.305 & -0.50 \\
\hline Schnell [88SC1] & 5.00 & & & 50.0 & 100 & 50 & 16.20 & 21.60 & 0.290 & -0.54 \\
\hline Schnell [88SC1] & 5.00 & & Rectangular & 50.0 & 100 & 50 & 28.00 & 35.00 & 0.344 & -0.63 \\
\hline Johnson [89JO1]-Ecodyne & 1.83 & 5.95 & Grid (T shape) & 8.5 & 305 & 76 & 5.74 & - & 0.469 & -0.35 \\
\hline Johnson [89JOl]-Ecodyne & 2.44 & 5.95 & Grid (T shape) & 8.5 & 305 & 76 & 5.74 & - & 0.416 & -0.35 \\
\hline [89JOl]-Ecodyne & 3.35 & 5.95 & Grid (T-shape) & 8.5 & 305 & 76 & 5.74 & - & 0.364 & -0.35 \\
\hline [89JO1]-ATPCD & 2.00 & 5.95 & & 4.5 & 203 & 81 & 6.41 & 6.44 & 0.502 & -0.42 \\
\hline [89JOl]-ATPCD & & 5.95 & & 4.5 & 203 & 81 & 6.41 & 6.44 & 0.424 & -0.42 \\
\hline Johnson [89JO1]-ATPCD & 3.40 & 5.95 & Grid (T shape) & 4.5 & 203 & 81 & 6.41 & 6.44 & 0.385 & -0.42 \\
\hline
\end{tabular}

where $d_{h}=\left(\frac{4}{a^{\prime}}\right)=\frac{4(\text { Volume available for airflow })}{\text { Area of packing }}$

This correlation is compared to the data listed in Table 2.1 for $\dot{M}_{a}=\dot{M}_{w}=2.78 \mathrm{~kg} / \mathrm{m}^{2} \mathrm{~s}$ in Figure 2.2. 


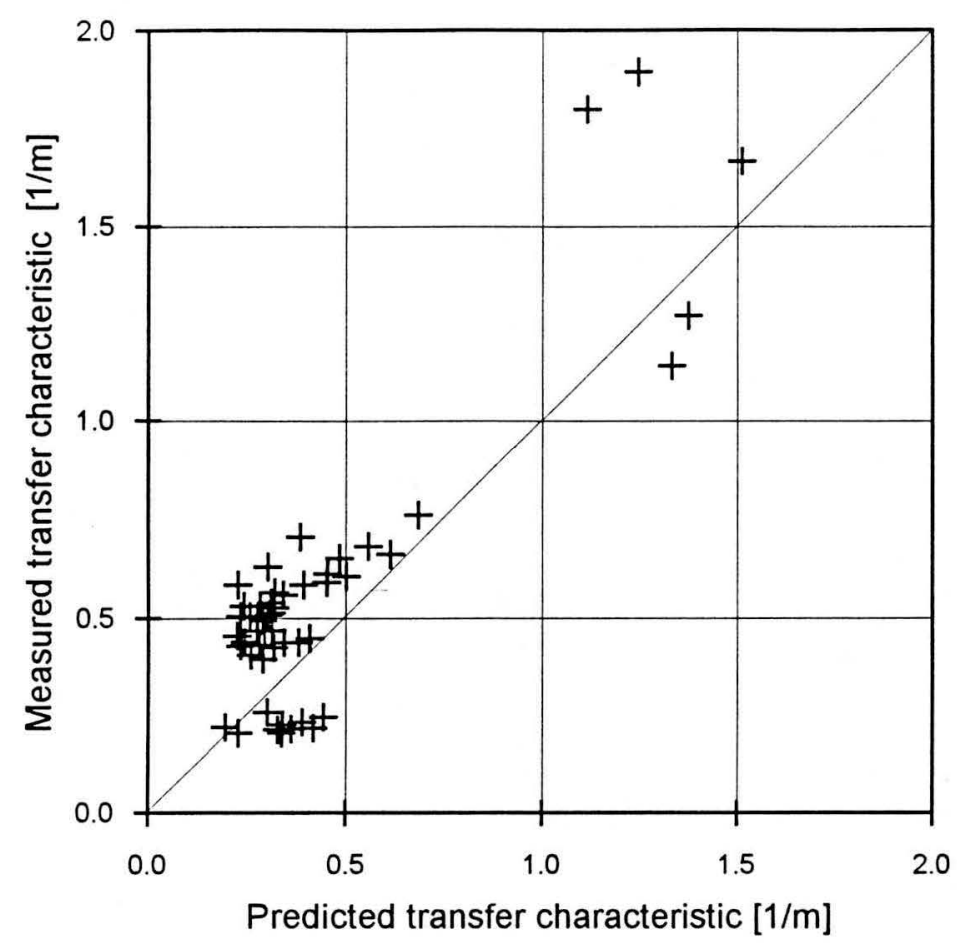

Figure 2.2 Comparison between the transfer characteristic correlation by Tezuka et al. ([73TE1],[75TE1],[86FU1]) and the data listed in Table 2.1.

\subsection{Mathematical modelling from basic principles}

Very little published literature on the modelling of cooling tower splash pack from first principles could be found. Several authors discussed the operating principles of splash packing material without proposing mathematical prediction models. Limited published literature is available on the mathematical modelling of heat/mass transfer from free-falling sprays of large drops. The modelling of the cooling/heating (and evaporation) of small drops, as found in fuel injection systems, has received more attention. The simpler models for describing drop cooling invariably assume that the drop distribution can be expressed by a single representative drop size, e.g. Sauter mean drop diameter. The Sauter mean diameter of a distribution of drop sizes is that drop diameter which has the same ratio of mass to surface area as the complete distribution. Symbolically, the Sauter mean diameter, $d_{32}$, can be expressed as

$$
d_{32}=\frac{\sum_{j} N_{j} d_{j}^{3}}{\sum_{j} N_{j} d_{j}^{2}}
$$

Similarly, the mass mean drop diameter is defined as 


$$
d_{30}=\left(\frac{\sum_{j} N_{j} d_{j}^{3}}{\sum_{j} N_{j}}\right)^{1 / 3}
$$

For small volatile drops $(\mathrm{d}<100 \mu \mathrm{m})$ evaporating at high temperatures, Alkidas [81AL1] and Aggarwal [88AG1] found that the Sauter mean diameter can be used to calculate the heating of a poly-disperse (distribution of drop sizes) spray. Hollands and Goel [76HOl] showed analytically that it is not generally possible to use a mean drop diameter to model the cooling/heating of a distribution of drops. They found that a mean drop size can be used in the following cases: (i) when the particles move through the heat/mass exchanger so rapidly that they do not change appreciably in temperature (low efficiency systems) or (ii) when the drops are very small and represent a small mass in comparison to the airstream (in which case the drops quickly reach the local air wet bulb temperature).

Nottage and Boelter [40NO1] developed a semi-graphical method to determine the cooling of mono-disperse sprays (sprays of uniform drop size) accelerating under gravity through an upward flowing airstream. The transfer in such a spray was described using a so-called "dynamic function", $\Psi$, and a "thermal function", $\Phi$. Lowe and Christie [62LO1] noted that the "thermal function" defined by Nottage and Boelter was in error. Lowe and Christie [62LO1] showed that the relation between the conventional cooling tower transfer characteristic and the "dynamic function" for drops at terminal velocity can be expressed as

$$
\frac{\mathrm{Ka}}{\dot{\mathrm{M}}_{\mathrm{w}}}=\frac{\mathrm{c}_{\mathrm{pw}} \mathrm{k}_{\mathrm{a}}}{\mathrm{c}_{\mathrm{pa}} \mathrm{k}_{\mathrm{w}}\left(\mathrm{v}_{\mathrm{T}}-\mathrm{v}_{\mathrm{a}}\right)} \Psi=\frac{\mathrm{c}_{\mathrm{pw}} \mathrm{k}_{\mathrm{a}}}{\mathrm{c}_{\mathrm{pa}} \mathrm{k}_{\mathrm{w}} \mathrm{v}_{\mathrm{rel}}} \Psi
$$

Berman [61BE1] described an approximate method to determine the relative contributions of the large drops dripping below the slats, the spray or splash drops and the films on the slats to the total air/water interface area and heat transfer rate for counterflow cooling tower splash packs. This model was based on simple experimental data regarding the volumes of splash drops formed by drops impacting on wooden slats, data on the size distribution of the drop dripping from below typical slats and information on the size distribution of the spray drops formed by splashing on the slats. Two different Sauter mean diameters were used to characterise the dripping drops and the spray drops. For a numerical example based on a typical splash pack with $a_{\text {slats }}=4.4 \mathrm{~m}^{2} / \mathrm{m}^{3}$, Berman found that, although the spray drops constituted only about $20 \%$ of the interface area, they were responsible for approximately $65 \%$ of the heat/mass transfer. Using his model, Berman concluded that increased airflow will result in higher transfer due to increased drop residence times and that increased water flow rates should increase the dripping and splash drop interface area slightly. 
Hollands [74HO1] modelled the operation of a spray tower (cooling tower without any packing material) mathematically using basic aerodynamic, hydrodynamic and heat/mass transfer information to describe the cooling of the drops falling down through the tower. He used the upper limit distribution function (first proposed by Mugele and Evans [51MU1]) to describe the distribution of drop sizes. The effect of drop deformation on the drag and the heat/mass transfer experienced by the drops were taken into account. In conventional cooling tower modelling using empirically determined volumetric transfer coefficients, it is assumed that the water at any height has a uniform temperature distribution. Using this assumption, Hollands [74HO1] proposed a second approximate model which uses a fixed drop size and does not require stepwise integration along the tower height. Factors to correct the simplified model predictions are given in graphical form for all the pertinent non-dimensional groups. For the best thermal performance, Hollands [74HO1] concluded that, for a given mean drop size, a uniform drop distribution will perform better than a wide (poly-disperse) spray. Warrington and Musselman [83WA1] reached the same conclusion in comparing the performance of a mono-disperse spray to that of a poly-disperse spray.

Benton and Rehberg [86BE1], Benocci et al. [86BE2], Hoffmann and Kröger [90HO1] and Conradie [93CO1] used numerical models to calculate the drop motion and cooling in the rain zones below the packing in large natural cooling towers. These models all use a single drop diameter to represent the distribution of actual drop sizes and, as expected, the solutions are very sensitive to the choice of drop size. Similar models have been used by Lefebvre [89LE1] and Mercker [93ME1] to calculate the performance of mono-disperse sprays of drops.

The available models for calculating the thermal performance of spray cooling ponds all use a single drop size (usually the Sauter mean diameter) to describe the dynamics and cooling of water drops sprayed upwards into the air above the pond. See Chen and Trezek [77CH1], Frediani and Smith [77FR1], Palaszewski et al. [81PA1] and Moussiopoulos and Ernst [87MO1].

\subsection{Other relevant information}

\subsubsection{Drag on spheres and drops}

Three forces act on a particle as it moves through a fluid: the gravitational force, the buoyancy force and a force due to the drag of the surrounding fluid. It is normal practice to express this last force using a drag coefficient, $\mathrm{C}_{\mathrm{D}}$, as defined by

$$
\mathrm{F}_{\text {drag }}=0.5 \rho_{\mathrm{f}} \mathrm{v}_{\text {rel }}^{2} \mathrm{~A}_{\mathrm{fr}} \mathrm{C}_{\mathrm{D}}
$$

The area is usually defined as the projected frontal area of the particle. For non-spherical particles, it is usual to use the equivalent spherical diameter, $\mathrm{d}_{\mathrm{e}}=(6 \mathrm{~V} / \pi)^{0.333}$, for the 
determination of the projected frontal area. From the force balance of a paricle moving through a fluid, it follows that

$$
C_{D}=\frac{m\left(\left(\left(\rho_{p}-\rho_{f}\right) g / \rho_{p}\right)-(\partial v / \partial t)\right)}{0.5 \rho_{f} v_{\text {rel }}^{2} A_{f r}}
$$

The drag coefficient, as defined above, is dependent on the acceleration of the particle. At terminal velocity the three forces are exactly balanced and the acceleration of the particle is zero. The drag coefficient of a particle at terminal velocity is referred to as the standard drag coefficient.

Clift et al. [78CL1] presented a set of 10 polynomial regressions to represent the available standard drag coefficient data over the complete range of Reynolds numbers. Turton and Levenspiel [86TU1] used the same data to obtain the following correlation which fits the data better than the set of equations proposed by Clift et al.,

$$
C_{D}=\frac{24}{\operatorname{Re}}\left(1+0.173 \operatorname{Re}^{0.657}\right)+\left(\frac{0.413}{1+16300 \operatorname{Re}^{-1.09}}\right)
$$

where

$$
\operatorname{Re}=\frac{\rho_{\mathrm{f}} \mathrm{v}_{\mathrm{rel}} \mathrm{d}}{\mu_{\mathrm{f}}}
$$

In systems where the particle and fluid densities are similar (liquid-liquid and liquid-solid systems), acceleration influences the drag coefficient strongly (see Equation (2.14)) and the "added mass" concept is often used to describe the effect of acceleration on particle drag (see Clift et al. [78CL1] and Renganathan et al. [89RE1]). The "added mass" concept takes into account the acceleration of some of the surrounding fluid due to the acceleration of the particle. There is some contradicting evidence in the literature regarding the effect of acceleration on particle drag coefficients in gas-solid systems. See Hughes and Gilliland [52HU1], Buzzard and Nedderman [67BU1], Clift and Gauvin [71CL1], Marchildon and Gauvin [79MA1] and Temkin and Metha [82TE1]. For systems with high density ratios $\left(\rho_{\mathrm{p}} / \rho_{\mathrm{f}}\right)$ and particle acceleration below $10 \mathrm{~m} / \mathrm{s}^{2}$, the standard drag curve can be assumed to approximate the drag coefficients of accelerating non-deformable particles reasonably well.

Evaporation (mass efflux) decreases the drag experienced by particles by reducing the skin friction and by increasing the pressure in the wake (resulting in decreased form drag). Various researchers, including Eisenklam et al. [67EI1], Renksizbulut and Yuen [83RE1] and Chuchottaworn and Asano [85CH1], studied this phenomenon. In the case of evaporating or 
burning fuel drops, the drag coefficient can be significantly influenced, but for water-air systems with temperature differences below $40^{\circ} \mathrm{C}$, evaporation will lower the drag coefficient very slightly. In a similar fashion, it can be expected that condensation (mass influx) would increase drag.

Clift and Gauvin [71CL1] and Clift et al. [78CL1] reviewed the available literature on the effect of free-stream turbulence on the drag of spheres. In general, drag coefficients increase with increasing free-stream turbulence in the sub-critical region $\left(\operatorname{Re}<3 \times 10^{5}\right)$, but this effect is small for turbulence intensities, i.e.

$$
\mathrm{Tu}=\left(\frac{\sqrt{\mathrm{v}^{2}}}{\overline{\mathrm{v}}_{\text {rel }}}\right) \leq 0.1
$$

According to Clift et al. [78CL1], the effect of turbulence macroscale on solid sphere drag is insignificant for the lower Reynolds number range $(\operatorname{Re}<1000)$.

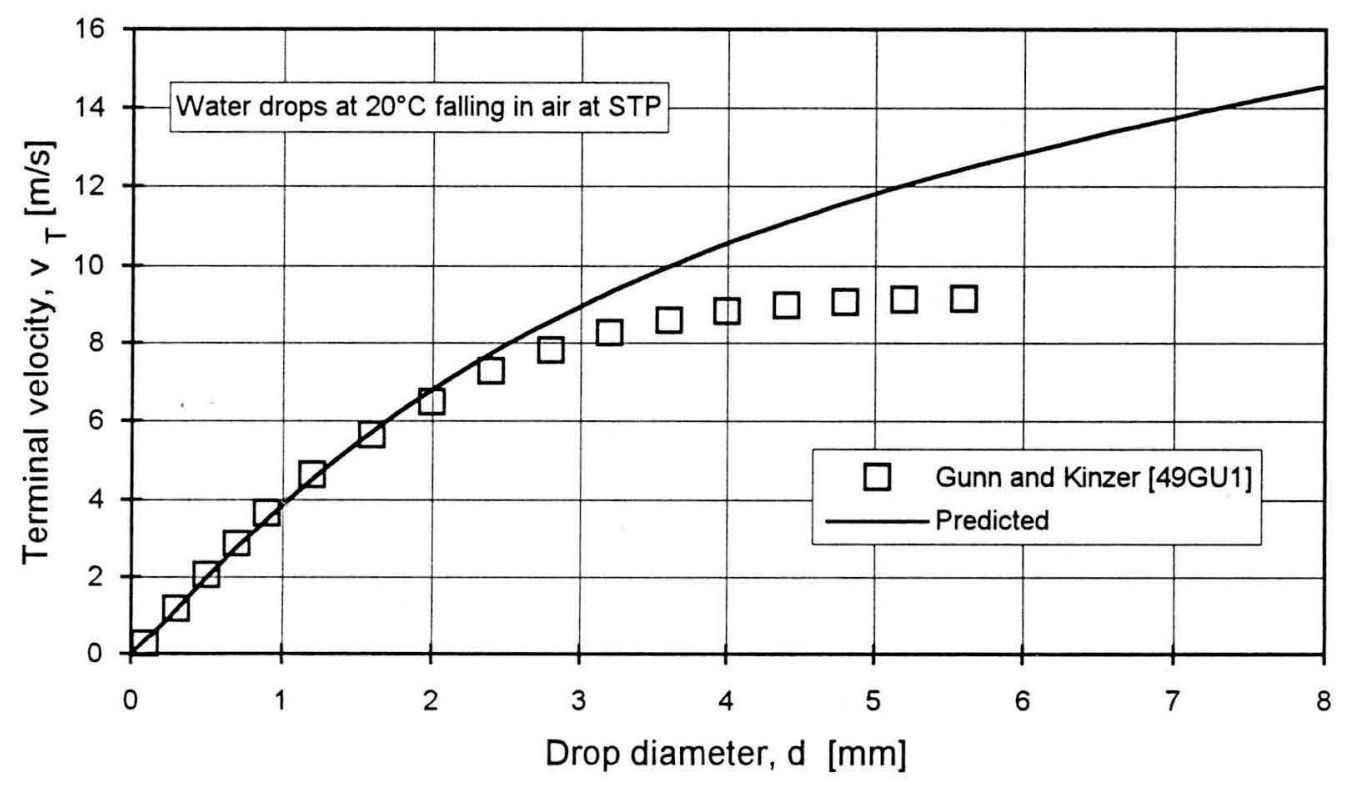

Figure 2.3 Comparison between the drop terminal velocity measured by Gunn and Kinzer and the predicted drop terminal velocity data obtained from the solid sphere correlation of Turton and Levenspiel [86TU1].

The foregoing discussion is applicable to solid particles moving through a continuous medium. Predictions made using solid sphere drag data do not agree with experimentally determined liquid drop velocities. Laws [41LA1] measured the acceleration of various water drop sizes in air. Gunn and Kinzer [49GU1], Beard and Pruppacher [69BE1] and Ryan [76RY1] measured the terminal velocities of water drops in air. The data of Gunn and Kinzer is shown in Figure 
2.3 together with the terminal velocities predicted using a solid sphere drag correlation. The experimentally determined terminal velocity of a $6 \mathrm{~mm}$ water drop falling in air at STP is approximately $9.1 \mathrm{~m} / \mathrm{s}$, which differs markedly from the terminal velocity of $12.9 \mathrm{~m} / \mathrm{s}$ predicted by using solid sphere drag correlation.

The drag experienced by liquid drops is influenced by internal circulation, drop oscillation and drop deformation. Several models have been proposed to correct solid sphere drag correlations to ensure that they correctly predict drop terminal velocity (see Hughes and Gilliland [52HU1], Beard [76BE1] and Clift et al. [78CL1]). These models typically use the drop deformation at terminal velocity to interpolate between experimentally determined correlations for the drag experienced by spheres and flat disks (or oblate spheroids). There remains some uncertainty whether these models will accurately predict the motion of accelerating liquid drops. The phenomena of internal circulation, oscillation and deformation and the acceleration of liquid drops are discussed in greater detail below.

\section{Internal circulation}

Working independently, Hadamard and Ryczynski (see Clift et al. [78CL1]) derived the following equation for the drag coefficient of a fluid sphere in creeping flow by solving the Navier-Stokes equations for both the surrounding fluid and the inside of the liquid drop,

$$
\mathrm{C}_{\mathrm{D}}=\frac{8}{\operatorname{Re}}\left(\frac{2+3\left(\mu_{\mathrm{p}} / \mu_{\mathrm{f}}\right)}{1+\left(\mu_{\mathrm{p}} / \mu_{\mathrm{f}}\right)}\right)
$$

The Stokes solution for creeping flow past a solid sphere $\left(C_{D}=24 / R e\right)$ can be obtained directly from Equation $(2.18)$ by setting $\left(\mu_{\mathrm{p}} / \mu_{\mathrm{f}}\right)=\infty$. The Hadamard-Ryczynski equation predicts drag coefficients up to $50 \%$ lower than that predicted by the Stokes law, which implies that spherical liquid drops fall faster than solid spheres of the same density. This is due to a reduction in skin friction experienced by liquid drops due to an internal circulation induced in the drop. At higher Reynolds numbers it is expected that the internal circulation will tend to move the separation point towards the rear stagnation point of the drop, resulting in a smaller wake and reduced form drag. In practice it has been found that internal circulation is very strongly influenced by the presence of surfactants in the drop and only very pure systems are observed to follow the Hadamard-Ryczynski law (see Levich [62LE1] and Clift et al. [78CL1]).

LeClair et al. [72LE1] numerically calculated drop surface circulation velocities for small (spherical) water drops at $\operatorname{Re}<400$. According to LeClair et al. [72LE1] and Gillaspy and Hoffer [83GI1], the circulation velocities level off at $\mathrm{Re}>800$. For free-falling water drops in air, Garner and Lane [59GA1] and Pruppacher et al. [70PR2] have observed much lower 
surface velocities than those predicted by numerical models. For water drops falling in air, the effect of contamination should be relatively small since the viscosity ratio of this combination is such that the internal circulating velocity should be small even for very pure systems. LeClair et al. [72LE1] found the contribution of internal circulation on the drag of a water drop in air to be less than $1 \%$.

In certain circumstances, internal circulation can also be the result of drop oscillation (see Trinh and Wang [82TR1]) or due to the action of the drop detachment on departure from a nozzle.

\section{Drop deformation}

The aspect ratio for an ellipsoidal particle is defined as

$$
E=\left(\frac{d_{a}}{d_{b}}\right)
$$

where $d_{b}$ and $d_{a}$ are described as shown in Figure 2.4. A drop is described as an oblate spheroid if it has an aspect ratio of less than unity, otherwise it is called prolate. At higher Reynolds numbers, liquid drops change to non-symmetrical ellipsoidal shapes (approximately oblate) due to increased hydrodynamic pressure at the forward stagnation point. Increasing oblateness of a drop tends to promote the formation of an attached wake and the onset of wake shedding, causing an increase in the drag coefficient.

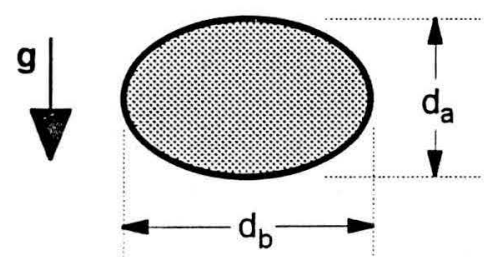

Figure 2.4 Approximate geometry of a deformed drop.

Various researchers, including Davies [45DA1], Pruppacher and Beard [70PR2], Ryan [76RY1], Chandrasekar et al. [88CH1] and Saviji et al. [90SA1], presented experimentally determined correlations for water drop deformation at terminal velocity in air. The Eotvos number, Eo, which is the ratio of the maximum hydrostatic pressure head inside the drop to the surface tension forces, is usually used as a correlating parameter to describe drop deformation. Pruppacher and Pitter [71PR1], Green [75GR1], Beard and Chuang [87BE1] and others calculated the drop deformation at terminal velocity numerically and found fair agreement with the available experimental data. LeClair et al. [72LE1] concluded that the effect of internal circulation on drop shape is negligible. This is confirmed by the fact that the models by Green [75GR1] and Beard and Chuang [87BE1], which do not include the effect of 
internal circulation, agree well with experimentally observed drop shapes. Pozrikidis [89PO1] found that increasing internal circulation of a drop results in decreasing oblateness (more spherical shape).

Hollands [74HO1], Beard [76BE1] and Clift et al. [78CL1] proposed models for calculating the drag of deformed drops. According to Clift et al. [78CL1], interpolation between the drag curve for a solid sphere and that of a deformed sphere (e.g. the drag correlation for a spheroid with $\mathrm{E}=0.5$ of Stringham et al. [69ST1]) can be used to determine the drag coefficient of any deformed sphere (with any E).

\section{Drop oscillation}

Rayleigh (see Clift et al. [78CL1]) derived a relation to predict the oscillating frequency of a liquid drop in a gas of negligible density. Lamb [45LA1] modified this model to include the effect of gas density and showed that the frequency of oscillation of a liquid drop after a small initial disturbance is given by

$$
f=\sqrt{\frac{2 \sigma(n-1) n(n+1)(n+2)}{\pi^{2} d_{e}^{3}\left((n+1) \rho_{p}+n \rho_{f}\right)}}
$$

where $\mathrm{n}$ is the mode of oscillation. The value of $\mathrm{n}=0$ corresponds to a radial oscillation mode which is not possible for incompressible liquids. A mode of $n=1$ represents the case of drop translation as a whole. The second mode, $n=2$, thus represents the first oscillatory mode of the drop (oblate-prolate type oscillations). Garner and Lane [59GA1], Nelson and Gokhale [72NE1], Srikrishna et al. [82SR1], Epema and Riezebos [84EP1] and Hijikata et al. [84HI1] found that this relation (with $\mathrm{n}=2$ ) predicts the oscillation frequency of liquid drops in gas reasonably well. Yao [74YAl] and Yao and Schrock [76YAl] found oscillation frequencies of water drops $\left(3<\mathrm{d}_{\mathrm{e}}<6 \mathrm{~mm}\right)$ falling in air from the tip of a drop generator to correspond closely to that predicted by the Lamb equation for modes 2 and 3. As the drops fell from a nozzle, it was observed that the oscillations decayed to negligible magnitude at a fall distance of 150 drop diameters.

The vortex shedding frequency of the drop wake has often been cited as a driving force for inducing drop oscillations in liquid-liquid systems. The frequency of oscillation of a water drop of $1 \mathrm{~mm}$ equivalent diameter corresponds to the frequency of vortex shedding for a sphere of this size moving at terminal velocity. In carefully conducted experiments, Beard et al. [83BE1], [89BE1] found that single drops in the size range 1 to $1.6 \mathrm{~mm}$, travelling at terminal velocity, undergo spontaneous oscillations which were thought to be the result of eddy shedding from the drop. Once drop oscillation has been initiated, the frequency of vortex shedding may shift as a result of the shape changes. Drop collisions can also result in drop 
oscillations of larger drops as shown by Beard et al. [83BE1], [89BE1]. In rainfall, large drops collide every few seconds, thus keeping the drops oscillating. The more numerous smaller drops do not undergo collisions frequently enough to maintain drop oscillation.

There is also some speculation that drop oscillation could be due to free-stream turbulence or turbulence due to wakes of other drops. Srikrishna et al. [82SR1] observed sustained oscillations for large water drops at terminal velocity in air. For drops of this size there is a poor match between the natural frequency of a drop and the vortex shedding frequency. Since this experimental work was conducted in a test section downstream from a centrifugal blower, the observed sustained oscillation of large water drops can be attributed to a high degree of free-stream turbulence.

For liquid-liquid systems, a plot of terminal velocity versus Reynolds number which shows a maximum, would indicate that the drop oscillates at the higher Reynolds numbers. According to Edge et al. [74ED1], Clift et al. [78CL1], Grace and Weber [82GR1] and Greene [88GR1], [91GR1], the peak in the curve corresponds to the onset of liquid oscillations.

Beard [77BE1] and Pruppacher and Klett [78PR1] concluded that the oscillation frequency of water drops is too high in comparison with the drop velocity relaxation time, $v_{T} /(2 g)$, for drop oscillation to have a noticeable effect on drop drag and terminal velocity (in the absence of air turbulence). This conclusion can also be reached by noting that a plot of terminal velocity versus Reynolds number of a water drop falling in air, does not show a maximum value which would indicate the onset of drop oscillation (as is found for liquid-liquid systems). The curve does flatten off for drops larger than $5 \mathrm{~mm}$, but this is due to drop deformation.

\section{Accelerating drops}

For liquid drops moving through a continuous medium, the problem of determining the drag coefficient during acceleration is complicated by the deformation of the drop. Wang and Pruppacher [77WA1] overcame the problem of finding the instantaneous drop shape during acceleration by assuming that the drag coefficient of an accelerating drop can be found from the experimentally determined standard drag coefficient curve (valid at terminal velocity) at the same Reynolds number. Beard [77BE1] questioned the validity of the drag coefficient assumption made by Wang and Pruppacher [77WA1]. Beard reasoned that this assumption would require the drop to change shape in just the right way to ensure that the shape of the instantaneous drag coefficient versus Reynolds number coincides with the drag coefficient curve for drops at terminal velocity. Beard [77BE1] presented a semi-empirical model based on the drop distortion model of Green [75GR1] to account for the effect of drop shape change on the drop drag during acceleration. This model by Beard predicts the terminal velocity of 
drops very well without resorting to the experimentally measured drop drag coefficients (empirical correlations for solid sphere drag coefficients are used) and it predicts slightly larger accelerations than the method by Wang and Pruppacher. Beard showed that the drag coefficient curve for an accelerating drop is indeed unique, but not exactly as predicted by Wang and Pruppacher.

\subsubsection{Heat and mass transfer from spheroids and drops}

This literature review is primarily concerned with the steady state external heat and mass transfer to/from particles. Since the governing dimensionless equations and boundary conditions for heat and mass transfer are of identical form, the solutions also have identical forms for low heat and mass transfer rates. (At high heat/mass fluxes, the heat and mass transfer processes influence one another.) This results in the same profiles of dimensionless heat and mass transfer. Consequently, the Nusselt and Sherwood numbers are similar functions of Reynolds, Prandtl and Schmidt numbers and time. It is thus possible to interchange the Nusselt and Prandtl numbers for heat transfer solutions and the Sherwood and Schmidt numbers for mass transfer solutions. For systems undergoing simultaneous heat and mass transfer, the Lewis factor, which is a function of the Lewis ratio $(\mathrm{Sc} / \mathrm{Pr})$, would control the heat and mass transfer (see summary by Dreyer [88DR1]). For air-water systems, it is often convenient to assume a Lewis factor of unity.

From work by Schlichting [87SC1], Yao [74YA1] concluded that the boundary layer around a drop accelerating in a fluid would attain a steady state in the time required for the drop to move a distance equal to its diameter. This assumption allowed Yao to split the transient cooling process of an accelerating drop into many small quasi-steady steps.

Sideman and Shabtai [64SI1], Clift et al. [78CL1] and Godfrey and Hanson [82GO1] summarised most of the relevant literature on heat/mass transfer to/from spherical and nonspherical solid and liquid bodies. Various correlations for heat/mass transfer to/from solid spheres at higher Reynolds numbers are available from the literature, i.e. Rowe et al. [65RO1], Whitaker [72WH1], Brauer and Sucker [78BR2] and Clift et al. [78CL1].

According to Raithby and Eckert [68RAl] and Clift et al. [78CL1], increasing turbulence intensity increases heat/mass transfer to/from spheres at all Reynolds numbers. Clift et al. [78CL1] presented a simple model to estimate the turbulence effect on heat and mass transfer. The effect of turbulence intensity, Tu, on the sphere Nusselt number can be expressed as

$$
\frac{\mathrm{Nu}}{\mathrm{Nu}_{\mathrm{Tu}=0}}=1+4.8 \times 10^{-4} \mathrm{Re}^{0.57}\left(\frac{\mathrm{Tu}}{0.077}\right) \text { for } \mathrm{Tu}<0.077
$$


For example, this correlation predicts an increase of $1.2 \%$ in heat transfer from a sphere at $\mathrm{Tu}=0.02$ and $\mathrm{Re}=3000$. According to Clift et al. [78CL1], the effect of turbulence intensity on the heat transfer from solid spheres is much more significant than the effects of turbulence macroscale or the turbulent energy spectrum.

High mass flux to/from a surface could have a significant effect on the heat transfer rate to/from a surface. Collier [81CO1] and Chuchottaworn et al. [84CH2] proposed corrections to account for these effects. For air-water systems at temperatures which are found in cooling towers $\left(\mathrm{T}_{\mathrm{w}}<60^{\circ} \mathrm{C}\right)$, the effect of mass flux on the heat transfer coefficient is normally small.

Skelland and Cornish [63SK1], Lochiel and Calderbank [64LO1] and Beg [75BE1] experimentally determined the heat/mass transfer from/to solid spheroids (oblate and prolate). Chuchottaworn and Asano [86CH2] determined the mass transfer from spheroids numerically for $\operatorname{Re}<200$ and found good agreement between their results and published data. Skelland and Cornish [63SK1] found that their data for mass transfer from an oblate spheroid could be calculated from any correlation for mass transfer from a sphere by using a Reynolds number based on an equivalent diameter equal to the surface area of the spheroid divided by its perimeter normal to the flow. Generally, the average Nusselt/Sherwood numbers increase slightly as the particle becomes more oblate. At an eccentricity of $E=0.5$, the Sherwood number for the spheroid is only $10 \%$ larger than that of a sphere.

Due to experimental difficulties, the heat and mass transfer rates from liquid drops has mostly been determined using drops suspended on thin wires or glass fibres. It can be expected that the suspension device has an influence on internal circulation and drop oscillation. Raithby and Eckert [68RA1] found that the position of the support holding an experimental sphere has a significant influence on the heat/mass transfer from a solid sphere. It can be assumed that this is also true for experiments conducted with pendant liquid drops as well.

The best known correlations for heat/mass transfer from small liquid drops are those presented by Frössling [38FR1]

$$
\mathrm{Sh}_{\mathrm{f}}=2+0.552 \mathrm{Re}_{\mathrm{f}}^{1 / 2} \mathrm{Sc}_{\mathrm{f}}^{1 / 3} \text { for } 2<\mathrm{Re}_{\mathrm{f}}<800
$$

and Ranz and Marshall [52RA1], [52RA2],

$$
\begin{aligned}
& \mathrm{Nu}_{\mathrm{f}}=2+0.6 \mathrm{Re}_{\mathrm{f}}^{1 / 2} \operatorname{Pr}_{\mathrm{f}}^{1 / 3} \text { for } 2<\mathrm{Re}_{\mathrm{f}}<200 \\
& \mathrm{Sh}_{\mathrm{f}}=2+0.6 \operatorname{Re}_{\mathrm{f}}^{1 / 2} \mathrm{Sc}_{\mathrm{f}}^{1 / 3} \text { for } 2<\operatorname{Re}_{\mathrm{f}}<200
\end{aligned}
$$


Both these studies were conducted using small drops $(\mathrm{d} \leq 1.1 \mathrm{~mm})$ suspended on thin fibres. These correlations are in good agreement with data for solid spheres. This indicates that the effects of drop oscillation and internal circulation were minimal during these experiments. Miura et al. [77MU1] showed that the Ranz and Marshall correlation is valid up to a Reynolds number of 2000. Kinzer and Gunn [51KI1] measured heat/mass transfer rates to/from relatively large water drops $\left(0.27<\mathrm{d}_{\mathrm{e}}<4.2 \mathrm{~mm}\right)$ falling at terminal velocity in air. They noted that their data was well correlated by the correlation of Frössling [38FR1]. The correlations by Beard and Pruppacher [71BE1], Srikrishna et al. [82SR1] and Wedding et al. [86WE1] are in good agreement with the correlations of Frössling [38FR1] and Ranz and Marshall [52RA1], [52RA2].

For solid oblate spheroids, the effect of deformation on the external heat/mass transfer was shown by Lochiel and Calderbank [64LO1] and Clift et al. [78CL1] to be small. Since large water drops falling in air have been found to have oblate, spheroidal shapes, the heat transfer from a deformed water drop could be approximated by using any of the heat/mass transfer correlations for spheres or spherical drops and the actual surface area of the drop.

Clift et al. [78CL1] derived a criterion to determine whether oscillation influences the heat/mass transfer from/to a drop based on the correlation for heat transfer from oscillating spheres presented by Gibert and Angelino [74GI1]. According to this criterion, the effect of surface oscillation on the external heat/mass transfer to/from a drop is negligible if

$$
\left(\frac{d_{e} f_{n}}{v}\right)<0.15
$$

According to this criterion, drop oscillations only influence the heat/mass transfer if the relative velocity between the drop and the airstream is less than the critical velocity specified by this criterion. Using this criterion, it can be shown that the heat/mass transfer from/to a water drop larger than $\mathrm{d}_{\mathrm{e}}=1 \mathrm{~mm}$, accelerating in still air, will only be influenced by oscillations for a very short time (until the drop velocity exceeds the critical velocity). For small drops $\left(\mathrm{d}_{\mathrm{e}}<1 \mathrm{~mm}\right)$, the terminal velocity may be smaller than the critical velocity, implying that drop oscillations will always play a role in the heat/mass transfer from/to a small drop. However, the initial amplitudes of small drops are expected to be small and the oscillations will be rapidly damped for small drops.

Due to the oscillatory motion of a liquid drop, the effective, time-averaged surface area of the drop is larger than the surface area of a non-oscillating drop having the same time-averaged shape. Using the model by Lamb [45LA1] to calculate drop oscillation frequencies (with $n=2$ ), it can be shown that during the first second after formation, a $6 \mathrm{~mm}$ oscillating drop with an 
initial amplitude of $A_{m} / d_{e}=1.1 d_{e}$ has an effective area only $2 \%$ larger than that of a $6 \mathrm{~mm}$ spherical drop. A $6 \mathrm{~mm}$ drop falling from rest will travel more than $4 \mathrm{~m}$ in 1 second. Note that a large drop falling through air becomes progressively more deformed. For a drop oscillating about a non-spherical, time-averaged shape $(\overline{\mathrm{E}}<1)$, the effective area ratio (compared to that of a spheroid with fixed deformation equal to $\bar{E}$ ) is larger than that of an undeformed drop $(\bar{E}=1)$ oscillating with the same initial amplitude. It can be shown that during the first second after formation, a $6 \mathrm{~mm}$ drop oscillating about a spheroidal shape with $\overline{\mathrm{E}}=0.5$ ( $\mathrm{n}=2$, $A_{m} / d_{e}=1.1 d_{e}$ ) has an effective area approximately $7 \%$ larger than that of a non-oscillating spheroidal drop with $\overline{\mathrm{E}}=0.5$ and $\mathrm{d}_{\mathrm{e}}=6 \mathrm{~mm}$.

The correlation of Srikrishna et al. [82SR1] for heat/mass transfer from freely falling water drops at terminal velocities, agrees very well with correlations of other researchers (i.e. Frössling [38FR1], Ranz and Marshall [52RA1], [52RA2], Beard and Pruppacher [71BE1] and Wedding et al. [86WE1]), regardless of the fact that Srikrishna et al. observed large oscillations on the surface of the drops during their experiments. The other researchers studied small and/or pendant drops for which very little oscillatory motion (only low amplitude, high frequency oscillations are expected for small drops with $\mathrm{d}_{\mathrm{e}} \approx 1 \mathrm{~mm}$ ) was expected. This, together with the previous discussion on small increases in effective area due to drop oscillations, leads to the conclusion that the effect of drop oscillation is of almost negligible importance in the heat/mass transfer behaviour of water drops in air.

The internal circulation in a drop due to drop detachment is expected to be fairly strong immediately after release of a drop. This, together with drop shape oscillations, is expected to result in good mixing on the inside of a drop (see Hijikata et al. [84HI1]). In air/water systems, drop oscillation and internal circulation are not expected to have a very large effect on the heat transfer rates since the air side thermal resistance is by far the controlling resistance.

Snyder [51SN1] measured the cooling rates of single water drops accelerating freely in air, and found fair agreement between his data and that for solid spheres for $\operatorname{Re}<1000$. At higher Reynolds numbers his data for 5 and $6 \mathrm{~mm}$ drops differ by up to $15 \%$ from the heat transfer data for solid spheres. Yao [74YA1] and Yao and Schrock [76YA1] presented the following correlation (based on that of Ranz and Marshall [52RA1], [52RA2]) for the external heat/mass transfer from/to oscillating water drops $\left(3<\mathrm{d}_{\mathrm{e}}<6 \mathrm{~mm}\right)$ released from a fixed nozzle in air in terms of the dimensionless fall distance, with $10<\left(\mathrm{z} / \mathrm{d}_{\mathrm{e}}\right)<600$ :

$$
\mathrm{Nu}_{\mathrm{f}}=2+\mathrm{g}_{\mathrm{YS}}\left(0.6 \operatorname{Re}_{\mathrm{f}}^{1 / 2} \operatorname{Pr}_{\mathrm{f}}^{1 / 3}\right)
$$

where 


$$
g_{Y S}=25\left(\frac{z}{d_{e}}\right)^{-0.7}
$$

The accuracy of this correlation was questioned by Chen and Trezek [77CH1], who found that this correlation failed to predict the correct trends in the cooling of drops in spray ponds. This correlation predicts Nusselt numbers approaching 2 at large fall distances. If the difference between this correlation and that of Ranz and Marshall can be attributed to the effect of drop oscillation on the external flow field, it is expected that the Nusselt number predicted by this correlation should approach the steady state Nusselt number (given by the Ranz and Marshall correlation) at large fall distances, which is not the case.

Mercker [93ME1] measured the heat/mass transfer from accelerating water drops, with $2.5<\mathrm{d}<6 \mathrm{~mm}$, falling downwards into an upward flowing airstream. Mercker correlated his data by

$$
g_{Y S}=1+66.54 M_{\ddot{z}}^{0.8}\left(\frac{d}{d_{m}}\right)^{-0.1}
$$

for $M_{\ddot{Z}}<3.0 \times 10^{-3}$. The maximum stable drop size was defined as

$$
d_{m}=\sqrt{\frac{16 \sigma}{g\left(\rho_{w}-\rho_{a}\right)}}
$$

Note that the correlation by Mercker approaches the Ranz and Marshall correlation when the drop approaches terminal velocity (i.e. when $M_{\ddot{z}} \rightarrow 0$ ).

\subsubsection{Aerodynamic break-up}

As a relatively large drop of liquid accelerates through another liquid or a gas, the drop becomes increasingly deformed and at a critical speed it becomes unstable and breaks into a number of fragments. The Weber number, which is the ratio of the disruptive hydrodynamic force to stabilising surface tension forces, can be expected to be of major importance in describing the aerodynamic break-up of drops. Free-stream turbulence is also thought to contribute to drop break-up. Criteria for the break-up of freely falling drops and drops suddenly exposed to high speed gas flows were presented by Lane [51LA1], Hinze [55HI1], Brodkey [67BR1], Borisov et al. [81BO1], Pilch and Erdman [88PI1], Kitscha and Kocamustafaogullari [89KI1] and Wierzba [90WI1].

Wierzba [90WI1] summarised most of the relevant aerodynamic drop break-up data in the open literature up to 1986 . Wierzba found that the available data for critical Weber numbers ranged between 2.2 and 99.6. In an experimental study it was found that drop break-up 
occurred in the range $11<\mathrm{We}_{\mathrm{c}}<14$. In this range of Weber numbers, five types of drop behaviour were observed: (i) flattening without break-up, (ii) vibrational type break-up (drop splitting), (iii) bag type break-up, (iv) initial characteristics of the vibrational type break-up, except that break-up does not occur and (v) initial characteristics of the vibrational type breakup, but continuing into a bag type break-up. In the bag type break-up, the drop flattens and thins in the centre section, forming a thick rim with the thin film inside the rim blowing open in the gas stream and eventually shattering into very small drops. The rim breaks into many larger drops. For a given drop size and a Weber number in this range, it was observed that the percentage of bag type drop break-ups increased with an increasing Weber number. Larger drops also tended to result in a larger percentage of bag type drop break-ups.

In order to determine the maximum stable drop size, taking the Weber number equal to the critical Weber number yields the following relation

$$
d_{m}=W e_{c}\left(\frac{\sigma}{\rho_{\mathrm{f}} \mathrm{v}^{2}}\right)
$$

Assuming a critical Weber number of 12 , this equation predicts a maximum water drop diameter (falling in air) of approximately $8.5 \mathrm{~mm}$. Lehrer [75LE1] and Grace et al. [78GR1] found that the maximum stable drop size can be described by

$$
d_{m}=c_{1} \sqrt{\frac{\sigma}{g\left(\rho_{p}-\rho_{f}\right)}}
$$

where $c_{1}=4$. For a pure water drop in air at STP, this equation predicts a maximum drop diameter of $10.8 \mathrm{~mm}$. Ryan [76RA1] found the maximum stable drop diameter to be proportional to $\sigma^{1 / 2}$ and observed a maximum stable water drop diameter of $9 \mathrm{~mm}$ (resulting in $\mathrm{c}_{1}=3.41$ ).

\subsubsection{Drop-drop collisions}

In a rain cloud where large and small drops fall together under the influence of gravity, the large drops have higher terminal velocities than the smaller drops. Due to the relative velocity between the large and small drops, there is a possibility of larger drops catching up and colliding with the smaller drops in their paths. Upon collision, the two interacting drops could bounce apart, coalesce permanently or coalesce temporarily and then break into smaller satellite drops. The evolution of the drop size distribution in clouds is governed by the collisions between drops. Cloud physicists have extensively studied the collision behaviour of cloud drops. Pruppacher and Klett [78PR1] and Rogers [79RO1] gave extensive reviews of the available literature up to the late 1970's. 
In cooling towers fitted with splash packing material it can be expected that drop-drop collisions will also occur. In the case of cooling towers, the falling water drops strike splash grids which then result in very small splash drops being formed, while large drops drip from below the grids. The grids have the effect of reducing the velocity of a fraction of the spray, which then results in increased relative velocity between the falling drops. This, together with the very high rain densities (in comparison to that of natural clouds), would result in a large number of drop-drop collisions occurring in cooling towers even though the drop residence time in a cooling tower is order of magnitude smaller than that of drops in a cloud.

If a large drop catches up with a smaller drop which has zero inertia, the smaller drop would be swept aside by the flow around the larger drop and no collision would occur. Whether or not a collision occurs between two drops depends on the relative importance of the inertia and aerodynamic forces and the distance between the drop centres. Collision efficiency, $\eta_{\text {coll }}$, is defined as the fraction of drops in the flight path of a given drop which are actually struck by the drop. Many researchers investigated the collision efficiencies of very small drops, $d_{e}<100$ $\mu m$, since this is of major importance in the modelling of rain drop growth from condensation nuclei. Pruppacher and Klett [78PR1] gave a review of the available literature on collision efficiencies. Collision efficiencies larger than unity can occur for drops of similar sizes. This can be attributed to wake interaction. Air turbulence increases the collision efficiency of very small drops $\left(\mathrm{d}_{\mathrm{e}}<30 \mu \mathrm{m}\right)$ but has little effect on larger drops due to their larger inertia. For drops of the sizes which are of importance in cooling towers, $\mathrm{d}_{\mathrm{e}}>500 \mu \mathrm{m}$, the inertia of the drops is expected to dominate the collision process. In this case, a fixed collision efficiency of $\eta_{\text {coll }}=1$ can be assumed to hold.

Upon the collision of two liquid drops, the following behaviour has been observed : (i) they may bounce apart, (ii) they may coalesce and remain permanently coalesced, (iii) they may coalesce temporarily and then separate, apparently maintaining their initial identities, (iv) they may coalesce temporarily and then break apart, forming satellite drops, and (v) spattering (or splashing) may occur during high energy impacts. Various researchers studied the collisions between water drops experimentally, i.e. Adam et al. [68AD1], List et al. [70LI1], BrazierSmith et al. [72BR1], [73BR1], Spengler and Gokhale [73SP1], McTaggart-Cowan and List [75Mcl], Bradley and Stow [78BR1], [79BR1], Low and List [82LO1], [82LO2], Podvysotsky and Shraiber [84PO1] and Dubrovsky et al. [92DU1]. Ashgriz and Givi [87AS1], [89AS1], Brenn and Frohn [89BR1], [89BR2] and Jiang et al. [92JI1] studied the collisions between fuel drops. The theoretical and experimental work of Park (see Pruppacher and Klett [78PR1]), Ashgriz and Poo [90AS1] and Jiang et al. [92JI1] shows good agreement with the 
following model by Brazier-Smith et al. [72BR1] for drop coalescence. Brazier-Smith et al. [72BR1] derived the following relation for coalescence efficiency:

$$
\eta_{\text {coal }}=\left(\frac{2.4}{W_{e}}\right) \frac{\left(1+\left(\frac{d_{L}}{d_{S}}\right)^{2}-\left(1+\left(\frac{d_{L}}{d_{S}}\right)^{3}\right)^{2 / 3}\right)\left(1+\left(\frac{d_{L}}{d_{S}}\right)^{3}\right)^{11 / 3}}{\left(\frac{d_{L}}{d_{S}}\right)^{6}\left(1+\left(\frac{d_{L}}{d_{S}}\right)\right)^{2}}
$$

This relation was found to predict their experimental data well for the ranges: $d_{L}$ and $d_{S}$ between 300 and $1500 \mu \mathrm{m}, 1<\left(\mathrm{d}_{\mathrm{L}} / \mathrm{d}_{\mathrm{S}}\right)<2.5,0.3<\left(\mathrm{v}_{\mathrm{L}}-\mathrm{v}_{\mathrm{S}}\right)<3 \mathrm{~m} / \mathrm{s}$ and $0<\mathrm{x}<\left(\left(\mathrm{d}_{\mathrm{L}}+\mathrm{d}_{\mathrm{S}}\right) / 2\right)$ where $\mathrm{x}$ is the perpendicular distance between the centre of one drop and the undeflected trajectory of the other. If complete coalescence does not occur, Brazier-Smith et al. [73BR1] found that on average three satellite drops were formed, each of which has a volume given by

$$
V=\frac{0.04 V_{L} V_{S}}{V_{L}+V_{S}}
$$

Many models exist to predict the evolution of rain drop size distributions and rain formation times. The continuous and stochastic growth models (ignoring the effects of aerodynamic drop break-up and drop collisions) were summarised by Pruppacher and Klett [78PR1]. Young [75YO1] and List and Gillespie [76LI1] found the effect of drop collisions to be of major importance in rain drop size distribution modelling. Most of the more recent studies on the evolution of drop size distributions in natural rain use the break-up distribution functions of Low and List [82LO1], [82LO2] for the determination of satellite drop sizes. The studies by Feingold et al. [88FE1], Tzivion et al. [89TZ1], List and McFarquhar [90LI1] and Brown [91BR1] describe the latest developments in this field.

\subsubsection{Non-contact drop interaction}

In the dense 'rain' falling through a cooling tower, the statistical average distance between the drops may be only a few times the drop diameter. For drops travelling at their terminal velocity with rain density of $10000 \mathrm{~kg} / \mathrm{m}^{2} \mathrm{hr}$, the average distance between $1 \mathrm{~mm}$ drops is approximately 10.4 times the drop diameter and for $6 \mathrm{~mm}$ drops the spacing is approximately 13.7 drop diameters. Decreasing the drop velocity decreases the relative spacing between drops, e.g. for a rain density of $10000 \mathrm{~kg} / \mathrm{m}^{2} \mathrm{hr}$ the relative drop spacing would be 6.6 (for any drop size) at a constant velocity of $1 \mathrm{~m} / \mathrm{s}$. Due to the proximity of other drops, a given drop may show an increased or decreased drag, while the heat/mass transfer can also be expected to be affected due to wake interactions and free-stream turbulence generated by the surrounding drops. 
Rowe and Henwood [61RE1], Arrowsmith and Foster [73AR1], Tsuji et al. [82TS1] and Taniguchi and Asano [89TA1] found decreased drag for solid spheres falling in-line for spacings of up to 10 diameters. Increased drag was observed for adjacent spheres at spacings less than 3 diameters. Tal and Sirignano [82TA1] conducted a numerical study on the wake interaction of spheres in a packed array with $\operatorname{Re}<100$. The most significant interactions occurred for spacings less than 2 diameters. For distances greater than 5 diameters, the interaction effects were found to be negligible.

Cataneo et al. [71CA1] found that a $0.75 \mathrm{~mm}$ drop was measurably influenced by a preceding drop which was up to 165 diameters downstream and up to 3 diameters offset (from the centreline). List and Hand [71LI1] measured wakes up to 10 diameters wide and 1340 diameters long behind $2.9 \mathrm{~mm}$ diameter water drops falling at $78 \%$ of their terminal velocity in air. Liu et al. [88LI1] used numerical and experimental methods to determine the drag coefficients of a drop in a chain of drops and found drag forces of up to an order of magnitude smaller than that of a single drop in an infinite fluid. Nguyen et al. [91NG1] observed that a freely falling drop following closely behind another freely falling drop of the same size, catches up with the first drop. They concluded that this was due to the reduction of drag experienced by the following drop. Poo and Ashgriz [91PO2] and Choi and Lee [92CH1] found significant drag reduction for drops spaced closer than 4 diameters.

In conclusion, the effect of neighbouring drops on drop drag is of importance in the calculation of drop motion only when the inter-drop spacing is less than about 6 diameters. Care should be taken to calculate the drag coefficient of a particular drop on the free-stream velocity experienced by the drop. This is especially important in the case of streams of drops moving in still air where airflow induced by the movement of the drops can decrease the actual velocity between the drop and the air significantly.

Waslo and Gal-Or [71WA1] and Labowski [76LA1] predicted reduced heat and mass transfer rates with increasing particle concentration. Miura et al. [77MI1], [80MI1] measured the evaporation rates of water drops (into moving air) in the proximity of glass beads. The effects of different diameters and spacings were investigated. It was found that the heat and mass transfer from the drops was significantly reduced with in-line inter-particle spacings of less than 6 drop diameters. For adjacent drops (not in-line) no influence on the heat and mass transfer was observed. The results of Skelland and Vasti [85SK1] for non-oscillating drops agree well with findings of Miura et al., but in the case of oscillating drops no interaction was observed, even for very small inter-drop spacings. Chiang and Kleinstrauer [91CH2] and Taniguchi et al. [91TAl] studied chains of closely spaced, monodisperse drops with blowing 
(evaporation). Evaporation and wake effects were found to reduce the drop drag and mass transfer for each interacting drop with increasing Reynolds number.

In the case of heat and mass transfer from arrays or groups of drops, it should be noted that the driving-potentials for mass and heat transfer are decreased by the proximity of other drops, which would lead to decreased heat/mass transfer. The actual transfer coefficients could, however, also be influenced by the interactions.

\subsubsection{Splashing and cutting}

This literature survey is mainly concerned with the impact of water drops travelling at speeds below $10 \mathrm{~m} / \mathrm{s}$ striking solid surfaces covered by thin water films (films thinne than the diameter of the impacting drop).

The splash sequence of a drop striking a deep pool of liquid is as follows: After impact, a thin film of liquid is thrown upwards and outwards from the point of impact. The height of the film increases as the drop penetrates deeper into the liquid. Small jets of liquid are formed at the upper rim of the film. These jets break up and form many small drops which leave the impact point at high velocity. Due to the physical appearance of this film, the jets and the small drops, this structure is often referred to as the "crown". While the "crown" is formed, a cavity is formed at the point of impact. As the "crown" disappears, the cavity starts to collapse as well. This results in the formation of a so-called Rayleigh jet rising out from the point of impact to above the liquid layer. The Rayleigh jet may break up to form one or more large drops. See Engel [66EN1], [67EN1], Hobbs et al. [67HO1], [67HO2], Macklin et al. [69MA1], [76MA1], Tuong and Painter [74TU1] and Allen [75AL1]. Upon reduction of the pool depth, the "crown" formed by an impacting drop becomes more unstable. The Rayleigh jet rises higher and more drops are formed from it as the water depth is decreased. Reducing the liquid layer depth beyond a critical depth sharply reduces the Rayleigh jet height and number of drops until the Rayleigh jet disappears completely for thin liquid layers as shown by Macklin and Hobbs [69MA1]. The numerical model by Harlow and Shannon [67HA1] also predicted the non-existence of the Rayleigh jet for splashing in thin layers. Mutchler and Hansen [70MU2] and Macklin and Metaxas [76MA1] presented experimental data describing the appearance of the crown formed by splashes in thin water layers.

The volume of splash drops leaving a thin liquid layer after impact by a liquid drop, travelling at terminal velocity, was determined by Mutchler [70MU1] and Mutchler and Larson [71MU1]. The splash volume was found to be a function of the incoming drop size and layer thickness only. According to Mutchler and Larson [71MU1], the splash volume' can be correlated by 


$$
\frac{\mathrm{m}_{\mathrm{s}}}{\mathrm{m}_{\mathrm{i}}}=\mathrm{c}_{1}+\mathrm{c}_{2}\left(\frac{\delta}{\mathrm{d}_{\mathrm{i}}}-\frac{\delta}{4}\right)
$$

with

$$
\begin{aligned}
& c_{1}=1+2.02 \exp (-2.56(\delta / 4))-3.02 \exp (-16(\delta / 4)) \\
& c_{2}=-0.2-13.6 \exp (-2.87(\delta / 4))+60 \exp (-18.63(\delta / 4))
\end{aligned}
$$

with $\delta$ in $\mathrm{mm}, 0.1<\delta<18 \mathrm{~mm}, 2.96<\mathrm{d}_{\mathrm{i}}<5.6 \mathrm{~mm}$. Stedman [79ST1] investigated the splashing of drops falling onto thin water films at velocities below terminal velocity. The splash volume was found to be reduced by inclining the target surface from the horizontal.

Mutchler [70MU1], [71MU2] found that the splash drops formed after the impact of a liquid drop (travelling at terminal velocity) in a thin liquid layer, were normally distributed and presented correlations for the median drop diameter, the standard deviation and the total number of splash drops formed. Other researchers, i.e. Gregory et al. [59GR1], Levin and Hobbs [71LE1] and Stow and Stainer [77ST1] found the splash drop diameters to be lognormally distributed. Stow and Stainer [77ST1] found that the number of splash drops formed is proportional to the kinetic energy of the drop at impact. The mean splash drop size was found to increase with increasing incoming drop size, surface roughness and depth of the liquid film. The mean splash drop size was found to decrease with increasing impact velocity and with a reduction of surface tension.

Scriven et al. [74SC1] developed a simple model to predict the size distribution of splash drops formed from the impact of liquid drops on thin liquid layers. By using the Helmholz instability theory for sheet break-up, a distribution function was determined which related the number and sizes of drops formed at a given instant after the initial impact. Scriven et al. [74SC1] and Ghadiri and Payne [88GH1] found the drops which were formed later in the splashing process to be larger and slower than splash drops formed directly after impact. The form of this distribution function is given as

$$
N\left(d_{s}\right)=c\left(\frac{\rho_{a} v_{i}^{2}\left(d_{i} / 2\right)}{\sigma}\right)\left(\frac{\left(d_{i} / 2\right)}{d_{s}^{2}}\right)
$$

Evidence regarding the bouncing (or non-coalescence) of small liquid drops impacting liquid films has been reported by Jayaratne and Mason [64JA1], Ching et al. [84CH1], [86CH1] and Lee and Hanratty [88LE1].

The impact of drops on dry surfaces has been studied by Levin and Hobbs [71LE1], Stow and Stainer [77ST1], Cheng [77CH2], Stow and Hadfield [81ST1], Yao et al. [88YA1] and 
Chandra and Avedisian [91CH1]. The smoothness of the dry surface and the impact velocity were found to influence the splash formation. The splashing on a dry, unpolished (rough) metal surface was found to be similar to that observed on a thin liquid film, while no or very little splashing was observed for drop impacts on perfectly smooth surfaces.

Various studies have been conducted on the spreading of pathogens on plant surfaces by the splashing action of rain drops, see Gregory et al. [59GR1], Fitt et al. [82FI1], [84FI1], Brennan et al. [85BR1], [85BR2] and Xang et al. [91XA1]. Xang et al. [91XA1] found that the distribution of splash drops formed after a rain drop impact on the surface of a strawberry can be described by the Weibull distribution function for which the smallest drop size is equal to zero. This type of Weibull distribution function is the same as the Rosin-Rammler distribution function which is commonly used to describe the drop distributions formed by spray nozzles.

Moss [89MO1] measured the mass of water remaining on a roughened metal plate after the impact of $3.6 \mathrm{~mm}$ and $5.1 \mathrm{~mm}$ drops. If the impact point was further than 3 or 4 drop diameters from the edge of the plate, the losses (drop mass minus the mass remaining on the plate) were between $20 \%$ and $25 \%$. As the drop impact neared the edge of the plate, the losses rose, reaching $80-90 \%$ at half a diameter from the edge. The edge was found to modify the impact splashing events more than the mere spilling of the crown over the edge.

Yao et al. [88YA1] studied the drop size distribution downstream from the thin reactor spacer grids during a simulated loss-of-coolant-accident (LOCA). The strips were typically thinner than the drop diameter which meant that the impacts were edge effect dominated. On the thin strips, the drop break-up mechanism was a combination of splashing and cutting (splitting of the impacting drop). Using high speed photography, downstream drop size distributions were found which agreed well with the data by Lee et al. [83LE1]. A balance of the incoming kinetic energy and the newly generated drop surface energy yielded a critical Weber number of 6 for impacts on an infinitely thin strip. For Weber numbers less than 6 , the surface tension would resist the cutting of the drop and for Weber numbers greater than 6 , a drop will always be cut by a strip of zero thickness. The average ejected drop size was found to be independent of the offset of impact on the thin strips and almost independent of the Weber number of the incoming drop.

Berman [61BE1], Young [61YO1] and Tooke [65TO1] described experimental work regarding the splashing of water (falling under gravity) on wooden slats. Berman [61BE1] and Young [61YO1] found that up to $80 \%$ of the incoming water (consisting mainly of large 6-8 $\mathrm{mm}$ drops) splashed from a slat on impact. As can be expected, the splash volumes decrease 
with decreasing impact velocity. In an actual cooling tower, the slat will be struck by many slower moving, smaller drops which will not result in splashes removing large volumes of water, but they will contribute to the growth of a water film on the upper surface of the slat. The thickness of the water film on the slat will be governed by the water removing action of drops with high kinetic energy, the addition of water by the low energy impacts and the dripping from below the slat. Young [61YO1] found that the splash volume is strongly influenced by the thickness of the water film on the surface. Berman [61BE1] reported measured splash drop sizes formed by the impact of water dripping from a water supply slat $0.35 \mathrm{~m}$ and $0.7 \mathrm{~m}$ above the test slat. Tooke [65TO1] used an external water supply to feed the films on the upper surfaces of his test slats. He found a significant increase in the number of splash drops smaller than $0.95 \mathrm{~mm}$ formed with increasing impact velocity of the incoming drop. He also observed that the incoming drops oscillated during their fall and that the drop deformation at impact influenced the number of small splash drops formed. Siscoe and Levin [71SI1] and Khaleeq-ur-Rahman and Saunders [89KH1] also observed influences on the splash due to surface waves in the water prior to the impact of a drop.

\subsubsection{Dripping below horizontal surfaces}

Using dimensional analysis, it can be shown that the group, $\sigma /\left(d^{2} g\left(\rho_{1}-\rho_{g}\right)\right)$, which is the ratio of the surface tension and gravity forces, will be of importance in the low mass flux dripping from an object. Rewriting this ratio yields the following relation, describing the size of drops dripping from an object

$$
d_{p}=C \sqrt{\frac{\sigma}{g\left(\rho_{1}-\rho_{g}\right)}}
$$

The value of the constant in Equation (2.37) is expected to be a function of the liquid flow rate and of the geometry of the surface from which the drop falls. Various researchers presented values for this constant at low liquid flow rates, as shown in Table 2.2.

Table 2.2 Dimensionless drop size constant.

\begin{tabular}{|l|l|c|c|}
\hline Reference & Geometry & $\mathbf{C}$ & $\mathbf{m}_{\mathbf{p}} /\left(\mathbf{m}_{\mathbf{p}}+\mathbf{m}_{\mathbf{s}} \mathbf{)}\right.$ \\
\hline Nottage and Boelter [40NO1] & horizontal surface & 3.29 & - \\
Shoukry et al. [75SH1] & horizontal surface & 3.31 & - \\
Yung et al. [80YU1] & $38.1 \mathrm{~mm}$ tube & 3.0 & 0.81 \\
Hozawa et al. [81HO2] & horizontal surface & $3.30-3.48$ & - \\
Walzer and Klaumünzer [81WA1] & horizontal surface & 2.7 & 0.86 \\
Nicol and Aidoun [84NI1] & $28 \mathrm{~mm}$ tube & 2.4 & - \\
Walzel [90WA1] & vertical plate & 2.7 & - \\
Walzel [90WA1] & horizontal surface & $2.9-3.3$ & - \\
\hline
\end{tabular}


Equation (2.37) is usually used to describe the large drops dripping from an object. Normally each large drop (primary drop) is followed by one or two smaller satellite drops. Yung et al. [80YU1] found that each primary drop falling from below a wetted tube would be followed by four or five smaller satellite drops. The sizes of these secondary drops were in the range

$$
0.24<\left(\frac{d_{s}}{d_{p}}\right)<0.46
$$

As mass flow rates increase, the dripping mode changes to the so-called column mode, characterised by the formation of a liquid column at each drop formation point. As the column accelerates downwards it breaks up into drops of roughly similar size. Yung et al. [80YU1] presented an empirical criterion to determine the flow rate at the onset of the column mode. The size of the drops formed by the break-up of the liquid columns can be calculated from the theory of liquid column instability developed by Rayleigh (see Clift et al. [78CL1]). Using this model, the diameters of the drops are expected to be approximately 1.89 times the diameter of the column from which they are formed. Nottage and Boelter [40NO1] reviewed some of the earlier literature on this subject, while Bogy [79BO1] and Chuech et al. [91CH3] reviewed the more recent literature.

Berman [61BE1] listed average drop sizes for dripping of water below flat bottomed and vshaped wooden slats at various liquid flow rates. As expected, the drops formed below the flat bottomed slat were larger than those formed below the v-shaped slat. With both slats, a reduction of mean drop sizes was observed at increased liquid flow rates.

\subsubsection{Flow through and heat/mass transfer from grids}

Cooling tower splash packing material usually consists of layers of grids packed inside the tower. The airflow through a grid is associated with a static pressure loss due to the blockage effect of the grid. The porosity of a grid is usually expressed as the ratio of open area to the total frontal area

$$
\beta=\frac{A_{\text {fr }}-A_{\text {fr, grid }}}{A_{f r}}=1-\left(\frac{A_{f r, \text { grid }}}{A_{f r}}\right)
$$

and the grid Reynolds number is expressed in terms of the grid (or wire) diameter, i.e.

$$
\operatorname{Re}_{\text {grid }}=\frac{\rho v_{\infty} d_{\text {grid }}}{\mu}
$$

The static pressure drop across a grid is usually given as 


$$
\mathrm{K}_{\Delta \mathrm{p}, \text { grid }}=\frac{\Delta \mathrm{p}_{\text {grid }}}{0.5 \rho v_{\infty}^{2}}=\mathrm{C}_{\text {grid }}\left(\frac{1-\beta^{2}}{\beta^{2}}\right)
$$

For round-edged grids, the value of $\mathrm{C}_{\text {grid }}$ generally decreases with increasing grid Reynolds number, e.g., Pinker and Herbert [67PIl] found that $\mathrm{C}_{\text {grid }}$ levels off at 0.52 for large $\mathrm{Re}_{\text {grid }}$ and Groth and Johansson [88GR2] found that $\mathrm{C}_{\text {grid }} \approx 0.45$ for $\mathrm{Re}_{\text {grid }}>300$.

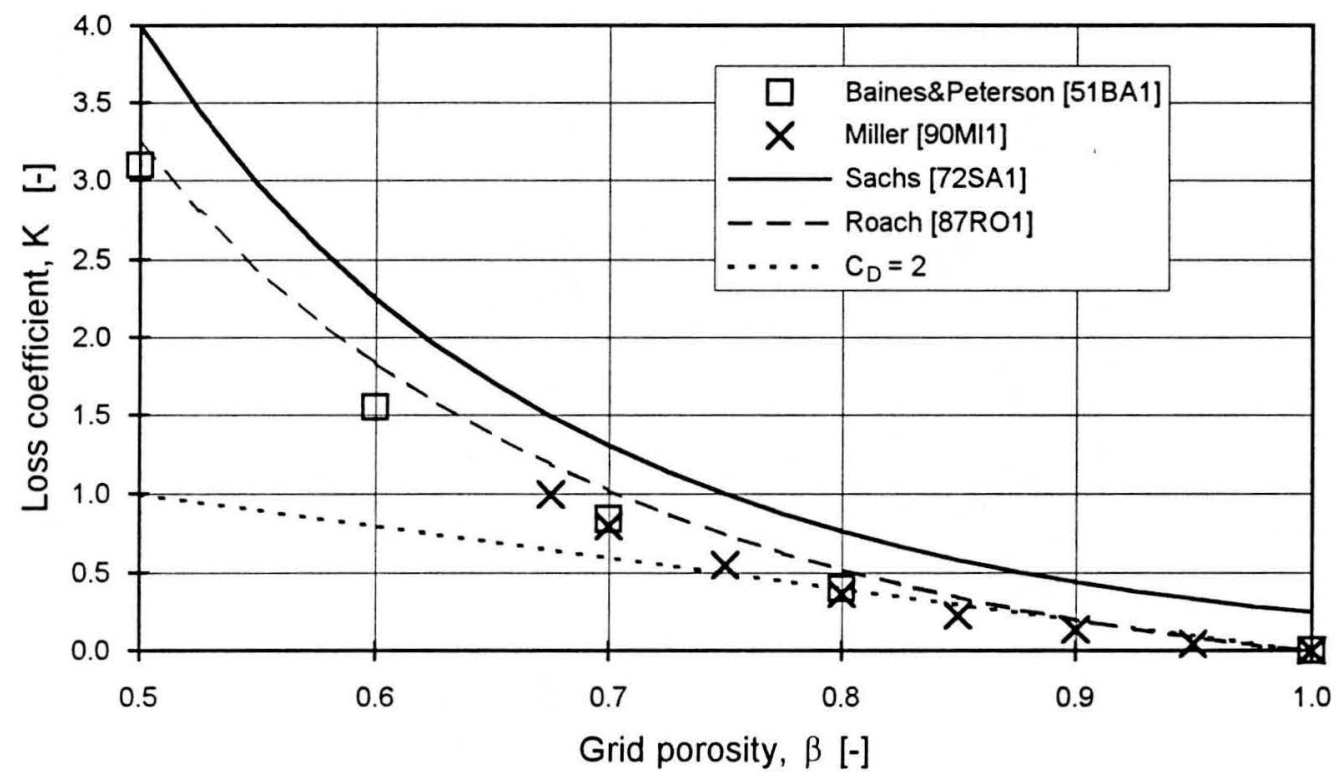

Figure 2.5 Loss coefficients for flow through sharp-edged grids.

Sachs [72SA1] and Roach [87RO1] gave the following correlations for the pressure loss coefficient for flow through sharp-edged meshes at high Reynolds numbers

$$
\mathrm{K}_{\Delta \mathrm{p}, \mathrm{grid}}=\left(\frac{1.5-\beta}{\beta}\right)^{2}
$$

and

$$
\mathrm{K}_{\Delta \mathrm{p}, \mathrm{grid}}=0.98\left(\left(\frac{1}{\beta}\right)^{2}-1\right)^{1.09}
$$

It is also possible to approximate the pressure loss across a coarse grid from the drag coefficient of the slats in the grid, assuming that there is no aerodynamic interaction between neighbouring slats. According to Sachs [72SA1], the pressure loss across a coarse mess can be calculated from the drag coefficient of a single slat, if $\beta>0.85$. The pressure drop across a coarse planar grid can then be expressed as 


$$
\Delta \mathrm{p}_{\text {grid }}=\left(0.5 \rho \mathrm{v}_{\infty}^{2}\right) \mathrm{C}_{\mathrm{D}}\left(\frac{\mathrm{W}}{\mathrm{P}}\right)
$$

yielding a loss coefficient of

$$
\mathrm{K}_{\Delta \mathrm{p}, \text { grid }}=\mathrm{C}_{\mathrm{D}}\left(\frac{\mathrm{W}}{\mathrm{P}}\right)=\mathrm{C}_{\mathrm{D}}(1-\beta)
$$

Figure 2.5 compares the different loss coefficient correlations for flow through sharp-edged grids with data by Baines and Peterson [51BA1] and Miller [90MI1]. The correlation by Sachs [72SAl] predicts significantly higher loss coefficients than that of the other correlations and data.

In a cooling tower fitted with splash packing material, the grids are expected to be covered by a thin water film which has an air/water interface area approximately the same as the surface area of the grids. The heat/mass transfer from the thin film on the grids cools the water film and this results in lowering the temperature of the drops dripping from below the grids. The surface area of the grids is of the same order of magnitude as that of the freely falling drops. The heat/mass transfer coefficients governing the transfer from the water films on the grids can be calculated from the correlations by Jakob [49JA1] or Gnielinski [83GN1] for forced convection from immersed bodies. Jakob presented different correlations for a number of slat cross sectional profiles (squares, hexagonals and thin plates), while Gnielinski used a hydraulic diameter based on the surface area of the slat to the wetted perimeter in the airflow direction to obtain the following general correlation which is applicable to any symmetrical slat profile,

$$
\mathrm{Nu}_{\mathrm{b}}=0.3+\sqrt{\left(0.664 \sqrt{\operatorname{Re}_{\mathrm{b}}} \operatorname{Pr}_{\mathrm{b}}^{1 / 3}\right)^{2}+\left(\frac{0.037 \operatorname{Re}_{\mathrm{b}}^{0.8} \operatorname{Pr}_{\mathrm{b}}}{1+2.443 \operatorname{Re}_{\mathrm{b}}^{-0.1}\left(\operatorname{Pr}_{\mathrm{b}}^{2 / 3}-1\right)}\right)^{2}}
$$

This correlation covers the complete range of Reynolds numbers between 10 and $10^{7}$. It is not certain whether this correlation by Gnielinski [83GN1] is applicable to slats with more complex cross sectional profiles such as $\mathrm{T}$-sections. (Slats with $\mathrm{T}$-shaped cross sectional profiles are commonly used in modern injection-moulded plastic splash pack grids).

\subsubsection{Free-stream turbulence}

In the discussion above, free-stream turbulence was found to influence the drag experienced by drops as well as the heat/mass transfer to/from drops. The free-stream turbulence can be expected to influence the cooling of the water films on the slats of splash packs as well. The drops moving through the airstream and the presence of the splash grids can influence the downstream free-stream turbulence in a cooling tower splash pack 
Based on data from the literature, Gore and Crowe [89GO1] found that the presence of particles in an airstream will increase the free-stream turbulence if the ratio of particle diameter to the turbulent length scale, $\left(\mathrm{d}_{\mathrm{p}} / \mathrm{l}_{\mathrm{t}}\right)$, is larger than a critical value of 0.1 . The turbulent length scale for flow in a pipe can be approximated by $l_{t} \approx 0.1 \mathrm{~d}$. For $\left(\mathrm{d}_{\mathrm{p}} / \mathrm{l}_{\mathrm{t}}\right)$ ratios less than the critical value, the presence of the particles will tend to reduce the free-stream turbulence. Hetsroni [89HE1] found that particles with $\mathrm{Re}>400$ tend to increase free-stream turbulence while the free-stream turbulence is reduced for particle Reynolds numbers $<400$.

Yuan and Michaelides [92YU1] and Schreck and Kleis [93SC1] proposed simple models to describe the modification of turbulence intensity by the presence of particles in dilute gasparticle systems. The model by Yuan and Michaelides [92YU1] includes terms for the two opposing effects of turbulence enhancement and reduction: energy dissipation due to particle acceleration tends to reduce the turbulence intensity, while the turbulence is enhanced by the combination of flow disturbance due to particle motion, wake formation and vortex shedding. With large particles, the change in particle velocity due to free-stream turbulence is small and subsequently the turbulence production term dominates. The inverse is true for small particles.

Fine grids and screens are often used in wind tunnels to reduce free-stream turbulence and to change the velocity profiles. Laws and Livesay [78LA1] and Groth and Johansson [88GR2] reviewed the literature on this subject. Coarse grids with Reynolds numbers larger than 80 contribute to the free-stream turbulence.

Table 2.3 Typical values for the constants describing turbulence decay behind a grid.

\begin{tabular}{|l|l|c|c|c|c|}
\hline Reference & Grid & $\boldsymbol{\beta}$ & $\mathbf{c}_{\mathbf{1}}$ & $\mathbf{z}_{\mathbf{0}} / \mathbf{M}$ & $\mathbf{c}_{\mathbf{2}}$ \\
\hline Comte-Bellot \& Corrsin [66CO1] & Bi-planar & 0.66 & $>20$ & & 1.25 \\
Raithby \& Eckert [68RA1] & Bi-planar & 0.56 & 10.1 & 3 & 1.29 \\
Raithby \& Eckert [68RA1] & Bi-planar & 0.66 & 24.3 & 6 & 1.27 \\
Raithby \& Eckert [68RA1] & Bi-planar & 0.77 & 35.4 & 9 & 1.38 \\
Laws \& Livesay [78LA1] & Bi-planar & 0.20 & $100 / \mathrm{K}_{\Delta \mathrm{p}, \text { grid }}$ & 10 & 1 \\
Laws \& Livesay [78LA1] & Planar & 0.20 & $50 / \mathrm{K}_{\triangle \mathrm{p}, \text { grid }}$ & 10 & 1 \\
Groth and Johansson [88GR2] & Planar & 0.58 & 25 & 6 & 1 \\
\hline
\end{tabular}

The turbulence intensity decay downstream of a grid is usually expressed as

$$
\left(\frac{1}{\mathrm{Tu}}\right)^{2}=\mathrm{c}_{1}\left(\frac{\mathrm{z}}{\mathrm{M}}-\frac{\mathrm{z}_{0}}{\mathrm{M}}\right)^{\mathrm{c}_{2}}
$$

Typical values of $\mathrm{c}_{1}, \mathrm{z}_{0} / \mathrm{M}$ and $\mathrm{c}_{2}$ are given in Table 2.3. Gad-el-Hak and Corrsin [74GA1] reviewed the earlier literature on this subject and tabulated the values for the constants in the 
equation above. For coarse grids with high porosity, such as typical cooling tower splash grids, the 'apparent origin' is small $\left(\mathrm{z}_{0} / \mathrm{M} \rightarrow 0\right)$ and $\mathrm{c}_{1} \sim 20$. For small $\left(\mathrm{z}_{0} / \mathrm{M}\right)$ values, the exponent in the equation above is approximately 1.3. At a given position behind a grid, Fourie and Kröger [87FO1] found the turbulence intensity to increase with increasing Reynolds number.

Figure 2.6 shows the typical curves of turbulence decay behind a grid with $\left(\mathrm{z}_{0} / \mathrm{M}\right)=0$ and $c_{2}=1.3$. In modern extruded plastic splash packs, the mesh size is in the order of $50 \mathrm{~mm}$ with $\beta$ $\approx 0.8$ and an inter-grid spacing of $200 \mathrm{~mm}$ to $600 \mathrm{~mm}$. Note from Figure 2.6, that for the case of $c_{1}=10$, the free-stream turbulence behind a grid is still above $5 \%$ at $(z / M)=10$ (or at $z=500$ $\mathrm{mm}$ for a grid with $\mathrm{M}=50 \mathrm{~mm}$ ). This implies that in a typical splash pack, the turbulence created by a previous (upstream) grid has not died down before the airflow reaches the second grid.

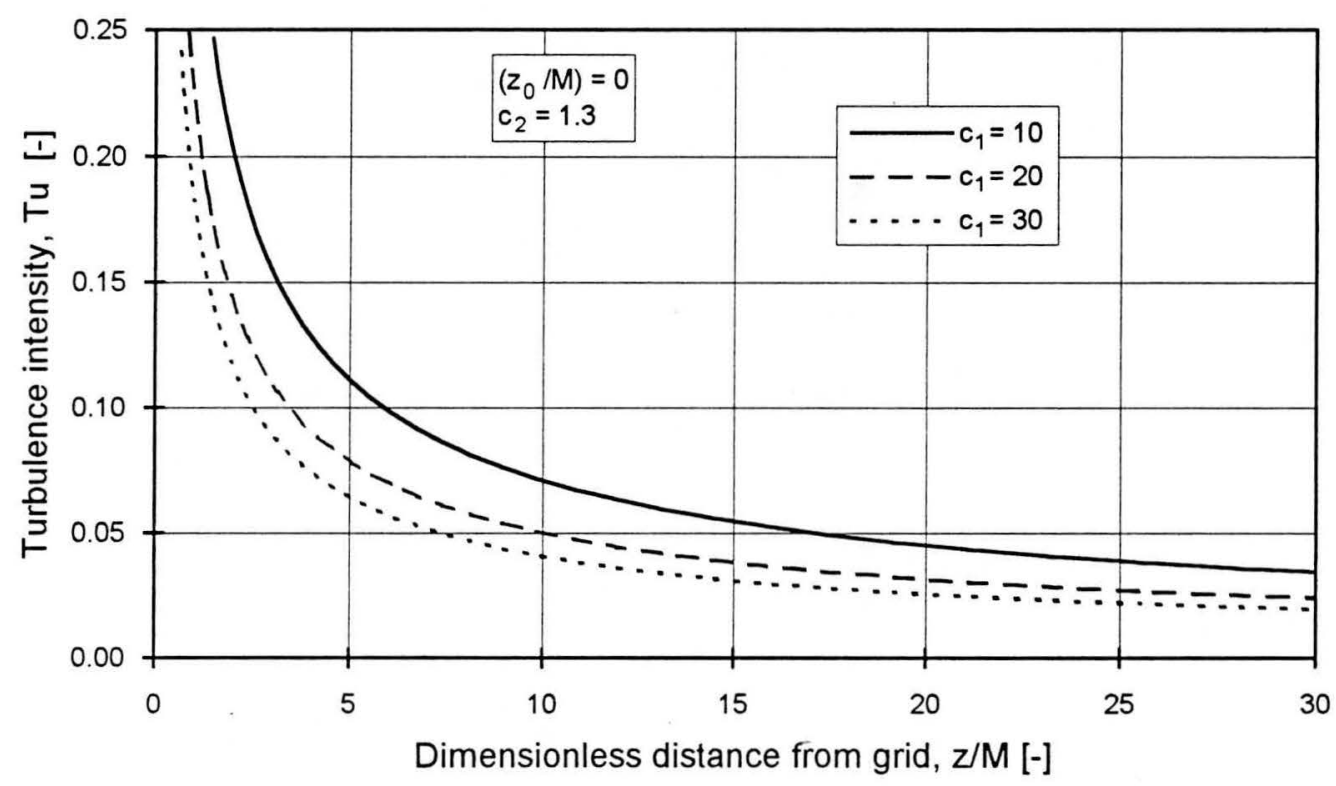

Figure 2.6 Turbulence decay behind a bi-planar grid.

Based on the foregoing discussion, the average turbulence intensity in a splash pack can be expected to be more than $10 \%$, which is high enough to influence the drop drag and the transport of heat and mass from falling drops.

The average turbulence intensity generated by a series of grids and the effect of a polydisperse spray of drops moving through the air simultaneously is beyond the prediction capabilities of any current theory on turbulence generation. This requires further investigation. 


\subsection{Discussion}

After reviewing the literature, the following conclusions can be made:

i) It is not generally possible to model the cooling of a poly-disperse spray with a single representative drop size. The mathematical modelling of the cooling of a poly-disperse spray of drops (of the sizes found in cooling tower splash pack) should be performed by dividing the water mass into different size zones and integrating each zone independently along the direction of the drop motion.

ii) Although empirically determined transfer and pressure drop data was obtained for many different types of splash packing material, it is difficult to draw accurate conclusions from the data since many important parameters such as initial drop size distribution and rain/spray zone depths above and below the packing are not available. The size of the test section used in the experimental work is known to have a strong influence on the measured transfer characteristics and subsequently, the applicability of data obtained in test sections with flow areas smaller than about $1 \mathrm{~m}^{2}$ is limited.

iii) There is uncertainty about the calculation of the drag of accelerating liquid drops due to difficulty in accounting for internal circulation, deformation and drop shape oscillations. It can be concluded that the internal circulation and drop oscillation effects are much less pronounced than the effect of drop deformation on the drag of liquid drops falling in gaseous surroundings.

iv) The heat/mass transfer coefficients experienced by freely falling drops are also expected to be influenced by internal circulation, deformation and drop oscillation. The effects of internal circulation and oscillation results in increased internal mixing of the drop which influences the heat and mass transfer potentials, while drop oscillation and deformation influence the interface surface area and the flow pattern around the drop.

v) There is an abundance of literature on splashing of liquid drops on solid or liquid surfaces, but most of these are limited to drops falling at terminal velocity onto large surfaces uninfluenced by edges. Most of the work in this field was done by researchers investigating soil erosion or the distribution of pathogens on plants by rain.

vi) Drops falling through upward flowing air and the presence of splash grids are expected to increase free-stream turbulence. This could influence aerodynamic drop break-up, noncontact drop interaction effects, drag and heat/mass transfer experienced by drops and the water films on slats.

vii) The literature review uncovered enough information to model the operation of countercurrent cooling tower splash pack from basic principles, but several gaps in the available information had to be investigated in more detail, using experimental work where needed. 


\section{CHAPTER 3 MODELLING APPROACH}

In this chapter the procedure of modelling the performance of cooling tower splash pack is described in general terms without specific reference to the computer algorithms required for simulation purposes. The information available in the literature on the physical processes occurring in cooling tower splash pack is described and shortcomings are highlighted. The algorithms required for the numerical simulation of splash pack and the simulation program are described in Chapter 5.

\subsection{Modelling principles}

In the modelling of the counterflow cooling tower splash pack it is assumed that the Merkel model (Merkel [26ME1]) can be used to describe the heat and mass transfer between the air and the water. This also implies that the change in water flow rate due to evaporation is negligible and that $\mathrm{Le}_{\mathrm{f}}=1$. It is assumed that the drop size distribution and the initial drop velocity at the water inlet side of the packing are known.

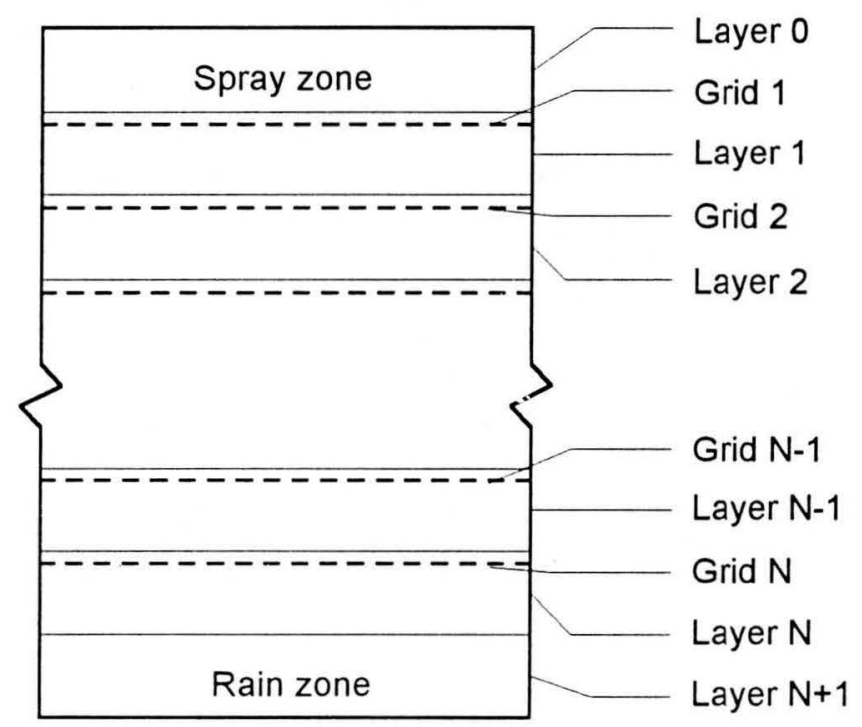

Figure 3.1 Layout of the imaginary integration elements along the packing height.

The packing zone is divided into a number of imaginary elements along its height (see Figure 3.1). It is possible to model the spray zone above the packing and the rain zone below the packing by taking these zones as being a part of the packing zone. For a typical element, the Merkel approach results in the following governing equation for the total heat transfer from the water to the air:

$$
\hat{\partial Q}=\mathrm{K} \partial \mathrm{A}\left(\mathrm{i}_{\text {asw }}-\mathrm{i}_{\mathrm{a}}\right)=\mathrm{Ka} \mathrm{A}_{\mathrm{fr}} \partial \mathrm{z}\left(\mathrm{i}_{\text {asw }}-\mathrm{i}_{\mathrm{a}}\right)
$$

An energy balance gives 


$$
\dot{\partial} \dot{\mathrm{Q}}=\dot{\mathrm{m}}_{\mathrm{da}} \partial \mathrm{i}_{\mathrm{a}}=\dot{\mathrm{m}}_{\mathrm{w}} \mathrm{c}_{\mathrm{pw}} \partial \mathrm{T}_{\mathrm{w}}
$$

These equations describe the cooling of the drops and the enthalpy increase of the air in an element. The integration process starts at the top of the packing zone since the drop size and velocity distributions are known there. By solving the equation of motion in every element for each drop size it is possible to calculate the drop temperature change through the whole packing zone. To actually follow the cooling of every drop would require an excessive number of calculations, but an efficient method of reducing the number of calculations by grouping drops of similar size, temperature and velocity together has been devised. This is described in more detail in Chapter 5 (Computer simulation). Note that the grids change the drop size and velocity distributions and that there is some transfer from the water films covering the grids. Upon reaching the bottom of the packing zone the mass mean drop temperature is used as the water outlet temperature for the calculation of the cooling tower transfer characteristic using a conventional cooling tower integration procedure or the Tchebycheff integration technique.

\subsection{Free fall zone evaluation}

As the drops fall through the open spaces between the horizontal splash grids, the drops experience (i) acceleration downwards due to gravity (note that some of the smaller drops may flow upwards with the airstream), (ii) cooling due to simultaneous heat and mass transfer between the water and the air, (iii) non-contact interaction effects, (iv) collisions with each other and (v) aerodynamic instability which could result in drop break-up. These phenomena are described in more detail below and shortcomings in the available data are highlighted.

\subsubsection{Drop motion}

The velocity at which the drops move through the packing in a cooling tower plays a major role in the energy transfer processes by influencing (i) the drop residence time (which in turn governs the interface area in a given packing volume), (ii) the transfer coefficients and (iii) the splashing process (higher velocity impacts on the slat generally result in larger splash volumes and smaller splash drop sizes). The drag forces on the drops (which area dependant on the drop velocity) together with the pressure loss across the grids constitutes the main parts of the total static pressure loss across the packing.

The motion of a drop in a counterflow cooling tower is governed by the following forces: drag, buoyancy and gravity. The drop acceleration or deceleration can be determined from the nett force balance on the drop using the following relation

$$
\mathrm{m}\left(\frac{\partial \mathrm{v}}{\partial \mathrm{t}}\right)=\mathrm{m}\left(\frac{\left(\rho_{\mathrm{w}}-\rho_{\mathrm{a}}\right) \mathrm{g}}{\rho_{\mathrm{w}}}\right)-0.5 \rho_{\mathrm{a}} \mathrm{v}_{\mathrm{rel}}^{2} \mathrm{~A}_{\mathrm{fr}} \mathrm{C}_{\mathrm{D}}
$$


Drops which flow upwards with the airstream could be: (i) lost in the airstream leaving the cooling tower, (ii) caught by a drift eliminator usually installed above the packing in a counterflow cooling tower, or (iii) they can be intercepted by the other drops falling downwards through the packing.

If drops are assumed to be solid spheres, the correlation by Turton and Levenspiel [86TU1] can be used to calculate the drag coefficient on every drop. This approach is known to overpredict the terminal velocities of large water drops (see Figure 2.3). A better model for calculating the drag coefficients experienced by liquid drops should account for the effects of drop deformation, oscillation and internal circulation. The effect of acceleration on the drag coefficients of solid spheres and liquid drops is not certain, as was noted in the literature review. These uncertainties required more investigation.

Assuming that drop deformation is the main reason for the increased drag experienced by liquid drops, it follows that

$$
\frac{C_{D}}{C_{D, \text { sphere }}}=f(E)
$$

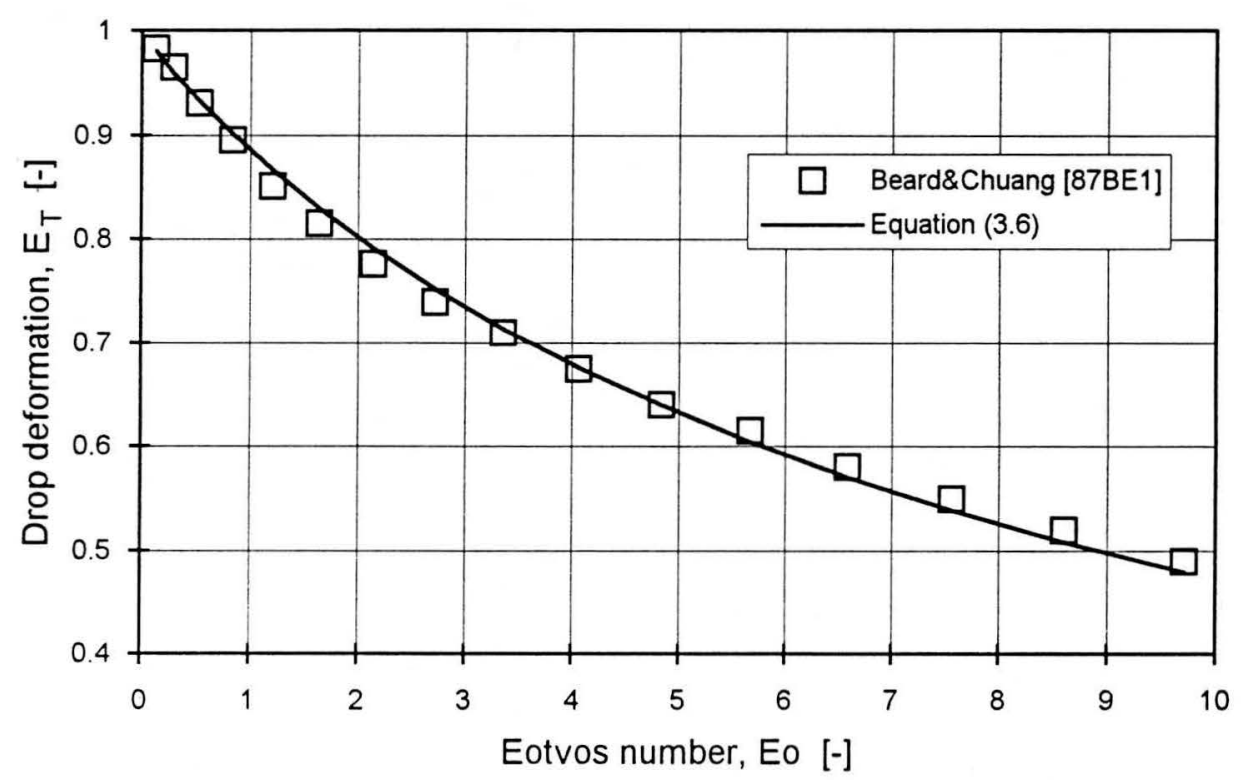

Figure 3.2 Drop deformation at terminal velocity versus Eotvos number.

Once the functional relation in Equation (3.4) is known, the drop deformation at any instant is required in order to calculate the drag experienced by an accelerating drop. Assuming that (i) drop deformation is a function of the square of velocity, (ii) the drop has a spherical shape at 
zero velocity and that, (iii) the drop deformation at terminal velocity is $\mathrm{E}_{\mathrm{T}}$, the deformation of an accelerating drop can be approximated as follows

$$
\mathrm{E}=1-\left(\frac{\mathrm{v}}{\mathrm{v}_{\mathrm{T}}}\right)^{2}\left(1-\mathrm{E}_{\mathrm{T}}\right)
$$

The drop deformation data by Beard and Chuang [87BE1] was correlated as (see Figure 3.2)

$$
\mathrm{E}_{\mathrm{T}}=\frac{1}{1+0.148 \mathrm{Eo}^{0.85}} \text { for all Eo }>0
$$

By using drop deformation data together with experimentally determined drop drag data, it is possible to find the relation between solid sphere and drop drag as a function of drop deformation. Using the relation for drop deformation at terminal velocity, the drop terminal velocity data of Gunn and Kinzer [49GU1] and the standard drag coefficient curve for solid spheres by Turton and Levenspiel [86TU1] the following correlation was obtained

$$
\left(\frac{C_{D}}{C_{D, \text { sphere }}}\right)=1.0-0.17185\left(1-E_{T}\right)+6.692\left(1-E_{T}\right)^{2}-6.605\left(1-E_{T}\right)^{3}
$$

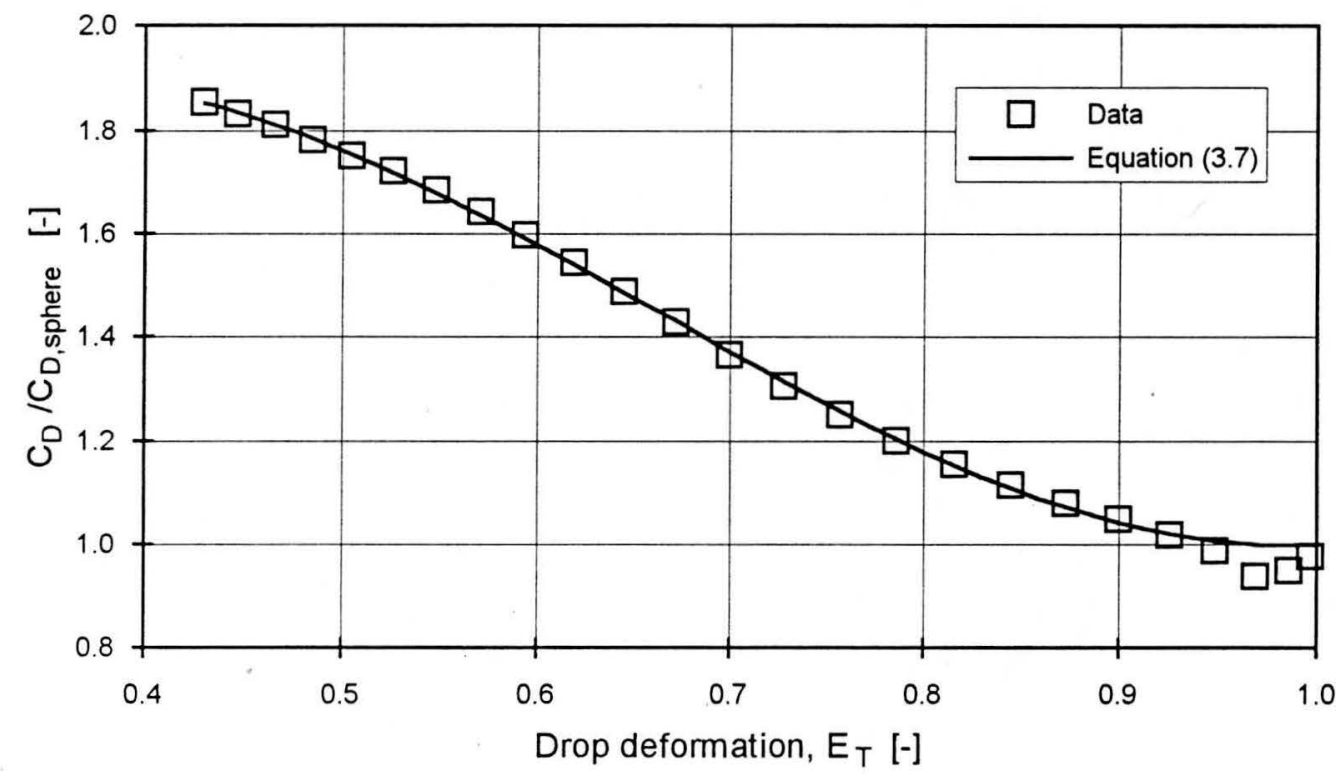

Figure 3.3 Variation of drop drag coefficient with drop deformation for drops travelling at terminal velocity.

Figure 3.3 shows the correlation above together with the data on which it was based. Note that the drop drag coefficient in the correlation above is based on the actual frontal area of the deformed drop $\left(d_{f r}=d_{e} E^{-(1 / 3)}\right)$ and that the drop Reynolds number is based on the equivalent 
spherical drop diameter. For undeformed drops, this correlation predicts the same drag coefficient as for a solid sphere. The discrepancy in data at deformation ratios close to unity could be attributed to internal circulation in the drops. In this deformation range, the correlation overpredicts the drag coefficient, which is expected to yield lower terminal velocities. It can be seen from Figure 2.3 that the drop terminal velocity data of Gunn and Kinzer [49GU1] fall slightly above the terminal velocity curve for a solid sphere at small drop diameters. This is due to the low drop drag found by Gunn and Kinzer at very small deformations.

Assuming that Equation (3.7) holds for drops travelling at velocities below terminal velocity, it can be used together with Equation (3.5) to predict the acceleration of deformable water drops.

\subsubsection{Heat and mass transfer from drops}

Any one of the correlations discussed in Chapter 2 can be used to calculate the average Nusselt number describing the transfer of heat and mass from any drop in every element. The mass transfer coefficient required to calculate the heat transfer rate from a drop due to heat and mass transfer (using Equation (3.1)) can be found from Nusselt number and the analogy between heat and mass transfer as follows

$$
K=\frac{h_{c}}{L e_{f} c_{p m}}=\frac{N_{f} k_{a f}}{L e_{f} c_{p m} d}
$$

The correlations by Frössling [38FR1] and Ranz and Marshall [52RA1], [52RA2] are often used to calculate heat/mass transfer from liquid drops moving in gaseous surroundings. These correlations were both obtained by measuring the heat/mass transfer from pendant drops in a steady airstream. Since acceleration influences the drag experienced by a falling drop, it can be expected that acceleration will also influence the heat/mass transfer from/to a drop. In the case of large drops accelerating in air, Yao [74YA1] and Yao and Schrock [76YA1] proposed the following correlation for the heat transfer from/to a drop

$$
\mathrm{Nu}_{\mathrm{f}}=2+\mathrm{g}_{\mathrm{YS}}\left(0.6 \operatorname{Re}_{\mathrm{f}}^{1 / 2} \operatorname{Pr}_{\mathrm{f}}^{1 / 3}\right) \text { for } \operatorname{Re}_{\mathrm{f}}<2500
$$

with

$$
g_{Y S}=25\left(\frac{z}{d}\right)^{-0.7} \text { for } 10<\left(\frac{z}{d}\right)<600
$$

The data of Yao [74YAl] was re-correlated (see Appendix A), using the drag model described above. The following correlations were obtained 


$$
g_{Y S}=2.32\left(\frac{z}{d}\right)^{-0.16} \text { for } 10<\left(\frac{z}{d}\right)<600
$$

or alternatively

$$
g_{Y S}=0.22+3.15 M_{\ddot{z}}^{0.2}\left(\frac{d}{d_{m}}\right)^{0.2} \text { for } M_{\ddot{z}}>5.0 \times 10^{-4}
$$

The maximum stable drop size is given by

$$
d_{m}=\sqrt{\frac{16 \sigma}{g\left(\rho_{w}-\rho_{a}\right)}}
$$

and the acceleration modulus is defined by

$$
M_{\ddot{z}}=\left(\frac{(\partial v / \partial t) d}{v^{2}}\right)
$$

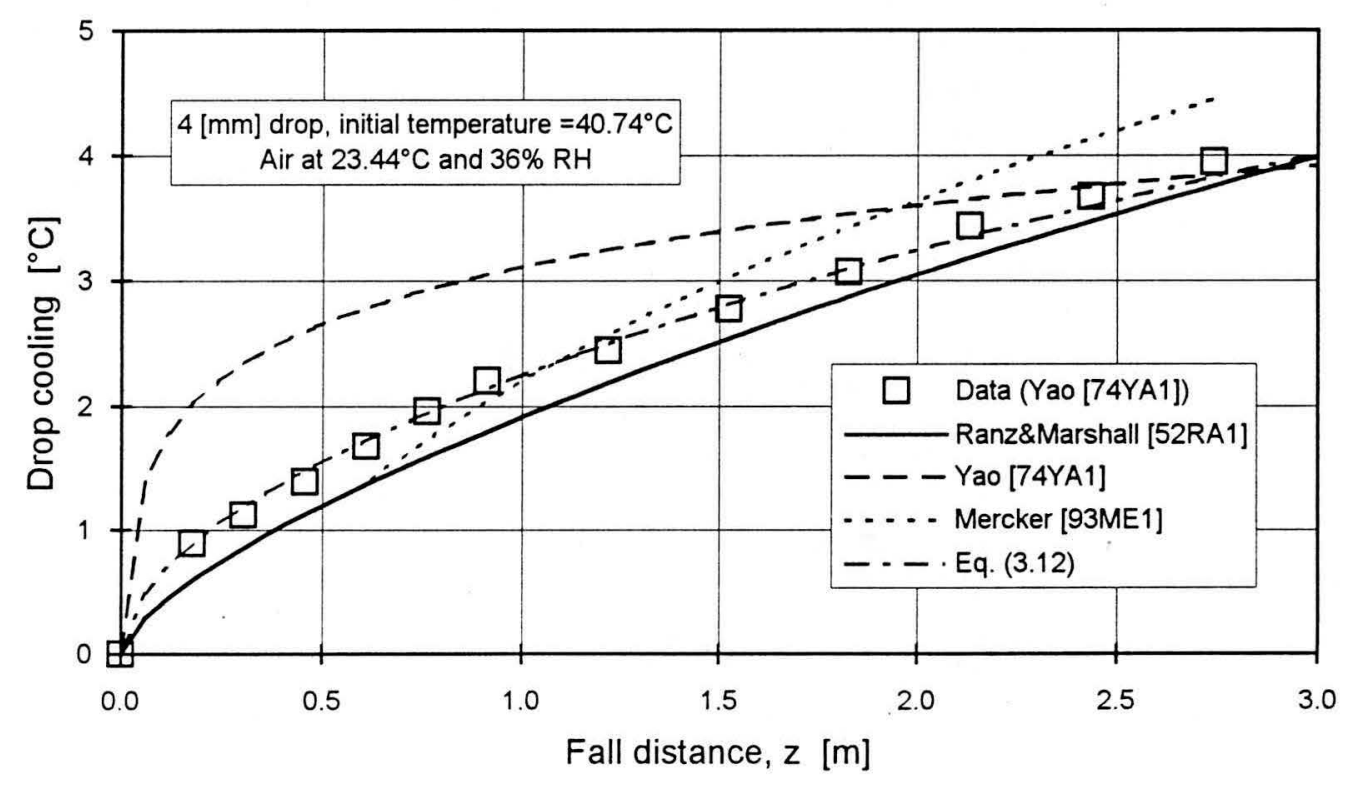

Figure 3.4 Comparison between the different models for predicting the heat/mass transfer from an accelerating drop and the data of Yao [74YA1].

The drop temperature variation predicted by the original correlation by Yao [74YAl], Equation (3.10), is compared to the data by Yao [74YAl] for a $4 \mathrm{~mm}$ water drop falling in still air with $\mathrm{T}_{w i}=40.74^{\circ} \mathrm{C}, \mathrm{RH}=36 \%$ and $\mathrm{T}_{\mathrm{adb}}=22.44^{\circ} \mathrm{C}$ in Figure 3.4. The predictions obtained by using the Ranz and Marshall correlation (Equation (3.9) with $g_{Y S}=1$ ), the new correlation based on the data by Yao, Equation (3.12), and the correlation by Mercker [93ME1], Equation (2.28), are also shown in Figure 3.4. It can be noted that the correlation 
by Yao differs significantly from the data, while the predictions based on Equation (3.12) and the correlation by Mercker [93ME1] agrees fairly well with the data of Yao [74YA1].

The correlations by Ranz and Marshall [52RA1], Yao [74YA1], Yao and Schrock [76YA1] and Mercker [93ME1] are assumed to be accurate enough for the purpose of this study. The effects of free-stream turbulence and non-contact drop interaction effects are expected to influence the heat and mass transfer coefficients governing the energy transfer between the air and water drops in a cooling tower. These effects have been discussed in more detail in Chapter 2.

\subsubsection{Aerodynamic drop break-up}

Large water drops accelerating in air, become unstable due to the aerodynamic forces acting on each drop. The aerodynamic break-up of large liquid drops moving in a gaseous environment has been studied exhaustively and, subsequently, it was concluded that as far as this study is concerned, this phenomenon did not warrant any further experimental work.

The model proposed by Wierzba [90WI1] was selected to model the aerodynamic break-up of large drops in the simulation of cooling tower splash pack. Wierzba found that a drop becomes unstable if the drop Weber number, $\rho_{\mathrm{a}} \mathrm{v}^{2} \mathrm{~d} / \sigma$, is greater than 11 . This can be incorporated into the model to predict the performance of splash pack by checking the Weber number of every drop in the model and to use some algorithm to describe the drop break-up when the Weber number is larger than the critical Weber number.

\subsubsection{Drop-drop collisions}

Due to the relative velocity between drops falling in a cooling tower, there is a probability of collisions between drops. For every drop in every element of the packing there exists a probability of colliding with the other drops moving around it. Assuming straight drop trajectories, the probability of a collision between a given drop and another drop per unit time and unit volume can be expressed as a function of the relative velocities and the sizes of the drops involved.

Upon collision coalescence, break-up or bouncing occurs. The simple model by Brazier-Smith et al. [72BR1], [73BR1] or the experimentally determined correlations by Low and List [82LO1], [82LO2] can be employed to determine which of these phenomena occur on impact and to describe the sizes of the drops formed during break-up. The effect of drop-drop collisions on the performance of cooling tower splash pack is expected to be of secondary importance and therefore it was assumed that these existing models are more than adequate for use in the cooling tower splash pack simulation. 


\section{$\underline{3.2 .5}$ Pressure drop}

The total static pressure drop across the packing is the sum of the pressure drop across the grids and the pressure drop due to the drag forces on the drops. The total static pressure drop across the packing due to the drop drag can be expressed as

$$
\Delta \mathrm{p}=\frac{\sum_{\mathrm{i}=1}^{\mathrm{N}_{\text {elements }}} \sum_{\mathrm{j}=1}^{\mathrm{N}_{\text {drops }}}\left(\mathrm{F}_{\mathrm{drag}, \mathrm{i}}\right)}{\mathrm{A}_{\mathrm{fr}}}
$$

\subsection{Grid evaluation}

In an effort to simplify the interaction between a drop falling onto a narrow slat, the following assumptions are made: (i) The incoming drops are assumed to travel in straight trajectories. This is justified by the relatively high inertia of the drops compared to the small deflecting drag forces due to the airflow around the grids (see Appendix E). (ii) The water falling through the packing is evenly distributed over the entire flow area.

Upon drop impact on the surface of the grid, the drop may splash if the impact energy is high enough (see Figure 3.5(a)), or the drop may experience a cutting or splitting action if the impact is close to the edge of a slat or if the slat width is small relative to the size of the incoming drop (see Figure 3.5(b)). Only a fraction of the water that impacts the grid is lost due to splashing or cutting, while the rest drips from below the grid as relatively large drops.
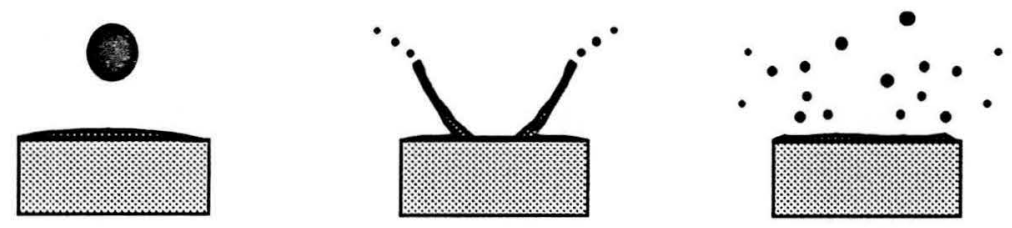

a) Splashing
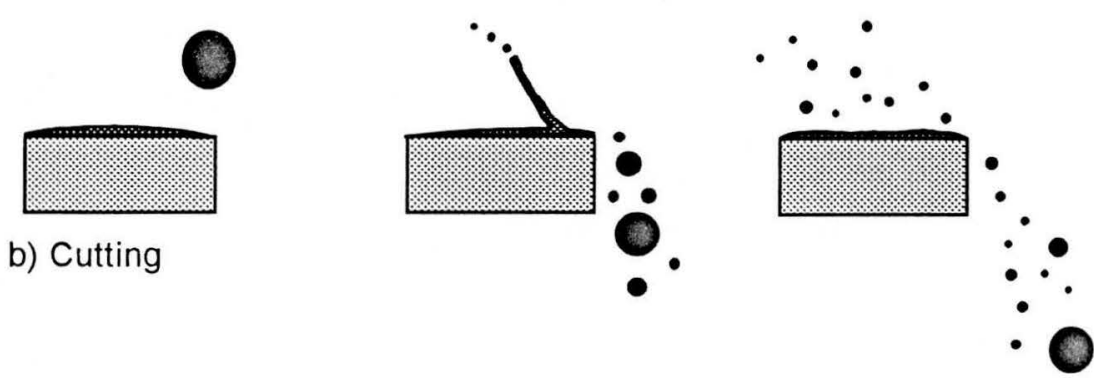

Figure 3.5 Schematic description of the splashing and cutting processes. 


\subsubsection{Splashing and cutting}

The mass of water leaving a grid upon drop impact and the distribution of the drops formed by the impact are expected to be of major importance in predicting the performance of a given splash pack accurately. Very little useful information on this subject is available in the literature. The following crude model was used as a starting point for evaluating the effect of splashing on the modelling of splash pack.

The correlation by Mutchler [70MU1] and Mutchler and Larson [71MU1] (see Chapter 2) can be used to calculate the mass of the liquid leaving the surface due to splashing caused by a drop, travelling at terminal velocity, striking a water film on a large hard smooth surface.

From data by Stedman [79ST1] (see Figure 3.6), it can be seen that the total mass of the splash drops formed by a particular impact is approximately a linear function of the impact velocity. From this observation, the mass of water leaving the point of impact for any drop impact velocity can be estimated from the available data for splash mass for a terminal velocity impact as follows

$$
\mathrm{m}_{\mathrm{s},{ }^{*}} \approx\left(\frac{\mathrm{v}_{\mathrm{i}}}{\mathrm{v}_{\mathrm{i}, \mathrm{T}}}\right) \mathrm{m}_{\mathrm{s},{ }^{*} \mathrm{~T}}
$$

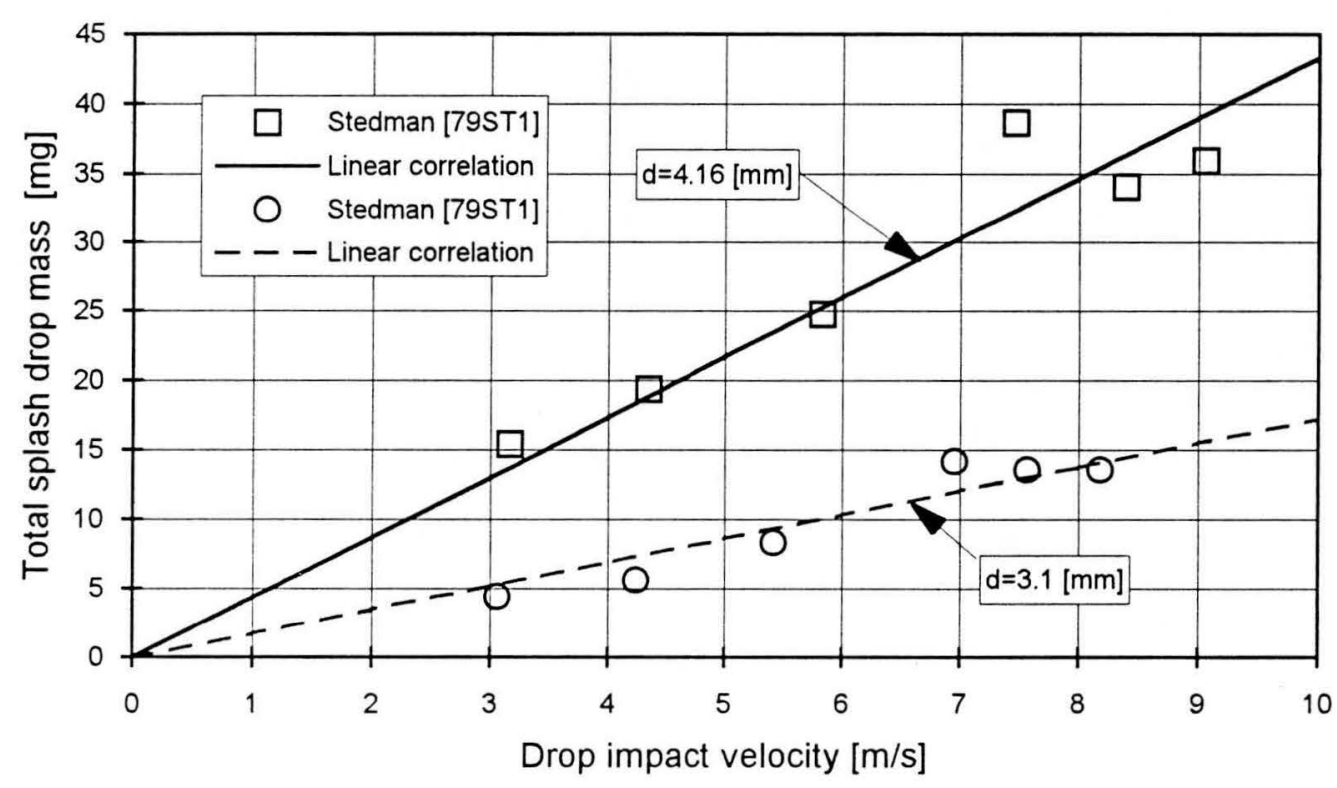

Figure 3.6 Variation of total splash drop mass with drop impact velocity determined by Stedman [79ST1].

Moss [89MO1] found that splashing is strongly influenced by the proximity of an edge near the point of impact of a falling drop. According to Yao et al. [88YA1], drops with an 
incoming Weber number larger than a critical value of $\mathrm{We}_{\text {crit }} \approx 6$, are expected to split on impact with an infinitely thin slat. Based on this, the following two phenomena can be distinguished when a drop strikes a narrow slat (i) splashing and (ii) cutting (or splitting). A drop impacting near the edge of a plate would undergo cutting more readily than a drop impacting further from the edge. Note that a slat can still be impacted by an approaching drop, of which the centre is not in line with the slat, albeit at a glancing angle only; therefore the effective width of the slat would be $\mathrm{W}+\mathrm{d}_{\mathrm{i}}$.

Assuming that the volume of water lost due to cutting is equal to the volume of the incoming drop which is not directly over the slat, as shown in Figure 3.7(a), the mass of water leaving the edge of a wide slat due to cutting can be expressed by

$$
\mathrm{m}_{\mathrm{c}}=\rho_{\mathrm{w}}\left(\frac{\pi \mathrm{b}^{2}}{3}\left(\frac{3 \mathrm{~d}_{\mathrm{i}}}{2}-\mathrm{b}\right)\right)
$$

where

$$
\mathrm{b}=\left(\frac{2 \mathrm{x}-\mathrm{W}}{2}\right)+\left(\frac{\mathrm{d}_{\mathrm{i}}}{2}\right)
$$

which is valid if $\mathrm{W}>\mathrm{d}_{\mathrm{i}}$ and $\mathrm{0}>\mathrm{b}>\mathrm{d}_{\mathrm{i}}$. For a drop grazing the edge of a slat, i.e. $\mathrm{b}=\mathrm{d}_{\mathrm{i}}$ or $\mathrm{x}=\left(\mathrm{W}+\mathrm{d}_{\mathrm{i}}\right) / 2$, the total drop mass will be lost due to cutting. In the case of a drop impacting with its centre on the edge, $x=(\mathrm{W} / 2), 50 \%$ of the drop will be lost due to cutting. No mass will be lost due to cutting for a drop falling at $\mathrm{x}=\left(\mathrm{W}-\mathrm{d}_{\mathrm{i}}\right) / 2$. On a narrow slat, with $\mathrm{W}<\mathrm{d}_{\mathrm{i}}$, it is possible that a given drop is cut by both edges of the slat as shown in Figure 3.7(b).

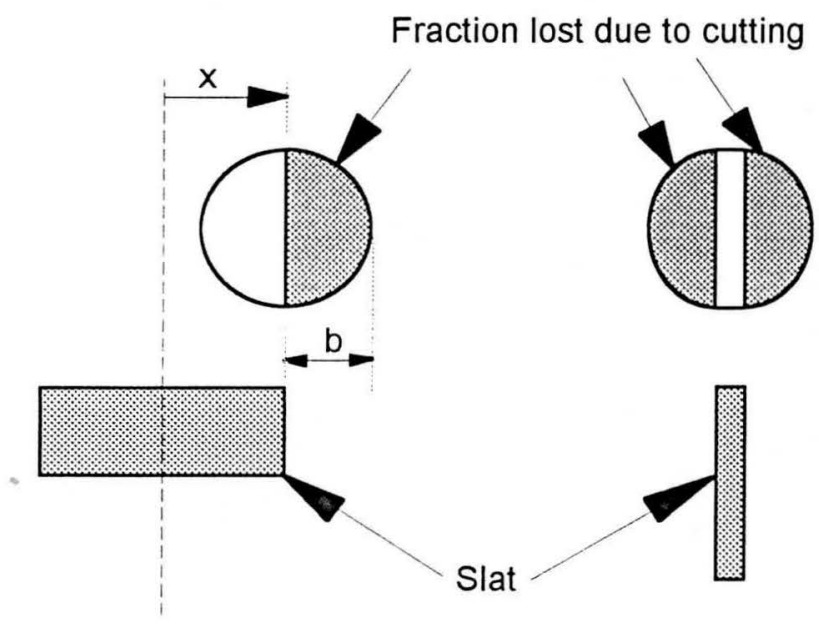

Figure 3.7 Schematic diagram to describe the cutting fraction.

By defining the cutting fraction as the ratio of the mass of water leaving the slat due to cutting to mass of the incoming drop striking the slat at position $\mathrm{x}$, i.e., 


$$
f_{c}(x)=\frac{m_{c}}{m_{i}}
$$

the average volume of water lost over the edges of a slat for a drop striking the slat at any position can be expressed as

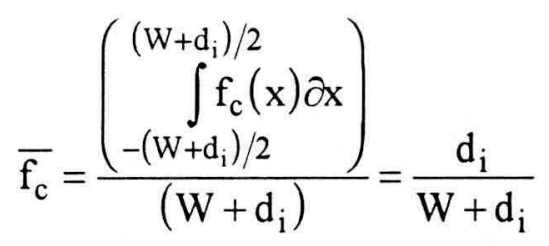

This relation is valid for any combination of drop diameter, $\mathrm{d}_{\mathrm{i}}$, and slat width, W. Figure 3.8 shows typical values for the average cutting fraction for different combinations of drop size and slat width. Note that the average cutting fraction, $\overline{f_{c}}$, approaches unity for impacts on a narrow slat since most impacts are effected by the proximity of the edges of the slat.

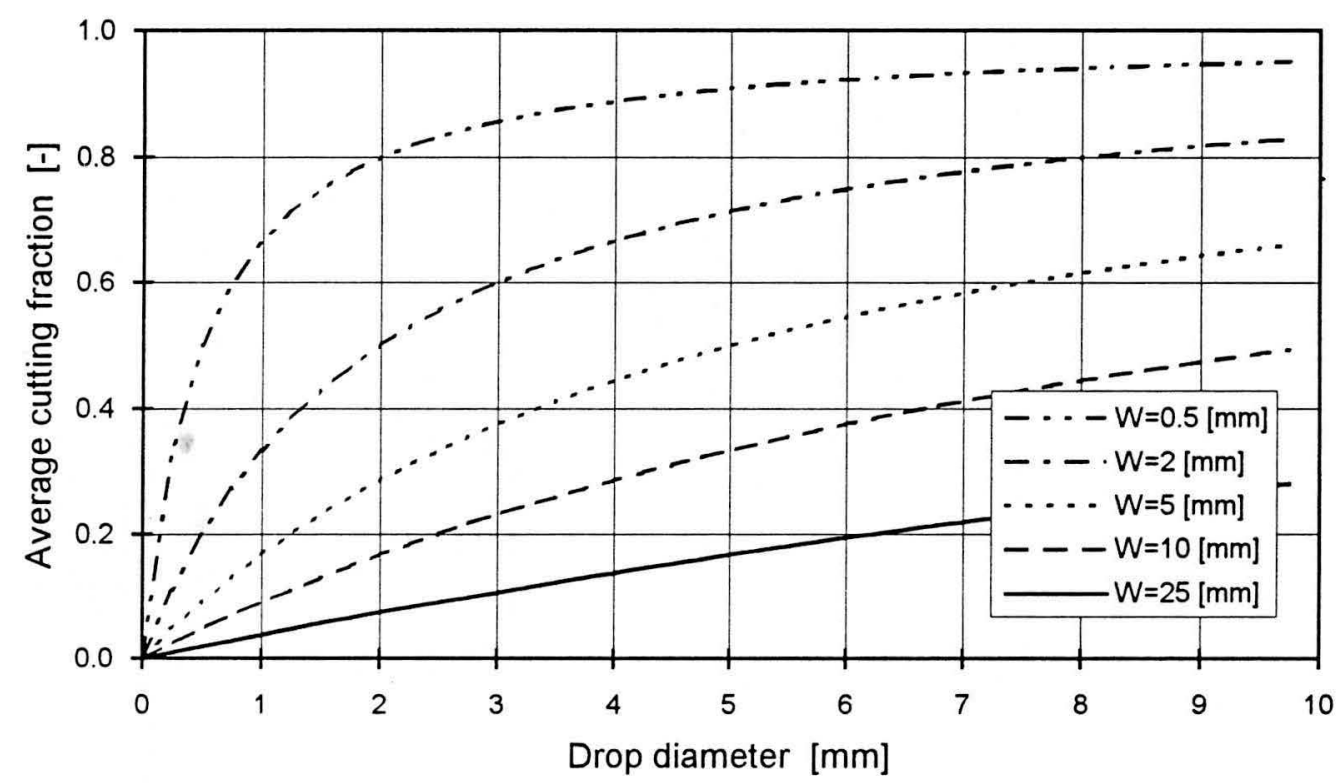

Figure 3.8 Average cutting fraction for various combinations of slat width and drop diameter as predicted by Equation (3.20).

The volume of water lost due to splashing can be described by the splash fraction, which is defined as the ratio of the mass of water leaving the slat due to the splashing phenomenon to the mass of the incoming water drop, i.e.

$$
f_{s}(x)=\frac{m_{s}}{m_{i}}
$$

Splash fractions larger than $100 \%$ imply that more liquid leaves the impact point as splash drops than the mass of the incoming drop. A drop striking a slat at $x=\left(W+d_{i}\right) / 2$ will not 
cause any splashing since all of the drop mass will be lost over the edge due to cutting. It can therefore be assumed that the splash fraction will vary with the position of the drop impact point as shown in Figure 3.9. The average splash fraction will also depend on the incoming drop diameter, drop velocity, slat width and the thickness of the water film on the upper surface of the slat. Due to the lack of suitable data in the literature, the average splash fraction had to be determined experimentally.
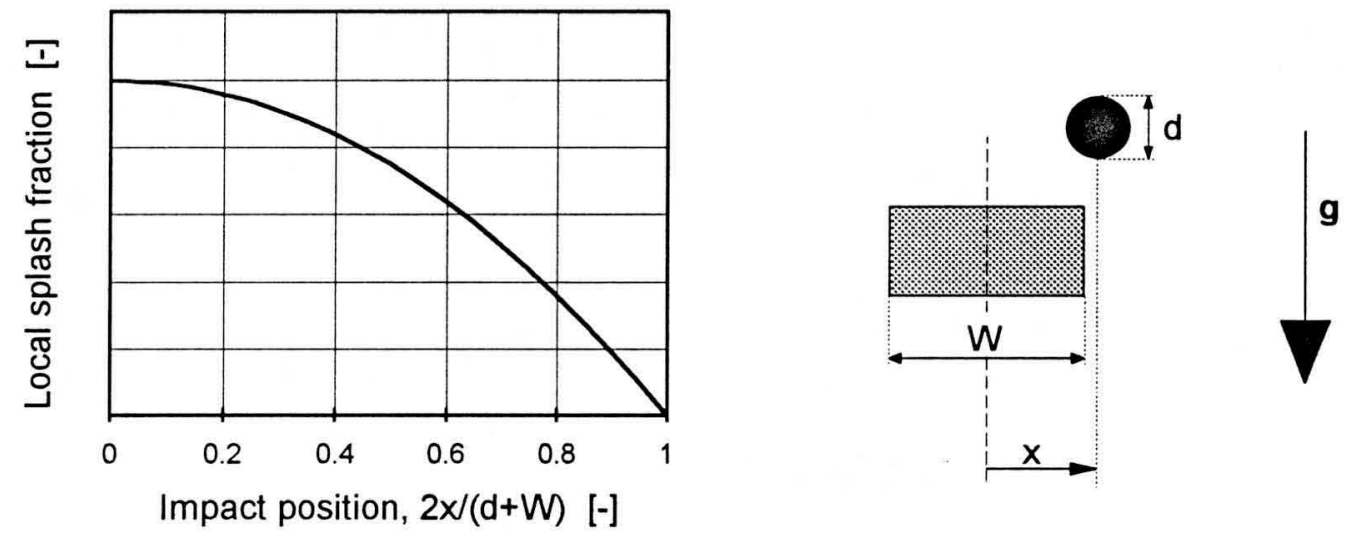

Figure 3.9 Variation of local splash fraction with drop impact position on a slat.

As a rough approximation it can be assumed that the splash fraction varies linearly between

$$
\mathrm{f}_{\mathrm{s}}=0 \text { at } \mathrm{x}=\left(\frac{\mathrm{W}+\mathrm{d}_{\mathrm{i}}}{2}\right)
$$

and

$$
\mathrm{f}_{\mathrm{s}}=\frac{\mathrm{m}_{\mathrm{s}, *}}{\mathrm{~m}_{\mathrm{i}}} \text { at } \mathrm{x}=0
$$

The average volume of water leaving the slat due to splashing can then be expressed as

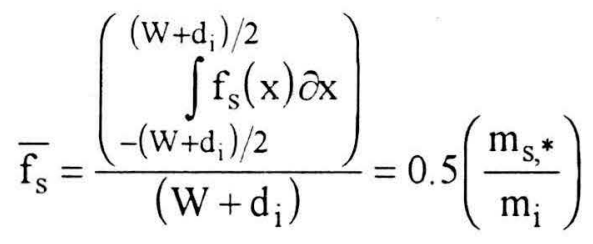

For a given drop impact on a slat, the mass of the drops formed by the splashing and cutting phenomena can be greater than the volume of the impacting drop; the extra water being contributed by the water film present on the slat. It is expected that many of the smaller drops (which could constitute a large fraction of the total water mass) would not result in any splash (or cutting) drops being thrown clear of the slat on impact. These low energy drops striking a slat will contribute to the film of water which is present on the top surface of each slat and to 
the volume of water dripping from below the slat. The mass flow rate of water dripping from below each slat can be obtained from the total mass flow rate of water striking a slat less the mass flow rate lost due to splashing and that lost to cutting. Mathematically this can be expressed as

$$
\dot{\mathrm{m}}_{\mathrm{d}}=\sum_{\mathrm{j}}\left(\dot{\mathrm{M}}_{\mathrm{w}} \mathrm{L}\left(\mathrm{W}+\mathrm{d}_{\mathrm{i}, \mathrm{j}}\right)\right)-\sum_{\mathrm{j}}\left(\overline{\mathrm{f}_{\mathrm{s}, \mathrm{j}}} \dot{\mathrm{M}}_{\mathrm{w}} \mathrm{L}\left(\mathrm{W}+\mathrm{d}_{\mathrm{i}, \mathrm{j}}\right)\right)-\sum_{\mathrm{j}}\left(\overline{\mathrm{f}_{\mathrm{c}, \mathrm{j}}} \dot{\mathrm{M}}_{\mathrm{w}} \mathrm{L}\left(\mathrm{W}+\mathrm{d}_{\mathrm{i}, \mathrm{j}}\right)\right)
$$

According to Mutchler [70MU1], Timmons et al. [71TI1] and Allen [88AL1] the splash drops formed by the splashing of a drop striking a thin film of liquid covering a smooth hard surface, consists of a mixture of water from the impacting drop and the water film. Mutchler [70MU1] found that up to $50 \%$ of the mass of the splash drops was contributed by the impacting drop when a drop strikes a thin liquid layers on a hard smooth surface at terminal velocity. Figure 3.10 , which is based on the findings of Mutchler, shows the variation of this ratio with drop size for different film thicknesses.

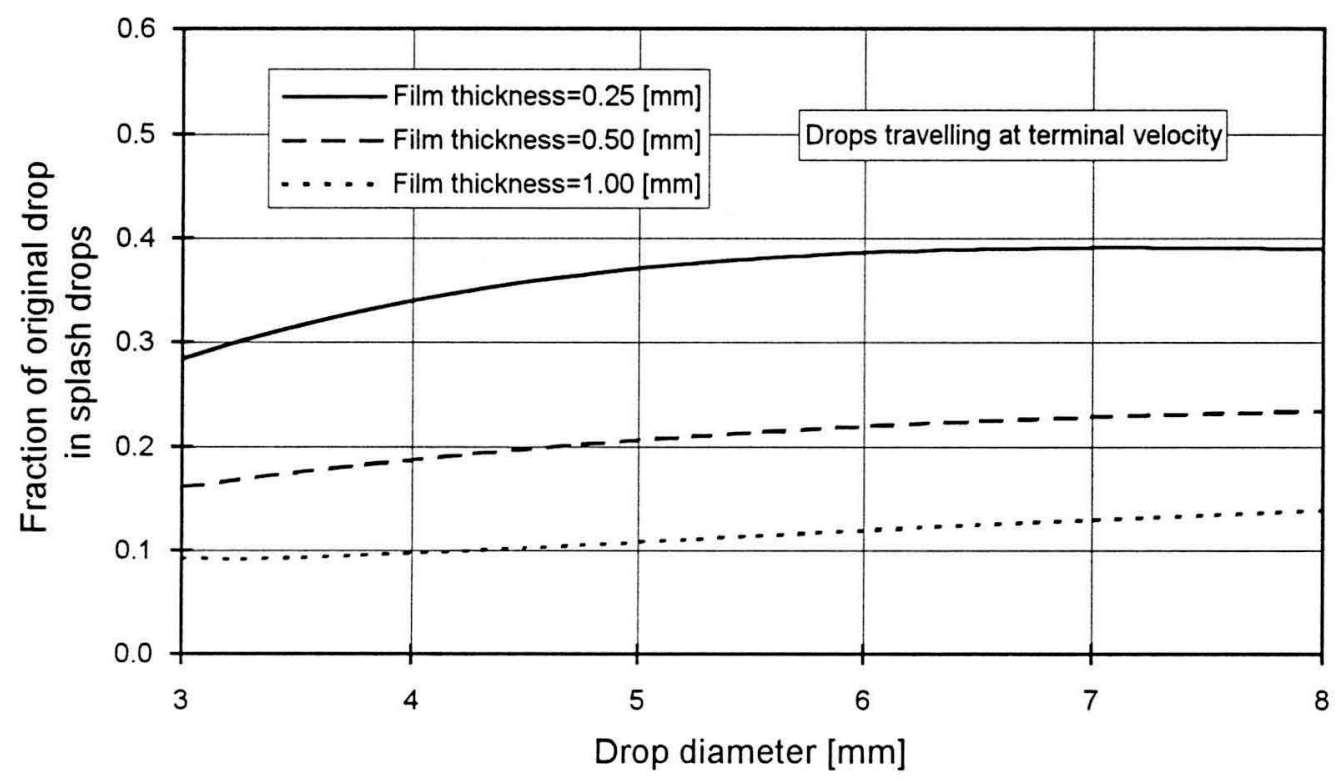

Figure 3.10 Fraction of the original drop mass contained in the splash drops.

As an approximation, it can be assumed that the initial temperature of the splash drops can be expressed as

$$
\mathrm{T}_{\mathrm{S}}=\phi \mathrm{T}_{\mathrm{f}}+(1-\phi) \mathrm{T}_{\mathrm{i}}
$$

The sensitivity of the solution for the chosen value of the mixing ratio, $\phi$, will be investigated in more detail in Chapter 6. 
The temperature of cutting drops are assumed to be the same as that of the incoming drop from which they were formed. The temperature of the water film on the upper surface on the slat can be calculated (iteratively) from the energy balance of an imaginary area around the top part of the slat,

$$
\begin{aligned}
\dot{\mathrm{m}}_{\mathrm{d}} \mathrm{T}_{\mathrm{f}} & =\sum_{\mathrm{j}}\left(\dot{\mathrm{M}}_{\mathrm{w}} \mathrm{L}\left(\mathrm{W}+\mathrm{d}_{\mathrm{i}, \mathrm{j}}\right) \mathrm{T}_{\mathrm{i}, \mathrm{j}}\right) \\
& -\sum_{\mathrm{j}}\left(\overline{\mathrm{f}_{\mathrm{c}, \mathrm{j}}} \dot{\mathrm{M}}_{\mathrm{w}} \mathrm{L}\left(\mathrm{W}+\mathrm{d}_{\mathrm{i}, \mathrm{j}}\right) \mathrm{T}_{\mathrm{i}, \mathrm{j}}\right)-\sum_{\mathrm{j}}\left(\overline{\mathrm{f}_{\mathrm{s}, j}} \dot{\mathrm{M}}_{\mathrm{w}} \mathrm{L}\left(\mathrm{W}+\mathrm{d}_{\mathrm{i}, \mathrm{j}}\right) \mathrm{T}_{\mathrm{s}, \mathrm{j}}\right)
\end{aligned}
$$

If a drop of diameter, $d$, is cut into two equally sized drops, each of the two resulting drops will have a diameter of approximately $0.79 \mathrm{~d}_{\mathrm{i}}$. For any incoming drop, with $\mathrm{d}_{\mathrm{i}}<\mathrm{W}$, striking a slat close to the edge it can be shown that the mean drop size of the drop formed by the part of the incoming drop lost over the edge can be expressed as

$$
\begin{aligned}
\mathrm{d}_{\mathrm{c}} & =\sqrt[3]{\frac{1}{2}\left(\frac{6}{\pi}\right)\left(\frac{\pi \mathrm{d}_{\mathrm{i}}^{3}}{6}\right)} \\
& \approx 0.79 \mathrm{~d}_{\mathrm{i}}
\end{aligned}
$$

Assuming that a drop striking a thin edge, with $\mathrm{d}_{\mathrm{i}}>\mathrm{W}$, will be cut into two parts, corresponding to parts A and B in Figure 3.11, it can be shown that the volume of each of the two parts can be calculated from

$$
\mathrm{V}=\frac{\pi \mathrm{b}^{2}}{3}\left(\frac{3 \mathrm{~d}_{\mathrm{i}}}{2}-\mathrm{b}\right)
$$

where $\mathrm{b}$ is defined in Figure 3.11.

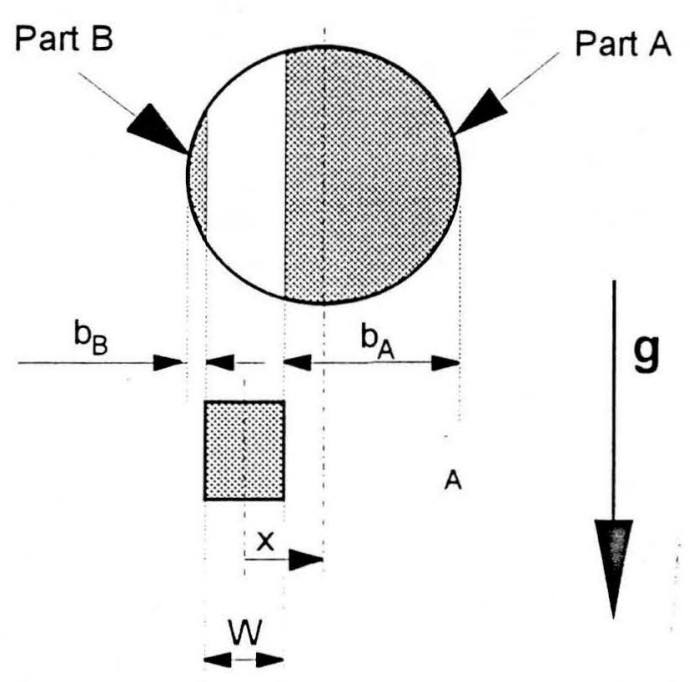

Figure 3.11 Diagram to describe the size of drops formed by cutting. 
For any drop impact between $\mathrm{x}=0$ and $\mathrm{x}=\left(\mathrm{W}+\mathrm{d}_{\mathrm{i}}\right) / 2$ the average volume of parts $\mathrm{A}$ and $\mathrm{B}$ can be obtained from (see Equation (3.18))

$$
\begin{aligned}
\overline{\mathrm{b}_{\mathrm{A}}} & =\mathrm{b}_{\mathrm{A}}\left(\frac{1}{2}\left(\frac{\mathrm{W}+\mathrm{d}_{\mathrm{i}}}{2}\right)\right) \\
& =\left(\frac{3 \mathrm{~d}_{\mathrm{i}}}{4}\right)-\left(\frac{\mathrm{W}}{4}\right)
\end{aligned}
$$

and

$$
\begin{aligned}
\overline{b_{B}} & =b_{B}\left(\frac{1}{2}\left(\frac{d_{i}-W}{2}\right)\right) \\
& =\left(\frac{d_{i}}{4}\right)-\left(\frac{W}{4}\right)
\end{aligned}
$$

From the graphical size distributions given by Yao et al. [88YA1], the drops formed by cutting can be roughly assumed to be normally distributed (based on drop mass) with the mean drop size given by

$$
\frac{\mathrm{d}_{\mathrm{c}}}{\mathrm{d}_{\mathrm{i}}} \approx 0.85
$$

and a standard deviation of approximately $0.16 \mathrm{~mm}$ (for $1.0<\mathrm{d}_{\mathrm{i}}<1.52 \mathrm{~mm}$ ). This is in rough agreement with the simplified model proposed above. Yao et al. [88YA1] found that there is some reduction in mean drop size with increasing Weber number which cannot be accounted for by the simplified cutting model described above.

As discussed in Chapter 2, most of the previous research regarding the distribution of splash drops after the impact of a large drop on a wet surface have concentrated on incoming drops travelling at terminal velocity and the size distributions were usually expressed as number distributions. Very little data is available for drop impacts at lower velocities and for impacts near edges of the impact targets. The normal, log-normal and Weibull (Rosin-Rammler) distribution functions have been used to described the splash drop size distributions. Many of the earlier splash drop number distributions showed a peak at $d_{s}<<1 \mathrm{~mm}$ and often the results were only shown for splash drop sizes up to $1 \mathrm{~mm}$. A single $1 \mathrm{~mm}$ drop has the same mass as 1000 drops of $d_{s}=0.1 \mathrm{~mm}$. In this study the mass (or volume) distribution of the splash drops is more important than the number distribution and some doubt exists regarding the degree to which some of the earlier number distributions can be extrapolated to the larger drops sizes. The distribution of splash drops and drops formed by cutting after a drop impact near the edge of a surface covered by a thin water film required more investigation. 
The initial velocity of the drops formed by cutting is expected to be approximately the same as the velocity of the incoming drop. The initial velocity of the drops formed by splashing are described in more detail below.

Due to the general lack of data describing the sizes of drops formed by the splashing and cutting of water drops on narrow slats and the importance of these phenomena on the performance of splash packing material, this required extensive experimental study.

\subsubsection{Interference between adjacent impacts}

Due to the high rain densities occurring in cooling towers, some interference between adjacent impacts (splashes) can be expected. Assuming a mono-disperse drop distribution, the time between drop impacts at a given position can be estimated from the size of a typical splash crown and the rain density in the cooling tower as follows

$$
\mathrm{t}_{\mathrm{i}} \approx \frac{\rho_{\mathrm{w}}(\pi / 6) \mathrm{d}_{\mathrm{i}}^{3}}{\dot{\mathrm{M}}_{\mathrm{w}}(\pi / 4) \mathrm{W}_{\text {crown }}^{2}}
$$

Mutchler [70MU1] gave the following correlation to describe the width of the crown formed by the impact of a drop, travelling at terminal velocity, striking a solid surface covered by a thin liquid film

$$
\frac{\mathrm{W}_{\text {crown }}}{\mathrm{d}_{\mathrm{i}}}=5.0+3.7 \exp \left(-1.3 \frac{\delta}{\mathrm{d}_{\mathrm{i}}}\right)-4.5 \exp \left(-4.5 \frac{\delta}{\mathrm{d}_{\mathrm{i}}}\right)
$$

Mutchler also gave the following correlation to describe the time from drop impact to the maximum crown size

$$
\frac{\mathrm{t}_{\max \text { crown size }}}{\mathrm{d}_{\mathrm{i}}^{2}}=0.05+0.25 \exp \left(-4.8 \frac{\delta}{\mathrm{d}_{\mathrm{i}}}\right)-0.3 \exp \left(-6.6 \frac{\delta}{\mathrm{d}_{\mathrm{i}}}\right)
$$

Assuming that the crown lifetime is approximately twice as long as the time required for maximum crown size, the interference ratio can be defined as the ratio of crown lifetime to the average time between drop impacts, i.e.

$$
I=\frac{2 t_{\max \text { crown size }}}{t_{i}}
$$

An interference ratio of larger than unity implies that on average every splash crown will be influenced by adjacent splash crowns and vice versa. Figure 3.12 shows the variation of the 
interference ratio with drop size based on the discussion above for a rain density of 10000 $\mathrm{kg} / \mathrm{m}^{2} \mathrm{hr}$.

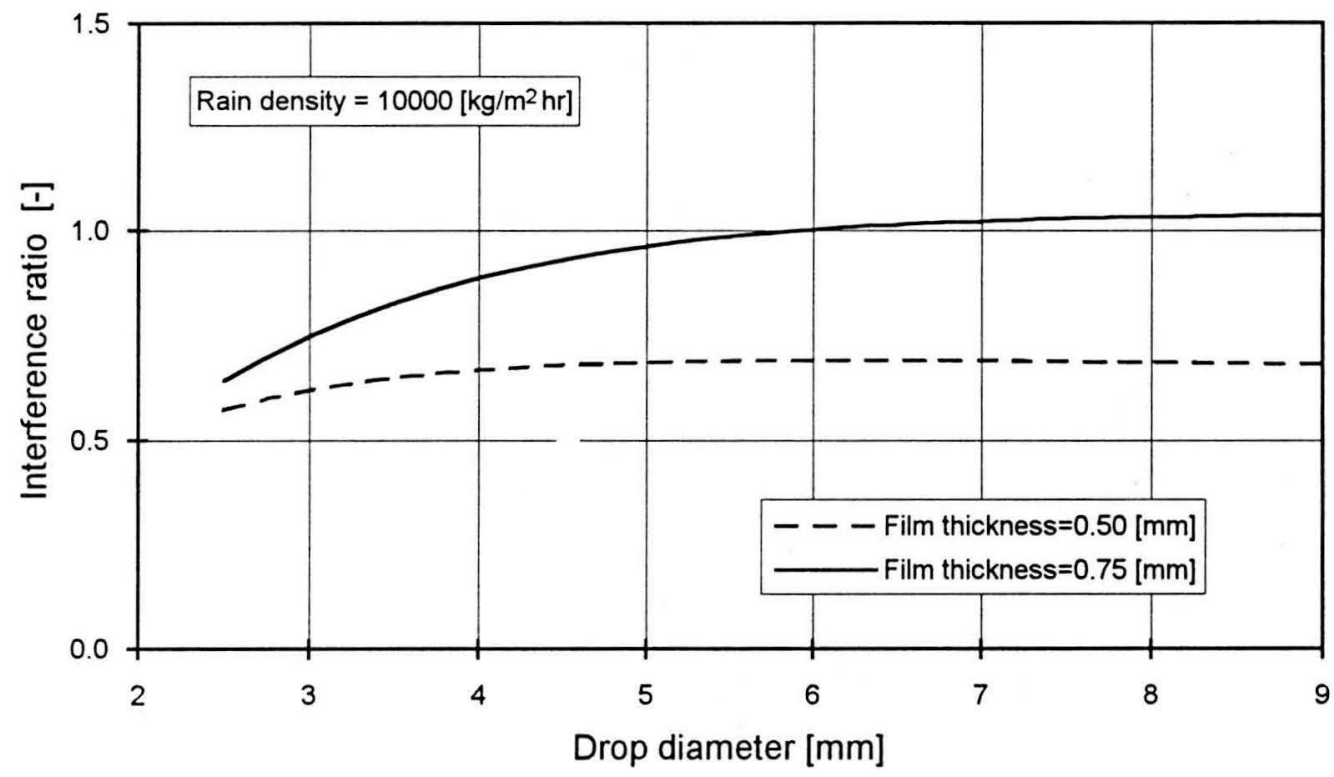

Figure 3.12 Variation of interference ratio with impacting drop size.

In the discussion above, it was assumed that the crown size is the same as the maximum crown size (as predicted by the Mutchler correlation), but the crown size will be smaller than the maximum crown size during the crown growth phase and during the crown break-up stage. If it can be assumed that the crown grows and decays linearly during the crown lifetime, the mean crown size will be $50 \%$ of the maximum crown size and consequently the interference ratio (based on maximum crown size) will be halved. It is expected that the crown lifetime will be shorter for drop impact velocities below terminal velocity, resulting in even lower interference ratios than those shown in Figure 3.12.

It can be concluded that the effect of splash interference could influence the crown formation and splashing phenomena significantly at the rain densities found in cooling towers.

\subsubsection{Cooling of upward moving splash drops}

When a water drop strikes a slat covered by a water film, the resulting splash drops leave the slat at various angles and with different initial velocities. As these drops travel through the air they experience some cooling which should be accounted for in a model attempting to predict the performance of splash packing material. The two-dimensional flight path of each splash drop can be calculated by solving the equation of motion for each drop through a method of numerical integration. Instead of finding the splash drop flight path by a time consuming numerical integration procedure it is possible to describe the drop flight path by analytical 
equations, after making some simplifying assumptions. A set of simplified equations to describe the splash drop motion is derived in Appendix D. The temperature change of each splash drop during its flight can be calculated once the flight path (and flight time) is known.

The following correlation by Mutchler [70MU1] and Mutchler and Hansen [70MU2] gives the splash angle for the splash crown formed by a drop, travelling at terminal velocity, striking a large surface covered by a thin water film:

$$
\theta=80-34 \exp \left(-6.3\left(\frac{\delta}{d_{i}}\right)\right)
$$

This correlation can be used as a rough guide to estimate the splash angles, but for splashes after impact of slower drops or drop impacts near the edges of solid objects, the splash angles of the splash drops had to be determined experimentally.

Ghadiri and Payne [78GH1] measured the initial velocities of splash drops after the impact of large drops travelling at terminal velocities into a thin liquid layer covering a sandy surface. Their data could be correlated as

$$
\mathrm{v}_{\mathrm{si}}=\exp \left(3.8260+0.3054 \mathrm{~d}_{\mathrm{si}}-2.2379 \sqrt{\mathrm{d}_{\mathrm{si}}}-0.09666\left(\frac{\mathrm{d}_{\mathrm{si}}}{\mathrm{KE}_{\mathrm{i}}}\right)^{0.4}\right)
$$

for $3.5<\mathrm{d}_{\mathrm{i}}<6.2 \mathrm{~mm}$ and with $\mathrm{v}_{\mathrm{si}}$ in $\mathrm{m} / \mathrm{s}, \mathrm{d}_{\mathrm{si}}$ in $\mathrm{mm}$ and $\mathrm{KE}_{\mathrm{i}}$ in Joule. The data on the initial velocity and initial splash angle of splash drops are far from adequate and this also required more experimental investigation.

\subsubsection{Dripping below slats}

For water dripping from below a surface at low flow rates, it is normally observed that one large drop (primary drop) and one or more satellite drops are formed as water drips at every dripping point. The size of the primary drop can be described by an equation such as

$$
d_{p}=C \sqrt{\frac{\sigma}{g\left(\rho_{w}-\rho_{a}\right)}}
$$

where the value of $\mathrm{C}$ varies from about 2.7 to 3.3 depending on the geometry of the object from which the drop is falling. Yung et al. [80YU1] found that there were 5 satellite drops ranging in size from $0.24 d_{p}$ to $0.46 d_{p}$ falling behind each primary drop.

At increased flow rates the primary drop size can be expected to decrease while fewer satellites can be expected. The water flow rate and the slat geometry are expected to influence 
the distribution of drop sizes falling from below the slats of splash packing material in a cooling tower, and subsequently this required more investigation.

\subsubsection{Film cooling on grids}

The cooling of the water film spilling over and dripping down from the bottom of the slats can contribute a substantial fraction of the total transfer occurring in the splash pack. The cooling of the film flowing down a slat can be approximated by

$$
\partial \dot{\mathrm{Q}}=\dot{\mathrm{m}}_{\mathrm{w}, \mathrm{film}} \mathrm{c}_{\mathrm{pw}} \partial \mathrm{T}_{\mathrm{w}} \approx \mathrm{KA}_{\text {slat }}\left(\mathrm{i}_{\mathrm{asw}}-\mathrm{i}_{\mathrm{a}}\right)
$$

The correlation by Gnielinski [83GN1], given in Chapter 2, can be used to calculate the forced convection heat transfer from immersed square, rectangular and round profiles. Some doubt existed concerning the applicability of this correlation to predict the heat and mass transfer from non-symmetrical shapes such as T-sections. The applicability of this correlation to such non-symmetrical profiles required experimental verification.

\subsubsection{Pressure drop}

The data by Baines and Peterson [51BA1] and Miller [90MI1] for the pressure loss coefficient for flow through sharp-edged grids could be correlated by

$$
\mathrm{K}_{\Delta \mathrm{p}, \text { grid }}=0.78\left(\left(\frac{1}{\beta}\right)^{2}-1\right)^{1.25}
$$

This correlation predicts significantly lower pressure loss coefficients than the other correlations listed in Chapter 2 (see Figure 2.5). The reason for this discrepancy is not clear and this required further experimental investigation since the pressure drop across the grids contributes significantly to the total pressure drop across splash packing material.

It should be noted that for the relatively rough grids usually employed as splash pack, the screen Reynolds number is usually larger than 80 , which indicates that the grids would contribute to free-stream turbulence. 


\section{CHAPTER 4 EXPERIMENTAL STUDY}

In the previous chapter various uncertainties and shortcomings in the data available in the literature, required for the modelling of cooling tower splash pack, were highlighted, e.g., insufficient data on the acceleration of deformable drops or the shortage of information regarding the effect of an edge on splash drop formation. Many of these uncertainties cannot be modelled analytically and subsequently experimental work was required to clarify these uncertainties. This chapter describes various experiments which were conducted in an attempt to fill the gaps in the available information.

\subsection{Determination of drop acceleration}

Although terminal velocity data for water drops falling in air abound, only one set of data on the acceleration of water drops in still air could be found in the literature (Laws [41LA1]). A simple measuring technique was employed to measure the instantaneous velocity of water drops after different fall heights in still air. The velocities of drops with diameters between 2.5 and $6.1 \mathrm{~mm}$ were measured after fall heights between 0 and $7 \mathrm{~m}$.

A water drop dripping from a nozzle and falling through air, is easily deflected by air currents or by slightly non-symmetrical break-off from the nozzle. Subsequently water drops dripping from a nozzle do not always fall straight downwards, but they are distributed around a point directly below the nozzle. An opto-electronic drop detector was designed to detect a falling water drop crossing a $30 \mathrm{~mm}$ wide, horizontal beam of light (see Prinsloo [92PR1]). Figure 4.1 shows the layout of such a drop detector. By using a small light bulb placed at the focal point of lens it was possible to create a beam of parallel light. Two knife edges were used to reduce the beam of parallel light to a thin, flat beam of light. This beam of light is directed at the open ends of a row of 20 plastic optic fibres. Any drops passing between the lens and the open ends of the optic fibres interrupt the light beam. Water drops act like miniature lenses, bending the light travelling through them; but by ensuring that the drop does not pass within $25 \mathrm{~mm}$ from the ends of the optic fibres, secondary effects due to this can be avoided. The 1 $\mathrm{mm}$ diameter optic fibres were mounted with their centres $1.5 \mathrm{~mm}$ apart in a solid PVC block to ensure that the open end each fibre is aligned with the incoming beam of light. A phototransistor (Motorola MRD701) was glued to the other end of each optic fibre. The signal from each photo-transistor was compensated for the intensity environmental light by subtracting from it the signal from an extra photo-transistor which monitors the light intensity of the environment. This ensured that the detector worked well in a well-lit room as well as in a dark room. The compensated output signal is then passed through a Schmidt trigger to ensure that the signal complies to TTL specifications (required for interfacing with digital electronics and 
personal computers) and to prevent multiple switching between the on/off states when a drop moves past the detector. The digital signal from each of the 20 photo-transistors was fed to a digital input/output card (Eagle Electric PC-14B) mounted in an IBM-compatible personal computer.

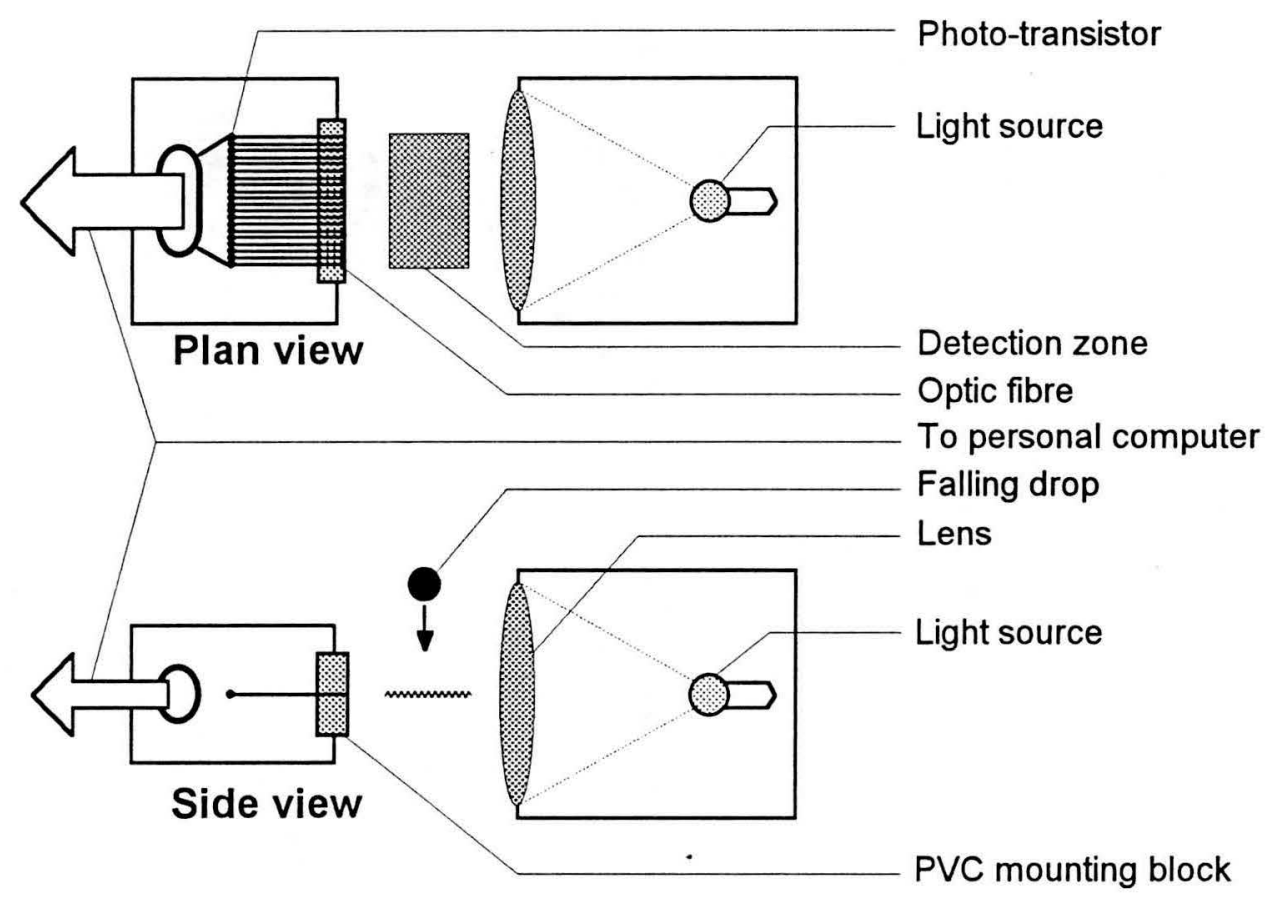

Figure 4.1 Layout of the opto-electronic drop detector used in strobe/flash timing, drop velocity measurement (in which two similar detectors were used) and drop position detection.

Two of these detectors were built and mounted at right angles with their optical planes approximately $50 \mathrm{~mm}$ apart (as shown in Figure 4.2). The time elapsed between the moment when a drop was detected by the upper detector and by the second detector was measured using the $2 \mathrm{MHz}$ oscillator and digital counters on the digital input/output card in the personal computer. By using two drop detectors it was possible to determine the exact location in the horizontal plane (xy) where a drop fell through the two sheets of light. The distance between the two planes was measured at various positions in the horizontal plane (xy) with a vernier height gauge. The elapsed time between detection at the first and second plane together with the known vertical distance between the detection planes (at the given co-ordinates where the drop was detected) allowed the calculation of the average velocity of the drop between the two planes. Different water drop sizes were obtained by dripping the water, from different sizes of ground glass nozzles and stainless steel needles. The drop sizes were determined by measuring the mass of a known number of drops. A Mettler P162 scale with a full scale reading of 160 grams and a 1 milligram resolution was used for these measurements. During the tests the surface tension of the water was measured with the drop weight method 
described in Appendix F. It was found that the surface tension of the water used in these experiments agreed well with the surface tension of pure distilled water.

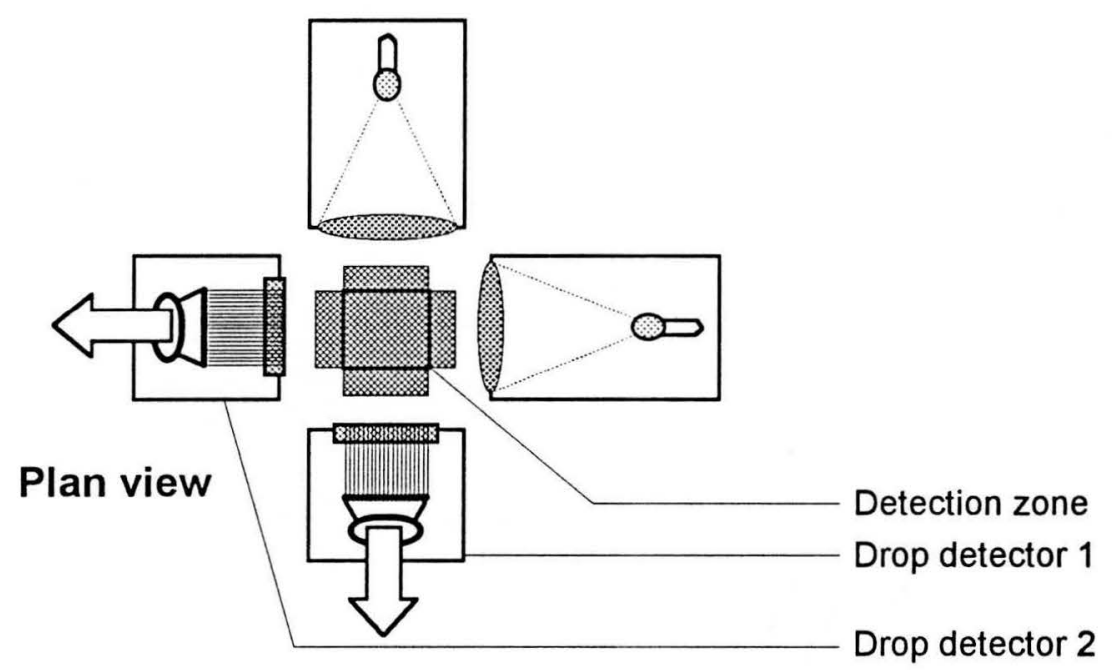

Figure 4.2 Layout of the drop detectors used in velocity measurements. Note that the one drop detector was mounted approximately $50 \mathrm{~mm}$ above the other.

For each combination of fall height and drop size, the local velocity of 10 or more drops were averaged. The atmospheric pressure and air conditions were also measured during these experiments. The data obtained with these experiments is listed in Appendix J.

The time delay measurement is estimated to be accurate to within $5 \mu \mathrm{s}$, while the distance between the two detection planes could be measured to within $0.5 \mathrm{~mm}$. It should be noted that the drops oscillate while they fall and this can influence the velocity measurements. For example, if a drop is prolate when activates the top detector and it is oblate when it passes the second detector, the velocity drop will be over-estimated. The effect of drop oscillation on the velocity measurement will largely be cancelled by the averaging of all the measurements taken for each case. The overall accuracy of the velocity measurements is expected to be within $2 \%$.

The calculation of the drop drag coefficient and the drop deformation was done as follows:

i) For every drop size, a correlation of the following form was determined from the experimental data by a least squares curve fitting procedure

$$
v=c_{1} z+c_{2} z^{1 / 2}
$$

ii) The acceleration of the drop at any position was determined from

$$
\frac{\partial v}{\partial t}=\left(\frac{\partial v}{\partial z}\right)\left(\frac{\partial z}{\partial t}\right)
$$


or

$$
\frac{\partial v}{\partial t}=\left(c_{1}+0.5 c_{2} z^{-1 / 2}\right)\left(c_{1} z+c_{2} z^{1 / 2}\right)
$$

iii) A force balance of the drop at every position, $\mathrm{z}$ yields

$$
C_{D}=\frac{m\left(\left(\left(\rho_{w}-\rho_{a}\right) g / \rho_{w}\right)-(\partial v / \partial t)\right)}{0.5 \rho_{\mathrm{a}} v^{2} A_{f r}}
$$

iv) The drag coefficient of a sphere can be determined at the local Reynolds number from the correlation by Turton and Levenspiel [86TU1].

v) The corresponding drop deformation was obtained from the correlation describing the ratio of drop drag to sphere drag as a function of drop deformation proposed in Chapter 3 , i.e.,

$$
\left(\frac{C_{D}}{C_{D, \text { sphere }}}\right)=1.0-0.17185(1-E)+6.692(1-E)^{2}-6.605(1-E)^{3} \quad \text { for } E>0.4
$$

vi) The terminal velocity of a given drop was determined by using the correlation above together with the correlation for drop deformation at terminal velocity described in Chapter 3, i.e.,

$$
\mathrm{E}_{\mathrm{T}}=\frac{1}{1+0.148 \mathrm{Eo}^{0.85}}
$$

where

$$
\text { Eo }=\frac{g\left(\rho_{w}-\rho_{a}\right) d^{2}}{\sigma}
$$

vii) Repeat steps (i) to (v) for all fall heights.

The following simple relation was proposed in Chapter 3, to describe the change of the drop deformation with increasing velocity

$$
E=1-\left(\frac{v}{v_{T}}\right)^{2}\left(1-E_{T}\right)
$$

The agreement between this relation and the drop deformation data calculated from the experimental data obtained during this study and the data by Laws [41LA1] is shown in Figure 4.3. It can be seen that there is fair agreement between the experimental drop deformation data and that predicted by proposed relation for drop deformation during acceleration. 


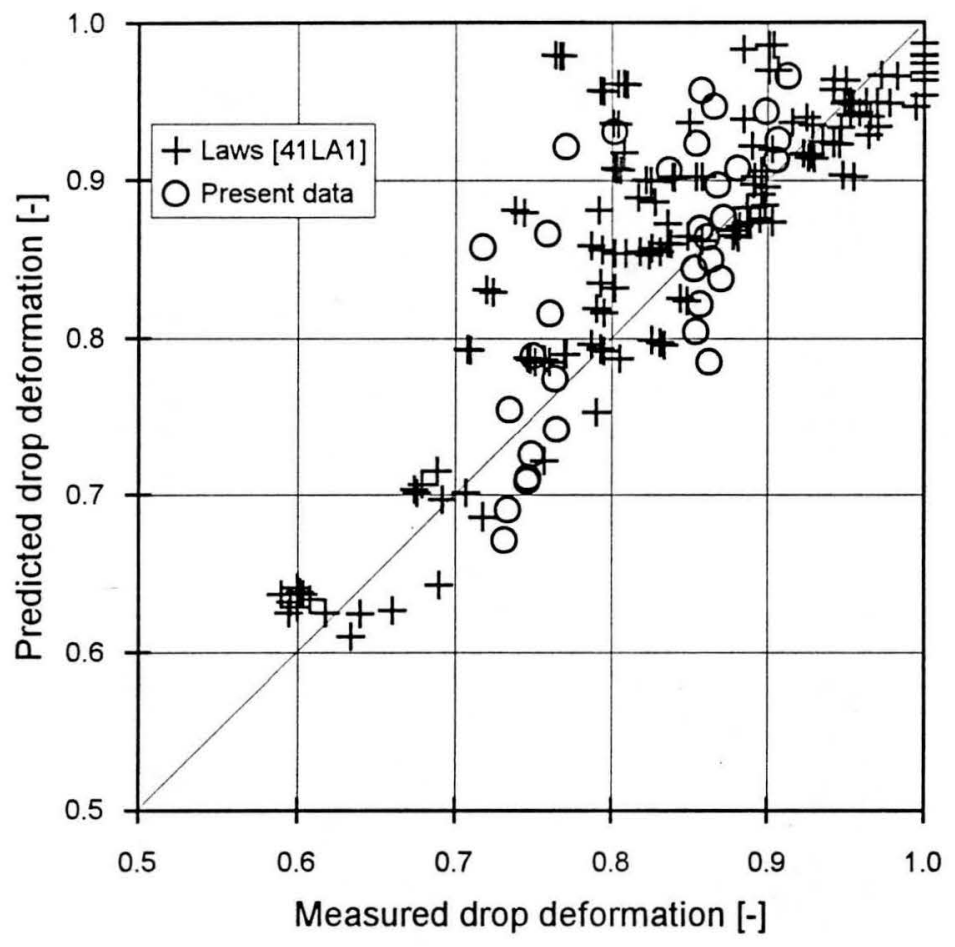

Figure 4.3 Comparison between the drop deformation obtained from the experimental data and the drop deformation predicted by Equation (4.8).

Figure 4.4 shows that near the drop terminal velocity, the correlation for drop deformation, Equation (4.6), agrees well with the drop deformation calculated from the experimental data.

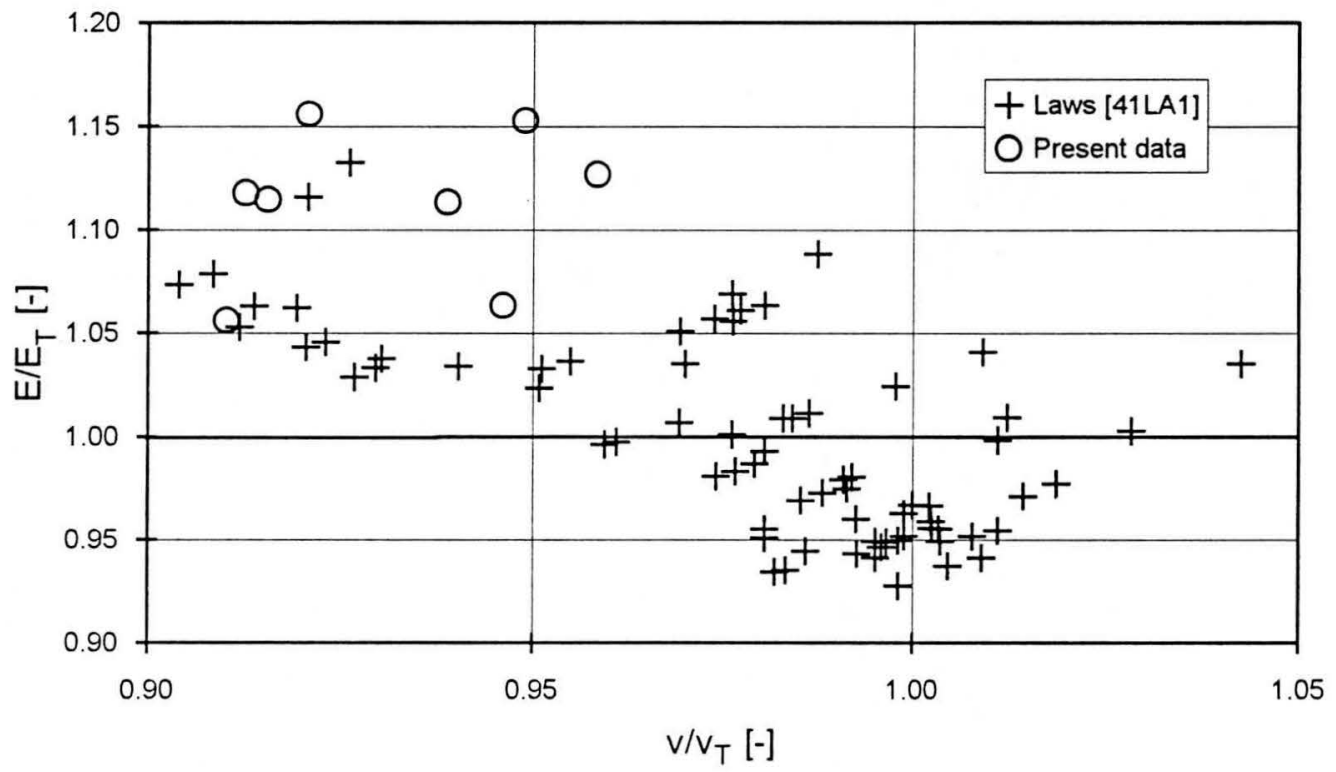

Figure 4.4 Measured versus predicted drop deformation near drop terminal velocity. 


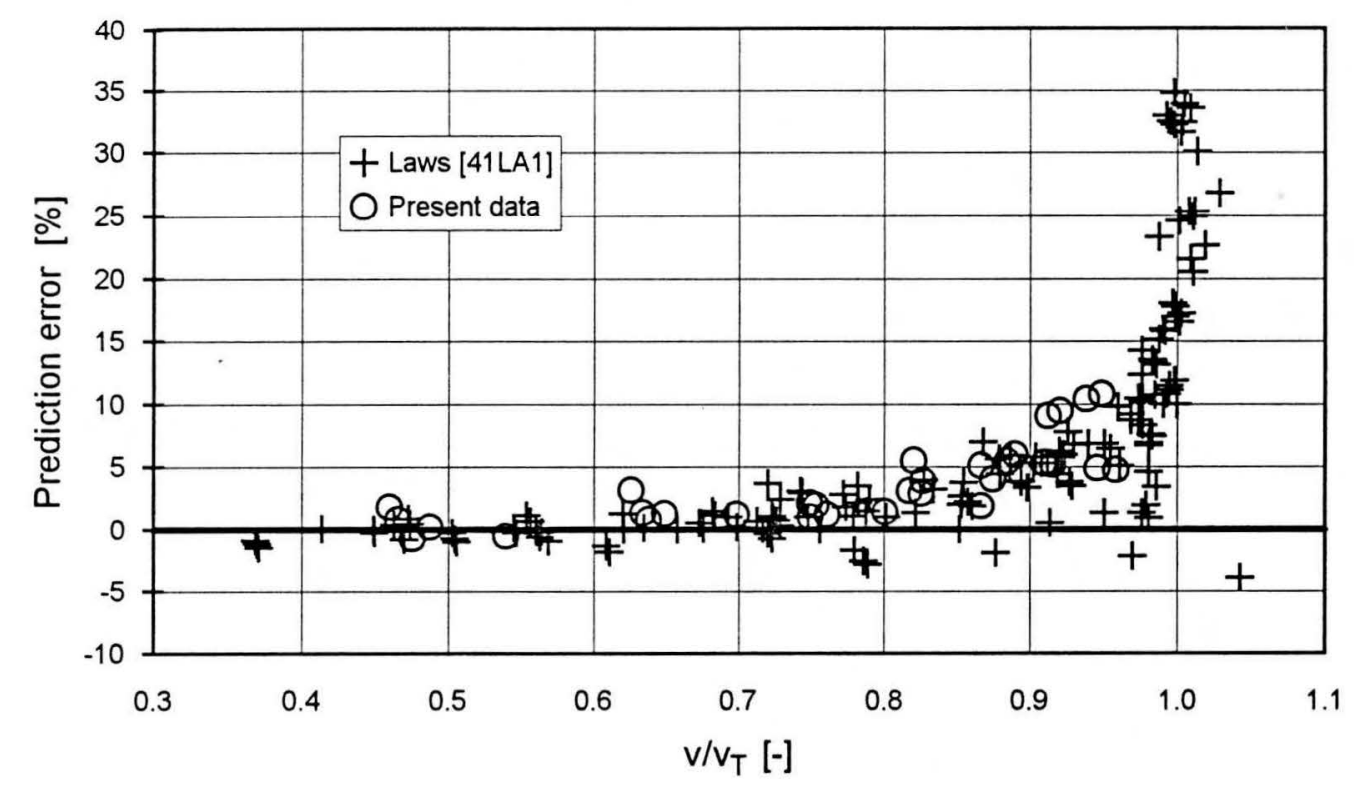

Figure 4.5 Comparison between measured drop velocity and the predicted drop velocity, assuming that drop drag can be calculated by the drag curve for a solid sphere.

If it is assumed that the drag experienced by water drops can be predicted by a drag correlation for solid spheres, the drop velocity is over-predicted for drop velocities approaching the drop terminal velocity, as shown in Figure 4.5. When using the proposed relationship describing the drop deformation at velocities below terminal velocity, Equation (4.8), the drop velocity prediction is much better.

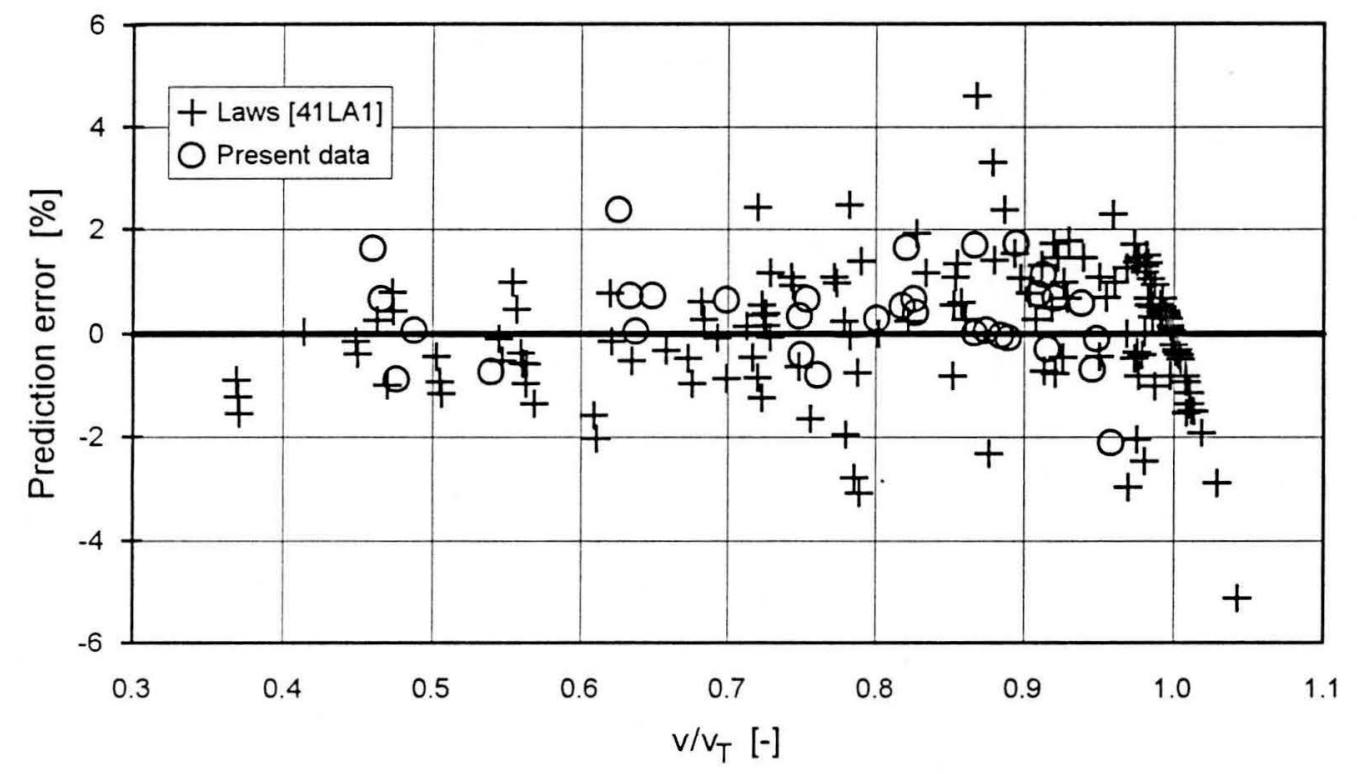

Figure 4.6 Comparison between measured drop velocity and the predicted drop velocity, calculated with the deforming drop drag model described above. 
Figure 4.6 shows that this proposed relationship allows one to predict the drop velocity during acceleration to within 3\%. As seen in Figure 4.7, the model for calculation of drop acceleration proposed by Beard [77BE1] predicts the acceleration of drops well, but it tends to overpredict the drop terminal velocity.

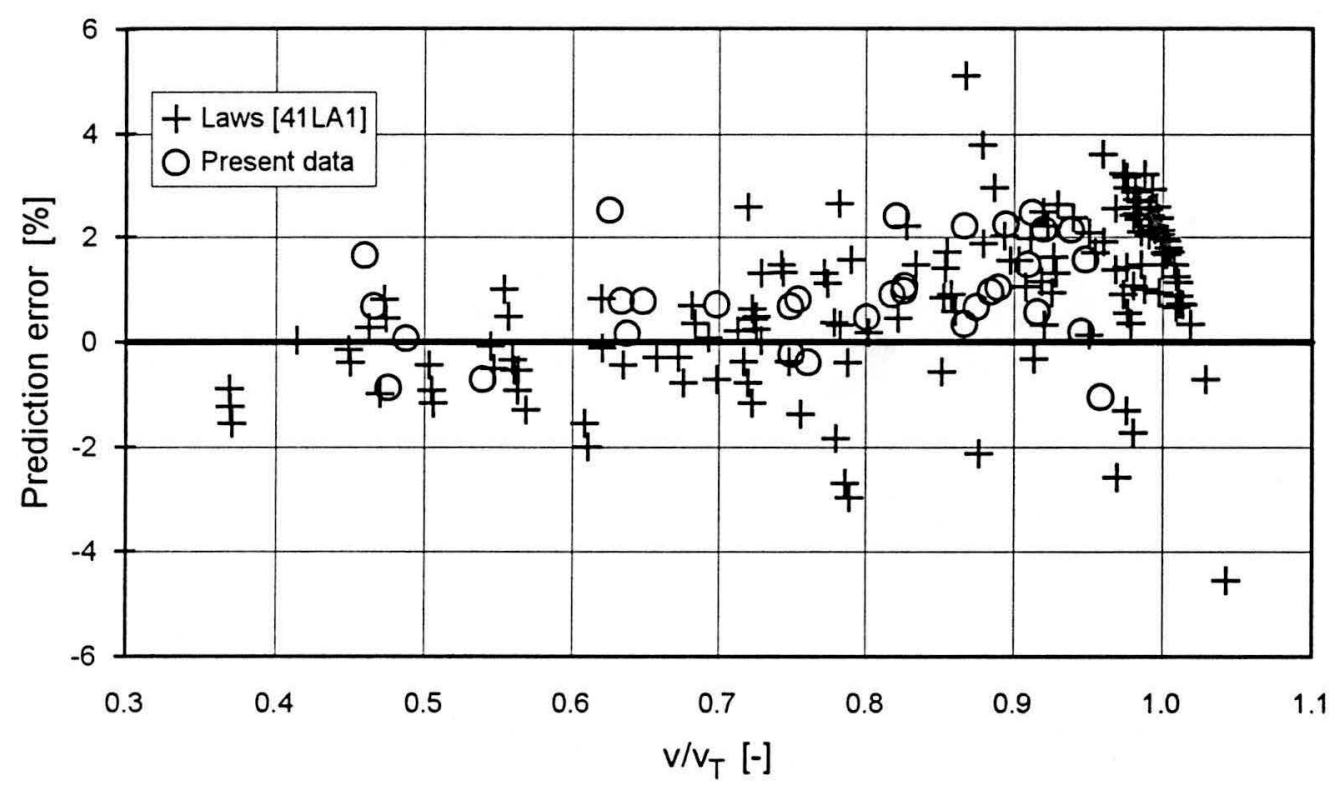

Figure 4.7 Comparison between measured drop velocity and the predicted drop velocity, when assuming that the drop drag can be calculated by the model of Beard [77BE1].

It can be concluded that the model for predicting the acceleration of deformable water drops proposed in Chapter 3 agrees well with the experimentally determined velocity for accelerating drops.

\subsection{Film thickness measurements on slats in a cooling tower}

When a water drop impacts on a solid surface covered by a water film, water from the original drop and from the liquid film is thrown clear of the surface as small splash drops. Mutchler [70MU1] and others have shown that the mass of liquid thrown clear of the point of impact and size distribution of the splash drops are significantly influenced by the thickness of the liquid film on the surface before the impact of the incoming drop. This can be assumed to be true for drops striking narrow slats covered by liquid films (as found in splash packs) as well. The water films on the slats of a splash grid are continuously being fed by small or slow moving drops falling from above without removing any of the existing water on the grid. Larger drops, travelling at higher velocities, tend to splash on impact with the slats, removing some of the water on the slat. The time-averaged film thickness is governed by the relative rates of water removal and addition as well as by the geometry of the slat (i.e., width and edge 
sharpness). Static menisci of water on sharp edged slats were found to be up to $4 \mathrm{~mm}$ thick (see Erens and Dreyer [93ER1]). Experiments have been planned to measure the mass of water splashing from narrow slats upon drop impact and the distribution of splash drop sizes formed. To cover the whole range of film thicknesses from 0 to $4 \mathrm{~mm}$ water film thicknesses would require a very large number of experiments. To reduce the number of experiments required, it was necessary to have an indication of the film thicknesses on the slats of an operational cooling tower. In this section, experiments are described in which the thicknesses of water films on various slats in an operational cooling tower were measured.

Table 4.1 Slats and probes used in the film thickness measurements on splash grids.

\begin{tabular}{|c|c|c|c|c|}
\hline Probe & $\begin{array}{c}\mathbf{W} \\
\mathrm{mm}\end{array}$ & $\begin{array}{c}\mathbf{d}_{\mathbf{p}} \\
\mathrm{mm}\end{array}$ & $\begin{array}{c}\mathbf{s} \\
\mathrm{mm}\end{array}$ & $\begin{array}{c}\mathbf{s} / \mathbf{d}_{\mathbf{p}} \\
-\end{array}$ \\
\hline 1 & 5 & 3.5 & 15 & 4.3 \\
2 & 10 & 5.5 & 20 & 3.6 \\
3 & 15 & 6.0 & 25 & 4.2 \\
4 & 25 & 6.0 & 25 & 4.2 \\
5 & 50 & 6.0 & 25 & 4.2 \\
\hline
\end{tabular}

A conductance measuring probe, as described in Appendix $\mathrm{C}$, was used to measure the film thicknesses on five different slats mounted below 10 layers of commercial splash packing material (with $\beta \approx 0.8$ ) in a cooling tower. The reference probe was mounted inside the cooling tower to ensure that any variances in water conductivity did not influence the accuracy of the film thickness readings. Table 4.1 shows the dimensions of the slats and the dimensions of the electrodes used in these experiments. The electrode set on each slat was calibrated as described in Appendix C. Since the centreline film thickness on each slat was used in the calibration of each set of electrodes, the film thicknesses measured on each slat refers to the centreline film thickness. According to Dreyer and Erens [93ER1], the shape of a steady liquid meniscus on a narrow slat can be accurately described if the centreline film thickness is known.

The probes were used to measure the instantaneous film thicknesses continuously. Figure 4.8 shows a typical variation of film thickness with time. Steady (mean) film thicknesses were measured by determining the average of more than 50000 instantaneous readings. For each of the slats the film thicknesses on the slats were measured at three water flow rates between 6400 and $12800 \mathrm{~kg} / \mathrm{m}^{2} \mathrm{hr}$ and three air flow velocities between 1 and $3 \mathrm{~m} / \mathrm{s}$. The results of these measurements are given in Appendix K. 


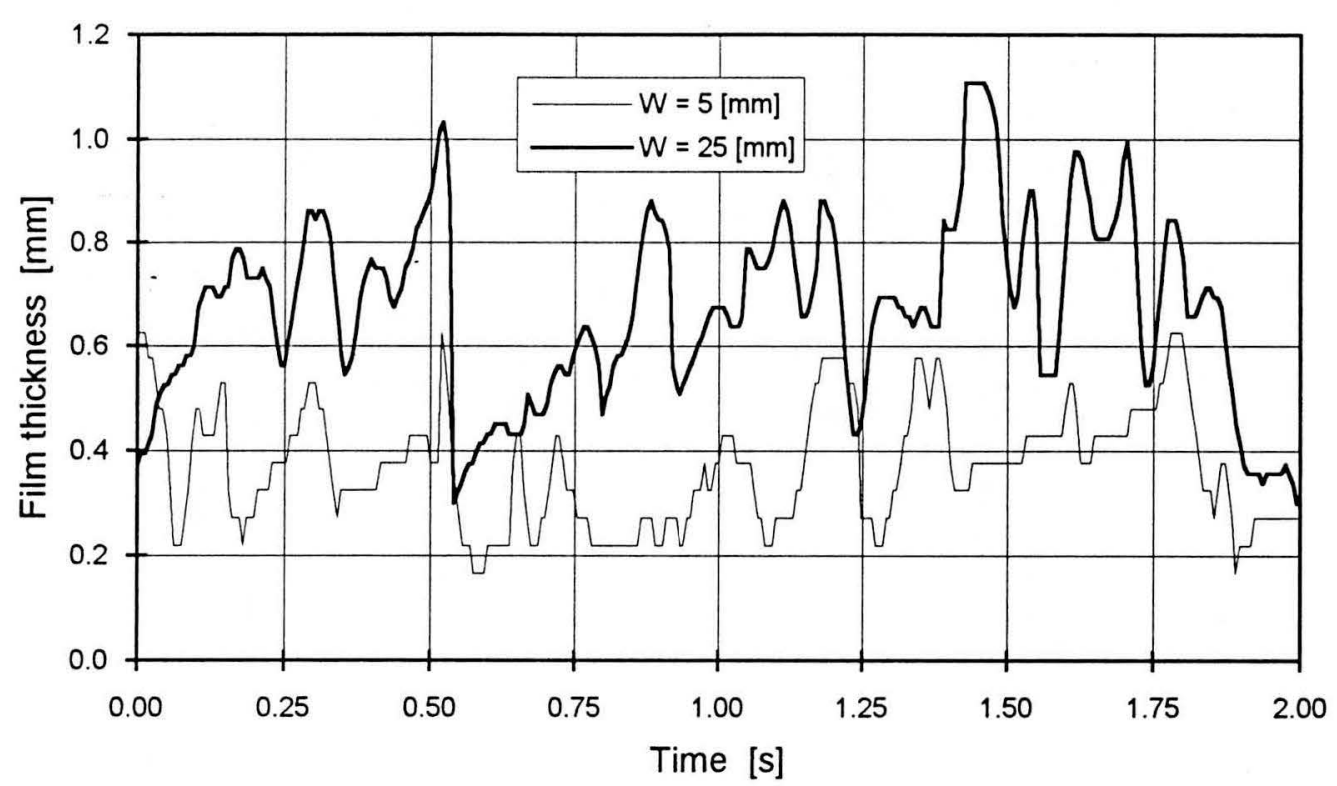

Figure 4.8 Variation of the water film thickness on different slats placed below 10 layers of splash grids in an operational cooling tower.

The following correlation was determined from the measured water film thicknesses on the slats, at different air and water flow rates,

$$
\begin{aligned}
\bar{\delta}= & 0.315-1.387 \times 10^{-2} \dot{\mathrm{M}}_{\mathrm{w}}-2.550 \times 10^{-3} \mathrm{v}_{\mathrm{a}}+1.575 \times 10^{-2} \mathrm{~W} \\
& -3.992 \times 10^{-4} \mathrm{~W}^{2}+1.916 \times 10^{-3}\left(\dot{\mathrm{M}}_{\mathrm{w}} \mathrm{W}\right)+2.188 \times 10^{-3}\left(\mathrm{v}_{\mathrm{a}} \mathrm{W}\right)
\end{aligned}
$$

where $\bar{\delta}$ and $\mathrm{W}$ is in $m m, \mathrm{v}_{\mathrm{a}}$ is in $\mathrm{m} / \mathrm{s}$ and $\dot{\mathrm{M}}_{\mathrm{w}}$ in $\mathrm{kg} / \mathrm{m}^{2} s$.

The correlation is shown in Figures 4.9 and 4.10 for a typical constant air velocity and a constant water flow rate respectively. For a fixed water flow rate it can be seen from that the water film thickness increases with increasing air velocity. This is to be expected since the average velocity of the drops striking the test slat is reduced by the increasing air flow velocity. At a constant air velocity, the film thicknesses increase with increasing water flow rate.

At a given air velocity and water flow rate the water films were generally found to be thinner on the narrower slats. Erens and Dreyer [93ER1] observed the same trend for static menisci on narrow slats. The fact that the films measured on the $50 \mathrm{~mm}$ wide slat were found to be thinner than the films on the $25 \mathrm{~mm}$ wide slat therefore appears surprising at first, but it can be attributed to the following: On a narrow slat, or in the case of a drop impact near the edge of a wide slat, a significant fraction of the kinetic energy of a large drop striking the slat is transferred to large drops glancing off the side of the slat. In the case of a drop striking the 
centre of a wide slat most of the kinetic energy of the incoming drop is transferred to the liquid film on the slat, resulting in larger volumes of water being removed from the slat by splashing which in turn results in reduced film thicknesses.

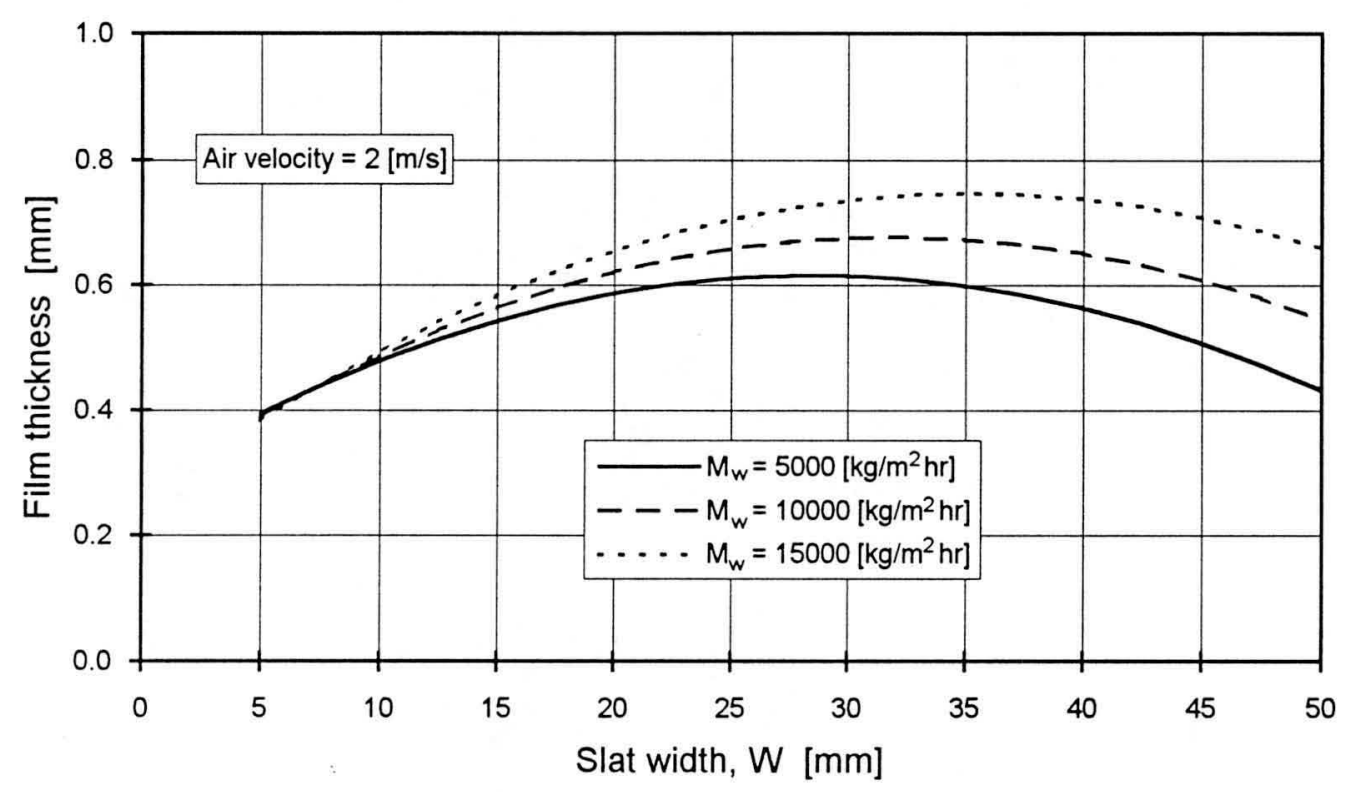

Figure 4.9 Predicted variation of the average water film thickness with slat width at a constant air velocity.

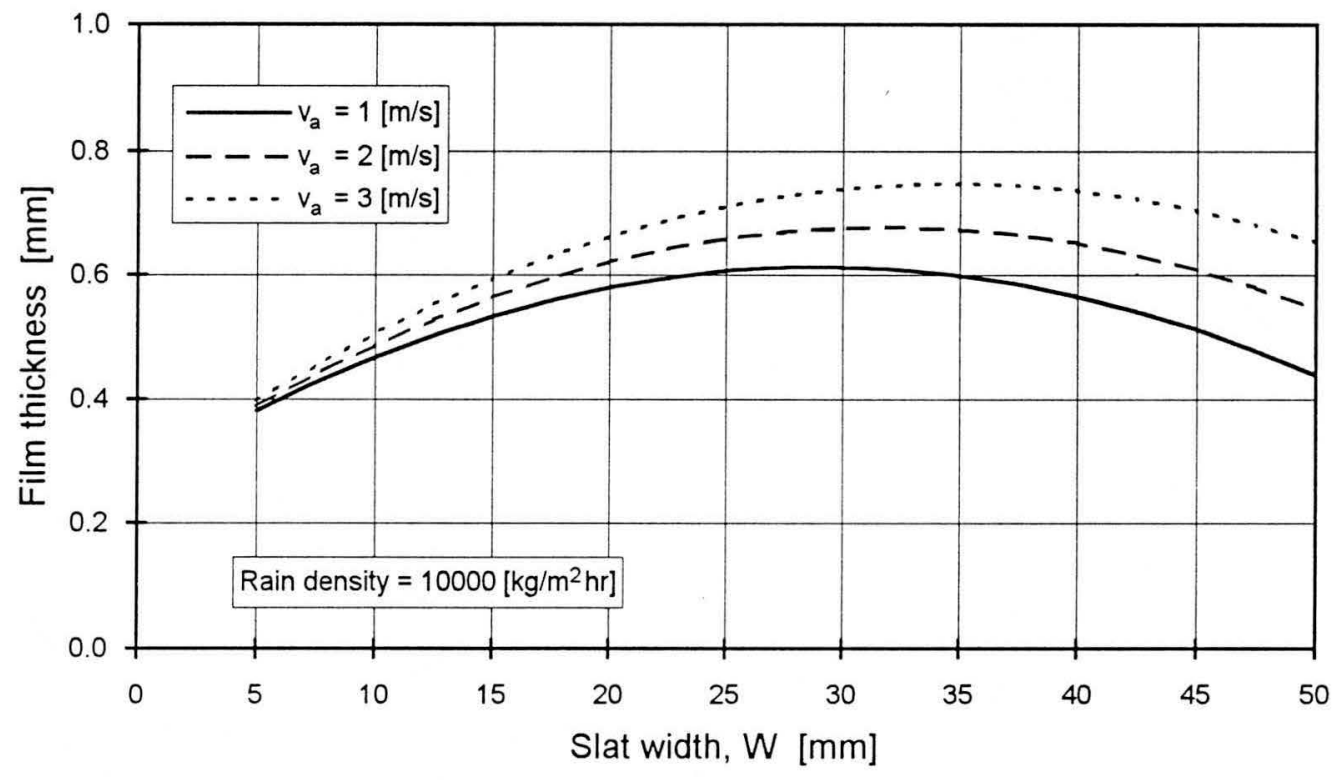

Figure 4.10 Predicted variation of the average water film thickness with slat width at a constant water mass flux. 
The correlation for the average film thickness given above is not intended to be used to describe the film thicknesses on the slats of all splash pack grids. It can be expected that the position of the test slat in the packing, the packing type (above the test slat) and the type of water distribution system will influence the film thicknesses on a given slat.

From these measurements of the typical film thicknesses on different slats in an operational cooling tower, it was concluded that the planned experiments to study the splashing of water drops on splash packing material should be limited to the range of film thicknesses between 0.3 and $1.0 \mathrm{~mm}$.

\subsection{Experimental investigation of the splashing and cutting processes}

In Chapters 2 and 3 the lack of information regarding the formation of splash drops during the impact of drops on slats was highlighted. Most of the data in the literature was obtained for water drops striking large flat surfaces far from any edges. The total volume of splash drops and the distribution of these drops are of major importance in predicting the thermal performance of splash packing material. A series of experiments was conducted to study the formation of splash drops during the impact of drops on narrow slats covered by thin liquid films.

The experimental set-up, shown in Figure 4.11, was relatively simple. Two $2.5 \mathrm{~m}$ long $40 \mathrm{~mm}$ diameter pipes were mounted vertically $25 \mathrm{~mm}$ from a wall. These pipes were used as the mountings for various devices used in the drop splashing experiments. The drop generating system consisted of a constant head tank with an overflow system and various stainless steel and glass nozzles. Gravity-fed water from the constant head tank was allowed to drip from the nozzles. The drip rate from each nozzle could be adjusted by adjusting a simple clamp on the plastic supply tube. The size of the drops formed by the nozzles are dependant on the nozzle diameter and the liquid flow rate (see Appendix F). It was possible to generate drops with diameters between 2.3 and $7.5 \mathrm{~mm}$ in this way. With the larger drops $(6.2$ and $7.5 \mathrm{~mm}$ ) satellite drops were formed behind each drop. The single satellite drop which formed behind the $6.2 \mathrm{~mm}$ drop represented less than $0.1 \%$ of the primary drop mass. More than one satellite drop was formed behind the $7.5 \mathrm{~mm}$ drop, representing up to $8 \%$ of the mass of the primary drop. The drop sizes were determined by weighing 10 to 50 drops on an electronic scale, which has a full scale reading of 160 grams and a 1 milligram resolution, and calculating the average drop size from the average mass of each drop. The sizes of the satellite drops falling behind the large primary drops $(6.2$ and $7.5 \mathrm{~mm})$ were determined from photographs. The drop generating system could slide up and down one of the two mounting pipes to conduct tests at different drop fall heights. In this way the distance from the nozzle to the test slat could be varied from 0 to $2.5 \mathrm{~m}$. 
A $2.5 \mathrm{~m}$ fall height is not enough for the drops to reach terminal velocity e.g. a $5 \mathrm{~mm}$ drop, which has a terminal velocity of approximately $9.1 \mathrm{~m} / \mathrm{s}$, will reach $6.5 \mathrm{~m} / \mathrm{s}$ (or $70 \%$ of its terminal velocity) after a $2.5 \mathrm{~m}$ fall in still air at STP. A $1 \mathrm{~mm}$ drop will reach $95 \%$ of its terminal velocity after a $2.5 \mathrm{~m}$ fall in still air. In a cooling tower the upward flowing air velocity in the cooling tower will result in lower relative velocities between the drops and the slats. For example, if the upward air velocity in the tower is $2 \mathrm{~m} / \mathrm{s}$ the terminal velocity of a 5 $m m$ drop relative to the grids will be only $7.1 \mathrm{~m} / \mathrm{s}$. A drop falling from a height of $2.5 \mathrm{~m}$ in still air reaches almost $92 \%$ of this velocity. Based on the above and the fact that the drops in a cooling tower seldom reach terminal velocity, it is clear that a test set-up, with a maximum fall distance of $2.5 \mathrm{~m}$, will be able to simulate most of the possible types of drop impacts occurring in an actual splash pack.

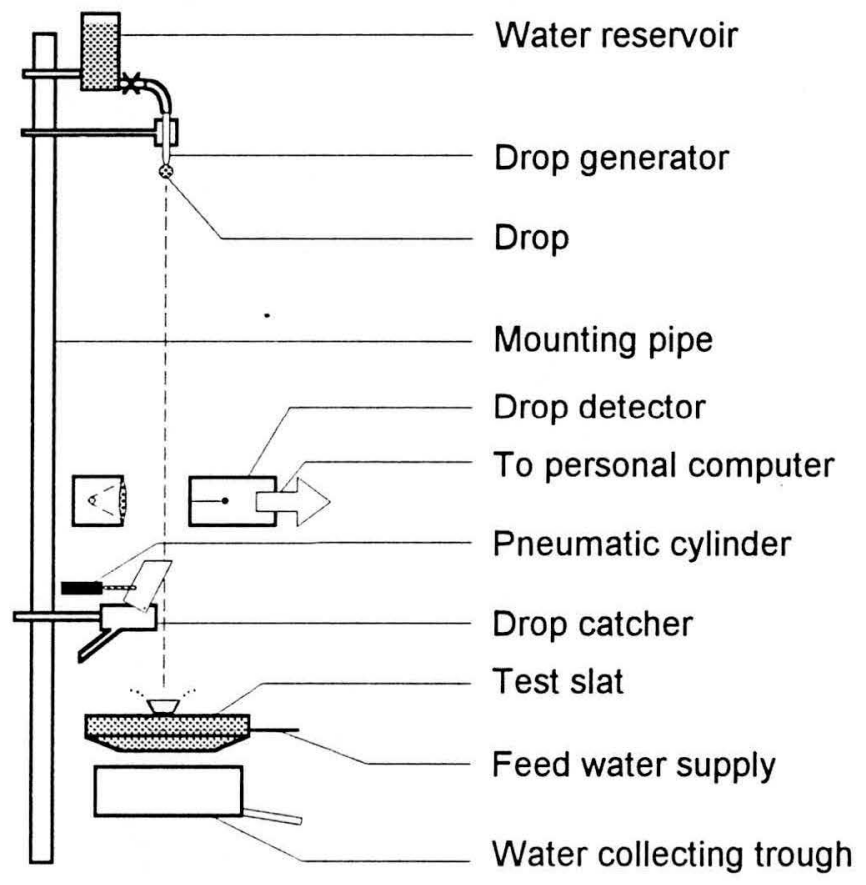

Figure 4.11 Layout of the experimental set-up used to study the splashing and cutting of single drops on narrow slats.

Small drops falling in air are very easily influenced by air currents. For drops with $\mathrm{d} \approx 1 \mathrm{~mm}$, the vortex shedding frequency agrees roughly with the natural oscillation frequency and the drops may experience flight path deviations due to interaction between drop and its wake. For larger drops, non-symmetrical break-off from a nozzle is the major cause of variations in the drop flight path. The same opto-electronic drop detector as that described in section 4.1 above, was used during the splashing tests to monitor drop deflection. The drop detector consists of a parallel light beam projected at a $30 \mathrm{~mm}$ wide horizontal row of light detectors. The digital signals from the photo-transistors were fed to a digital input/output card (Eagle Electric PC14B) mounted in an IBM-compatible personal computer. As a drop falls past the row of 
photo-detectors a binary image of the drop could be reconstructed in real time on the computer. The centroid of the binary image could then be calculated as each drop fell past the drop detector. The horizontal position of the centroid of the binary image was assumed to correspond to the centroid of the actual drop. The drop detector was also used to synchronise the photography of the splashing drops.

Various test slats were used in the experiments to quantify the splashing process. A typical test slat is shown in Figure 4.12. The slats were made from Perspex coated with PVA paint to ensure good wetting of the surface. Water from a second constant head tank could be supplied to the upper surface of each slat to replenish the water film on the slat. The thickness of the layer of water on each slat could be measured using the conductance measuring technique described in section 4.2 above (and in Appendix C). The electrodes on each of the test slats were calibrated individually using a vernier height gauge. The top surface of roughly one third of the length of each slat sloped towards the end of the slat as shown in Figure 4.12, ensuring that thin films $(\delta<1 \mathrm{~mm})$ could be maintained over the electrodes of the film thickness measuring probes. If a completely square, flat slat was used it was not possible to maintain such thin films on the slats due to "damming" on the slat caused by surface tension effects at the sharp edges. During the experiments the incoming drops were directed at the area of the slat between the electrodes since this is where the water film thickness was measured. The slats were mounted on an adjustable frame below the drop generator, drop detector and drop catcher as shown in Figure 4.11.

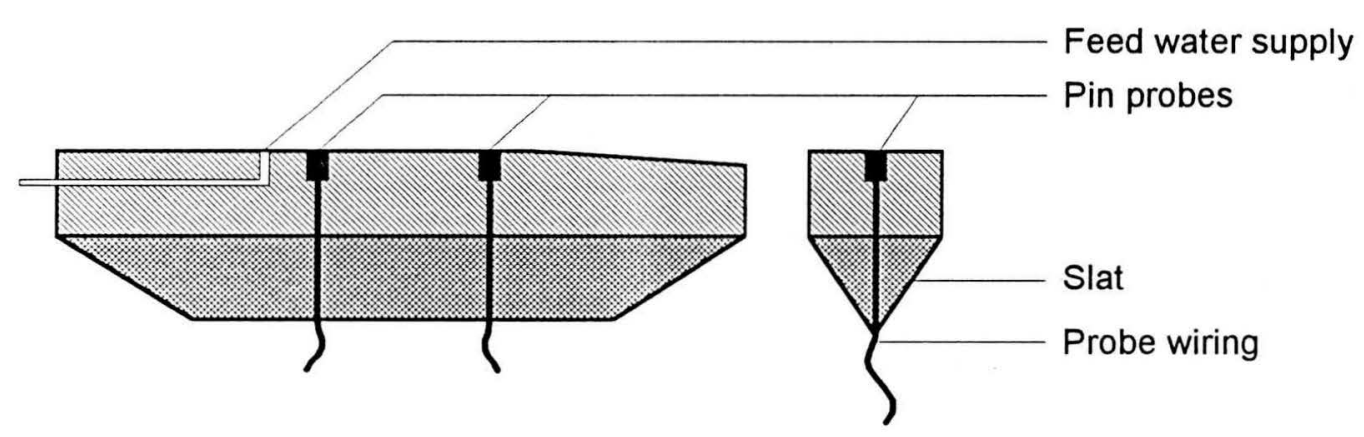

Figure 4.12 Layout of a typical test slat showing the feed water supply and the film thickness measuring probe.

The water supply rate to the upper surface of each slat was measured using calibrated capillary tube flow meters made from stainless steel tubes with a $1.6 \mathrm{~mm}$ inner diameter. The water flow rate was regulated by clamping the plastic feed pipe between two metal plates; in this way it was possible to obtain a very steady flow rate even at very small flow rates. The excess water from the overflow of the constant head reservoir was ducted through the reference probe of the film thickness measuring device to cancel any changes in the water conductivity. 
In some of the experiments it was necessary to allow only a fixed number of drops to fall onto the test slat or to allow only the drops which will impact the test slat at a given position to fall onto the slat. A drop catcher was therefore designed to remove unwanted drops before they could strike the slat. The drop catcher was designed to have very short reaction times to minimise the distance between the drop detector and the test slat. Figure 4.13 shows the layout of the drop catcher. If a 'skew' drop is detected by the drop detector, a digital signal from the personal computer activates a solenoid operated pneumatic valve which in turn controls a small the pneumatic cylinder. When activated, the pneumatic cylinder moves a drop catching shutter into the flight path of the drop and keeps it there until the drop has been intercepted before the shutter is returned to its normal position. The small pressure chambers at the inlet port of the solenoid valve and at the outlet port of the cylinder reduce the reaction time of the drop catcher by action as 'buffers' for the air entering and exiting the cylinder. The drop catcher could be in the 'out' position within $80 \mathrm{~ms}$ of detecting a 'skew' drop. Water from drops which have been intercepted is ducted away to the splash tray below the test slat. The drop catcher was mounted on the second mounting tube to prevent vibrations from the drop catcher from influencing the drop generation system (which was mounted on the other tube).

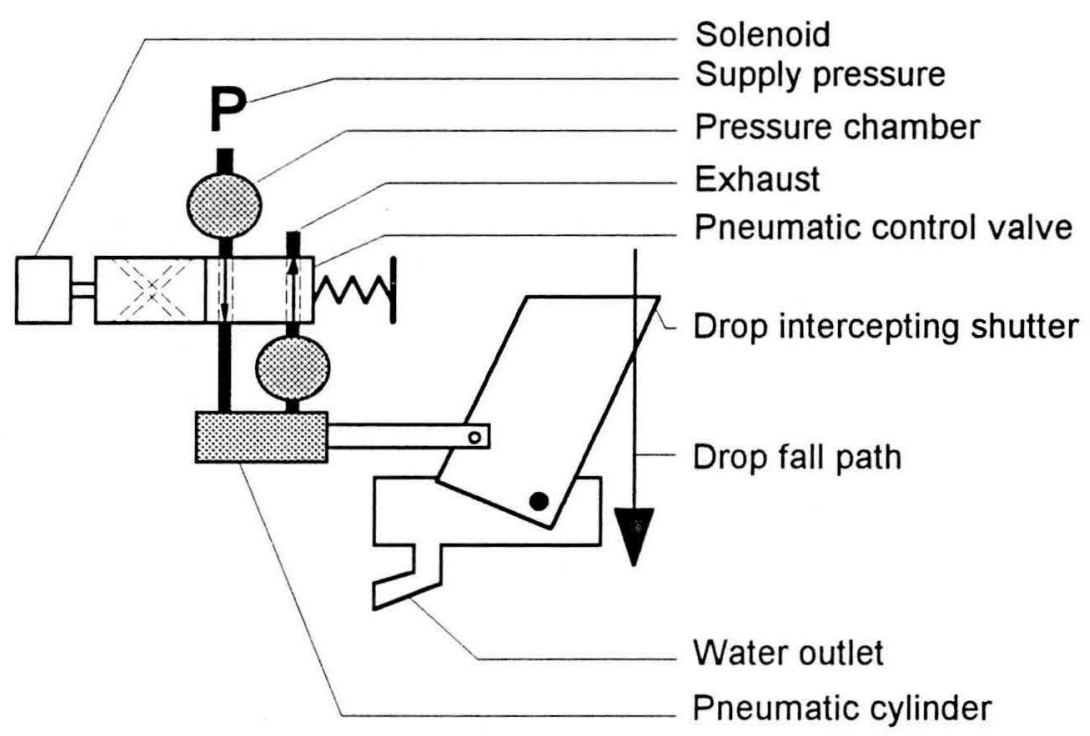

Figure 4.13 Layout of the drop catcher used to intercept "skew" drops.

\subsubsection{Splash photography}

The formation of the splash crown and splash drops after the impact of a drop on the slat can be visualised in a dark room by activating a high speed strobe at the correct moments. A computer program was written in Turbo Pascal to control the operation of the strobe. The digital $\mathrm{I} / \mathrm{O}$ card in the personal computer which is used to read the drop detector output, incorporates a digital counter which can be used for strobe timing. The $\mathrm{I} / \mathrm{O}$ card was configured to use an external $100 \mathrm{kHz}$ pulse from a stabilised signal generator to control the 
counting. Before the drop detector senses a drop a given delay count value is entered into the counter; once a drop is detected, the count value is decremented on every timing pulse from the signal generator. Once the counter value reaches zero, a hardware interrupt is generated and the software triggers the strobe once. Since the operation of the strobe is controlled by software, it is relatively easy to advance the delay time between drop detection and the activation of the strobe after the illumination of each drop. This allows one to observe the whole sequence of the splash process by actually observing successive drops at slightly different delay times.

Photographs of the splash process were taken in a dark room using a camera with an open shutter and activating a high intensity flash with a short flash duration time at the correct moment. The software used to operate the strobe was adapted to control the photography of splashing drops. This program uses the same procedure to control the delay time between drop detection and the moment at which the photograph is to be taken. Signals from the computer I/O card switched small reed relays, which in turn operated the motor drive of the camera and the triggered the flash. If a drop impacting on a slat is to be photographed at a given delay after detection, the camera shutter is opened just after the detection of the drop. At the moment when the preset count value reaches zero the flash is activated and shortly afterwards the shutter is closed again. A Hasselblad ELM camera and a $135 \mathrm{~mm}$ macro lens with bellows were used to photograph the impacting drops. Ilford FP4 black and white film was used. A photographic flashlight with a flash duration time of between 20 and $30 \mu s$ was used to illuminate a white polystyrene sheet behind the splash impact point. This backlighting technique, referred to as shadowgraphing, results in the shadows of the drops being photographed. The layout of the photographic system is given in Figure 4.14.

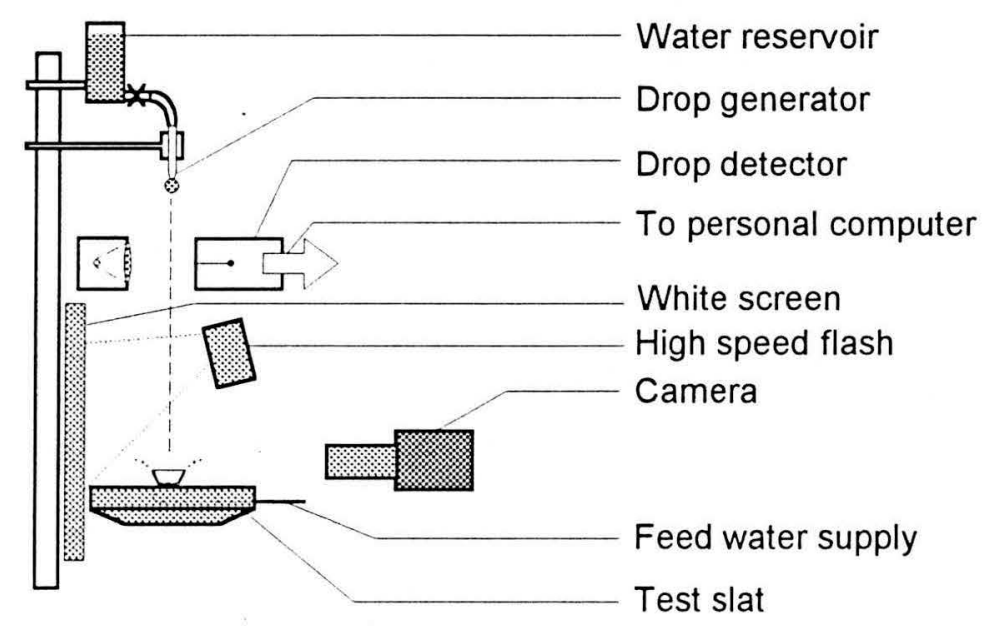

Figure 4.14 Layout of the set-up used for the photography of the splashing and cutting phenomena. 
A series of photographs was taken, using the technique described above, to gain more insight into the splashing and cutting phenomena occurring on slats. Table 4.2 lists the ranges of drop sizes, slat widths, impact positions, fall heights and film thicknesses which were covered in the photographic study. It is convenient to express the drop impact position on the slat in terms of a dimensionless impact parameter. The dimensionless impact position is defined as

$$
\chi=\frac{\mathrm{x}}{\left(\left(\mathrm{W}+\mathrm{d}_{\mathrm{i}}\right) / 2\right)} \text { with }\left(-\frac{\left(\mathrm{W}+\mathrm{d}_{\mathrm{i}}\right)}{2}\right) \leq \mathrm{x} \leq\left(\frac{\left(\mathrm{W}+\mathrm{d}_{\mathrm{i}}\right)}{2}\right)
$$

where $\mathrm{x}$ is the horizontal distance between the impact point and the centreline of the slat.

Table 4.2 Ranges of variables covered by the photographic study.

\begin{tabular}{|l|l|}
\hline Variable parameter & Range \\
\hline Drop diameter, d & 2.3 to $7.5 \mathrm{~mm}$ \\
Slat width, W & 5 to $25 \mathrm{~mm}$ \\
Dimensionless impact position, $\chi$ & 0 to 1 \\
Fall height, $z$ & 0.5 to $2.5 \mathrm{~m}$ \\
Centreline film thickness, $\delta$ & 0.3 to $1.0 \mathrm{~mm}$ \\
\hline
\end{tabular}

For each case a sequence of four to ten photographs was taken at different times after the impact of the drop. To ensure that these sequences of photographs, in which different drops were photographed at slightly different delay times after the initial impact time, match closely, the computer program only activated the camera and flash when both the following conditions were met : (i) the centroid of the drop, as observed by the drop detector, falls in a very narrow zone about a preset position, and (ii) when the water film thickness was in a very narrow range about a preset mean value.

Figure 4.15 shows a typical sequence of photographs of a $5.6 \mathrm{~mm}$ drop striking the centre of a $25 \mathrm{~mm}$ wide slat covered by a $0.7 \mathrm{~mm}$ thick water film after a $2.5 \mathrm{~m}$ fall. Note the change in the sizes of the splash drops and initial angle of the splash drops leaving the edge of the crown with time. The angle at which the splash drops leave the point of impact relative to the horizontal plane is referred to as the splash angle. The drops formed immediately after impact are very small, $\mathrm{d}_{\mathrm{s}}<0.1 \mathrm{~mm}$, and they leave the impact point at a very shallow splash angle, $\theta$ $<30^{\circ}$. Later in the life of the crown the splash drops are much larger and they leave the crown at a much greater angle. Figure 4.16 shows a similar sequence of photographs for a $5.6 \mathrm{~mm}$ drop striking the centre of $5 \mathrm{~mm}$ wide slat covered by a $0.7 \mathrm{~mm}$ thick water film. Note that the crown formed in this case is much smaller than before and it is non-symmetrical due to the proximity of the slat edges. 

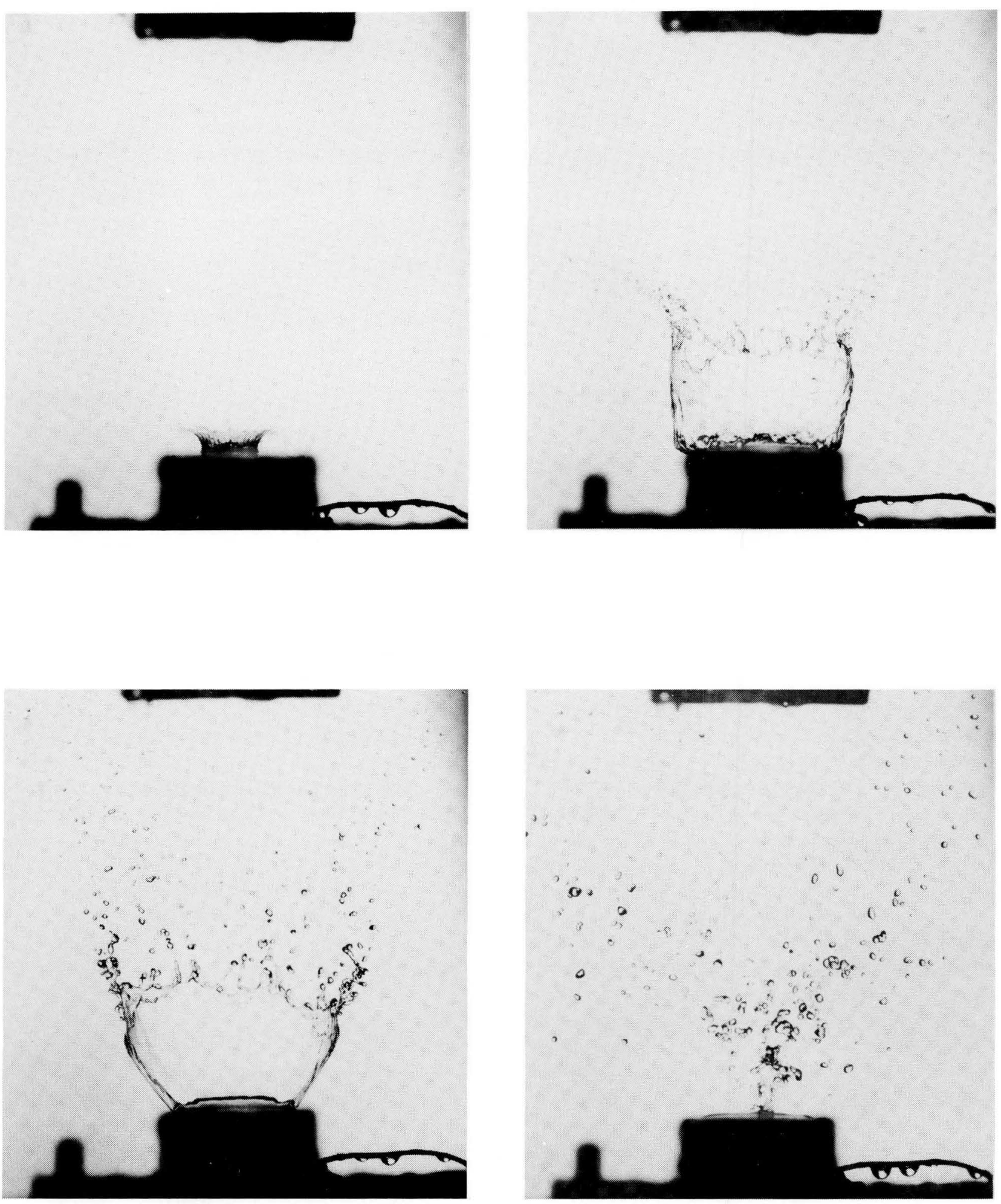

Figure 4.15 Sequence of photographs showing the crown and the splash drops at various delay times after the impact of a $5.6 \mathrm{~mm}$ water drop striking the centre of a 25 $\mathrm{mm}$ wide slat covered by a $1.0 \mathrm{~mm}$ thick water layer. 

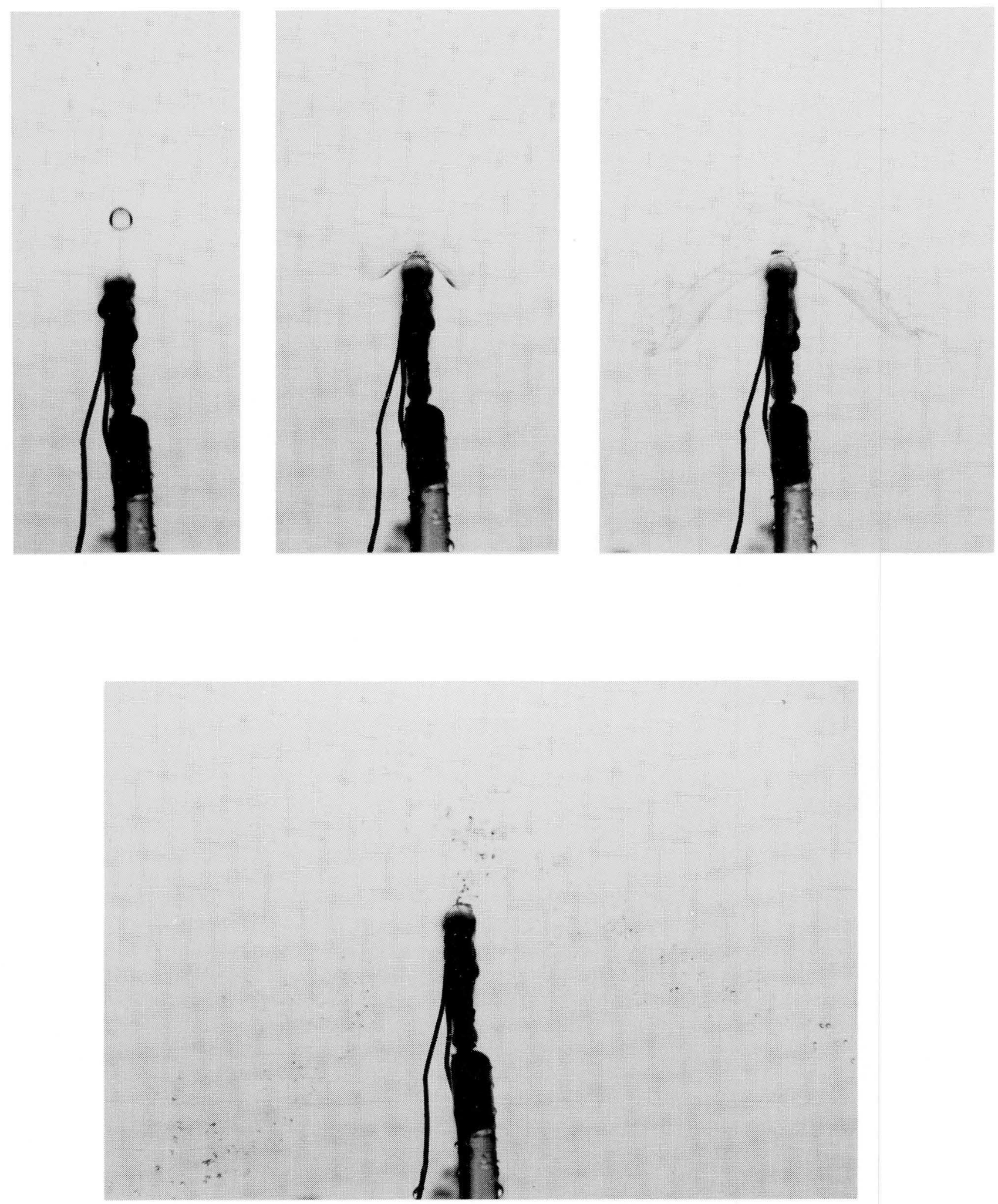

Figure 4.16 Sequence of photographs showing the crown and the splash drops at various delay times after the impact of a $5.6 \mathrm{~mm}$ water drop striking the centre of a $5 \mathrm{~mm}$ wide slat covered by a $0.7 \mathrm{~mm}$ thick water layer. 
Table 4.3 Splash angle and crown growth time data obtained from the splash photographs.

\begin{tabular}{|l|c|c|c|c|c|c|c|c|}
\hline Source & $\mathbf{d}_{\mathbf{i}}$ & $\mathbf{Z}$ & $\mathbf{\mathbf { v } _ { \mathbf { i } }}$ & $\mathbf{W e}$ & $\boldsymbol{\delta}$ & $\boldsymbol{\delta} / \mathbf{d}_{\mathbf{i}}$ & $\boldsymbol{\theta}$ & $\mathbf{t}_{\text {crown }}$ \\
& $\mathrm{mm}$ & $\mathrm{m}$ & $\mathrm{m} / \mathrm{s}$ & - & $\mathrm{mm}$ & - & $\mathrm{deg}$ & $\mathrm{ms}$ \\
\hline Present data & 3.3 & 0.50 & 2.1 & 202 & 0.4 & 0.121 & 40 & \pm 5 \\
Present data & 3.3 & 0.50 & 2.1 & 202 & 0.7 & 0.212 & - & $5-10$ \\
Present data & 3.3 & 0.50 & 2.1 & 202 & 1.0 & 0.303 & - & $5-10$ \\
Present data & 3.3 & 1.25 & 4.3 & 847 & 0.4 & 0.121 & 50 & \pm 5 \\
Present data & 3.3 & 1.25 & 4.3 & 847 & 0.7 & 0.212 & 55 & $5-10$ \\
Present data (side view) & 3.3 & 1.25 & 4.3 & 847 & 0.7 & 0.212 & 60 & $5-10$ \\
Present data & 3.3 & 1.25 & 4.3 & 847 & 1.0 & 0.303 & 60 & $5-10$ \\
Present data & 3.3 & 2.50 & 6.0 & 1645 & 0.4 & 0.121 & 60 & \pm 5 \\
Present data & 3.3 & 2.50 & 6.0 & 1645 & 0.7 & 0.212 & 60 & $5-10$ \\
Present data & 3.3 & 2.50 & 6.0 & 1645 & 1.0 & 0.303 & - & $5-10$ \\
Mutchler [70MU1] & 3.3 & $\mathrm{v}_{\mathrm{T}}$ & 8.0 & 2933 & 0.4 & 0.121 & 64.2 & 6.0 \\
Mutchler [70MU1] & 3.3 & $\mathrm{v}_{\mathrm{T}}$ & 8.0 & 2933 & 0.7 & 0.212 & 71.1 & 7.2 \\
Mutchler [70MU1] & 3.3 & $\mathrm{v}_{\mathrm{T}}$ & 8.0 & 2933 & 1.0 & 0.303 & 75.0 & 7.3 \\
Present data & 6.2 & 0.50 & 2.2 & 410 & 0.4 & 0.066 & 50 & - \\
Present data & 6.2 & 0.50 & 2.2 & 410 & 0.7 & 0.115 & 55 & - \\
Present data & 6.2 & 0.50 & 2.2 & 410 & 1.0 & 0.164 & 60 & - \\
Present data & 6.2 & 1.25 & 4.6 & 1793 & 0.4 & 0.066 & 55 & \pm 12 \\
Present data & 6.2 & 1.25 & 4.6 & 1793 & 0.7 & 0.115 & 65 & \pm 15 \\
Present data (side view) & 6.2 & 1.25 & 4.6 & 1793 & 0.7 & 0.115 & 70 & \pm 15 \\
Present data & 6.2 & 1.25 & 4.6 & 1793 & 1.0 & 0.164 & 70 & \pm 16 \\
Present data & 6.2 & 2.50 & 6.5 & 3580 & 0.4 & 0.066 & 65 & \pm 12 \\
Present data & 6.2 & 2.50 & 6.5 & 3580 & 0.7 & 0.115 & 67.5 & \pm 15 \\
Present data & 6.2 & 2.50 & 6.5 & 3580 & 1.0 & 0.164 & 70 & \pm 17 \\
Mutchler [70MU1] & 6.2 & $\mathrm{v}_{\mathrm{T}}$ & 9.1 & 6970 & 0.4 & 0.066 & 57.5 & 14.7 \\
Mutchler [70MU1] & 6.2 & $\mathrm{v}_{\mathrm{T}}$ & 9.1 & 6970 & 0.7 & 0.115 & 63.5 & 20.9 \\
Mutchler [70MU1] & 6.2 & $\mathrm{v}_{\mathrm{T}}$ & 9.1 & 6970 & 1.0 & 0.164 & 67.9 & 24.4 \\
\hline
\end{tabular}

Table 4.3 lists the measured splash angles and the time required for the crown to reach maximum size for various impacts on the centreline of a $25 \mathrm{~mm}$ wide slat. The predicted splash angle data and crown growth time data for drops striking a thin water film at terminal velocity, obtained with the correlations by Mutchler [70MU1] (see Equations (3.35) and (3.37)), are included in Table 4.3 for comparison. Figure 4.17 shows the splash angle data graphically. The time required for the crown to reach maximum size was found to be approximately half of the crown lifetime. 

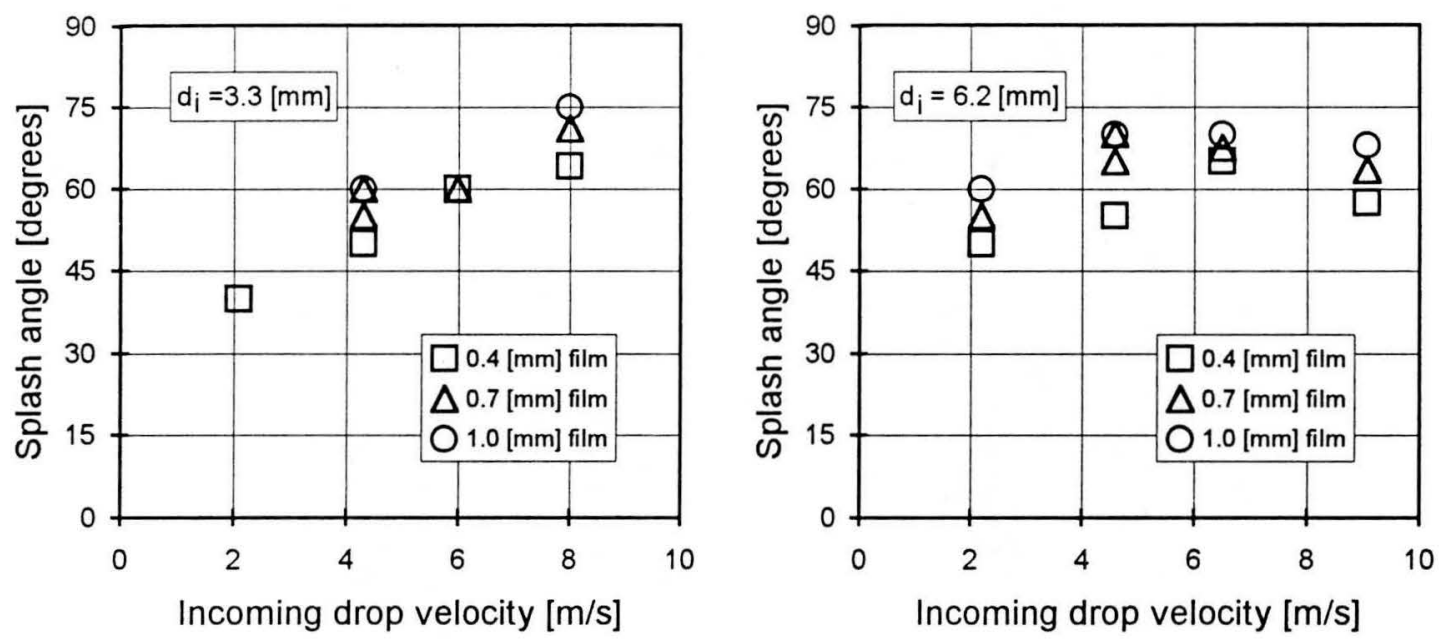

Figure 4.17 Summary of splash angle data.

Figure 4.18 describes the observed splash/cutting interaction for drop impacts near and on the edges of the solid slats.

a)

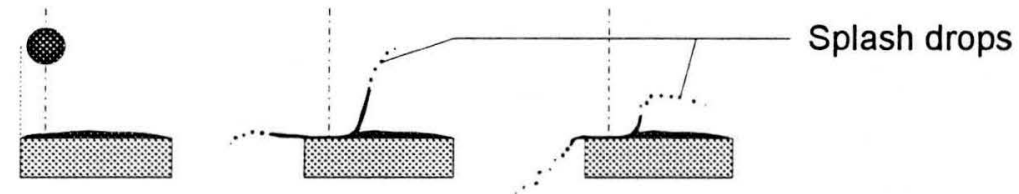

b)

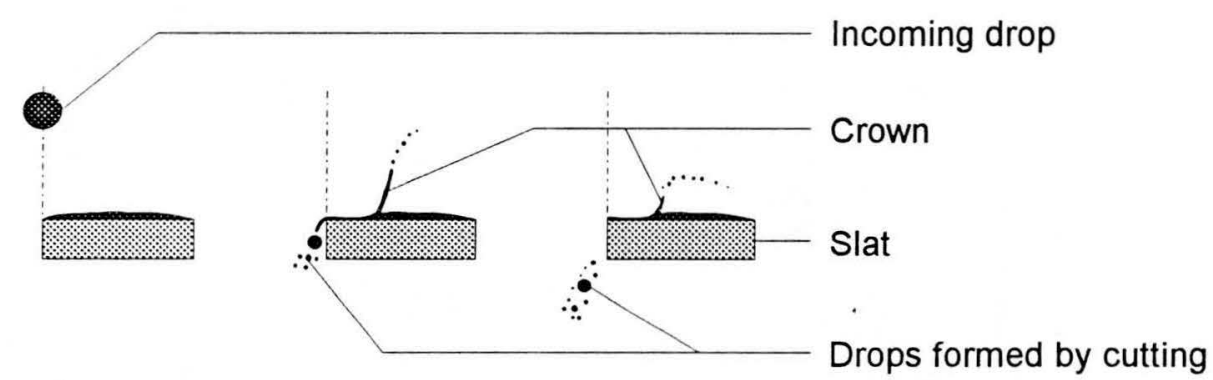

c)
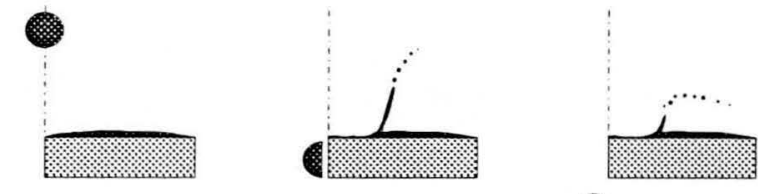

(2)

Increasing time

Figure 4.18 Graphical description of the cutting/splashing phenomena for drop impacts (a) near an edge, (b) impacts on an edge at high Weber numbers and (c) impacts on an edge at low Weber numbers.

\subsubsection{Splash fractions}

When a drop hits the surface of a slat covered by a water film some of the incoming drop mass and some of the liquid from the film leave the point of impact and is flung clear of the slat. For 
drops impacting far from the edges, all the water leaving the slat is lost due to the splashing action, but for drops impacting near the edge of a slat some of the water leave the slat at a glancing angle. The process by which water is lost over the edge of a slat in this way is referred to as cutting. Figure 3.5 shows the splashing and cutting processes schematically. The total volume of water leaving the slat following the impact of a drop near the edge of the slat, is made up of water lost due to splashing and due to cutting (see definitions in Chapter 3).

By installing a small trough below the test slat, as shown in Figure 4.11, it is possible to measure the mass flow rate of the water dripping from below the test slat. For a given drop size, fall height, impact point, slat width and film thickness, the total mass of water lost due to cutting and splashing can be measured by keeping all the variable parameters constant and dripping a large number of drops, say 250 , onto the slat. The combined splash and cutting fraction can be calculated from the incoming drop mass flow rate, the slat feed rate and the measured water flow rate from the bottom of the slat as follows

$$
\mathrm{f}_{\mathrm{s}}+\mathrm{f}_{\mathrm{c}}=\frac{\left(\dot{\mathrm{m}}_{\mathrm{i}}+\dot{\mathrm{m}}_{\text {feed }}\right)-\dot{\mathrm{m}}_{\mathrm{d}}}{\dot{\mathrm{m}}_{\mathrm{i}}}
$$

A series of experiments was conducted to measure the volume of water leaving the test slat when the slat is struck by a water drop falling from above. The ranges in which these experiments were conducted are given in Table 4.4 .

Table 4.4 Ranges of variable parameters covered in splash fraction measurements.

\begin{tabular}{|l|l|}
\hline Variable parameter & Range \\
\hline Drop diameter, $\mathrm{d}_{\mathrm{i}}$ & 2.3 to $7.5 \mathrm{~mm}$ \\
Slat width, W & 5,10 and $25 \mathrm{~mm}$ \\
Dimensionless impact position, $\chi$ & -1 to 1 \\
Fall height, $z$ & 0.25 to $2.5 \mathrm{~m}$ \\
Centreline film thickness, $\delta$ & 0.3 to $1.0 \mathrm{~mm}$ \\
\hline
\end{tabular}

The drop catcher and its controlling computer software was used to allow a fixed number of drops to strike the slat at a given position. The drop catcher intercepted all the incoming drops for which one or both of the following conditions were met: (i) the film thickness is not in the prescribed range and (ii) the drop will not impact at the prescribed position on the slat. The mass of water dripping below the slat in this time, the average drop size (obtained by measuring the mass of a known number of drops) and the slat feed rate were measured. In all the experiments the drops were set up to strike the slat between the film thickness measuring electrodes to ensure the accurate measurement of the film thickness. The average film thickness and impact position (determined by the drop detector) during each experiment were also recorded. 
Figure 4.19 shows the typical variation of film thickness with time during the impact of a water drop on the film. Initially the film thickness reduces rapidly after the point of impact, but then the film builds up to its steady state value again. The dripping frequency was set to ensure that the film thickness returns to the flat part of the curve before the next drop impact. This ensured that the film thickness (at the moment of drop impact) stays constant throughout a test. A sharp spike could be observed in the film thickness readings when the slat was struck by a large drop. This could be attributed the decreased resistance measured by the conductance probe when the large drop provides a low resistance conduction path between the film thickness measuring probes.
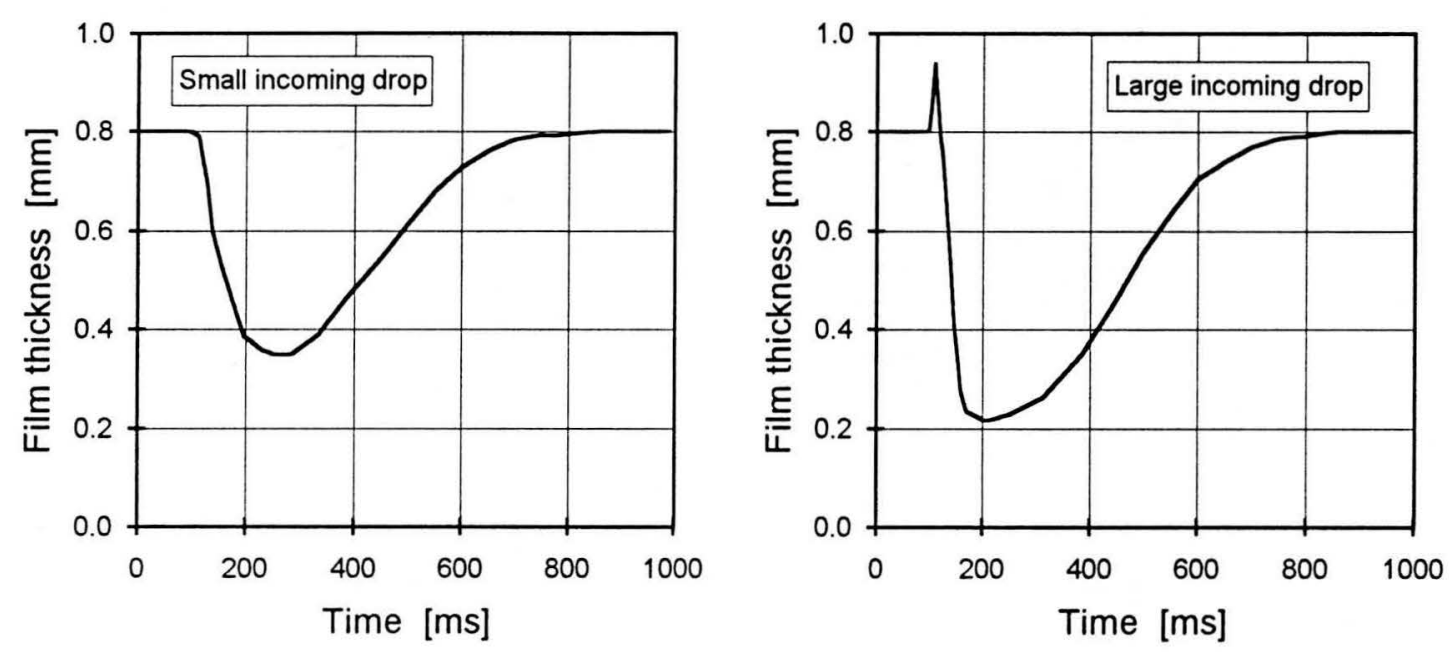

Figure 4.19 Typical instantaneous water film thicknesses on a slat before, during and after being struck by a falling drop.

Upon changing any of the variable parameters, the experimental set-up was left to run undisturbed for about 5 minutes before the counting of drops and the measuring of the flow rates commenced. This allowed the dripping flow rate below the slat to reach a steady state.

The experimental data, given in Appendix L, was used to obtain the average splash fraction for a drop impacting a given slat at any position as follows:

i) For each data point the effect of cutting was cancelled by subtracting the predicted fraction of mass which would be lost due to cutting (given by Equation (3.17)) from the measured volume of mass lost (due to cutting and splashing).

ii) Curves of the local splash fraction versus impact position were constructed for each set of data with the same fall height and drop size. For the $25 \mathrm{~mm}$ slat these curves were of the form 
$f_{s}(x)=\left(c_{1}\left(x-\left(\frac{W+d_{i}}{2}\right)\right)+c_{2}\left(x-\left(\frac{W+d_{i}}{2}\right)\right)^{c_{3}}\right)\left(c_{4}+c_{5} \delta\right)$

while they were assumed to be of the form

$f_{s}(x)=\left(x-\left(\frac{w+d_{i}}{2}\right)\right)\left(c_{1}+c_{2} \delta\right)$

for the $10 \mathrm{~mm}$ and $5 \mathrm{~mm}$ slats. Note that both these curves predict $\mathrm{f}_{\mathrm{s}}\left(\left(\mathrm{W}+\mathrm{d}_{\mathrm{i}}\right) / 2\right)=0$. The data for the $5 \mathrm{~mm}$ wide slat was used to approximate the average splash fraction for a 2 $m m$ slat.

iii) The average splash fraction could then obtained by integration, i.e.,

$$
\overline{f_{s}}=\frac{\int_{0}^{\left(w+d_{i}\right) / 2} f_{s}(x) \partial x}{\left(w+d_{i}\right) / 2}
$$

The average splash fraction data for the $25 \mathrm{~mm}$ slat was calculated as described above, and the results were correlated by an equation of the form

$$
\begin{array}{r}
\overline{f_{s}}=0.01\left(c_{1}+\left(\frac{\delta}{d_{i}}\right)\left(c_{2}+c_{3}\left(\frac{W e}{W_{\text {ref }}}\right)+c_{4}\left(\frac{W e}{W_{\text {ref }}}\right)^{0.4}\right)\right) \\
\left(c_{5}+c_{6}\left(\frac{W e}{W e_{\text {ref }}}\right)^{c_{7}}\right)\left(c_{8}+c_{9}\left(\frac{\delta}{d_{i}}\right)^{c_{10}}\right)
\end{array}
$$

and the average splash fraction for the 2,5 and $10 \mathrm{~mm}$ wide slats was correlated by equations of the form

$$
\overline{f_{s}}=0.01\left(c_{1}+c_{2}\left(\frac{\delta}{W}\right)\right)\left(c_{3}+c_{4}\left(\left(\frac{W e}{W e_{\text {ref }}}\right)\left(\frac{d_{i}}{W}\right)^{2}\right)^{c_{5}}\right)\left(c_{6}+c_{7}\left(\frac{d_{i}}{W}\right)^{c_{8}}\right)
$$

where

$$
\mathrm{We}=\frac{\rho_{\mathrm{w}} \mathrm{v}^{2} \mathrm{~d}_{\mathrm{i}}}{\sigma}
$$

The reference Weber number is defined as the Weber number of the maximum stable drop size at terminal velocity at STP, i.e. 


$$
W e_{\text {ref }}=\frac{\rho_{\mathrm{w}} v_{T}^{2} d_{m}}{\sigma}
$$

where

$$
d_{m}=\sqrt{\frac{16 \sigma}{g\left(\rho_{w}-\rho_{a}\right)}}
$$

The constants in these correlations and the correlation coefficients are given in Table 4.5. Figures 4.20 (a) and 4.20 (b) shows the experimentally determined splash fractions versus the predicted values.

Table 4.5 Constants in the correlations for the average splash fraction.

\begin{tabular}{|c|c|c|c|c|}
\hline Constant & $\begin{array}{c}\mathbf{2 5} \mathbf{m m} \text { slat } \\
\text { Eq. } 4.15\end{array}$ & $\begin{array}{c}\mathbf{1 0} \mathbf{m m} \text { slat } \\
\text { Eq. } 4.16\end{array}$ & $\begin{array}{c}\mathbf{5} \mathbf{~ m m} \text { slat } \\
\text { Eq. } 4.16\end{array}$ & $\begin{array}{c}\mathbf{2} \mathbf{m m} \text { slat } \\
\text { Eq. } 4.16\end{array}$ \\
\hline $\mathrm{c}_{1}$ & -1.930 & 4.882 & 6.613 & 10.737 \\
$\mathrm{c}_{2}$ & 67.471 & 22.930 & 8.200 & 6.805 \\
$\mathrm{c}_{3}$ & 101.876 & -2.301 & -12.550 & -12.996 \\
$\mathrm{c}_{4}$ & -298.003 & 3.242 & 13.700 & 14.766 \\
$\mathrm{c}_{5}$ & -62.009 & $5.327 \times 10^{-2}$ & $1.628 \times 10^{-2}$ & $3.578 \times 10^{-2}$ \\
$\mathrm{c}_{6}$ & 36.996 & 8.061 & $5.506 \times 10^{-1}$ & $1.210 \times 10^{-1}$ \\
$\mathrm{c}_{7}$ & $9.383 \times 10^{-2}$ & 2.598 & 12.551 & 15.575 \\
$\mathrm{c}_{8}$ & $4.466 \times 10^{-1}$ & -2.977 & -1.792 & -2.148 \\
$\mathrm{c}_{9}$ & -4.454 & - & - & - \\
$\mathrm{c}_{10}$ & 2.086 & - & - & - \\
$\mathrm{R}^{2}$ & 0.969 & 0.965 & 0.956 & 0.963 \\
\hline
\end{tabular}
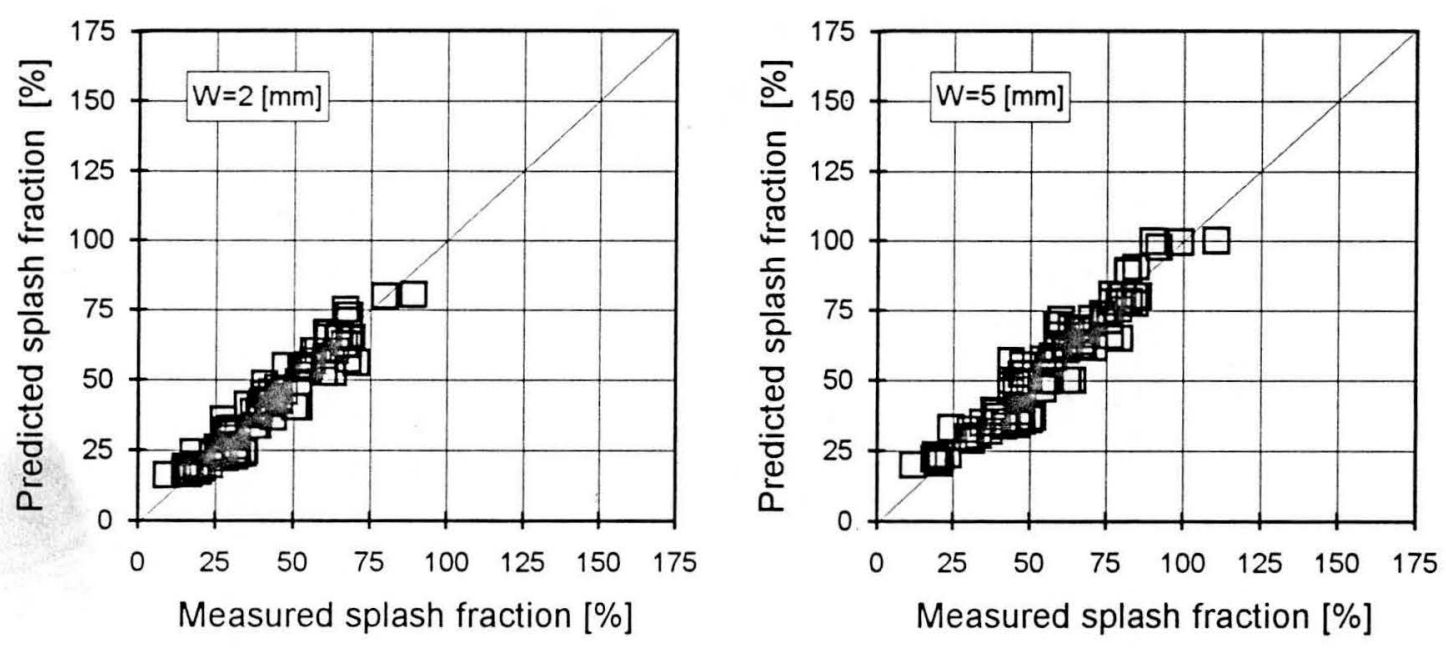

Figure 4.20(a) Accuracy of the correlations for the average splash fraction on narrow slats. 

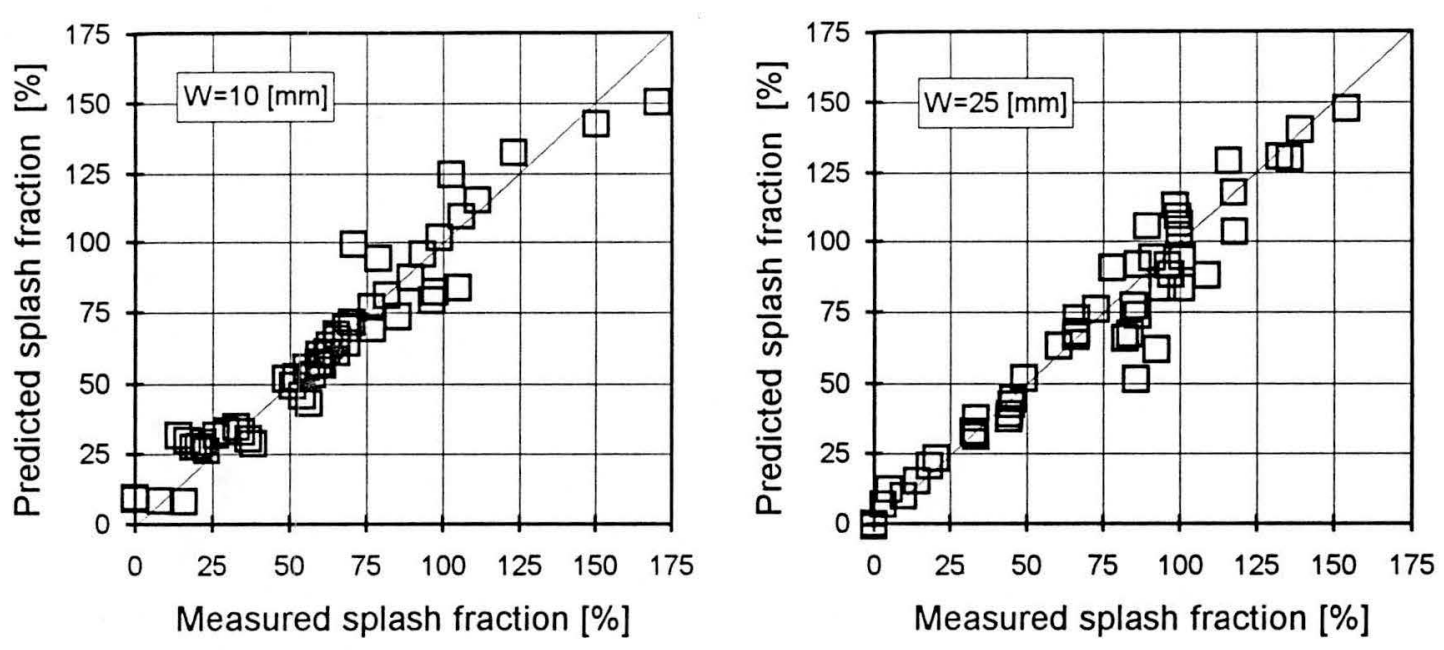

Figure 4.20(b) Accuracy of the correlations for the average splash fraction on wide slats.

Figures $4.21,4.22,4.23$ and 4.24 show the predicted variation of the average splash fraction on the four slats listed in Table 4.5 for the case where each slat is covered by a $0.5 \mathrm{~mm}$ thick water film. Generally, it can be seen that the average splash fraction data shows a peak at drop diameters between 2 and $3 \mathrm{~mm}$. The kinetic energy of smaller drops is absorbed by the water film without the formation of any splash drops. For large drops striking a slat, the contribution of the film to the volume of water splashed from the slat increases as the slat width increases. With narrow slats, a larger fraction of the large drops is lost due to cutting and consequently not all the kinetic energy of the incoming drop contributes to splashing.

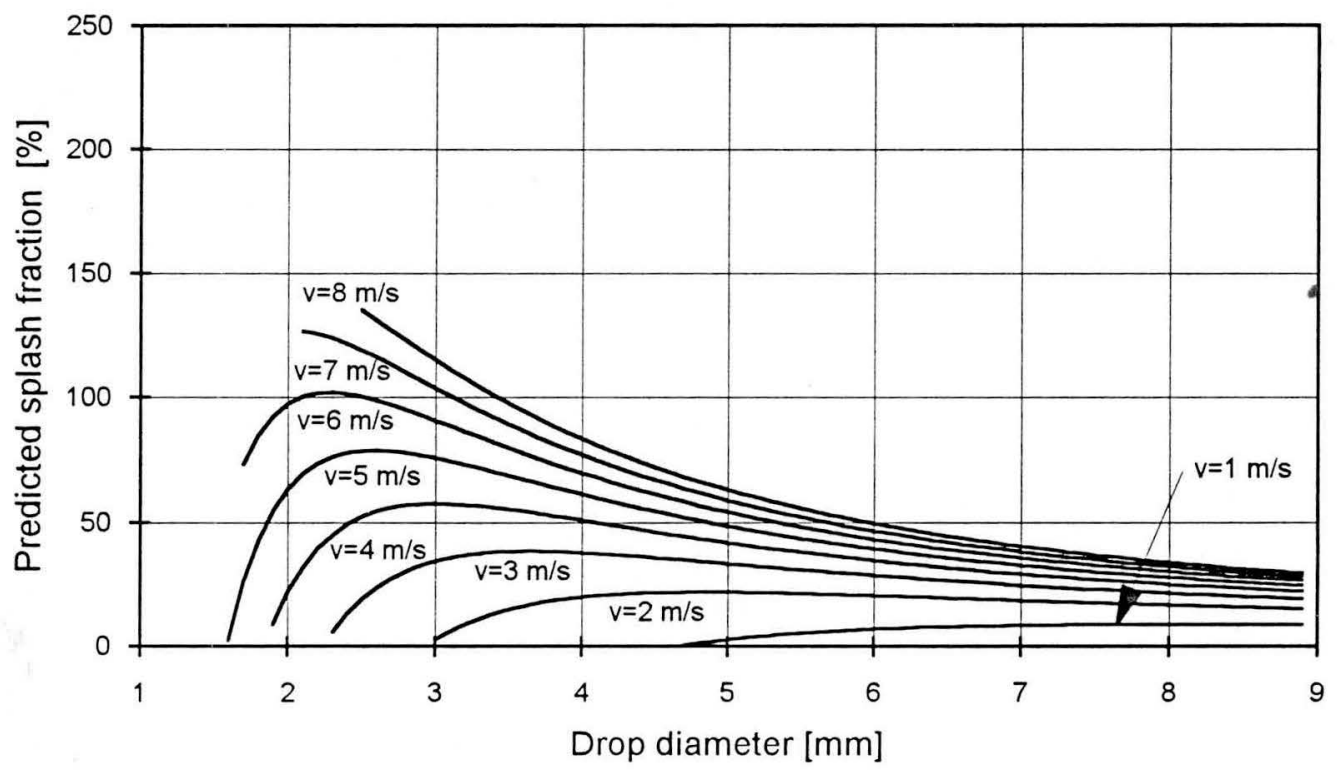

Figure 4.21 Average splash fractions on a $2 \mathrm{~mm}$ wide slat covered by a $0.5 \mathrm{~mm}$ thick water film for various combinations of drop velocity and drop size. 


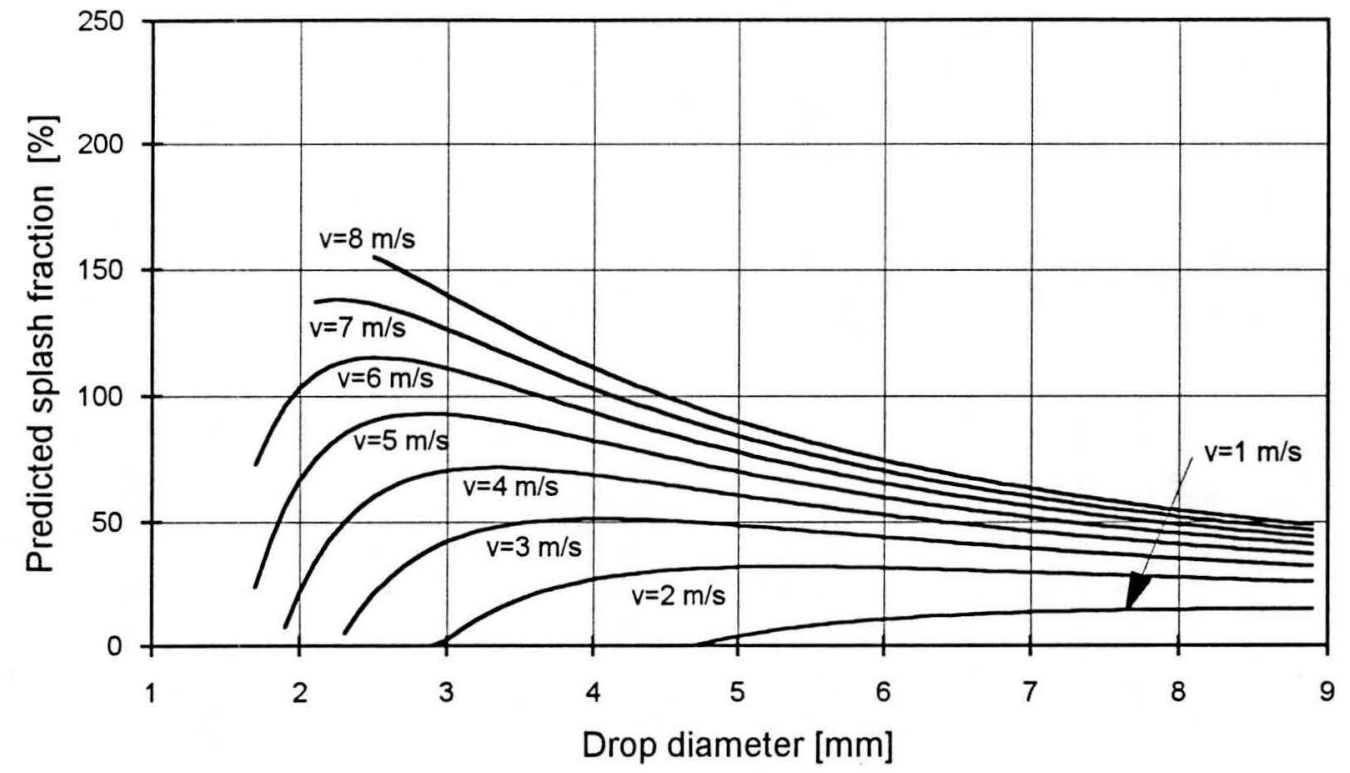

Figure 4.22 Average splash fractions on a $5 \mathrm{~mm}$ wide slat covered by a $0.5 \mathrm{~mm}$ thick water film for various combinations of drop velocity and drop size.

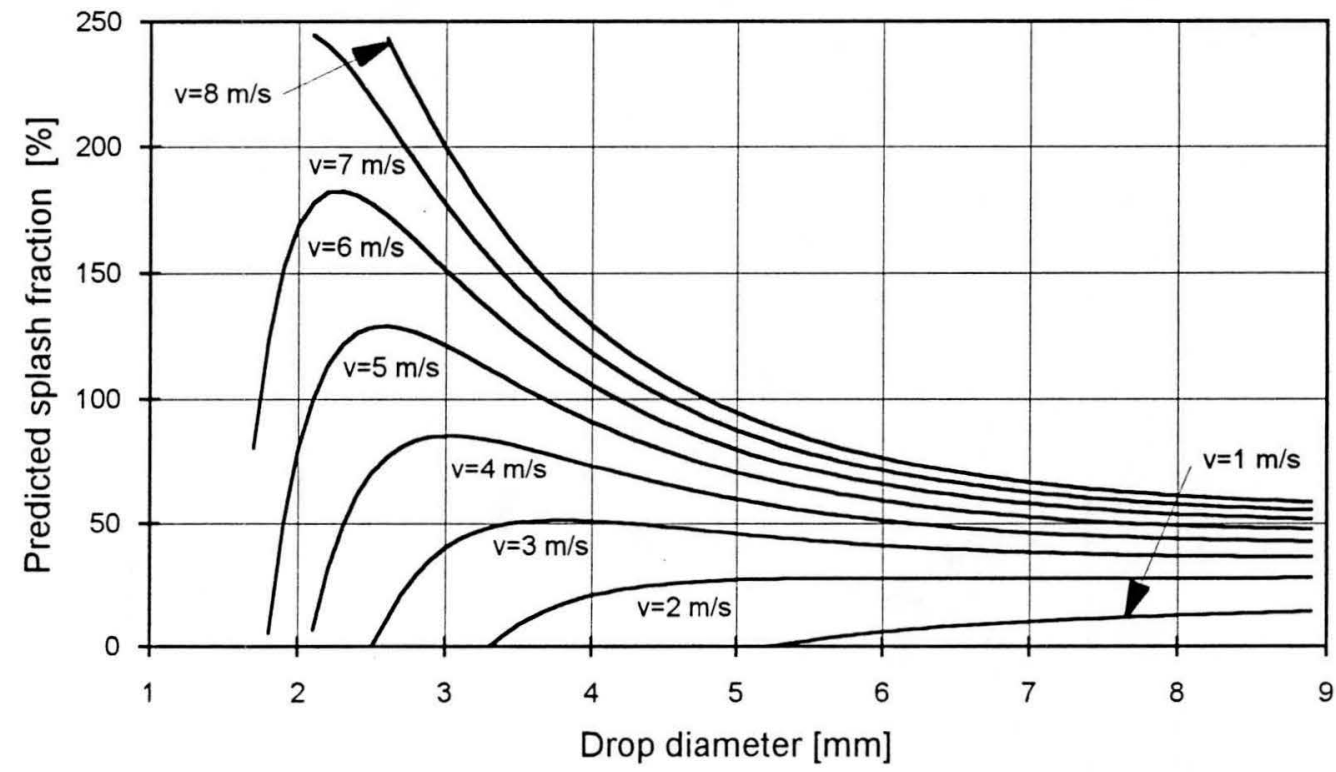

Figure 4.23 Average splash fractions on a $10 \mathrm{~mm}$ wide slat covered by a $0.5 \mathrm{~mm}$ thick water film for various combinations of drop velocity and drop size. 


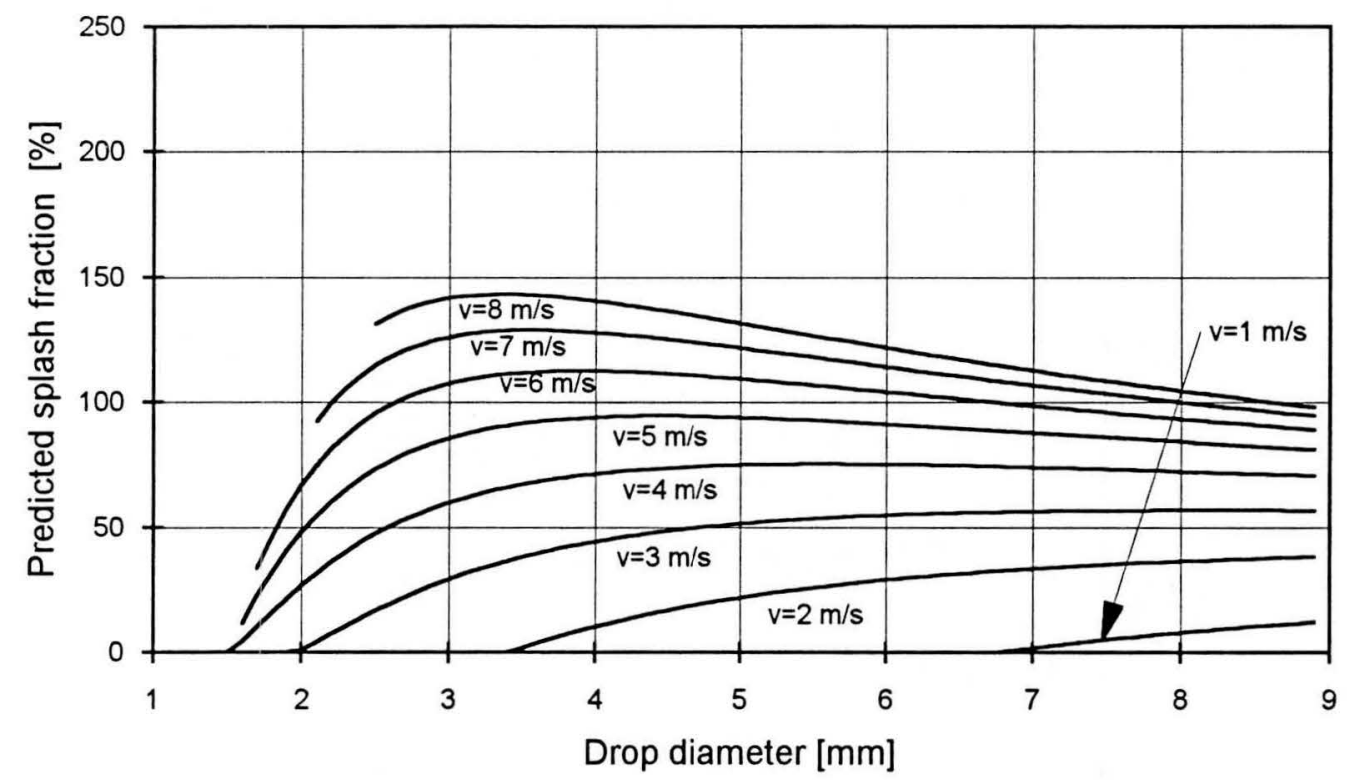

Figure 4.24 Average splash fractions on a $25 \mathrm{~mm}$ wide slat covered by a $0.5 \mathrm{~mm}$ thick water film for various combinations of drop velocity and drop size.

The average splash fractions for the wider slats are more dependant on the film thickness than that for the narrow slats. Figure 4.25 shows the variation of average splash fraction for a 25 $m m$ slat covered by a $1 \mathrm{~mm}$ thick water film. Note that small drops which splashed when the water film was $0.5 \mathrm{~mm}$ thick (see Figure 4.24) do not splash at all when the film is $1 \mathrm{~mm}$ thick.

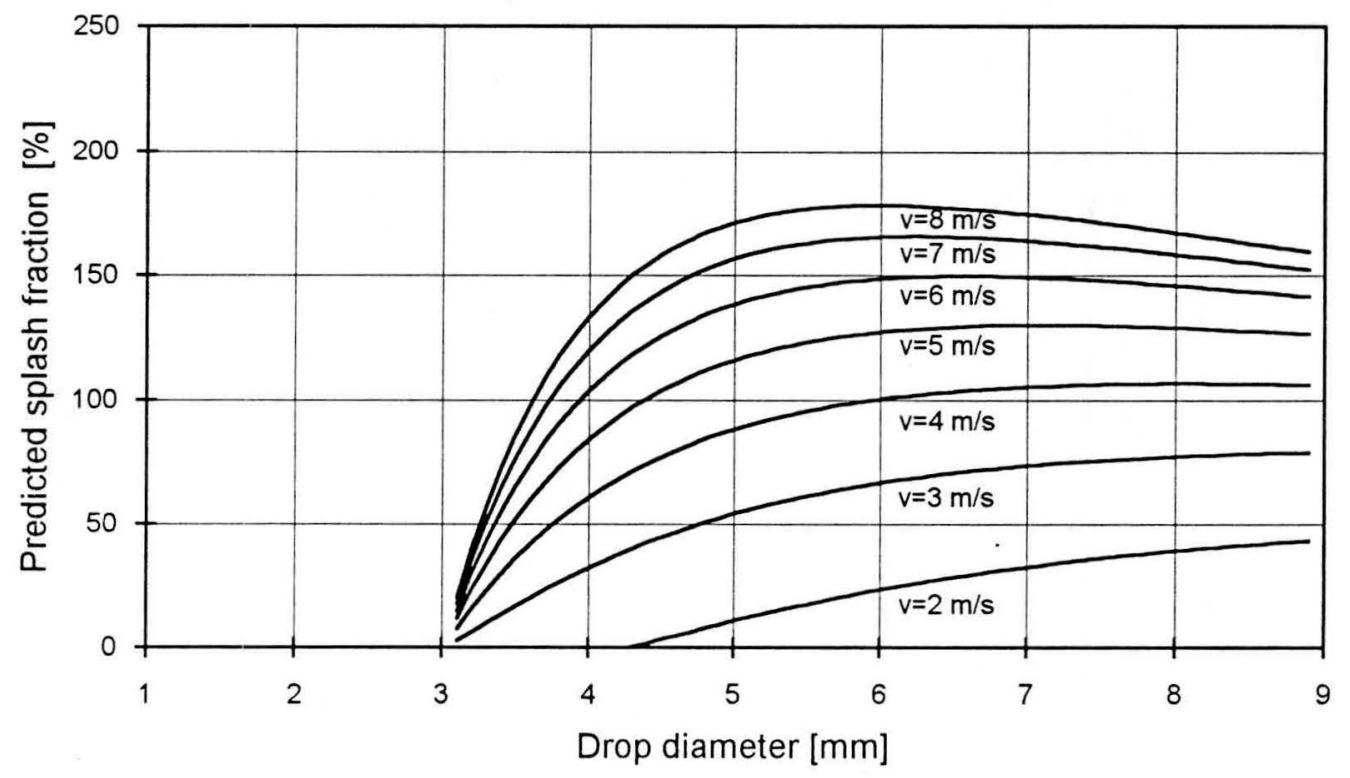

Figure 4.25 Average splash fractions on a $25 \mathrm{~mm}$ wide slat covered by a $1 \mathrm{~mm}$ thick water film for various combinations of drop velocity and drop size. 
Linear interpolation can be used to determine the average splash fractions for other slat widths. Extrapolation beyond slat widths of $25 \mathrm{~mm}$ is expected to overpredict the splash fraction for low energy impacts (small drops or low velocities) since a large fraction of the splash drops is expected to fall back onto the wide slat for impacts far from the edges of the slat.

\subsubsection{Splash and cutting drop size distributions}

The distribution of the drops leaving the impact point after the impact of a drop on a narrow slat covered by a thin film of water, is very important in determining the thermal performance of cooling tower splash pack. Due to the lack of suitable data to describe the distribution of drops formed in this manner, it was deemed necessary to obtain suitable data experimentally.

The following technique, based on the method used by Eigel and Moore [83EI1], was developed to measure the size distribution of the drops formed by cutting/splashing: (i) small square Petri dishes $(100 \times 100 \times 20 \mathrm{~mm}$ or $120 \times 120 \times 20 \mathrm{~mm})$ filled with silicone oil were placed at various positions around the impact point on the slat, (ii) a number of drops, of a known size, were allowed to strike the slat at a given position while the liquid film thickness on the slat was held at a fixed value, (iii) the splash drops caught in the silicone oil was then photographed using a light box shown in Figure 4.26, and (iv) finally the photographs are enlarged, scanned and analysed digitally to count and measure the individual drops using the technique described in Appendix B.

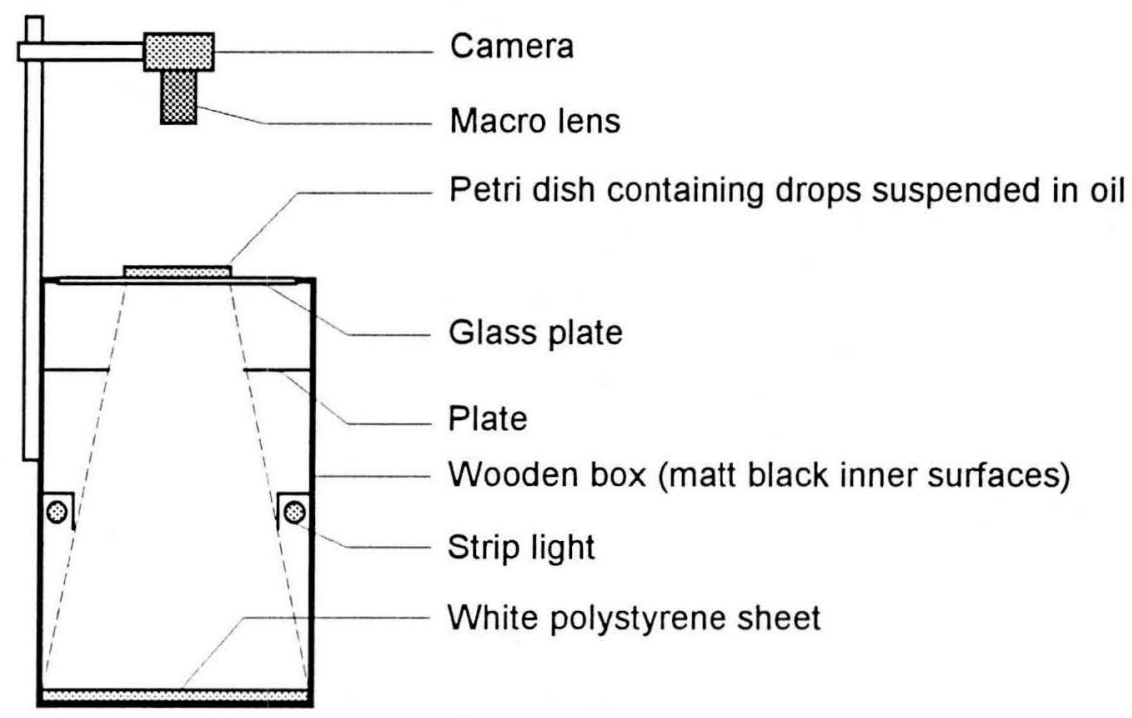

Figure 4.26 Layout of the light box used to photograph the water drops caught in the oil filled Petri dishes.

The photography of the drops required some trial-and-error experimentation to obtain the optimum contrast between the drops and the background of the photographs. By using the so- 
called shadowgraphing method, in which the shadows of the drops are photographed, the outer perimeters of the drops in the oil show up as dark circles on a light background. The photographs were taken in a dark room with the oil filled trays containing the drops which were to be photographed, placed on a light box. The light box, shown in Figure 4.26, provided almost parallel light illuminating the drops from behind. A Pentax Spotmatic F $35 \mathrm{~mm}$ single lens reflex camera with a $100 \mathrm{~mm}$ Takumar macro lens and 40 ASA Technical Pan film was used to photograph the drops. The rest of apparatus used in these experiments, i.e. the test slats, drop generating system, the drop detector and the drop catcher, were the same as that described in the previous part of this chapter.

This method of measuring the drop sizes has the following advantages:

i) The method requires no calibration since the drops stay perfectly spherical in the thick layer of silicone oil. After a few hours in the oil, the larger water drops tend to wet the bottom of the Petri dish and deform.

ii) By using silicone oil with a kinematic viscosity of $200 \mathrm{cSt}\left(200 \times 10^{-6} \mathrm{~m}^{2} / \mathrm{s}\right)$ and a density of $970 \mathrm{~kg} / \mathrm{m}^{3}$ at $25^{\circ} \mathrm{C}$, it was observed that large water drops do not break upon impact with the surface of the oil even at velocities approaching the terminal velocity of the particular drop size. This is not always the case if water-sensitive paper or photographic paper is used to intercept the splash drops.

iii) The photographs form a permanent record of the drops for further processing.

iv) This method of drop size measurement does not require the use of complex and/or expensive apparatus, except for a camera.

The main disadvantage of this method is the time consuming manual post-processing required. This disadvantage was partially offset by the digital image processing technique used to count the drops.

Very small drops, $\mathrm{d}_{\mathrm{s}}<0.1 \mathrm{~mm}$, tend to bounce on the surface of the oil without penetrating leading to the loss of some of the small drop over the edge of the Petri dish. Many of these small drops submerge near the edges of the Petri dishes after bouncing on the oil surface and colliding with the protruding edge of the Petri dish. Many of these drops near the edges of the Petri dishes were not recorded by the scanner since the only the centre portion of the image was digitised. This is not a very serious disadvantage since drops smaller than $0.1 \mathrm{~mm}$ represents a very small fraction of the total splash drop mass. The digital image processing system is capable of detecting drops smaller than $0.1 \mathrm{~mm}$. The $300 \mathrm{dpi}$ (dots per inch) resolution of the digital image scanner translates to a pixel size of $0.05 \times 0.05 \mathrm{~mm}^{2}$, assuming that the photographic images of the drops were enlarged by a factor of 1.75 . A 50 and a $100 \mu$ $m$ drop corresponds roughly to one and four pixels, respectively, on the digital image. 
There is no clear transition between the cutting and splashing phenomena during drop impacts on narrow slats. On very narrow slats, $d_{i} / W>3$, the cutting phenomenon dominates the drop break-up, while pure splashing can only be observed during drop impacts far from the edges of a slat, i.e. in the centre of a wide slat.

\section{Cutting}

A series of experiments was conducted to investigate the spectrum of drop sizes formed by pure cutting. These experiments were conducted by allowing water drops to fall onto narrow metal slats and measuring the sizes of the drops formed with the drop-in-oil technique described above. Two slats were used, one $0.3 \mathrm{~mm}$ wide and the other $2 \mathrm{~mm}$ wide. The data obtained during these experiments is given in Appendix M.

Figure 4.27 shows the typical drop size distribution data for central drop impacts $(\chi=0)$ on the $0.3 \mathrm{~mm}$ wide slat, obtained in these experiments together with data by Yao et al. [88YA1]. It can be observed from Figure 4.27 that more than $50 \%$ of the mass of water falls in the size zone $0.7 d_{i}$ to $0.9 d_{i}$, which is in rough agreement with the simplified model proposed in Chapter 3 .

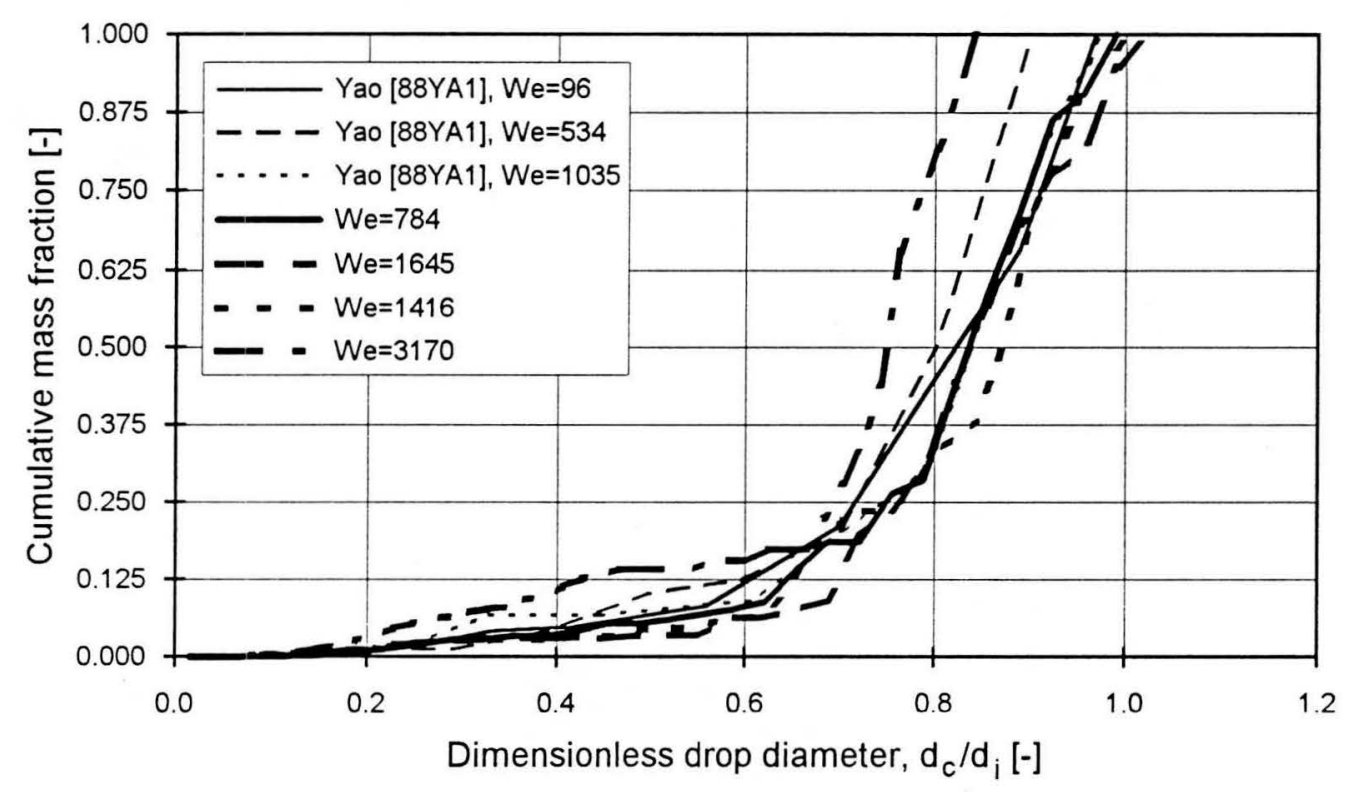

Figure 4.27 Drop size distributions formed after drop impact on narrow slats, $d_{i} / W>3$.

At very low Weber numbers (corresponding to drop fall heights of around $0.25 \mathrm{~m}$ ) it was observed that a drop impacting the $0.3 \mathrm{~mm}$ wide split in two equal halves which then recoalesced after falling past the slat to form one large drop of approximately the same size as the incoming drop. Yao et al. [88YA1] did not observe this, probably due to the fact that their 
experiments were conducted using thin slats which were heated to above the Leidenfrost temperature. (The Leidenfrost temperature is the critical temperature at which a hot surface in contact with a liquid will just wet. At higher temperatures a thin vapour film will form between the surface and the liquid).

Table 4.6 Summary of Sauter mean drop sizes of drop distributions formed by cutting.

\begin{tabular}{|c|c|c|c|r|c|c|}
\hline Source & $\boldsymbol{\chi}$ & $\begin{array}{c}\mathbf{d}_{\mathbf{i}} \\
m m\end{array}$ & $\begin{array}{c}\mathbf{W} \\
m m\end{array}$ & $\begin{array}{c}\mathbf{W e} \\
-\end{array}$ & $\begin{array}{c}\mathbf{d}_{\mathbf{i}} / \mathbf{W} \\
-\end{array}$ & $\begin{array}{c}\mathbf{d}_{\mathbf{3 2}} / \mathbf{d}_{\mathbf{i}} \\
-\end{array}$ \\
\hline Yao et al. [88YA1] & -1 to 1 & 1.27 & 0.33 & 96 & 3.8 & 0.74 \\
Yao et al. [88YA1] & -1 to 1 & 1.27 & 0.33 & 534 & 3.8 & 0.57 \\
Yao et al. [88YA1] & -1 to 1 & 1.27 & 0.33 & 862 & 3.8 & 0.52 \\
Yao et al. [88YA1] & -1 to 1 & 1.52 & 0.33 & 1035 & 4.6 & 0.42 \\
Yao et al. [88YA1] & -1 to 1 & 1.00 & 0.18 & 317 & 5.7 & 0.80 \\
Yao et al. [88YA1] & -1 to 1 & 1.00 & 0.18 & 1322 & 5.7 & 0.80 \\
Present data & 0 & 3.30 & 0.30 & 183 & 11.0 & 0.943 \\
Present data & 0 & 3.30 & 0.30 & 784 & 11.0 & 0.752 \\
Present data & 0 & 3.30 & 0.30 & 1645 & 11.0 & 0.766 \\
Present data & 0 & 5.60 & 0.30 & 373 & 18.7 & 0.862 \\
Present data & 0 & 5.60 & 0.30 & 1416 & 18.7 & 0.751 \\
Present data & 0 & 5.60 & 0.30 & 3170 & 18.7 & 0.595 \\
Present data & 0.51 & 5.60 & 0.30 & 1416 & 18.7 & 0.736 \\
Present data & 0.51 & 5.60 & 0.30 & 3170 & 18.7 & 0.596 \\
Present data & 0 & 6.10 & 2.00 & 802 & 3.1 & 0.519 \\
Present data & 0 & 6.10 & 2.00 & 1553 & 3.1 & 0.282 \\
Present data & 0 & 6.10 & 2.00 & 2255 & 3.1 & 0.225 \\
Present data & 0 & 6.10 & 2.00 & 2902 & 3.1 & 0.213 \\
Present data & 0 & 6.10 & 2.00 & 3492 & 3.1 & 0.177 \\
\hline
\end{tabular}

It can also be seen from Figure 4.27 that there is a general trend towards smaller drop sizes at higher Weber numbers. Table 4.6 lists the available data on the Sauter mean drop size of the drops formed by cutting for a range of incoming drop sizes, slat widths, Weber numbers etc. . The data listed in Table 4.6 was correlated by the following relation

$$
\left(\frac{d_{32}}{d_{i}}\right)=\left(0.958+9.921\left(d_{i} / W\right)^{-0.662}\right) W e^{\left(-0.113-0.808\left(d_{i} / W\right)^{-0.895}\right)}
$$

where

$$
W e=\frac{\rho_{w} v^{2} d_{i}}{\sigma}
$$

The predicted and experimental data is compared graphically in Figure 4.28. It can be concluded that the correlation is a fair fit of the data, although there are two points which differ almost $30 \%$ from the experimental data. 


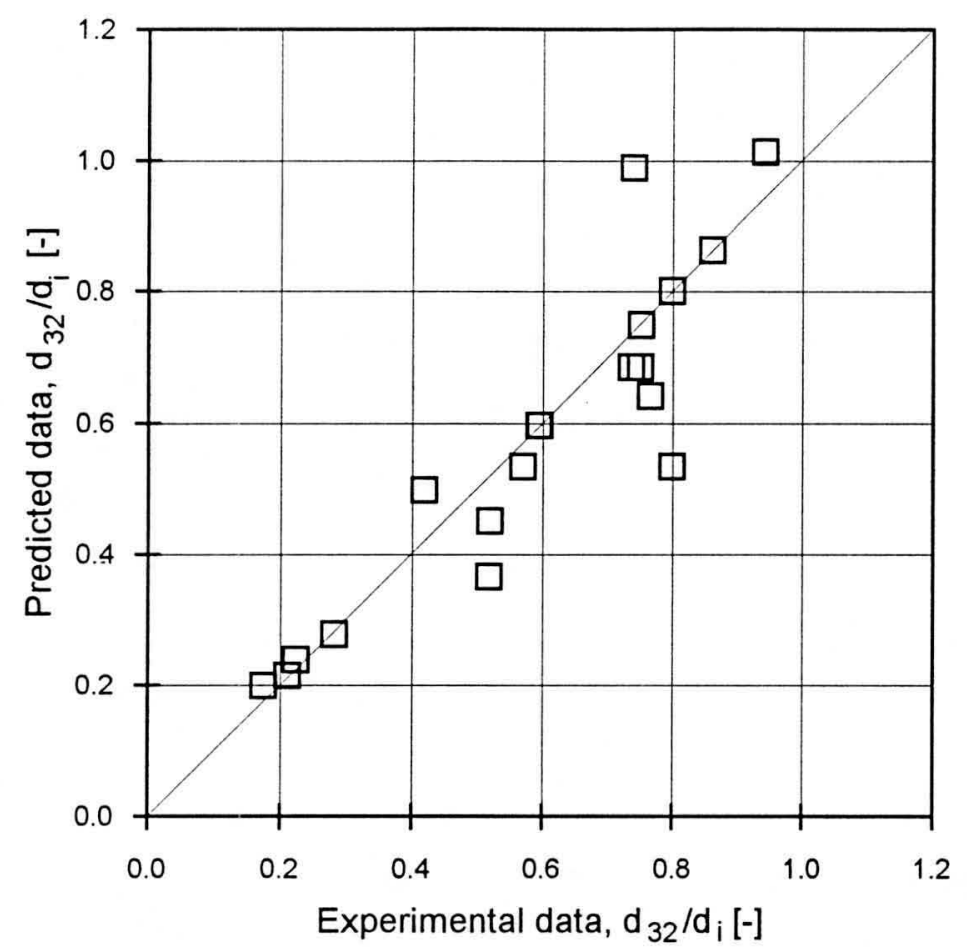

Figure 4.28 Correlation accuracy for the size of the drops formed by cutting.

The variation of dimensionless Sauter mean diameter with slat width and Weber number is given in Figure 4.29. The decrease in drop size with increasing Weber number can be attributed to the higher impact kinetic energy which results in more violent drop break-up.
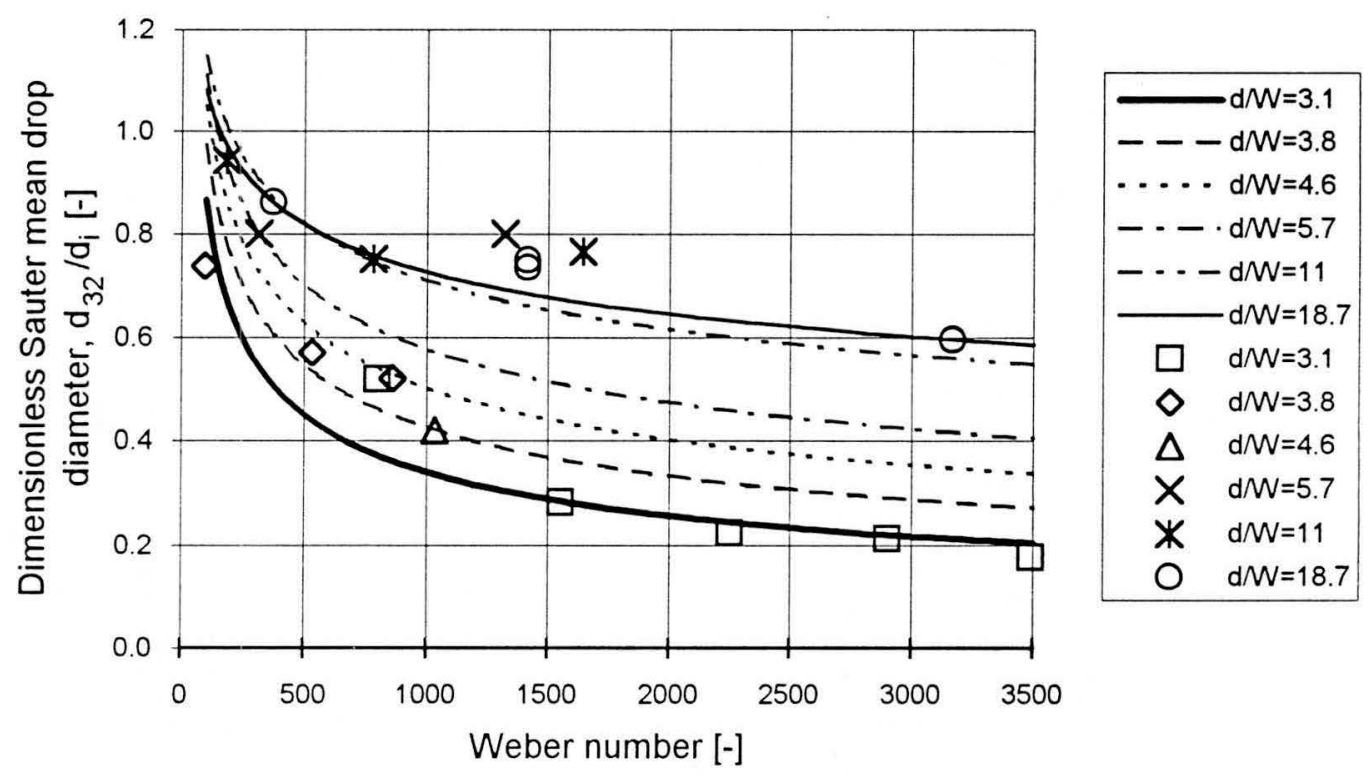

Figure 4.29 Comparison between the experimental data and correlations for the Sauter mean drop size after impact on a narrow slat with increasing Weber number. 
At a constant Weber number the impacts on narrow slats yield much larger drops than the impacts on wider slats. The simplified model proposed in Chapter 3 holds approximately for low Weber number impacts $(\mathrm{We}<1000)$ on narrow slats $\left(\mathrm{d}_{\mathrm{i}} / \mathrm{W}>10\right)$.

\section{Splashing}

A series of experiments was conducted to investigate the distribution of drop sizes formed during drop impact on a hard smooth surface covered by a thin water layer. To eliminate any drops formed by cutting, the drops were allowed to fall onto the centre of a $25 \mathrm{~mm}$ wide slat. The splash drop sizes were measured with the drop-in-oil technique described above at different positions around the impact point. The incoming drop size was varied between 3.3 and $7.5 \mathrm{~mm}$; the water film thickness was varied between 0.3 and $0.85 \mathrm{~mm}$, while the drop fall height was varied between $0.25 \mathrm{~m}$ and $2.5 \mathrm{~m}$. The data obtained during these experiments is given in Appendix M. Figure 4.30 shows a sample of the drop distribution data obtained in these experiments.

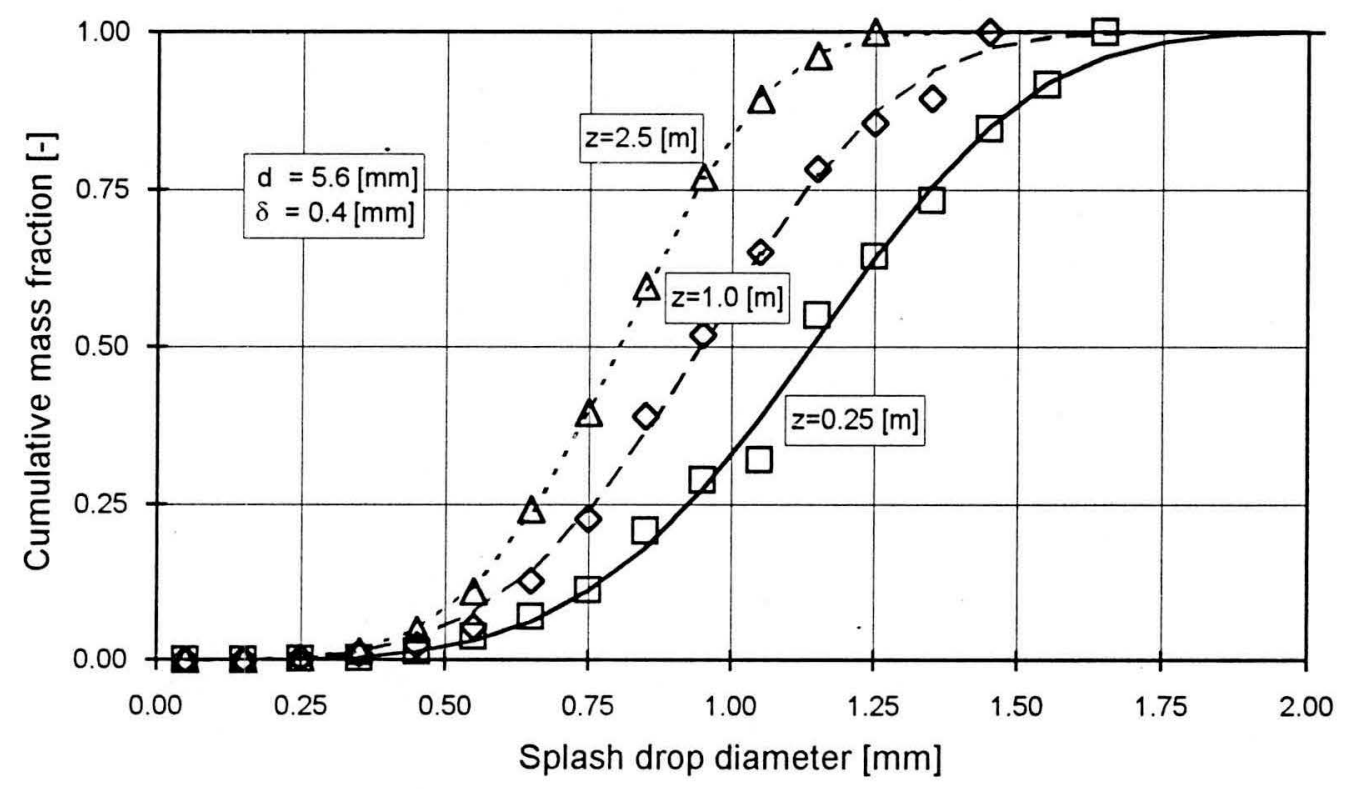

Figure 4.30 Typical splash drop size distribution data and the corresponding Rosin-Rammler correlations for different drop fall heights.

It was found that the cumulative mass fraction data for the drops formed by splashing could be correlated by the Rosin-Rammler distribution function. The Rosin-Rammler distribution function expresses the mass fraction of drops smaller than a given size as

$$
R(d)=1-\exp \left(-\left(\frac{d}{d_{R R}}\right)^{n_{R R}}\right)
$$


with

$$
\mathrm{d}_{\mathrm{RR}}=\mathrm{d}_{50}\left(0.6931^{-\left(1 / \mathrm{n}_{\mathrm{RR}}\right)}\right)
$$

Table 4.7 shows the Rosin-Rammler parameters, $d_{R R}$ and $n_{R R}$, which were calculated for each of the data sets obtained in this study and the relevant data from the thesis of Mutchler [70MU1].

Table 4.7 Rosin-Rammler distribution data describing the mass distribution of splash drops.

\begin{tabular}{|c|c|c|c|c|c|c|c|}
\hline Source & $\mathbf{Z}$ & $\mathbf{d}_{\mathbf{i}}$ & $\boldsymbol{\delta}$ & $\mathbf{v}_{\mathbf{i}}$ & $\mathbf{d}_{\mathbf{5 0}}$ & $\mathbf{n}_{\mathbf{R R}}$ & $\mathbf{d}_{\mathbf{R R}}$ \\
& $m$ & $m m$ & $m m$ & $m / s$ & $\dot{m} m$ & - & $m m$ \\
\hline Present data & 1.00 & 3.32 & 0.37 & 4.14 & 0.777 & 4.242 & 0.847 \\
Present data & 2.50 & 3.29 & 0.38 & 5.99 & 0.775 & 4.758 & 0.838 \\
Present data & 1.00 & 3.32 & 0.83 & 4.14 & 1.238 & 4.552 & 1.342 \\
Present data & 2.50 & 3.29 & 0.84 & 5.99 & 1.271 & 4.657 & 1.375 \\
Present data & 0.25 & 5.56 & 0.37 & 2.19 & 1.141 & 4.218 & 1.244 \\
Present data & 1.00 & 5.56 & 0.40 & 4.27 & 0.947 & 3.945 & 1.039 \\
Present data & 2.50 & 5.61 & 0.46 & 6.38 & 0.803 & 4.475 & 0.872 \\
Present data & 0.25 & 5.56 & 0.78 & 2.19 & 1.689 & 5.124 & 1.815 \\
Present data & 1.00 & 5.56 & 0.85 & 4.27 & 1.372 & 4.517 & 1.488 \\
Present data & 2.50 & 5.61 & 0.80 & 6.38 & 1.231 & 4.157 & 1.344 \\
Present data & 0.25 & 7.48 & 0.41 & 2.20 & 1.131 & 3.395 & 1.260 \\
Present data & 1.00 & 7.70 & 0.38 & 4.31 & 1.195 & 3.534 & 1.325 \\
Present data & 2.50 & 7.48 & 0.46 & 6.50 & 1.025 & 3.701 & 1.131 \\
Present data & 0.25 & 7.53 & 0.83 & 2.20 & 1.854 & 3.239 & 2.076 \\
Present data & 1.00 & 7.70 & 0.83 & 4.31 & 1.826 & 3.250 & 2.043 \\
Present data & 2.50 & 7.48 & 0.75 & 6.50 & 1.489 & 2.818 & 1.696 \\
Mutchler [70MU1] & - & 2.96 & 0.50 & 7.96 & 1.112 & 3.005 & 1.256 \\
Mutchler [70MU1] & - & 2.96 & 1.00 & 7.96 & 1.054 & 2.713 & 1.207 \\
Mutchler [70MU1] & - & 4.23 & 0.50 & 8.82 & 0.953 & 2.977 & 1.078 \\
Mutchler [70MU1] & - & 4.23 & 1.00 & 8.82 & 1.627 & 2.322 & 1.905 \\
Mutchler [70MU1] & - & 5.59 & 0.50 & 9.07 & 0.960 & 3.144 & 1.079 \\
Mutchler [70MU1] & - & 5.59 & 1.00 & 9.07 & 1.281 & 2.720 & 1.466 \\
Mutchler [70MU1] & - & 3.51 & 0.50 & 8.45 & 1.092 & 3.220 & 1.224 \\
Mutchler [70MU1] & - & 4.80 & 0.50 & 8.97 & 0.919 & 3.125 & 1.034 \\
\hline
\end{tabular}

The Rosin-Rammler shape parameter, $\mathrm{n}_{\mathrm{RR}}$, data and the mass median diameter, $\mathrm{d}_{50}$, data could be correlated by

$$
\mathrm{n}_{\mathrm{RR}}=\left(24.532-75.174\left(\frac{\delta}{\mathrm{d}_{\mathrm{i}}}\right)\left(\frac{\mathrm{We}_{\mathrm{i}}}{\mathrm{We}_{\text {ref }}}\right)^{0.74}\right)\left(0.149+6.801 \times 10^{-4}\left(\frac{\mathrm{KE}}{\mathrm{KE}_{\text {ref }}}\right)^{-0.76}\right)
$$

and 


$$
\begin{aligned}
\left(\frac{d_{50}}{d_{m}}\right)= & 3.08 \times 10^{-2}+\left(-0.163+4.560 \times 10^{-2}\left(\frac{\delta}{d_{m}}\right)^{-0.34}\right) \times \\
& \left(-0.804-0.619\left(\frac{W_{e}}{W_{\text {ref }}}\right)^{-0.27}\right)\left(-1.738+1.980\left(\frac{\delta}{d_{i}}\right)^{-0.17}\right)
\end{aligned}
$$

where $\mathrm{d}_{\mathrm{m}}$, We and $\mathrm{We}_{\mathrm{ref}}$ are defined as in section 4.3.2 above, and

$$
\mathrm{KE}_{\mathrm{ref}}=0.5\left(\frac{\rho_{\mathrm{w}} \pi \mathrm{d}_{\mathrm{m}}^{3}}{6}\right) \mathrm{v}_{\mathrm{T}}^{2}
$$

The "goodness of fit" of the correlations for $n_{R R}$ and $d_{50}$ for the distribution of the splash drops are given in Figures 4.31(a) and 4.31(b) respectively.
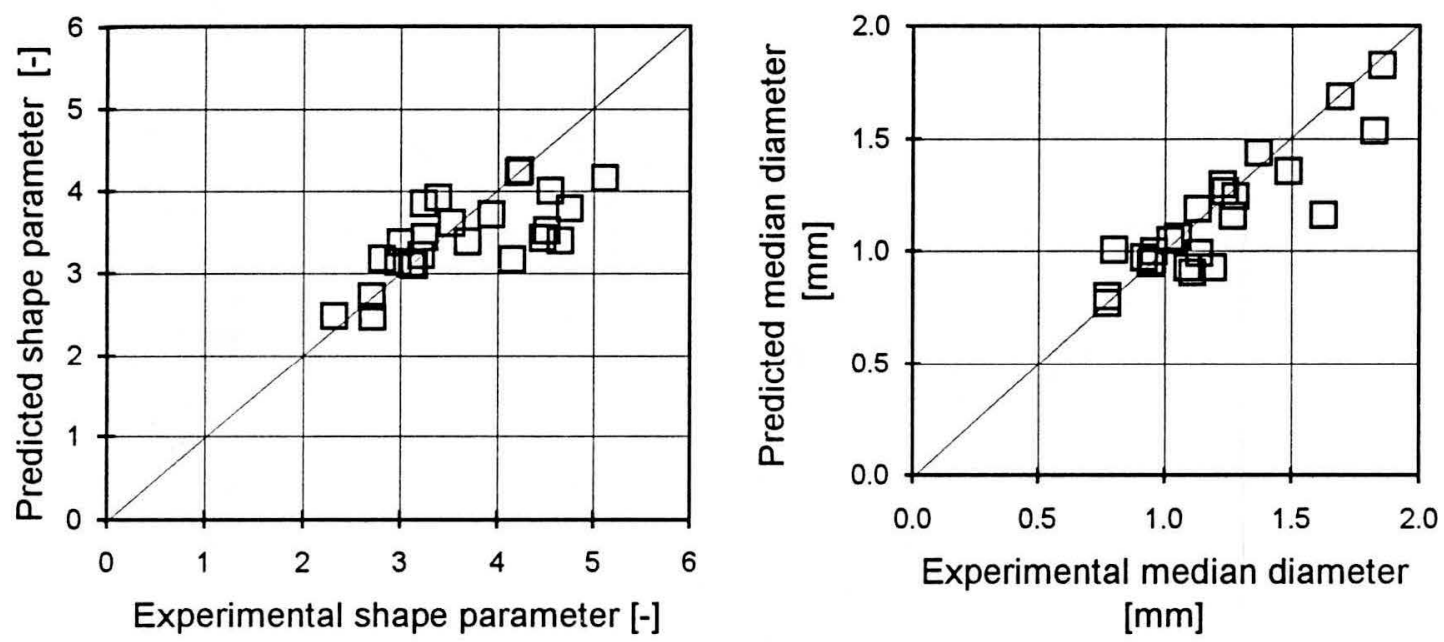

Figure 4.31 Accuracy of the correlations for Rosin-Rammler shape parameter and mass median drop diameter describing the distribution of drops formed by splashing.

The travel distance of each drop from the impact point was known from the position of the oil filled Petri dish and the position of each drop in each Petri dish. Note that the Petri dishes were placed on a horizontal plane approximately $100 \mathrm{~mm}$ below the upper surface of the slat. By employing the approximate splash angle data of Mutchler [70MU1] (or the splash angles observed on the high speed photographs taken of the splash events) together with the known drop position, it was possible to calculate the approximate initial velocity of the splash drops. Assuming a constant splash angle of $60^{\circ}$, the initial splash drop velocity was calculated for each of the cases listed in Table 4.7. The data showed a large degree of scatter, which can be attributed to the assumption of a constant splash angle. During a typical splash, as seen in section 4.3.1, the splash angle varies significantly from the moment of impact until the end of the crown life. Figure 4.32 shows the variation of the average splash drop initial velocity with 
splash drop size. Note that this figure shows that the initial splash drop velocity is not very different for the different incoming drop sizes and impact velocities.

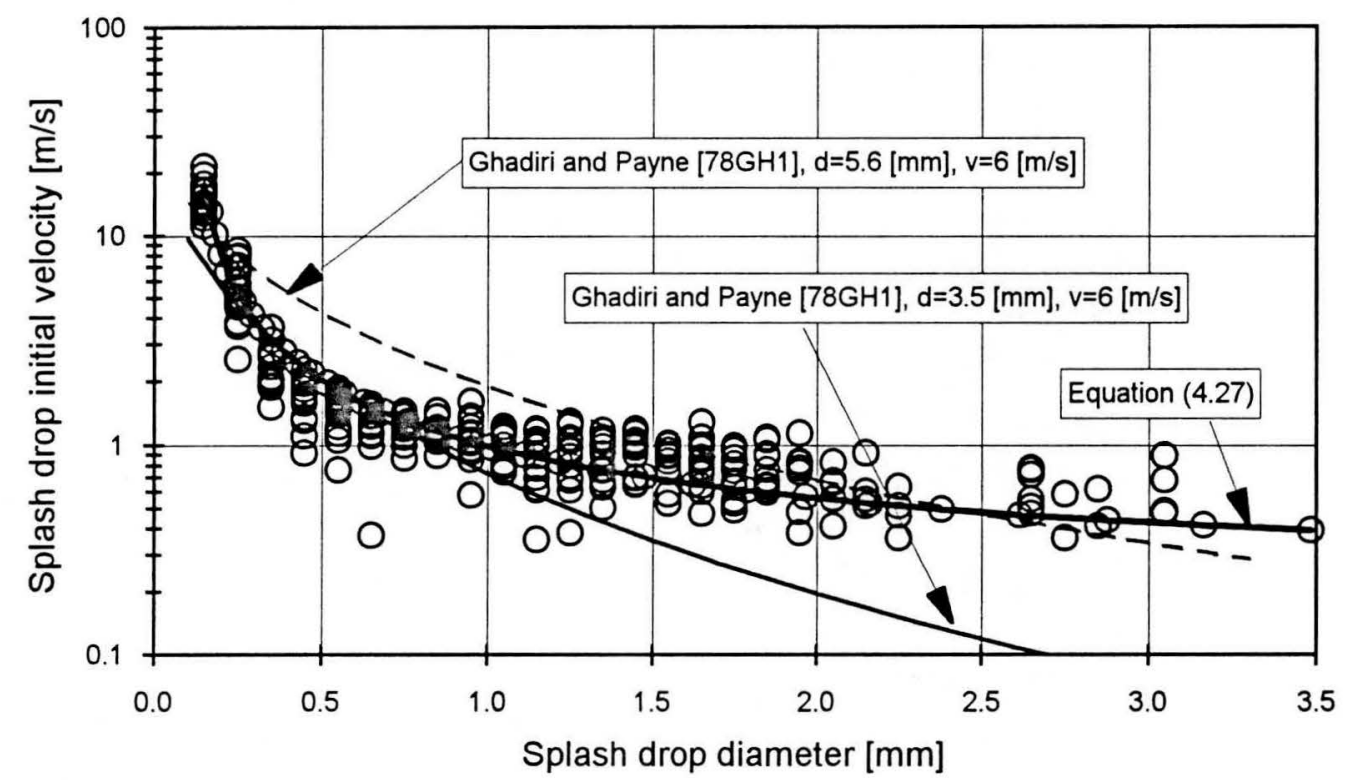

Figure 4.32 Comparison between the experimentally determined mean initial splash drop velocity and the correlation based on the work by Ghadiri and Payne [78GH1].

The initial splash drop velocity data shown in Figure 4.32 could be correlated by

$$
\left(\frac{\mathrm{v}_{\mathrm{si}}}{\mathrm{v}_{\mathrm{T}}\left(\mathrm{d}_{\mathrm{m}}\right)}\right)=1.925 \times 10^{-2}+\left(\frac{7.372 \times 10^{-3}}{\left(\mathrm{~d}_{\mathrm{s}} / \mathrm{d}_{\mathrm{m}}\right)-0.01}\right)
$$

\section{Combined cutting and splashing}

It was not attempted to determine the splash drop distributions and the distribution of drops formed by cutting in the cases where cutting and splashing occurred simultaneously.

A series of experiments was conducted in which water drops were allowed to strike a $5 \mathrm{~mm}$ slat at various positions on the slat. In all the cases the slat was covered by a thin water film. As before the splash/cutting drop sizes were determined by catching the drops in silicone oil and then measuring the drops from photographs taken of the drops in the oil. Figures 4.33 and 4.34 shows typical results obtained in this manner. The curves showing the predicted drop distributions were obtained by combining the splashing and cutting models proposed in Chapter 3 and in the previous part of this section. For the case of drop impacts on the edge of the slat, $x=(W / 2)$, one would expect that the fraction of mass contained in drops formed by cutting would be $50 \%$ of the total mass of drops formed, but as can be seen from the figures 
below this fraction is less than $50 \%$. This is due to the fact that the local splash fraction for a drop impact on the edge of a slat can be more than $50 \%$ (See sections 3.3.1 and 4.3.2).

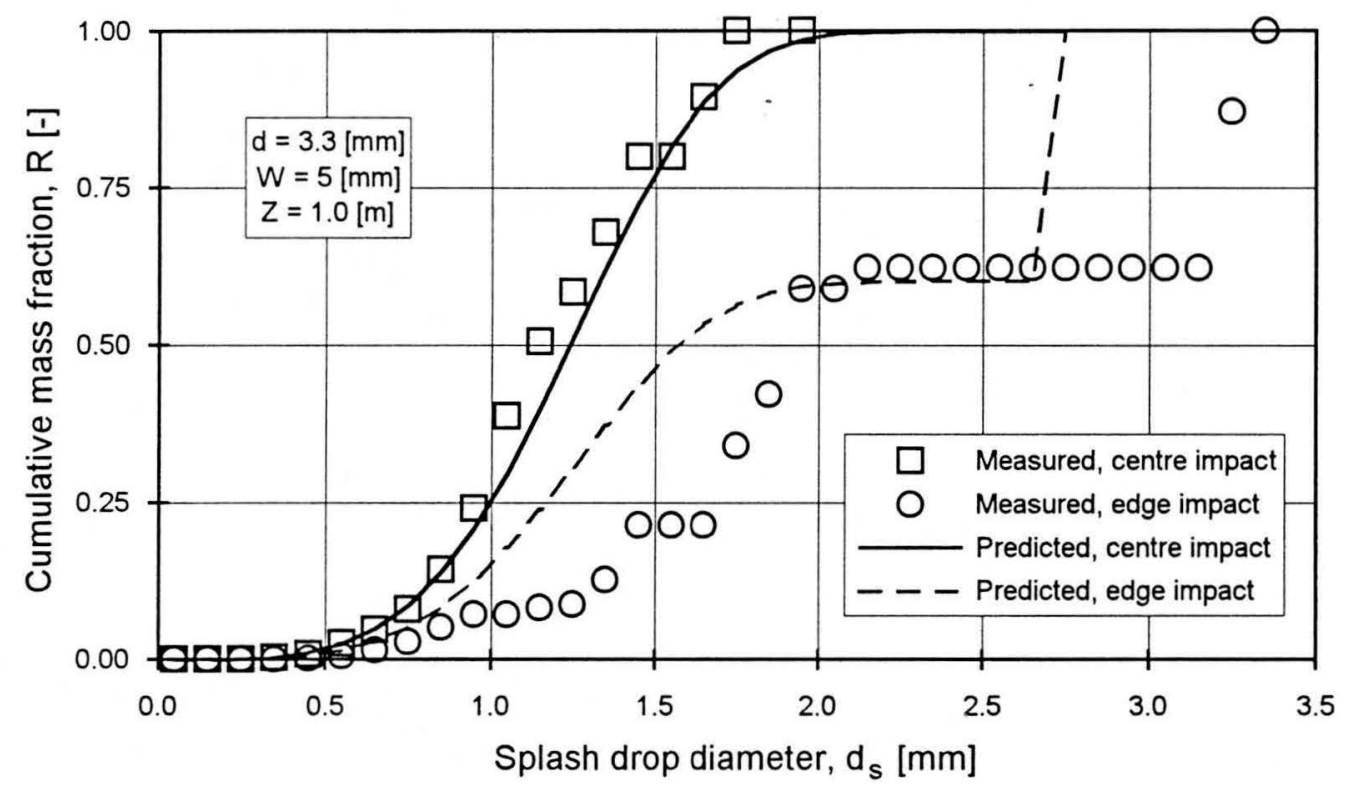

Figure 4.33 Comparison between measured and predicted splash and cutting drop size distribution (small incoming drop).

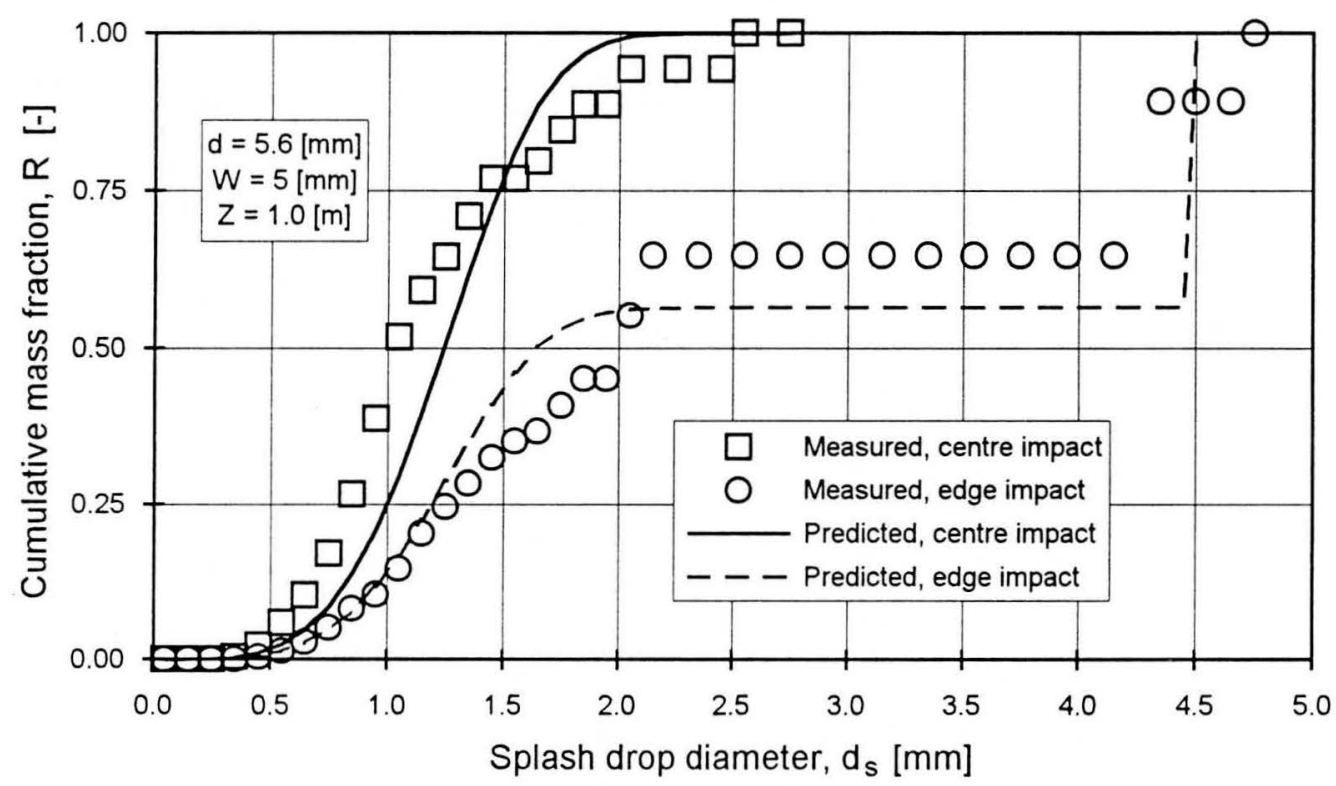

Figure 4.34 Comparison between measured and predicted splash and cutting drop size distributions (large incoming drop).

From these figures it can be concluded that by combining the splashing and cutting models proposed in Chapter 3 and in the previous part of this section, it is possible to approximate the drop size distributions due to combined (simultaneous) splashing and cutting. 


\subsection{Dripping drop size distribution measurements}

Since the water drops dripping from below the slats in the splash pack represent a significant fraction of the total water mass flow rate past each layer of slats, the distribution of dripping drop sizes are of major significance in determining the thermal performance of splash pack. A series of experiments was conducted to measure the distribution of drop sizes dripping from five different types of slat for a range of liquid flow rates up to $140 \mathrm{~g} / \mathrm{ms}$.
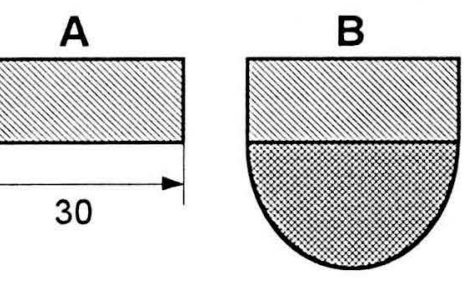

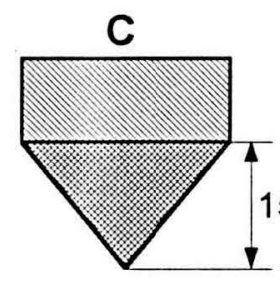

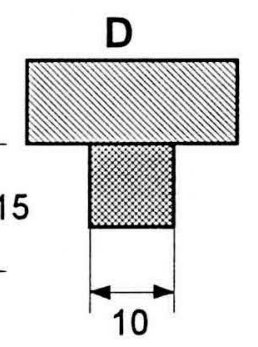

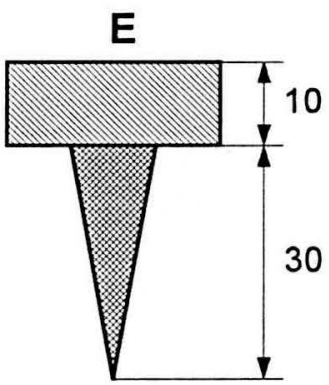

Figure 4.35 Cross sectional profiles of the slats used in the experiments to measure dripping drop sizes. Dimensions in $\mathrm{mm}$.

Figure 4.35 shows the five different slats which were evaluated. The slats which were manufactured from wood, varied from a flat bottomed slat, A, to a sharp edged slat, E. The slats were mounted horizontally below a water distributor as shown in Figure 4.36. The water distributor consisted of a wooden strip with a supply pipe mounted in a groove along the top length of the wooden strip. A water supply pipe with a row of small diameter holes along its length was installed with the holes at the bottom of the groove in the wood strip as shown in Figure 4.36. Two different rotameters were used to measure the water flow rates.
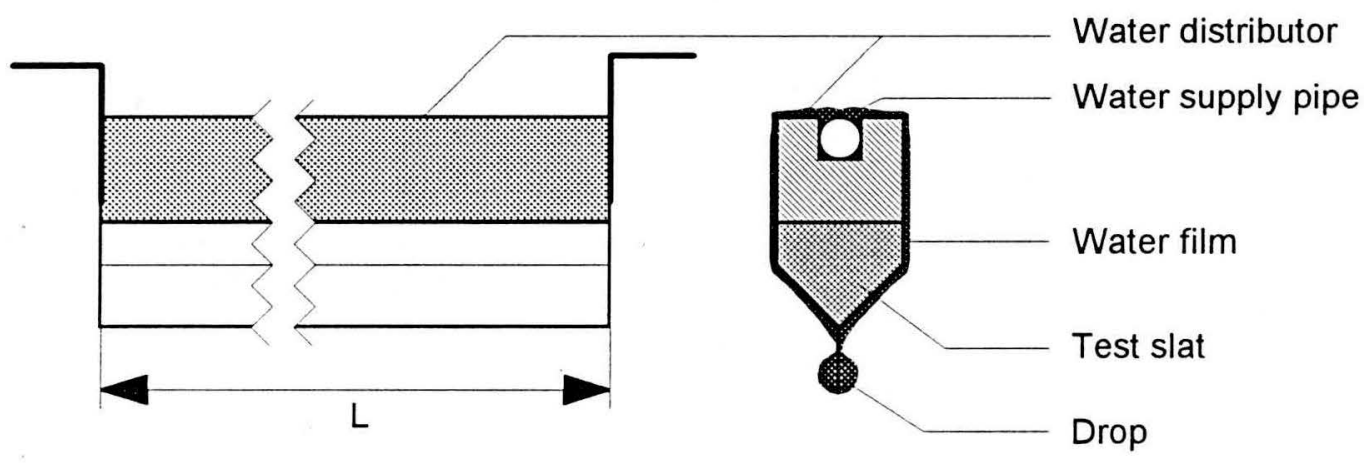

Figure 4.36 Water distributor used to supply water to the slats.

The drops falling from below each slat were photographed at different water flow rates. In order to capture the transparent water drops on the photographic film a photographic technique called shadowgraphing was used. When using this technique the background is evenly illuminated and the shadows of the drops are actually photographed. The layout of the photographic set-up is shown in Figure 4.37. A $35 \mathrm{~mm}$ Pentax Spotmatic F camera fitted with 
a $100 \mathrm{~mm}$ Takumar macro lens and Ilford FP4 black-and-white film was used. To ensure that the moving drops are 'frozen' on the photographs, a flash with a flash duration time in the order of 20 to $30 \mu \mathrm{s}$ was used to illuminate a sheet of white polystyrene behind the test slat.

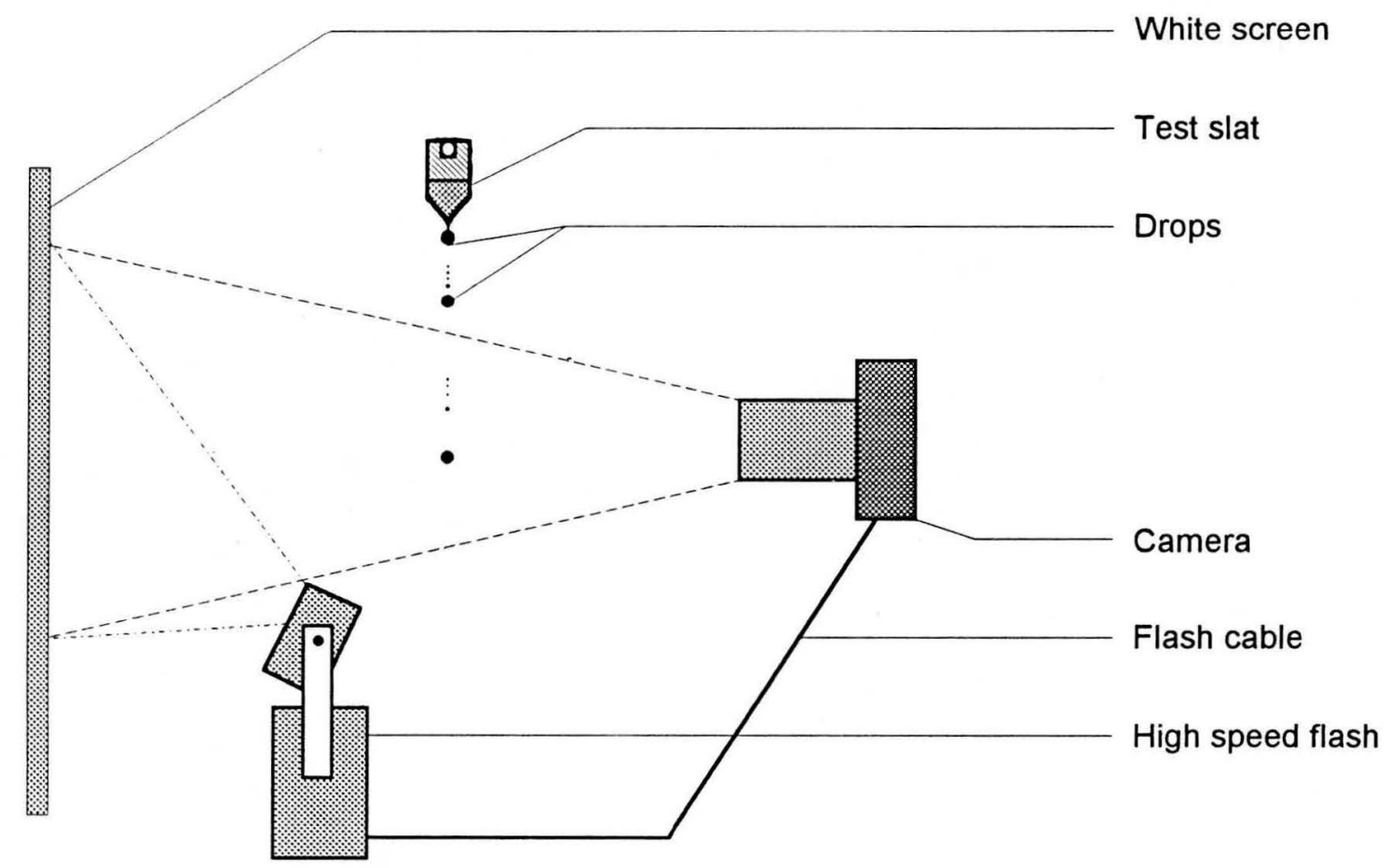

Figure 4.37 Layout of the photographic system used to photograph the drops dripping from below the test slats.

The tests were all conducted using tap water at approximately $15^{\circ} \mathrm{C}$. The surface tension of the water was measured using the drop weight method described in Appendix $F$ and it was find to agree very closely with the surface tension of pure water.

A series of photographs was taken for each combination of water flow rate and slat type. Since the photographs were taken at random times (i.e. the photographs were not synchronised with the dripping of the drops below the slat), it was decided to take more photographs of each case at the low liquid flow rates due to the low drop density. Fewer photographs were taken at the higher liquid flow rates. At the low liquid flow rates 24 photographs were taken at each combination of water flow rate and slat type and at the high liquid flow rates only 6 photographs were taken of each case. Five water flow rates below 140 $\mathrm{g} / \mathrm{ms}$ were used on each slat except on slat A, for which an extra water flow rate was evaluated. Figures 4.38 and 4.39 show typical photographs obtained for low and high liquid flow rates below slat $\mathrm{A}$. 


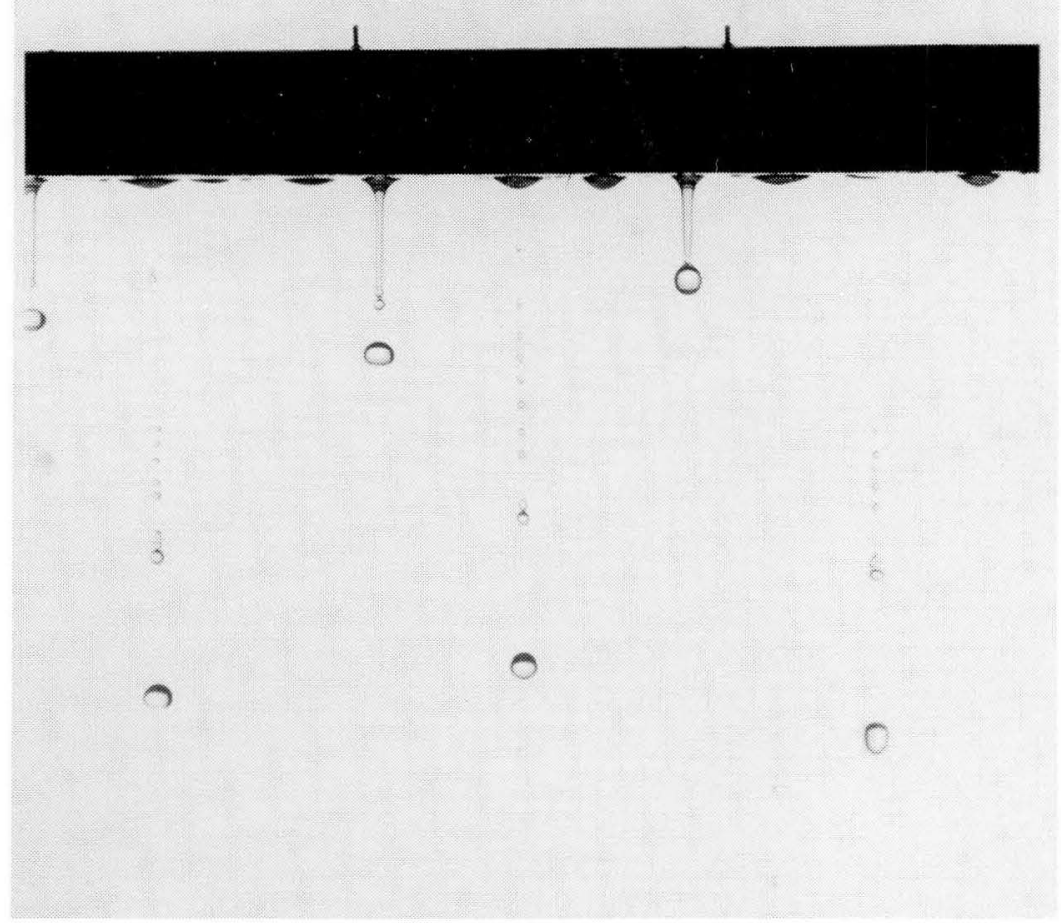

Figure 4.38 Typical photograph of water drops dripping below slat $A$ at a low water flow rate $(\Gamma \approx 16 \mathrm{~g} / \mathrm{ms})$.

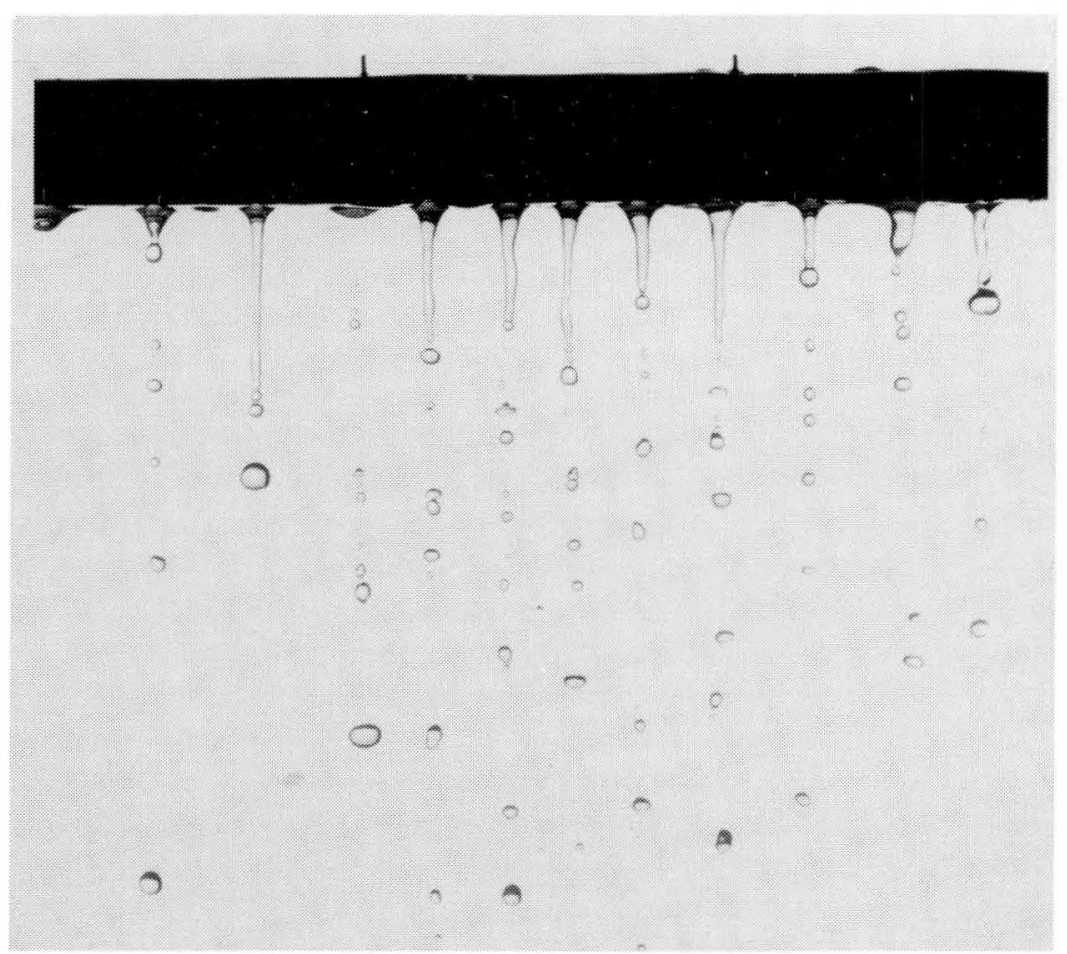

Figure 4.39 Typical photograph of water drops dripping below slat $\mathrm{A}$ at a high water flow rate $(\Gamma \approx 64 \mathrm{~g} / \mathrm{ms})$ 
The photographs were enlarged, edited, scanned and processed by the digital image analysis program described in Appendix B. This program was able to count and measure all the drops on more than 300 photographs in less than 2 hours on a $50 \mathrm{MHz} 80486$ based IBM compatible personal computer.

The measured drops were divided into 30 size classes representing drop diameters between 0 and $15 \mathrm{~mm}$. The relative frequency and cumulative mass distributions for each slat and flow rate are given in tabulated form in Appendix $\mathrm{N}$. The relative mass frequency distributions of the drops dripping below slats $\mathrm{A}$ and $\mathrm{E}$ are summarised in Figures 4.40 and 4.41.

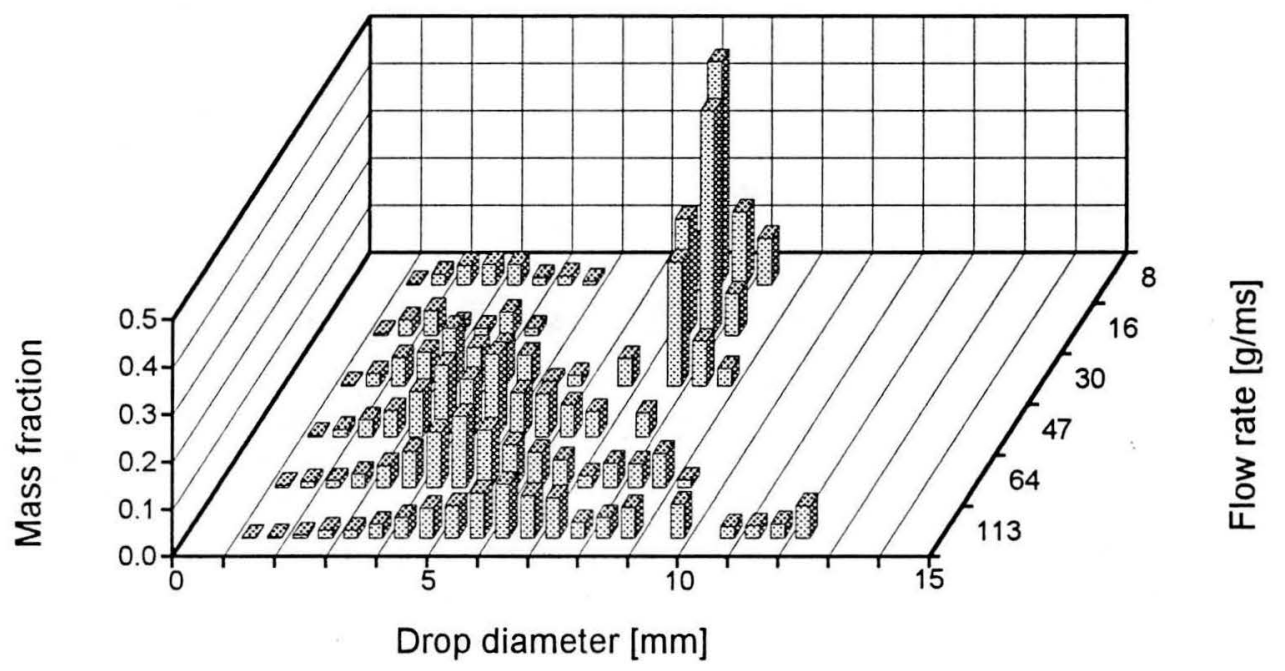

Figure 4.40 Relative mass fraction distribution for dripping below slat A.

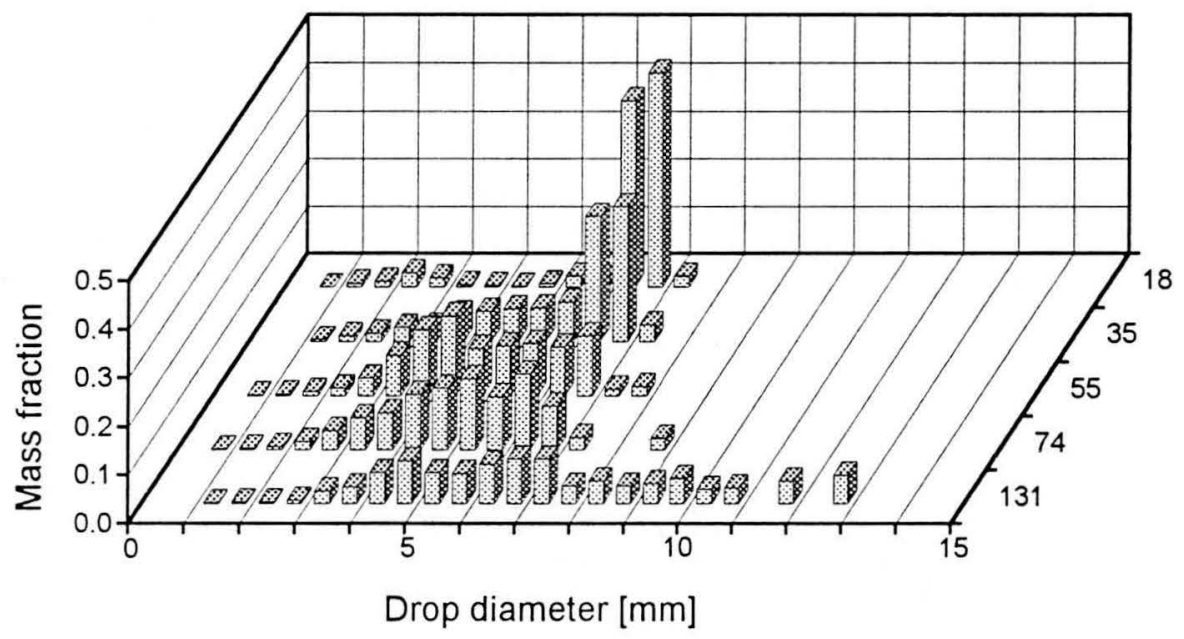

Figure 4.41 Relative mass fraction distribution for dripping below slat E. 
At low water flow rates the dripping of the liquid below all five slats was characterised by the formation of large primary drops leaving the slats at fixed dripping points below the slats. This mode of operation is referred to as the drop mode. Each primary drop was closely followed by a number of smaller satellite drops formed by the break-up of the thin water column which initially connects the primary drop to the water film on the lower surface of the slat. Table 4.8 summarises the data obtained for dripping of water below the test slats at the lowest water flow rate.

Table 4.8 Summary of drop distribution data at lowest liquid flow rate $(\Gamma \approx 18 \mathrm{~g} / \mathrm{ms})$.

\begin{tabular}{|c|c|c|c|c|c|}
\hline Slat & $\mathbf{d}_{\mathbf{p}}$ & $\mathbf{N}_{\mathbf{s}}$ & $\mathbf{m}_{\mathbf{p}} /\left(\mathbf{m}_{\mathbf{p}}+\mathbf{m}_{\mathbf{s}}\right)$ & $\mathbf{S}$ & $\mathbf{C}$ \\
- & $m m$ & - & - & - \\
\hline A & 7.66 & 8.9 & 0.81 & 1.00 & 2.83 \\
B & 7.28 & 5.3 & 0.84 & 0.98 & 2.69 \\
C & 6.61 & 3.1 & 0.93 & 0.87 & 2.44 \\
D & 7.31 & 6.0 & 0.82 & 0.97 & 2.70 \\
E & 6.47 & 3.5 & 0.92 & 0.72 & 2.39 \\
\hline
\end{tabular}

From Table 4.8 it can be seen that the primary drops dripping below the flat bottomed slat, slat A, are larger than the primary drops below the narrow, sharp-edged slat, Slat E. This difference in drop size can be attributed to the difference in geometry of the bottom of the slats. A similar phenomenon is observed for liquid drops dripping from a cylindrical nozzle, where the drop size is very strongly dependent on the diameter of the nozzle (see Appendix F). In an attempt to describe the geometry or the 'sharpness' of the lower edge of a slat the following scheme is proposed: Imagine a sphere placed with its centre on the bottom edge of the slat, as shown in Figure 4.42. The circumference of the vertical projection of the interpenetration curve formed between the sphere and the slat gives one an indication of the sharpness of the shape. For a flat bottomed slat the circumference of this curve will be the same as the circumference of the sphere at its centreline. For a thin vertical plate the circumference of the vertical projection of the interpenetration line will be equal to double the diameter of the sphere. Based on this, it is possible to define a 'shape factor' to describe the sharpness of the slat as

$$
\mathrm{S}=\frac{\text { Circumference of the vertical projection of the interpenetration line on slat }}{\text { Circumference of the sphere }}
$$

The shape factor for a large flat horizontal surface is $S=1$ and for a thin vertical plate it can be shown that $S \approx 2 / \pi$. It was found that by assuming an imaginary sphere size of $12.5 \mathrm{~mm}$ diameter, it was possible to correlate the calculated non-dimensional primary drop diameter for each of the slats with the shape factor for each slat. 


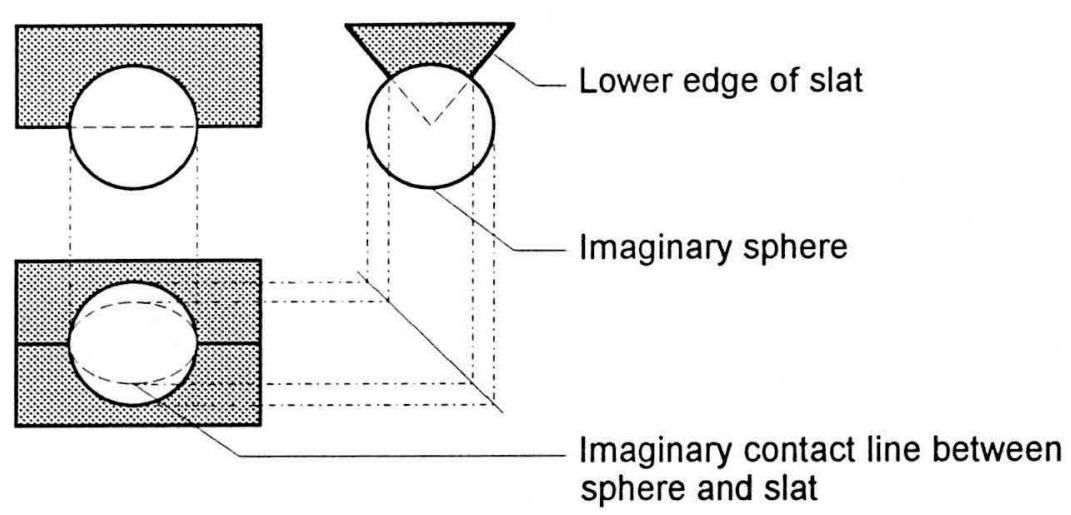

Figure 4.42 Explanatory diagram to define the shape factor.

The following relation is often used to describe the size of drops under the influence of gravity and surface tension forces

$$
d_{p}=C \sqrt{\frac{\sigma}{g\left(\rho_{w}-\rho_{a}\right)}}
$$

The constant in this correlation can be seen as a non-dimensional drop diameter. The sizes of the primary drops dripping from the test slats at low liquid flow rate (see Table 4.8) could be correlated as

$$
\mathrm{C}=2.206+\left(\frac{0.0597}{1.1-\mathrm{S}}\right)
$$

The data and the correlation for the non-dimensional primary drop diameter are shown in Figure 4.43 .

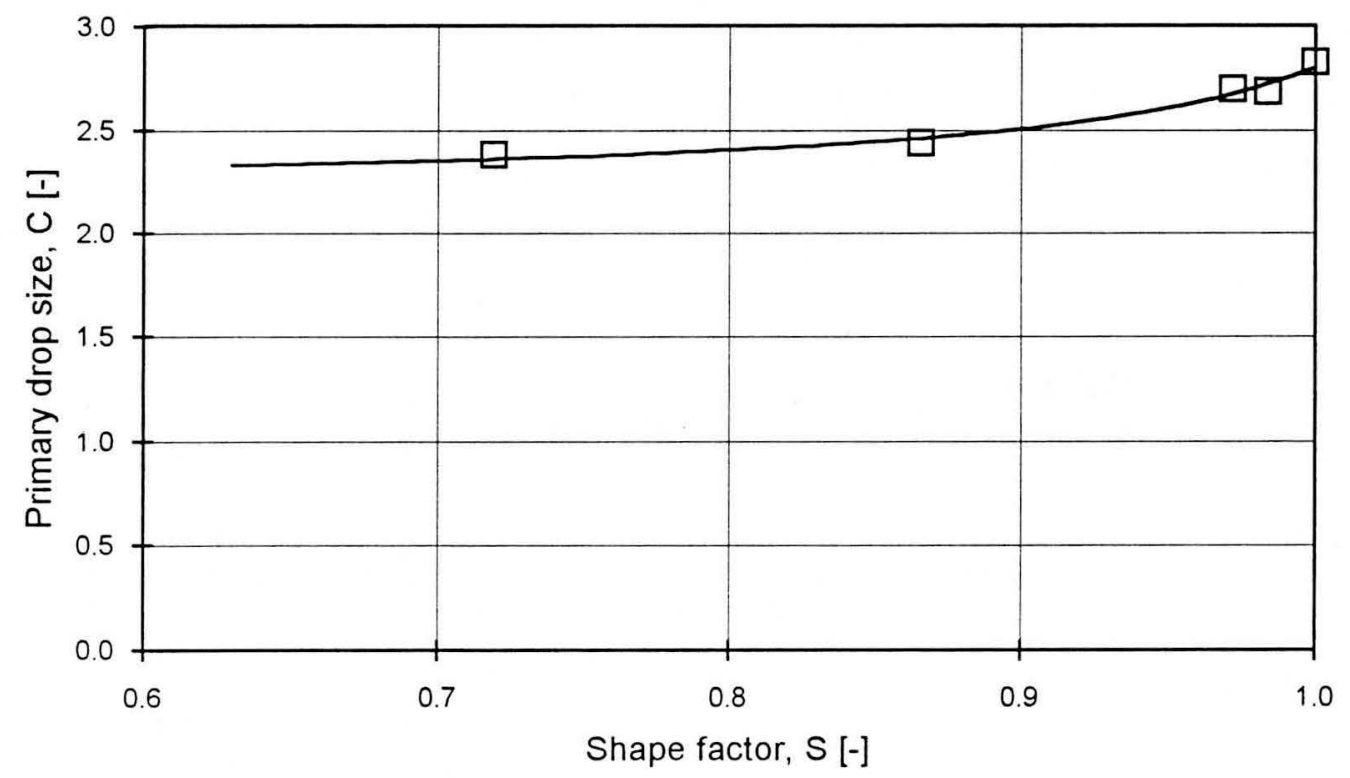

Figure 4.43 Data and correlation for non-dimensional primary drop size. 
At higher flow rates the average drop sizes are reduced due to momentum effects during the formation of the drops. Columns (or jets) of water are formed at certain positions while drop mode dripping occurs at other positions on the slat at the same time. The columns break up and form drops which have diameters approximately two times the diameter of the column as predicted by Rayleigh (see Clift et al. [78CL1]). At the highest water flow rates it is not possible to distinguish the drop mode any more since the column mode now dominates.

Through interpolation, it is possible to use the experimental data obtained in this study to approximate the size distribution of dripping drops below any type of slat with $(2 / \pi) \leq S \leq 1$, at any water flow rate below $140 \mathrm{~g} / \mathrm{ms}$. Although the experiments were conducteu at only one temperature, the drop distribution at other temperatures can be estimated by employing Equation (4.29) as follows

$$
d \approx d_{\exp } \sqrt{\left(\frac{\sigma}{\sigma_{\exp }}\right)\left(\frac{\left(\rho_{\mathrm{w}}-\rho_{\mathrm{a}}\right)_{\exp }}{\left(\rho_{\mathrm{w}}-\rho_{\mathrm{a}}\right)}\right)}
$$

\subsection{Heat/mass transfer from grids}

The cooling of the water films on the surface of the slats of cooling tower splash pack can contribute significantly to the thermal performance of the splash pack. The heat and mass transfer from the water film flowing over a slat can be calculated using the analogy between heat and mass transfer in conjunction with a correlation for the heat transfer coefficient on the outside of the slat. In Chapter 3 doubt was expressed about the applicability of the following correlation by Gnielinski [ $83 \mathrm{GN} 1]$ to calculate the heat transfer coefficient of immersed bodies of complex shapes such as T-sections,

$$
\mathrm{Nu}_{\mathrm{b}}=0.3+\sqrt{\left(0.664 \sqrt{\mathrm{Re}_{\mathrm{b}}} \operatorname{Pr}_{\mathrm{b}}^{1 / 3}\right)^{2}+\left(\frac{0.037 \mathrm{Re}_{\mathrm{b}}^{0.8} \mathrm{Pr}_{\mathrm{b}}}{1+2.443 \mathrm{Re}_{\mathrm{b}}^{-0.1}\left(\mathrm{Pr}_{\mathrm{b}}^{2 / 3}-1\right)}\right)^{2}}
$$

The Reynolds number is based on a hydraulic diameter which is defined as the ratio of the total surface area of the body to the maximum perimeter perpendicular to the flow, while the fluid properties are calculated at the local film temperature.

A simple experiment was set up to determine the external convection heat transfer coefficient of various tubular sections in an airstream. The geometry of the tubular sections evaluated are listed in Table 4.9. The experimental layout, shown in Figure 4.44, consists of a steam generator connected to a length of tube mounted in the inlet section of a horizontal wind 
tunnel. The wind tunnel has a variable speed fan which allows one to set the free-stream air velocity in the $2 \times 2 \mathrm{~m}^{2}$ test section at any constant value up to $3 \mathrm{~m} / \mathrm{s}$. The heat transfer rate from each tube could be determined accurately by measuring the rate of condensate flow dripping from the open end of the tube. To ensure that condensation takes place inside the tube along its complete length, the power input to the steam generator was set to generate an excess steam mass flow rate. The heat transfer rate from each tube was measured at six air velocities. The ambient pressure and air temperature were also measured during each test. The data obtained during these experiments is given in Appendix $\mathrm{O}$.

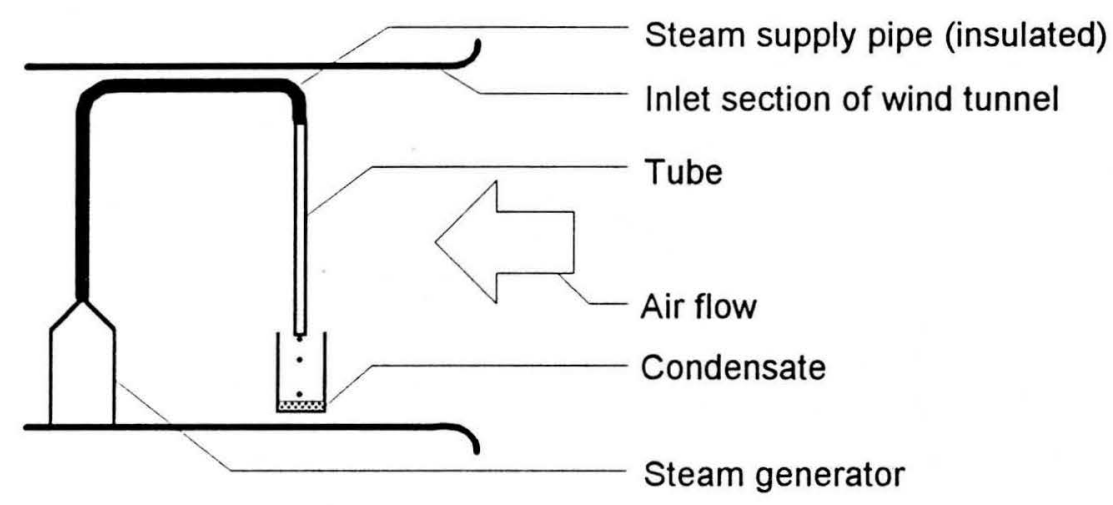

Figure 4.44 Experimental apparatus used to determine the heat transfer from different tubes.

Table 4.9 Tube profiles used in the experiments to measure the external heat transfer coefficient.

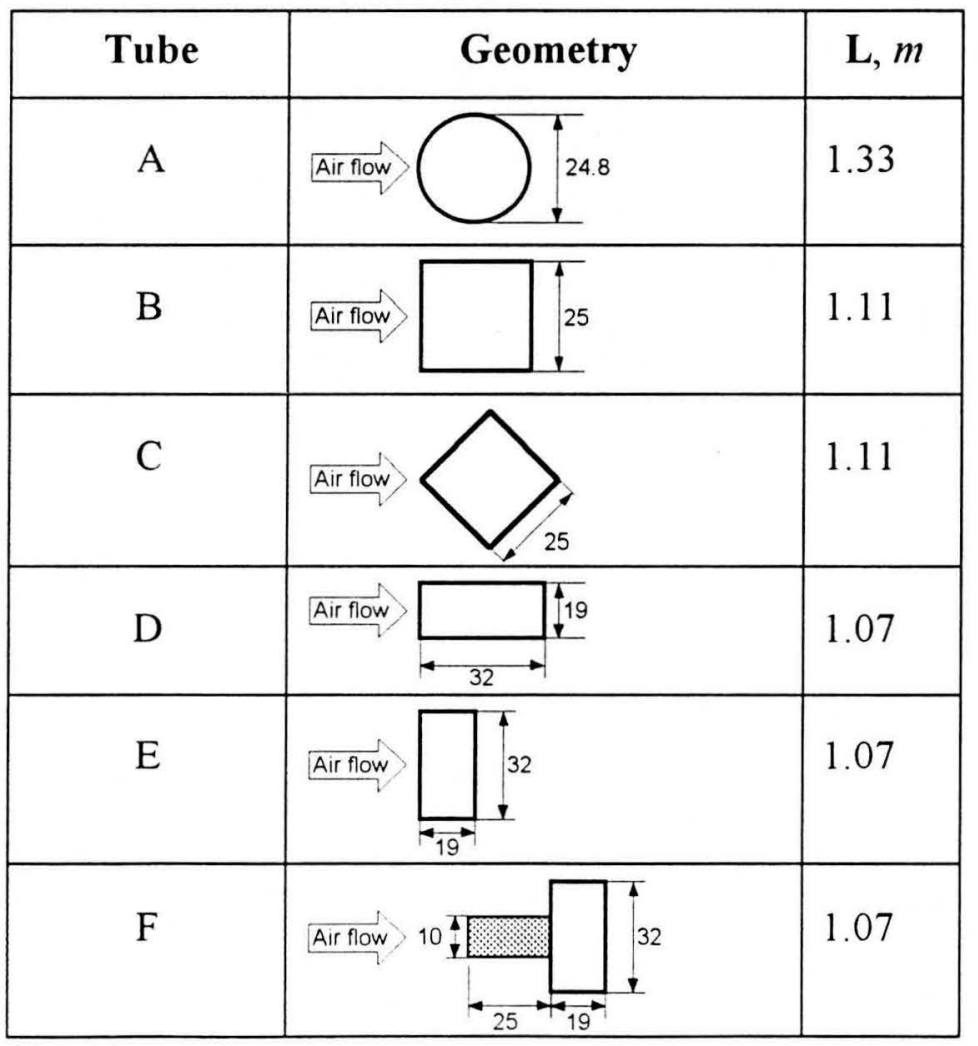


The calculation of the air-side convection heat transfer coefficients from the experimental data proceeded as follows:

i) The steam temperature corresponding to the atmospheric pressure was calculated.

ii) The total heat transfer rate could be expressed as

$$
\dot{\mathrm{Q}}_{\text {total }}=\dot{\mathrm{m}}_{\mathrm{c}} \mathrm{i}_{\mathrm{fg}}
$$

iii) The heat transfer rate due to natural and forced convection was obtained by subtracting the heat transfer rate due to thermal radiation from the total heat transfer rate

$$
\begin{aligned}
\dot{\mathrm{Q}}_{\mathrm{nc}+\mathrm{fc}} & =\dot{\mathrm{Q}}_{\text {total }}-\dot{\mathrm{Q}}_{\mathrm{rad}} \\
& =\dot{\mathrm{Q}}_{\text {total }}-\varepsilon \sigma_{\mathrm{rad}} \mathrm{A}\left(\left(\mathrm{T}_{\mathrm{s}}+273.15\right)^{4}-\left(\mathrm{T}_{\mathrm{a}}+273.15\right)^{4}\right)
\end{aligned}
$$

In the case of the $\mathrm{T}$-shape tube (tube $\mathrm{F}$ ) the effective area for radiation is not the total outer surface of the tube since the some of the sides 'see' each other. From geometrical considerations it can be shown that $85 \%$ of the actual surface area take part in the radiation process.

iv) The total heat coefficient due to natural and forced convection was expressed by

$$
\mathrm{h}_{\mathrm{nc}+\mathrm{fc}}=\frac{\dot{\mathrm{Q}}_{\mathrm{nc}+\mathrm{fc}}}{\mathrm{A}\left(\mathrm{T}_{\mathrm{s}}-\mathrm{T}_{\mathrm{a}}\right)}
$$

Note that the equation above implies that the condensation heat transfer coefficient is large in comparison with the heat transfer coefficients on the outside of the tube and that the temperature gradient through the tube wall is negligible. The T-shaped tube was constructed by welding a solid steel strip to the side of a rectangular hollow section. This strip forming the leg of the $T$ profile can be assumed to act as a fin. Calculations indicated that this fin has a fin efficiency approaching 100\%, which implies that the fin can be assumed to be at the same temperature as the tube wall (which is assumed to be equal to the steam temperature).

v) The natural convection heat transfer coefficient was calculated from the following simplified relations given by Holman [81HO1]

$$
\begin{aligned}
& h_{n c, l}=1.42\left(\frac{T_{s}-T_{a}}{L}\right)^{0.25} \quad \text { for } \operatorname{Gr} \operatorname{Pr}<10^{9} \\
& \text { or } \\
& h_{n c, t}=0.95\left(T_{s}-T_{a}\right)^{1 / 3} \quad \text { for } \operatorname{Gr} \operatorname{Pr} \geq 10^{9}
\end{aligned}
$$

vi) Churchill $[83 \mathrm{CH} 1]$ found that the combined effect of natural convection and forced convection could be found from 


$$
N u_{n c+f c}=\left(N u_{n c}^{3}+N u_{f c}^{3}\right)^{1 / 3}
$$

Based on this, the transfer coefficient due to forced convection was found from

$$
h_{\mathrm{fc}}=\left(h_{\mathrm{nc}+\mathrm{fc}}^{3}-h_{\mathrm{nc}}^{3}\right)^{1 / 3}
$$

vii) The Reynolds number and Nusselt number based on the film temperature was then calculated for each test,

$$
\begin{aligned}
& \operatorname{Re}_{\mathrm{b}}=\frac{\rho_{\mathrm{b}} \mathrm{v}_{\mathrm{a}} \mathrm{d}_{\mathrm{h}}}{\mu_{\mathrm{b}}} \\
& \text { and } \\
& \mathrm{Nu}_{\mathrm{b}}=\frac{\mathrm{h}_{\mathrm{fc}} \mathrm{d}_{\mathrm{h}}}{\mathrm{k}_{\mathrm{b}}}
\end{aligned}
$$

The experimentally determined Nusselt numbers are shown graphically in Figure 4.45 together with the correlation by Gnielinski [ $83 \mathrm{GN} 1]$. It can be seen that the all experimental data agrees very well with the correlation by Gnielinski. It can therefore be assumed that the correlation of Gnielinski is also valid for the calculation of the forced convection heat transfer from more complex tubular sections such as $\mathrm{T}$-sections.

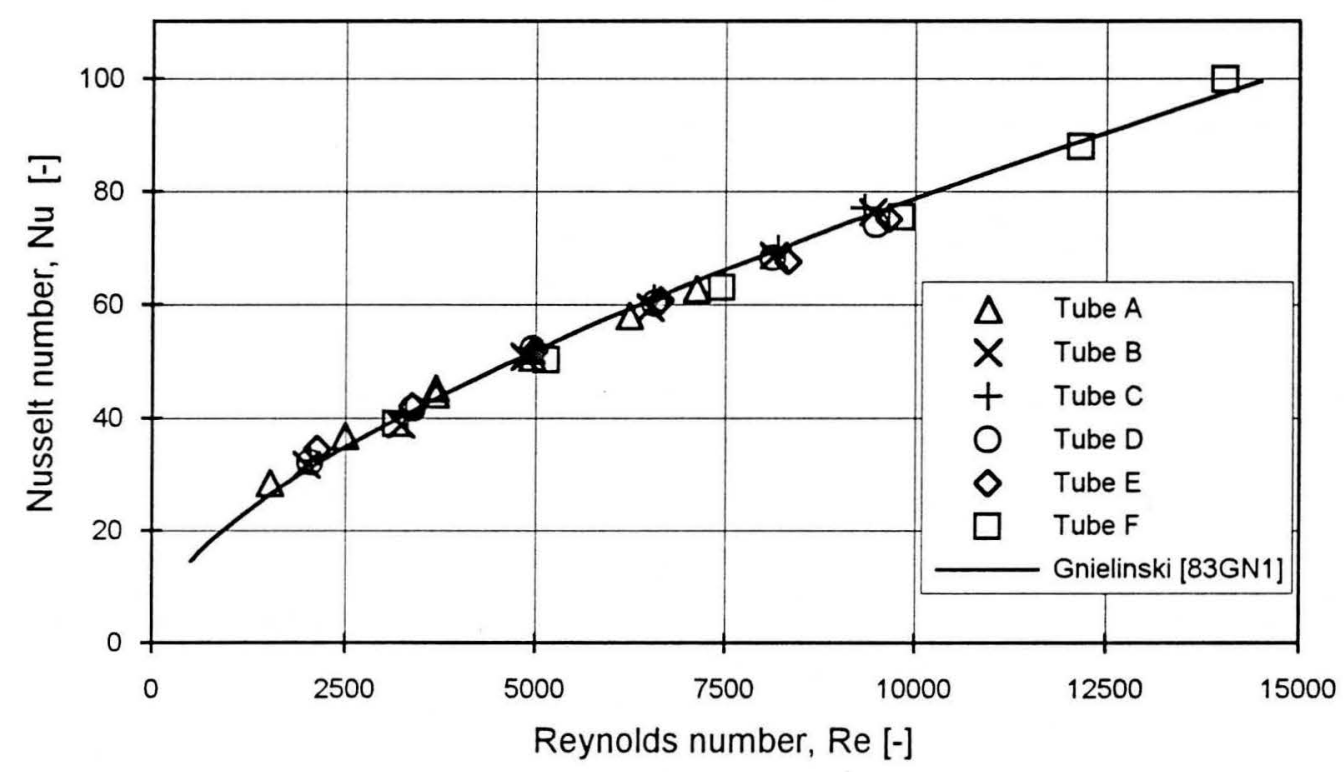

Figure 4.45 Comparison between the experimental data and the correlation of Gnielinski.

\subsection{Cooling tower packing performance tests}

The wet/dry cooling test facility at the Department of Mechanical Engineering at the University of Stellenbosch was designed for the evaluation of cooling tower packing material and spray-cooled heat exchangers. The $1.5 \times 1.5 \mathrm{~m}^{2}$ vertical test section, shown in Figure 4.46, 
was designed to evaluate counterflow cooling tower packing material. A centrifugal fan with a variable speed motor is used to supply the air flow through the test section at up to $4.5 \mathrm{~m} / \mathrm{s}$. The air mass flow rate to the test section is calculated from the pressure drop measured across a set of ASHRAE 51-75 elliptical flow nozzles.

To minimise the effect of water accumulating and cooling on the walls of the test section, a series of deflector plates, as shown in Figure 4.46, was installed around the inner perimeter of the test section at $300 \mathrm{~mm}$ intervals. These deflector plates remove the water film from the wall of the test section and redistributes the water in the packing zone. To reduce heat loss to the environment, the outside of the vertical test section was insulated with a $25 \mathrm{~mm}$ thick closed-cell polyurethane foam layer.

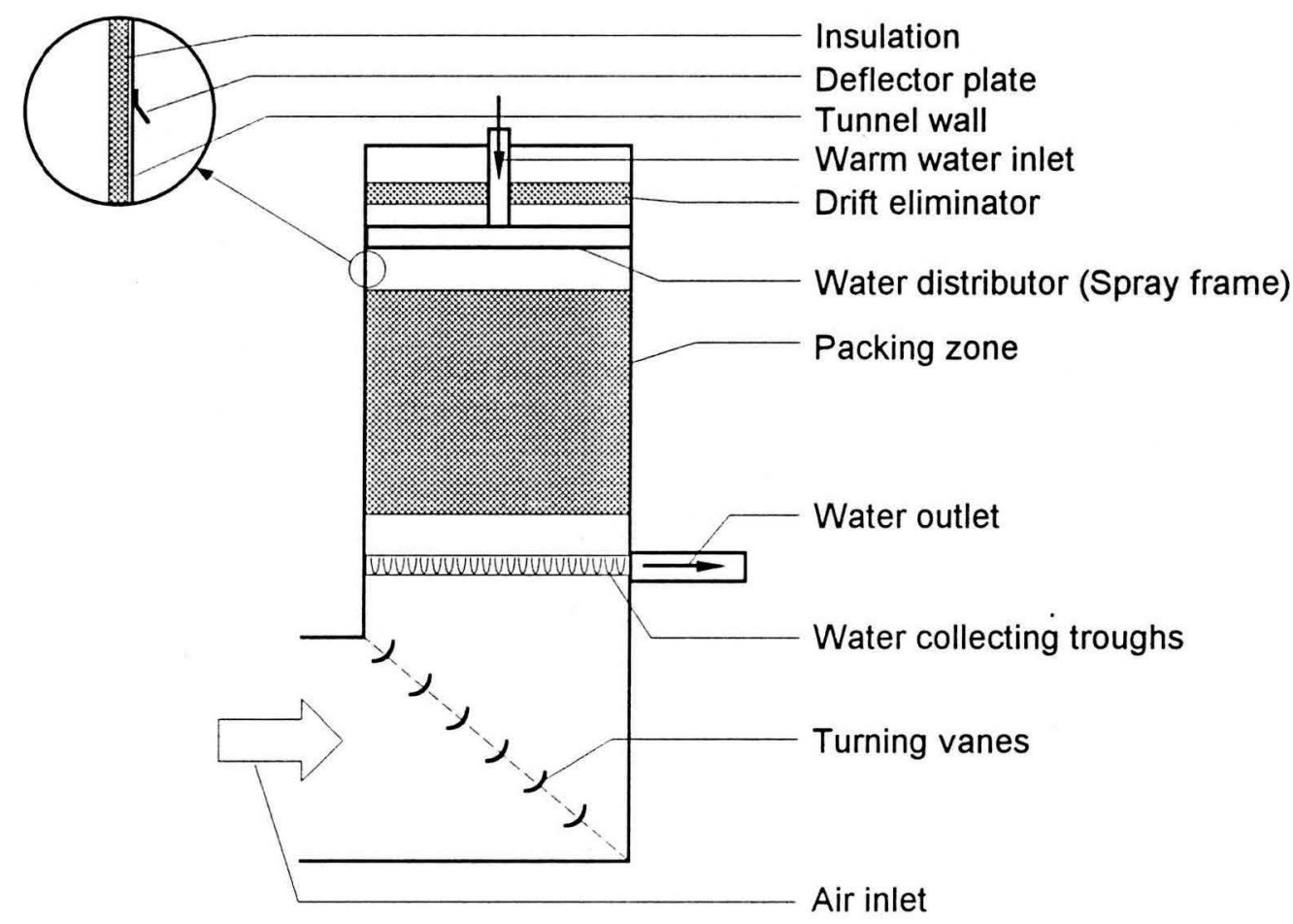

Figure 4.46 Counterflow cooling tower packing material test facility.

The water is supplied to the test section by a distributing spray frame as shown in Figure 4.47. The spray frame consists of 20 drip plates, each $1.5 \mathrm{~m}$ long, which are supplied with water by water jets issuing from small openings in the distribution pipes as shown in Figure 4.47. The lower edge of each plate was cut to form a $45^{\circ}$ sawtooth pattern. This set-up ensures a good distribution of water since the water drips from the points of the sawtooth. The water flow rate is calculated from the pressure drop across an orifice plate designed according to the BS 1042 standard. To minimise the size of the rain zone below the packing in a typical cooling tower packing test, the water below the packing material is removed from the test section through a double set of collection troughs. The airflow velocity through the gaps between the 
troughs is high enough to ensure that drops will not fall through the gaps, but instead the drops are forced to fall into the troughs.

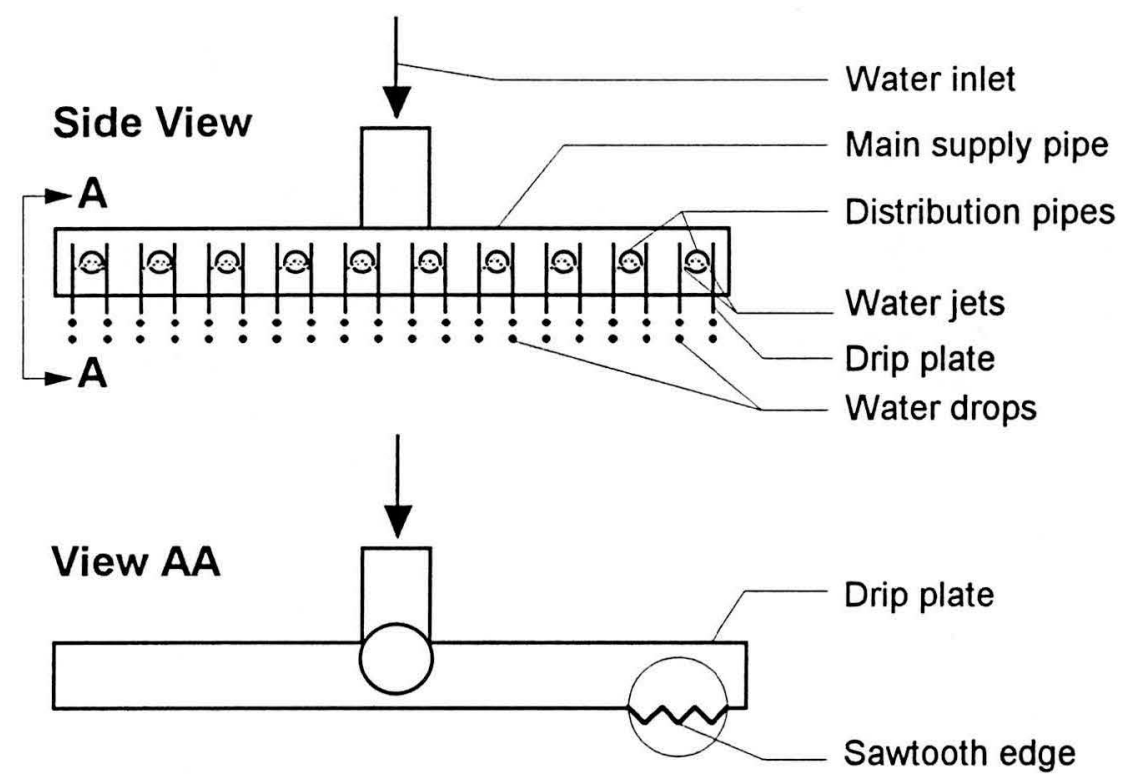

Figure 4.47 Water distributing spray frame.

To cope with the large thermal capacity of cooling tower packing tests in a test section of this size, water in a $40 \mathrm{~m}^{3}$ underground reservoir is preheated to the required water inlet temperature by circulating the water through a $100 \mathrm{~kW}$ diesel burning water heater. During a test, water from the top of the reservoir is pumped to the test section, and after passing through the test section, the water is fed back through a narrow slot at the bottom of the reservoir. This ensures that stratification occurs in the reservoir and subsequently the water supply temperature to the test section stays almost constant during a test.

The temperatures are measured using calibrated copper-constantan thermocouples. The calibration data of each thermocouple was obtained from its reading at the melting point of ice and the boiling temperature at ambient pressure. The air dry-bulb and wet-bulb temperatures are measured at four positions upstream and four positions downstream of the test section using ventilated psychrometric probes as shown in Figure 4.48. All the thermocouples are directly connected to a Schlumberger data logging system.

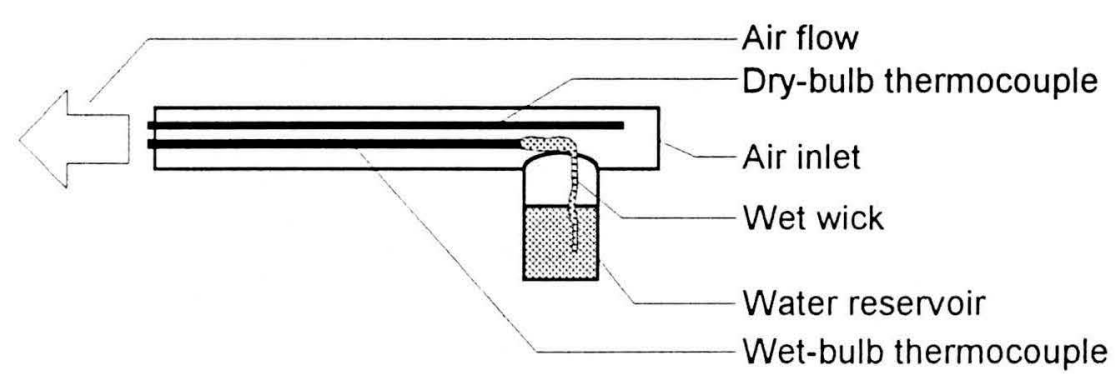

Figure 4.48 Psychrometric probe. 
The pressure readings (for determining the air and water mass flow rates) are obtained from differential pressure transducers connected to the data logging system. The pressure transducers were individually calibrated. The ambient pressure is obtained from a mercury barometer. The pressure drop across the packing zone is measured with a Betz micromanometer. Due to the high water mass flux and the slight over-pressure, conventional wall pressure tappings cannot be used to measure the local static pressure in the test section. Special pressure measuring probes, shown in Figure 4.49, are used to measure the local static pressure in the test section. These probes consist of two flat plastic plates connected by a 20 $\mathrm{mm}$ plastic tube. The static pressure point is located at the top of this tube. These probes were found to be insensitive to slight inclinations relative to the air flow direction. Any water entering the tube runs out the other side without wetting the pressure point.

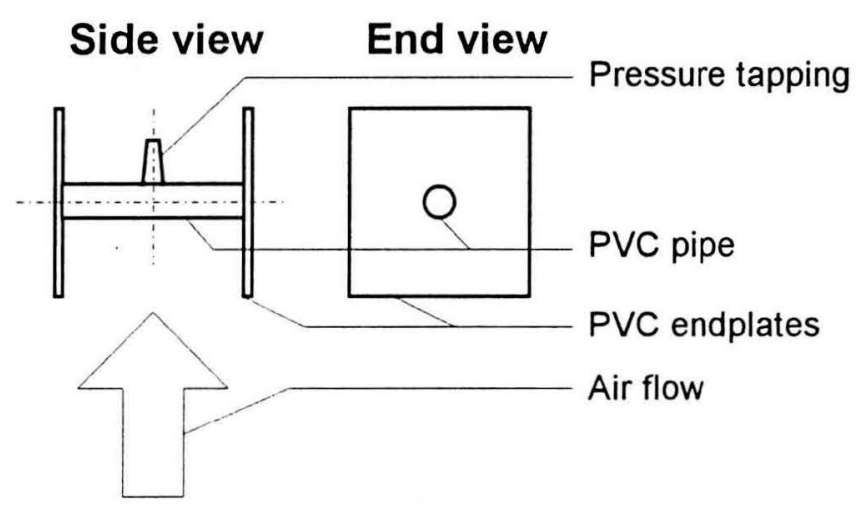

Figure 4.49 Pressure measuring probes.

The Schlumberger data loggers, which are capable of reading all the channels once per second, are connected to a personal computer. A Turbo Pascal program is used to read the data from the data loggers and to process it immediately. The program can also display time traces of the temperatures, energy balance, mass flow rates and transfer characteristics. The real-time processing of the test data allows one to continue a test until steady-state is reached. Once steady state conditions are reached the data could be stored on magnetic disk further processing.

\subsubsection{Initial drop size distribution}

The distribution of drop sizes formed below the drop distribution spray frame is of major importance when the data obtained from full scale cooling tower tests (or packing tests) are to be compared to that obtained with the splash pack simulation program. The spray frame, described in more detail above, was designed to handle water flow rates of up to $10 \mathrm{~kg} / \mathrm{s}$, or (10/30) $\mathrm{kg} / \mathrm{ms}$ down the side of each drip plate. A representative full-scale model of a section of the water distributor, shown in Figure 4.50, was built to facilitate the measurement of the drop size distribution below the drop distributor. The model consisted of a single $0.5 \mathrm{~m}$ long 
section of drip plate supplied with water from water jets from a single supply pipe, aimed at the one side of the plate. To cover the same range of water flow rates as that of the complete spray frame, the model was supplied with water flow rates up to $0.170 \mathrm{~kg} / \mathrm{s}$.

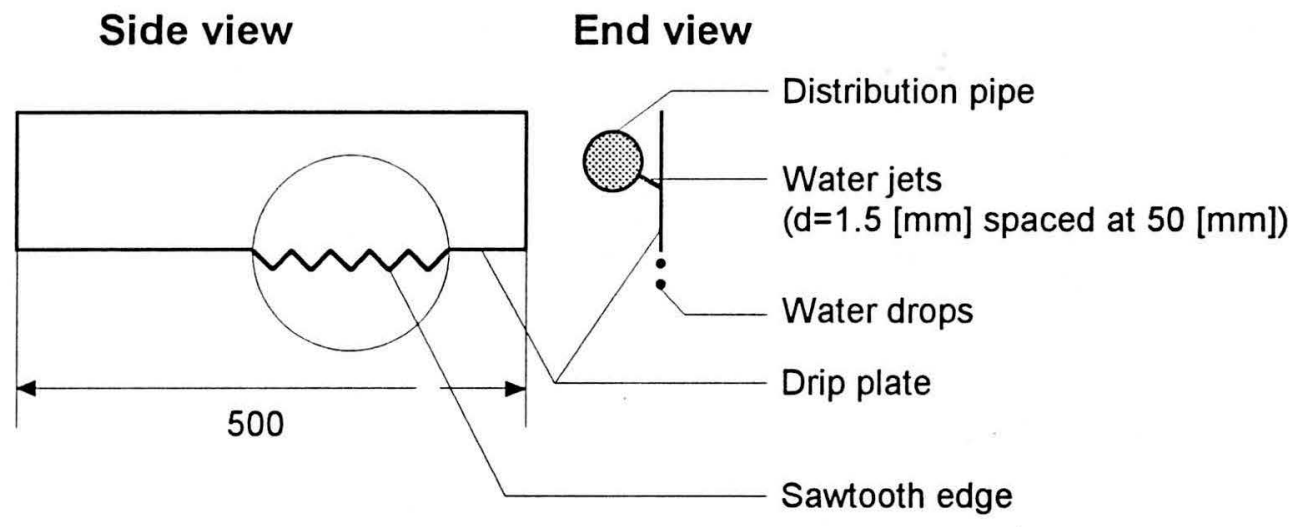

Figure 4.50 Model of the water distributing spray frame.

A similar experimental technique to that described above for the determination of the drop sizes dripping from the slats in a splash pack, was used to determine drop distribution below the spray frame. The experimental set-up was similar to that shown in Figure 4.37. As before, the shadowgraphing technique was used to obtain photographs of the falling drops. The drops were photographed at six water flow rates. Approximately eight photographs were taken at each flow rate. At high liquid flow rates the water flowing from the drip plate formed large wavy streams of water which broke up into drops only after about $300 \mathrm{~mm}$ of free fall. Two sets of photographs were taken to cover a wide range of fall distances below the slat. The first series covered the first $300 \mathrm{~mm}$ of the free fall zone (series A) and the second series covered the next $300 \mathrm{~mm}$ (series B).

The photographs were enlarged, digitised and analysed using the procedure described in Appendix B. The drop size distributions of series A and series B were found to be almost identical except that in the case of series A, a few very large drops (>15 $\mathrm{mm}$ diameter) were measured at higher water flow rates. These large drops seem to be crude agglomorations of parts of the wavy streams of water breaking away from the drip plate. Although it can be expected that these large drops will break up relatively quickly, they deform the measured cumulative mass distribution significantly (due to their large mass in comparison to that of the smaller drops) and it was therefore decided only to use the data of series B for further investigations. The experimental drop size distribution for series $\mathrm{B}$ is given in tabular form in Appendix P. The measured cumulative mass fraction data is shown in Figure 4.51. Note that the water flow rates were scaled up to that of the full scale spray frame. 


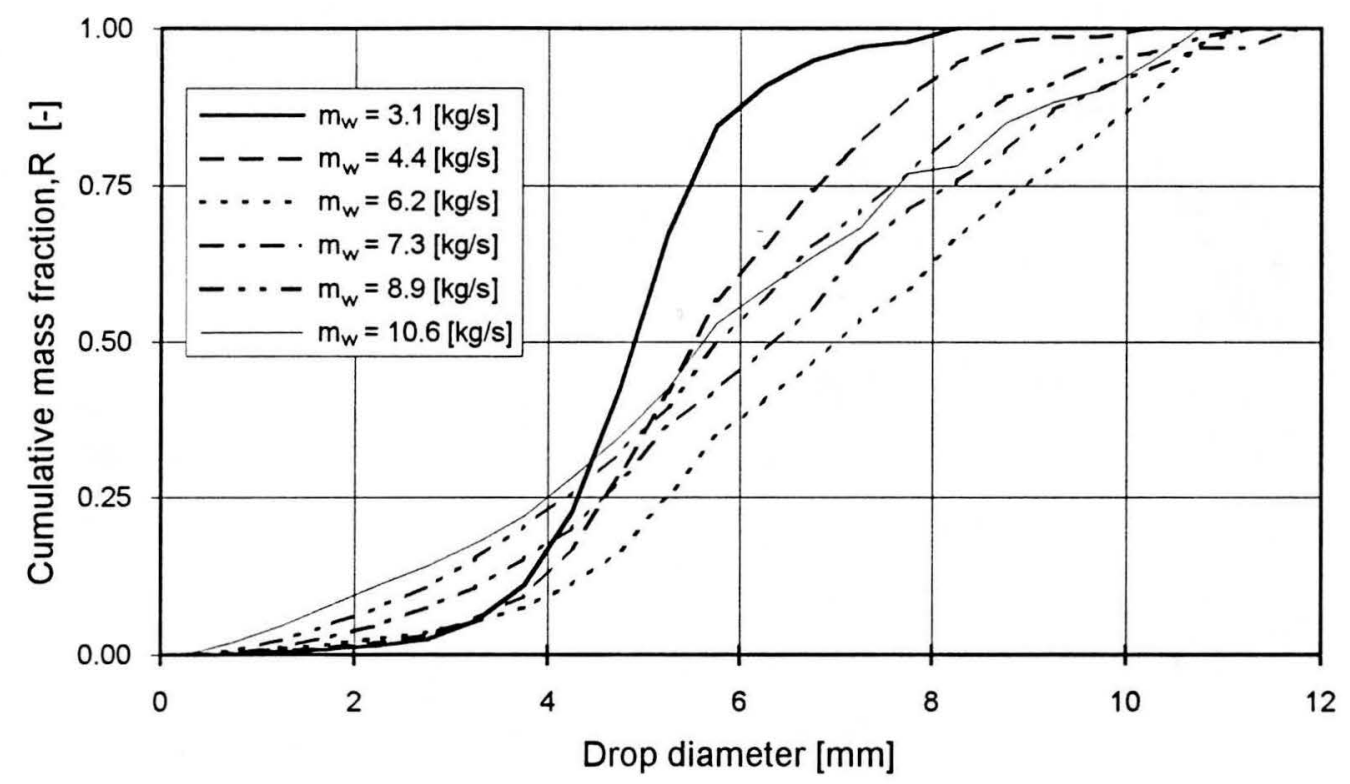

Figure 4.51 Measured cumulative mass distribution for the spray frame model.

The cumulative mass fraction data at each water flow rate was found to be expressed reasonable well by the Rosin-Rammler distribution function. The Rosin-Rammler distribution function gives the mass fraction of drops smaller than a given size as

$$
R(d)=1-\exp \left(-\left(\frac{d}{d_{R R}}\right)^{n_{R R}}\right)
$$

with

$$
\mathrm{d}_{\mathrm{RR}}=\mathrm{d}_{50}\left(0.6931^{-\left(\mathrm{l} / \mathrm{n}_{\mathrm{RR}}\right)}\right)
$$

The two parameters describing the Rosin-Rammler distribution for each of the six liquid flow rates are listed in Table 4.10.

Table 4.10 Rosin-Rammler parameters describing the spray frame drop size distribution.

\begin{tabular}{|c|c|c|c|}
\hline $\begin{array}{c}\dot{\mathbf{m}}_{\mathbf{w}} \text { (full scale) } \\
\mathrm{kg} / \boldsymbol{s}\end{array}$ & $\begin{array}{c}\mathbf{d}_{\mathbf{5 0}} \\
\mathrm{mm}\end{array}$ & $\begin{array}{c}\mathbf{d}_{\mathbf{R R}} \\
\mathbf{m m}\end{array}$ & $\begin{array}{c}\mathbf{n}_{\mathbf{R R}} \\
-\end{array}$ \\
\hline 3.1 & 4.92 & 5.22 & 6.14 \\
4.4 & 5.63 & 6.17 & 3.98 \\
6.2 & 7.03 & 7.89 & 3.16 \\
7.3 & 6.28 & 7.18 & 2.75 \\
8.3 & 5.78 & 6.63 & 2.68 \\
10.6 & 5.68 & 6.65 & 2.33 \\
\hline
\end{tabular}


These parameters were correlated as a function of water flow rate, yielding the following two equations

$$
\mathrm{d}_{50}=1.804+1.306 \dot{\mathrm{m}}_{\mathrm{w}}-0.0910 \dot{\mathrm{m}}_{\mathrm{w}}^{2}
$$

and

$$
\mathrm{n}_{\mathrm{RR}}=1.607+\left(\frac{7.091}{\dot{\mathrm{m}}_{\mathrm{w}}-1.5}\right)
$$

where $\mathrm{d}_{50}$ is expressed in $\mathrm{mm}$ and $\dot{\mathrm{m}}_{\mathrm{w}}$ in $\mathrm{kg} / \mathrm{s}$. The data and correlations are shown in Figure 4.52 .
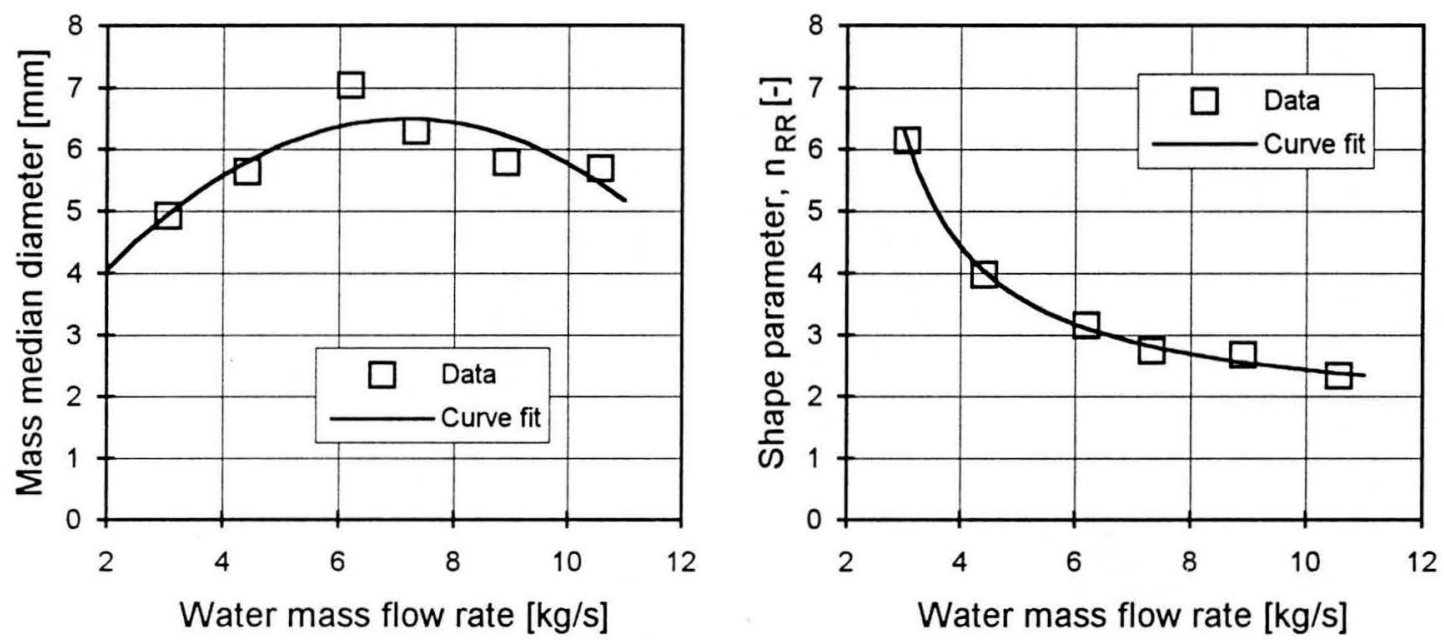

Figure 4.52 Rosin-Rammler distribution parameters describing the spray frame drop distribution.

By using the Rosin-Rammler distribution correlations above or by interpolation from the experimental data directly, it is possible to approximate the size distribution of the drops formed below the spray frame at any water flow rate. Although the experiments were conducted at only one water temperature, i.e. $20^{\circ} \mathrm{C}$, the drop distribution at other temperatures can be estimated by employing Equation (4.29) as follows

$$
\mathrm{d} \approx \mathrm{d}_{\exp } \sqrt{\left(\frac{\sigma}{\sigma_{\exp }}\right)\left(\frac{\left(\rho_{\mathrm{w}}-\rho_{\mathrm{a}}\right)_{\exp }}{\left(\rho_{\mathrm{w}}-\rho_{\mathrm{a}}\right)}\right)}
$$

\subsubsection{Empty tower tests}

In the packing material test section it can be expected that some water will strike the walls and flow down the sides of the tunnel as thin films. The deflector plates mounted on the wall, as 
shown in Figure 4.46, remove the water film and reintroduce the water back into the test section. The wall area per unit volume of the test section is approximately $2.67 \mathrm{~m}^{2} / \mathrm{m}^{3}$; this is of a similar order of magnitude of the surface area of the coarse drops generated by the spray frame, shown in Figure 4.47. It can be assumed that there is some cooling of the films on the drip plates of the water distribution spray frame. Similarly cooling of water in the drop collection troughs occur by heat transfer (or combined heat and mass transfer if some liquid flows over the edges of the troughs) from the upstream sides of the troughs. Some splashing of drops striking the top edges of the troughs and the water in the troughs is also expected to occur, and the corresponding formation of small splash drops will contribute to the overall transfer. To quantify the contributions of these effects on the overall transfer characteristic measured for a given packing material, a series of empty tower tests was conducted. Four different empty tower depths (the distance between drop generator and the collecting troughs) were tested, i.e., $\mathrm{Z} \approx 0.0,1.3,2.0$ and $2.6 \mathrm{~m}$. At each depth the thermal performance of the empty tower was measured for a range of air and water flow rates.

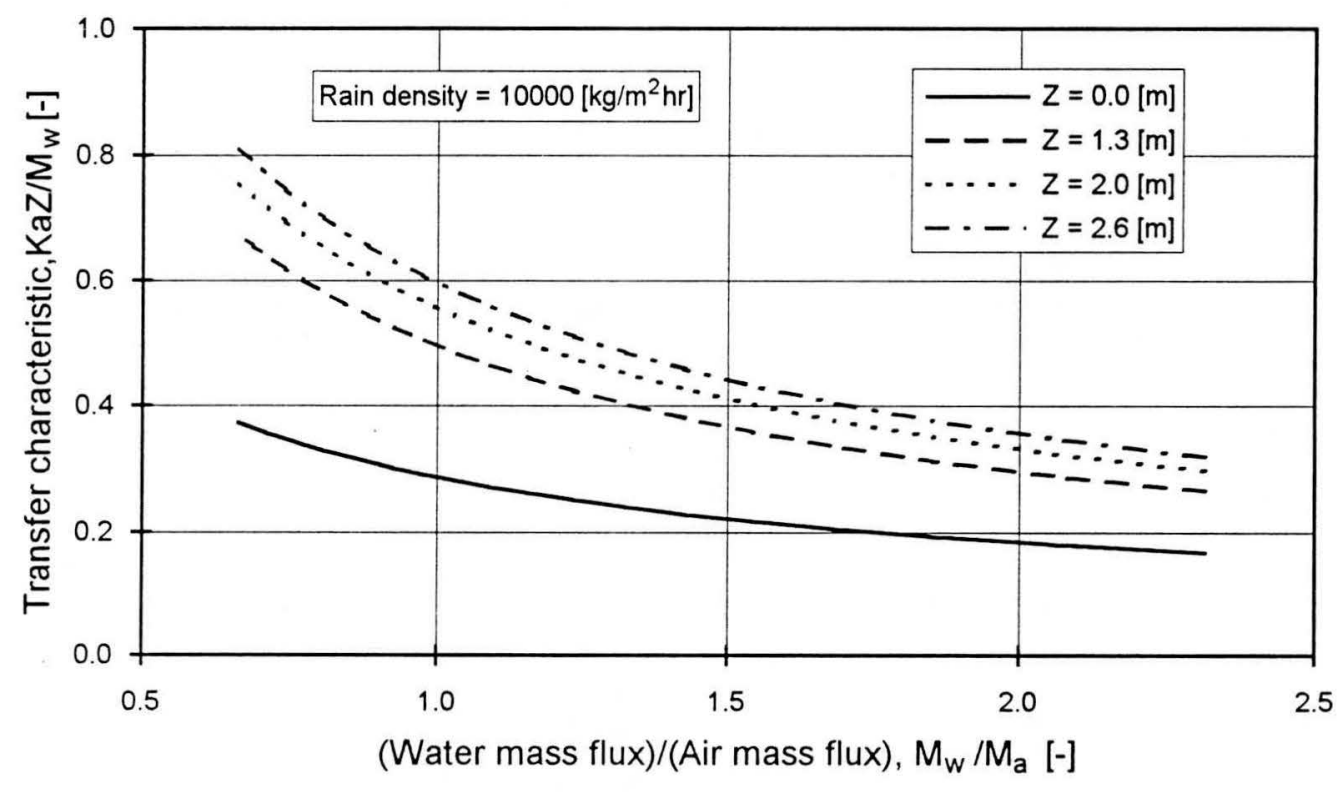

Figure 4.53 Empty tower transfer characteristics.

The thermal transfer characteristic data for the empty tower with $Z \approx 0.0 \mathrm{~m}$ was correlated by

$$
\frac{\mathrm{KaZ}}{\dot{\mathrm{M}}_{\mathrm{w}}}=0.125 \dot{\mathrm{M}}_{\mathrm{w}}^{0.044} \dot{\mathrm{M}}_{\mathrm{a}}^{0.666}
$$

The transfer characteristic data for $\mathrm{Z}=1.3,2.0$ and $2.6 \mathrm{~m}$ was correlated as

$$
\frac{\mathrm{KaZ}}{\dot{\mathrm{M}}_{\mathrm{w}}}=0.167 \mathrm{Z}^{0.268} \dot{\mathrm{M}}_{\mathrm{w}}^{0.250} \dot{\mathrm{M}}_{\mathrm{a}}^{0.745}
$$


Figure 4.53 shows variation of these transfer characteristic correlations with varying air flow rate, graphically.

\subsubsection{Splash pack performance tests}

As shown in Chapter 2, there is a large amount of transfer characteristic data available for various types of counterflow splash pack. Since the initial drop size and velocity distributions are not known for any of this data, it is not possible to use this data for direct comparison with the predictions of the mathematical model describing the performance of cooling tower splash pack. A series of tests was conducted on two different sets of adjustable splash grids mounted in the packing material test facility shown in Figure 4.46, to obtain transfer characteristic and pressure drop data for comparison purposes.

Adjustable splash grids were manufactured from $9 \mathrm{~mm}$ and $25 \mathrm{~mm}$ wide treated wood strips. Galvanised threaded rod, washers and nuts were used mount the wood strips as $1.5 \times 1.5 \mathrm{~m}^{2}$ grids as shown in Figure 4.54. With this layout it was possible to vary the slat spacing by removing or adding slats to/from each grid and by adjusting the spacing of the strips accordingly. Table 4.11 summarises the different splash grid configurations which were tested. Each configuration was evaluated at four water flow rates and five air flow rates between 3 and $9 \mathrm{~kg} / \mathrm{s}$ and 1 to $3 \mathrm{~m} / \mathrm{s}$, respectively. Note that the total pressure drop was measured across the packing zone only, and not across the spray and rain zones above and below the packing.

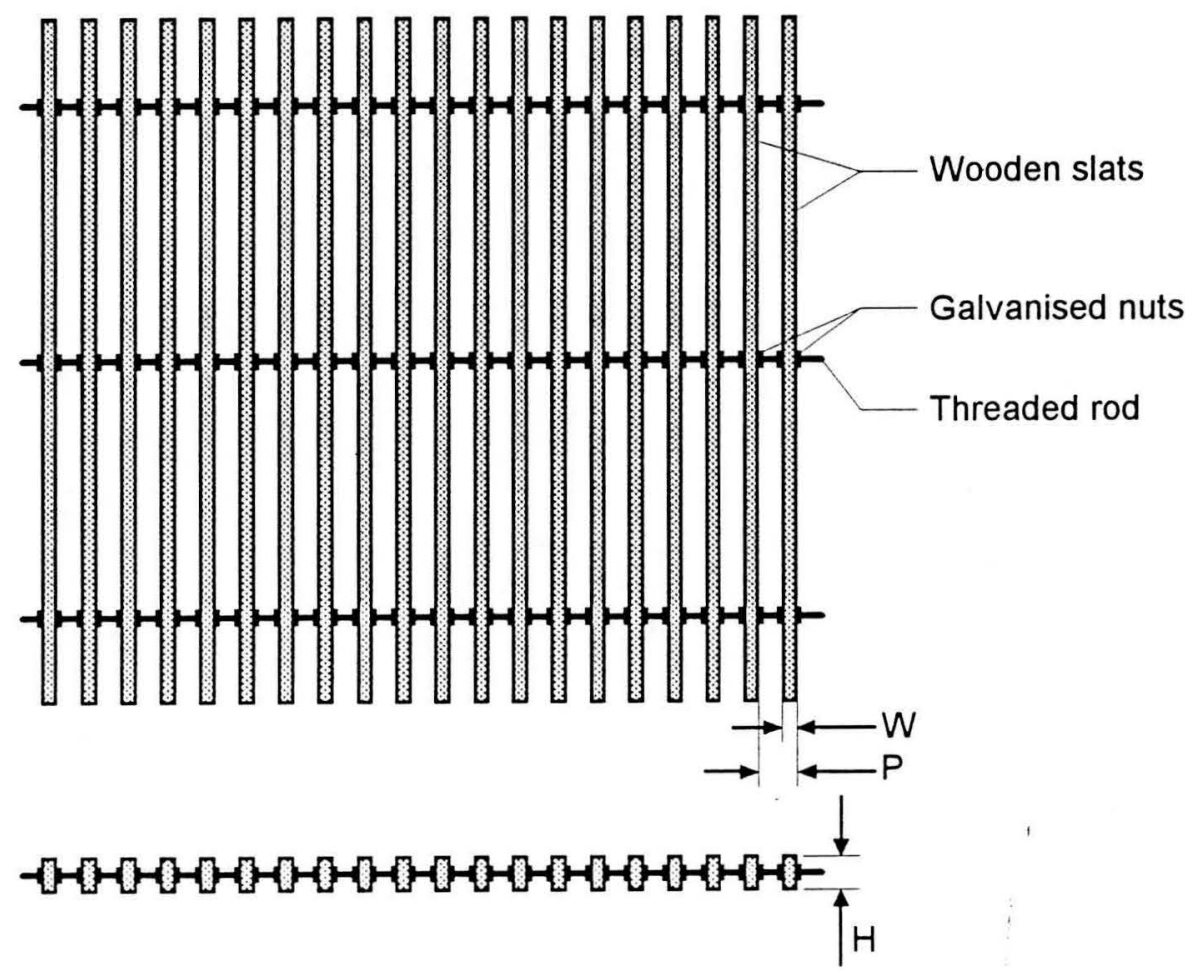

Figure 4.54 Layout of the experimental splash grids. 
Table 4.11 Splash grid layouts used in splash pack tests.

\begin{tabular}{|c|c|c|c|c|c|c|c|c|c|c|c|}
\hline Layout & $\mathbf{Z}_{\text {spray }}$ & $\mathbf{Z}_{\text {pack }}$ & $\mathbf{Z}_{\text {rain }}$ & $\mathbf{N}_{\text {slats }}$ & $\mathbf{N}_{\text {grids }}$ & $\begin{array}{c}\mathbf{P}_{\mathbf{v}} \\
m\end{array}$ & $\begin{array}{c}\mathbf{W} \\
m\end{array}$ & $\begin{array}{c}\mathbf{H} \\
m\end{array}$ & $\begin{array}{c}\mathbf{a} \\
m^{2} m^{3}\end{array}$ & $\begin{array}{c}\boldsymbol{\beta} \\
-\end{array}$ & $\begin{array}{c}\boldsymbol{\beta}^{*} \\
-\end{array}$ \\
\hline A & 1.3 & 1.0 & 0.2 & 30 & 10 & 100 & 9 & 22 & 12.4 & 0.82 & 0.81 \\
B & 0.5 & 2.0 & 0.0 & 30 & 10 & 200 & 9 & 22 & 6.2 & 0.82 & 0.81 \\
C & 0.4 & 2.1 & 0.0 & 30 & 7 & 300 & 9 & 22 & 4.1 & 0.82 & 0.81 \\
D & 1.3 & 1.0 & 0.2 & 18 & 10 & 100 & 9 & 22 & 7.4 & 0.89 & 0.88 \\
E & 0.5 & 2.0 & 0.0 & 18 & 10 & 200 & 9 & 22 & 3.7 & 0.89 & 0.88 \\
F & 0.4 & 2.1 & 0.0 & 18 & 7 & 300 & 9 & 22 & 2.5 & 0.89 & 0.88 \\
G & 1.3 & 1.0 & 0.2 & 20 & 10 & 100 & 25 & 31 & 14.9 & 0.67 & 0.66 \\
H & 0.5 & 2.0 & 0.0 & 20 & 10 & 200 & 25 & 31 & 7.5 & 0.67 & 0.66 \\
I & 0.4 & 2.1 & 0.0 & 20 & 7 & 300 & 25 & 31 & 5.0 & 0.67 & 0.66 \\
J & 1.3 & 1.0 & 0.2 & 12 & 10 & 100 & 25 & 31 & 9.0 & 0.80 & 0.79 \\
K & 0.5 & 2.0 & 0.0 & 12 & 10 & 200 & 25 & 31 & 4.5 & 0.80 & 0.79 \\
L & 0.4 & 2.1 & 0.0 & 12 & 7 & 300 & 25 & 31 & 3.0 & 0.80 & 0.79 \\
\hline
\end{tabular}

* including the frontal area of the threaded rods, washers and nuts.

The transfer characteristics were calculated from the measured temperature and mass flow rate data and correlated by the following two types of correlating equation

$$
\frac{\mathrm{KaZ}_{\mathrm{total}}}{\dot{\mathrm{M}}_{\mathrm{w}}}=\mathrm{c}_{\mathrm{l}}\left(\frac{\dot{\mathrm{M}}_{\mathrm{w}}}{\dot{\mathrm{M}}_{\mathrm{a}}}\right)^{\mathrm{c}_{2}}
$$

and

$$
\frac{\mathrm{KaZ}_{\text {total }}}{\dot{\mathrm{M}}_{\mathrm{w}}}=\mathrm{c}_{3} \dot{\mathrm{M}}_{\mathrm{w}}^{\mathrm{c}_{4}} \dot{\mathrm{M}}_{\mathrm{a}}^{\mathrm{c}_{5}}
$$

where

$$
Z_{\text {total }}=Z_{\text {spray }}+Z_{\text {pack }}+Z_{\text {rain }}
$$

The pressure loss coefficient for each packing configuration was correlated by an equation of the form

$$
\mathrm{K}_{\Delta \mathrm{p}, \text { fill }}=\frac{\left(\Delta \mathrm{p}_{\text {fill }} / \mathrm{Z}_{\text {fill }}\right)}{0.5 \rho_{\mathrm{a}} \mathrm{v}_{\mathrm{a}}^{2}}=\mathrm{c}_{6} \dot{\mathrm{M}}_{\mathrm{w}}^{\mathrm{c}_{7}} \dot{\mathrm{M}}_{\mathrm{a}}^{\mathrm{c}_{8}}
$$

Table 4.12 lists the values for the coefficients $c_{1}$ to $c_{8}$ obtained for each of the packing configurations. Surface tension measurements on the water used during these packing tests showed the surface tension to be with in $1 \%$ of the surface tension of distilled water at the corresponding temperature. 
Table 4.12 Correlation coefficients for the experimental splash pack.

\begin{tabular}{|c|c|c|c|c|c|c|c|c|c|c|c|}
\hline Layout & $\mathbf{c}_{\mathbf{1}}$ & $\mathbf{c}_{\mathbf{2}}$ & $\mathbf{R}^{\mathbf{2}}$ & $\mathbf{c}_{\mathbf{3}}$ & $\mathbf{c}_{\mathbf{4}}$ & $\mathbf{c}_{\mathbf{5}}$ & $\mathbf{R}^{\mathbf{2}}$ & $\mathbf{c}_{\mathbf{6}}$ & $\mathbf{c}_{\mathbf{7}}$ & $\mathbf{c}_{\mathbf{8}}$ & $\mathbf{R}^{\mathbf{2}}$ \\
\hline A & - & - & - & - & - & - & & $1 / \boldsymbol{m}$ & - & - & - \\
B & 0.845 & -0.511 & 0.81 & 0.678 & -0.359 & 0.587 & 0.85 & 6.557 & 0.387 & -0.508 & 0.71 \\
C & 0.908 & -0.522 & 0.88 & 0.742 & -0.344 & 0.614 & 0.93 & 3.969 & 1.020 & -0.908 & 0.92 \\
D & 0.805 & -0.453 & 0.80 & 0.729 & -0.287 & 0.524 & 0.85 & 3.576 & 0.827 & -0.881 & 0.96 \\
E & 0.804 & -0.417 & 0.75 & 0.536 & -0.126 & 0.591 & 0.96 & 3.050 & 0.915 & -0.924 & 0.97 \\
F & 0.765 & -0.434 & 0.77 & 0.505 & -0.145 & 0.599 & 0.96 & 2.725 & 0.978 & -1.027 & 0.98 \\
G & 0.898 & -0.465 & 0.86 & 0.668 & -0.262 & 0.577 & 0.95 & 9.732 & 0.433 & -0.000 & 0.79 \\
H & 1.024 & -0.489 & 0.87 & 0.760 & -0.283 & 0.605 & 0.95 & 5.130 & 0.596 & -0.155 & 0.75 \\
I & 0.951 & -0.486 & 0.87 & 0.687 & -0.273 & 0.590 & 0.95 & 3.863 & 0.655 & -0.251 & 0.83 \\
J & 0.761 & -0.461 & 0.83 & 0.518 & -0.190 & 0.605 & 0.98 & 8.057 & 0.472 & -0.546 & 0.92 \\
K & 0.811 & -0.510 & 0.89 & 0.599 & -0.297 & 0.626 & 0.97 & 4.462 & 0.650 & -0.616 & 0.96 \\
L & 0.821 & -0.446 & 0.83 & 0.570 & -0.193 & 0.587 & 0.98 & 3.471 & 0.747 & -0.674 & 0.94 \\
\hline
\end{tabular}

The measured overall transfer characteristic data is influenced by the water flowing down the walls of the test tower, as well as entrance effects due to the transfer taking place at the water distribution system and at the water collecting troughs below the packing. The wall effect is expected to be reduced by the deflecting plates which were installed on the inner walls of the test facility (see Figure 4.46). The effect of the water collecting troughs and the water distribution system can be accounted for by subtracting the empty tower transfer characteristic data, with $Z \approx 0 m$, from the packing test data. Note that this correction is only an approximation since the splashing on the water collecting troughs is expected to be influenced by the drop velocity and size distribution, which is different for every geometry.

Figure 4.55 compares the experimental data (corrected for entrance effects) to the general splash pack transfer characteristic correlation by Tezuka et al. [73TE1],[75TE1],[86FU1] (see Equation (2.8)) for a range of water and air flow rates. Although the Tezuka correlation generally overpredicts the transfer characteristics, the prediction accuracy of this correlation is fairly good for the less dense packings (with lower transfer characteristics per metre). 


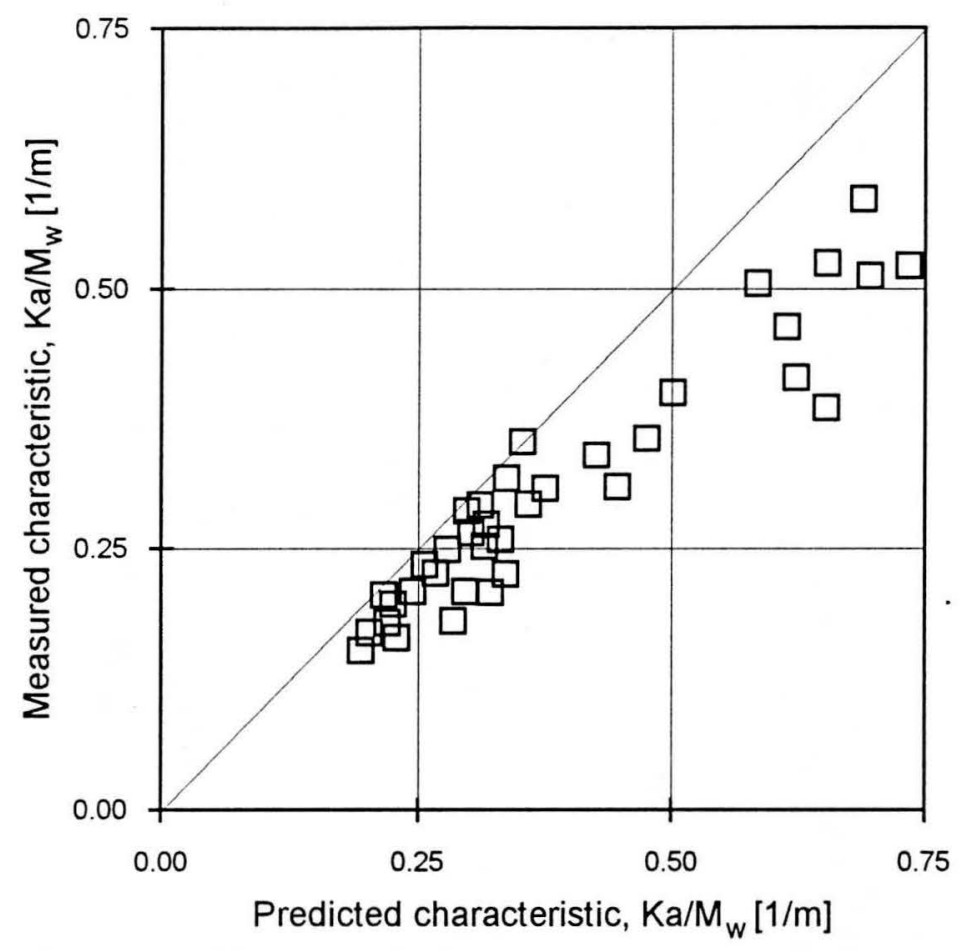

Figure 4.55 Comparison between experimental data and the general correlation by Tezuka et al. [73TE1],[75TE1],[86FU1].

\subsubsection{Drop size measurements in a cooling tower}

Drop size distribution measurements in a cooling tower fitted with splash packing material would be useful when comparing the accuracy of splash pack simulation model with the measured splash pack performance data. To obtain such data, a spring-operated shutter mechanism, as shown in Figure 4.56 was constructed to allow sampling of the drops falling through the experimental splash packing material. The shutter opening moved across a Petri dish containing silicone oil in less than a tenth of a second.

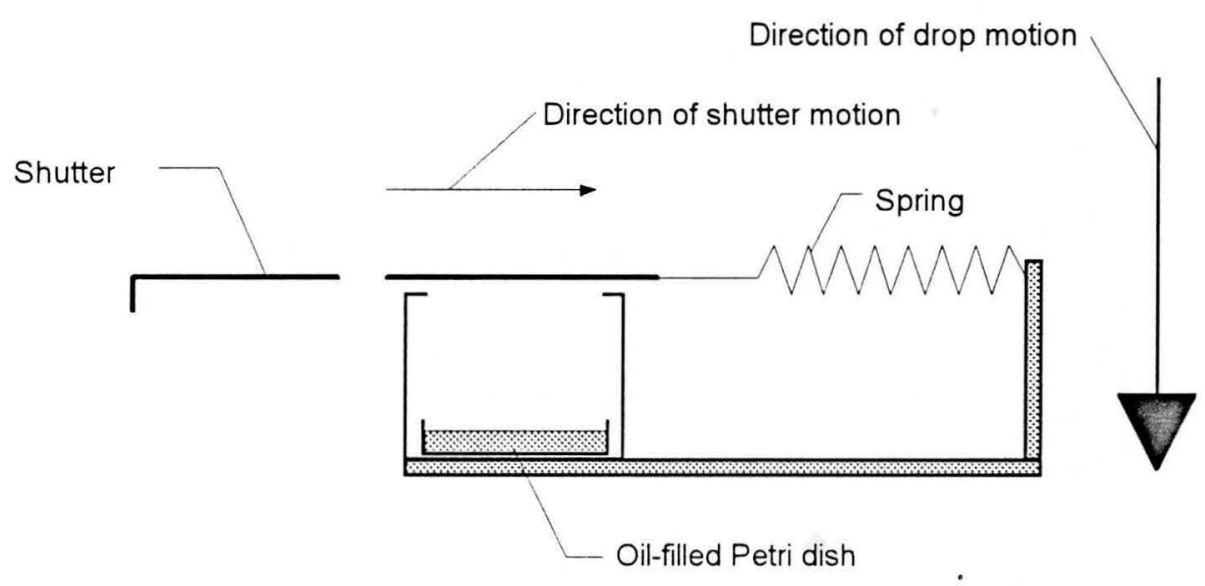

Figure 4.56 Layout of the shutter used to catch a sample of drops in a cooling tower. 
Photographs were taken of the drops which were caught in the oil. The drops on the photographs were then measured and counted using the method described in Appendix B. The drop size distributions below the packing of layouts D and I, where sampled at two water flow rates $\dot{\mathrm{M}}_{\mathrm{w}}=1.8$ and $3 \mathrm{~kg} / \mathrm{m}^{2} \mathrm{~s}$. The air flow velocity was kept constant at $1.5 \mathrm{~m} / \mathrm{s}$. Ten different photographs were taken in each case and the shutter position (in the horizontal plane) was different for every photograph to ensure a large random statistical sample. The results of these measurements are given in Appendix Q and Figure 4.57.

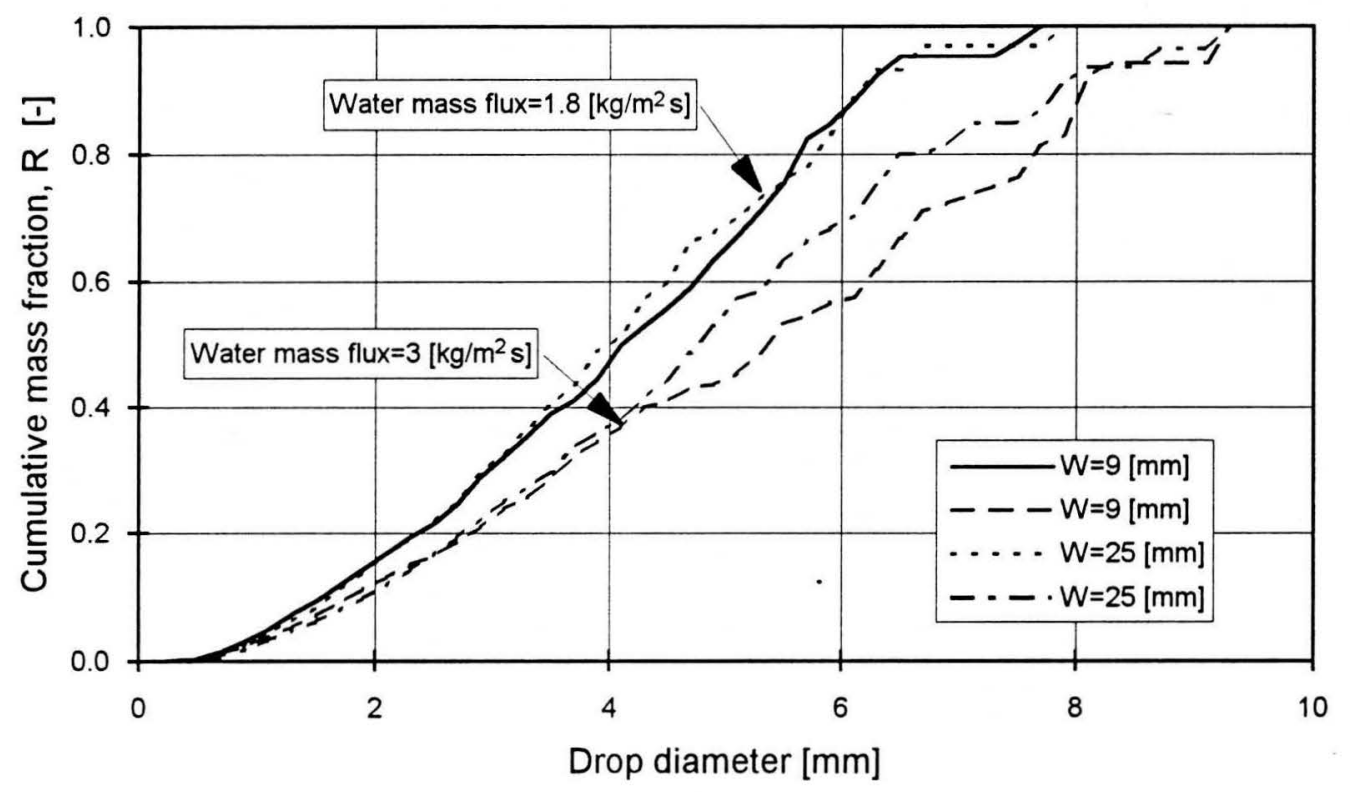

Figure 4.57 Measured drop size distribution data.

\subsubsection{Pressure drop across the splash grids in dry operation}

The pressure drop across the splash grids during dry operation was measured in an attempt to resolve the uncertainty regarding the loss coefficient for flow through sharp-edged grids. The pressure drop data is given in Appendix $\mathrm{R}$.

It can be seen from Figure 4.58, that the loss coefficient data obtained from the experimental data is in good agreement with the correlation based on the data by Baines and Peterson [51BA1] and Miller [90MI1], Equation (3.41).

It was found that the loss coefficients for the layouts with $200 \mathrm{~mm}$ grid spacing were slightly lower than that for the corresponding cases with grid spacings at $100 \mathrm{~mm}$ and $300 \mathrm{~mm}$. In all the cases the measured loss coefficients were found to increase slightly with increasing grid Reynolds number. The grid Reynolds numbers were much higher than 80 in all the cases listed in Appendix R, implying that the grids always contribute to the free stream turbulence. 


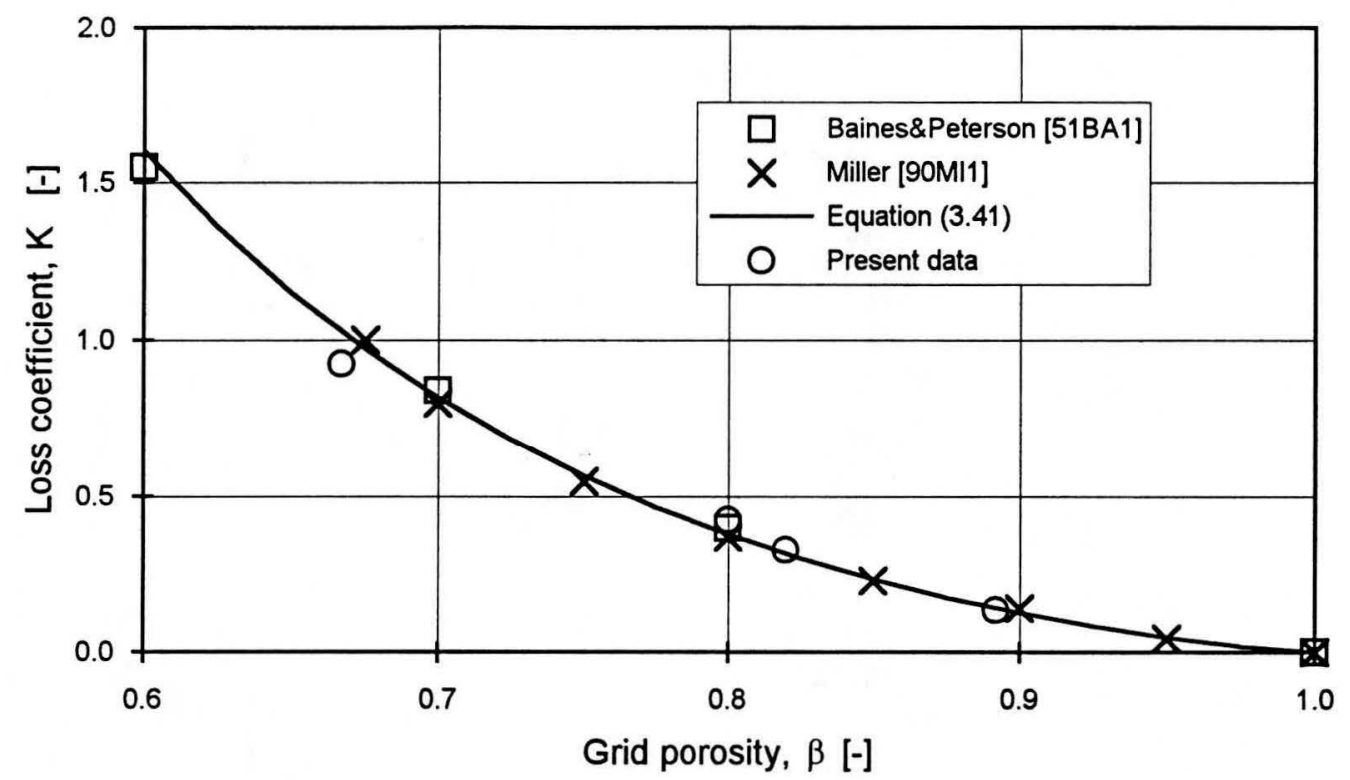

Figure 4.58 Experimentally determined pressure loss coefficients for flow through sharpedged grids. 


\section{CHAPTER 5 COMPUTER SIMULATION}

In this chapter the computer program for the simulation of cooling tower splash packing material is described with specific reference to the algorithms required. The different options for modelling drop acceleration, heat/mass transfer from drops, splash drop distribution, dripping drop formation below the slats, etc., based on information from the literature and from the experimental investigation of this study, are described.

The simulation program, SPSIM (Splash Pack SIMulation), was written in Borland Turbo Pascal on an IBM compatible personal computer. The source code for the simulation program is given in Appendix S. The Turbo Pascal code was written in such a manner to allow easy conversion to standard Pascal, to facilitate the execution of the simulation program on other (faster) computers.

\subsection{Mathematical model}

The following assumptions are made in the mathematical modelling of counterflow cooling tower splash pack:

i) The enthalpy potential model for simultaneous heat and mass transfer, proposed by Merkel [26ME1], is valid. This implies that $\mathrm{Le}_{\mathrm{f}}=1$ and that evaporation is negligible.

ii) The air is thoroughly mixed, i.e. the air enthalpy is constant in any given horizontal plane,

iii) Radiation effects are negligible,

iv) The initial drop size distribution and drop velocities are known at the water inlet side,

v) The transient problem of modelling accelerating drops may be approximated as a succession of steady states (see Yao [74YA1]),

vi) The drop drag coefficients and heat/mass transfer coefficients experienced by each drop in the splash pack is not influenced by the proximity of other drops (see discussion on interaction effects in Chapter 2).

vii) The effect of free stream turbulence on the drag of individual drops is assumed to be negligible. The effect of free stream turbulence on the heat and mass transfer from the drops is taken into account.

For integration purposes, the packing zone is divided into a number of layers. The number of layers corresponds to the number of splash grids. These imaginary layers in the packing are selected in such a way as to ensure that every grid (if any) falls on the boundary of a layer. If a rain and spray zone are to be evaluated as well, they each represent another layer (layers 0 and $\mathrm{N}_{\text {grids }}+1$ ), as shown in Figure 5.1. Every layer is subdivided into a number of elements, each with a thickness of $\partial z$. 


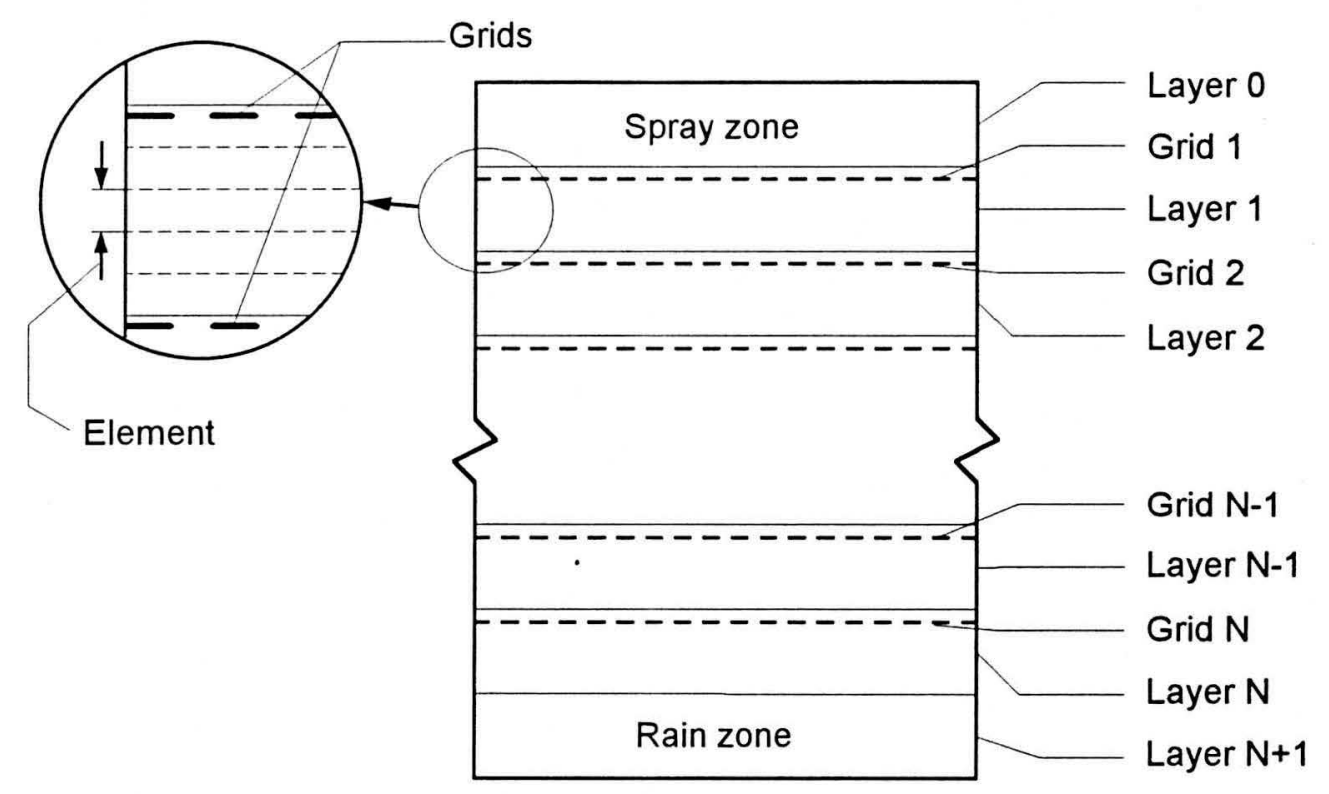

Figure 5.1 Layout of the imaginary integration elements along the packing height.

For a typical element, the following governing equation for the total heat transfer from the water to the air can be derived from the Merkel theory:

$$
\partial \dot{\mathrm{Q}}=\mathrm{K} \partial \mathrm{A}\left(\mathrm{i}_{\text {asw }}-\mathrm{i}_{\mathrm{a}}\right)=\mathrm{Ka} \mathrm{A}_{\mathrm{fr}} \partial \mathrm{z}\left(\mathrm{i}_{\text {asw }}-\mathrm{i}_{\mathrm{a}}\right)
$$

An energy balance gives

$$
\partial \dot{\mathrm{Q}}=\dot{\mathrm{m}}_{\mathrm{da}} \partial \mathrm{i}_{\mathrm{a}}=\dot{\mathrm{m}}_{\mathrm{w}} \mathrm{c}_{\mathrm{pw}} \partial \mathrm{T}_{\mathrm{w}}
$$

The temperature drop of the water and the air enthalpy gain in an element can be calculated from the equations above.

In cooling tower design calculations, the value of $\mathrm{K}$ (or $\mathrm{Ka}$ ) in Equation (5.1) is usually known from experimental data and the solution of the governing equations in each element is relatively straightforward. In this study, however, the equation of motion of a given drop falling through an element is solved to determine the average velocity of the drop through the element. The drop velocity is then used to calculate the heat and mass transfer coefficients. From these coefficients, the cooling rate of the drop in the element is calculated. As the drops fall through the packing, they strike the slats and this changes the drop size distribution. Small drops are formed by the splashing action on top of the slats, while relatively large drops drip from below the slats. Since the average drop velocity just below a slat is lower than that just above the slat, the net effect of the slat is to reduce the overall drop velocity and consequently to increase the drop residence time in an element. Both the smaller average drop size and the lower overall drop velocity tend to increase the air/water interface area. 


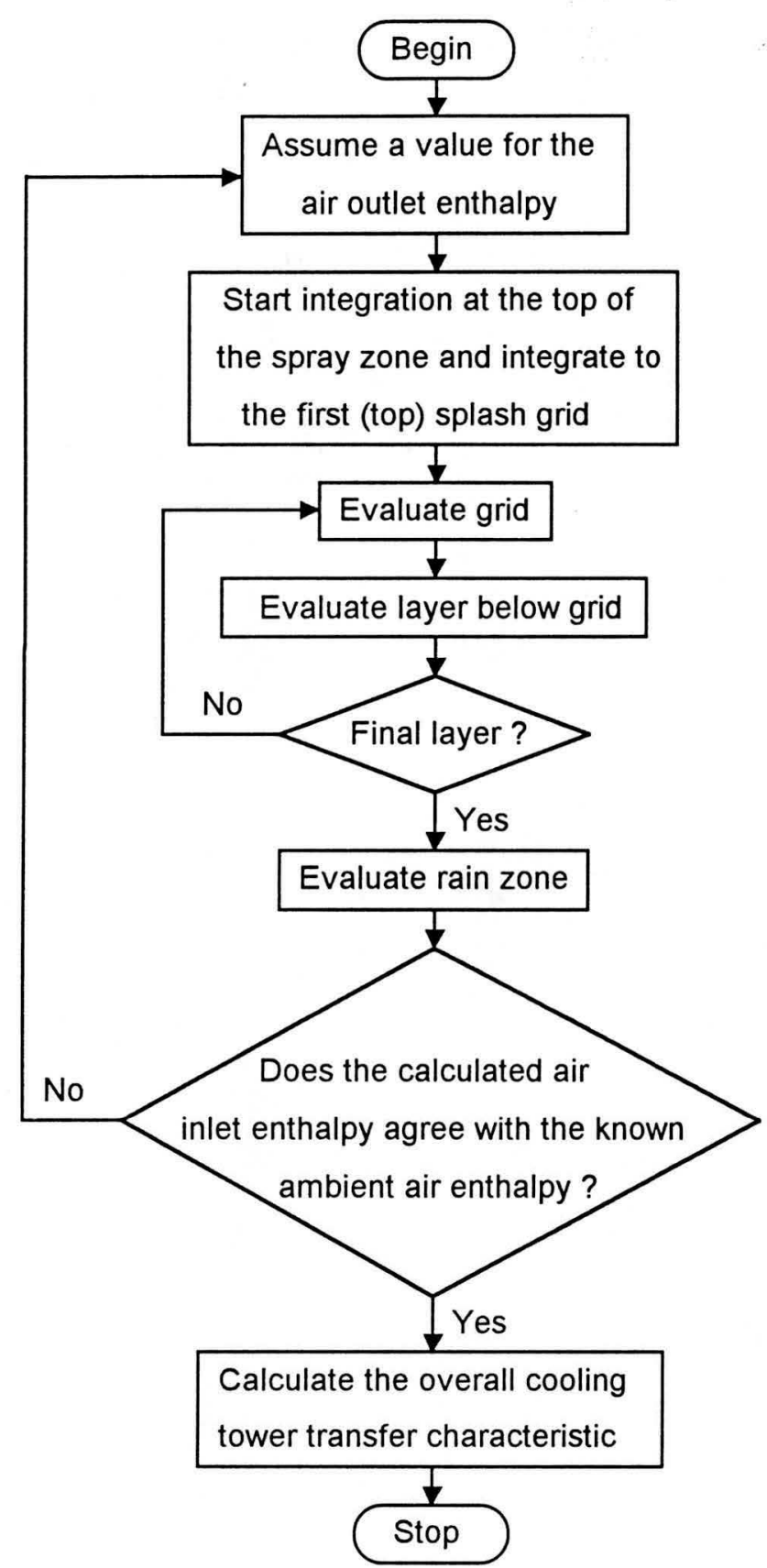

Figure 5.2 Flow diagram showing the main calculation steps in the splash pack simulation program.

It is convenient to start the integration process at the top of the packing zone (at the water inlet side) since the initial drop size and velocity distributions are known there. The outlet air enthalpy, $\mathrm{i}_{\mathrm{ao}}$, is not known and an initial value of $\mathrm{i}_{\mathrm{ao}}$ has to be assumed. The initial choice of air outlet enthalpy should be less than the smaller of $i_{a s}\left(T_{w i}\right)$ and

$$
\mathrm{i}_{\mathrm{ao}, \text { max }}=\mathrm{i}_{\mathrm{ai}}+\frac{\dot{\mathrm{m}}_{\mathrm{w}} \mathrm{c}_{\mathrm{pw}}\left(\mathrm{T}_{\mathrm{wi}}-\mathrm{T}_{\text {aiwb }}\right)}{\dot{\mathrm{m}}_{\mathrm{da}}}
$$


After the integration downwards through the packing, the calculated air inlet enthalpy should correspond with the ambient air enthalpy (if the initial choice of $i_{a o}$ was correct). If it does not agree, a new value of air outlet enthalpy has to be assumed and the integration process repeated until a solution is reached. The interval halving or the secant methods are usually employed to find the correct value of the air outlet enthalpy. Upon completion of the integration process (after reaching the air inlet side with the correct choice of outlet air enthalpy), the average outlet water temperature can be calculated. The overall transfer characteristic, or $\mathrm{KaZ} / \dot{\mathrm{M}}_{\mathrm{w}}$, of the packing can then be calculated by using any conventional cooling tower integration procedure, e.g., the Tchebycheff integration method (see Cale [82CA1] or Johnson [89JO1]).

The calculation procedure is shown in Figure 5.2. The algorithms required for the evaluation of the free fall zones and the influence of the splash grids are described in more detail below.

\subsection{Packet concept}

To simplify and reduce the number of calculations required to evaluate a given element, the collection of drops in each element is divided into discrete packets. These packets allow drops of similar diameter, temperature and velocity to be lumped together. Each packet has a unique combination of drop size, velocity and temperature. To specify the number of drops per packet, the mass flow rate represented by each packet is used. In the computer model, each packet is represented by a unique number. Four one-dimensional arrays (vectors) are used to store the values of drop diameter, velocity, temperature and mass flow rate (per packet).

Any drop size distribution can be represented by distributing the drops in a range of fixed drop size intervals (or classes). Since large water drops are inherently unstable in free fall, it is assumed that the drop distribution has an upper limit, $\mathrm{d}_{\max }$. The drop size intervals can be linearly or logarithmically spaced between $d_{\min }(0 \mathrm{~mm})$ and $d_{\max }$. If it is assumed that $d_{\max }=10$ $\mathrm{mm}$ and that there are 20 equal-sized classes, it follows that the first drop class is represented by drops with diameters of $0.25 \mathrm{~mm}$, the next $0.75 \mathrm{~mm}$, etc.. At the top of the range, the ratio of class width to drop size, $\Delta \mathrm{d} / \mathrm{d}$, is $0.05(0.5 / 9.75)$, while it is equal to $2(0.5 / 0.25)$ at the low end of the size range. In order not to lose resolution at the low end of the range, the drop size classes can be logarithmically distributed.

The discrete packet modelling approach has the following advantages: (i) the equation of motion and the heat/mass transfer is only evaluated once per element per packet, thus reducing the number of calculations significantly and (ii) since the numbers of packets are relatively small (compared to the number of drops) in a given element, the computer memory required to store the packet information is not excessive. 


\subsection{Free fall zone evaluation}

The major part of the total energy transfer in a splash pack takes place in the free fall zones between the grids. The drop residence times and velocities in a particular free fall zone are governed by the upward air velocity, the initial drop velocities and the drag forces on the individual drops. The drop velocity is not only important in controlling the drop cooling, but it controls the splashing phenomenon occurring on the grids below.

Figure 5.3 shows the sequence of steps used in the simulation program to describe the heat/mass transfer, drop motion, etc. for each packet of water drops falling between the grids in a coolı1g tower splash pack. The modelling of the individual steps is described in more detail below.

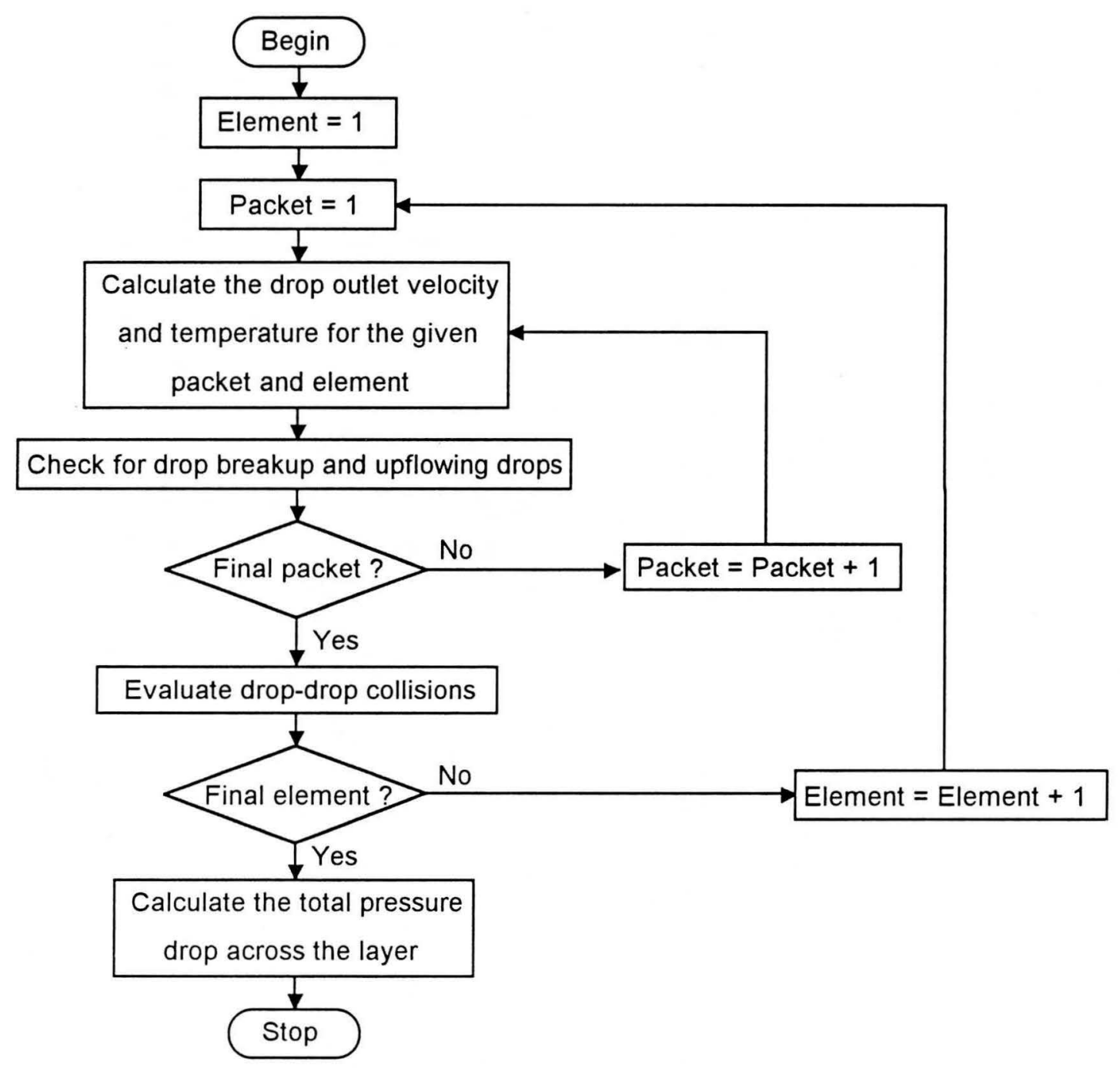

Figure 5.3 Flow diagram showing the calculation steps required to evaluate a typical free fall zone between grids. 


\subsubsection{Drop motion}

For a drop falling vertically downwards through an upward flowing airstream, the upward drag force can be expressed as

$$
\mathrm{F}_{\mathrm{up}}=0.5 \rho_{\mathrm{a}}\left(\mathrm{v}+\left|\mathrm{v}_{\mathrm{a}}\right|\right)^{2} \mathrm{~A}_{\mathrm{fr}} \mathrm{C}_{\mathrm{D}}
$$

Note that positive direction is chosen as vertically downwards, thus a falling drop has a positive velocity and an upward flowing drop has a negative velocity. Since the airflow is always upwards, it is convenient to assume $\mathrm{v}_{\mathrm{a}}=\left|\mathrm{v}_{\mathrm{a}}\right|$ and to implement it as such in the governing equations. Also note that the velocities are always expressed relative to a fixed reference frame unless specifically stated otherwise. The effect of gravity and buoyancy is given by

$$
F_{\text {down }}=\left(\frac{\pi d^{3}}{6}\right)\left(\rho_{w}-\rho_{a}\right) g
$$

The procedure to determine the outlet (leaving) velocity of a drop entering an element of thickness, $\partial z$, at a velocity of $v_{i}$, is described in Figure 5.4 .

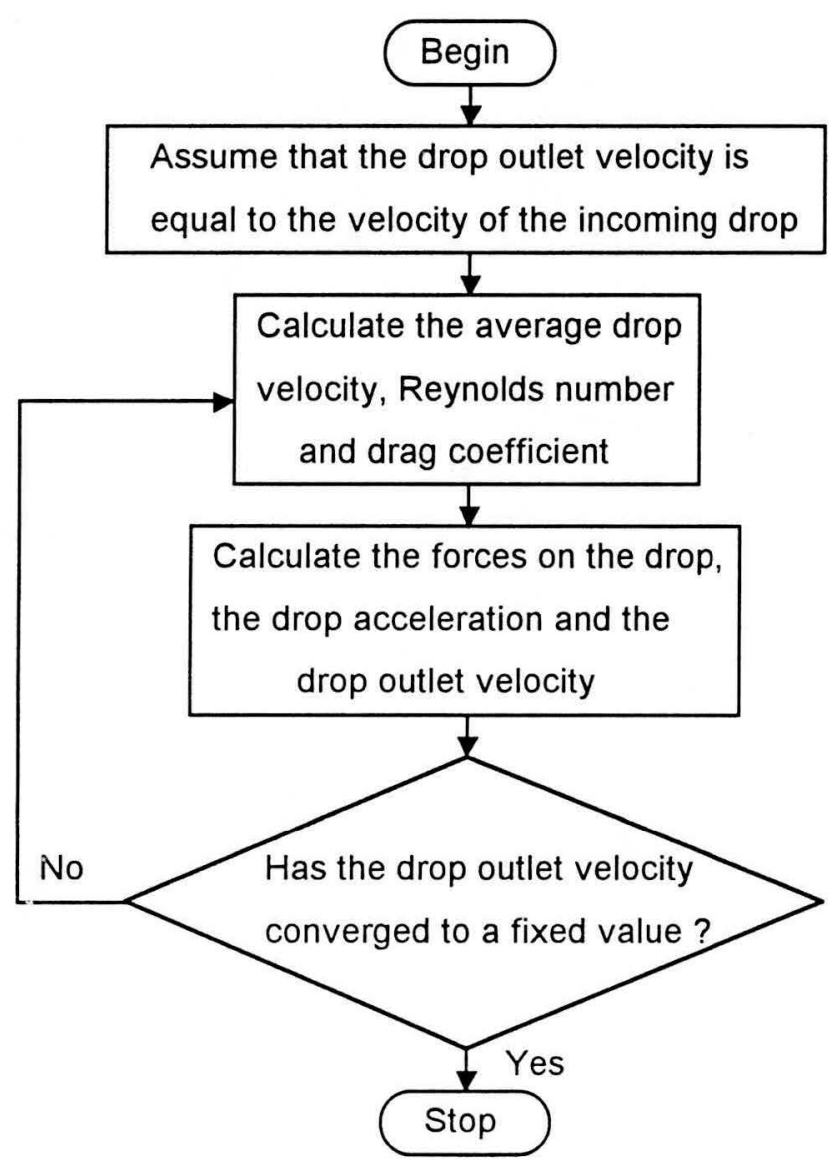

Figure 5.4 Calculation procedure to find the drop velocity change in an integration element. 
Since very small drops can be dragged upwards by the airstream, it may be necessary to divide the packing into smaller elements to prevent numerical problems in the element where a small drop starts moving upwards.

The simulation program allows the user to select one of three drag models i.e. (i) no drag $\left(C_{D}=0\right)$, (ii) drop drag calculated from solid sphere drag data and (iii) drop drag calculated using the model which takes drop deformation into account. For solid spheres, the drag coefficient is calculated using the correlation by Turton and Levenspiel [86TU1] given in Chapter 2. The effect of acceleration on the drag experienced by a solid sphere is assumed to be negligible. The model developed in Chapters 3 and 4 is used to calculate the drag experienced by deformable water drops during acceleration.

\subsubsection{Heat and mass transfer from drops}

From the analogy between heat and mass transfer, it follows that

$$
K=\frac{h_{c}}{L e_{f} c_{p m}}=\frac{N_{u} k_{a}}{L e_{f} c_{p m} d}
$$

The average Nusselt number can be calculated from the known drop size, the average velocity relative to the airstream, etc.. The simulation program allows the user to select any one of the Nusselt number correlations listed in Chapters 2 and 3, i.e.: (i) the Ranz and Marshall [52RA1], [52RA2] correlation, (ii) the Yao [74YA1] correlation, (iii) the correlation based on the recalculated data by Yao [74YAl] and (iv) the correlation given by Mercker [93ME1].

The number of drops of any given packet with drop diameter, $d$, and velocity, $v$, in an element can be found from

$$
\begin{aligned}
\mathrm{N} & =(\text { number of drops per second })(\text { residence time }) \\
& =\left(\frac{\dot{\mathrm{m}}_{\mathrm{w}}}{\rho_{\mathrm{w}} \mathrm{V}_{\mathrm{d}}}\right)\left(\frac{\partial \mathrm{z}}{\mathrm{v}_{\mathrm{m}}}\right)
\end{aligned}
$$

The total surface area of the drops belonging to a particular packet in an element can be calculated from the known number of these drops in the element. The procedure to calculate the temperature change of a drop across an element, is shown in Figure 5.5. 


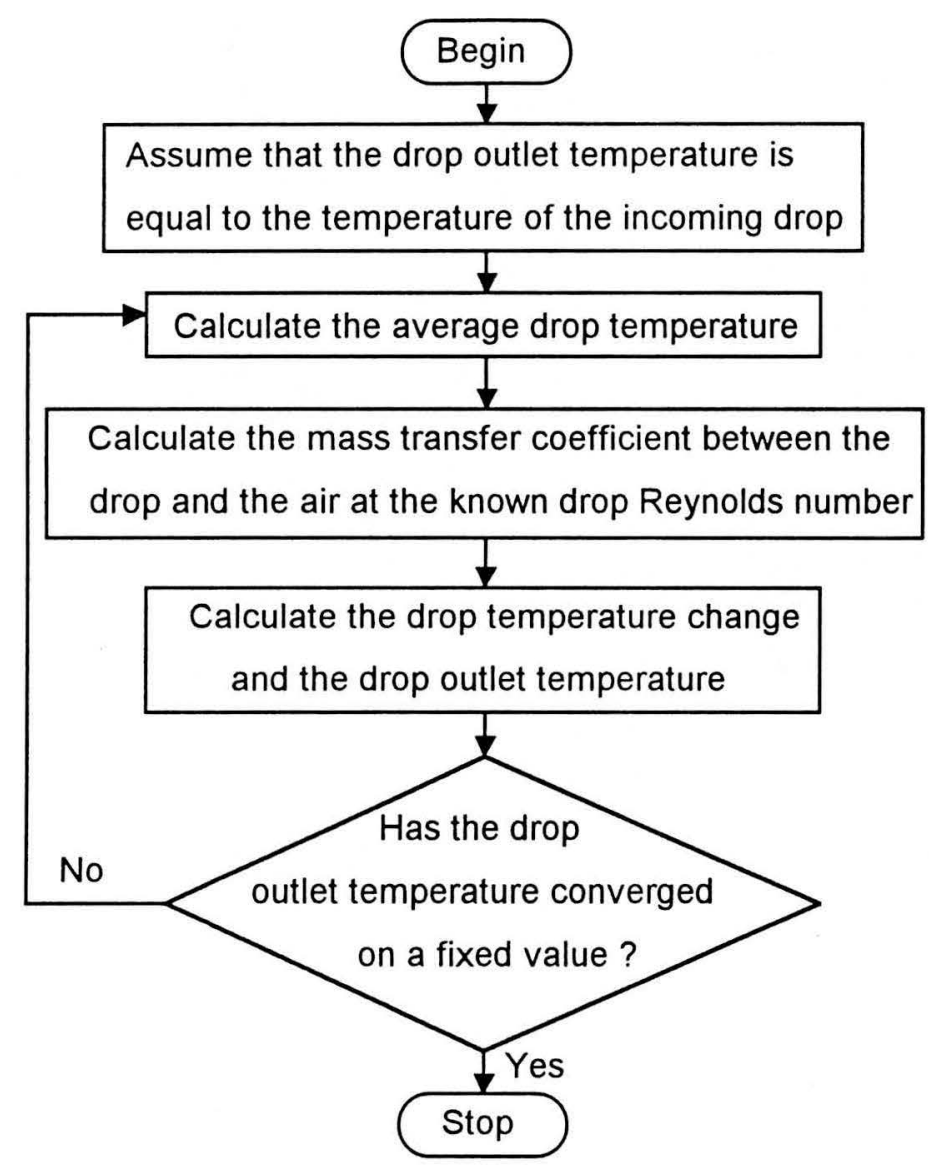

Figure 5.5 Calculation procedure to find the drop temperature change in an integration element.

For very small drops, or drops with terminal velocities very close to the velocity of the upward flowing airstream, the total surface area of the drops in an element may be very large, resulting in very large $\partial \mathrm{T}_{\mathrm{w}}$ values for the element, which results in a numerical instability in the calculation procedure described above. To cure this, the value of $\partial z$ must be decreased by subdividing each element into even smaller steps. For each of the smaller steps, a stable value of $\partial \mathrm{T}_{\mathrm{w}}$ can be found. In some cases where $\mathrm{t}_{\mathrm{res}} \rightarrow \infty$ (drops moving down very slowly), an excessive number of steps would be required to achieve a stable solution for $T_{w o}$. In these cases, the drop temperature approaches the adiabatic saturation temperature at the local air conditions and in the simulation model it is assumed that $\mathrm{T}_{\mathrm{wo}}=\mathrm{T}_{\text {sat }}$.

\subsubsection{Aerodynamic drop break-up}

Large water drops accelerating in air, become unstable due to the aerodynamic forces acting on each drop. The various criteria for aerodynamic drop break-up are discussed in Chapter 2 . The splash pack simulation program uses the criterion specified by Wierzba [90WI1] to determine whether a given drop is unstable. According to this criterion the drop will be unstable if the drop Weber number, $\rho_{\mathrm{a}} \mathrm{v}^{2} \mathrm{~d} / \sigma$, is greater than 11 , or when the drop velocity (relative to the packing) is greater than $v_{\text {crit }}$ where 


$$
\mathrm{v}_{\mathrm{crit}}=\sqrt{\frac{11 \sigma}{\rho_{\mathrm{a}} \mathrm{d}}}-\left|\mathrm{v}_{\mathrm{a}}\right|
$$

The following simple algorithm is used to model the aerodynamic break-up of drops: If a particular drop is found to be unstable, the drop is divided into two identical, smaller drops. Each smaller drop has (i) $50 \%$ of the volume of the parent drop, (ii) the same temperature and (iii) the same velocity as the parent drop.

\subsubsection{Drop-drop collisions}

As discussed in Chapter 2, drop-drop collisions occur in rain since large and small drops fall at different velocities in still air. In the case of drops falling through splash packing material, the drops normally travel at velocities below the terminal velocity. Slow moving drops are formed at each grid and it creates the possibility that a fast moving, small drop may catch up with a slower moving, larger drop. This, together with the very high rain densities found in cooling towers, results in large numbers of drop/drop collisions occurring in the packing zone of cooling towers fitted with splash packing material.

The complete stochastic modelling of drop-drop collisions would require excessive computing time. The following simplified algorithm, assuming straight drop trajectories $\left(\eta_{\text {coll }}=1\right)$, is employed to evaluate the drop-drop collisions in the splash pack simulation program. The number of collisions between drops in two packets (say A and B) can be calculated from the probability of collision between the drops. Consider a drop of diameter, $\mathrm{d}_{\mathrm{A}}$, falling at a given velocity, $\mathrm{v}_{\mathrm{A}}$, through a cloud of slower moving drops belonging to packet $\mathrm{B}$. During a unit time, this drop sweeps out the following volume,

$$
\dot{\mathrm{V}}=\left(\frac{\pi}{4}\right)\left(\mathrm{d}_{\mathrm{A}}+\mathrm{d}_{\mathrm{B}}\right)^{2}\left|\mathrm{v}_{\mathrm{A}}-\mathrm{v}_{\mathrm{B}}\right|
$$

Assuming that there are $\mathrm{N}_{\mathrm{A}}$ drops in packet $\mathrm{A}$ and $\mathrm{N}_{\mathrm{B}}$ drops in packet $\mathrm{B}$ per unit volume and assuming a collision efficiency of $\eta_{\text {coll }}$, the number of collisions occurring between these two packets per unit volume per unit time can be expressed as

$$
\dot{\mathrm{N}}_{\text {coll }}=\left(\frac{\pi}{4}\right)\left(\mathrm{d}_{\mathrm{A}}+\mathrm{d}_{\mathrm{B}}\right)^{2}\left|\mathrm{v}_{\mathrm{A}}-\mathrm{v}_{\mathrm{B}}\right| \eta_{\text {coll }} \mathrm{N}_{\mathrm{A}} \mathrm{N}_{\mathrm{B}}
$$

where

$$
\mathrm{N}_{\mathrm{A}}=\left(\frac{\dot{\mathrm{N}}_{\mathrm{A}}}{\mathrm{A}_{\mathrm{fr}} \mathrm{v}_{\mathrm{A}}}\right) \text { with } \dot{\mathrm{N}}_{\mathrm{A}}=\left(\frac{\dot{\mathrm{m}}_{\mathrm{A}}}{\rho_{\mathrm{w}}\left(\pi \mathrm{d}_{\mathrm{A}}^{3} / 6\right)}\right)
$$


and

$$
\mathrm{N}_{\mathrm{B}}=\left(\frac{\dot{\mathrm{N}}_{\mathrm{B}}}{\mathrm{A}_{\mathrm{fr}} \mathrm{v}_{\mathrm{B}}}\right) \text { with } \dot{\mathrm{N}}_{\mathrm{B}}=\left(\frac{\dot{\mathrm{m}}_{\mathrm{B}}}{\rho_{\mathrm{w}}\left(\pi \mathrm{d}_{\mathrm{B}}^{3} / 6\right)}\right)
$$

Assuming a collision efficiency of unity, the number of collisions per second between the drops in the two packets in an imaginary volume of thickness $\partial \mathrm{z}$ and frontal area of $\mathrm{A}_{\mathrm{fr}}$ can be expressed as

$$
\dot{\mathrm{N}}_{\text {coll, } \mathrm{A}, \mathrm{B}}=\dot{\mathrm{N}}_{\text {coll }} \mathrm{A}_{\text {fr }} \partial \mathrm{z}
$$

The following simple algorithm is used in the simulation program to model the drop-drop collisions between all the packets of drops in a single element:

i) For each pair of packets, the collision probabilities, $\left(\dot{\mathrm{N}}_{\text {coll }, A, B} / \dot{\mathrm{N}}_{A}\right)$ and $\left(\dot{\mathrm{N}}_{\text {coll }, A, B} / \dot{\mathrm{N}}_{B}\right)$, are calculated. To ensure that the drop collisions are evaluated correctly, the collision probabilities are calculated using the conditions (drop diameter, velocity and drop temperature) of the packets leaving the previous element. The packets resulting from the collisions are stored in temporary arrays. This means that the effect of collisions between packets $\mathrm{A}$ and $\mathrm{B}$ does not reduce the number of drops in packet $\mathrm{A}$, which could influence the number of collisions between packets $\mathrm{A}$ and $\mathrm{C}$, etc.

ii) Only if one (or both) of the collision probabilities for a pair of colliding drop packets is larger than a predetermined fraction, is the collision evaluated to determine the existence and composition of any new packet(s) which may be formed. By only considering collisions which have a large effect on the total drop distribution, the possibility of forming single-drop packets is eliminated and the program execution is speeded up significantly since the total number of packets is kept small.

Upon collision, it is assumed that only coalescence or bouncing occurs. The simulation program employs the relation by Brazier-Smith et al. [72BR1] to determine the coalescence efficiency. The number of colliding drops which coalesce after collision is given by $\left(\dot{\mathrm{N}}_{\text {coll }, \mathrm{A}, \mathrm{B}} \eta_{\text {coal }}\right)$. The coalescing drops are removed from their parent packets and form a new packet. The diameter, velocity, temperature, and mass flow rate of the drops in the new packet are determined from the laws of energy, momentum and mass conservation.

In actual collisions between liquid drops, it is expected that satellite drops would form (see Chapter 2). This has not been incorporated in the current mathematical model in order to prevent excessively large numbers of new packets from forming. This simplification is not expected to have a significant influence on the predicted splash pack performance as the 
current simple drop coalescence model was found to have a very small influence on the predicted splash pack performance (see Chapter 6).

\subsubsection{Handling of upward flowing drops}

If the velocity of the upward flowing airstream is higher than the terminal velocity of a given drop, the drop will move upwards with the airstream. In a cooling tower most of these upflowing drops will be intercepted by the drops falling through the packing or by the drift eliminators installed above the packing (in most cooling towers). Only very small drops will leave the cooling tower through the drift eliminator. The effect of this water loss on the performance of splash packing material is usually negligible due to the very small mass flow rate which these drops represent.

This phenomenon is handled in one of three ways in the simulation program:

i) The up-flowing drop mass is assumed to be lost, i.e. it is removed from the calculation.

ii) All the up-flowing drops originating in a given element are assumed to be caught by the falling drops in the element. The mass, momentum and temperature of these drops are redistributed among the falling drops according to the probability of collision between the up-flowing drops and the down-flowing drops.

iii) The up-flowing drops are assumed to collect on the drift eliminator which is usually installed above the packing in a counterflow cooling tower. These drops are assumed to cool to the local air wet-bulb temperature at the tower outlet and then drip down as large drops. In the simulation program it is assumed that these drops (at the air wet-bulb temperature at the air outlet side) can be included in determination the average water outlet temperature. In actual fact, these large drops could undergo further cooling and/or splashing as they fall towards the pond below the packing, but attempting to include these effects in the simulation would complicate matters significantly. This simplification was found to be of lesser importance since the effect of drift loss on the thermal performance of the splash pack was found to be insignificant (see Chapter 6).

\subsubsection{Pressure drop}

The static pressure drop across the free fall zones (i.e. excluding the pressure drop across the splash grids) can be expressed as

$$
\Delta \mathrm{p}_{\text {drops }}=\frac{\sum_{\mathrm{i}=1}^{\mathrm{N}_{\text {elements }}} \sum_{\mathrm{j}=1}^{N_{\text {packets }}}\left(F_{\text {drag }, \mathrm{i}, \mathrm{j}}\right)}{\mathrm{A}_{\mathrm{fr}}}
$$

This equation states that the total static pressure drop across a drop free fall zone is equal to the sum of the drag forces experienced by all the drops, divided by the air flow area. 


\subsection{Grid evaluation}

Grids are placed in the fall path of the drops moving through a cooling tower to decrease the average drop size, redistribute the water (large drops, which are normally warmer due to their relative small surface area and short residence times, splash and form smaller splash drops which cool much more efficiently) and to reduce the average drop velocity in the cooling tower. The surface area of the grid can also have a noticeable effect on the overall performance of the packing, due to the cooling of the water film covering the grids. Upon drop impact on the surface of a grid, the drop may splash if the impact energy is high enough, or the drop may experience a cutting or splitting action if the impact is close to the edge of a slat. Only a fraction of the water which impacts a grid is lost due to splashing and/or cutting, while the rest drips from below the grid as relatively large drops.

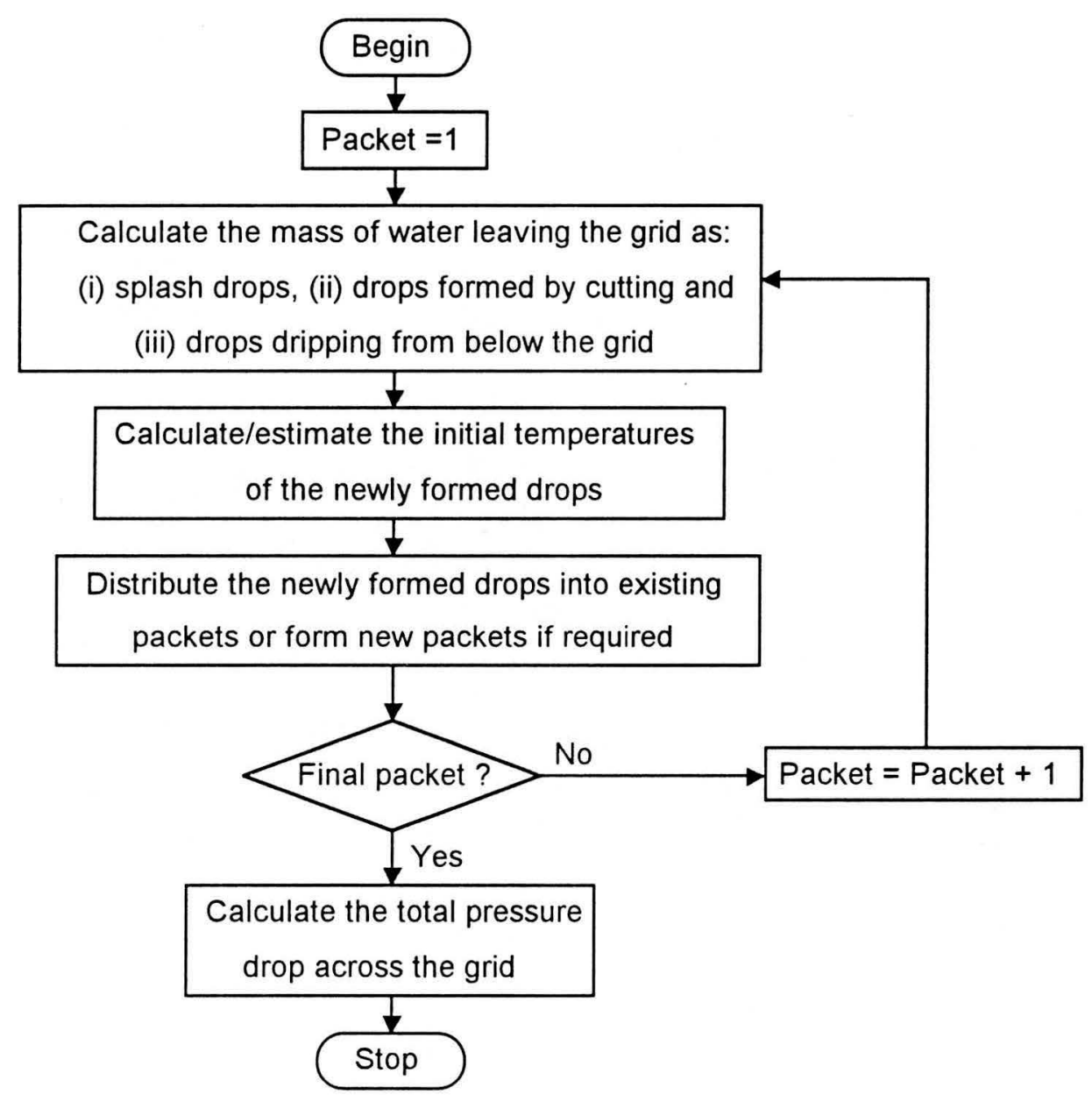

Figure 5.6 Flow diagram showing the calculation steps required to evaluate the influence of a grid on the drops falling through the packing. 
In the splash pack simulation, the imaginary elements in the packing are selected in such a way as to ensure that every grid (if any) falls on the boundary of an element. The following assumptions are made:

i) The incoming drops are assumed to travel in straight trajectories. This is justified by the relatively high inertia of the drops compared to the small deflecting drag forces due to the airflow around the grids. (see Appendix E)

ii) The drop packets are evenly distributed over the entire flow area.

iii) The drop impacts on the slats and the resulting crown formation are not influenced by neighbouring impacts. This assumption is discussed in more detail in Chapter 6.

The sequence of steps used to describe the grid influence on the packets of water drops falling through cooling tower splash pack is shown in Figure 5.6. The modelling of the individual steps are described in more detail below.

\subsubsection{Splashing and cutting}

\section{Splashing}

The total mass of water splashing from the surface of a slat when it is struck by an incoming drop is given by

$$
\mathrm{m}_{\mathrm{s}}=\overline{\mathrm{f}_{\mathrm{s}}}\left(\rho_{\mathrm{w}}\left(\frac{\pi \mathrm{d}_{\mathrm{i}}^{3}}{6}\right)\right)
$$

Similarly, the total mass flow rate of water splashing from a slat when it is struck by the drops of a packet, say packet $A$, is given by

$$
\dot{\mathrm{m}}_{\mathrm{s}, \mathrm{A}}=\overline{\mathrm{f}_{\mathrm{s}, \mathrm{A}}}\left(\mathrm{W}+\mathrm{d}_{\mathrm{i}, \mathrm{A}}\right) \dot{\mathrm{LM}}_{\mathrm{w}, \mathrm{A}}
$$

The mean splash fraction is dependent on the film thickness on the slat before the drop impact, the size of the incoming drop and the velocity of the incoming drop. The empirical correlations for the mean splash fraction described in Chapters 3 and 4 can be used to determine the mean splash fraction for each packet. The empirical correlations in Chapter 4 were determined for four slat widths i.e. $\mathrm{W}=2,5,10$ and $25 \mathrm{~mm}$. Since there is a very gradual transition between these correlations for a given film thickness, drop size and drop velocity, linear interpolation can be used to find the mean splash fractions for slat widths between 2 and $25 \mathrm{~mm}$. For slats slightly wider that $25 \mathrm{~mm}$, the mean splash fraction is roughly the same as that for the $25 \mathrm{~mm}$ slat, but for very wide slats, $W>50 \mathrm{~mm}$, the mean splash fraction will be less than that for the $25 \mathrm{~mm}$ wide slat. This can be attributed to the fact that for drops impacting near the centre of a wide slat, a large fraction of the resulting splash drops will fall back onto the slat. Assuming 
that the splash fraction is zero on an infinite narrow slat, the mean splash fractions for drop impacts on slats narrower than $2 \mathrm{~mm}$ can be approximated by

$$
\overline{f_{s}}=\left(\frac{W}{2}\right) \overline{f_{s, W=2}}
$$

where $\mathrm{W}$ is expressed in $\mathrm{mm}$. Note that by using the mean splash fraction in the equations above, the mass of the drops splashing from a slat is independent of the impact position on the slat. These equations express the splash drop mass as the average mass leaving the slat for any drop impact position between $-\left(\mathrm{W}+\mathrm{d}_{\mathrm{i}}\right) / 2$ and $\left(\mathrm{W}+\mathrm{d}_{\mathrm{i}}\right) / 2$.

Two models for predicting the splash drop size distribution are incorporated in the simulation program:

i) The splashing drops are assumed to be distributed according to the model proposed by Scriven et al. [74SC1], where the number of drops of size $d$ is given by

$$
N\left(d_{s}\right)=c_{1}\left(\frac{\rho_{a} v_{i}^{2}\left(d_{i} / 2\right)}{\sigma}\right)\left(\frac{\left(d_{i} / 2\right)}{d_{s}^{2}}\right)
$$

Only one value of $c_{1}$ will result in a splash drop distribution which has the same total volume as that of the actual splash volume. This value of $c_{1}$ is found iteratively from Equation (5.18). This model predicts the existence of splash drops of all sizes smaller than the incoming drop size. This differs significantly from the experimentally observed upper limit on splash drop diameter of approximately $2.5 \mathrm{~mm}$.

ii) The splash drop sizes are assumed to be distributed according to the Rosin-Rammler distributions described in Chapter 4. The experimentally determined correlations are used to determine the Rosin-Rammler shape parameter and the mass mean drop diameter for every combination of incoming drop size and velocity.

One of two models is employed to determine the initial downward velocity and the initial temperature of the drops formed by splashing, i.e :

i) The first model ignores the upward motion of the splash drops due to the splashing action. In this case the splash drops are assumed to start from zero velocity from the slat position, and the initial splash drop temperatures are calculated using the mixing model proposed in Chapter 3.

ii) In the second model, the upward motion of the splash drops is taken into account when determining the initial splash drop velocity and temperature. The splash drop motion and the corresponding drop cooling can be calculated using the simplified analytical model described in Appendix D. The initial drop velocity (for the splash drops falling downwards from the slat) is also determined from the analytical drop trajectory 
calculations. The initial temperature of the splash drops at the start of the upward splash drop motion can be determined from the mixing model proposed in Chapter 3 . The initial upward velocity of the splash drops can be determined from the correlation based on the data by Ghadiri and Payne [78GH1], Equation (3.38), or from Equation (4.27), which is based on the experimental work of this study.

A large number of new packets, containing the drops which are formed by splashing, are formed by each packet striking a given slat. The packet combination algorithm described below is employed to combine packets which are very similar.

\section{Cutting}

The average mass of water lost due to cutting for a given drop impact can expressed by

$$
\mathrm{m}_{\mathrm{c}}=\overline{\mathrm{f}_{\mathrm{c}}}\left(\rho_{\mathrm{w}}\left(\frac{\pi \mathrm{d}_{\mathrm{i}}^{3}}{6}\right)\right)
$$

As before, the total mass flow rate of water in a packet, say packet $A$, which is lost over the edge of the slat due to cutting, is given by

$$
\dot{\mathrm{m}}_{\mathrm{c}, \mathrm{A}}=\overline{\mathrm{f}_{\mathrm{c}, \mathrm{A}}}\left(\mathrm{W}+\mathrm{d}_{\mathrm{i}, \mathrm{A}}\right) \dot{\mathrm{LM}}_{\mathrm{w}, \mathrm{A}}
$$

From the definition of the mean splash fraction (see Chapter 3), i.e.,

$$
\overline{f_{c, A}}=\left(\frac{d_{i, A}}{W+d_{i, A}}\right)
$$

it follows that

$$
\dot{\mathrm{m}}_{\mathrm{c}, \mathrm{A}}=\mathrm{d}_{\mathrm{i}, \mathrm{A}} \mathrm{LM}_{\mathrm{w}, \mathrm{A}}
$$

The simulation program uses the simple cutting model described in Chapter 3 , to determine the sizes of the drops formed by cutting. Note that each impacting drop does not result in one drop formed by cutting, but that the total mass of drops of diameter, $\mathrm{d}_{\mathrm{i}}$, which are cut on impact with the grid, is redistributed as cut drops.

It is assumed that the new packets, containing drops formed by the cutting action, have initial velocities and initial temperatures equal to the velocity and temperature, respectively, of the packet from which they were formed. 


\subsubsection{Heat/mass transfer from grids}

The water films covering the surface of the slats are cooled by the air flowing through the grids. The initial temperature of the film on a slat, $\mathrm{T}_{\mathrm{fi}}$ (see Figure 5.7), can be calculated from the energy balance between the water entering and leaving an imaginary control volume around the water film on the upper surface of the slat (see Equation (3.27)).

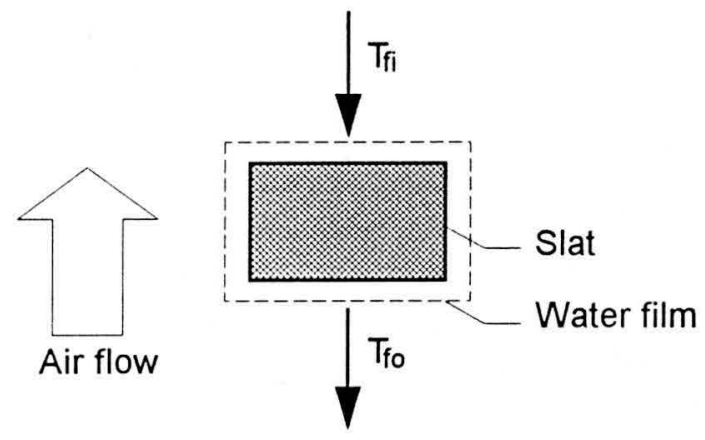

Figure 5.7 Schematic layout of the control volume used to model the cooling of the water film flowing over the slats.

The water film flowing down over the surface of a slat is cooled by simultaneous heat and mass transfer to the air. The temperature of the water film dripping from below a slat, $\mathrm{T}_{\mathrm{fo}}$, can be calculated from

$$
\mathrm{T}_{\mathrm{fo}}=\mathrm{T}_{\mathrm{fi}}-\left(\frac{\mathrm{KA}_{\text {slat }}\left(\mathrm{i}_{\mathrm{asw}}-\mathrm{i}_{\mathrm{a}}\right)}{\dot{\mathrm{m}}_{\mathrm{d}} \mathrm{c}_{\mathrm{pw}}}\right)
$$

The mass transfer coefficient can be determined from the analogy between heat and mass transfer and the convective heat transfer coefficient around a slat (see Equation (5.6)). The applicability of the correlation by Gnielinski [83GN1], for the calculation of the convective heat transfer coefficient experienced around any type of slat, was confirmed experimentally (see Chapter 4).

The correlation of Gnielinski is strictly only valid for determining the heat transfer coefficient around a single slat, but it is assumed that this correlation can be used to approximate the average heat transfer coefficient experienced around a bi-planar (2D) grid as well. To account for the blockage effect of the grid, the grid Reynolds number is based on the air velocity in the smallest air flow area between the grids.

\subsubsection{Dripping below grids}

Once the splashing and cutting of all the packets striking on a particular grid have been evaluated, the mass flow rate of the water dripping below the grid can be determined from the mass balance of water striking and leaving the grid, as shown in Chapter 3, i.e.. 


$$
\dot{\mathrm{m}}_{\mathrm{d}}=\sum_{\mathrm{j}}\left(\dot{\mathrm{M}}_{\mathrm{w}} \mathrm{L}\left(\mathrm{W}+\mathrm{d}_{\mathrm{i}, \mathrm{j}}\right)\right)-\sum_{\mathrm{j}}\left(\overline{\mathrm{f}_{\mathrm{s}, \mathrm{j}}} \dot{\mathrm{M}}_{\mathrm{w}} \mathrm{L}\left(\mathrm{W}+\mathrm{d}_{\mathrm{i}, \mathrm{j}}\right)\right)-\sum_{\mathrm{j}}\left(\overline{\mathrm{f}}_{\mathrm{c}, \mathrm{j}} \dot{\mathrm{M}}_{\mathrm{w}} \mathrm{L}\left(\mathrm{W}+\mathrm{d}_{\mathrm{i}, \mathrm{j}}\right)\right)
$$

The simulation program uses two models to describe the distribution of the dripping water mass flow rate into new packets:

i) The first model, which is based on the model proposed by Yung et al. [80YU1], assumes that the dripping water leaves the slat as groups of drops consisting of one large primary drop and 5 smaller satellite drops. The primary drop size can be described by

$$
d_{p}=C \sqrt{\frac{\sigma}{g\left(\rho_{w}-\rho_{a}\right)}}
$$

where the dimensionless constant, $\mathrm{C}$, varies between 2.5 and 3.3. Based on the findings of Yung et al., the secondary drops are assumed to be linearly spaced between $d_{s, 1}=0.24 d_{p}$ and $d_{s, 5}=0.46 d_{p}$. As described in Chapters 2 and 4 , the constant, $C$, in this equation is dependent on the profile of the bottom of the slats and on the water flow rate. Equation (4.30) can be employed to approximate the value of $\mathrm{C}$ for different slat shapes. The formation of individual primary and secondary drops at low water flow rates is replaced by the formation of more evenly sized drops formed by 'jetting' at higher liquid flow rates. Due to the lack of suitable data, this model does not account for the reduction in primary drop size with increasing flow rate.

ii) The second model uses a double linear interpolation technique to determine the dripping drop mass distribution from the experimental data described in Chapter 4 (also see Appendix N). This model is expected to be more accurate than the previous model since it takes the effects of slat profile and liquid flow rate into account. The experimental data was obtained from a photographic study of the drop size distribution of drops dripping below single slats. It is assumed that the experimentally determined drop size distributions are valid for dripping below bi-planar (2D) grids as well. The effect of varying liquid properties can be accounted for by employing Equation (4.31).

The temperature of the dripping drops can be obtained from the energy balance of the water flowing over the grid and the cooling of the water film on the surface of the grid, as described in section 5.4 .2 above. The drops which drip from below the grid obviously start from zero velocity.

\subsubsection{Pressure drop}

The static pressure drop across the grids in the packing can be expressed as

$$
\Delta \mathrm{p}_{\text {grids }}=\mathrm{N}_{\text {grids }} \mathrm{K}_{\Delta \mathrm{p}}\left(0.5 \rho_{\mathrm{a}} \mathrm{v}_{\mathrm{a}}^{2}\right)
$$


where the pressure loss coefficient, $\mathrm{K}_{\Delta \mathrm{p}}$, is calculated from any of the applicable correlations discussed in Chapters 2 or 3 .

The static pressure drop due to the splash drop motion above a grid can be calculated from the following relation,

$$
\Delta \mathrm{p}_{\text {drops }}=\frac{\sum_{\mathrm{j}=1}^{\mathrm{N}_{\text {packets }}\left(F_{\text {drag }, \mathrm{j}}\right)}}{\mathrm{A}_{\mathrm{fr}}}
$$

Note that in some cases, the initial (upward) velocities of the splash drops could be larger than the air velocity which results in reduced pressure drop due to the fast moving drops dragging the air upwards.

\subsection{Optimisation}

The packet concept works very well in the modelling of splash packing material, but the splashing action on the grids and the drop/drop collisions result in the formation of very large numbers of new packets. This creates two problems, i.e. excessive execution time and large amounts of computer memory storage space. The following section describes methods which are employed to prevent the formation of unnecessary packets and to reduce the overall number of packets.

\subsubsection{Combination of similar packets}

After integration through an element, every packet is compared to every other packet to determine if the two packets are similar enough to be combined into a single packet. If all the following criteria are met, the two packets, say A and B, are assumed to be similar enough to be combined into a single element :

i) The drops must belong to the same size class, or

$$
\mathrm{d}_{\mathrm{A}}=\mathrm{d}_{\mathrm{B}}
$$

ii) The drop velocities must be within a specified range from each other, or

$$
\left|v_{A}-v_{B}\right|<v_{\text {crit }}
$$

iii) The drop temperatures must be similar, or

$$
\left|\mathrm{T}_{\mathrm{A}}-\mathrm{T}_{\mathrm{B}}\right|<\mathrm{T}_{\text {crit }}
$$

The following critical values, viz. $\mathrm{v}_{\text {crit }}=0.25 \mathrm{~m} / \mathrm{s}$ and $\mathrm{T}_{\text {crit }}=0.25^{\circ} \mathrm{C}$, have been found to reduce the number of packets significantly without oversimplifying the calculations. 
If two packets, $\mathrm{A}$ and $\mathrm{B}$, are combined, the total water mass flow rate of the new packet, $\mathrm{C}$, is equal to the sum of the water mass flow rates of the two packets which are combined, i.e.,

$$
\dot{\mathrm{m}}_{\mathrm{w}, \mathrm{C}}=\dot{\mathrm{m}}_{\mathrm{w}, \mathrm{A}}+\dot{\mathrm{m}}_{\mathrm{w}, \mathrm{B}}
$$

The temperature and velocity of the drops represented by the new packet are determined from the energy and momentum conservation laws, i.e.

$$
\mathrm{T}_{\mathrm{C}} \approx\left(\frac{\dot{\mathrm{m}}_{\mathrm{w}, \mathrm{A}} \mathrm{T}_{\mathrm{A}}+\dot{\mathrm{m}}_{\mathrm{w}, \mathrm{B}} \mathrm{T}_{\mathrm{B}}}{\dot{\mathrm{m}}_{\mathrm{w}, \mathrm{C}}}\right)
$$

and

$$
\mathrm{v}_{\mathrm{w}, \mathrm{C}} \approx\left(\frac{\dot{\mathrm{m}}_{\mathrm{w}, \mathrm{A}} \mathrm{v}_{\mathrm{A}}+\dot{\mathrm{m}}_{\mathrm{w}, \mathrm{B}} \mathrm{v}_{\mathrm{B}}}{\dot{\mathrm{m}}_{\mathrm{w}, \mathrm{C}}}\right)
$$

\subsubsection{Distribution of splashing/cutting/dripping drops}

The method of combining similar packets described above is only used after the integration through an element. However, if an element contains a grid, the number of packets formed by splashing, cutting and dripping in the element could become excessive during the evaluation of the element. A packet combination algorithm (similar to that described above) is employed in the routine which creates new packets for the drops formed by the splashing, cutting and dripping action. Since drops formed by splashing and dripping often have the same initial velocity (zero) and the same initial temperature, many of these drops can be lumped together in single packets. 


\section{CHAPTER 6 RESULTS AND DISCUSSION}

In this chapter the simulation program, SPSIM, is employed to evaluate the transfer characteristics and pressure drop of various types of counterflow cooling tower splash pack material. The sensitivity of SPSIM for various parameters and modelling options such as film thickness, drop drag and drop-drop collisions is investigated. The experimentally determined splash pack transfer characteristic and pressure drop data are compared to the predictions by SPSIM. In an attempt to find the optimum splash pack layout, SPSIM is used to find trends in splash pack performance with varying slat width, slat shape and slat pitch.

\subsection{Typical simulation results}

\subsubsection{Empty tower with mono-disperse drop distribution}

SPSIM was used to determine the cooling rates and pressure drop across sprays of monodisperse water drops in counterflow with an airstream, in the absence of any packing material. Two cases were evaluated for comparison: (i) the drops were assumed to travel at their terminal velocity and (ii) the drops were assumed to accelerate from zero velocity at $\mathrm{z}=0 \mathrm{~m}$ to a final position at $\mathrm{z}=3 \mathrm{~m}$. The drops were assumed to be solid spheres for the drag calculations and the heat/mass transfer from the drops was calculated using the correlation of Ranz and Marshall [52RA1], [52RA2].

In the case of a mono-disperse spray it is not necessary to use an iterative process to calculate the local drop temperatures in order to find the outlet water temperature and the corresponding overall transfer characteristic. Note that in the case of a mono-disperse spray, all the drops at a given horizontal plane have the same temperature, implying that the driving force for heat and mass transfer is the same for every drop in this plane. It is possible to calculate the transfer characteristic by calculating the drop motion numerically and summing the local transfer in every element as follows:

$$
\frac{\mathrm{KaZ}_{\text {spray }}}{\dot{\mathrm{M}}_{\mathrm{w}}}=\sum_{\mathrm{i}}\left(\frac{\mathrm{Ka} \partial \mathrm{z}}{\dot{\mathrm{M}}_{\mathrm{w}}}\right)
$$

The average pack densities of the mono-disperse water sprays are shown in Figure 6.1. Note that the pack density is higher for the accelerating drops, due to the closer drop spacing at the lower drop velocities. In both cases the drop surface area decreases with increasing drop size due to increasing drop velocity and decreasing specific drop area (area per unit mass). 

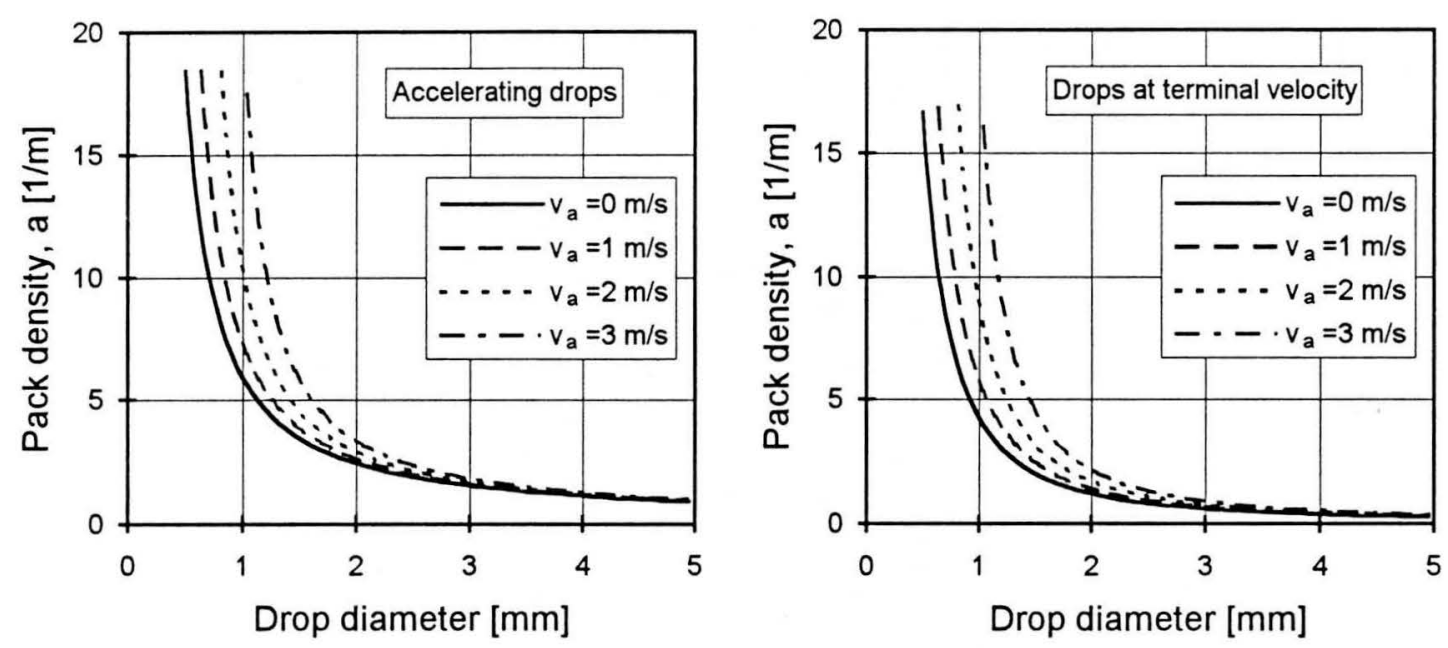

Figure 6.1 Pack density for mono-disperse spray systems (with no packing).

The cooling tower transfer characteristics and pressure drop per metre for each of the two cases are shown in Figures 6.2 and 6.3. The increase in transfer characteristic with decreasing drop size is due to the increased pack density with decreasing drop size. The increase in pressure drop is due to the increase in specific drag force on the drops with decreasing drop size.
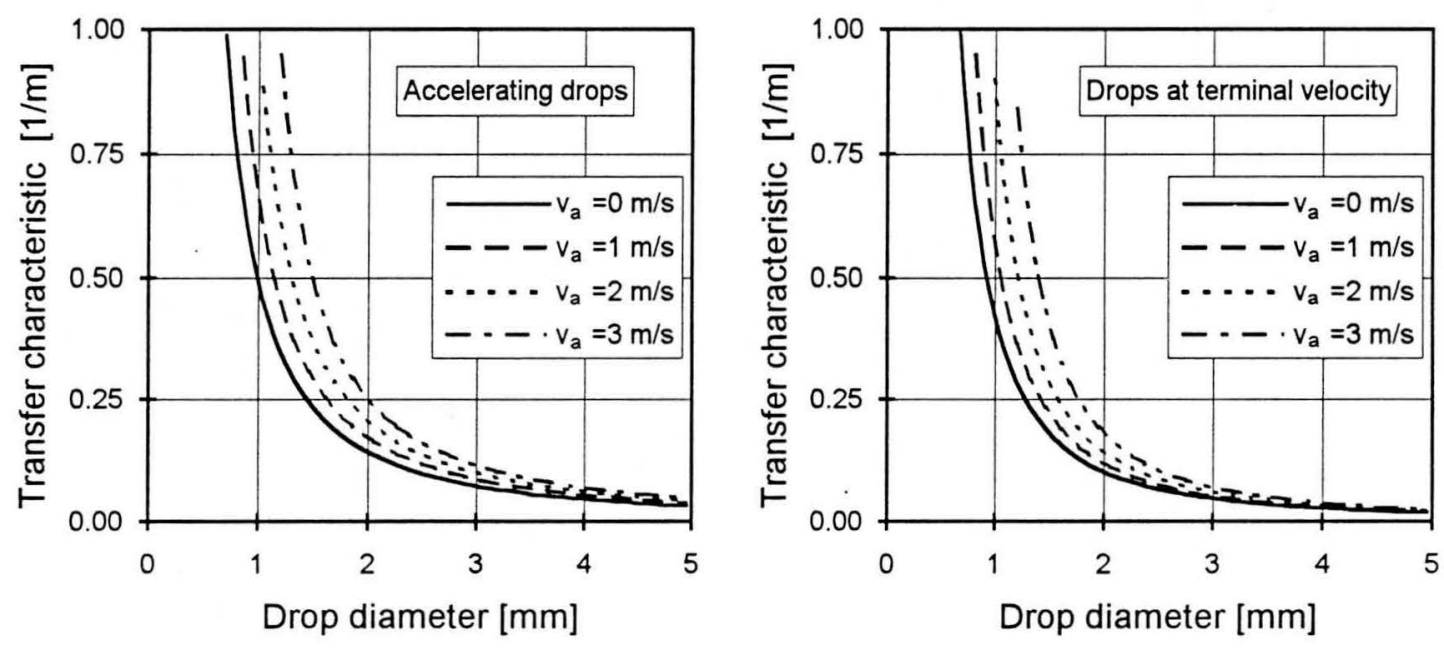

Figure 6.2 Transfer characteristics for mono-disperse spray systems (with no packing).

Note that the pressure drop across the accelerating spray is less than that across the spray of drops at terminal velocity. This difference in pressure drop, which is more pronounced at the larger drop sizes, is due to the larger relative velocity between the drops and the air in the case of the drop travelling at terminal velocity. Smaller drops, $\mathrm{d}<1 \mathrm{~mm}$, reach their terminal velocity in a much shorter time than larger drops and consequently the correspondence between the pressure drop for the two cases is good for small drops. 

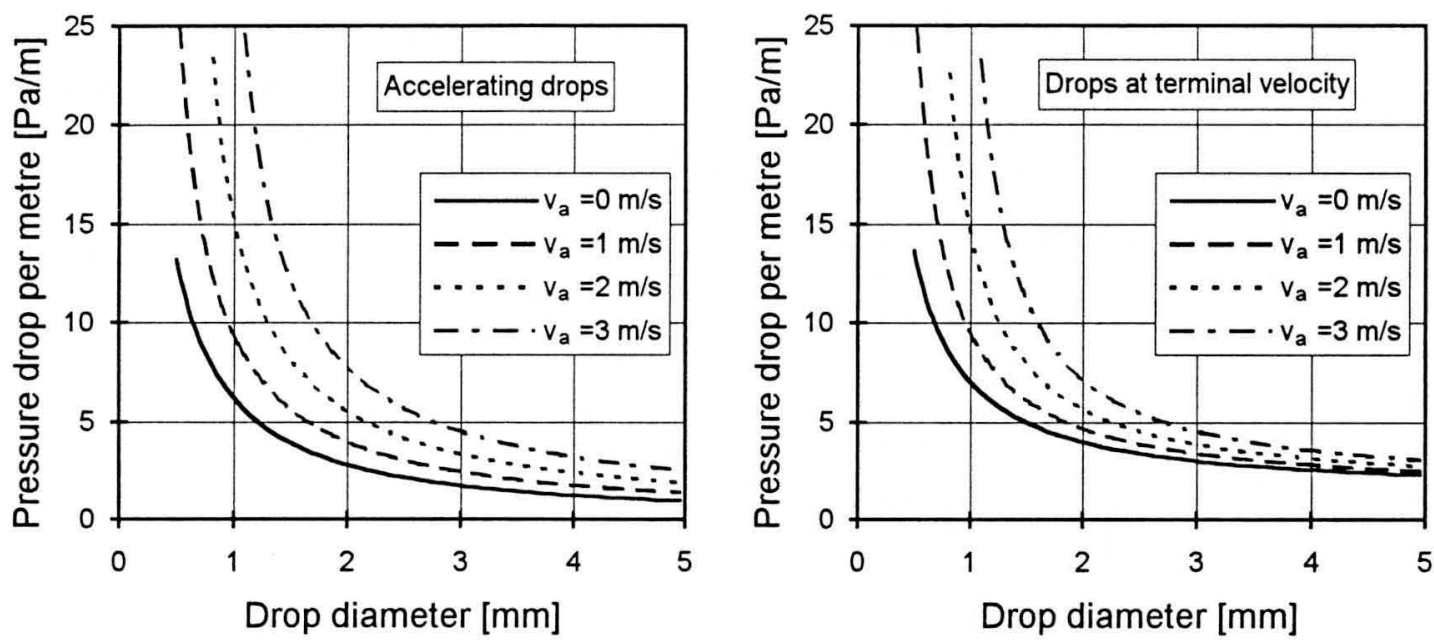

Figure 6.3 Pressure drop per metre for mono-disperse spray systems (with no packing).

The efficiency of a mono-disperse spray of drops can be expressed in two ways, (i) the ratio of transfer characteristic per metre to the pressure drop per metre, i.e.

$$
\eta_{1}=\frac{\left(\mathrm{Ka} / \dot{\mathrm{M}}_{\mathrm{w}}\right)}{(\Delta \mathrm{p} / \mathrm{Z})} \quad\left[P a^{-1}\right]
$$

or (ii) as the ratio of transfer characteristic per metre to the pressure loss coefficient per metre, i.e.

$$
\eta_{2}=\frac{\left(\mathrm{Ka} / \dot{\mathrm{M}}_{\mathrm{w}}\right)}{\left(\frac{(\Delta \mathrm{p} / \mathrm{Z})}{0.5 \rho_{\mathrm{a}} \mathrm{v}_{\mathrm{a}}^{2}}\right)}
$$

Note that the first definition of spray efficiency is not dimensionless. By definition the pressure loss coefficient, $\mathrm{K}_{\Delta \mathrm{p}}$, is infinite at zero air velocity, and consequently the corresponding efficiency defined by Equation (6.3), $\eta_{2}$, would be zero. At zero air velocity, there is no real pressure loss across the spray zone, but a vertical pressure gradient builds up in the air due to the drop drag.

Figures 6.4 and 6.5 show the variation of spray efficiency versus drop size for different air velocities. The curves of $\eta_{1}$ for the different air velocities fall exactly on each other for the spray drops travelling at terminal velocity. These curves show that the optimum drop size is the smallest drop that is not dragged upwards by the airstream. The spray efficiencies of the accelerating drops are higher than that of the drops at terminal velocity due to the high initial pack density of the accelerating drops. The spray efficiency based on Equation (6.2) decreases 
with increasing air velocity, while the spray efficiency based on Equation (6.3) increases with increasing air velocity, for a fixed drop size.
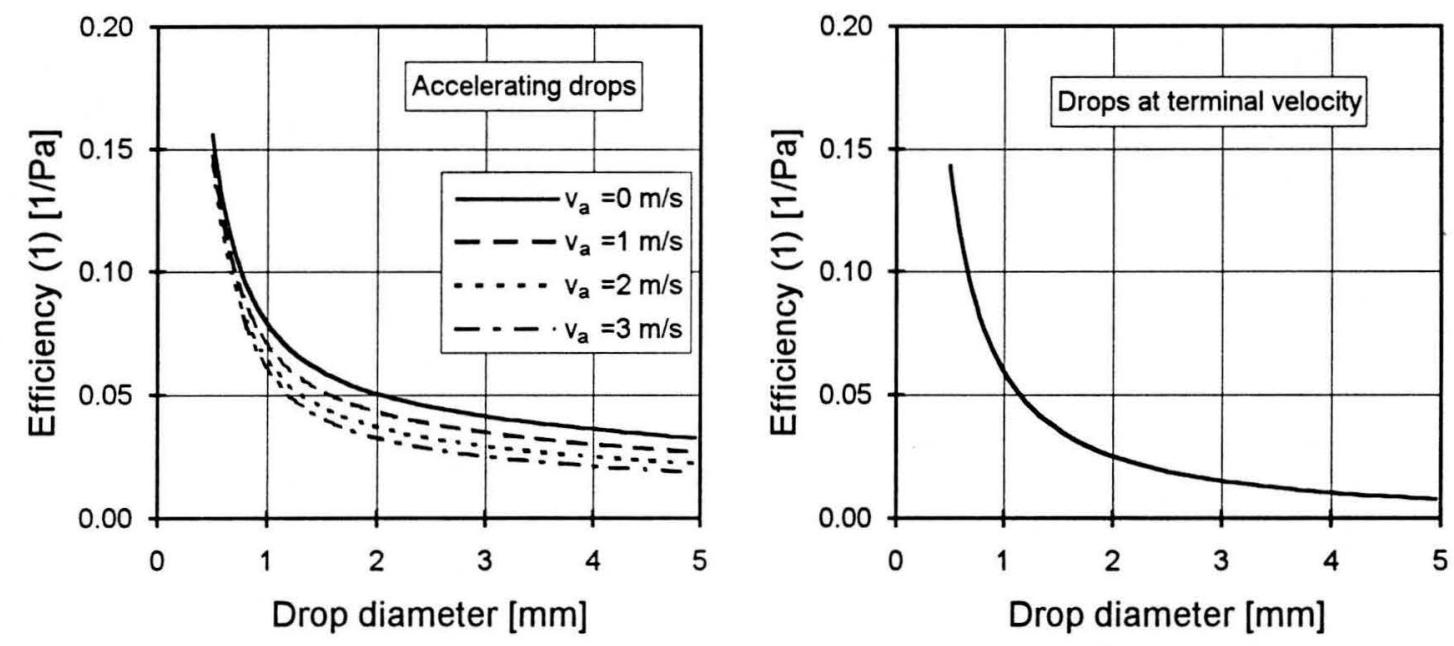

Figure 6.4 Spray efficiencies for mono-disperse spray systems with no packing, according to Equation (6.2).
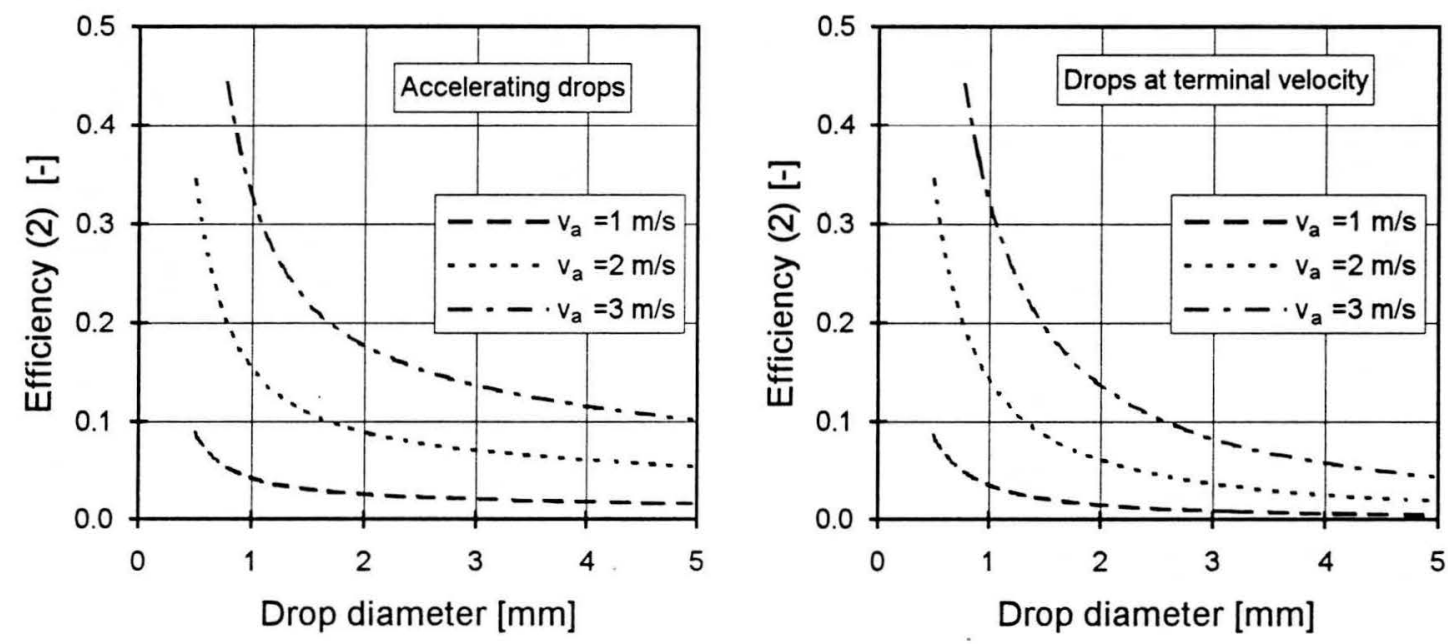

Figure 6.5 Spray efficiencies for mono-disperse spray systems with no packing, according to Equation (6.3).

The transfer characteristics for mono-disperse sprays of drops predicted by SPSIM, is compared to that predicted by the model proposed by Lowe and Christie [62LO1] (see Equation (2.12)) in Figure 6.6. This model by Lowe and Christie uses the steady state "dynamical function" data ("dynamical function" for drops falling at terminal velocity) tabulated by Nottage and Boelter [40NO1]. The drops were assumed to be travelling at terminal velocity. The drop drag calculations in SPSIM were performed using the model for drop deformation proposed in Chapters 3 and 4. 
There is good agreement between the trends predicted by these two methods. Generally, the transfer characteristics predicted by the model of Nottage and Boelter [40NO1] fall slightly above that predicted by SPSIM.

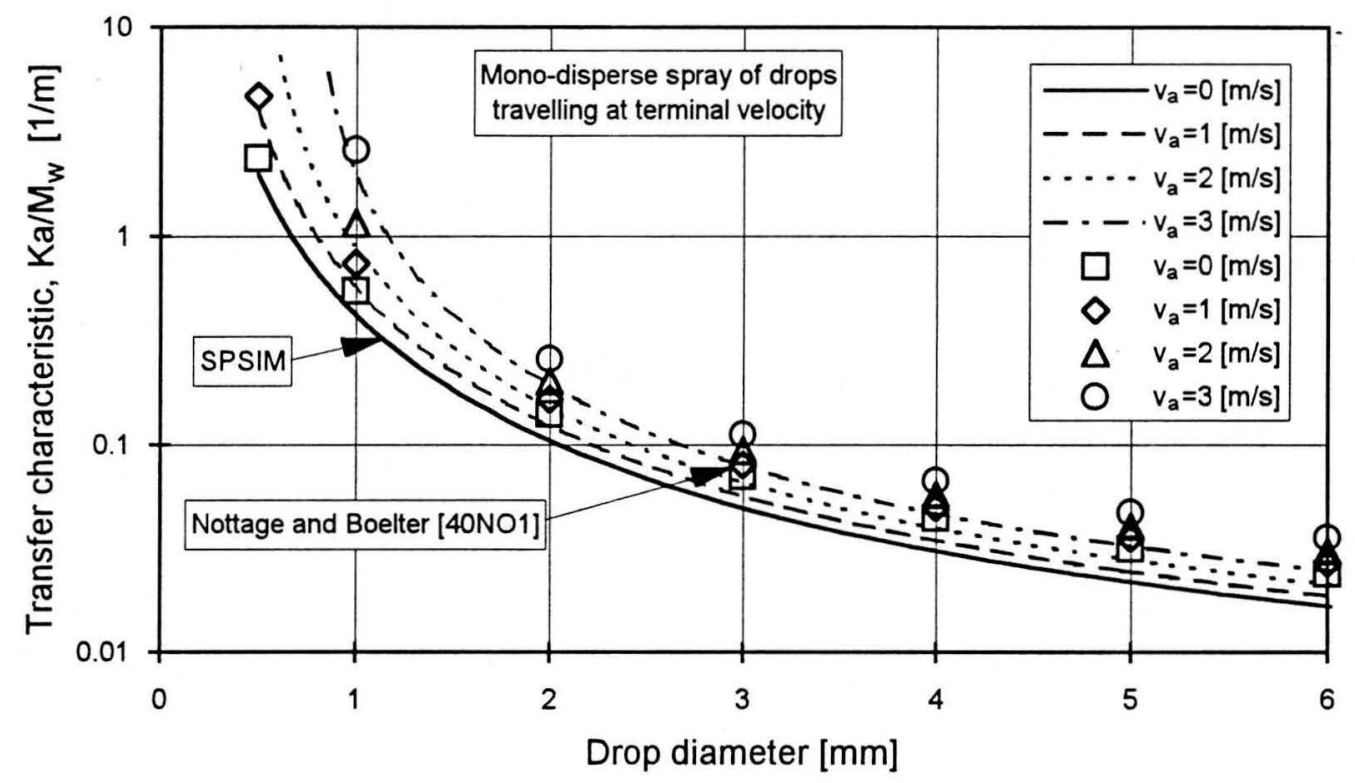

Figure 6.6 Comparison between the transfer characteristics based on the model of Nottage and Boelter [40NO1] and the predicted values obtained with SPSIM.

\subsubsection{Empty tower with poly-disperse drop distribution}

The simulation program, SPSIM, was used to determine the cooling rates and pressure drop across sprays of mono-disperse and poly-disperse water drops in counterflow with an airstream, in the absence of any packing material.

Four different distributions were evaluated, i.e. one mono-disperse distribution and three Rosin-Rammler distributions with different shape factors. In all the cases the mass median drop diameter was assumed to be $3 \mathrm{~mm}$. The three Rosin-Rammler distributions are shown in Figure 6.7. Note that the Rosin-Rammler distributions were modified to ensure that all the drops were larger than $1 \mathrm{~mm}$, to prevent numerical problems with up-flowing drops. The drops were assumed to accelerate from zero velocity at $z=0 \mathrm{~m}$ to a final position at $\mathrm{z}=3 \mathrm{~m}$. They were also assumed to be solid spheres for the drag calculations. The heat/mass transfer from the drops was calculated using the correlation of Ranz and Marshall [52RA1], [52RA2]. In all the cases the water flow rate was held constant at $10000 \mathrm{~kg} / \mathrm{m}^{2} \mathrm{hr}$. 


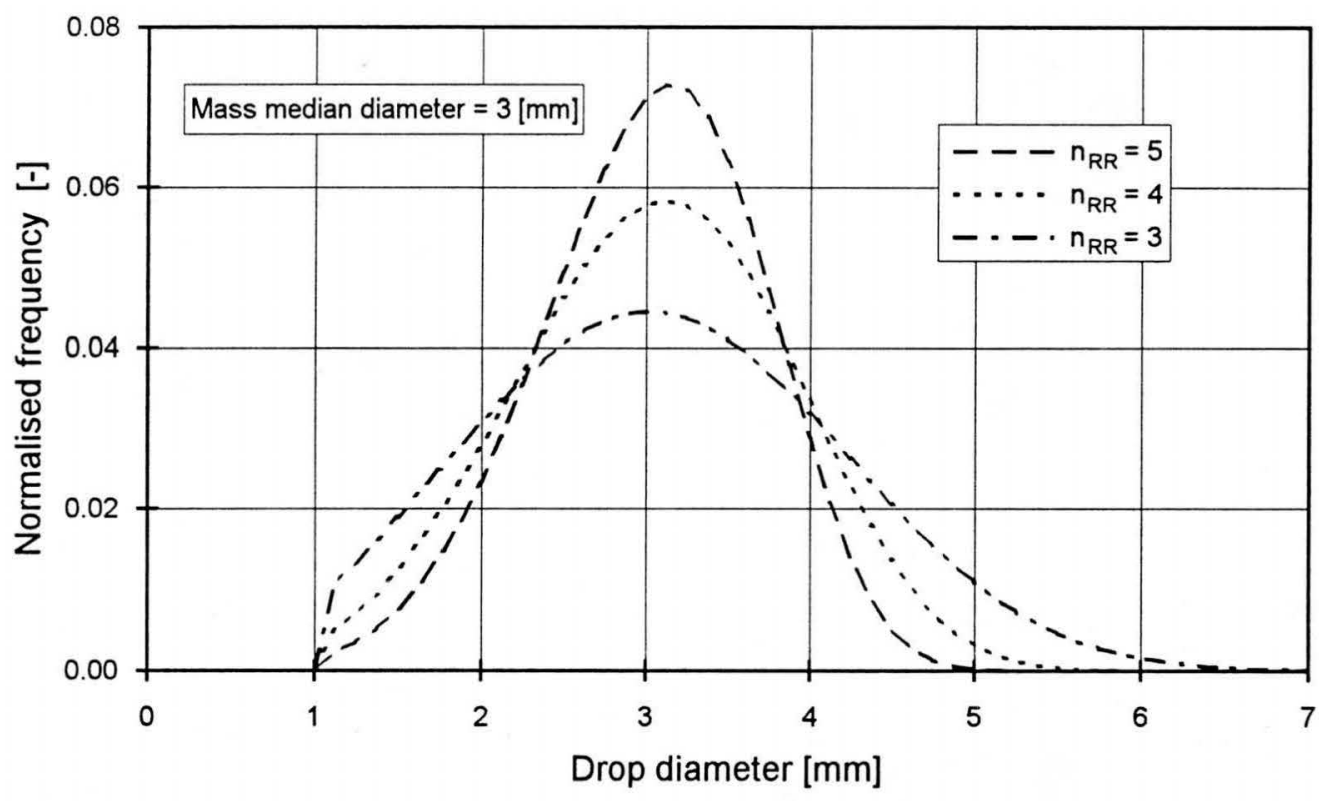

Figure 6.7 Modified Rosin-Rammler distributions used in poly-disperse spray calculations.

Figures 6.8 and 6.9 show the variation of transfer characteristics and pressure drop across poly-disperse sprays in a counterflow airstream. The transfer characteristics and the pressure drop of the poly-disperse sprays are higher than that of the mono-disperse spray.

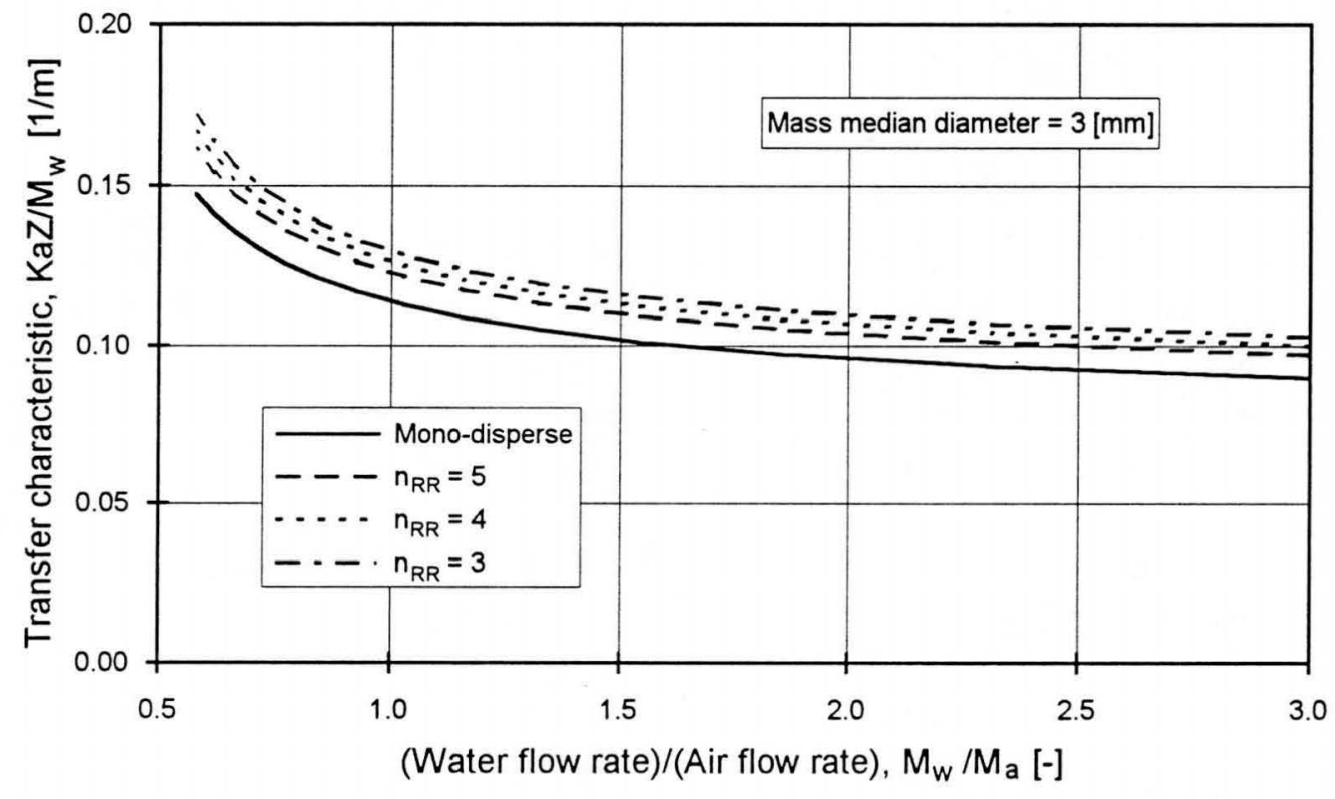

Figure 6.8 Predicted transfer characteristics for poly-disperse sprays in an empty tower. 


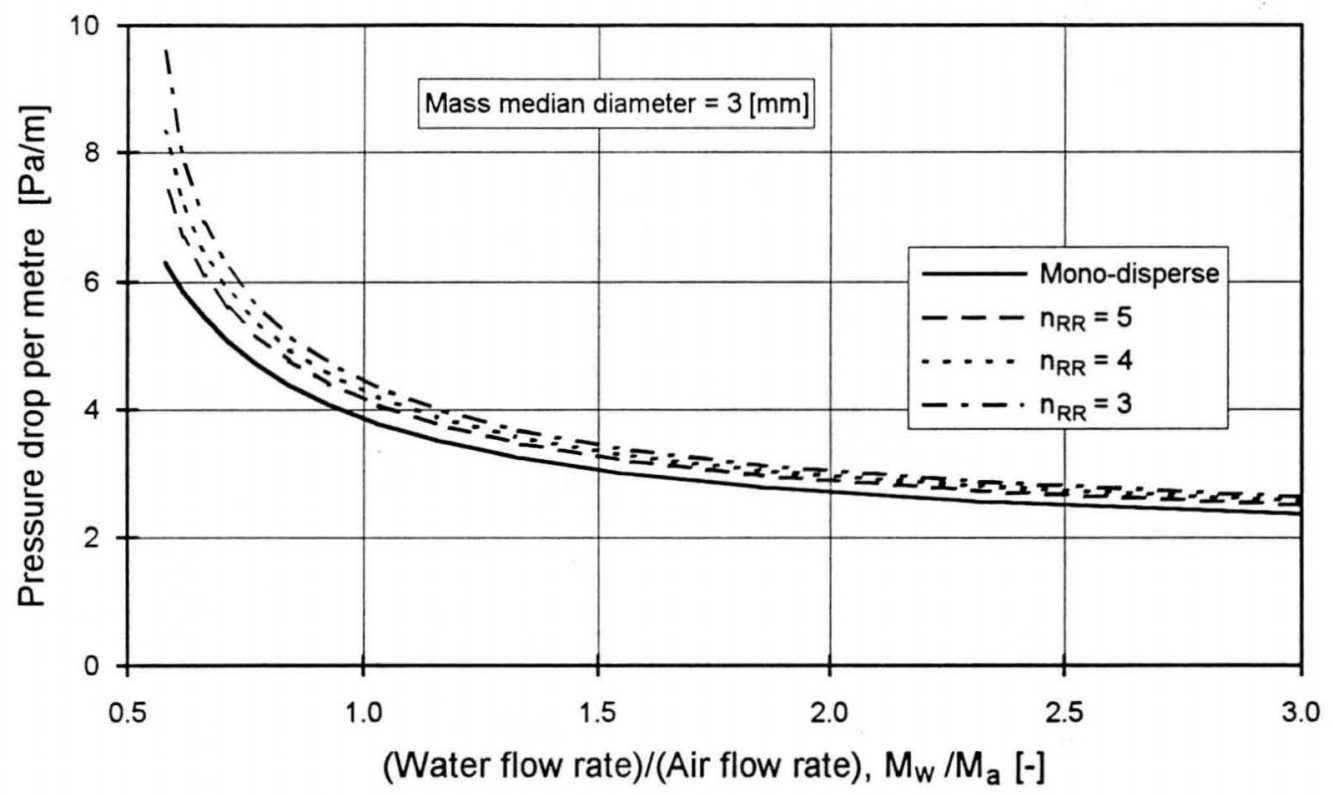

Figure 6.9 Predicted pressure drop for poly-disperse sprays in an empty tower.

Figure 6.10 shows the variation of the efficiency of the different sprays (defined by Equation (6.2)) with the ratio between water and air flow rates. There is very little difference between the efficiencies of the different distributions. The efficiency of the mono-disperse spray is higher than that of the poly-disperse sprays at the higher air-flow rates and vice versa. The efficiency of the spray (mono- and poly-disperse) increases with increasing $\left(\dot{\mathrm{M}}_{\mathrm{w}} / \dot{\mathrm{M}}_{\mathrm{a}}\right)$ ratios.

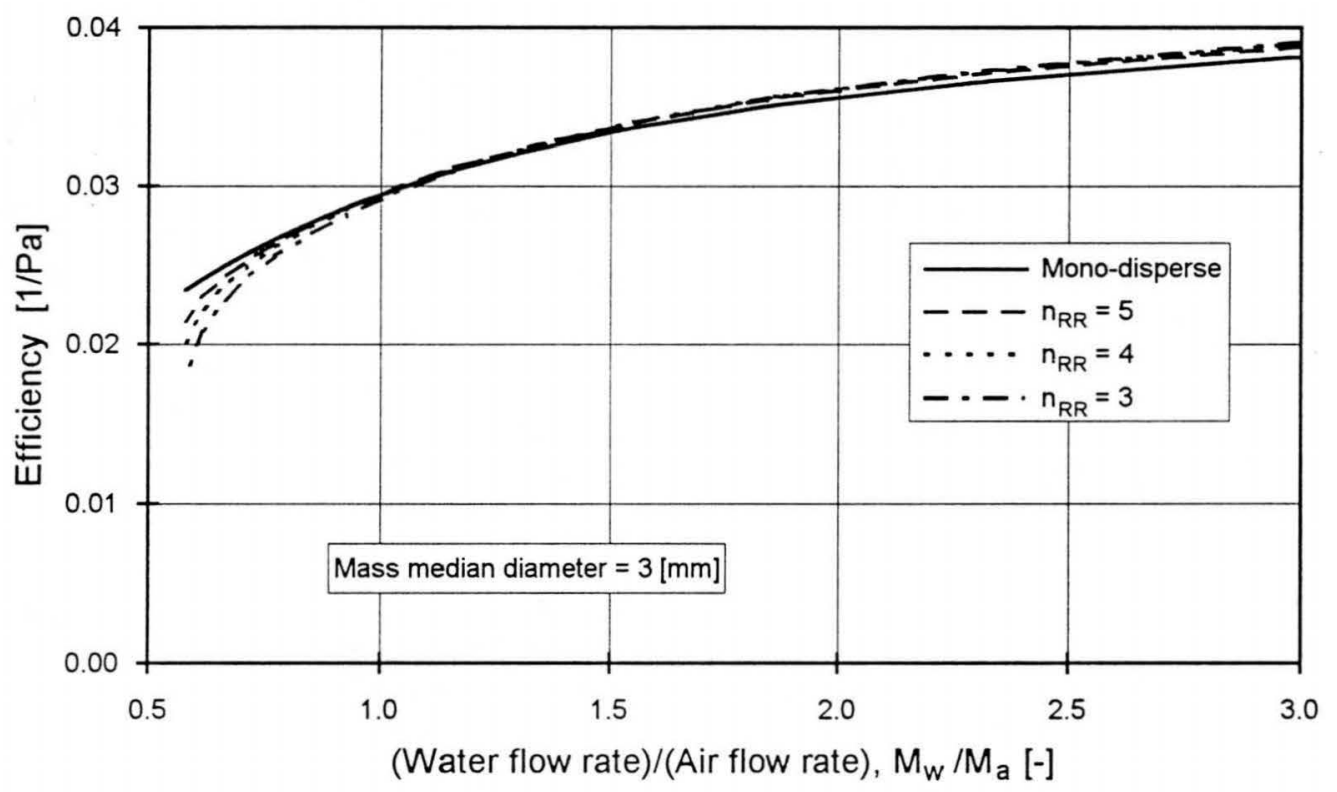

Figure 6.10 Predicted efficiency of poly-disperse sprays in an empty tower. 


\subsubsection{Splash pack simulation sensitivity analysis}

The splash pack simulation program, SPSIM, was used to predict the performance of a typical splash pack. For the reference case, the splash pack geometry, the modelling options and the input parameters were set at fixed values. The sensitivity of the simulation program for the different parameters and options was then determined by changing these options one at a time.

The following simulation program options were used as the reference case:

i) Layout: The packing material was made up of 10 splash grids consisting of $9 \mathrm{~mm}$ wide slats spaced at $50 \mathrm{~mm}$. The vertical spacing between the grids was taken to be $200 \mathrm{~mm}$. It was assumed that there was a spray zone of $0.5 \mathrm{~m}$ above the top grid and a rain zone of $0.0 \mathrm{~m}$ below the packing. (Note that the packing height is $2 \mathrm{~m}$ measured downwards from the top grid, although the distance between the top and bottom grids is only $1.8 \mathrm{~m}$ ). The splash pack used in the reference case has the same geometry as splash pack B used in the experimental study (Table 4.11).

ii) Input: Atmospheric pressure, $\mathrm{P}_{\text {atm }} \quad: 101325 \mathrm{~Pa}$

Ambient dry-bulb temperature, $\mathrm{T}_{\mathrm{aidb}}: 20^{\circ} \mathrm{C}$

Ambient wet-bulb temperature, $\mathrm{T}_{\text {aiwb }}: 14^{\circ} \mathrm{C}(\mathrm{RH}=50 \%)$

Inlet water temperature, $\mathrm{T}_{\mathrm{wi}} \quad: 40^{\circ} \mathrm{C}$

Water mass flux, $\dot{\mathrm{M}}_{\mathrm{w}} \quad: 10000 \mathrm{~kg} / \mathrm{m}^{2} \mathrm{hr}\left(2.78 \mathrm{~kg} / \mathrm{m}^{2} \mathrm{~s}\right)$

The air velocity was varied between 0.75 and $3 \mathrm{~m} / \mathrm{s}$.

iii) Options: The initial drop size distribution was calculated from the experimental data obtained for the water distribution system in the counterflow test facility (see section 4.6.1),

Ranz and Marshall correlation was used to find heat/mass transfer coefficients, Free-stream turbulence effects on the transfer from drops were ignored, Drag correlation for solid spheres was used to model drop drag,

Pressure drop across the grids was calculated using Equation (3.41),

Drop-drop collisions were not taken into account,

Splash drop temperature changes during upwards splashing were calculated,

Initial splash drop velocities were calculated using Equation (4.27),

Aerodynamic drop break-up was not taken into account,

Total splash drop volume was calculated using the correlations from Chapter 4,

Splash drop size distributions were calculated using correlations from Chapter 4 ,

Cutting drops were modelled using simple model proposed in Chapter 3,

Minimum/Maximum drop sizes $\quad: 0$ and $10 \mathrm{~mm}$

Number of drop size classes $\quad: 30$

Film thickness on the slats, $\delta \quad: 0.5 \mathrm{~mm}$ (see Figures 4.9 and 4.10)

Mixing ratio, $\phi \quad: 0.5$ (see Equation (3.26)) 


\section{Correlations for drop heat/mass transfer coefficients}

The effect of using different correlations to calculate the heat/mass transfer coefficients around the drops on the predicted splash pack performance is shown in Figure 6.11. Note that the correlations which include terms to account for drop acceleration predicts slightly higher transfer coefficients than the reference case. The pressure drop across the splash pack is not influenced by the choice of heat/mass transfer correlation.

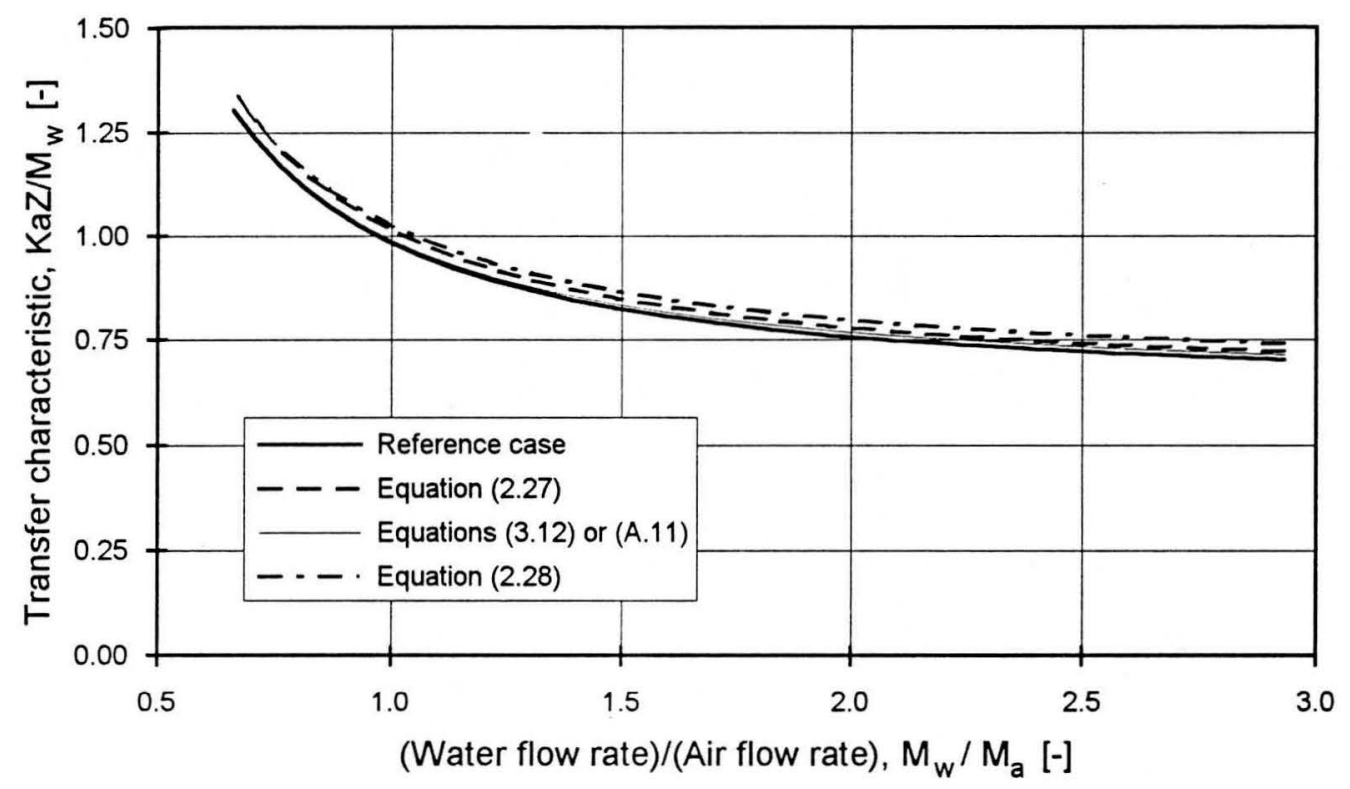

Figure 6.11 Predicted transfer characteristics with different correlations for heat/mass transfer from the drops.

\section{Drop drag models}

Figures 6.12 and 6.13 shows the predicted transfer characteristics and pressure drop for the sample splash pack when using the more accurate drop drag model, which takes the drop deformation into account. There is very little difference between these predictions and that of the reference case (which used a solid sphere drag correlation to approximate drop drag).

The increase in computer simulation time is significant when using the accurate deforming drop drag model. For this reason and in view of the small difference in the predicted splash pack performance, the use of the solid sphere drag correlation can be justified. For very deep splash packs, the larger drops, which are significantly influenced by drop deformation, will approach their terminal velocities and the drag model taking drop deformation into account will increase the prediction accuracy of the simulation. 


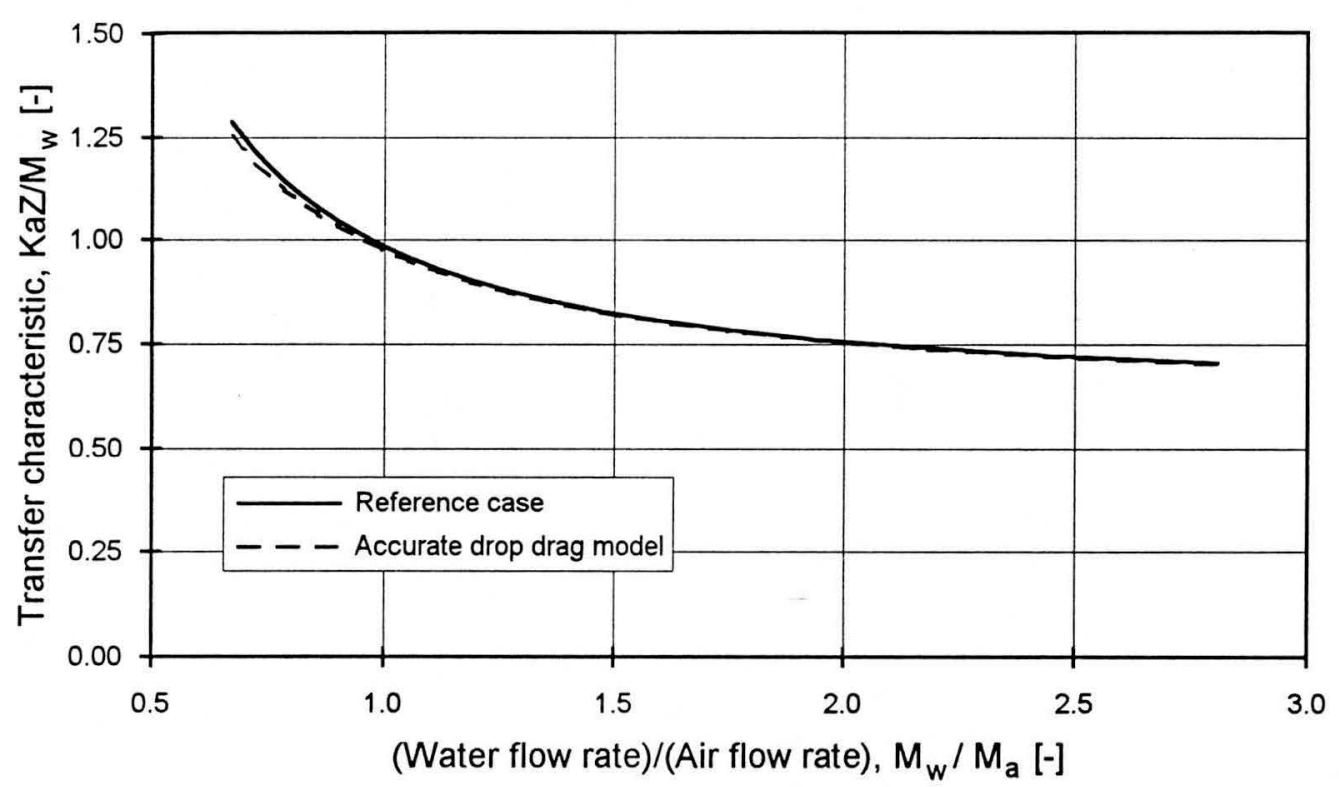

Figure 6.12 Predicted transfer characteristics with different drop drag models.

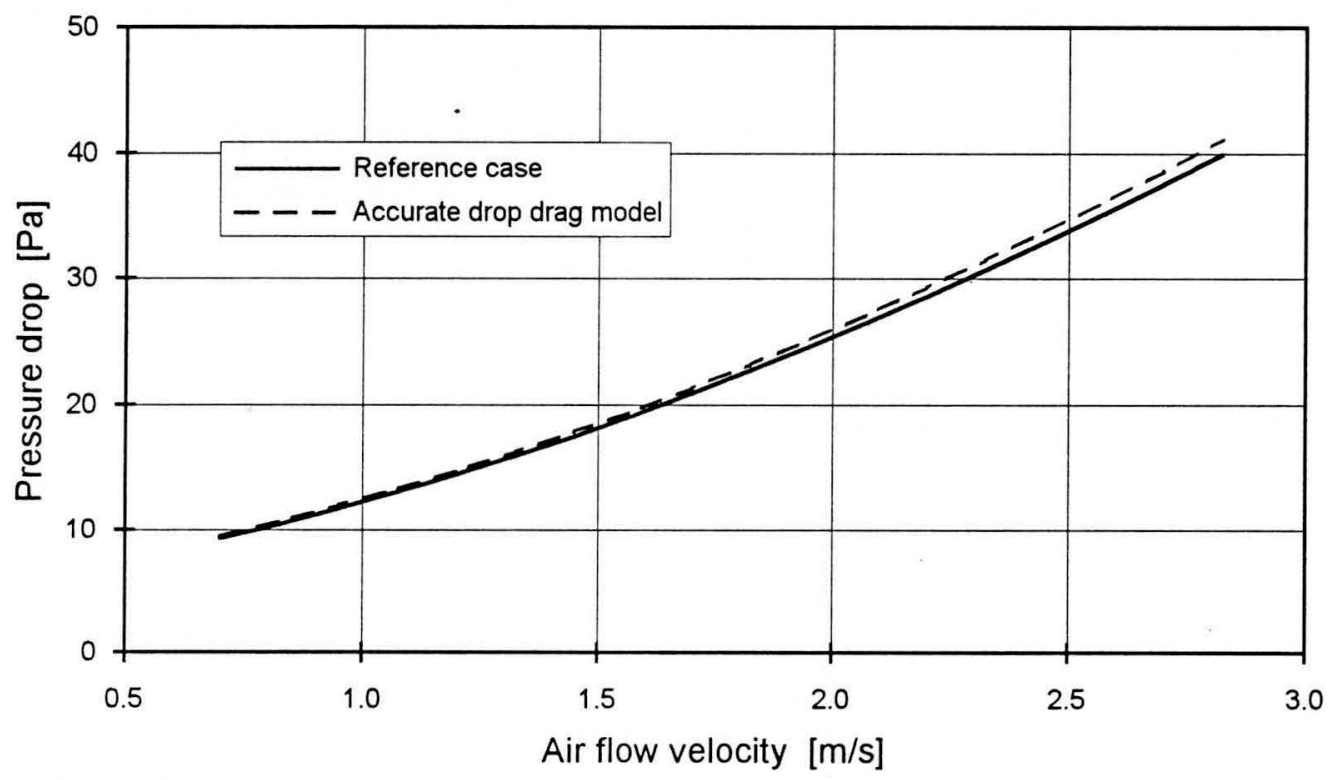

Figure 6.13 Predicted pressure drop with different drop drag models.

\section{Film thicknesses on the slats}

Figures 6.14 and 6.15 show the variation in the predicted splash pack performance for different film thicknesses. The effect of three different fixed film thicknesses (i.e. $\delta=0.4,0.5$ and $0.6 \mathrm{~mm}$ ) on the splash pack simulation is shown. The fourth curve on each graph shows the predictions obtained by employing Equation (4.9) to calculate the film thickness on the slats for every combination of air and water flow rate. The curves for varying film thickness agree well with that of the reference case. From these figures it can be concluded that the 
simulation program is very sensitive to film thickness. A reduction in the film thickness results in a sharp increase in the predicted transfer characteristic.

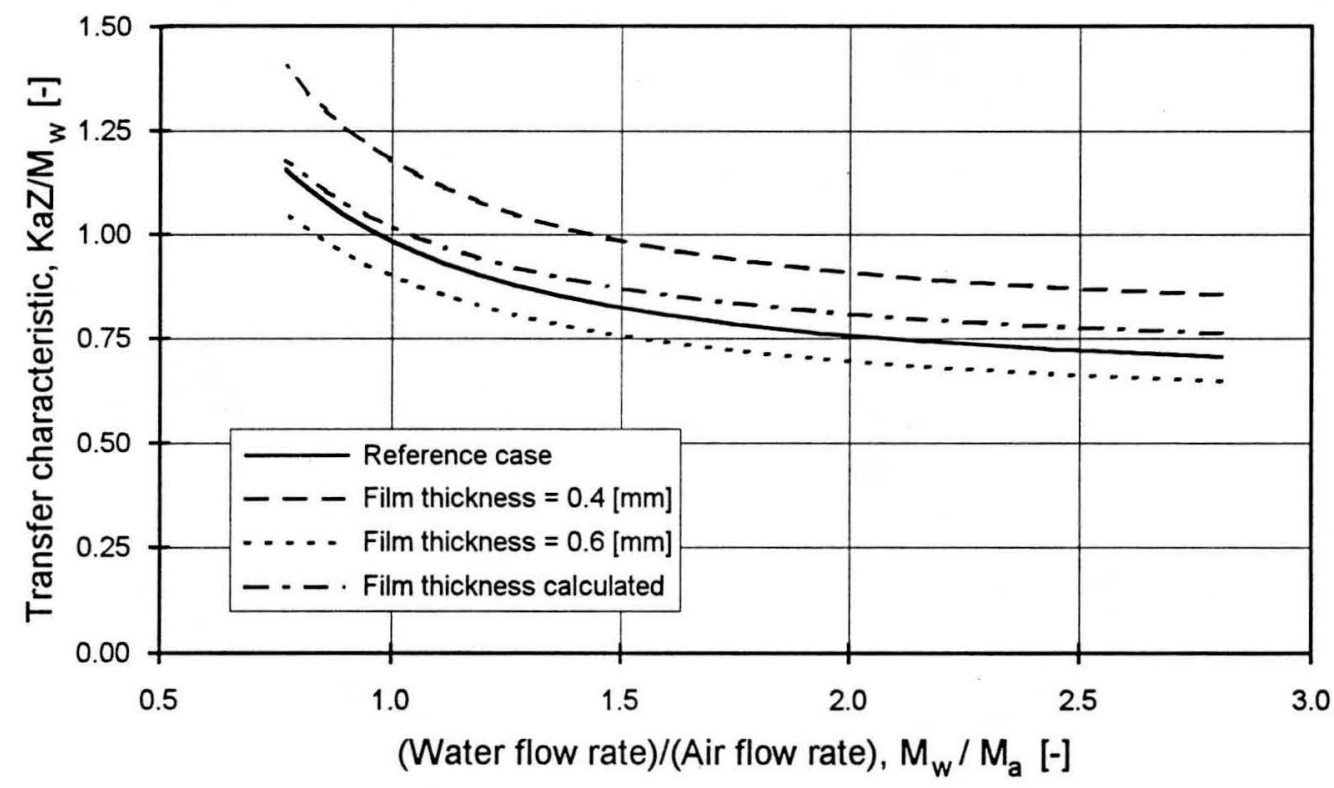

Figure 6.14 Predicted transfer characteristics with different film thicknesses.

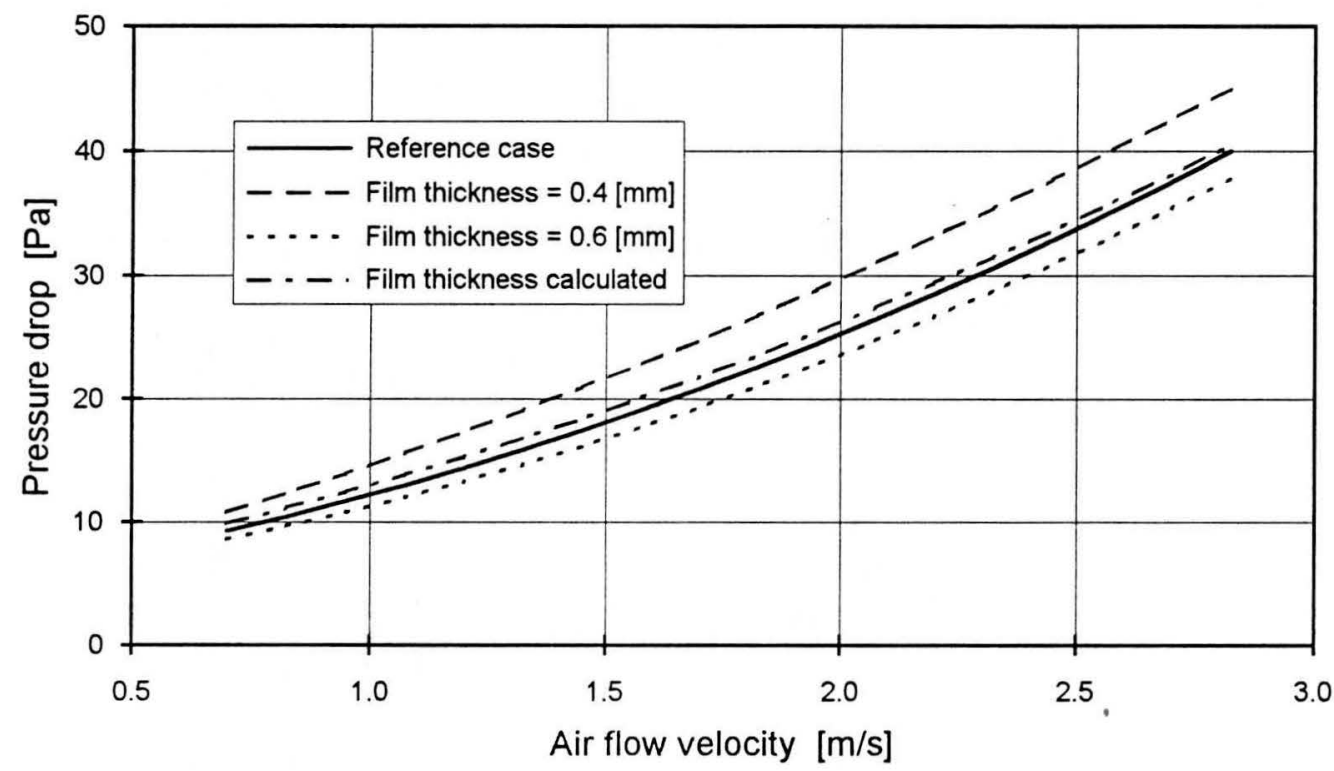

Figure 6.15 Predicted pressure drop with different film thicknesses.

From measurements of the film thicknesses on slats inside a cooling tower, it is known that there is a large variation in the film thickness about the average value due to the large number of water drops striking the slats per unit area in a given time interval. Splash fractions obtained by using the mean film thicknesses was found to agree closely to the mean of the splash fractions calculated at the instantaneous film thicknesses. The surface waves on the liquid film 
are expected to influence the actual splashing phenomenon and this would require further investigation (see Khaleeq-ur-Rahman and Saunders [89KH1]).

\section{Rain density}

Figures 6.16 and 6.17 show the variation in the predicted splash pack performance for different rain densities. The simulation program predicts a decrease in transfer characteristic with increasing rain density, which is in agreement with the trends observed from experimental evaluation of splash pack performance.

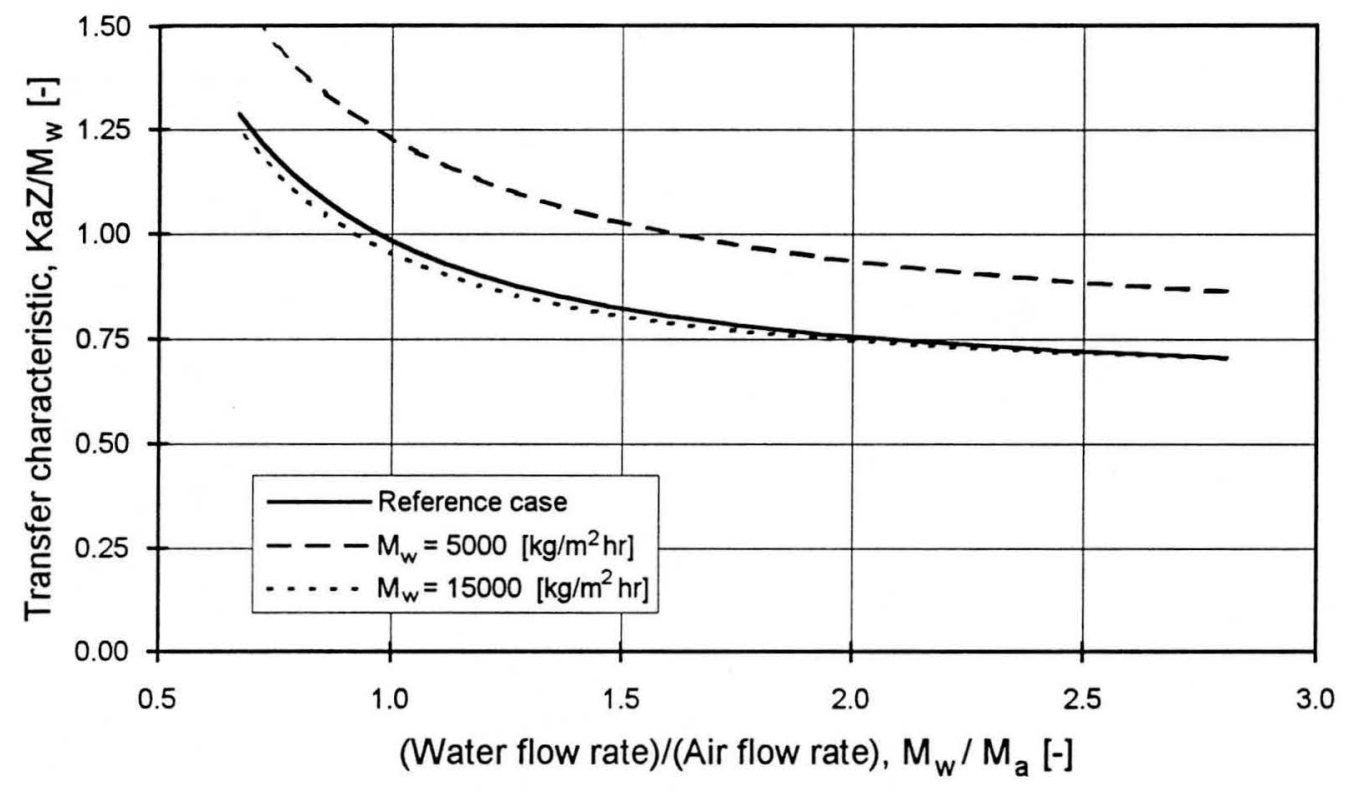

Figure 6.16 Predicted transfer characteristics with different rain densities.

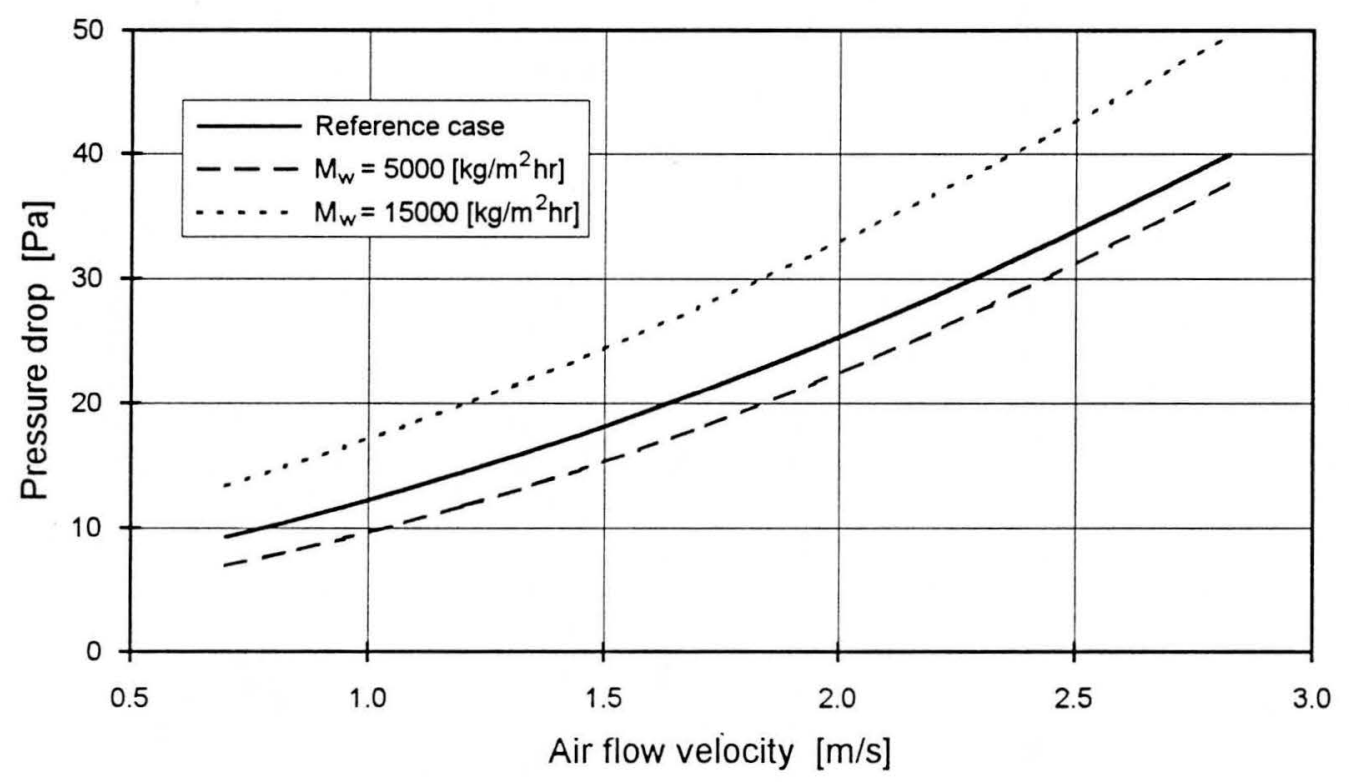

Figure 6.17 Predicted pressure drop with different rain densities. 


\section{Alternative splash fraction models}

The simplified splash fraction model based on the data by Mutchler [70MU1] and Stedman [79ST1] described in Chapter 3, is compared to the experimentally determined splash fraction data (used in the reference case) in Figures 6.18 and 6.19. The simplified splash fraction model predicts significantly lower transfer characteristics and pressure drops than the reference case.

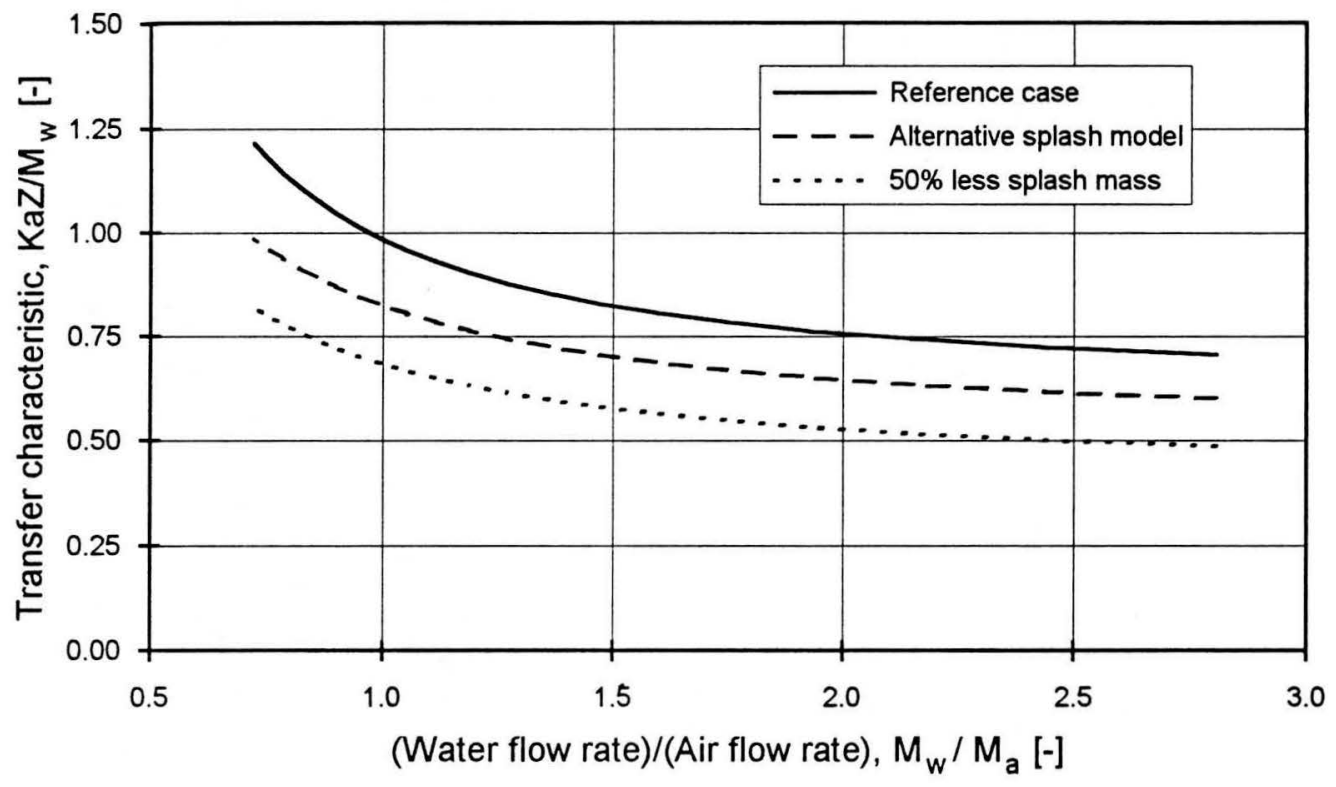

Figure 6.18 Predicted transfer characteristics with different splash mass models.

It was shown in Chapter 3, that interaction effects due the proximity of other splash crowns at a given point can be expected play a role in the splashing of water drops on the splash grids in a cooling tower. These interactions are expected to influence the total mass of water splashing from the slats and/or the distribution of drops formed by splashing. In an attempt to quantify this effect on the predicted splash pack performance, the experimentally determined splash fractions were artificially reduced by employing an interference factor, $f_{i}$, i.e.

$$
\left(\overline{f_{s}}\right)_{\text {corrected }}=f_{i} \bar{f}_{s}
$$

where $f_{i}$ has a value of less than unity. An interference factor of 0.5 was found to reduce the predicted pressure drop and transfer characteristics significantly, as shown in Figures 6.18 and 6.19. The interference effect is expected to be dependent on the water mass flux, the air flow velocity, the incoming drop size and velocity distributions and the film thickness on the slat before drop impact (see Figure 3.12). At higher water mass fluxes the interference effects are expected to be more severe. At lower air velocities, the relative velocities between the drops and the slats will increase, resulting in higher energy impacts and larger crowns (with longer lifetimes). This implies that the interference effects will increase with decreasing air velocity. It 
can be concluded that interference effects will tend to reduce the transfer characteristics more at the high $\left(\dot{\mathrm{M}}_{\mathrm{w}} / \dot{\mathrm{M}}_{\mathrm{a}}\right)$ ratios than at the lower $\left(\dot{\mathrm{M}}_{\mathrm{w}} / \dot{\mathrm{M}}_{\mathrm{a}}\right)$ ratios.

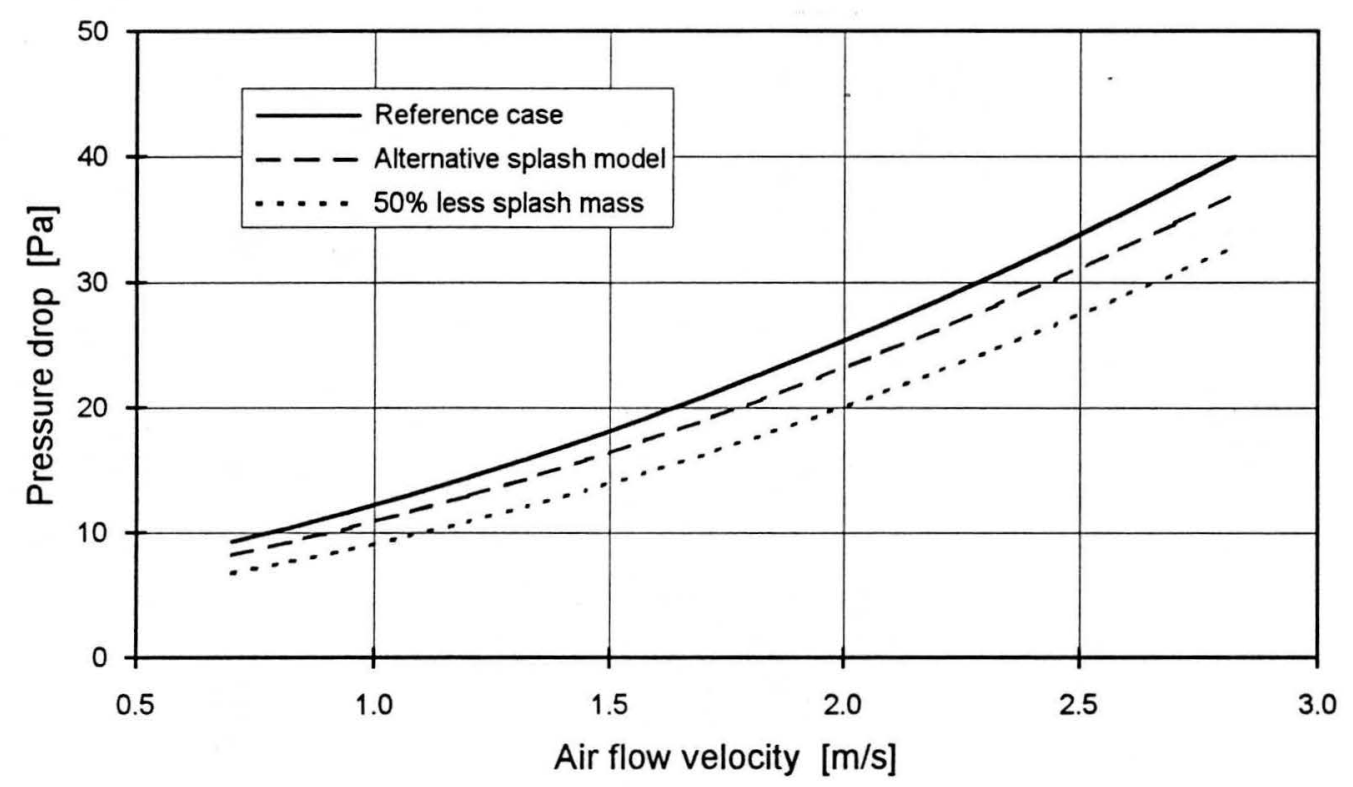

Figure 6.19 Predicted pressure drop with different splash mass models.

\section{Up-splashing from slats}

The sensitivity of the simulation for the cooling of the splash drops during the splash drop motion above the level of the grids was investigated by comparing the reference case with a similar case which ignores the upward splash motion of the splash drops.

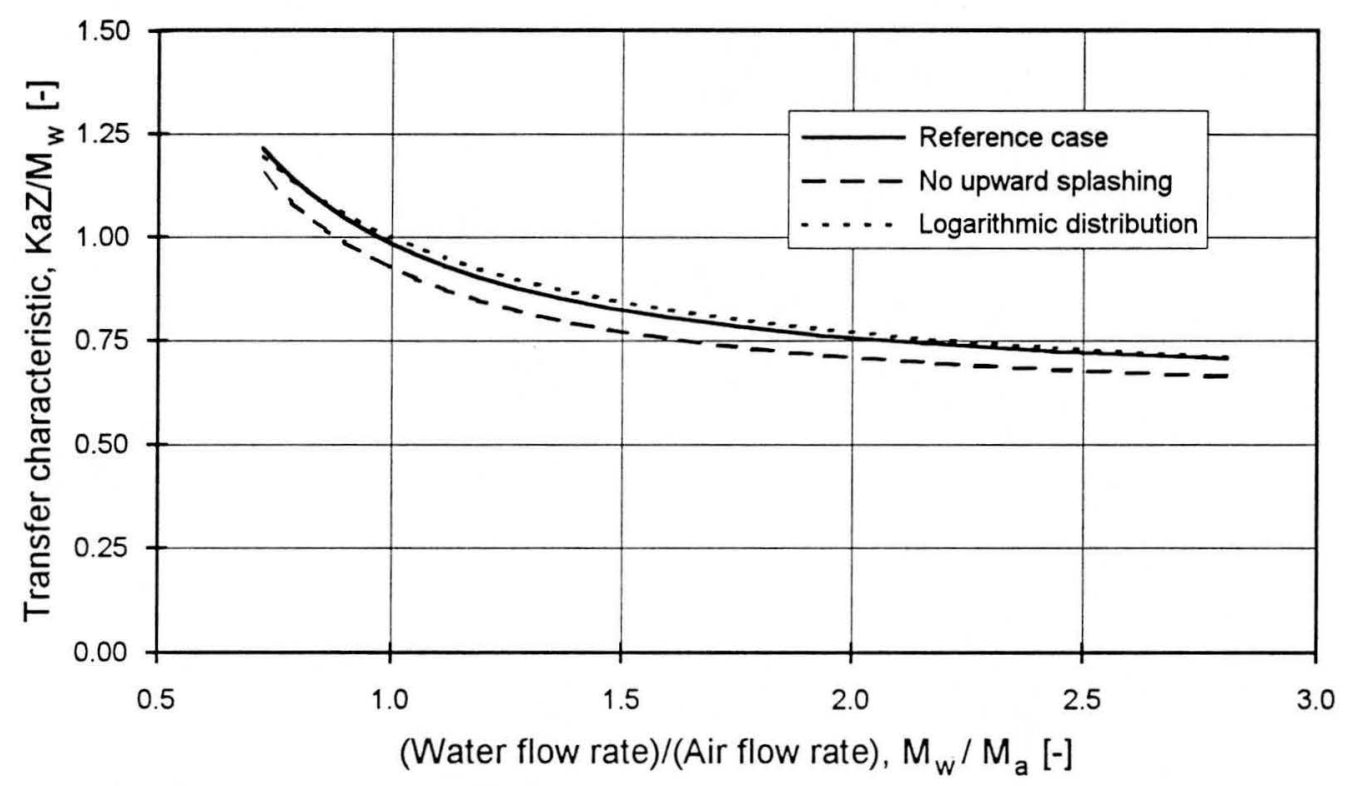

Figure 6.20 Predicted transfer characteristics when ignoring upward splash drop motion and when using a logarithmic drop size zone distribution. 
Figure 6.20 shows that the predicted transfer characteristics are reduced by ignoring the upward splash drop motion. The program execution time is halved when the upward drop splashing is ignored. Ignoring the upward splash drop motion has a pronounced effect on the predicted pressure drop across the splash pack as seen from Figure 6.21.

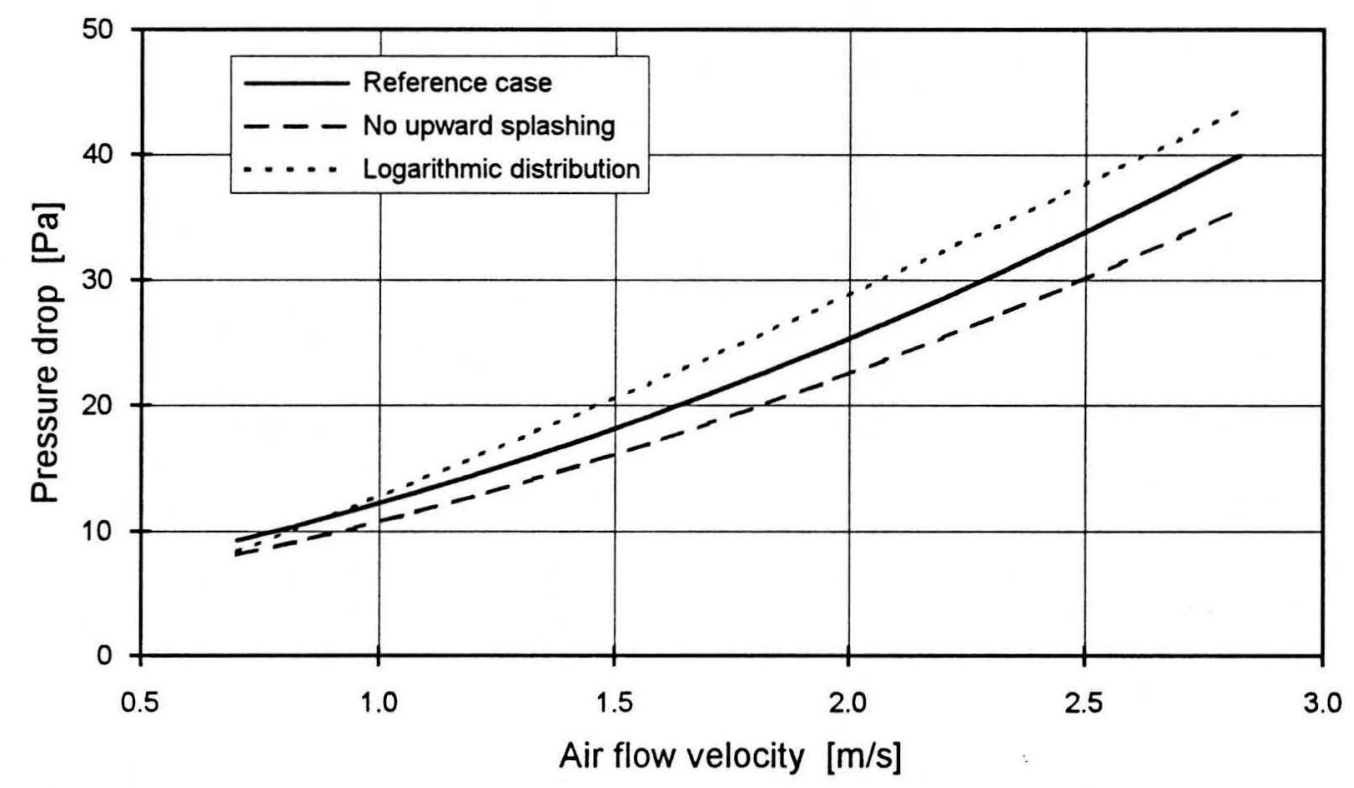

Figure 6.21 Predicted pressure drop when ignoring upward splash drop motion and when using a logarithmic drop size zone distribution.

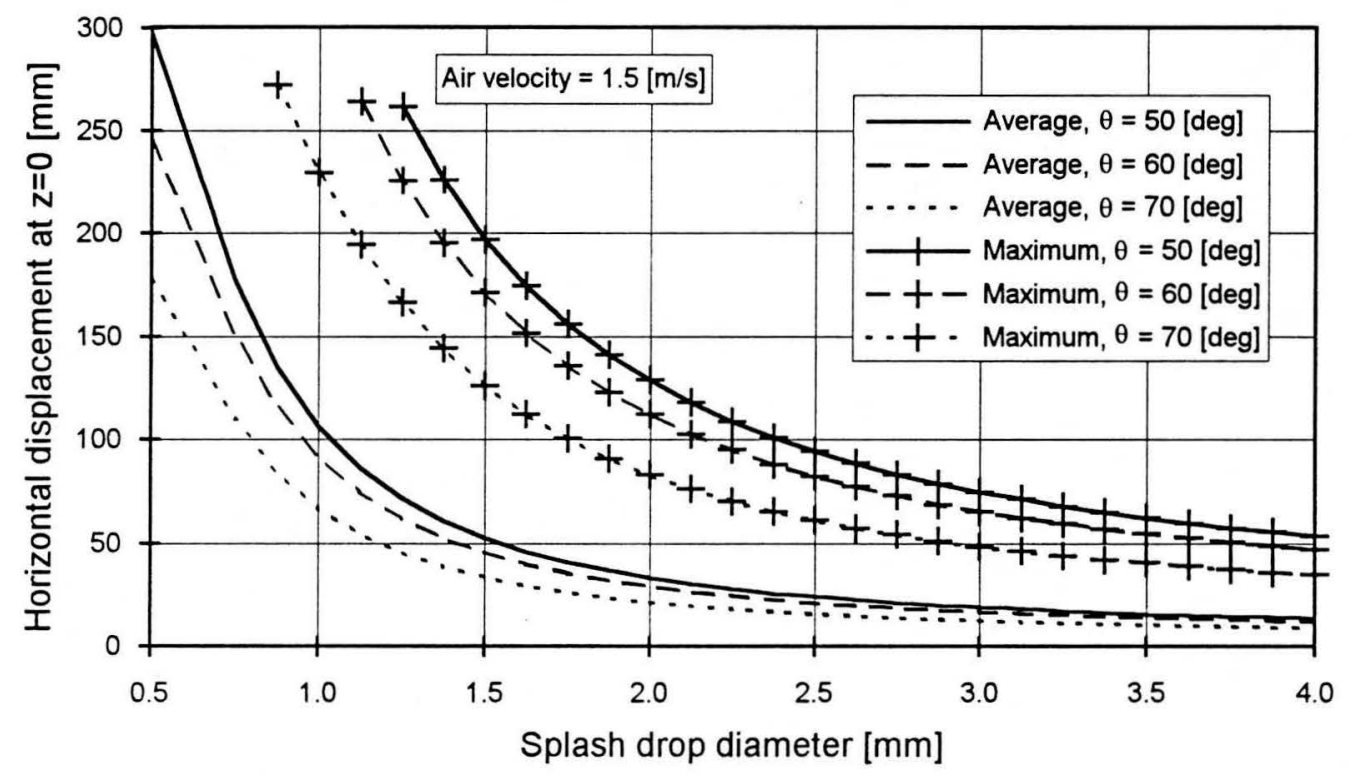

Figure 6.22 Predicted radial displacement of splash drops from the point of impact.

There is a possibility that splash drops which are flung from the point of impact on a given slat could strike another slat in the same horizontal plane. Since drops formed by splashing are 
usually smaller than $2 \mathrm{~mm}$, splash drops are not expected to splash on impact with the other slat, but instead they will join the water dripping from below the slat. Figure 6.22 shows the typical horizontal displacement of splash drops from the point of impact based on the initial splash drop velocity correlation, Equation (4.27). This correlation estimates the average initial splash drop velocity, but some drops may have initial velocities of double that predicted by this correlation, resulting in much larger horizontal displacements.

The splash drops leave the point of impact, losing speed up to the inflection point and then fall down past the initial plane of departure at a very steep angle as shown in Figure 6.23. The very small drops lose all their horizontal momentum and fall down vertically. Although the horizontal displacement of the splash drops is certainly large enough to ensure that many of the splash drops could strike other slats in the same plane, the number splash drops with initial splash angles of around $60^{\circ}$, which actually strike the sides of neighbouring slats is fairly low since the drops fall almost vertically downwards. If only vertical splash drop motion is considered, the fraction of splash drops striking a slat upon return will be much less than $(1-\beta)$ for grids with wide slat spacing since most of the returning drops will not travel as far as the closest neighbouring slat.

The horizontal distance travelled by splash drops (above the level of impact) and the angle at which these drops return are strongly influenced by the air velocity and the splash angle. The splash angle is close to zero for drop impacts very close to the edges of slats, which could result in large fractions of splash drops striking the neighbouring slats if the slats are closely spaced.

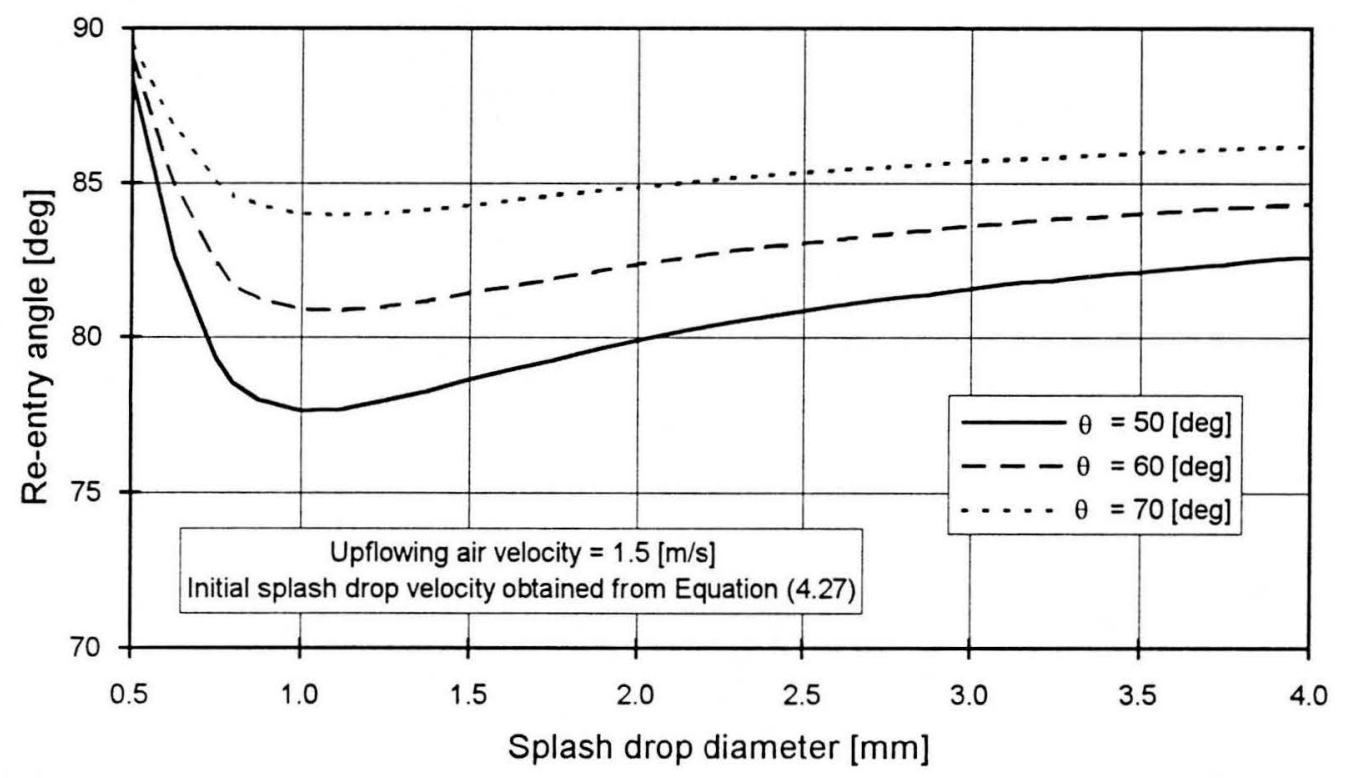

Figure 6.23 Predicted re-entry angles of the splash drops at $z=0$. 


\section{Logarithmic drop size zone distribution}

As described in Chapter 5, the use of a logarithmic drop size distribution can be used to improve the resolution of the smaller drop size classes. As shown in Figures 6.20 and 6.21, the use of a logarithmic size zone distribution (with 30 drop size classes) has very little effect on the predicted transfer characteristics and pressure drop. The use of the logarithmic drop size distribution increases the program execution time significantly, since many more packets are formed than with the reference case.

\section{Cooling of water film on slats}

The contribution of the cooling on the water films covering the surface of the slats is found to contribute significantly to the overall transfer as shown in Figure 6.24.

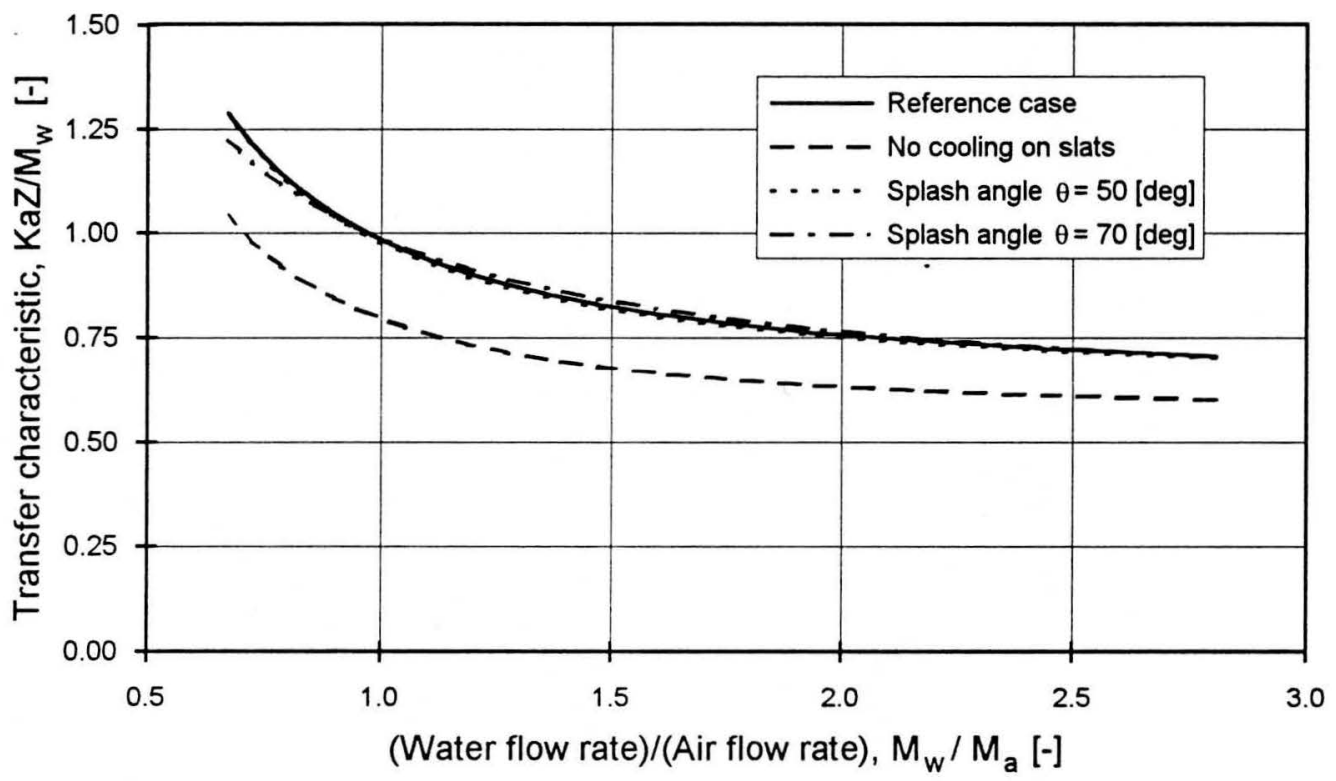

Figure 6.24 Predicted transfer characteristics showing the influence of the cooling of the water film covering the slats and the crown splash angle.

\section{Crown splash angle}

The predicted transfer characteristics were found to be insensitive to variation in the crown splash angle as shown in Figure 6.24. These variations in splash angle had a negligible effect on the predicted pressure drop across the splash pack.

\section{Mixing ratio}

The effect of the value of the mixing ratio, $\phi$, on the predicted splash pack performance was found to be very small. Varying the mixing ratio, $\phi$, between 0.4 and 0.6 resulted in changes in 
the predicted transfer characteristics of less than $0.1 \%$. Any variation in the mixing ratio does not influence the predicted pressure drop.

\section{Dripping drop size distribution model}

The dripping drop model based on that by Yung et al. [80YU1] described in Chapter 3, was used to describe the size distribution of the drops dripping below the slats. This resulted in negligible changes in the predicted transfer characteristics and pressure drop across the splash pack. At very high water flow rates, the model by Yung et al. is expected to over-predict the dripping drop size.

\section{Drop-drop collision modelling}

The effect of drop-drop collisions on the predicted transfer characteristics and pressure drop was found to be negligible, although the program execution time increased by up to 10 times. Most of the collisions that do occur usually involve one packet containing very small drops (due to the large number of these small drops). In such a collision between a packet containing very small drops and another packet containing larger drops, the resulting packet formed by coalescence will be very similar to the original packet containing the larger drops. Note that SPSIM uses a simple drop coalescence model and that it does not account for drop break-up upon collision.

\section{Initial drop size distribution}

The effect of the initial (spray) drop distribution was investigated by comparing the reference case to the predictions obtained using the following three initial drop size distributions: (i) mono-disperse initial drop distribution with $\mathrm{d}_{50}=7 \mathrm{~mm}$ (note that the mass median diameter of the drops produced by the experimental water distribution system is $7 \mathrm{~mm}$ at $\dot{\mathrm{M}}_{\mathrm{w}}=10000$ $\mathrm{kg} / \mathrm{m}^{2} \mathrm{hr}$ ), (ii) Rosin-Rammler drop distribution with $\mathrm{d}_{50}=7 \mathrm{~mm}$ and $\mathrm{n}_{\mathrm{RR}}=3$, and (iii) RosinRammler drop distribution with $\mathrm{d}_{50}=3.5 \mathrm{~mm}$ and $\mathrm{n}_{\mathrm{RR}}=3$.

The results of these calculations are shown in Figures 6.25 and 6.26. The predicted transfer characteristics and the pressure drop across the splash pack with the mono-disperse initial drop distribution are lower than that of the reference case. The case with a Rosin-Rammler initial drop distribution with $\mathrm{d}_{50}=7 \mathrm{~mm}$, agrees very closely with the predictions of the reference case. The predicted transfer characteristics and pressure drop across the splash pack with the Rosin-Rammler initial drop distribution with $\mathrm{d}_{50}=3.5 \mathrm{~mm}$, are higher than that of the reference case. It can be concluded that the type of water distribution system can have a significant effect on the measured transfer characteristics of a splash pack. 


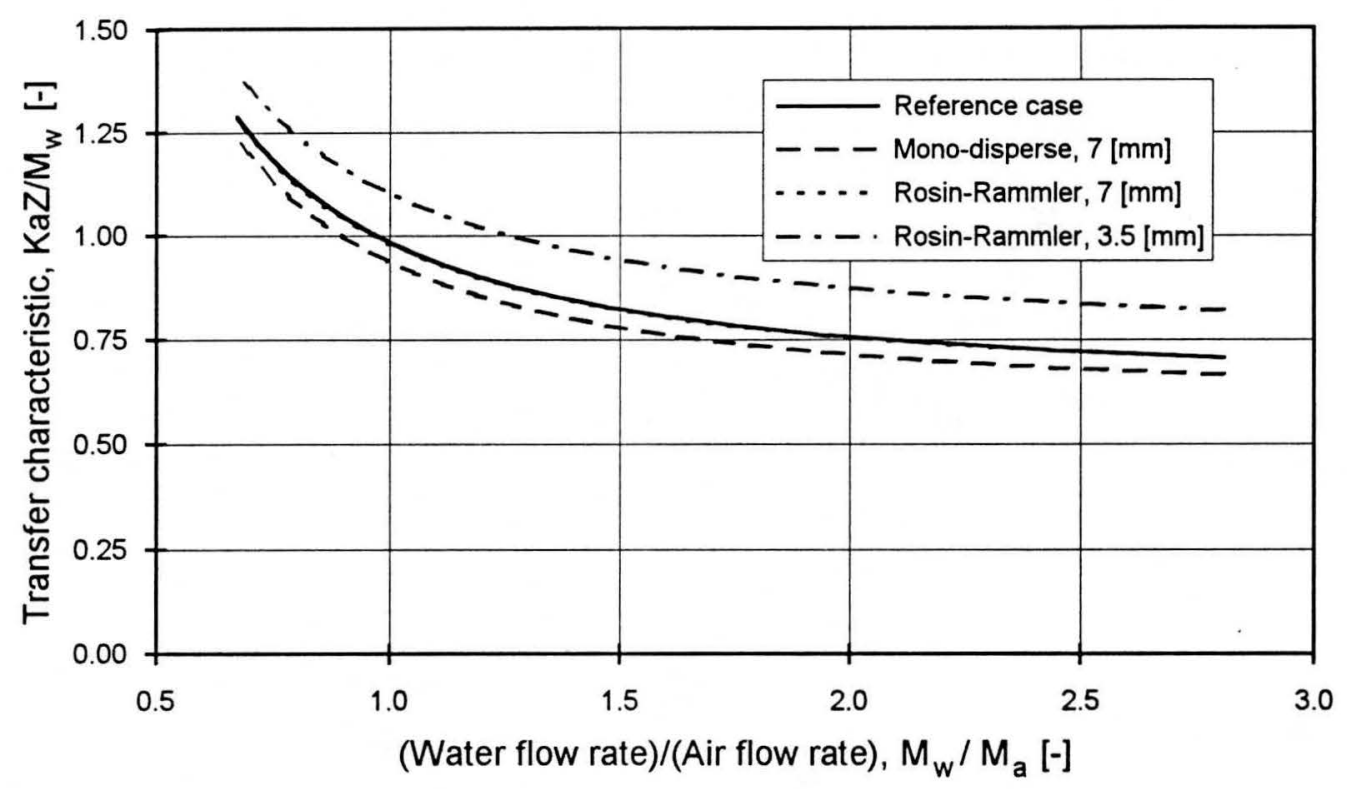

Figure 6.25 Predicted transfer characteristics with different initial drop size distributions.

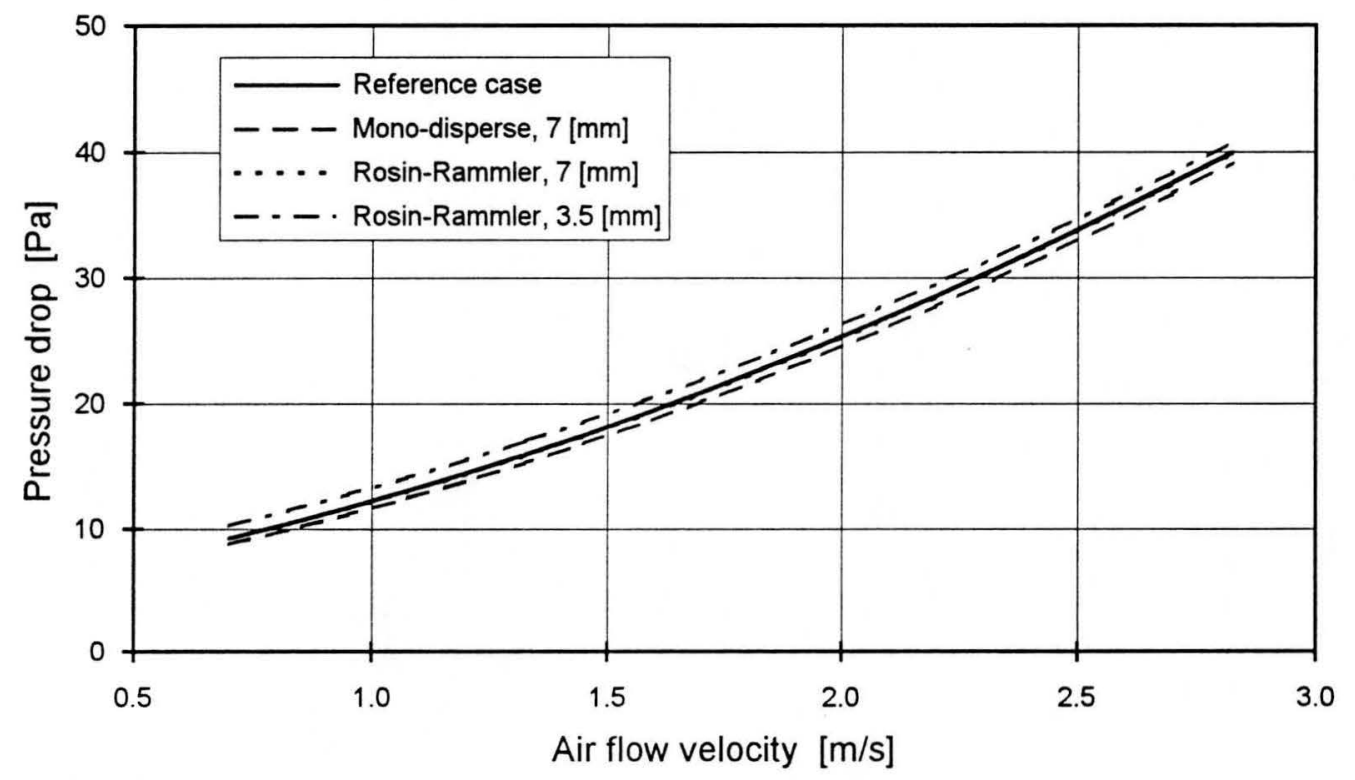

Figure 6.26 Predicted pressure drop with different initial drop size distributions.

\section{Optimisation parameters}

The predicted transfer characteristics and pressure drop were found to be insensitive to changes in the packet-reducing parameters, $\mathrm{v}_{\text {crit }}$ and $\mathrm{T}_{\text {crit }}$. Changing these parameters from $0.25 \mathrm{~m} / \mathrm{s}$ and $0.25^{\circ} \mathrm{C}$ to $0.5 \mathrm{~m} / \mathrm{s}$ and $0.5^{\circ} \mathrm{C}$ had a negligible effect on the predicted transfer characteristics and pressure drop. The program execution time was reduced by approximately $15 \%$ by using the less stringent packet-reducing parameters. 


\subsection{Comparison with measured splash pack performance}

The predicted transfer characteristics and pressure drop data obtained with the simulation program, SPSIM, is compared to the experimentally determined splash pack performance data for the twelve different splash pack geometries described in Table 4.11. The simulation program options used to obtain the predicted data was the same as that for the reference case described under the Sensitivity Analysis above (except for the splash pack geometry, air and water flow rates and the film thicknesses on the slats). The film thicknesses on the slats were assumed to be constant at $0.5 \mathrm{~mm}$ on the $9 \mathrm{~mm}$ wide slats and $0.65 \mathrm{~mm}$ on the $25 \mathrm{~mm}$ wide slats (see Figures 4.9 and 4.10).

\subsubsection{Thermal performance}

Figure 6.27 shows a typical comparison between the measured and the predicted transfer characteristic data for splash pack $I$ in Table 4.11 at three water flow rates, i.e. 5000, 10000 and $15000 \mathrm{~kg} / \mathrm{m}^{2} \mathrm{hr}$. The agreement between the measured and the predicted data is fairly good at the higher air flow rates (i.e. at low water flux to air flux ratios). The simulation program over-predicts the transfer characteristics at the lower air flow rates. Note that the experimental data was not corrected for inlet and outlet effects (due to heat/mass transfer on the water distribution system and the water collecting troughs) as discussed in Chapter 4 . If the inlet and outlet corrections are applied, the predicted data fall approximately $25 \%$ higher than the experimental data. This will be discussed in more detail below.

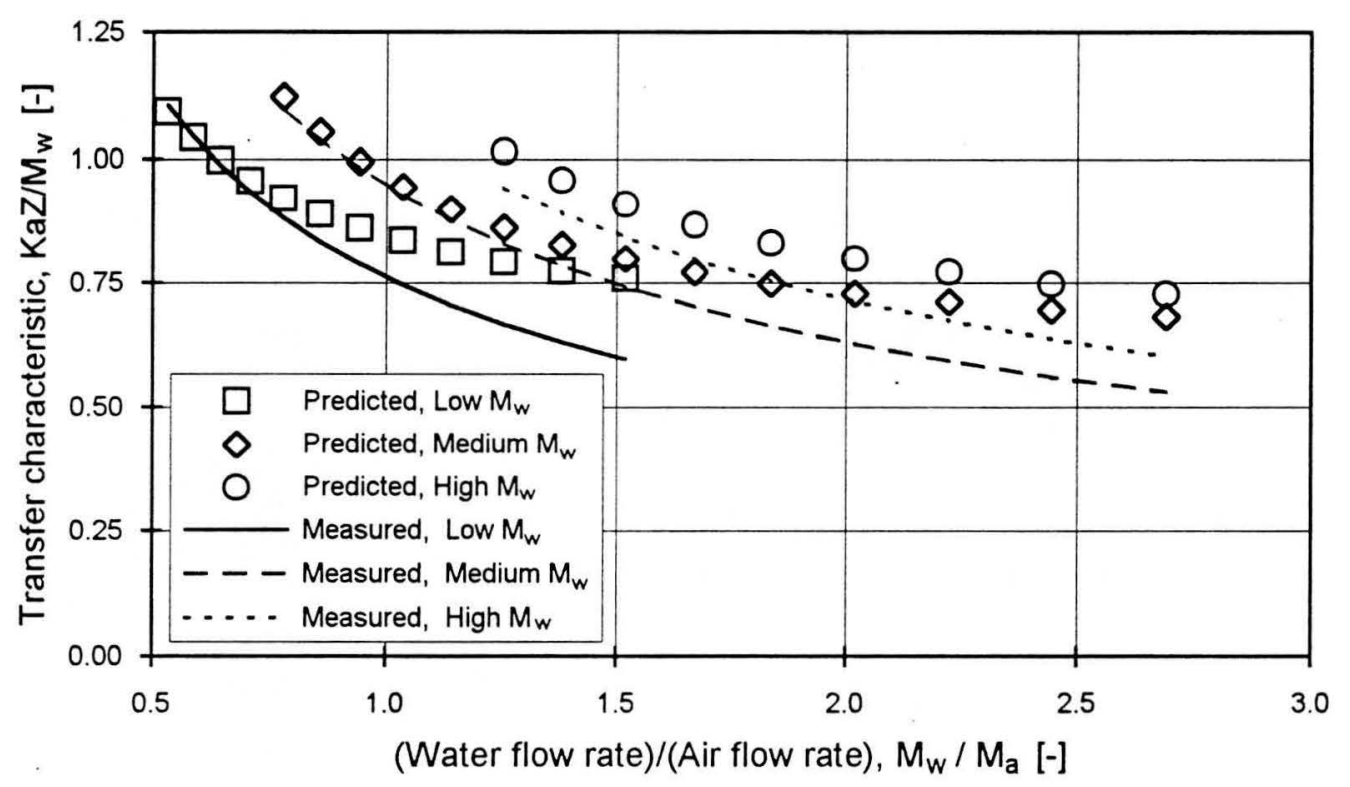

Figure 6.27 Comparison between predicted and measured transfer characteristic for experimental splash pack I. 
If interference effects between neighbouring splashes are taken into account, the predicted transfer characteristics are expected to be reduced at high $\left(\dot{\mathrm{M}}_{\mathrm{w}} / \dot{\mathrm{M}}_{\mathrm{a}}\right)$ ratios (See the discussion in the Sensitivity Analysis above). This will result in improved agreement between the predicted and measured trends of the transfer characteristics shown in Figure 6.27.

The predicted and uncorrected measured transfer characteristics for all twelve of the experimental splash packs are compared graphically in Figure 6.28. Each packing was evaluated at three water flow rates, i.e. 5000,10000 and $15000 \mathrm{~kg} / \mathrm{m}^{2} \mathrm{hr}$, and six air flow rates between 0.75 and $3.25 \mathrm{~m} / \mathrm{s}$. There is good agreement between the predicted and the measured data.

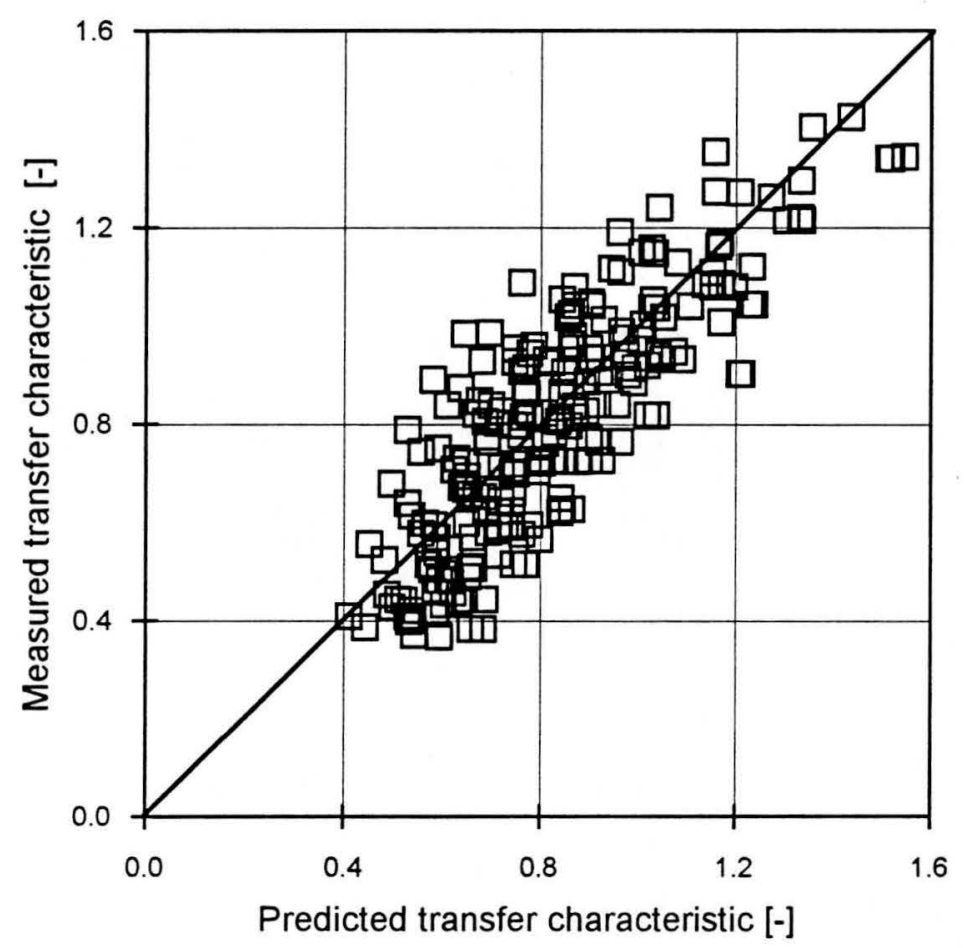

Figure 6.28 Correlation between predicted and uncorrected experimentally determined transfer characteristics.

The measured transfer characteristics were corrected by subtracting from it the empty tower transfer characteristic data (Equation (4.47)) to account for heat and mass transfer occurring on the water distribution system and the water collecting troughs used in the experimental investigation. The predicted and corrected measured transfer characteristics for all twelve the experimental splash packs are compared graphically in Figure 6.29. The predicted transfer characteristics fall approximately $25 \%$ above the corresponding corrected measured data. This can be attributed to one or more of the following:

i) The corrected experimentally determined transfer characteristics are too low due to the over-estimation of the inlet/outlet correction applied to the measured data. 
ii) The experimentally determined transfer characteristics are too low due to the influence of water bypassing the packing material by flowing down the side walls of the test facility (see Fabre and Legrand [88FA1]). In a typical test the following values were measured: $\mathrm{T}_{\mathrm{wi}}=40^{\circ} \mathrm{C}, \mathrm{T}_{\mathrm{wo}}=30^{\circ} \mathrm{C}, \mathrm{i}_{\mathrm{ai}}=65 \mathrm{~kJ} / \mathrm{kg}$ and $\dot{\mathrm{m}}_{\mathrm{w}}=\dot{\mathrm{m}}_{\mathrm{a}}=6.25 \mathrm{~kg} / \mathrm{s}$. These measured values yield an overall transfer characteristic of 0.945 . If $5 \%$ of the water flowed down the walls and entered the collecting troughs at $35^{\circ} \mathrm{C}$, the actual water temperature leaving the packing material was $29.7^{\circ} \mathrm{C}$, which yields a transfer characteristic of 1.01 . The actual transfer characteristic is thus approximately $7 \%$ higher than that based on the mixed outlet water temperature. According to Fabre and Legrand [88FA1] the actual transfer characteristics of the packing material can be between 20 and $50 \%$ higher than that based on the mean outlet water temperature and the total water flow leaving the test section.

iii) The possibility that the simulation program over-predicts the performance of the splash pack due to its inability to account for the interaction effects between neighbouring splash crowns (see discussion under the Sensitivity Analysis above).

From the discussion below it is known that the simulation program over-predicts the pressure drop across the packing by about $25 \%$. This over-prediction of the pressure drop seems to substantiate the possibility that the simulation program over-predicts the thermal performance of the splash pack.

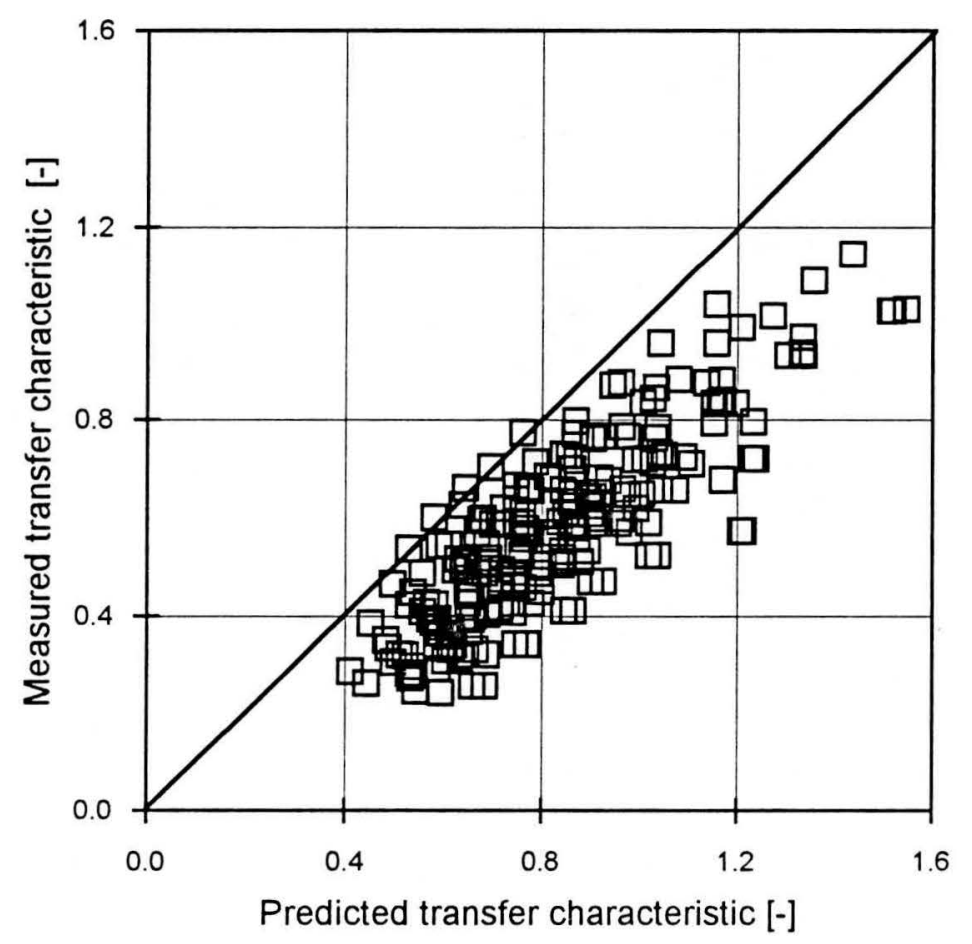

Figure 6.29 Correlation between predicted and corrected experimentally determined transfer characteristics. 


\subsubsection{Pressure drop}

Figure 6.30 compares the measured and the predicted pressure drop across splash pack I (see Table 4.11) at three water flow rates, i.e. 5000,10000 and $15000 \mathrm{~kg} / \mathrm{m}^{2} \mathrm{hr}$. The agreement between the measured and the predicted data is fair although the pressure drop is generally over-predicted.

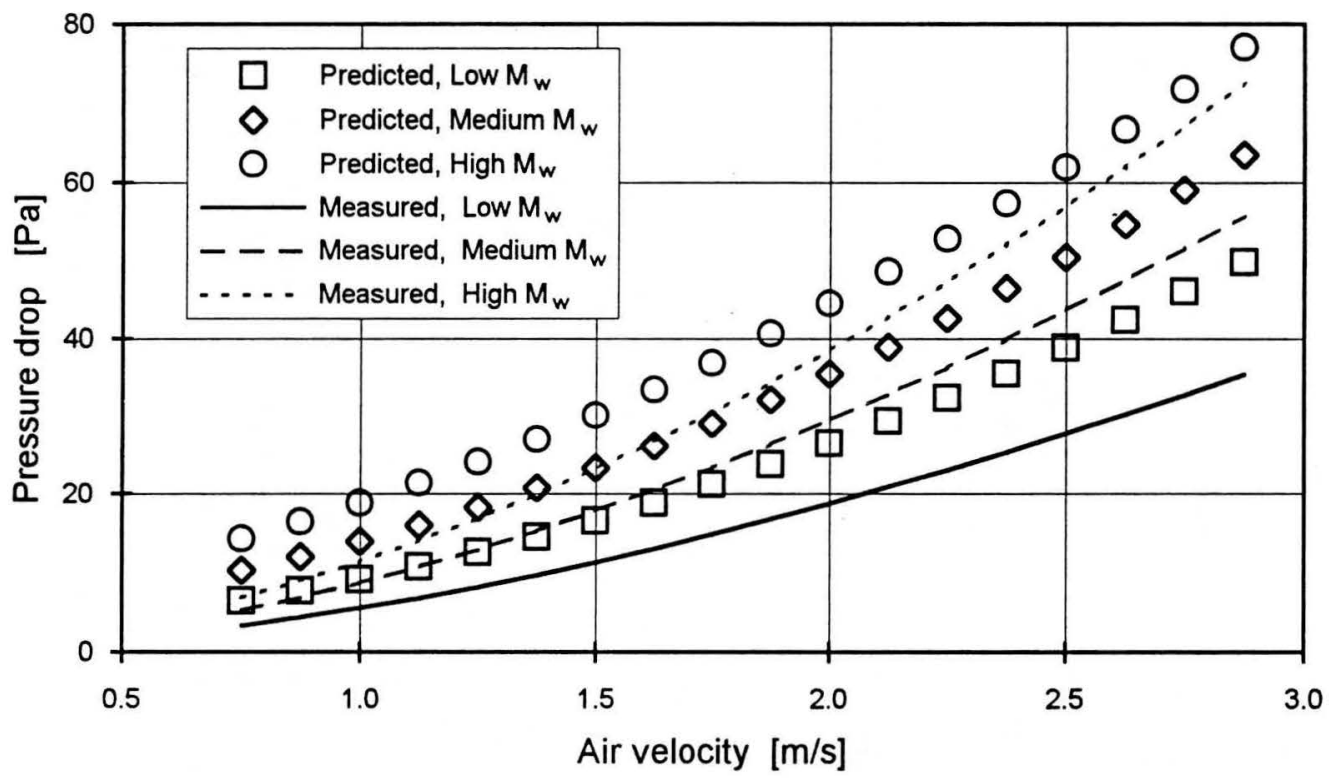

Figure 6.30 Comparison between predicted and measured pressure drop across experimental splash pack I.

Figure 6.31 shows the correlation between the predicted and measured pressure drop across the twelve splash packs which were tested. The predicted pressure drop data were generated for air flow rates between 0.75 and $2.75 \mathrm{~m} / \mathrm{s}$ and water flow rates between 5000 and 15000 $\mathrm{kg} / \mathrm{m}^{2} \mathrm{~s}$. It can be seen that the pressure drop is over-predicted by about $25 \%$. From the discussion below it can be concluded that this over-prediction is due to the simulation program over-predicting the number of splash drops. The splash drops which are usually smaller than 2 $\mathrm{mm}$ is expected to result in an over-prediction of the pressure drop across the splash pack since smaller drops contribute more to the pressure drop than larger drops (see Figure 6.3 above).

It should be borne in mind that the simulation program does not account for the expected increased pressure drop across each grid due to the increased blockage resulting from the water films flowing down the sides of the slats. The water hanging below the slats is expected to reduce the pressure drop across each grid by streamlining the leading edge of each slat, but since this effect is not easy to quantify, it is not incorporated in the simulation program SPSIM. 


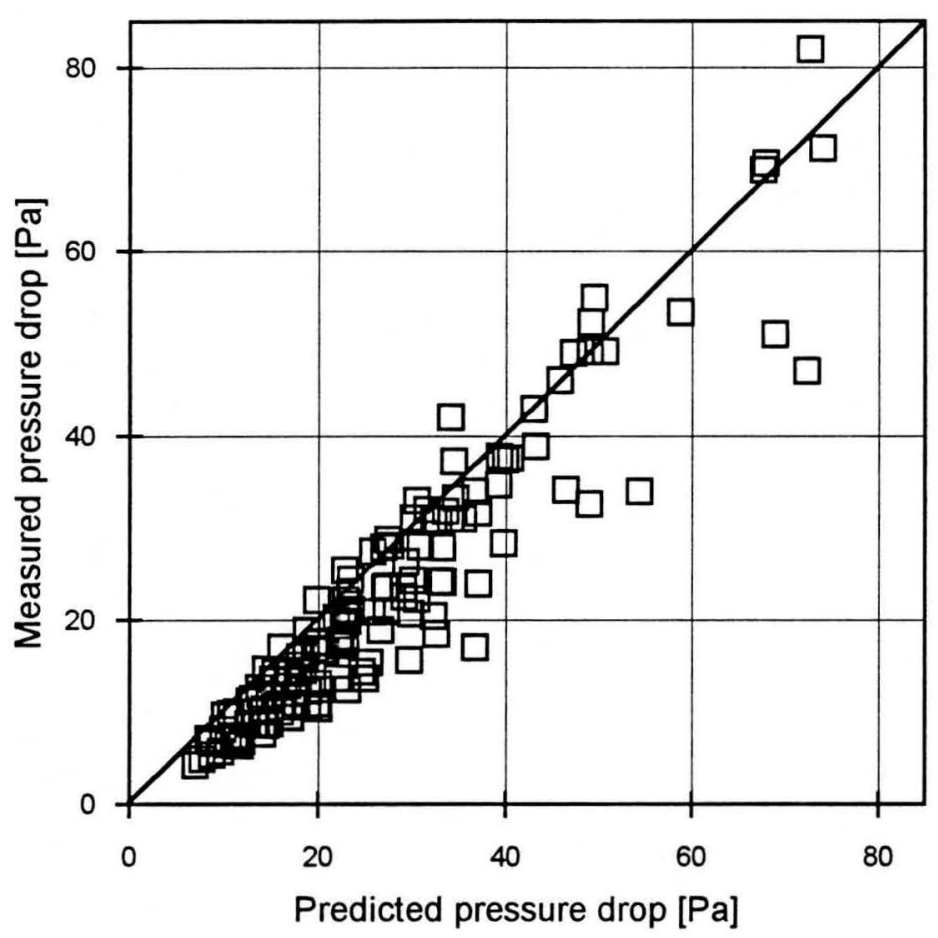

Figure 6.31 Correlation between predicted and experimentally determined pressure drop.

\subsubsection{Drop size}

The measured drop size distribution below the experimental splash pack I is compared to the drop distribution predicted by SPSIM in Figure 6.32. The predicted drop distribution shows a large fraction of the total water mass to be concentrated in drops smaller than $2 \mathrm{~mm}$, while this is not the case with the measured drop distribution. In the simulation program small drops of this size are mainly produced by splashing which tends to indicate that the simulation program over-predicts the mass of drops formed by splashing.

The over-prediction of splashing drops can be due to the failure of the prediction program to account for interaction between adjacent splash crowns. In Chapter 3 , it was shown that the average time between drop impacts at a given point is of the same order of magnitude as the crown lifetimes, i.e. the interference factor, I, is of the order unity. This implies that, on average, a splash crown will not have disappeared before the next drop impact in the direct proximity of the original splash crown. The discrepancy between the measured and predicted drop size distribution for drops smaller than $2 \mathrm{~mm}$ largely disappears when artificially reducing the splash fractions in SPSIM by $50 \%$. Such a reduction in splash fraction will result in reduction in the pressure drop and transfer characteristic data predicted by the simulation program as shown in the Sensitivity Analysis above. 
The splash crown interactions can also be expected to influence the splash drop size distribution, the initial splash drop velocities and the splash angles. None of these effects have been accounted for in the current version of SPSIM and this could be the subject of further investigation.

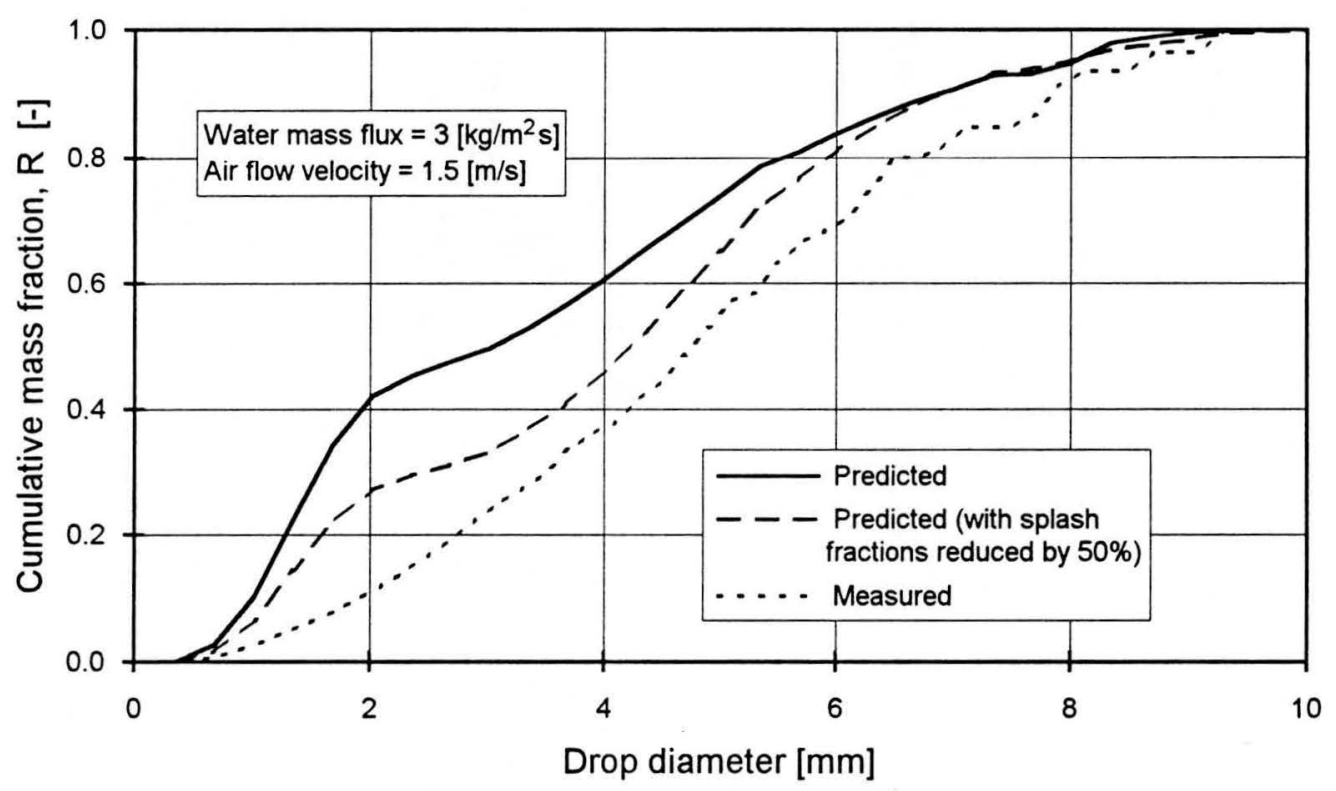

Figure 6.32 Comparison between measured and predicted drop size distributions below experimental splash pack I.

\subsection{Design trends for splash pack optimisation}

The simulation program, SPSIM, was used to calculate the transfer characteristics and pressure drop across typical splash packs. The effect of slat width on the performance of the splash pack was investigated by varying the slat widths between 2.5 and $25 \mathrm{~mm}$ while keeping the grid porosity constant at $70 \%, 80 \%$ or $90 \%$. In all the cases the same simulation options as in the reference case of the Sensitivity Analysis was used. The slat circumference was assumed to be fixed at $60 \mathrm{~mm}$.

The effect of cutting becomes more important than splashing in the case of narrow slats $(\mathrm{W}<5$ $\mathrm{mm}$ ) and since the simulation program uses a very simple model to describe the cutting phenomenon, care should be taken when using the simulation program to model splash packs with such narrow slats.

Figure 6.33 shows the variation of the predicted transfer characteristics when the cooling of the films on the surface of the slats is taken into account. 


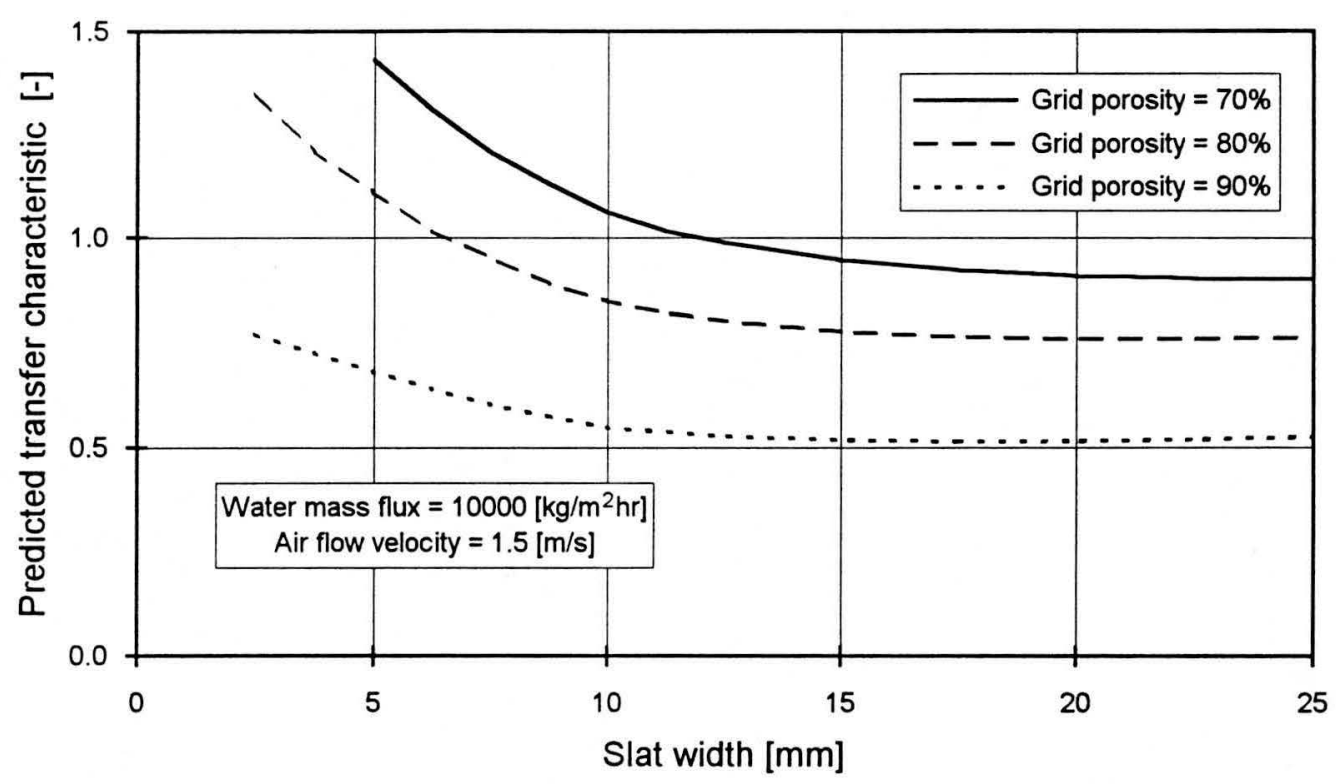

Figure 6.33 Predicted transfer characteristic variation with slat width when cooling of the films covering the slats is taken into account.

Since the slat circumference was taken as a constant, the contribution of the film cooling on the slats tend to flatter the predicted performance of the splash packs with smaller slat widths and lower grid porosities. Figure 6.34 shows the predicted performance of the splash packs when the cooling of the films on the slats is neglected.

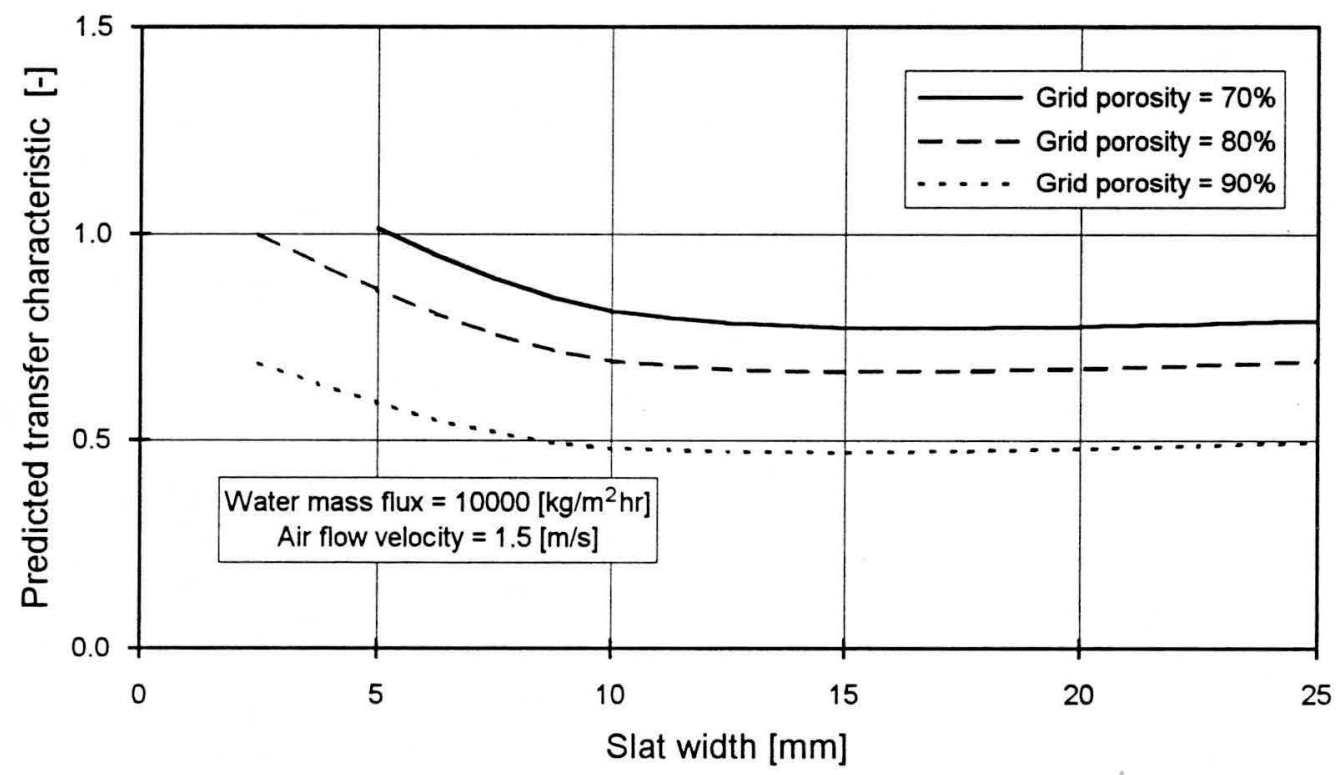

Figure 6.34 Predicted transfer characteristic variation with slat width when cooling of the films covering the slats is NOT taken into account. 
The predicted pressure drop across the splash pack is shown in Figure 6.35. As expected, the pressure drop across the grids with the higher porosities (more open area) is less than that across grids with lower porosities. The packing with the highest transfer characteristics also yields the highest pressure drop.

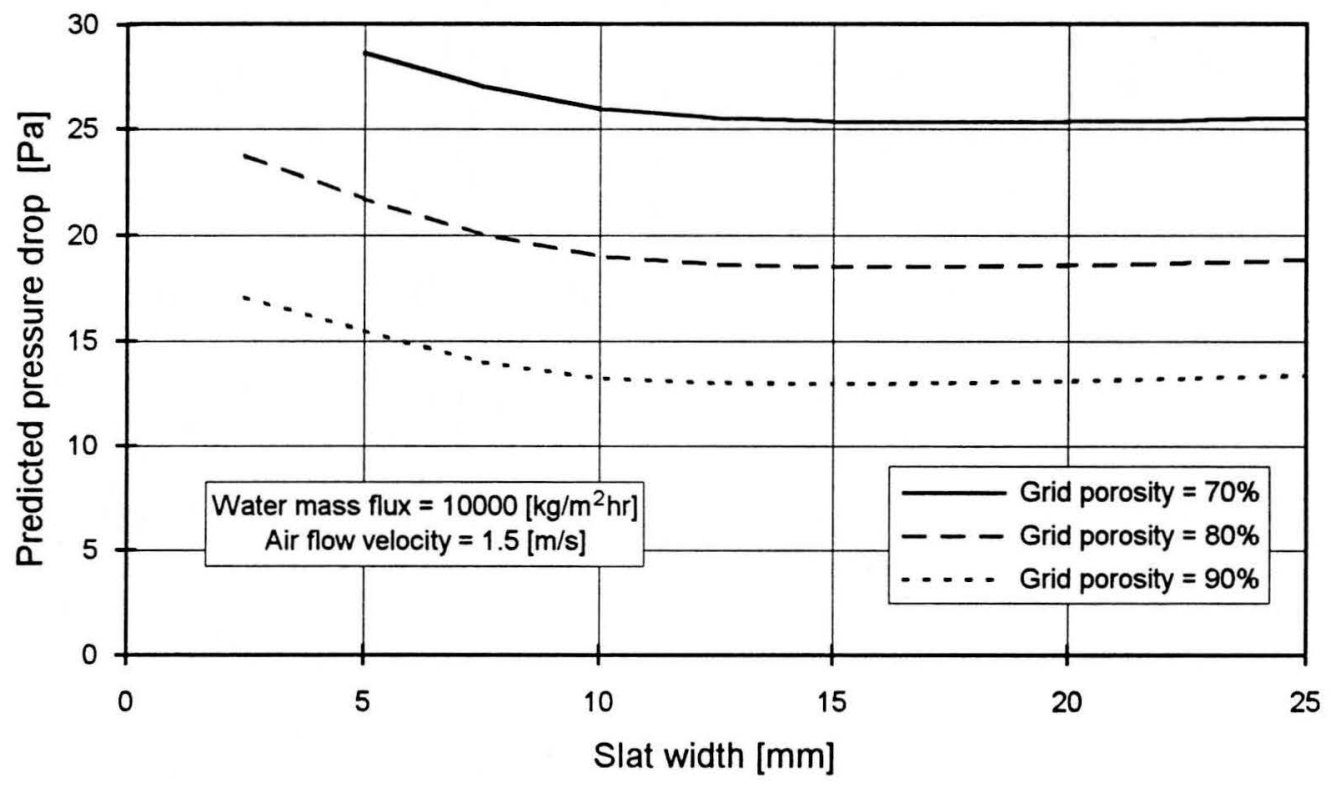

Figure 6.35 Predicted pressure drop variation with slat width.

The packing efficiency, which is the ratio of the overall transfer characteristics to the pressure drop across the packing (see Equation (6.2)), can be used to compare the different packing materials on a common base. Figures 6.36 and 6.37 show the packing efficiencies for the cases when the film cooling is taken into account and when it is ignored, respectively. It can be seen from the figures below that the packing thermal efficiency increases with decreasing slat width. In both cases shown below, the packing with $80 \%$ porosity shows the highest efficiencies for slat widths of less than $10 \mathrm{~mm}$. The efficiencies of the splash packs evaluated here typically lie at approximately $\eta_{1} \approx 0.04 \mathrm{~Pa}^{-1}$ when the cooling of the films on the slats is ignored. As can be seen from Figure 6.4, this corresponds to the efficiencies found in mono-disperse spray towers with drops diameters between 1 and $2 \mathrm{~mm}$. Note that the initial drop distribution used in this simulation is the same as that measured for the spray system used in the experimental study (Chapter 4). The mass median drop diameter of this distribution is approximately $7 \mathrm{~mm}$. This means that through the use of splash grids it was possible to obtain the same cooling from a very coarse spray of drops as one would obtain from a much finer spray in an empty tower. The production of such a fine spray by a pressure nozzle would require a high water pressure, which in turn would require far more pumping power than would be required to generate a coarse spray such as that used in the splash pack simulation. 


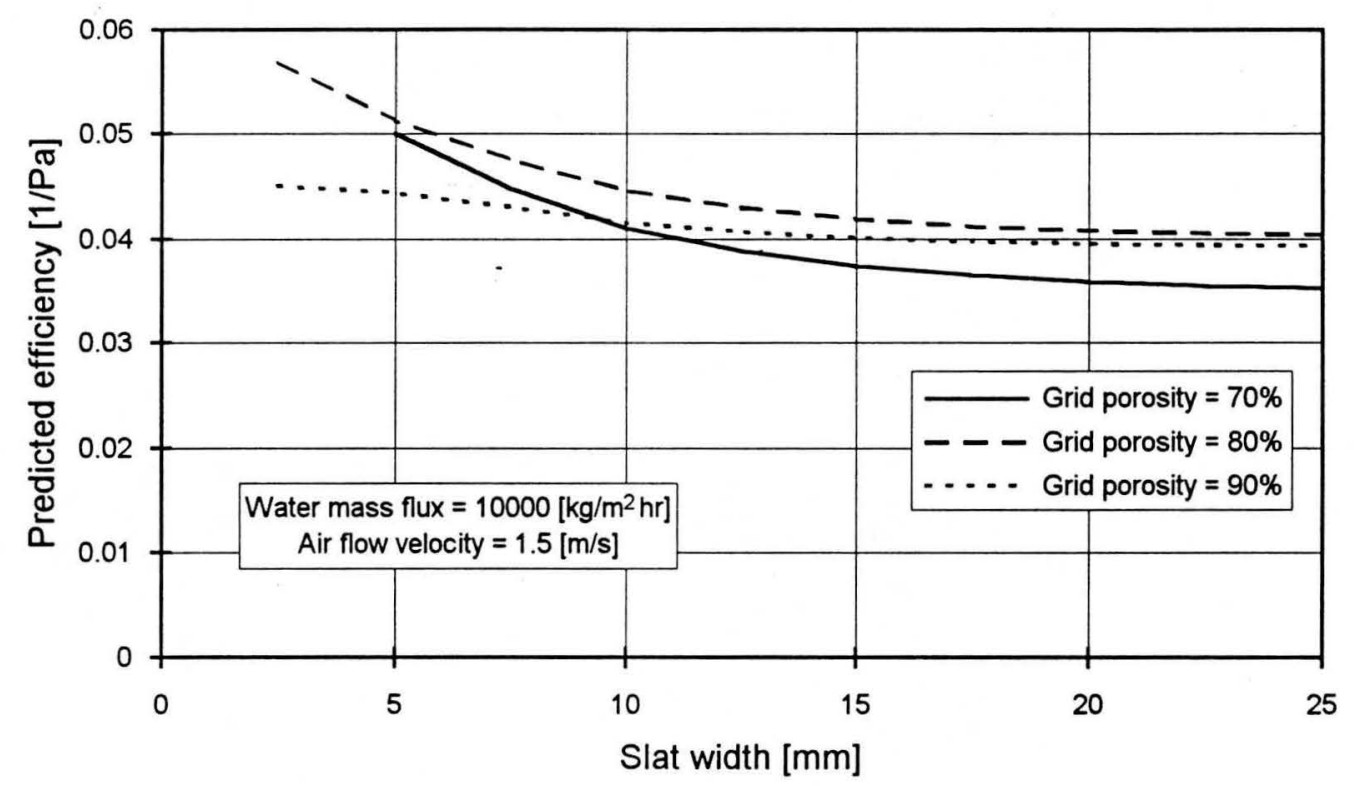

Figure 6.36 Predicted packing efficiency variation with slat width when cooling of the films covering the slats is taken into account.

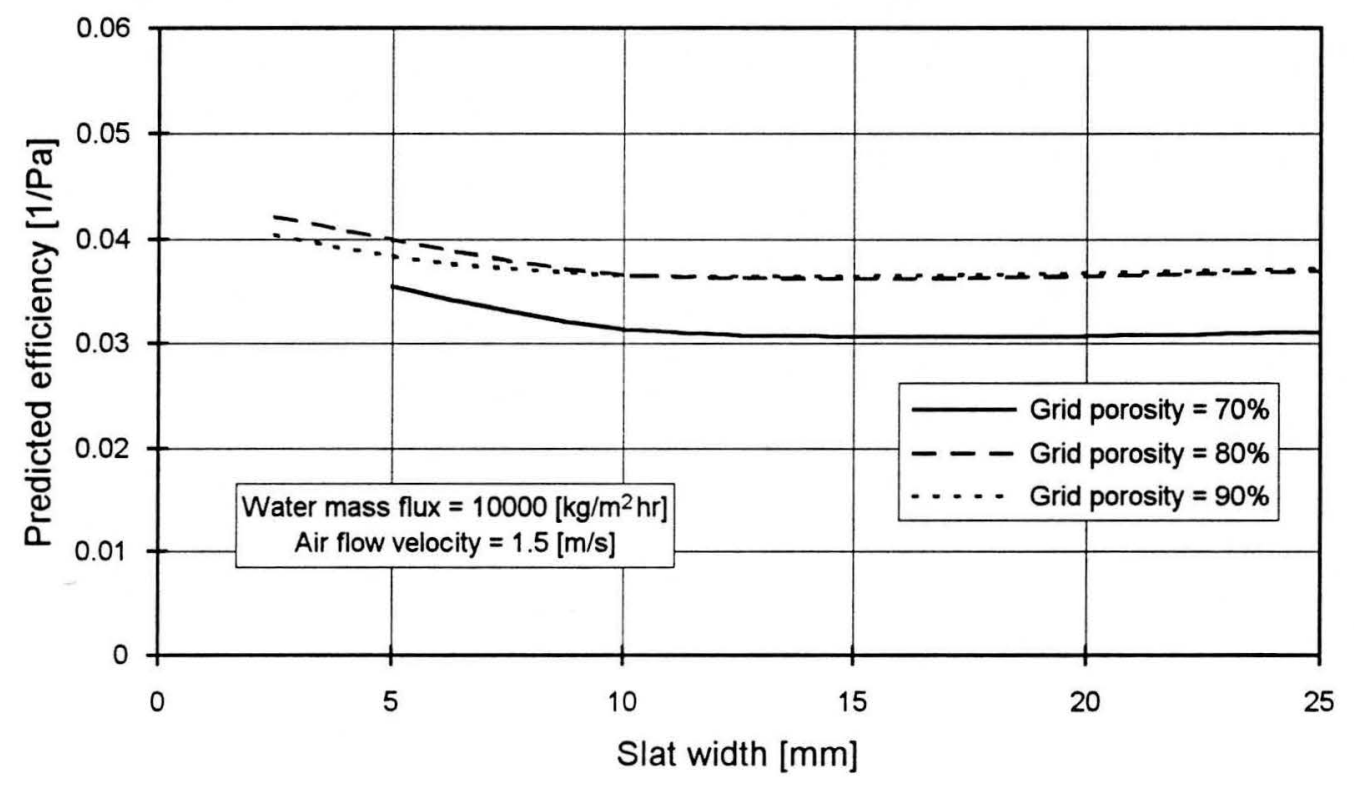

Figure 6.37 Predicted packing efficiency variation with slat width when cooling of the films covering the slats is NOT taken into account.

The performance of cooling tower splash pack could be enhanced by the reduction of the water surface tension with a chemical additive. Ryan [76RY1] found that the addition of minute amounts of fluorochemical surfactants reduced the surface tension of water by up to $75 \%$. The reduced surface tension results in smaller drops being formed through splashing and dripping, which in turn influences the thermal performance of the splash pack. Figure 6.38 shows the predicted performance of the reference splash pack (see the Sensitivity analysis 
above) with and without a $50 \%$ reduction in the water surface tension. Both the predicted transfer characteristics and the pressure drop across the packing were increased by the reduction in water surface tension. The overall efficiency of the pack is slightly increased by the reduction in surface tension.

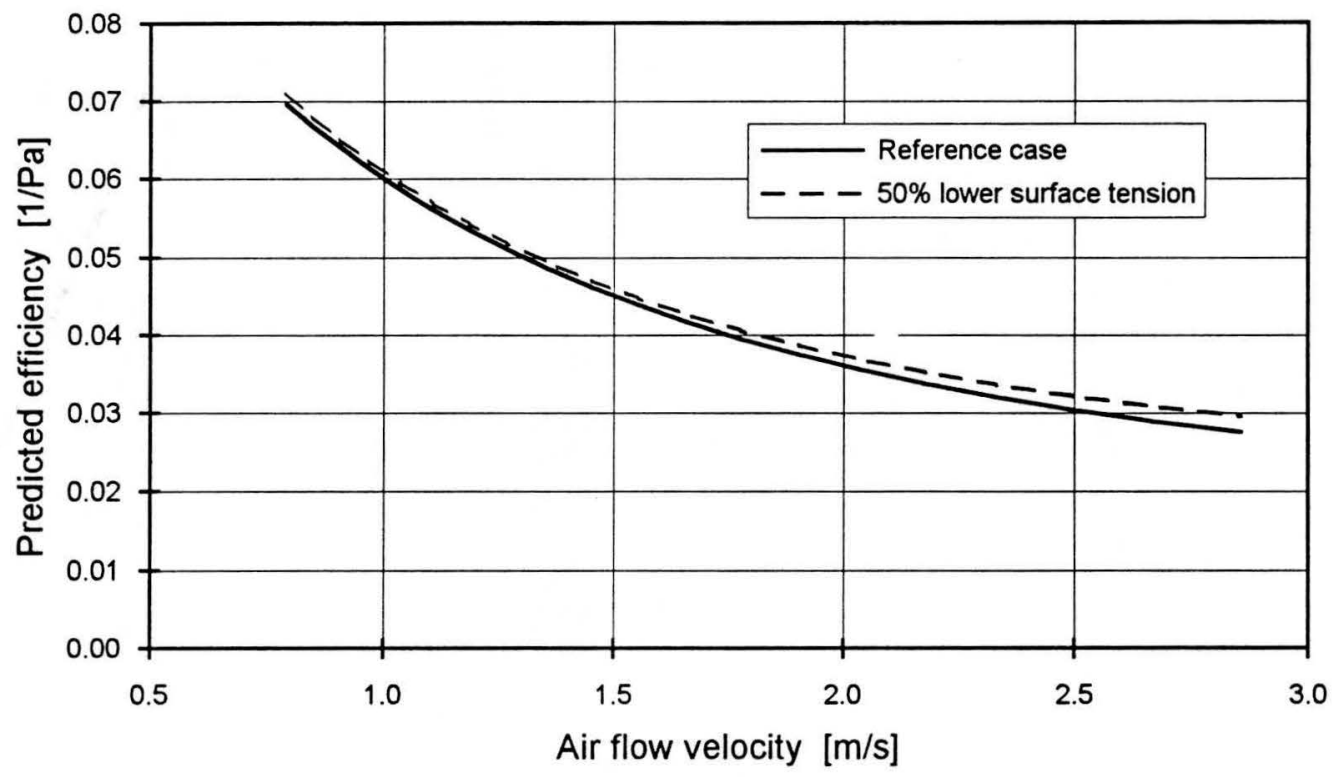

Figure 6.38 Predicted splash pack efficiency with and without surface tension reducing additives.

A large fraction of the pressure drop across a typical splash pack is due to the pressure drop across the grids. Figure 6.39 shows that for experimental splash pack I, the pressure drop due to the grids often contribute more than $50 \%$ of the total pressure drop across the splash pack. At low water flow rates and high air flow rates, the pressure drop across the grids contribute up to $75 \%$ of the total pressure drop across the splash pack.

If the pressure drop across the grids can be reduced this could result in a significant reduction in the total pressure drop across splash packing material. The pressure drop across a roundedged grid with $\beta \approx 0.656$ is approximately $60 \%$ of that across a sharp-edged grid of the same porosity (see Chapter 2). For a splash pack with sharp-edged grids in which the pressure drop across the grids accounts for $50 \%$ of the total pressure drop, rounding the leading (lower) edges of the grids could reduce the total pressure drop across the splash packing by approximately $20 \%$.

Rounding the lower edges of the slats could also result in a reduction of the sizes of the drops dripping below the slats, if the shape factor of the lower surface of the slats is reduced. 


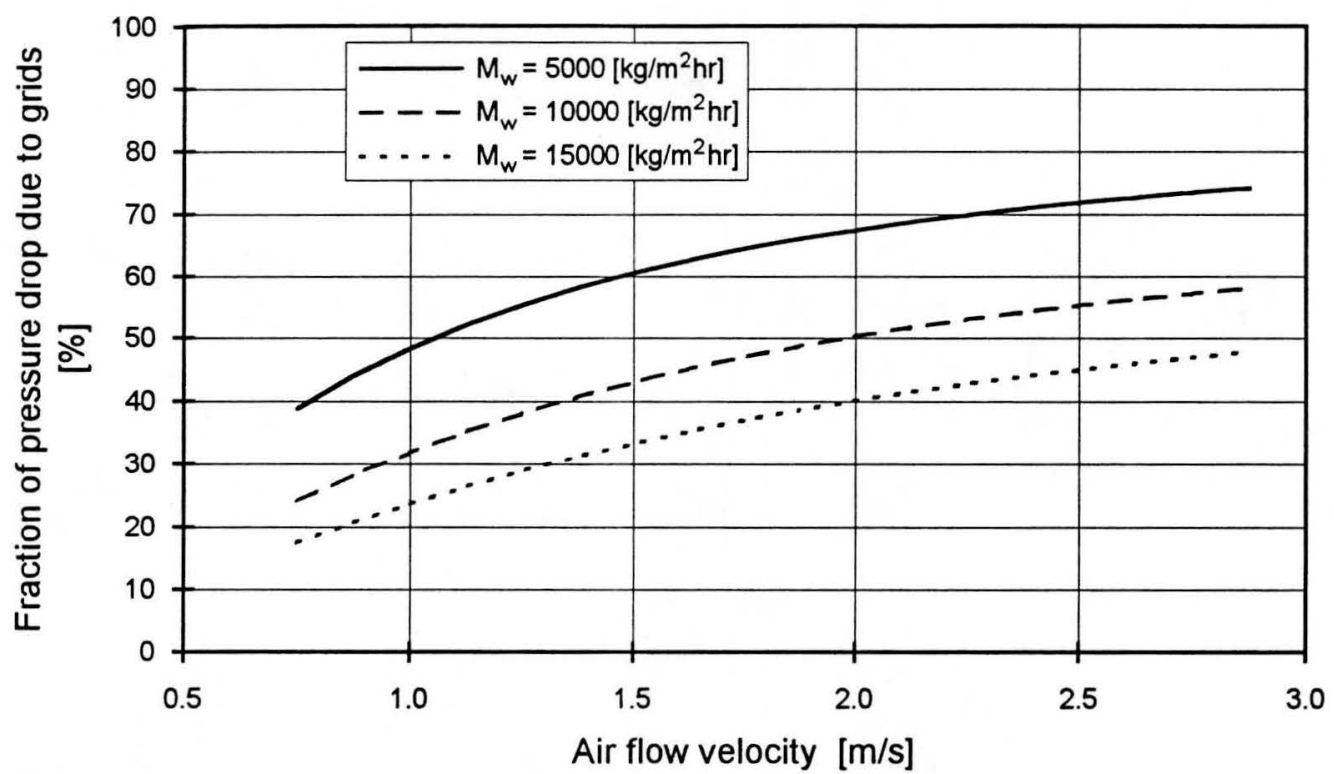

Figure 6.39 Contribution of pressure drop across the grids to the total predicted pressure drop across splash pack I. 


\section{CHAPTER 7 CONCLUSIONS AND RECOMMENDATIONS}

A mathematical model and a computer simulation program have been developed to predict the performance of counterflow cooling tower splash packing material. This program can be used to evaluate different splash pack designs without making use of empirical data from splash pack tests. The program uses empirical data relating to the grid characteristics, drop dynamics and drop formation by splashing, cutting and dripping. The use of the packet concept to group similar drops together, works well in reducing the number of calculations required. Without the use of the packet concept the amount of computer memory required to store all the drop size, velocity and temperature data would become excessive.

The counterflow cooling tower splash pack simulation program, SPSIM, was found to predict the correct trends in transfer characteristics and pressure drop with varying air and water flow rates. The predicted pressure drop data agreed well with the experimentally determined data for air and water flow rates in the ranges used in cooling towers.

The actual transfer characteristic values were over-predicted by up to $25 \%$ at high water flow rates when the simulation program is compared to experimentally data obtained in a packing material test facility with a $1.5 \times 1.5 \mathrm{~m}^{2}$ test section. In such a small test section, the effect of water bypassing the packing by flowing down the walls of the test tunnel, is expected to yield low transfer characteristic data. Some uncertainty exists regarding the size of the correction made to correct the experimental transfer characteristic data for inlet and outlet effects. The simulation program can be used to compare different packing geometries and operating conditions, regardless of the fact that it may not predict the exact packing characteristics.

The results obtained with the simulation program were found to be sensitive to the following parameters :

i) the thickness of the water films covering the slats,

ii) the model to calculate the volume of water splashing from a slat upon a drop impact,

iii) the distribution of drop sizes formed by splashing,

iv) the initial drop size distribution, and

v) the amount of cooling of the water films flowing down the slats.

The main limitations of the of current model are the following:

i) The water film thickness covering the slats has to be specified. The thickness of the films on the slats cannot be determined analytically at this stage. The film thickness is expected 
to depend on the air flow rate, the water flow rate, the drop size distribution, the drop velocities, surface tension and grid geometry.

ii) The inability to predict the interaction effects between neighbouring splash crowns on the slats. These interactions are expected to influence the total mass of water splashing from the slats and/or the distribution of drops formed by splashing. At high water flow rates these interaction effects are expected to become more pronounced. The interaction effects could also be influenced by the air velocity through the packing material; at low air velocities the relative velocity between the drops and the slats increases, resulting in larger splash crowns being formed, which in turn implies more interaction between neighbouring splashes.

Through the use of the simulation program, the following general guidelines regarding the optimum layout of cooling tower splash pack could be determined:

i) Optimise the surface area of the slats. In splash packs the wetted surface areas of the slats contribute significantly to the total interface area between the air and water.

ii) A grid with $80 \%$ open area $(\beta \approx 80 \%)$ and a slat width of less than $10 \mathrm{~mm}$ represents the best combination between transfer characteristics and pressure drop.

iii) Rounding of the leading (lower) edges of the slats can lead to significant reduction in pressure drop across the splash pack. This is expected to be more important when using slats wider than $10 \mathrm{~mm}$ since the water hanging below the narrow slats will tend to streamline the flow about the slats anyway. The mean drop size of the dripping drops can also be reduced by using slats with sharper lower edges.

iv) The shape of the upper surface of the slats could influence the thickness of the water layer covering the upper surface of the slats. This in turn could influence the total mass of water splashing from each slat and the distribution of the splash drops. This could be the subject of further investigation.

v) It was found that the reduction of the surface tension of the circulating water results in increased cooling capacity and increased pressure drop across a splash pack due to reduced drop sizes. The overall improvement (ratio of transfer characteristics to total pressure drop across the packing) was found to be small even for a $50 \%$ reduction in water surface tension.

Suggestions for further work include the following:

i) Analytical determination of the film thicknesses on the upper surfaces of the slats.

ii) The modelling of the interaction effects between splash crowns which are expected to influence the total mass of water splashing from the impact point and the distribution of the splash drop sizes. 
iii) The modelling of the splashing on the upper surface of the slats taking the varying film thicknesses and the wave action in the thin films covering the slats into account.

iv) Determining the effect of the shape of the upper surfaces of the slats on the total volume of water leaving the surface of the slats due to splashing and the distribution of splash drops.

v) The analytical modelling or experimental determination of the size distribution of drops dripping below bi-planar grids.

vi) Investigating the interaction between in-line grids. There is some evidence that the splashing phenomenon is influenced by the shape of the drop (i.e. prolate or oblate) upon impact with the slat. If this is true, there could be a critical distance between in-line slats at which the drops dripping from the upper slat will reach the lower slat at exactly the right instant to maximise the splash volumes.

vii) The modelling of turbulence effects on the drop drag and the heat/mass transfer from the drops. The relatively coarse grids used in splash pack can be assumed to increase the free-stream turbulence in the splash pack, but the combined effect of multiple grids on the free-stream turbulence needs to be investigated more thoroughly.

viii) The modelling of non-contact interaction effects in a poly-disperse spray of drops (with different velocities). These effects are expected to result in lower drop drag and a corresponding decrease in heat/mass transfer.

ix) The development of testing procedures to improve splash pack performance evaluation in small-scale test facilities by minimising the bypassing effect due to water running down the sides of the walls of the test tunnel. 


\section{REFERENCES}

[26ME1] Merkel, F., Verdunstungskühlung, VDI-Zeitschrift, Vol. 70, No. 4, pp. 123-128, January 1926.

[38FR1] Frössling, N., Über die Verdunstung Fallender Tropfen, Gerlands Beitrage Zur Geophysik, Vol. 52, pp. 170-216, 1938.

[40NO1] Nottage, H.B., and Boelter, L.M.K., Dynamic and thermal behaviour of water drops in evaporative cooling processes, ASHVE, Research Report, No. 1146, 10EEE, pp. 41-82, 1940.

[41LAl] Laws, J.O., Measurement of the fall velocity of water drops and raindrops, Transactions of the American Geophysical Union, Part III, 22nd Annual Meeting, pp. 709-721, 1941.

[43LI1] Lichtenstein, J., Performance and selection of mechanical-draft cooling towers, Transactions of the ASME, Vol. 65, pp. 779-787, October 1943.

[45DA1] Davies, C.N., Definitive equations for the fluid resistance of spheres, Proceedings of the Physics Society of London, Vol. A57, pp. 259-270, 1945.

[45LA1] Lamb, H., Hydrodynamics, 6th Edition, Cambridge University Press, London, 1945.

[49GU1] Gunn, R., and Kinzer, G.D., The terminal velocity of fall for water droplets in stagnant air, Journal of Meteorology, Vol. 6, pp. 243-248, August 1949.

[49JA1] Jakob, M., Heat Transfer, Vol. 1, John Wiley and Sons, Inc., New York, 1949.

[51BA1] Baines, W.D., and Peterson, E.G., An investigation of flow through screens, Transactions of the ASME, Vol. 73., No.1, pp. 467-480, 1951.

[51KI1] Kinzer, G.D., and Gunn, R., The evaporation, temperature and thermal relaxation-time of freely falling waterdrops, Journal of Meteorology, Vol. 8, No. 2, pp. 71-83, April 1951.

[5lLAl] Lane, W.R., Shatter of drops in streams of air, Industrial and Engineering Chemistry, Vol. 43, No. 6 , pp. 1312-1317, June 1951.

[51MU1] Mugele, R.A., and Evans, H.D., Droplet size distribution in sprays, Industrial and Engineering Chemistry, Vol. 43, No. 6, pp. 1317-1324, 1951.

[51SN1] Snyder, R.W., The cooling of a freely falling water-drop, IMechE, ASME Proceedings of the General Discussion on Heat Transfer, pp. 160-166, September 1951.

[52HU1] Hughes, R.R., and Gilliland, E.R., The mechanics of drops, Chemical Engineering Progress, Vol. 48, No. 10, pp. 497-504, 1952.

[52RA1] Ranz, W.E., and Marshall Jr., W.R., Evaporation from drops, Part I, Chemical Engineering Progress, Vol. 48, No. 3, pp. 141-146, March 1952.

[52RA2] Ranz, W.E., and Marshall Jr., W.R., Evaporation from drops, Part II, Chemical Engineering Progress, Vol. 48, No. 4, pp. 173-180, April 1952.

[54Mcl] McDonald, J.E., The shape of raindrops. Scientific American, Vol. 190, No. 2, pp. 64-68, 1954.

[55HI1] Hinze, J.O.. Fundamentals of the hydrodynamic mechanism of splitting in dispersion processes, AIChE Journal, Vol. 1, No. 3, pp. 289-295, September 1955. 
[56KE1] Kelly, N.W., and Swenson, L.K., Comparative performance of cooling tower packing arrangements, Chemical Engineering Progress, Vol. 52., No. 7, pp. 263-268, 1956.

[56SM1] Smith, W.W., Study of three redwood cooling tower packings, Heating, Piping and Air Conditioning, pp. 139-142, 1956.

[59GAl] Garner, F.H., and Lane, J.J., Mass transfer to drops of liquid suspended in a gas stream. Part II : Experimental work and results, Transactions of the IChemE, Vol. 37, pp. 162-172, 1959.

[59GR1] Gregory, P.H., Guthrie, E.J., and Bunce, M.E., Experiments on splash dispersal of fungus spores, Journal of General Microbiology, Vol. 20, pp. 328-354, 1959.

[60AD1] Adamson, A.W., Physical Chemisty of Surfaces, Interscience Publishers Inc., New York, 1960.

[61BA1] Baker, D.R., and Shryock, H.A., A comprehensive approach to the analysis of cooling tower performance, ASME Journal of Heat Transfer, pp. 339-350, 1961.

[61BE1] Berman, L.D., Evaporative cooling of circulating water, Translated from Russian by R. Hardbottle, Edited by H. Sawistowski, Pergamon Press, Oxford, 1961.

[61RO1] Rowe, P.N., and Henwood, G.A., Drag forces in a hydraulic model of a fluidized bed part 1, Transactions of the IChemE, Vol. 39, pp. 43-54, 1961.

[61YO1] Young, G.A.J., Cooling tower splash packings, British Hydromechanics Research Association Report PR 707, October 1961.

[62LE1] Levich, V.G., Physicochemical Hydrodynamics, Prentice-Hall, Eaglewood Cliffs, New Jersey, 1962.

[62LO1] Lowe, H.J., and Christie, D.G., Heat transfer and pressure drop on cooling tower packings, and model studies of the resistance of natural draft towers to airflow, Proceedings of the 2nd International Heat Transfer Conference, Boulder, Colorado, Part V, Paper 113, pp. 933-950, 1962.

[63IZ1] Izard, J.A.W., Cavers, S.D., and Forsyth, J.S., Production of liquid drops by discontinuous injection, Chemical Engineering Science, Vol. 18, pp. 467-469, 1963.

[63SK1] Skelland, A.H.P., and Cornish, A.R.H., Mass transfer from spheroids to an air stream, AIChE Journal, Vol. 9, No. 1, pp. 73-76, January 1963.

[64JA1] Jayaratne, O.W., and Mason, B.J., The coalescence and bouncing of water drops at an air/water interface, Proceedings of the Royal Society of London, Series A, Vol. 280, pp. 545-565, 1964.

[64LO1] Lochiel, A.C., and Calderbank, P.H., Mass transfer from the continuous phase around axisymmetric bodies of revolution, Chemical Engineering Science, Vol. 19, pp. 471-484, 1964.

[64SI1] Sideman, S., and Shabtai, H., Direct-contact heat transfer between a single drop and an immiscible liquid medium, The Canadian Journal of Chemical Engineering, pp. 107-117, 1964.

[65RO1] Rowe, P.N., Claxton, K.T., and Lewis, J.B., Heat and mass transfer from a single sphere in an extensive flowing fluid, Transactions of the IChemE, Vol. 43, pp. T14-T31, 1965.

[65TO1] Tooke, S., The impact of water drops on cooling tower splash-bar packings, Central Electricity Research Laboratories, Lab. Note No. RD/L/N 108/65, November 1965.

[65UC1] Uchida, H., Muneoka, H., Tezuka, S., Performance of cooling tower packing, Transactions of the SHASE, Vol. 2, pp. 29-37, 1965. 
[66CO1] Comte-Bellot, G.V., and Corrsin, S., The use of a contraction to improve the isotropy of gridgenerated turbulence, Journal of Fluid Mechanics, Vol. 25, pp. 657-682, 1966.

[66EN1] Engel, O.G., Crater depth in fluid impacts, Journal of Applied Physics, Vol. 37, No. 4, pp. 17981808, March 1966.

[67BR1] Brodkey, R.S., The phenomena of fluid motions, Addison-Wesley Publishing Company, Reading, Massachusetts, 1967.

[67BU1] Buzzard, J.L., and Nedderman, R.M., The drag coefficients of liquid droplets accelerating through air, Chemical Engineering Science, Vol. 22, pp. 1577-1586, 1967.

[67EI1] Eisenklam, P., Arunachalam, S.A., and Weston, J.A., Evaporation rates and drag resistance of burning drops, 11th International Symposium on Combustion, pp. 715-727, 1967.

[67EN1] Engel, O.G., Initial pressure, ini..al flow velocity, and the time dependence of crater depth in fluid impacts, Journal of Applied Physics, Vol. 38, No. 10, pp. 3935-3940, 1967.

[67HAl] Harlow, F.H, and Shannon, J.P., The splash of a liquid drop, Journal of Applied Physics, Vol. 38, No. 10, pp. 3855-3866, September 1967.

[67HA2] Harlow, F.H., and Shannon, J.P., Distortion of a splashing liquid drop, Science, Vol. 157, pp. 547550,1967

[67HO1] Hobbs, P.V., and Kezweeny, A.J., Splashing of a water drop, Science, Vol. 155, pp. 1112-1114, March 1967.

[67HO2] Hobbs, P.V., and Osheroff, T., Splashing of drops on shallow liquids, Science, Vol. 158, pp. 11841186, December 1967.

[67PI1] Pinker, R.A., and Herbert, M.V., Pressure loss associated with compressible flow through squaremesh wire gauzes, Journal of Mechanical Engineering Science, Vol. 9, pp. 11-23, 1967.

[68ADI] Adam, J.R., Lindblad, N.R., and Hendricks, C.D., The collision, coalescence, and disruption of water droplets, Journal of Applied Physics, Vol. 39, No. 11, pp. 5173-5180, October 1968.

[68RA1] Raithby, G.D., and Eckert, E.R.G., The effect of turbulence parameters and support position on the heat transfer from spheres, International Journal of Heat and Mass Transfer, Vol. 11, pp. 1233$1252,1968$.

[69BE1] Beard, K.V., and Pruppacher ,H.R., A determination of the terminal velocity and drag of small water drops by means of a wind tunnel, Journal of the Atmospheric Sciences, Vol. 26, pp. 10661072, September 1969.

[69MA1] Macklin, W.C., and Hobbs, P.V., Subsurface phenomena and the splashing of drops on shallow liquids, Science, Vol. 166, pp. 107-108, October 1969.

[69ST1] Stringham, G.E., Simons, D.B., and Guy, H.P., The behaviour of large particles falling in quiescent liquids, Geological Survey Professional Paper 562-C, US Government Printing Office, Washington, 1969.

[70LI1] List, R., MacNeil, C.F., and McTaggart-Cowan, J.D., Laboratory investigations of temporary collisions of raindrops, Journal of Geophysical Research, Vol. 75, No. 36, pp. 7573-7580, 1970.

[70MU1] Mutchler, C.K., Travel and composition of droplets formed by water drop splash on thin water layers, Ph.D dissertation, University of Minnesota, Minneapolis, Minnesota, 1970. 
[70MU2] Mutchler, C.K., and Hansen, L.M., Splash of a waterdrop at terminal velocity, Science, Vol. 169, pp. 1311-1312, September 1970 .

[70PR1] Pruppacher, H.R., LeClair, B.P., and Hamielec, A.E., Some relations between the drag and flow pattern of viscous flow past a sphere and a cylinder at low and intermediate Reynolds numbers, Journal of Fluid Mechanics, Vol. 44, pp. 781-790, 1970.

[70PR2] Pruppacher, H.R., and Beard, K.V., A wind tunnel investigation of the internal circulation and shape of water drops falling at terminal velocity in air, Quarterly Journal of the Royal Meteorological Society, Vol. 96, pp. 247-256, 1970.

[71BE1] Beard, K.V., and Pruppacher, H.R., A wind tunnel investigation of the rate of evaporation of small water drops falling at terminal velocity in air, Journal of the Atmospheric Sciences, Vol. 28, pp. 1455-1464, November 1971.

[71CA1] Cataneo, R., Adam, J.R., and Semonin, R.G., Interactions between equal-sized droplets due to the wake effect, Journal of the Atmospheric Sciences, Vol. 28, pp. 416-418, 1971.

[71CL1] Clift, R., and Gauvin, W.H., Motion of entrained particles in gas streams, The Canadian Journal of Chemical Engineering, Vol. 49, pp. 439-448, August 1971.

[71LE1] Levin, Z., and Hobbs, P.V., Splashing of water drops on solid and wetted surfaces: hydrodynamics and charge separation, Philosophical Transactions of the Royal Society of London, Series A, Vol. 269, pp. 555-585, 1971.

[71LI1] List, R., and Hand, M.J., Wakes of freely falling water drops, The Physics of Fluids, Vol. 14, No. 8, pp. 1648-1655, 1971.

[71MU1] Mutchler, C.K., and Larson, C.L., Splash amounts from waterdrop impacts on a smooth surface, Water Resources Research, Vol. 7, No. 1, pp. 195-200, February 1971.

[71MU2] Mutchler, C.K., Splash droplet production by waterdrop impact, Water Resources Research, Vol. 7, No. 4, pp. 1024-1030, August 1971.

[71PR1] Pruppacher, H.R., and Pitter, R.L., A semi-empirical determination of the shape of cloud and rain drops, Journal of the Atmospheric Sciences, Vol. 28, pp. 86-94, 1971.

[71SI1] Siscoe, G.L., and Levin, Z., Water-drop surface-wave interactions, Journal of Geophysical Research, Vol. 76, No. 21, pp. 5112-5116, July 1971.

[71TI1] Timmons, D.R., Mutchler, C.K., and Sherstad, E.M., Use of fluorescein to measure the composition of waterdrop splash, Water Resources Research, Vol. 7, No. 4, pp. 1020-1023, August 1971.

[71WA1] Waslo, S., and Gal-Or, B., Boundary layer for mass and heat transfer in clouds of moving drops, bubbles and particles, Chemical Engineering Science, Vol. 26, pp. 829-838, 1971.

[72BR1] Brazier-Smith, P.R., Jennings, S.G., and Latham, J., The interaction of falling water drops: coalescence, Proceedings of the Royal Society of London, Series A, Vol. 326, pp. 393-408, 1972.

[72LE1] LeClair, B.P., Hamielec, A.E., Pruppacher, H.R., and Hall, W.D., A theoretical study of the internal circulation in water drops falling at terminal velocity in air, Journal of the Atmospheric Sciences, Vol. 29, pp. 728-740, 1972.

[72NEI] Nelson, A.R., and Gokhale, N.R., The oscillation frequencies of freely suspended waterdrops, Journal of Geophysical Research, Vol. 77, pp. 2724-2727, 1972. 
[72SA1] Sachs, P., Wind forces in engineering, Pergamon Press, Oxford, 1972.

[72WH1] Whitaker, S., Forced convection heat transfer correlations for flow in pipes, past flat plates, single cylinders, single spheres, and for flow in packed beds and tube bundles, AIChE Journal, Vol. 18, No. 2, pp. 361-371, 1972.

[73AR1] Arrowsmith, A., and Foster, P.J., The motion of a stream of monosized liquid drops in air, The Chemical Engineering Journal, Vol. 5, pp. 243-250, 1973.

[73BR1] Brazier-Smith, P.R., Jennings, S.G., and Latham, J., Raindrop interactions and rainfall rates within clouds, Quarterly Journal of the Royal Meteorological Society, Vol. 99, pp. 260-272, 1973.

[73CO1] Coney, M.W.E., The theory and application of conductance probes for the measurement of liquid film thickness in two-phase flow, Journal of Physics E: Scientific Instruments, Vol. 6, pp. 903-910, 1973.

[73OH1] Ohta, R., and Hojoh, K., A study of a counterflow type cooling tower, Journal of the SHASE Japan, Vol. 47, pp. 33-38 and 91-95, 1973 (Translated by K. Hojoh).

[73SP1] Spengler, J.D., and Gokhale, N.R., Drop impactions, Journal of Applied Meteorology, Vol. 12, pp. 316-321, March 1973.

[73TE1] Tezuka, S, Performance of aqueous-film-type packing of cooling tower, Heat Transfer-Japanese Research, Vol. 2-3, pp. 40-52, 1973.

[74ED1] Edge, R.M., Flatman, A.T., Grant, C.D., and Kalafatoglu, I.E., The formation of freely falling drops immediately after formation, IChemE Symposium Series No. 38, Multi-Phase Flow Systems, Paper C3, 1974.

[74GAl] Gad-el-Hak, M., and Corrsin, S., Measurements of the nearly isotropic turbulence behind a uniform jet grid, Journal of Fluid Mechanics, Vol. 62, pp. 115-143, 1974.

[74GI1] Gibert, H., and Angelino, H., Transferts de matiere entre une sphere soumise a des vibrations et un liquide en mouvement (Mass transfer between a vibrating sphere and liquid flow), International Journal of Heat and Mass Transfer, Vol. 17, pp. 625-632, 1974.

[74HO1] Hollands, K.G.T., An analysis of a counterflow spray cooling tower, International Journal of Heat and Mass Transfer, Vol. 17, pp. 1227-1239, 1974.

[74SC1] Scriven, R.A., Morton, V.M., and Newlands, A.G., The production of airborne droplets below eliminator level in natural draft cooling towers, Atmospheric Environment, Vol. 8, pp. 337-347, 1974.

[74TU1] Tuong, T.P., and Painter, D.J., The interaction between a raindrop and a shallow body of water, 5th Australasian Conference on Hydraulics and Fluid Mechanics, University of Canterbury, Christchurch, New Zealand, pp. 96-102, December 1974.

[74YA1] Yao, S.C., Investigation on falling drop heat-mass transfer and drift elimination in wet cooling systems, Ph.D. dissertation, University of California, Berkeley, 1974.

[75AL1] Allen, R.F., The role of surface tension in splashing, Journal of Colloid and Interface Science, Vol. 51, No. 2, pp. 350-351, May 1975.

[75BE1] Beg. S.A., Forced convection mass transfer studies from spheroids, Wärme- und Stoffübertragung, Vol. 8, pp. 127-135, 1975. 
[75BU1] Bulanina, E.V., Morozov, V.A., and Sukhov, YE. A., High-capacity counterflow cooling towers, Heat Transfer-Soviet Research, Vol. 7, No. 3, pp. 44-54, May-June 1975.

[75GR1] Green, A.W., An approximation for the shapes of large raindrops, Journal of Applied Meteorology, Vol. 14, pp. 1578-1583, December 1975.

[75LE1] Lehrer, I.H., On bubble and drop deformation and break-up, Israel Journal of Technology, Vol. 13, pp. 246-252, 1975.

[75Mcl] McTaggart-Cowan, J.D., and List, R., Collision and breakup of water drops at terminal velocity, Journal of the Atmospheric Sciences, Vol. 32, pp. 1401-1411, 1975.

[75SH1] Shoukry, E., Hafez, M., Hartland, S., Separation of drops from wetted surfaces, Journal of Colloid and Interface Science, Vol. 53, No. 2, pp. 261-270, 1975.

[75TE1] Tezuka, S., and Nakamura, T., Performance of multitubular-type cooling tower packing, Transactions of the Society of Heating, Air-Conditioning and Sanitary Engineers of Japan, Vol. 13, pp. 44-52, 1975.

[75YO1] Young, K.C., The evolution of drop spectra due to condensation, coalescence and break-up, Journal of the Atmospheric Sciences, Vol. 32, pp. 966-973, 1975.

[76BE1] Beard, K.V., Terminal velocity and shape of cloud and precipitation drops aloft, Journal of the Atmospheric Sciences, Vol. 33, pp. 851-864, May 1976.

[76HOl] Hollands, K.G.T., and Goel, K.C., Mean diameters in parallel-flow and counter-flow aerosol systems, ASME Journal of Heat Transfer, pp. 297-302, May 1976.

[76LA1] Labowski, M., The effects of nearest neighbor interactions on the evaporation rate of cloud particles, Chemical Engineering Science, Vol. 31, pp. 803-813, 1976.

[76LI1] List, R., and Gillespie, J.R., Evolution of raindrop spectra with collision-induced breakup, Journal of the Atmospheric Sciences, Vol. 33, pp. 2007-2013, 1976.

[76MA1] Macklin, W.C., and Metaxas, G.J., Splashing of drops on liquid layers, Journal of Applied Physics, Vol. 47, No. 9, pp. 3963-3970, September 1976.

[76RY1] Ryan, R.T., The behaviour of large, low-surface tension drops falling at terminal velocity in air, Journal of Applied Meteorology, Vol. 15, pp. 157-165, February 1976.

[76YAl] Yao, S.C., and Schrock, V.E., Heat and mass transfer from freely falling drops, ASME Journal of Heat Transfer, pp. 120-126, February 1976.

[77BE1] Beard, K.V., On the acceleration of large water drops to terminal velocity, Journal of Applied Meteorology, Vol. 16, pp. 1068-1071, October 1977.

[77CH1] Chen, K.C., and Trezek, G.J., The effect of heat transfer coefficient, local wet bulb temperature and droplet size distribution function on the thermal performance of sprays, ASME Journal of Heat Transfer, Vol. 99, pp. 381-385, 1977.

[77CH2] Cheng, L., Dynamic spreading of drops impacting onto a solid surface, Industrial and Engineering Chemistry - Process Design and Development, Vol. 16, No. 2, pp. 192-197, 1977.

[77FR1] Frediani, H.A., and Smith, N., Mathematical model for spray cooling systems, ASME Journal of Engineering for Power, Vol. 99, No. 2, pp. 279-283, April 1977. 
[77MII] Miura, K., Miura, T. and Ohtani, S., Heat and mass transfer to and from droplets, AIChE Symposium Series, Vol. 73, No. 163, pp. 95- 102, 1977.

[77ST1] Stow, C.D., and Stainer, R.D., The physical products of a splashing water drop, Journal of the Meteorological Society of Japan, Vol. 55, No. 5, pp. 518-531, October 1977.

[77WA1] Wang, P.K., and Pruppacher, H.R., Acceleration to terminal velocity of cloud and raindrops, Journal of Applied Meteorology, Vol. 16, pp. 275-280, March 1977.

[78BR1] Bradley, S.G., and Stow, C.D., Collisions between liquid drops, Philosophical Transactions of the Royal Society of London, Series A, Vol. 287, pp. 635-675, 1978.

[78BR2] Brauer, H., and Sucker, D., Heat and mass transfer during flow about plates, cylinders and spheres, International Chemical Engineering, Vol. 18, No. 3, pp. 375-380, July 1978.

[78CL1] Clift, R., Grace, J.R., and Weber, M.E., Bubbles, drops and particles, Academic Press, New York, 1978 .

[78GH1] Ghadiri, H., and Payne, D., A study of soil splash using cine-photography, pp. 185-192, Assessment of Erosion, Eds. De Boodt and Gabriels, Wiley, Chicester, 1978.

[78GR1] Grace, J.R., Wairegi, T., and Brophy, J., Break-up of drops and bubbles in stagnant media, Canadian Journal of Chemical Engineering, Vol. 56, pp. 3-8, February 1978.

[78LA1] Laws, E.M, and Livesay, J.L., Flow through screens, Annual Review of Fluid Mechanics, Vol. 10, pp. 247-266, 1978.

[78PR1] Pruppacher, H.R., and Klett, J.D., Microphysics of clouds and precipitation, D. Reidel Publishing Company, Dordrecht, Holland, 1978.

[78WE1] Weszka, J.S., A survey of threshold selection techniques, Computer Graphics and Image Processing, Vol. 7, pp. 259-265, 1978.

[79BO1] Bogy, D.B., Drop formation in a circular liquid jet, Annual Review of Fluid Mechanics, Vol. 11, pp. 207-228, 1979.

[79BR1] Bradley, S.G., and Stow, C.D., On the production of satellite droplets during collisions between water drops falling in still air, Journal of the Atmospheric Sciences, Vol. 36, pp. 494-500, March 1979.

[79MA1] Marchildon, E.K., and Gauvin, W.K., Effects of acceleration, deceleration and particle shape on single-particle drag coefficients in still air, AIChE Journal, Vol. 25, No. 6, pp. 938-948, November 1979.

[79RO1] Rogers, R.R., A short course in cloud physics, Second Edition, Pergamon Press, Oxford, 1979.

[79ST1] Stedman, O.J., Patterns of unobstructed splash dispersal, Annual Applied Biology, Vol. 91, pp. 271$285,1979$.

[80MI1] Miura, T., Ohtani, S., and Maeda, S., Heat and mass transfer to and from sprays, Drying '80, Vol. 1, Developments in Drying, Ed. A.S. Majumdar, Hemisphere Publishing Corporation, pp. 351-356, 1980.

[80YU1] Yung, D., Lorenz, J.J., and Ganic, E.N., Vapour/liquid interaction and entrainment in falling film evaporators. ASME Journal of Heat Transfer, Vol. 102, pp. 20-25, February 1980. 
[81AL1] Alkidas, A.C., The influence of size-distribution parameters on the evaporation of polydisperse dilute sprays, International Journal of Heat and Mass Transfer, Vol. 24, No. 12, pp. 1913-1923, 1981.

[81BO1] Borisov, A.A., Gel'fand, B.E., Natanson, M.S., and Kossov, O.M., Droplet breakup regimes and criteria for their existence, Journal of Engineering Physics, Translated from Inzhenerno-Fizicheskii Zhurnal, Vol. 40, No. 1, pp. 44-49, July 1981.

[81COI] Collier, J.G., Convective Boiling and Condensation, 2nd Edition, McGraw-Hill International Book Company, London, 1982.

[81HOI] Holman, J.P., Heat Transfer, 5th Edition, McGraw-Hill International Book Company, Auckland, 1981.

[81HO2] Hozawa, M., Tsukada, T., Imaishi, N., and Fujinawa, K., Effect of wettability on static drop formation from a hole in horizontal flat plate, Journal of Chemical Engineering of Japan, Vol. 14, No. 5, pp. 358-364, 1981.

[81PA1] Palaszewski, S.J., Jiji, L.M., and Weinbaum, S., A three-dimensional air-vapor-droplet local interaction model for spray units, ASME Journal of Heat Transfer, Vol. 103, No. 3, pp. 514-521, 1981.

[81ST1] Stow, C.D., and Hadfield, M.G., An experimental investigation of fluid flow resulting from the impact of a water drop with an unyielding dry surface, Proceeding of the Royal Society of London, Series A, Vol. 373, pp. 419-441, 1981.

[81WA1] Walzer, P., and Klaumünzer, U., Flow regimes on horizontal porous plates, German Chemical Engineering, Vol. 4, pp. 154-160, 1981.

[82CA1] Cale, S.A., Development of evaporative cooling packing, Commission of European Communities, Report EUR 7709 EN, 1982.

[82FI1] Fitt, B.D.L., Lysandrou, M., and Turner, R.H., Measurement of spore carrying splash droplets using photographic film and an image-analysing computer, Plant Pathology, Vol. 31, pp. 19-24, 1982.

[82GOl] Godfrey, J.C., and Hanson, C., Liquid-liquid systems, Section 4.2 in Handbook of Multiphase Systems edited by Hetsroni, G., McGraw-Hill Book Company, New York, 1982.

[82GR1] Grace, J.R., and Weber, M.E., Hydrodynamics of drops and bubbles, Section 1.3.9 in Handbook of Multiphase Systems edited by Hetsroni, G., McGraw-Hill Book Company, New York, 1982.

[82HE1] Hewitt, G.F., Measurement of liquid-film thickness, Section 10.2.2.2, pp. 10:67-10:112, Handbook of Multiphase Systems, Ed. G. Hetsroni, McGraw-Hill Book Company, New York, 1982.

[82LO1] Low, T.B., and List, R., Collision, coalescence and breakup of raindrops. Part I: Experimentally established coalescence efficiencies and fragment size distributions in breakup, Journal of the Atmospheric Sciences, Vol. 39, pp. 1591-1606, 1982.

[82LO2] Low, T.B., and List, R., Collision, coalescence and breakup of raindrops. Part II: Parameterization of fragment size distribution, Journal of the Atmospheric Sciences, Vol. 39, pp. 1607-1618, 1982.

[82SR1] Srikrishna, M., Sivaji. K., and Narasimhamurty, G.S.R., Mechanics of liquid drops in air, The Chemical Engineering Journal, Vol. 24, pp. 27-34, 1982. 
[82TA1] Tal, R., and Sirignano, W.A., Cylindrical cell model for the hydrodynamics of particle assemblages at intermediate Reynolds numbers, AIChE Journal, Vol. 28, No. 2, pp. 233-237, 1982.

[82TE1] Temkin, S., and Metha, H.K., Droplet drag in an accelerating and decelerating flow, Journal of Fluid Mechanics, Vol. 116, pp. 297-313, 1982.

[82TR1] Trinh, E., and Wang, T.G., Large amplitude free and driven drop shape oscillation: experimental observations, Journal of Fluid Mechanics, Vol. 122, pp. 315-338, 1982.

[82TS1] Tsuji, Y., Morikawa, Y., and Terashima, K., Fluid-dynamic interaction between two spheres, International Journal of Multiphase Flow, Vol. 8, No. 1, pp. 71-82, 1982.

[83BE1] Beard, K.V., Johnson, D.B., and Jameson, A.R., Collisional forcing of raindrop oscillations, Journal of the Atmospheric Sciences, Vol. 40, pp. 455-462, February 1983.

[83BO1] Bourillot, C., TEFERI, Numerical model for calculating the performance of an evaporative cooling tower, EPRI Special Report CS-3212-SR, August 1983 (Translated by J.A. Bartz).

[83CH1] Churchill, S.W., Combined free and forced convection around immersed bodies, Heat Exchanger Design Handbook, Hemisphere Publishing Corporation, Section 2.5.9, 1983.

[83EI1] Eigel, J.D., and Moore, I.D., A simplified technique for measuring raindrop size and distribution, Transactions of the ASAE, pp. 1079-1084, 1983.

[83GI1] Gillaspy, P.H., and Hoffer, T.E., Experimental measurements of the effect of viscosity on drag for liquid drops, AIChE Journal, Vol. 29, No. 2, pp. 229-236, 1983.

[83GNI] Gnielinski, V., Forced convection around immersed bodies, Section 2.5.2 in Heat Exchanger Design Handbook, Hemisphere Publishing Corporation, 1983.

[83LE1] Lee, S.L., Sheen, H.J, Cho, S.K., Issapour, I., and Hua, S.G., Measurement of grid spacer's enhanced droplet cooling under reflood conditions in a PWR by LDA, presented at the 11th Water Reactor Safety Meeting, Garthersburg, MD, 24-28 October 1983.

[83RE1] Renksizbulut, M., and Yuen, M.C., Experimental study of droplet evaporation in a high-temperature air stream, ASME Journal of Heat Transfer, Vol. 105, pp. 384-388, May 1983.

[83SU1] Sutherland, J.W., Analysis of mechanical-draught counterflow air/water cooling towers, ASME Journal of Heat Transfer, Vol. 105, pp. 576-583, August 1983.

[83WA1] Warrington, R.O., and Musselman, R.L., Analysis of liquid/gas direct contact heat exchanger concept, Journal of Energy, Vol. 7, No. 6, pp. 732-734, 1983.

[84CH1] Ching, B., Golay. M.W., and Johnson. T.J., Drop impacts upon liquid surfaces, Science, Vol. 226, pp. 535-537, November 1984.

[84CH2] Chuchottaworn, P., Fujinami, A., and Asano, K., Experimental study of evaporation of a volatile pendant drop under high mass flux condition, Journal of Chemical Engineering of Japan, Vol. 17, No. 1, pp. 7-13, 1984.

[84EPI] Epema. G.F., and Riezebos, H.T., Drop shape and erosivity, Part 1: Experimental set up, theory, and measurements of drop shape, Earth Surface Processes and Landforms, Vol. 9, pp. 567-572, 1984.

[84FII] Fitt. B.D.L.. and Lysandrou. M., Studies on mechanisms of splash dispersal of spores, using Pseudocercosporella herpotrichoides spores. Phytopathology Zeitschrift, Vol. 111, pp. 323-331, 1984. 
[84HI1] Hijikata, K., Mori, Y., and Kawaguchi, S., Direct condensation of vapour to falling cooled droplets, International Journal of Heat and Mass Transfer, Vol. 27, No. 9, pp. 1631-1640, 1984.

[84NI1] Nicol, A.A., and Aidoun, Z.L., Simulated condensate film thickness and drainage studies, Proceedings of the 1st UK National Heat Transfer Conference, Vol. 1, pp. 649-658, 1984.

[84PO1] Podvysotsky, A.M., and Shraiber, A.A., Coalescence and breakup of drops in two phase flows, International Journal of Multiphase Flow, Vol. 10, pp. 195-209, 1984.

[85BR1] Brennan, R.M., Fitt, B.D.L., Taylor, G.S., and Colhoun, J., Dispersal of Septoria nodorum Pycnidiospores by simulated raindrops in still air, Phytopathology Zeitschrift, Vol. 112, pp. 281-290, 1985.

[85BR2] Brennan, R.M., Fitt, B.D.L., Taylor, G.S., and Colhoun, J., Dispersal of Septoria nodorum Pycnidiospores by simulated rain and wind, Phytopathology Ze ‘schrift, Vol. 112, pp. 291-297, 1985.

[85BR3] Bright, A., Minimum drop volume in liquid jet breakup, IChemE Chemical Engineering Research and Development, Vol. 63, pp. 59-66, 1985.

[85CH1] Chuchottaworn, P., and Asano, K., Calculation of drag coefficients of an evaporating or a condensing droplet, Journal of Chemical Engineering of Japan, Vol. 18, No. 1, pp. 91-94, 1985.

[85HA1] Haralick, R.M., and Shapiro, L.G., Image segmentation techniques, Computer Vision, Graphics, and Image Processing, Vol. 29, pp. 100-132, 1985.

[85SK1] Skelland, A.H.P., and Vasti, N.C., Effects of interaction between circulating or oscillating droplets on drop formation, free fall, and mass transfer, The Canadian Journal of Chemical Engineering, Vol. 63, pp. 390-398, June 1985.

[86BE1] Benton, D.J., and Rehberg, R.L., Mass transfer and pressure drop in sprays falling in a freestream at various angles, Proceedings of the 5th IAHR Cooling Tower Workshop, Monterey, California, September 1986.

[86BE2] Benocci, C., Buchlin, J-M., and Weinacht, P., Prediction of the air-droplet interaction in the inlet section of a natural draught cooling tower, Proceedings of the 5th IAHR Cooling Tower Workshop, Monterey, California, September 1986.

[86CH1] Ching, B., Golay, M.W., and Johnson, T.J., Rebounding and absorption of droplets impacting upon liquid surfaces such as upon cooling tower drift eliminators, submitted to the IAHR Symposium on Cooling Towers, Monterey, California, September 1986.

[86CH2] Chuchottaworn, P., and Asano, K., Numerical analysis of drag coefficients and the heat and mass transfer from spheroidal drops, Journal of Chemical Engineering of Japan, Vol. 19, No. 3, pp. 208$214,1986$.

[86FU1] Fujita, T., and Tezuka, S., Calculations on thermal performance of mechanical draft cooling towers, ASHRAE Transactions No. 2952, pp. 274-287, 1986.

[86TU1] Turton, R., and Levenspiel, O., A short note on the drag correlation for spheres, Powder Technology, Vol. 47, pp. 83-86, 1986.

[86WE1] Wedding, J.B., Kim. Y.J., and Dennison, R.S., Mass transfer from water droplets under simulated free-fall conditions, Atmospheric Environment, Vol. 20, No. 5, pp. 1039-1045, 1986. 
[87AS1] Ashgriz, N., and Givi, P., Binary collision dynamics of fuel droplets, International Journal of Heat and Fluid Flow, Vol. 8, No. 3, pp. 205-210, 1987.

[87BE1] Beard, K.V., and Chuang, C., A new model for the equilibrium shape of raindrops, Journal of the Atmospheric Sciences, Vol. 44, No. 11, pp. 1509-1524, 1987.

[87BE2] Beukman, J., Elektroniese evaluasie van die druppel spektrum op bespuite blare (Electronic evaluation of the drop (size) spectrum on sprayed leaves), B.Eng. project, University of Stellenbosch, Stellenbosch, South Africa, 1987.

[87FO1] Fourie, J.G., and Kröger, D.G., Influence of turbulence on performance of circular helically finned tubes, Proceedings of the 17th Congress for Refrigeration, Vienna, Vol. B, pp. 856-863, 1987.

[87HOl] Horn, B.K.P., Robot vision, 3rd Printing, The MIT Press, Cambridge, Massachusetts, 1987.

[87MO1] Moussiopoulos, N., and Ernst, G., Thermal performance of spray cooling ponds at zero wind velocity, ASME Journal of Heat Transfer, Vol. 109, pp. 212-217, 1987.

[87RO1] Roach, P.E., The generation of nearly isotropic turbulence by means of grids, The International Journal of Heat and Fluid Flow, Vol. 8, No. 2, pp. 82-92, 1987.

[87SC1] Schlichting, H., Boundary-Layer Theory, 7th edition, McGraw-Hill Book Company, New York, 1987.

[88AG1] Aggarwal, S.K., Ignition and flame propagation in dilute polydisperse sprays; Importance of $d_{32}$ and $\mathrm{d}_{20}$, Journal of Propulsion, Vol. 4, No. 1, pp. 14-21, January-February 1988.

[88AL1] Allen, R.F., The mechanics of splashing, Journal of Colloid and Interface Science, Vol. 124, pp. 309-316, 1988.

[88CH1] Chandrasekar, V., Cooper, W.A., and Bringi, V.I., Axis ratios and oscillations of raindrops, Journal of the Atmospheric Sciences, Vol. 45, No. 8, pp. 1323-1333, 1988.

[88DR1] Dreyer, A.A., Analysis of evaporative coolers and condensers, M.Eng. thesis, University of Stellenbosch, Stellenbosch, South Africa, 1988.

[88FAl] Fabre, L., and Legrand, G., MISTRAL, New test bench for cooling tower components, Proceedings of the International Cooling Tower Conference, Pisa, Italy, pp. 1.5.1-1.5.19, 1988.

[88FE1] Feingold, G., Tzivion, S., Levin, Z., Evolution of raindrop spectra. Part I : Solution to the stochastic collection/breakup equation using the method of moments, Journal of the Atmospheric Sciences, Vol. 45, No. 22, pp. 3387-3399, 1988.

[88GH1] Ghadiri, H., and Payne, D., The formation and characteristics of splash following raindrop impact on soil, Journal of Soil Science, Vol. 39, pp. 563-575, 1988.

[88GR1] Greene, G.A., Personal communication, 1988.

[88GR2] Groth, J., and Johansson, A.V., Turbulence reduction by screens, Journal of Fluid Mechanics, Vol. 197, pp. 139-155, 1988.

[88LE1] Lee, M.M., and Hanratty, T.J., The inhibition of droplet deposition by the presence of a liquid wall film, International Journal of Multiphase Flow, Vol. 14, No. 2. pp. 129-140, 1988.

[88LI1] Liu, D.Y.. Anders, K., and Frohn, A., Drag coefficients of single droplets moving in an infinite droplet chain on the axis of a tube, International Journal of Multiphase Flow. Vol. 14, No. 2, pp. 217-232. 1988. 
[88PI1] Pilch, M., and Erdman, C.A., Use of breakup time data and velocity history data to predict the maximum size of stable fragments for acceleration-induced breakup of a liquid drop, International Journal of Multiphase Flow, Vol. 13, No. 6, pp. 741-757, 1988.

[88SC1] Schnell, H., Rückkühlwerke und Verdunstungsverflüssiger, Handbuch der Kältetechnik, Ed. R. Planck, Sechster Band, Teil B, Springer Verlag, Berlin, pp. 492-535, 1988.

[88WE1] Webb, R.L., A critical evaluation of cooling tower design methodology, Heat Transfer Equipment Design, Eds. R.K. Shah, E.C. Subbarao and R.F. Mashelkar, Hemisphere Publishing Corporation, New York, pp. 547-558, 1988.

[88YAl] Yao, S.C., Hochreiter, L.E., and Cai, K.Y., Dynamics of droplets impacting on thin strips, ASME Journal of Heat Transfer, Vol. 110, pp. 214-220, February 1988.

[89AS1] Ashgriz, N., and Givi, P., Coalescence efficiencies of fuel droplets in binary collisions, International Communications in Heat and Mass Transfer, Vol. 16, pp. 11-20, 1989.

[89BE1] Beard, K.V., Ochs, H.T., and Kubesh, R.J., Natural oscillations of small raindrops, Nature, Vol. 342, pp. 408-410, November 1989.

[89BR1] Brenn, G., and Frohn, A., Collision and coalescence of droplets of various liquids, Journal of Aerosol Science, Vol. 20, No. 8, pp. 1027-1030, 1989.

[89BR2] Brenn, G., and Frohn, A., Collision and merging of two equal droplets of propanol, Experiments in Fluids, Vol. 7, pp. 441-446, 1989.

[89GO1] Gore, R.A., and Crowe, C.T., Effect of particle size on modulating turbulence intensity, International Journal of Multiphase Flow, Vol. 15, No. 2, pp. 279-285, 1989.

[89HE1] Hetsroni, G., Particles-turbulence interaction, International Journal of Multiphase Flow, Vol. 15, No. 5, pp. 735-746, 1989.

[89JO1] Johnson, B.M., Cooling tower performance prediction and improvement, Vols. 1 and 2, EPRI Report GS-6370, 1989.

[89KHI] Khaleeq-ur-Rahman, M., and Saunders, C.P.R., Effects of surface waves on corona from splashing water drops, Annales Geophysicae, Vol. 7, No. 1, pp. 47-52, 1989.

[89KI1] Kitscha, J., and Kocamustafaogullari, G., Breakup criteria for fluid particles, International Journal of Multiphase Flow, Vol. 15, No. 4, pp. 573-588, 1989.

[89KO1] Koskie, J.E., Mudawar, I., and Tiederman, W.G., Parallel-wire probes for measurement of thick liquid films, International Journal for Multiphase Flow, Vol. 15, No. 4, pp. 521-530, 1989.

[89LE1] Lefebvre, A.H., Atomization and sprays, Hemisphere Publishing Corporation, New York, 1989.

[89LU1] Lu, S.M, and Huang, Y.Y., Drop formation in air prior to jetting, AIChE Journal, Vol. 35, No. 9, pp. 1572-1574, September 1989.

[89MO1] Moss, A.J., Impact droplets and the protection of soils by plant covers, Australian Journal of Soil Research, Vol. 27, pp. 1-16, 1989.

[89PO1] Pozrikidis, C., Invicid drops with internal circulation, Journal of Fluid Mechanics, Vol. 209, pp. 7792, 1989.

[89RE1] Renganathan, K., Turton, R., and Clark, N.N., Accelerating motion of geometric and spherical particles in a fluid. Powder Technology, Vol. 58, pp. 279-284, 1989. 
[89TA1] Tanaguchi, I., and Asano, K., Calculation of drag coefficients and mass transfer of two adjacent spheres of various diameter ratios, Journal of Chemical Engineering of Japan, Vol. 22, No. 6, pp. 701-703, 1989.

[89TZ1] Tzivion, S., Feingold, G., and Levin, Z., Evolution of raindrop spectra. Part II : Collisional collection/breakup and evaporation in a rainshaft, Journal of the Atmospheric Sciences, Vol. 46, No. 21, pp. 3312-3327, 1989.

[90AS1] Ashgriz, N., and Poo, J.Y., Coalescence and separation in binary collisions of liquid drops, Journal of Fluid Mechanics, Vol. 221, pp. 183-204, 1990.

[90HOl] Hoffmann, J.E., and Kröger, D.G., Analysis of heat mass and momentum transfer in the rain zone of a natural draft counterflow cooling tower, Paper 19-EN-19, Proceedings of the 9th International Heat Transfer Conference, Jerusalem, pp. 227-231, August 1990.

[90LI1] List, R., and McFarquhar, G.M., The role of breakup and coalescence in the three-peak equilibrium distribution of raindrops, Journal of the Atmospheric Sciences, Vol. 47, No. 19, pp. 2274-2292, 1990 .

[90MI1] Miller, D.S., Internal Flow Systems, 2nd Edition, BHRA (Information Services), Cranfield, 1990.

[90MO1] Mori, Y.H., Harkins-Brown correction factor for drop formation, AIChE Journal, Vol. 36, No. 8, pp. 1272-1274, August 1990.

[90PE1] Peregrine, D.H., Shoker, G., and Symon, A., The bifurcation of liquid bridges, Journal of Fluid Mechanics, Vol. 212, pp. 25-39, 1990.

[90SA1] Saviji, K., Srikrishna, M., and Narasimhamurty, G.S.R., Shape of liquid drops falling in air, International Journal of Engineering Fluid Mechanics, Vol. 3, No. 1, pp. 91-94, 1990.

[90WA1] Walzel, P., Zerstäuben von Flüssigkeiten, Chemie Ingenieur Technik, Vol. 62, No. 12, pp. 983-994, 1990.

[90WI1] Wierzba, A., Deformation and breakup of liquid drops in a gas stream at nearly critical Weber numbers, Experiments in Fluids, Vol. 9, pp. 59-64, 1990.

[91BR1] Brown, P.S., Parameterization of the evolving drop-size distribution based on analytical solution of the linearized coalescence-breakup equation, Journal of the Atmospheric Sciences, Vol. 48, No. 1, pp. 200-210, 1991.

[91CH1] Chandra, S., and Avedisian, C.T., On the collision of a droplet with a solid surface, Proceedings of the Royal Society of London, Series A, Vol. 432, pp. 13-41, 1991.

[91CH2] Chiang, H., and Kleinstrauer, C., Convection heat transfer of colinear interaction droplets with surface mass transfer, International Journal of Heat and Fluid Flow, Vol. 12, No. 3, pp. 233-239, September 1991.

[91CH3] Chuech, S.G., Przekwas, A.J., and Singhal, A.K., Numerical modeling for primary atomization of liquid jets, Journal of Propulsion, Vol. 7, No. 6, pp. 879-886, November-December 1991.

[91FE1] Feltzin, A.E., and Benton, D.J., A more nearly exact representation of cooling tower theory, Cooling Tower Institute Journal, Vol. 12, No. 2, pp. 8-26, 1991. 
[91GR1] Greene, G.A., Heat, mass and momentum transfer in a multifluid bubbling pool, in Advances in Heat Transfer, Eds. Hartnett, J.P., Irvine, T.F., and Cho, Y.I., Academic Press, Vol. 21, pp. 277$346,1991$.

[91MA1] Marseille, T.J., Schliesing, J.S., Bell, D.M., and Johnson, B.M., Extending cooling tower thermal performance prediction using a liquid-side film resistance model, Heat Transfer Engineering, Vol. 12, No. 3, pp. 19-30, 1991.

[91NG1] Nguyen, Q.-V., Rangel, R.H., and Dunn-Rankin, D., Measurement and prediction of trajectories and collision of droplets, International Journal of Multiphase Flow, Vol. 7, No. 2, pp. 159-177, 1991.

[91PO1] Poppe, M., and Rögener, H., Berechnung von Rückkühlwerken, VDI-Wärmeatlas, pp. Mil-Mil5, 1991.

[91PO2] Poo, J.Y., and Ashgriz, N., Variation of drag coefficients in an interacting drop stream, Experiments in Fluids, Vol. 11, pp. 1-8, 1991.

[91TA1] Taniguchi, I., Kuriyama, T. and Asano, K., Simultaneous measurement of drag coefficients and mass transfer of a chain of uniform volatile drops, Journal of Chemical Engineering of Japan, Vol. 24, No. 2, pp. 232-237, 1991.

[91XA1] Xang, X., Madden, L.V., Reichard, D.L., Fox, R.D., and Ellis, M.A., Motion analysis of drop impaction on a strawberry surface, Agricultural and Forest Meteorology, Vol. 56, pp. 67-92, 1991.

[92BR1] Brink, A.D., Thresholding of digital images using a correlation measure, Proceedings of the First South African Workshop on Pattern Recognition, pp. 125-127, 1992.

[92CH1] Choi, K.J., and Lee, H.J., Experimental studies on the thermodynamics and evaporation of tandem liquid drops in a hot gas flow, International Journal of Heat and Mass Transfer, Vol. 35, No. 11, pp. 2921-2929, 1992.

[92DU1] Dubrovsky, V.V., Podvysotsky, A.M., and Shraiber, A.A., Particle interaction in three phase polydisperse flows, International Journal of Multiphase Flow, Vol. 18, No. 3, pp. 337-352, 1992.

[92JI1] Jiang, Y.J., Umemura, A., and Law, C.K., An experimental investigation on the collision behaviour of hydrocarbon droplets, Journal of Fluid Mechanics, Vol. 234, pp. 171-190, 1992.

[92KAl] Kang, H.C., and Kim, M.H., The development of a flush-wire probe and calibration method for measuring liquid film thickness, International Journal of Multiphase Flow, Vol. 18, No. 3, pp. 423437, 1992.

[92MO1] Mori, Y.H., and Mochizuki, T., Explicit expressions for volume of drops released from submerged nozzles: their derivation from semiempirical implicit correlations, International Journal of Multiphase Flow, Vol. 18, No. 1, pp. 141-144, 1992.

[92PR1] Prinsloo, M., Waterdruppel detektor (Water drop detector), B.Eng. project, University of Stellenbosch, Stellenbosch, South Africa, November 1992.

[92YU1] Yuan, Z., and Michaelides, E.E., Turbulence modulation in particulate flows - A theoretical approach, International Journal of Multiphase Flow, Vol. 18, No. 5, pp. 779-785, 1992.

[93CO1] Conradie, C.F.G., Die verkoelingsvermoë van nat koeltorings en droë/nat stelsels by kragstasies (The cooling capacity of wet cooling towers and dry/wet systems at power stations), M.Eng. thesis, University of Stellenbosch, Stellenbosch, South Africa, 1993. 


\section{1}

[93ER1] Erens, P.J., and Dreyer, A.A., Free surface menisci on narrow horizontal surfaces, submitted for publication in the International Journal of Heat and Mass Transfer, 1993.

[93ME1] Mercker, J.H., Heat and mass transfer from accelerating water drops, M.Eng. thesis, University of Stellenbosch, Stellenbosch, South Africa, 1993.

[93SC1] Schreck, S., and Kleis, S., Modification of grid-generated turbulence by solid particles, Journal of Fluid Mechanics, Vol. 249, pp. 665-688, 1993. 


\section{APPENDIX A Re-correlation of the data by Yao [74YA1] for heat $/ \mathrm{mass}$ transfer from free-falling, accelerating water drops}

Yao [74YA1] (also see Yao and Schrock [76YA1]) conducted a series of experiments to determine the heat/mass transfer coefficients governing the cooling of large $(3<\mathrm{d}<6 \mathrm{~mm})$ water drops accelerating from rest in still air. The drop temperatures were measured at various fall distances from the point of release using a purpose-built calorimeter.

Twelve sets of data for drop temperature versus fall distance were measured by Yao [74YA1]. The data covered the following ranges: $3<\mathrm{d}<6 \mathrm{~mm}, 29<\mathrm{RH}<100 \%$ and $0.18<\mathrm{z}<2.9 \mathrm{~m}$. To ensure steady air conditions, a very low air volume flow rate $\left(\mathrm{v}_{\mathrm{a}}=0.05 \mathrm{~m} / \mathrm{s}\right)$ was induced from the top to the bottom of the test section. The air temperature was held between 21.3 and $23.6^{\circ}$ $C$, while the incoming water temperature was kept at approximately $40.7^{\circ} \mathrm{C}$.

Yao [74YA1] and Yao and Schrock [76YA1] correlated their data for freely falling water drops using the following modified form of the well-known Ranz and Marshall correlation for heat/mass transfer from drops, i.e.

$$
\mathrm{Nu}_{\mathrm{f}}=2+\mathrm{g}_{\mathrm{YS}}\left(0.6 \mathrm{Re}_{\mathrm{f}}^{1 / 2} \operatorname{Pr}_{\mathrm{f}}^{1 / 3}\right) \text { for } \operatorname{Re}_{\mathrm{f}}<2500
$$

where the term $g_{Y S}$ was introduced to account for acceleration, drop deformation, internal circulation and drop oscillation effects. They correlated their data as

$$
g_{Y S}=25\left(\frac{z}{d}\right)^{-0.7} \text { for } 10<\left(\frac{z}{d}\right)<600
$$

The asymptotic values of the Nusselt numbers predicted by this correlation seems dubious. When using this correlation, it was not possible to reproduce the measured drop temperatures as determined by Yao et al. [74YA1], [76YA1]. Chen and Trezek [77CH1] also expressed their doubts about this correlation. There is a severe lack of other experimental data on the cooling of large freely falling water drops, and therefore it was decided to re-correlate the data of Yao [74YAl].

The following calculation procedure was used to determine the transfer coefficients from the data by Yao:

i) For each set of data giving drop temperature versus fall distance, a smooth analytical curve was obtained using a least squares data fitting procedure. Assuming that the drop Nusselt number can be expressed by a relation such as 
$\mathrm{Nu}_{\mathrm{f}}=2+\mathrm{c}_{1} \operatorname{Re}_{\mathrm{f}}^{\mathrm{c}_{2}} \operatorname{Pr}_{\mathrm{f}}^{1 / 3}$

and that the drop velocity of at any fall height can be described by a functional relation of the form

$\mathrm{v}=\mathrm{c}_{3} \mathrm{z}^{\mathrm{c}_{4}}$

the following relation can be used to correlate the drop temperature data

$$
\Delta \mathrm{T}_{\mathrm{w}}=\left(\mathrm{T}_{\mathrm{w}, \mathrm{z}=0}-\mathrm{T}_{\mathrm{w}, \mathrm{z}}\right)=\mathrm{c}_{5} \mathrm{z}^{\left(1-\mathrm{c}_{4}\right)}+\mathrm{c}_{6} \mathrm{z}^{\left(1-\left(\mathrm{c}_{2} \mathrm{c}_{4}\right)\right)}
$$

The follow:ng constant values were used for the recalculation of the data: $c_{2}=0.5$ and $c_{4}=0.45$. Note that a value of $c_{2}=0.5$ agrees with the exponent that Ranz and Marshall [52RA1], [52RA2] obtained in their correlation for the heat/mass transfer from drops.

ii) For each set of data the drop velocity was calculated at different fall heights using the drag model described in Chapters 2 and 3. A smooth curve, of the form given by Equation (A.4), was then determined to fit the calculated points.

iii) The mass transfer coefficient was then determined at different fall heights by considering a small element $\partial Z$ at every position. The cooling rate of the drop at every position can then be determined from

$$
\dot{\mathrm{Q}}=\frac{\mathrm{m}_{\mathrm{d}} \mathrm{c}_{\mathrm{pw}}\left(\Delta \mathrm{T}_{\mathrm{z}+0.5 \partial \mathrm{z}}-\Delta \mathrm{T}_{\mathrm{Z}-0.5 \partial \mathrm{z}}\right)}{\left(\partial \mathrm{z} / \mathrm{v}_{\mathrm{d}}\right)}
$$

and by employing the Merkel theory for simultaneous heat and mass transfer, the mass transfer coefficient can be expressed as

$$
\mathrm{K}=\frac{\dot{\mathrm{Q}}}{\mathrm{A}_{\mathrm{d}}\left(\mathrm{i}_{\text {asw }}-\mathrm{i}_{\mathrm{a}}\right)}
$$

iv) The use of the Merkel theory in the previous step implies that $\mathrm{Le}_{\mathrm{f}}=1$. With this in mind, the Nusselt number can be calculated from

$$
\mathrm{Nu}_{\mathrm{f}}=\frac{\mathrm{h}_{\mathrm{c}} \mathrm{d}}{\mathrm{k}_{\mathrm{af}}}=\left(\mathrm{Le}_{\mathrm{f}} \mathrm{c}_{\mathrm{pm}} \mathrm{K}\right) \frac{\mathrm{d}}{\mathrm{k}_{\mathrm{af}}}=\frac{\mathrm{c}_{\mathrm{pm}} \mathrm{Kd}}{\mathrm{k}_{\mathrm{af}}}
$$

The Sherwood number can be calculated in a similar fashion.

Since Yao [74YA1] did not report the value of the atmospheric pressure during his experimental work, it was assumed that the atmospheric pressure was $101325 \mathrm{~Pa}$. It was also assumed that the air was still and that the air conditions were constant along the drop fall paths. 
The recalculated results are shown in Figure A.1 together with the correlation of Yao [74YA1]. The deviation of Yao's correlation from the data is quite clear, with the correlation of Yao severely over-predicting the Nusselt numbers at small fall distances and underpredicting the Nusselt numbers at large fall distances. The discrepancy between the recalculated data and the correlation of Yao can be attributed to the fact that Yao used interpolations of the drop acceleration data by Laws [41LA1] to calculate the drop velocity at every position without accounting for the effects of differences in air density, viscosity, humidity, and drop surface tension. The calculated Nusselt numbers were found to be very sensitive to the form of the curve used to describe the drop temperatures versus falling distances (step (i) in the stepwise description above). The recalculated values of the correction factor, $g_{Y S}$, are all close to unity, indicating good agreement with the correlation of Ranz and Marshall [52RA1], [52RA2].

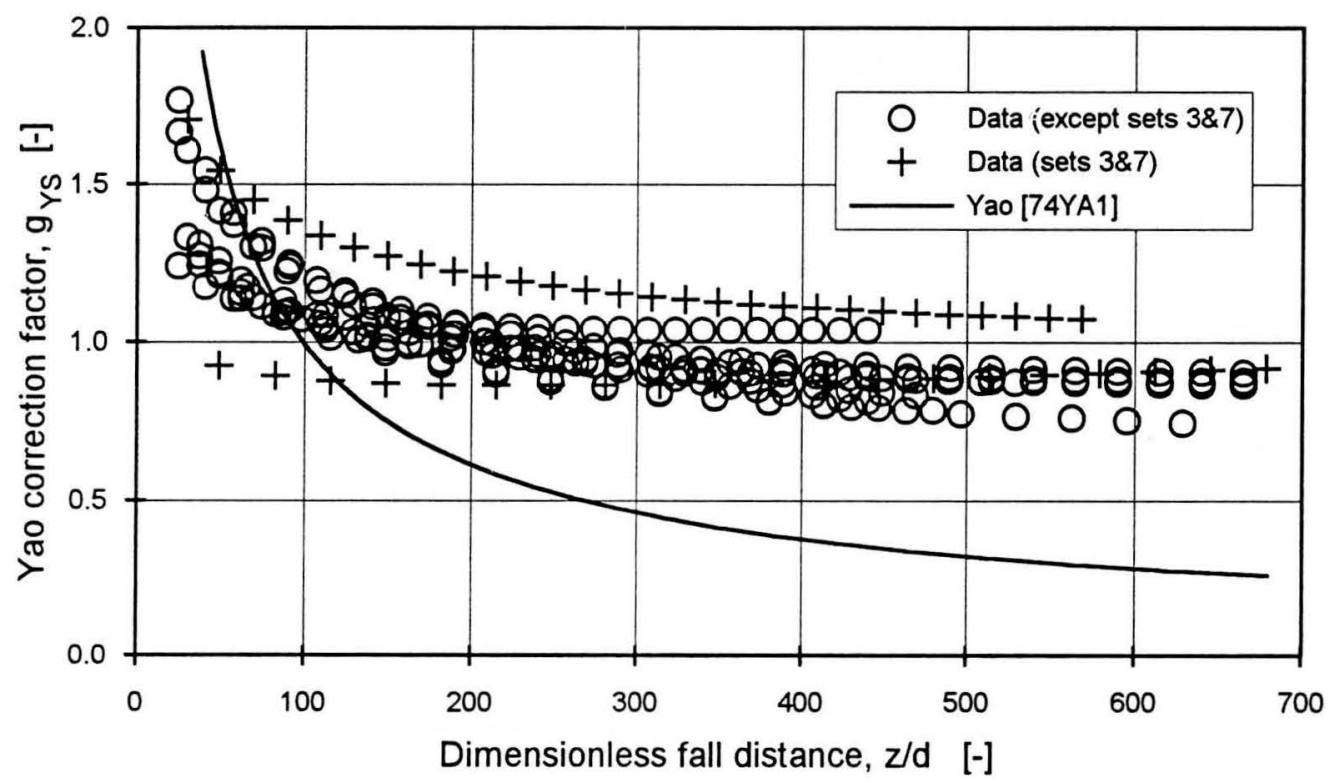

Figure A.1 Comparison between the recalculated Yao correction factor data and the correlation by Yao [74YA1].

The recalculated correction factor data was correlated as

$$
g_{Y S}=2.32\left(\frac{z}{d}\right)^{-0.16}
$$

When modelling the performance of cooling tower splash pack, it is inconvenient to use a Nusselt number correlation which accounts for the acceleration effects by a fall distance term. Since the up-flowing air in a cooling tower influences the acceleration of the drops, it is 
therefore more convenient to express the correction factor, $\mathrm{g}_{\mathrm{YS}}$, as a function of the dimensionless modulus of acceleration,

$$
M_{\ddot{z}}=\frac{(\partial v / \partial t) d_{e}}{v_{\text {rel }}^{2}}
$$

The following correlation, employing the dimensionless modulus of acceleration, was obtained from the recalculated data of Yao [74YA1] and Yao and Schrock [76YA1]

$$
\mathrm{g}_{\mathrm{YS}}=0.22+3.15 \mathrm{M}_{\ddot{\mathrm{Z}}}^{0.2}\left(\frac{\mathrm{d}}{\mathrm{d}_{\mathrm{m}}}\right)^{0.2} \text { for } \mathrm{M}_{\ddot{\mathrm{z}}}>5.0 \times 10^{-4}
$$

where the maximum stable drop size, $d_{m}$, is given by

$$
d_{m}=\sqrt{\frac{16 \sigma}{g\left(\rho_{w}-\rho_{a}\right)}}
$$

The results of two of the twelve sets of data (sets 3 and 7) were excluded in the determination of the correlations because of their general disagreement with the trends shown by the other data. Figure 3.4 shows the typical temperature variation with fall distance for one of the data sets of Yao together with predictions obtained using the Ranz and Marshall correlation, the correlation by $\mathrm{Yao}$ and that of the correlation above. It is obvious that the new correlation (Equation (A.11)) fits the data better than the correlation of Yao. The correlation of Ranz and Marshall under-predicts the cooling rate slightly.

It can be concluded that the data by Yao confirms the applicability of the Ranz and Marshall correlation to determine the Nusselt numbers of freely falling drops. It was found that the effect of acceleration is only of importance for a very short time after initial release (while the acceleration modulus is large). At large distances from the point of release, the Nusselt numbers based on the recalculated data of Yao lies slightly below that predicted by the Ranz and Marshall correlation. 


\section{APPENDIX B Description of the digital image processing technique used for the measurement of drop sizes from photographs}

The manual counting and measuring of drops from photographs are exceedingly timeconsuming and allows for human error. Commercial digital image analysis systems are usually very expensive. A relatively simple and inexpensive image analysis procedure was devised to measure drop sizes from photographic records.

The main steps of this procedure are:

i) Obtain a high quality black-and-white photograph with good contrast. With transparent objects such as water drops, a lighting technique called shadowgraphing can be used to illuminate the drops. With shadowgraphing the background is illuminated and the shadows of the drops are photographed. This ensures that the outline of the drops show up as dark circles on the photographs. Figures 4.38 and 4.39 are typical examples of photographs obtained with this lighting technique.

ii) Enlarge the photograph to an A4-size using a photocopying machine. This step saves the cost of using large photographic prints and it allows one to edit the images using a black pen and white correctional fluid. The computer algorithm (see step (iv)) used to measure the drop from the scanned images, requires that each image of a drop consists of a closed circle.

iii) Digitise and save the image to magnetic disk using an image scanner linked to a personal computer. It is preferable to scan the image using 256 grey scales, but if there is sufficient contrast between the light areas (background) and the darker areas (drops), a 16 level grey scale image will be good enough. In this study the images were scanned using a HP ScanJet scanner to digitise the photographs as 16 grey scale images with 300 dpi (dots per inch) resolution. This resolution implies that the smallest object which can be seen on an image that has been enlarged by a factor of 2 (through photography and photocopying) is approximately $42 \mu \mathrm{m}$ by $42 \mu \mathrm{m}$.

iv) Analyse the image file by computer. The following steps are used in the program to analyse the image-file: (i) the shaded (grey scale) image is segmented into a binary image showing the drops as black and the background as white, (ii) the drops are detected and the large drops, which show up as closed circles, are filled, (iii) the drop sizes are calculated from the number of black pixels making up each drop, and (iv) the results are stored on magnetic disk for further processing. The algorithms used for segmentation, drop detection and drop shape and size measurement are described in detail by Weszka [78WE1], Haralick and Shapiro [85HA1], Beukman [87BE2], Horn [87HO1] and Brink [92BR1]. The computer time required to analyse a typical image consisting of 1500 by 2000 pixels is approximately 2 minutes on a $25 \mathrm{MHz} 80386$ based IBM compatible personal computer. 


\section{APPENDIX C Description of apparatus for measuring water film thicknesses}

Conductance and capacitive probes are used extensively to measure liquid film thicknesses in various types of experimental apparatus. A conductance probe measures the liquid film thickness by measuring the conductance (or resistance) between two electrodes. Coney [73CO1], Hewitt [82HE1], Koskie et al. [89KO1] and Kang and Kim [92KA1] described different types of electrodes which can be used. The flush mounted pin probes (see Figure C.1) described by Hewitt [ $82 \mathrm{HE} 1]$ are easy to manufacture and they do not influence the flow of the liquid. A conductance measuring technique using flush mounted pin electrodes was developed to measure the thickness of thin water films on the upper surfaces of the slats used in the experimental work.

\section{Plan view}
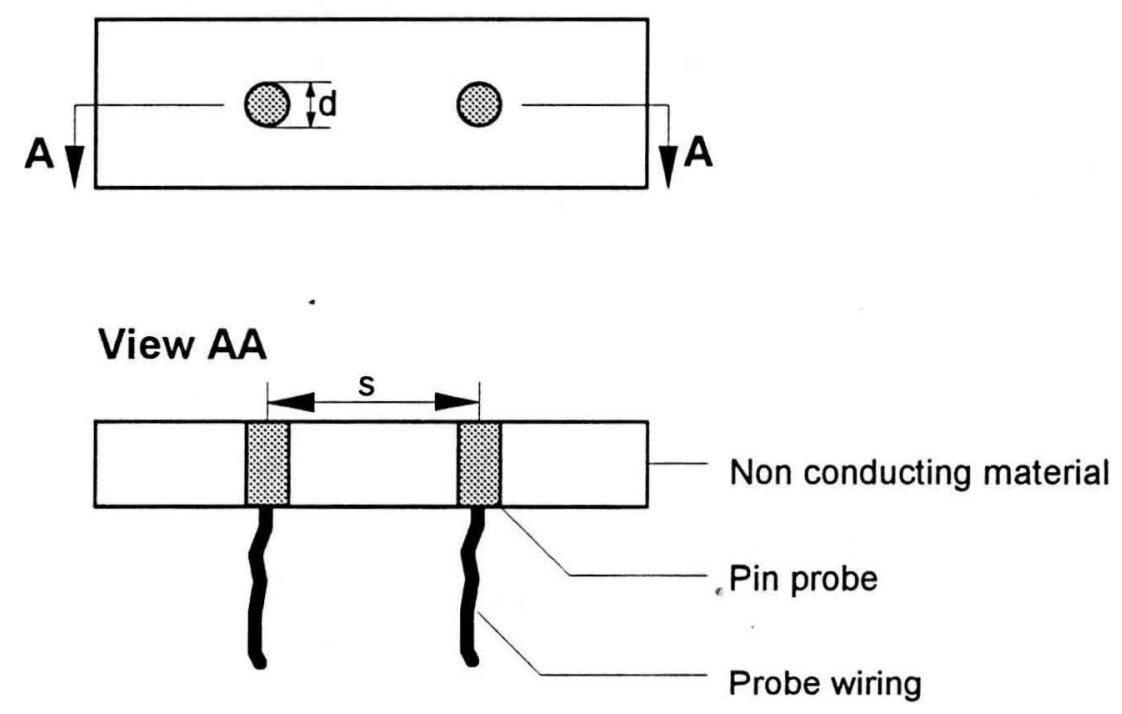

Figure C.1 Flush mounted pin electrodes used in water film thickness measurements.

By selecting the electrode dimensions carefully, it is possible to construct a probe which will give a linear response with varying liquid film thickness. Hewitt [82HE1] found that a probe using pin electrodes with an electrode spacing of 4 times the electrode diameter, or $\left(\mathrm{s} / \mathrm{d}_{\text {electrode }}\right)=4$, gives a linear response up to a dimensionless film thickness of 0.15 , or $(\delta / s)=0.15$. To construct a linear response probe (with $(\mathrm{s} / \mathrm{d})=4$ ) to measure film thicknesses of up to $3 \mathrm{~mm}$ would require an electrode spacing of at least $20 \mathrm{~mm}$ and electrodes with d=5 $\mathrm{mm}$.

A block diagram of the electronic circuit required to drive the probes and to analyse the output is shown in Figure C.2. An alternating voltage from a signal generator is supplied to the one electrode via a voltage follower with a high input resistance. The voltage follower (or buffer) 
ensures that the signal generator supply voltage is not influenced by the probe resistance. The signal is supplied to the electrode as an alternating voltage with a voltage swing of approximately $10 \mathrm{~V}$ alternating at a frequency of $1 \mathrm{kHz}$. The signal from the electrodes is amplified and passed to a full-wave rectifier before entering a low-pass Butterworth filter. The rectifier and filter stages remove the alternating component of the supply voltage from the output signal. The filter stage was designed to have a corner frequency of $100 \mathrm{~Hz}$. This means that the probe will be sensitive to changes in film thickness which occur at a rate of less 100 $H z$.

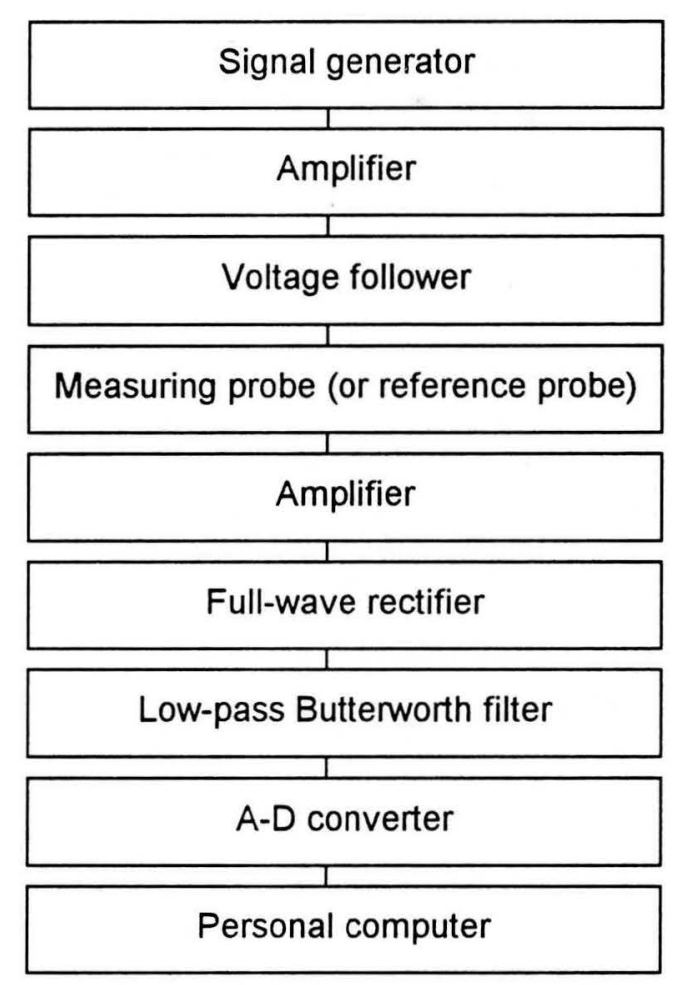

Figure C.2 Block diagram of the electronic device used to measure liquid film thicknesses.

To compensate for changes in the conductivity of the water, each set of electrodes was calibrated relative to a fixed geometry reference cell which could be connected to the circuit instead of the measuring electrodes. Water from the same source was supplied to the reference cell and to the electrodes mounted on the experimental slats. By adjusting the amplifier gain, the output signal from the reference cell could be kept at a fixed value of $3.5 \mathrm{~V}$ during calibration and experimental work. The electrode sets mounted on the test slats were calibrated by measuring the output voltage and the corresponding centreline film thickness on the test slat (using a vernier height gauge). By repeating this procedure at various film thicknesses it was possible to obtain a functional relationship between the probe output and the centreline film thickness on the slat. 
It was convenient to use a personal computer to read and process the output voltage from the film measuring probes. The probe output voltage was converted to a digital representation by an inexpensive eight bit analogue-to-digital (A-D) converter (National Semiconductor ADC0841). The A-D converter was wired to convert the incoming voltage to a digital format continuously. The eight bits of digital data was read in an un-synchronised fashion using eight single input lines from a digital I/O (input/output) card installed in the personal computer. The sampling rate of the computer was higher than the conversion rate of the A-D converter and this ensured that no data was lost during the transfer to the computer. For voltages varying between 0 and $5 \mathrm{~V}$, the resolution of an eight bit A-D converter is better than $20 \mathrm{mV}$. If a probe output voltage of $5 \mathrm{~V}$ corresponds to a film thickness of $3 \mathrm{~mm}$, an output voltage change of $20 \mathrm{mV}$ corresponds to a change in film thickness of less than $12 \mu \mathrm{m}$. 


\section{APPENDIX D Derivation of simplified analytic equations to describe the motion of splash drop above the impact plane}

Consider a drop of size, $d_{i}$, impacting a solid surface covered by a thin liquid film of depth, $\delta$, as shown in Figure D.1. The slat is mounted in an airstream flowing upwards at a velocity, $v_{a}$. Upon impact the so-called crown is formed and small splash drops are flung away from the edge of the crown. The initial angle at which splash drops leave the crown, the initial splash drop velocity, the splash drop size and the air velocity are all expected to influence the trajectory of the splash drop after release from the crown. It would be relatively simple, but time consuming, to calculate the drop trajectory and drop cooling by using a stepwise numerical integration technique.

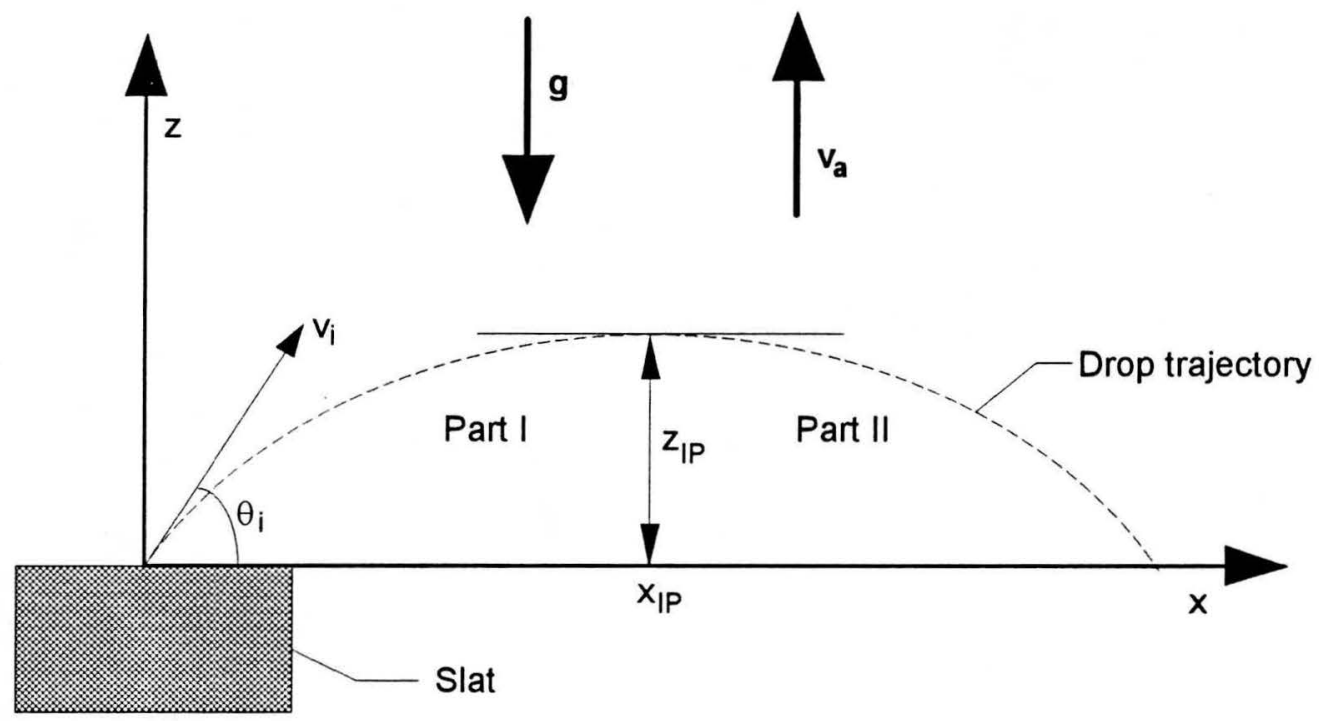

Figure D.1 Schematic description of splash drop trajectory from impact point.

In the splash pack simulation program it would be too time consuming to calculate the flight path of each splash drop in order to determine its cooling rate. Instead, this problem can be simplified as follows:

i) Small splash drops, with terminal velocities below the air velocity, will be lost upwards and carried away by the airstream. Larger drops will travel upwards, turn around and then fall downwards into the oncoming airstream. The splash drop motion and cooling above the plane of the slat $(z=0)$ can be approximated using the simplified analytical model described below.

ii) The results of the calculations in the previous step, i.e., the temperature and downward velocity at the point when a drop falls downwards past the plane of the slat $(z=0)$, can be used as the initial conditions of the splash drop for the integration process proceeding downwards along the height of the packing zone. 
Note that this simple two-step model takes the horizontal motion of the splash drops into account only while the drop is travelling above the slat. Once the drop falls down past the plane of the slat $(z=0)$ only the vertical drop velocity is used in further calculations.

Note The symbols A, B, C, D, E, G defined below are not listed in the Nomenclature.

These symbols are only used in this Appendix.

\section{Vertical motion}

The vertical motion of a splash drop of size, $d_{s}$, leaving the impact points at a velocity of $v_{v, i}$ can be described by the following equation

$$
-\frac{\partial v_{\text {rel }}}{\partial \mathrm{t}}=-\mathrm{G}+A \mathrm{v}_{\text {rel }}\left|\mathrm{v}_{\text {rel }}\right|
$$

where

$$
\begin{aligned}
& G=\left(\frac{g\left(\rho_{w}-\rho_{a}\right)}{\rho_{w}}\right) \\
& A=\left(\frac{3 \rho_{a} C_{D}}{4 \rho_{w} d_{s}}\right)
\end{aligned}
$$

where

$$
\mathrm{v}_{\mathrm{rel}}=\mathrm{v}_{\mathrm{a}}-\mathrm{v}_{\mathrm{v}}
$$

Assuming that the drag coefficient is constant during the flight of the splash drop, it is possible to solve the equation of motion analytically. The effect of this assumption will later be discussed in more detail. Two different solutions are possible depending on the sign of $\mathrm{v}_{\text {rel }}$.

\section{Case 1: $v_{\text {rel }}>\mathbf{0}\left(\right.$ or $\left.v_{a}>v_{v}\right)$}

For this case, one finds by the separation of variables that the drop travel time can be expressed by

$$
t=\frac{1}{A \sqrt{G / A}} \tanh ^{-1}\left(\frac{v_{\text {rel }}}{\sqrt{G / A}}\right)+C
$$

Assuming that the initial relative velocity between the drop and the air is $\mathrm{v}_{\mathrm{rel}, \mathrm{i}}=\mathrm{v}_{\mathrm{a}}-\mathrm{v}_{\mathrm{v}, \mathrm{i}}$, it follows that 


$$
t=\frac{1}{2 A \sqrt{G / A}} \ln \left(\left(\frac{\sqrt{G / A}+v_{\text {rel }}}{\sqrt{G / A}-v_{\text {rel }}}\right)\left(\frac{\sqrt{G / A}-v_{\text {rel, },}}{\sqrt{G / A}+v_{\text {rel, }, i}}\right)\right)
$$

The flight time to the inflection point is determined by setting $\mathrm{v}_{\mathrm{rel}}=0$ into the equation above, which yields

$$
t_{I P}=\frac{1}{2 A \sqrt{G / A}} \ln \left(\left(\frac{\sqrt{G / A}+v_{a}}{\sqrt{G / A}-v_{a}}\right)\left(\frac{\sqrt{G / A}-v_{\text {rel,i }}}{\sqrt{G / A}+v_{\text {rel, }, i}}\right)\right)
$$

The upward displacement of the splash drop can be described as

$$
\mathrm{z}_{\mathrm{IP}}=\int_{0}^{\mathrm{t}_{\mathrm{IP}}} \mathrm{v}_{\mathrm{a}} \partial \mathrm{t}-\int_{0}^{\mathrm{t}_{\mathrm{IP}}} \mathrm{v}_{\mathrm{rel}} \partial \mathrm{t}
$$

The equation for flight time required to reach the inflection point can be rewritten in terms of relative velocity; after substitution of the resulting relation for relative velocity into the equation above and integration, it follows that the maximum upward displacement can be expressed as

$$
\mathrm{z}_{\mathrm{IP}}=\mathrm{v}_{\mathrm{a}} \mathrm{t}_{\mathrm{IP}}-\frac{\ln \left(\left(1+\mathrm{B} \exp \left(-2 \mathrm{~A} \sqrt{\frac{\mathrm{G}}{\mathrm{A}}} \mathrm{t}_{\mathrm{IP}}\right)\right)\left(\mathrm{B}+\exp \left(2 \mathrm{~A} \sqrt{\frac{\mathrm{G}}{\mathrm{A}}} \mathrm{t}_{\mathrm{IP}}\right)\right)\right)-\ln \left((\mathrm{B}+1)^{2}\right)}{2 \mathrm{~A}}
$$

where

$$
B=\left(\frac{\sqrt{G / A}-v_{\text {rel,i }}}{\sqrt{G / A}+v_{\text {rel,i }}}\right)
$$

The downward motion of the drop from the inflection point can now be described. Note that the relative velocity will always be positive for the downward motion. Equation (D.5) can therefore also be used to describe the downward motion. The drop travel time from the inflection point can be obtained by noting that the relative velocity is equal to the air velocity $\left(\mathrm{v}_{\mathrm{rel}}=\mathrm{v}_{\mathrm{a}}\right)$ at the inflection point $(\mathrm{t}=0)$ and substituting this into Equation (D.5), yielding

$$
t=\frac{1}{2 A \sqrt{G / A}} \ln \left(\left(\frac{\sqrt{G / A}+v_{\text {rel }}}{\sqrt{G / A}-v_{\text {rel }}}\right)\left(\frac{\sqrt{G / A}-v_{a}}{\sqrt{G / A}+v_{a}}\right)\right)
$$

The downward displacement of the splash drop from the inflection point can be described by

$$
z=\int v_{\mathrm{a}} \partial \mathrm{t}-\int \mathrm{v}_{\mathrm{rel}} \partial \mathrm{t}
$$


Rewriting Equation (D.10) yields the following relation for relative velocity

$$
v_{\text {rel }}=\left(\sqrt{\frac{G}{A}}\right)\left(\frac{\exp (2 A \sqrt{G / A t})-D}{\exp (2 A \sqrt{G / A t})+D}\right)
$$

where

$$
D=\left(\frac{\sqrt{G / A}-v_{a}}{\sqrt{G / A}+v_{a}}\right)
$$

Substituting this relation into Equation (D.11), results in the following equation describing the downward motion of the splash drop from the inflection point

$$
\mathrm{z}=\mathrm{v}_{\mathrm{a}} \mathrm{t}-\frac{\ln \left(\left(1+\mathrm{D} \exp \left(-2 \mathrm{~A} \sqrt{\frac{\mathrm{G}}{\mathrm{A}} \mathrm{t}}\right)\right)\left(\mathrm{D}+\exp \left(2 \mathrm{~A} \sqrt{\frac{\mathrm{G}}{\mathrm{A}} \mathrm{t}}\right)\right)\right)-\ln \left((1+\mathrm{D})^{2}\right)}{2 \mathrm{~A}}
$$

The time required for the drop to fall from the inflection point to $z=0, \Delta t_{I P \rightarrow 0}$, can be calculated from this equation by setting $z=z_{\mathbb{I P}}$. The relative velocity reached by a drop accelerating from the inflection point to $z=0$ can be found from Equation (D.12) by setting $\mathrm{t}=\Delta \mathrm{t}_{\mathrm{IP} \rightarrow 0}$.

\section{Case 2: $\mathbf{v}_{\text {rel }}<0$ (or $\left.\mathbf{v}_{\mathrm{a}}<\mathrm{v}_{\mathrm{v}}\right)$}

Assuming an initial relative velocity between the drop and the air of $\mathrm{v}_{\mathrm{rel}, \mathrm{i}}=\mathrm{v}_{\mathrm{a}}-\mathrm{v}_{\mathrm{v}, \mathrm{i}}$ and solving the equation of motion by the separation of variables, it follows that the drop travel time to the point of inflection can be expressed by

$$
t=\frac{1}{A \sqrt{G / A}}\left(\tan ^{-1}\left(\frac{v_{\text {rel }}}{\sqrt{G / A}}\right)-\tan ^{-1}\left(\frac{v_{\text {rel, }}}{\sqrt{G / A}}\right)\right)
$$

The flight time to the inflection point can be determined by setting $\mathrm{v}_{\mathrm{rel}}=0$ into the equation above, which yields

$$
t_{I P}=\frac{1}{A \sqrt{G / A}}\left(\tan ^{-1}\left(\frac{v_{a}}{\sqrt{G / A}}\right)-\tan ^{-1}\left(\frac{v_{\text {rel,i }}}{\sqrt{G / A}}\right)\right)
$$

Rewriting Equation (D.14) yields

$$
v_{\text {rel }}=\sqrt{\frac{G}{A}} \tan \left(\left(A \sqrt{\frac{G}{A}} t\right)+\tan ^{-1}\left(\frac{v_{\text {rel }, i}}{\sqrt{G / A}}\right)\right)
$$


The upward displacement of the splash drop can be obtained from

$$
z_{I P}=\int_{0}^{t_{I P}} v_{a} \partial t-\int_{0}^{t_{I P}} v_{\text {rel }} \partial t
$$

Substituting Equation (D.15) into the equation above and integrating yields the following relation for the height of the inflection point

$$
\mathrm{z}_{\mathrm{IP}}=\mathrm{v}_{\mathrm{a}} \mathrm{t}_{\mathrm{IP}}-\left(\frac{1}{\mathrm{~A}}\right) \ln \left(\frac{\cos (\mathrm{E})}{\cos \left(\mathrm{E}+\mathrm{A} \sqrt{\mathrm{G} / \mathrm{A}} \mathrm{t}_{\mathrm{IP}}\right)}\right)
$$

where

$$
E=\tan ^{-1}\left(\frac{v_{\text {rel,i }}}{\sqrt{G / A}}\right)
$$

For the downward motion of a splash drop from the inflection point the relative velocity will be larger than $0\left(v_{\text {rel }}>0\right)$ and the solution of the equation of motion will be the same as that described for Case 1.

\section{Horizontal motion}

The horizontal motion of a splash drop of size, $d_{s}$, leaving the impact points at a velocity of $\mathrm{v}_{\mathrm{h}, \mathrm{i}}$ can be described by the following equation

$$
\frac{\partial v_{h}}{\partial t}=-A v_{h}^{2}
$$

where

$$
A=\left(\frac{3 \rho_{a} C_{D}}{4 \rho_{w} d_{s}}\right)
$$

Through integration of this equation and by noting that $\mathrm{v}=\mathrm{v}_{\mathrm{hi}}$ at $\mathrm{t}=0$, it follows that

$$
v=\left(\frac{v_{h, i}}{1+A v_{h, i} t}\right)
$$

The horizontal distance reached by the drop after a given time can be found by integration of this equation yielding

$$
x=\frac{\ln \left(1+A v_{h, i} t\right)}{A}+C
$$


By considering the horizontal motion between $\mathrm{t}=0$ and $\mathrm{t}=\mathrm{t}_{\mathrm{IP}}$ it follows that $\mathrm{C}=0$ in the equation above. If only drop motion for $\mathrm{t} \geq \mathrm{t}_{\mathrm{IP}}$ is considered, the equations for horizontal velocity and displacement become

$$
v=\left(\frac{v_{h, I P}}{1+A v_{h, \mathbb{P}}\left(t-t_{\mathbb{P}}\right)}\right)
$$

and

$$
x=x_{\mathbb{P}}+\left(\frac{1}{A}\right) \ln \left(\frac{1+A v_{h, \mathbb{P}} t}{1+A v_{h, \mathbb{P}} t_{I P}}\right)
$$

Note that it is also possible to obtain an analytical solution for the horizontal drop motion by assuming that the drop drag can be expressed by $C_{D}=\left(10 / \mathrm{Re}^{0.5}\right)$ (which is a fair approximation of the drag curve up to $\mathrm{Re}=500$ ). Considering the other approximations (i.e. the assumption of a constant drag coefficient during the vertical motion) made to simplify the drop trajectory calculations, the increased accuracy afforded by this approach cannot be justified by the extra complexity this would introduce.

\section{Solution procedure}

In the mathematical analysis above the horizontal and vertical motion of a splash drop were considered to be independent and the drag coefficients were considered to be constant. The following procedure, employing the analytical solutions above, can then be used to approximate the flight path of a splash drop. The drop flight path can be divided into two separate sections as seen in Figure D.1: (i) a part where the splash drop is moving upwards and (ii) a part in which the drop is moving downwards relative to the fixed reference.

The main steps in the solution for the first part of the flight (upward motion relative to the slat) can be summarised as follows:

i) Assume a value for $v_{h, I P}$ and calculate the mean relative velocity between the drop and the air from

$$
\mathrm{v}_{\mathrm{rel}, \mathrm{m}}=0.5\left(\sqrt{\mathrm{v}_{\mathrm{rel}, \mathrm{i}}^{2}+\mathrm{v}_{\mathrm{h}, \mathrm{i}}^{2}}+\sqrt{\mathrm{v}_{\mathrm{a}}^{2}+\mathrm{v}_{\mathrm{h}, \mathrm{IP}}^{2}}\right)
$$

where

$$
\mathrm{v}_{\mathrm{rel}, \mathrm{i}}=\mathrm{v}_{\mathrm{a}}-\mathrm{v}_{\mathrm{i}} \sin \left(\theta_{\mathrm{i}}\right)
$$

and 
$\mathrm{v}_{\mathrm{h}, \mathrm{i}}=\mathrm{v}_{\mathrm{i}} \cos \left(\theta_{\mathrm{i}}\right)$

ii) Calculate $C_{D}$ based on this velocity. The drag coefficients for the vertical and horizontal parts of the motion can be approximated as

$$
\begin{aligned}
& C_{D, v}=C_{D}\left(\frac{\sin \left(\theta_{\text {rel }, \mathrm{i}}\right)+\sin \left(\theta_{\text {rel, } \mathrm{PP}}\right)}{2}\right) \\
& \mathrm{C}_{\mathrm{D}, \mathrm{h}}=\mathrm{C}_{\mathrm{D}}\left(\frac{\cos \left(\theta_{\text {rel }, \mathrm{i}}\right)+\cos \left(\theta_{\text {rel, }, \mathrm{P}}\right)}{2}\right)
\end{aligned}
$$

where $\theta_{\text {rel, } \mathrm{i}}$ and $\theta_{\text {rel, }, \mathbb{P}}$ are defined as

$$
\begin{aligned}
& \theta_{\text {rel }, \mathrm{i}}=\tan ^{-1}\left(\frac{\mathrm{v}_{\text {rel }, \mathrm{i}}}{\mathrm{v}_{\mathrm{h}, \mathrm{i}}}\right) \\
& \theta_{\mathrm{rel}, \mathbb{P}}=\tan ^{-1}\left(\frac{\mathrm{v}_{\mathrm{a}}}{\mathrm{v}_{\mathrm{h}, \mathrm{P}}}\right)
\end{aligned}
$$

iii) Calculate $t_{\mathbb{I P}}, \mathrm{z}_{\mathrm{IP}}, \mathrm{x}_{\mathrm{IP}}$ and $\mathrm{v}_{\mathrm{h}, \mathrm{IP}}$ from the analytical relations derived above for either $\mathrm{v}_{\text {rel, }}>0$ or $\mathrm{v}_{\text {rel, }}<0$.

iv) Repeat from step (i) until $v_{h, I P}$ converges.

The second part of the drop flight between the inflection point and the plane of the slat at $\mathrm{z}=0$, (downward motion relative to the slat), can be solved by executing the following steps:

i) Assume values for $v_{\text {rel, }, \mathrm{z}=0}$ and $\mathrm{v}_{\mathrm{h}, \mathrm{x}=0}$; calculate the mean relative velocity between the drop and the air from

$$
\mathrm{v}_{\mathrm{rel}, \mathrm{m}}=0.5\left(\sqrt{\mathrm{v}_{\mathrm{a}}^{2}+\mathrm{v}_{\mathrm{h}, \mathrm{IP}}^{2}}+\sqrt{\mathrm{v}_{\mathrm{rel}, \mathrm{z}=0}^{2}+\mathrm{v}_{\mathrm{h}, \mathrm{z}=0}^{2}}\right)
$$

ii) Calculate $C_{D}$ based on this velocity. The drag coefficients for the vertical and horizontal parts of the motion can be approximated as

$$
\begin{aligned}
& C_{\mathrm{D}, \mathrm{v}}=\mathrm{C}_{\mathrm{D}}\left(\frac{\sin \left(\theta_{\mathrm{rel}, \mathrm{IP}}\right)+\sin \left(\theta_{\mathrm{rel}, \mathrm{z}=0}\right)}{2}\right) \\
& \mathrm{C}_{\mathrm{D}, \mathrm{h}}=\mathrm{C}_{\mathrm{D}}\left(\frac{\cos \left(\theta_{\mathrm{rel}, \mathrm{IP}}\right)+\cos \left(\theta_{\mathrm{rel}, \mathrm{z}=0}\right)}{2}\right)
\end{aligned}
$$


where $\theta_{\text {rel, }, \mathrm{P}}$ and $\theta_{\mathrm{rel}, \mathrm{z}=0}$ are defined as

$$
\begin{aligned}
& \theta_{\mathrm{rel}, \mathbb{P}}=\tan ^{-1}\left(\frac{\mathrm{v}_{\mathrm{a}}}{\mathrm{v}_{\mathrm{h}, \mathrm{P}}}\right) \\
& \theta_{\mathrm{rel}, \mathrm{z}=0}=\tan ^{-1}\left(\frac{\mathrm{v}_{\mathrm{rel}, \mathrm{z}=0}}{\mathrm{v}_{\mathrm{h}, \mathrm{z}=0}}\right)
\end{aligned}
$$

iii) Calculate $t_{\mathrm{IP} \rightarrow \mathrm{z}=0}, \mathrm{v}_{\mathrm{rel}, \mathrm{z}=0}, \mathrm{x}_{\mathrm{z}=0}$ and $\mathrm{v}_{\mathrm{h}, \mathrm{z}=0}$ from the analytical relations derived above.

iv) Repeat from step (i) until the values of $v_{\mathrm{rel}, \mathrm{z}=0}$ and $\mathrm{v}_{\mathrm{h}, \mathrm{z}=0}$ converge.

The cooling of the drop during it flight above the plane of the slat can be estimated by calculating the cooling of a drop travelling in air, at a velocity equal to the average relative velocity between the drop and the air during its flight, for a time equal to the total drop travel time. The rate equations for heat/mass transfer from the drop are similar to those described in Chapter 3.

It is important to use small time increments to ensure convergence of the calculation to determine the drop cooling rate. The critical time increments can be determined following a procedure similar to that used in finite-difference calculations of non-steady conduction heat transfer. The energy balance of a single drop can be expressed as

$$
-\frac{\mathrm{m}_{\mathrm{s}} \mathrm{c}_{\mathrm{pw}}(\mathrm{T}(\mathrm{t}+\Delta \mathrm{t})-\mathrm{T}(\mathrm{t}))}{\Delta \mathrm{t}}=\mathrm{KA}\left(\mathrm{i}_{\text {asw }}-\mathrm{i}_{\mathrm{a}}\right)
$$

Assuming that the air saturation enthalpy can be expressed as

$$
\mathrm{i}_{\text {asw }}=\mathrm{c}+\left(\frac{\partial \mathrm{i}_{\text {asw }}}{\partial \mathrm{T}}\right) \mathrm{T}_{\mathrm{w}}
$$

and using a similar argument as that by Holman [81HOl] for non-steady state heat transfer yields the following criteria for numerical stability

$$
\Delta \mathrm{t} \leq \frac{\mathrm{m}_{\mathrm{s}} \mathrm{c}_{\mathrm{pw}}}{\left(\partial \mathrm{i}_{\text {asw }} / \partial \mathrm{T}\right) \mathrm{KA}}
$$

By using time increments smaller than those specified by the equation above, the convergence of the drop cooling calculation is ensured.

The temperature of different sized splash drops at the end of their splash trajectories (at $z=0$ ) as predicted by a numerical integration procedure and by the simplified analytical solution are 
shown in Figure D.2. It can be seen that the simplified model closely predicts the drop temperatures predicted by the accurate numerical integration technique.

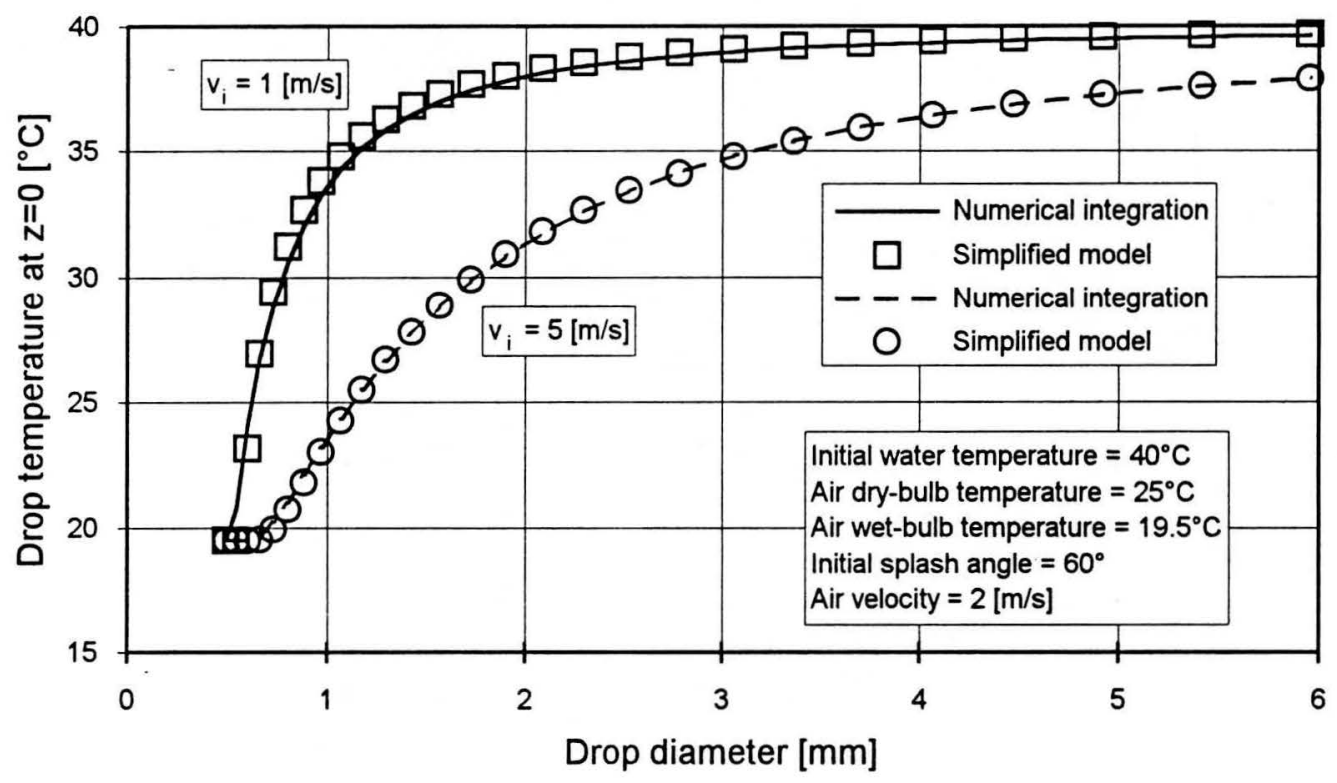

Figure D.2 Comparison between the drop temperatures predicted by the accurate numerical integration procedure and by the simplified model.

The use of the simplified model to calculate the splash drop trajectory above the slat as described in this appendix results in significant time savings when compared to a numerical integration procedure to calculate the drop trajectory. The simplified model requires between one and two orders of magnitude less computer execution time. 


\section{APPENDIX E Drop deflection around slats}

Consider the layout of a long cylindrical slat with air flowing around the slat from below as shown in Figure E.1. The air flowing around a slat diverges upstream of the slat and converges behind the slat. This convergence of the airflow behind the slat can be expected to influence small drops falling from above towards the slat, i.e., more drops could strike the slat than those vertically above the slat.

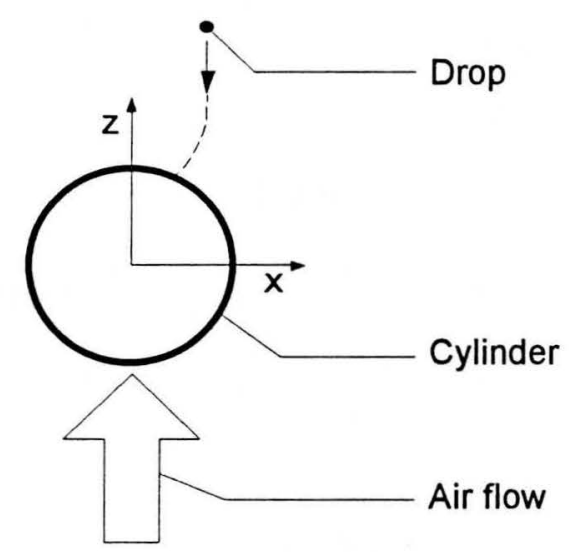

Figure E.1 Drop collection on the rear surface of a cylinder.

As a rough approximation, it can be assumed that the flow about the slat can be described by potential flow around a cylinder. Furthermore, the effect of the drops on the airflow is assumed to be negligible and that the drop trajectories can be calculated by simple superposition. The potential flow solution for the flow velocities around a cylinder of diameter, $d_{c y l}$, gives the components of the flow velocities in the $\mathrm{x}$ and $\mathrm{z}$ directions as

$$
v_{x}=-v_{\infty}\left(\frac{d_{\text {cyl }}}{2}\right)\left(\frac{x z}{\left(x^{2}+z^{2}\right)^{2}}\right)
$$

and

$$
v_{z}=v_{\infty}\left(1+\left(\frac{d_{c y l}}{2}\right)^{2} \frac{\left(x^{2}-z^{2}\right)}{\left(x^{2}+z^{2}\right)^{2}}\right)
$$

The drop collection on the top of the cylinder can be described by a collection efficiency, defined as

$$
\eta_{\text {coll }}=\frac{\dot{\mathrm{m}}_{\text {striking slat }}}{\dot{\mathrm{M}}_{\mathrm{w}}\left(\mathrm{d}_{\text {cyl }}+\mathrm{d}_{\mathrm{i}}\right) \mathrm{L}}
$$


This equation defines the collection efficiency as the ratio of the number of drops striking the cylinder to the number of drops directly in-line with the slat far above the slat. A collection efficiency larger than unity would therefore indicate that extra drops collected on the slat due to the deflection of air around the slat.

Figure E.2 shows typical collection efficiencies calculated for airflow around a cylinder $\left(\mathrm{v}_{\mathrm{a}}=1.5 \mathrm{~m} / \mathrm{s}\right)$. The drops were assumed to start falling at terminal velocity far from the cylinder. It can be seen that only a very narrow range of drop sizes is influenced by the presence of the cylinder. Drops with diameters smaller than $0.39 \mathrm{~mm}$ have terminal velocities smaller than $1.5 \mathrm{~m} / \mathrm{s}$ and will consequently be carried upwards by the airstream. Large drops are less deflected by the presence of the cylinder due to their larger mass and higher velocities. Drops deflect more in the presence of large cylinders. At higher air velocities the drop collection efficiencies will be even smaller than those shown in Figure E.2, since only larger drops with higher terminal velocities (which are influenced less by the airstream) will move downwards, towards the slat.

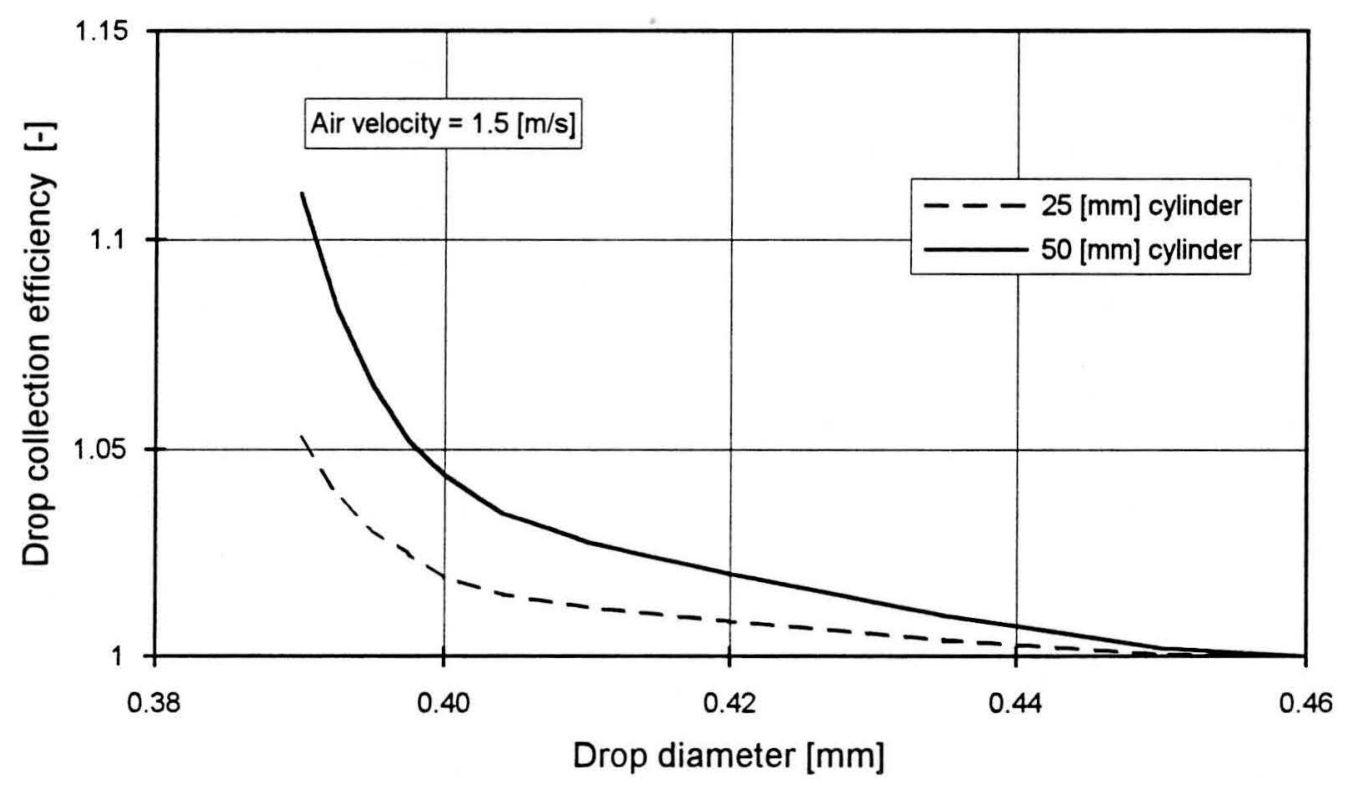

Figure E.2 Drop collection efficiency on the rear surface of a cylinder.

The actual flow pattern around a sharp-edged slat is expected to differ significantly from the potential flow solution, but the effect of drop deflection by the airflow behind the slat is expected only to affect a narrow range of drop sizes, with terminal velocities slightly higher than the air flow velocity. Based on the foregoing, it can be concluded that the effect of increased drop collection efficiency due to the airflow around the rear of a slat will generally be negligible. 


\section{APPENDIX F Low flow rate drop formation from cylindrical orifices (and surface tension measurement)}

The formation of drops from single orifices or capillary tubes is governed by surface tension, gravity and momentum effects. Clift et al. [78CL1], Godfrey and Hanson [82GO1], Mori [90MO1] and Mori and Mochizuki [92MO1] presented literature surveys on the dripping phenomena occurring at low flow rates (at which momentum effects are negligible). At increased liquid flow rates (but still prior to jetting), the drop sizes produced by dripping are influenced by momentum effects as well as gravity and surface tension (See $\mathrm{Lu}$ and Huang [89LU1] and Mori and Mochizuki [92MO1]). At high flow rates jetting and the associated jet break-up is observed. The formation of drops from a liquid jet was first described by Rayleigh (see Clift et al. [78CL1]). Bogy [79BO1], Bright [85BR3] and Chuech et al. [91CH3] reviewed the literature on the subject of jet unstability and break-up.

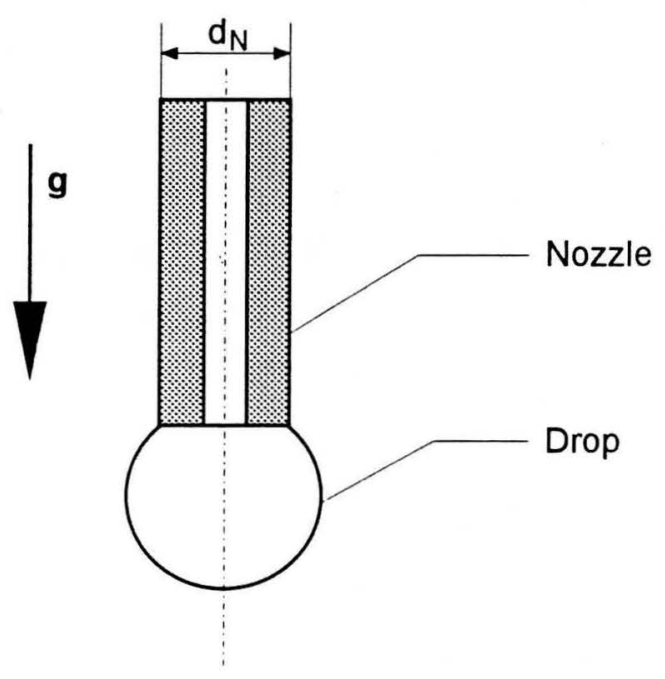

Figure F.1 Drop hanging below a square-edged cylindrical nozzle.

By balancing the surface tension and gravity forces on a drop at the moment of departure from the end of a cylindrical nozzle, as shown in Figure F.1, the following relations for theoretical drop volume and diameter at low liquid flow rates can be obtained:

$$
\mathrm{V}_{\text {theo }}=\frac{\pi \sigma \mathrm{d}_{\mathrm{N}}}{\mathrm{g} \rho_{\mathrm{l}}}
$$

and

$$
\mathrm{d}_{\text {theo }}=\sqrt[3]{\frac{6 \sigma \mathrm{d}_{\mathrm{N}}}{\mathrm{g} \rho_{1}}}
$$


In practice, dripping drops are found to be smaller than those predicted by these equations. In 1919 Harkins and Brown (see Adamson [60AD1]) defined the following correction to account for this discrepancy

$$
\varphi=\frac{\mathrm{V}}{\mathrm{V}_{\text {theo }}}
$$

Mori [90MO1] correlated the experimental data of Harkins and Brown, for dripping at very low flow rates, by

$$
\varphi=0.6+0.4\left(1-\left(\frac{\mathrm{d}_{\mathrm{N}}}{1.4}\right) \mathrm{V}_{\text {theo }}^{-1 / 3}\right)^{2.2}
$$

Under certain circumstances, one or more satellite drops are formed behind the large drop primary nozzles dripping from an orifice (Izard et al. [63IZ1] and Peregrine et al. [90PE1]).

It is possible to measure the surface tension of liquid by measuring the mass of a drop dripping from a square edged, circular nozzle at a very low flow rate. The surface tension can then be calculated by employing the Harkins-Brown correction factor together with the theoretical drop size as described above. 


\section{APPENDIX G Properties of air, water and air-water mixtures}

Note that the symbol, $\mathrm{T}$, is used in this Appendix to signify temperatures in degrees Kelvin.

The thermophysical properties of dry air from $220 \mathrm{~K}$ to $380 \mathrm{~K}$

Density

$$
\rho_{\mathrm{da}}=\frac{\mathrm{p}_{\mathrm{abs}}}{\mathrm{RT}}, \mathrm{kg} / \mathrm{m}^{3}
$$

where $\mathrm{R}=287.08 \mathrm{~J} / \mathrm{kgK}$

Specific heat

$$
\mathrm{c}_{\mathrm{pda}}=\mathrm{a}+\mathrm{bT}+\mathrm{cT}^{2}+\mathrm{dT}^{3}, \quad J / k g K
$$

where

$\mathrm{a}=1.045356 \times 10^{3}$

$\mathrm{b}=-3.161783 \times 10^{-1}$

$\mathrm{c}=7.083814 \times 10^{-4}$

$\mathrm{d}=-2.705209 \times 10^{-7}$

\section{Dynamic viscosity}

$$
\mu_{\mathrm{da}}=\mathrm{a}+\mathrm{bT}+\mathrm{cT}^{2}+\mathrm{dT}^{3}, \quad \mathrm{~kg} / \mathrm{ms}
$$

where

$\mathrm{a}=2.287973 \times 10^{-6}$

$\mathrm{b}=6.259793 \times 10^{-8}$

$c=-3.131956 \times 10^{-11}$

$d=8.150380 \times 10^{-15}$

Thermal conductivity

$$
\mathrm{k}_{\mathrm{da}}=\mathrm{a}+\mathrm{bT}+\mathrm{cT}^{2}+\mathrm{dT}^{3}, W / m K
$$

where

$$
\begin{aligned}
& a=-4.937787 \times 10^{-4} \\
& b=1.018087 \times 10^{-4} \\
& c=-4.627937 \times 10^{-8} \\
& d=1.250603 \times 10^{-11}
\end{aligned}
$$


The thermophysical properties of saturated water vapor from $273.15 \mathrm{~K}$ to $380 \mathrm{~K}$

\section{Vapor pressure}

$$
\begin{aligned}
\mathrm{p}_{\mathrm{v}} & =10^{\mathrm{z}}, P a \\
\mathrm{z} & =\mathrm{a}(1-\mathrm{x})+\mathrm{b} \log _{10}(\mathrm{x})+\mathrm{c}\left(1-10^{\mathrm{d}((1 / \mathrm{x})-1)}\right)+\mathrm{e}\left(10^{\mathrm{f}(1-\mathrm{x})}-1\right)+\mathrm{g} \\
\mathrm{x} & =273.16 / \mathrm{T}
\end{aligned}
$$

where

$$
\begin{aligned}
& a=1.079586 \times 10 \\
& b=5.028080 \\
& c=1.504740 \times 10^{-4} \\
& d=-8.296920 \\
& e=4.287300 \times 10^{-4} \\
& f=4.769550 \\
& g=2.786118
\end{aligned}
$$

\section{Specific heat}

$$
\mathrm{c}_{\mathrm{pv}}=\mathrm{a}+\mathrm{bT}+\mathrm{cT}^{5}+\mathrm{dT}^{6}, \quad J / k g K
$$

where

$$
\begin{aligned}
& a=1.360500 \times 10^{3} \\
& b=2.313340 \\
& c=-2.467840 \times 10^{-10} \\
& d=5.913320 \times 10^{-13}
\end{aligned}
$$

\section{Dynamic viscosity}

$$
\mu_{\mathrm{v}}=\mathrm{a}+\mathrm{bT}+\mathrm{cT}^{2}+\mathrm{dT}^{3}, \quad k g / m s
$$

where

$$
\begin{aligned}
& a=2.562435 \times 10^{-6} \\
& b=1.816683 \times 10^{-8} \\
& c=2.579066 \times 10^{-11} \\
& d=-1.067299 \times 10^{-14}
\end{aligned}
$$




\section{5}

\section{Thermal conductivity}

$$
\mathrm{k}_{\mathrm{v}}=\mathrm{a}+\mathrm{bT}+\mathrm{cT}^{2}+\mathrm{dT}^{3}, W / m K
$$

where

$$
\begin{aligned}
& a=1.304600 \times 10^{-2} \\
& b=-3.756191 \times 10^{-5} \\
& c=2.217964 \times 10^{-7} \\
& d=-1.111562 \times 10^{-10}
\end{aligned}
$$

\section{$\underline{\text { Vapour density }}$}

$$
\rho_{\mathrm{v}}=\mathrm{a}+\mathrm{bT}+\mathrm{cT}^{2}+\mathrm{dT}^{3}+\mathrm{eT}^{4}+\mathrm{fT}^{5}, \quad \mathrm{~kg} / \mathrm{m}^{3}
$$

where

$$
\begin{aligned}
& a=-4.062329 \\
& b=0.102770 \\
& c=-9.763004 \times 10^{-4} \\
& d=4.475241 \times 10^{-6} \\
& e=-1.004597 \times 10^{-8} \\
& f=8.915490 \times 10^{-12}
\end{aligned}
$$


The thermophysical properties of mixtures of dry air and water vapour

Density

$$
\rho_{\mathrm{a}}=(1+\mathrm{w})\left(1-\frac{\mathrm{w}}{(\mathrm{w}+0.62198)}\right)\left(\frac{\mathrm{p}_{\mathrm{abs}}}{\mathrm{RT}}\right), \mathrm{kg} / \mathrm{m}^{3}
$$

where $\mathrm{R}=287.08 \mathrm{~J} / \mathrm{kgK}$

Specific heat

$$
\mathrm{c}_{\mathrm{pa}}=\frac{\left(\mathrm{c}_{\mathrm{pda}}+\mathrm{wc}_{\mathrm{pv}}\right)}{(1+\mathrm{w})}, \mathrm{J} / \mathrm{kgK}
$$

\section{Dynamic viscosity}

$$
\mu_{\mathrm{a}}=\frac{\left(\mathrm{X}_{\mathrm{da}} \mathrm{M}_{\mathrm{da}}^{0.5} \mu_{\mathrm{da}}+\mathrm{X}_{\mathrm{v}} \mathrm{M}_{\mathrm{v}}^{0.5} \mu_{\mathrm{v}}\right)}{\left(\mathrm{X}_{\mathrm{da}} \mathrm{M}_{\mathrm{da}}^{0.5}+\mathrm{X}_{\mathrm{v}} \mathrm{M}_{\mathrm{v}}^{0.5}\right)}, \mathrm{kg} / \mathrm{ms}
$$

\section{Thermal conductivity}

$$
\mathrm{k}_{\mathrm{a}}=\frac{\left(\mathrm{X}_{\mathrm{da}} \mathrm{M}_{\mathrm{da}}^{0.33} \mathrm{k}_{\mathrm{da}}+\mathrm{X}_{\mathrm{v}} \mathrm{M}_{\mathrm{v}}^{0.33} \mathrm{k}_{\mathrm{v}}\right)}{\left(\mathrm{X}_{\mathrm{da}} \mathbf{M}_{\mathrm{da}}^{0.33}+\mathrm{X}_{\mathrm{v}} \mathrm{M}_{\mathrm{v}}^{0.33}\right)}, W / m K
$$

where

$$
\begin{aligned}
& \mathrm{M}_{\mathrm{a}}=28.97 \mathrm{~kg} / \text { mole } \\
& \mathrm{M}_{\mathrm{v}}=18.016 \mathrm{~kg} / \text { mole } \\
& \mathrm{X}_{\mathrm{da}}=1 /(1+1.608 \mathrm{w}) \\
& \mathrm{X}_{\mathrm{v}}=\mathrm{w} /(\mathrm{w}+0.622)
\end{aligned}
$$

\section{Humidity ratio}

$$
\begin{aligned}
\mathrm{w}= & \left(\frac{2501.6-2.3263\left(\mathrm{~T}_{\mathrm{wb}}-273.15\right)}{2501.6+1.8577\left(\mathrm{~T}_{\mathrm{db}}-273.15\right)-4.184\left(\mathrm{~T}_{\mathrm{wb}}-273.15\right)}\right)\left(\frac{0.62509 \mathrm{p}_{\mathrm{vwb}}}{\mathrm{p}_{\mathrm{abs}}-1.005 \mathrm{p}_{\mathrm{vwb}}}\right) \\
& -\left(\frac{1.00416\left(\mathrm{~T}_{\mathrm{db}}-\mathrm{T}_{\mathrm{wb}}\right)}{2501.6+1.8577\left(\mathrm{~T}_{\mathrm{db}}-273.15\right)-4.184\left(\mathrm{~T}_{\mathrm{wb}}-273.15\right)}\right), \quad \mathrm{kg} / \mathrm{kg}
\end{aligned}
$$


The thermophysical properties of saturated water liquid from $273.15 \mathrm{~K}$ to $380 \mathrm{~K}$

Density

$$
\rho_{\mathrm{w}}=\left(\mathrm{a}+\mathrm{bT}+\mathrm{cT}^{2}+\mathrm{dT}^{6}\right)^{-1}, \mathrm{~kg} / \mathrm{m}^{3}
$$

where

$$
\begin{aligned}
& a=1.493430 \times 10^{-3} \\
& b=-3.716400 \times 10^{-6} \\
& c=7.097820 \times 10^{-9} \\
& d=-1.90321 \times 10^{-20}
\end{aligned}
$$

\section{Specific heat}

$$
\mathrm{c}_{\mathrm{pw}}=\mathrm{a}+\mathrm{bT}+\mathrm{cT}^{2}+\mathrm{dT}^{6}, \quad J / k g K
$$

where

$\mathrm{a}=8.155990 \times 10^{3}$

$\mathrm{b}=-2.806270 \times 10$

$c=5.112830 \times 10^{-2}$

$d=-2.175820 \times 10^{-13}$

\section{Dynamic viscosity}

$$
\mu_{\mathrm{w}}=\mathrm{a} 10^{\mathrm{b} /(\mathrm{T}-\mathrm{c})}, \mathrm{kg} / \mathrm{ms}
$$

where

$\mathrm{a}=2.414 \times 10^{-5}$

$\mathrm{b}=247.8$

$c=140$

\section{Thermal conductivity}

$$
\mathrm{k}_{\mathrm{w}}=\mathrm{a}+\mathrm{bT}+\mathrm{cT}^{2}+\mathrm{dT}^{4}, W / m K
$$

where

$$
\begin{aligned}
& a=-6.142550 \times 10^{-1} \\
& b=6.996200 \times 10^{-3} \\
& c=-1.010750 \times 10^{-5} \\
& d=4.747370 \times 10^{-12}
\end{aligned}
$$




\section{Latent heat of vaporization}

$$
\mathrm{i}_{\mathrm{fg}}=\mathrm{a}+\mathrm{bT}+\mathrm{cT^{2 }}+\mathrm{dT}^{3}, \quad J / k g
$$

where

$$
\begin{aligned}
& a=3.483181 \times 10^{6} \\
& b=-5.862770 \times 10^{3} \\
& c=1.213957 \times 10 \\
& d=-1.402904 \times 10^{-2}
\end{aligned}
$$

\section{Critical pressure}

$$
\mathrm{p}_{\mathrm{w}, \text { crit }}=22.09 \times 10^{6}, \mathrm{~Pa}
$$

\section{Surface tension}

$$
\sigma=\mathrm{a}+\mathrm{bT}+\mathrm{cT}^{2}+\mathrm{dT}^{3}, \quad N / m
$$

where

$$
\begin{aligned}
& a=5.148103 \times 10^{-2} \\
& b=3.998714 \times 10^{-4} \\
& c=-1.472187 \times 10^{-6} \\
& d=1.214053 \times 10^{-9}
\end{aligned}
$$




\section{APPENDIX H Tchebycheff numerical integration method}

The four point Tchebycheff numerical integration method is often used to approximate the integral in the Merkel equation (Equation (2.3)). According to this method an integral can be approximated by

$$
\int_{a}^{b} f(x) \partial x \approx\left(\frac{b-a}{4}\right)\left(f\left(x_{1}\right)+f\left(x_{2}\right)+f\left(x_{3}\right)+f\left(x_{4}\right)\right)
$$

where

$$
\begin{aligned}
& \mathrm{x}_{1}=\mathrm{a}+0.102673(\mathrm{~b}-\mathrm{a}) \quad \mathrm{x}_{1} \approx \mathrm{a}+0.1(\mathrm{~b}-\mathrm{a}) \\
& \mathrm{x}_{2}=\mathrm{a}+0.406204(\mathrm{~b}-\mathrm{a}) \text { or simply } \mathrm{x}_{2} \approx \mathrm{a}+0.4(\mathrm{~b}-\mathrm{a}) \\
& \mathrm{x}_{3}=\mathrm{a}+0.593796(\mathrm{~b}-\mathrm{a}) \text { or simply } \mathrm{x}_{3} \approx \mathrm{a}+0.6(\mathrm{~b}-\mathrm{a}) \\
& \mathrm{x}_{4}=\mathrm{a}+0.897327(\mathrm{~b}-\mathrm{a}) \quad \mathrm{x}_{4} \approx \mathrm{a}+0.9(\mathrm{~b}-\mathrm{a})
\end{aligned}
$$

Using the Tchebycheff integration procedure the Merkel equation can be approximated by

$$
\begin{aligned}
\frac{\mathrm{KaZ}}{\dot{\mathrm{M}}_{\mathrm{w}}} & =\int_{\mathrm{Z}} \frac{\mathrm{c}_{\mathrm{pw}} \partial \mathrm{T}_{\mathrm{w}}}{\left(\mathrm{i}_{\mathrm{asw}}-\mathrm{i}_{\mathrm{a}}\right)} \\
& \approx\left(\frac{\mathrm{c}_{\mathrm{pw}}\left(\mathrm{T}_{\mathrm{wi}}-\mathrm{T}_{\mathrm{wo}}\right)}{4}\right)\left(\frac{1}{\left(\mathrm{i}_{\text {asw }}-\mathrm{i}_{\mathrm{a}}\right)_{1}}+\frac{1}{\left(\mathrm{i}_{\text {asw }}-\mathrm{i}_{\mathrm{a}}\right)_{2}}+\frac{1}{\left(\mathrm{i}_{\text {asw }}-\mathrm{i}_{\mathrm{a}}\right)_{3}}+\frac{1}{\left(\mathrm{i}_{\text {asw }}-\mathrm{i}_{\mathrm{a}}\right)_{4}}\right)
\end{aligned}
$$

where

$\left(\mathrm{i}_{\text {asw }}\right)_{1}$ is evaluated at $\mathrm{T}_{\mathrm{w}}=\mathrm{T}_{\mathrm{wo}}+0.1\left(\mathrm{~T}_{\mathrm{wi}}-\mathrm{T}_{\mathrm{wo}}\right)$

$\left(\mathrm{i}_{\text {asw }}\right)_{2}$ is evaluated at $\mathrm{T}_{\mathrm{w}}=\mathrm{T}_{\mathrm{wo}}+0.4\left(\mathrm{~T}_{\mathrm{wi}}-\mathrm{T}_{\mathrm{wo}}\right)$

$\left(\mathrm{i}_{\text {asw }}\right)_{3}$ is evaluated at $\mathrm{T}_{\mathrm{w}}=\mathrm{T}_{\mathrm{wo}}+0.6\left(\mathrm{~T}_{\mathrm{wi}}-\mathrm{T}_{\mathrm{wo}}\right)$

$\left(\mathrm{i}_{\text {asw }}\right)_{4}$ is evaluated at $\mathrm{T}_{\mathrm{w}}=\mathrm{T}_{\mathrm{wo}}+0.9\left(\mathrm{~T}_{\mathrm{wi}}-\mathrm{T}_{\mathrm{wo}}\right)$

and

$$
\begin{aligned}
& \left(\mathrm{i}_{\mathrm{a}}\right)_{1}=\mathrm{i}_{\mathrm{ai}}+0.1\left(\mathrm{i}_{\mathrm{ao}}-\mathrm{i}_{\mathrm{ai}}\right) \\
& \left(\mathrm{i}_{\mathrm{a}}\right)_{2}=\mathrm{i}_{\mathrm{ai}}+0.4\left(\mathrm{i}_{\mathrm{ao}}-\mathrm{i}_{\mathrm{ai}}\right) \\
& \left(\mathrm{i}_{\mathrm{a}}\right)_{3}=\mathrm{i}_{\mathrm{ai}}+0.6\left(\mathrm{i}_{\mathrm{ao}}-\mathrm{i}_{\mathrm{ai}}\right) \\
& \left(\mathrm{i}_{\mathrm{a}}\right)_{4}=\mathrm{i}_{\mathrm{ai}}+0.9\left(\mathrm{i}_{\mathrm{ao}}-\mathrm{i}_{\mathrm{ai}}\right)
\end{aligned}
$$




\section{APPENDIX I Numerical sample calculation}

The following numerical example shows the calculation procedure used in the simulation program. At first it is shown how a set of experimental data is used to calculate the transfer characteristic and then the experimental data is used as input data in a sample calculation showing the calculation procedure used in the splash pack simulation program, SPSIM.

\section{Evaluation of experimental data}

The following set of data represents a typical set of measurements obtained during the testing of splash pack B (see Table 4.11) in the counterflow packing material test facility described in Chapter 4 :

Inlet water temperature, $\mathrm{T}_{\mathrm{wi}}$ :

$42.70^{\circ} \mathrm{C}$

Outlet water temperature, $\mathrm{T}_{\mathrm{wo}}$ :

$31.33^{\circ} \mathrm{C}$

Inlet air dry bulb temperature, $\mathrm{T}_{\text {aidb }}: 17.77^{\circ} \mathrm{C}$

Inlet air wet bulb temperature, $\mathrm{T}_{\text {aiwb }}: 15.34{ }^{\circ} \mathrm{C}$

Dry air mass flow rate, $\dot{\mathrm{m}}_{\mathrm{da}}$ :

$3.948 \mathrm{~kg} / \mathrm{s}$

Inlet water mass flow rate, $\dot{\mathrm{m}}_{\mathrm{wi}}$ :

$6.198 \mathrm{~kg} / \mathrm{s}$

Atmospheric pressure, $\mathrm{p}_{\mathrm{atm}}$ :

$100.4 \mathrm{kPa}$

Pressure drop across packing, $\Delta \mathrm{p}$ :

$17.5 \mathrm{~Pa}$

From the correlations in Appendix G, the inlet air enthalpy, $\mathrm{i}_{\mathrm{ai}}$, and the inlet air humidity ratio, $\mathrm{w}_{\mathrm{ai}}$, can be found to be $43.412 \mathrm{~kJ} / \mathrm{kg}$ and $0.01007 \mathrm{~kg} / \mathrm{kg}$ respectively. The specific heat at the mean water temperature, $\mathrm{c}_{\mathrm{pw}}$, is found to be $4176.9 \mathrm{~J} / \mathrm{kgK}$. By employing an energy balance of the cooling tower, the outlet air enthalpy is found as follows

$$
\begin{aligned}
\mathrm{i}_{\mathrm{ao}} & =\mathrm{i}_{\mathrm{ai}}+\left(\frac{\dot{\mathrm{m}}_{\mathrm{wi}} \mathrm{c}_{\mathrm{pw}}\left(\mathrm{T}_{\mathrm{wi}}-\mathrm{T}_{\mathrm{wo}}\right)}{\dot{\mathrm{m}}_{\mathrm{da}}}\right) \\
& =43.412+\left(\frac{6.198 \times 4176.9(42.70-31.33)}{3.948 \times 1000}\right) \\
& =117.968 \mathrm{~kJ} / \mathrm{kg}
\end{aligned}
$$

From Equations (H.4) and (H.5) it follows that

$$
\begin{aligned}
& \left(\mathrm{i}_{\text {asw }}\right)_{1}=114.640 \mathrm{~kJ} / \mathrm{kg} \text { at } \mathrm{T}_{\mathrm{w}}=\mathrm{T}_{\mathrm{wo}}+0.1\left(\mathrm{~T}_{\mathrm{wi}}-\mathrm{T}_{\mathrm{wo}}\right)=32.47^{\circ} \mathrm{C} \\
& \left(\mathrm{i}_{\text {asw }}\right)_{2}=136.498 \mathrm{~kJ} / \mathrm{kg} \text { at } \mathrm{T}_{\mathrm{w}}=\mathrm{T}_{\mathrm{wo}}+0.4\left(\mathrm{~T}_{\mathrm{wi}}-\mathrm{T}_{\mathrm{wo}}\right)=35.88^{\circ} \mathrm{C} \\
& \left(\mathrm{i}_{\text {asw }}\right)_{3}=153.131 \mathrm{~kJ} / \mathrm{kg} \text { at } \mathrm{T}_{\mathrm{w}}=\mathrm{T}_{\mathrm{wo}}+0.6\left(\mathrm{~T}_{\mathrm{wi}}-\mathrm{T}_{\mathrm{wo}}\right)=38.15^{\circ} \mathrm{C} \\
& \left(\mathrm{i}_{\text {asw }}\right)_{4}=181.820 \mathrm{~kJ} / \mathrm{kg} \text { at } \mathrm{T}_{\mathrm{w}}=\mathrm{T}_{\mathrm{wo}}+0.9\left(\mathrm{~T}_{\mathrm{wi}}-\mathrm{T}_{\mathrm{wo}}\right)=41.56^{\circ} \mathrm{C}
\end{aligned}
$$




$$
\begin{aligned}
& \left(\mathrm{i}_{\mathrm{a}}\right)_{1}=\mathrm{i}_{\mathrm{ai}}+0.1\left(\mathrm{i}_{\mathrm{ao}}-\mathrm{i}_{\mathrm{ai}}\right)=50.868 \mathrm{~kJ} / \mathrm{kg} \\
& \left(\mathrm{i}_{\mathrm{a}}\right)_{2}=\mathrm{i}_{\mathrm{ai}}+0.4\left(\mathrm{i}_{\mathrm{ao}}-\mathrm{i}_{\mathrm{ai}}\right)=73.234 \mathrm{~kJ} / \mathrm{kg} \\
& \left(\mathrm{i}_{\mathrm{a}}\right)_{3}=\mathrm{i}_{\mathrm{ai}}+0.6\left(\mathrm{i}_{\mathrm{ao}}-\mathrm{i}_{\mathrm{ai}}\right)=88.146 \mathrm{~kJ} / \mathrm{kg} \\
& \left(\mathrm{i}_{\mathrm{a}}\right)_{4}=\mathrm{i}_{\mathrm{ai}}+0.9\left(\mathrm{i}_{\mathrm{ao}}-\mathrm{i}_{\mathrm{ai}}\right)=110.512 \mathrm{~kJ} / \mathrm{kg}
\end{aligned}
$$

The transfer characteristic can be calculated from Equation (H.3) as

$$
\begin{aligned}
\frac{\mathrm{KaZ}}{\dot{\mathrm{M}}_{\mathrm{w}}} & \approx\left(\frac{\mathrm{c}_{\mathrm{pw}}\left(\mathrm{T}_{\mathrm{wi}}-\mathrm{T}_{\mathrm{wo}}\right)}{4}\right)\left(\frac{1}{\left(\mathrm{i}_{\text {asw }}-\mathrm{i}_{\mathrm{a}}\right)_{1}}+\frac{1}{\left(\mathrm{i}_{\text {asw }}-\mathrm{i}_{\mathrm{a}}\right)_{2}}+\frac{1}{\left(\mathrm{i}_{\mathrm{asw}}-\mathrm{i}_{\mathrm{a}}\right)_{3}}+\frac{1}{\left(\mathrm{i}_{\text {asw }}-\mathrm{i}_{\mathrm{a}}\right)_{4}}\right) \\
& \approx\left(\frac{4176.9(42.70-31.33)}{4 \times 1000}\right)\left(\frac{1}{(114.640-50.868)}+\frac{1}{(136.498-73.234)}+\right. \\
& \approx 0.723
\end{aligned}
$$

The calculated transfer characteristic can be corrected for inlet and outlet effects (due to heat and mass transfer on the water distribution system and the water collecting troughs) by subtracting the transfer characteristic for the empty tower at $Z=0 \mathrm{~m}$. From Equation (4.47) the empty tower transfer characteristic at $Z=0 m$ is

$$
\begin{aligned}
\frac{\mathrm{KaZ}}{\dot{\mathrm{M}}_{\mathrm{w}}} & =0.125 \dot{\mathrm{M}}_{\mathrm{w}}^{0.044} \dot{\mathrm{M}}_{\mathrm{a}}^{0.666} \\
& =0.125 \times\left(\frac{\dot{\mathrm{m}}_{\mathrm{wi}}}{\mathrm{A}_{\mathrm{fr}}}\right)^{0.044} \times\left(\frac{\dot{\mathrm{m}}_{\mathrm{da}}\left(1+\mathrm{w}_{\mathrm{ai}}\right)}{\mathrm{A}_{\mathrm{fr}}}\right)^{0.666} \\
& =0.125 \times\left(\frac{6.198}{2.25}\right)^{0.044} \times\left(\frac{3.948(1+0.01007)}{2.25}\right)^{0.666} \\
& =0.191
\end{aligned}
$$

The corrected transfer characteristic of the splash pack is

$$
\begin{aligned}
\frac{\mathrm{KaZ}}{\dot{\mathrm{M}}_{\mathrm{w}}} & =\left(\frac{\mathrm{KaZ}}{\dot{\mathrm{M}}_{\mathrm{w}}}\right)_{\text {measured }}-\left(\frac{\mathrm{KaZ}}{\dot{\mathrm{M}}_{\mathrm{w}}}\right)_{\mathrm{z}=0} \\
& =0.723-0.191 \\
& =0.532
\end{aligned}
$$




\section{Mathematical evaluation of splash pack}

From Table 4.11 the layout of experimental splash pack B is as follows :

Slat width, W

Slat height, $\mathrm{H}$

Slat pitch, $\mathrm{P}$

Grid pitch (vertical), $\mathrm{P}_{\mathrm{v}}$

Total grid surface area, $A_{\text {grid }}$

Number of grids, $\mathrm{N}_{\text {grids }}$

Air flow area (or $\mathrm{L}_{\text {grid }} \times \mathrm{W}_{\text {grid }}$ ), $\mathrm{A}_{\mathrm{fr}}$

Spray zone depth, $Z_{\text {spray }}$

Packing depth, $Z_{\text {pack }}$

Rain zone depth, $Z_{\text {rain }}$
$9 \mathrm{~mm}$

$22 \mathrm{~mm}$

$50 \mathrm{~mm}$

$200 \mathrm{~mm}$

$2.79 \mathrm{~m}^{2}$ (per grid)

10

$2.25 \mathrm{~m}^{2}$

$500 \mathrm{~mm}$

$2000 \mathrm{~mm}$

$0 \mathrm{~mm}$

From the known ambient conditions, the following air properties can be calculated from the correlations in Appendix G (note that the properties are those of the air and vapour mixture):

Inlet air enthalpy, $\mathrm{i}_{\text {ai }}$

Inlet air humidity ratio, $\mathrm{w}_{\mathrm{ai}}$

Inlet air density, $\rho_{\mathrm{ai}}$

Inlet air dynamic viscosity, $\mu_{\mathrm{ai}}$

Inlet air specific heat, $\mathrm{c}_{\text {pai }}$

Inlet air thermal conductivity, $\mathrm{k}_{\mathrm{ai}}$

$43.412 \mathrm{~kJ} / \mathrm{kg}$
$0.01007 \mathrm{~kg} /(\mathrm{kg}$ dry air)
$1.195 \mathrm{~kg} / \mathrm{m}^{3}$
$1.7945 \times 10^{-5} \mathrm{~kg} / \mathrm{ms}$
$015.349 \mathrm{~J} / \mathrm{kgK}$
$0.025415 \mathrm{~W} / \mathrm{mK}$

Assume the air outlet enthalpy to be $\mathrm{i}_{\mathrm{ao}}=121.232 \mathrm{~kJ} / \mathrm{kg}$. Assuming the outlet air to be saturated the following air properties can be calculated from the correlations in Appendix G:

Outlet air temperature, $\mathrm{T}_{\mathrm{ao}}$

Outlet air humidity ratio, $\mathrm{w}_{\mathrm{ao}}$

Outlet air density, $\rho_{\mathrm{ao}}$

Outlet air dynamic viscosity, $\mu_{\mathrm{ao}}$

Outlet air specific heat, $\mathrm{c}_{\text {pao }}$

Outlet air thermal conductivity, $\mathrm{k}_{\mathrm{ao}}$
$33.557{ }^{\circ} \mathrm{C}$

$0.03409 \mathrm{~kg} /(\mathrm{kg}$ dry air)

$1.118 \mathrm{~kg} / \mathrm{m}^{3}$

$1.8423 \times 10^{-5} \mathrm{~kg} / \mathrm{ms}$

$1036.394 \mathrm{~J} / \mathrm{kgK}$

$0.02640 \mathrm{~W} / \mathrm{mK}$

The mean air properties can be found by averaging the inlet and outlet properties :

Mean air humidity ratio, $\mathrm{w}_{\mathrm{am}}$

Mean air density, $\rho_{\text {am }}$

Mean air dynamic viscosity, $\mu_{\mathrm{am}}$

Mean air specific heat, $\mathrm{c}_{\text {pam }}$

Mean air thermal conductivity, $\mathrm{k}_{\mathrm{am}}$

Mean air Prandtl number, $\operatorname{Pr}_{\mathrm{am}}$
$0.02208 \mathrm{~kg} /(\mathrm{kg}$ dry air)

$1.157 \mathrm{~kg} / \mathrm{m}^{3}$

$1.8184 \times 10^{-5} \mathrm{~kg} / \mathrm{ms}$

$1025.871 \mathrm{~J} / \mathrm{kgK}$

$0.02591 \mathrm{~W} / \mathrm{mK}$

0.720 
The mean air velocity can be calculated as

$$
\begin{aligned}
\mathrm{v}_{\mathrm{am}} & =\left(\frac{\dot{\mathrm{m}}_{\mathrm{da}}\left(1+\mathrm{w}_{\mathrm{am}}\right)}{\rho_{\mathrm{am}} \mathrm{A}_{\mathrm{fr}}}\right) \\
& =\left(\frac{3.948(1+0.02208)}{1.157 \times 2.25}\right) \\
& =1.550 \mathrm{~m} / \mathrm{s}
\end{aligned}
$$

The initial drop size distribution formed by the water distribution system in the test facility can be found by interpolation from Appendix P. The temperature correction described by Equation (4.31) is used to scale the distribution data to account for the difference in water temperature used during the experimental work and that used in the current calculation. Assuming 36 drop size classes linearly spaced between 0 and $12 \mathrm{~mm}$, it follows that there are 36 packets at the top of the spray zone. Packet 1, representing $0.166 \mathrm{~mm}$ drops, contains $0.074 \%$ of the total water mass flow rate at the top of the spray zone, i.e. $0.00457 \mathrm{~kg} / \mathrm{s}$. Packet 14 represents a drop size of $4.5 \mathrm{~mm}$ and a water mass flow rate of $0.21564 \mathrm{~kg} / \mathrm{s}$. The initial velocity of the drops leaving the water distribution system is zero and all the drops initially have the same temperature, $\mathrm{T}_{\text {wi }}$.

\section{Drop motion and cooling}

To illustrate the integration procedure through the splash pack only packet 14 is considered. The cooling and acceleration of the drops in the other packets can be calculated in a similar fashion. In order to calculate the air inlet enthalpy for every element, the total heat and mass transfer rate of all the drops in all the packets in the element must be used.

The spray zone above the packing is subdivided into 4 elements each with $\partial \mathrm{z}=0.125 \mathrm{~m}$. Assume the drop velocity (of the drops in packet 14) after the first element to be $\mathrm{v}_{\mathrm{do}}=1.521$ $\mathrm{m} / \mathrm{s}$. The mean drop velocity in the element is thus $\mathrm{v}_{\mathrm{dm}}=(0+1.521) / 2=0.761 \mathrm{~m} / \mathrm{s}$. The drop Reynolds number based on the relative velocity between the drop and the air can be found as

$$
\begin{aligned}
\operatorname{Re} & =\frac{\rho_{\mathrm{am}}\left(\mathrm{v}_{\mathrm{am}}+\mathrm{v}_{\mathrm{dm}}\right) \mathrm{d}}{\mu_{\mathrm{am}}} \\
& =\frac{1.157(1.550+0.761) 0.0045}{1.8184 \times 10^{-5}} \\
& =661.60
\end{aligned}
$$

The drag coefficient experienced by the falling drop can be calculated using Equation (2.15), 


$$
\begin{aligned}
C_{D} & =\frac{24}{\operatorname{Re}}\left(1+0.173 \operatorname{Re}^{0.657}\right)+\left(\frac{0.413}{1+16300 \mathrm{Re}^{-1.09}}\right) \\
& =\frac{24}{661.60}\left(1+0.173 \times 661.60^{0.657}\right)+\left(\frac{0.413}{1+16300 \times 661.60^{-1.09}}\right) \\
& =0.512
\end{aligned}
$$

The water density at the water inlet temperature can be found from the correlation in Appendix $\mathrm{G}$ as $\rho_{\mathrm{w}}=991.28 \mathrm{~kg} / \mathrm{m}^{3}$. The downward force experience by the drop can be found from Equation (5.5):

$$
\begin{aligned}
F_{\text {down }} & =\left(\pi d^{3} / 6\right)\left(\rho_{w}-\rho_{a m}\right) g \\
& =\left(\pi \times 0.0045^{3} / 6\right)(991.28-1.157) 9.8 \\
& =4.630 \times 10^{-4} \mathrm{~N}
\end{aligned}
$$

and the upward drag force experienced by the drop can calculated from Equation (5.4) as

$$
\begin{aligned}
F_{\text {up }} & =0.5 \rho_{\mathrm{am}}\left(\mathrm{v}_{\mathrm{am}}+\mathrm{v}_{\mathrm{dm}}\right)^{2} \mathrm{~A}_{\mathrm{fr}, \mathrm{d}} \mathrm{C}_{\mathrm{D}} \\
& =0.5 \times 1.157(1.550+0.761)^{2}\left(\pi \times 0.0045^{2} / 4\right) 0.512 \\
& =2.514 \times 10^{-5} \mathrm{~N}
\end{aligned}
$$

From the force balance, it follows that the average drop acceleration in the element is

$$
\begin{aligned}
a & =\frac{\left(F_{\text {down }}-F_{\text {up }}\right)}{m_{d}} \\
& =\frac{\left(4.630 \times 10^{-4}-2.514 \times 10^{-5}\right)}{991.28\left(\pi \times 0.0045^{3} / 6\right)} \\
& =9.257 \mathrm{~m} / \mathrm{s}^{2}
\end{aligned}
$$

The velocity of the drop leaving the element can be found from

$$
\begin{aligned}
\mathrm{v}_{\mathrm{do}} & =\sqrt{\mathrm{v}_{\mathrm{di}}^{2}+2 \mathrm{a} \partial \mathrm{z}} \\
& =\sqrt{0^{2}+2 \times 9.257 \times 0.125} \\
& =1.521 \mathrm{~m} / \mathrm{s}
\end{aligned}
$$

Since the terminal velocity of the drops in packet $1(\mathrm{~d}=0.166 \mathrm{~mm})$ is lower than the upward air velocity, the drops in packet 1 will travel upwards. The mass of drops in packet 1 is redistributed among the remaining 35 packets based on the probability of collision between the drops in every other packet with the drops in packet 1 . After redistributing the water mass flow rate of packet 1 , packet 14 now represents a mass flow rate of $0.21584 \mathrm{~kg} / \mathrm{s}$. Due to the 
very small mass flow rate represented by packet 1 , the velocity and temperature of the drops in packet 14 is not noticeably influenced.

The total number of drops in packet 14 in the element can be calculated from Equation (5.7) as

$$
\begin{aligned}
\mathrm{N} & =\left(\frac{\dot{\mathrm{m}}_{\mathrm{w}}}{\rho_{\mathrm{w}} \mathrm{V}_{\mathrm{d}}}\right)\left(\frac{\partial \mathrm{z}}{\mathrm{v}_{\mathrm{dm}}}\right) \\
& =\left(\frac{0.21584}{991.28\left(\pi \times 0.0045^{3} / 6\right)}\right)\left(\frac{0.125}{0.761}\right) \\
& =749.95
\end{aligned}
$$

The pressure drop due to the drops in packet 14 in the element can be calculated as follows

$$
\begin{aligned}
\Delta \mathrm{p} & =\frac{\mathrm{NF}_{\mathrm{up}}}{\mathrm{A}_{\mathrm{fr}}} \\
& =\frac{749.95 \times 2.514 \times 10^{-5}}{2.25} \\
& =8.380 \times 10^{-3} \mathrm{~Pa}
\end{aligned}
$$

The heat transfer coefficient on the outside of the drop can be found from the Ranz and Marshall correlation, Equation (2.23), i.e.

$$
\begin{aligned}
\mathrm{Nu} & =2+0.6 \operatorname{Re}^{1 / 2} \operatorname{Pr}^{1 / 3} \\
& =2+0.6(661.60)^{1 / 2}(0.720)^{1 / 3} \\
& =15.832
\end{aligned}
$$

The mass transfer coefficient can be found from the analogy between heat and mass transfer, Equation (5.6) as

$$
\begin{aligned}
K & =\frac{N u k_{a m}}{L e_{f} c_{\text {pam }} d} \\
& =\frac{15.832 \times 0.02591}{1.0 \times 1025.871 \times 0.0045} \\
& =0.0889 \mathrm{~kg} / \mathrm{m}^{2} s
\end{aligned}
$$

Using the correlations in Appendix G, the air saturation enthalpy at the water temperature can be found to be $\mathrm{i}_{\text {asw }}=192.560 \mathrm{~kJ} / \mathrm{kg}$ and the water specific heat at the inlet water temperature can be found to be $c_{p w}=4176.981 \mathrm{~J} / \mathrm{kgK}$. The temperature change of the drops in packet 14 in the element can be found as follows from Equations (3.1) and (3.2) 


$$
\begin{aligned}
\partial \mathrm{T}_{\mathrm{w}} & =\frac{\mathrm{K}\left(\mathrm{N} \pi \mathrm{d}^{2}\right)\left(\mathrm{i}_{\mathrm{asw}}-\mathrm{i}_{\mathrm{a}}\right)}{\mathrm{c}_{\mathrm{pw}} \dot{\mathrm{m}}_{\mathrm{w}}} \\
& =\frac{0.0889\left(749.95 \times \pi \times 0.0045^{2}\right)(192.560-121.232) 1000}{4176.981 \times 0.21584} \\
& =0.335^{\circ} \mathrm{C}
\end{aligned}
$$

The temperature of the drops leaving the element can now be calculated as

$$
\begin{aligned}
\mathrm{T}_{\mathrm{wo}} & =\mathrm{T}_{\mathrm{wi}}-\partial \mathrm{T}_{\mathrm{w}} \\
& =42.70-0.335 \\
& =42.365^{\circ} \mathrm{C}
\end{aligned}
$$

Continuing with the integration process to the top splash grid $(0.5 \mathrm{~m}$ from the start of the spray zone), it can be shown the drops in packet 14 cool down to $42.023{ }^{\circ} \mathrm{C}$, the air enthalpy at the first grid is $118.023 \mathrm{~kJ} / \mathrm{kg}$ and the drop velocity is $2.963 \mathrm{~m} / \mathrm{s}$. The water density and surface tension at $42.023^{\circ} \mathrm{C}$ can be found to be $991.55 \mathrm{~kg} / \mathrm{m}^{3}$ and $0.0692 \mathrm{~N} / \mathrm{m}$, respectively.

\section{Grid interaction}

Upon reaching the first splash grid, after integration through the $0.5 \mathrm{~m}$ spray zone, only a fraction of the drops in packet 14 would strike the grid. From the grid geometry the water mass flow rate (in packet 14) striking the grid can be found as

$$
\begin{aligned}
\dot{\mathrm{m}}_{\text {wi,grid }} & =\dot{\mathrm{m}}_{\mathrm{wi}}\left(\frac{\mathrm{W}+\mathrm{d}_{\mathrm{i}}}{\mathrm{P}}\right) \\
& =0.21584\left(\frac{0.009+0.0045}{0.05}\right) \\
& =0.05827 \mathrm{~kg} / \mathrm{s}
\end{aligned}
$$

The mass of water splashing from the test grid can be calculated from the empirical correlations listed in Chapter 4. Interpolation between the splash fractions for impacts on 5 and $10 \mathrm{~mm}$ slats is required to find the splash fraction for the impact on the $9 \mathrm{~mm}$ slat. From Figures (4.9) and (4.10) it can be concluded that the film thickness on the slats of the splash grids would be approximately $0.5 \mathrm{~mm}$ at the current air and water mass fluxes. The maximum stable drop diameter at STP can be found from Equation (4.19) to be $10.85 \mathrm{~mm}$ and the terminal velocity of such a drop can be found to be $\mathrm{v}_{\mathrm{T}, \mathrm{dm}}=9.075 \mathrm{~m} / \mathrm{s}$. The Weber number of the incoming drops and the reference Weber number can be found from Equations (4.17) and (4.18) as 
$\mathrm{We}=\frac{\rho_{\mathrm{w}} \mathrm{v}_{\mathrm{i}}^{2} \mathrm{~d}_{\mathrm{i}}}{\sigma}=\frac{991.55 \times 2.963^{2} \times 0.0045}{0.0692}=565.69$

and

$\mathrm{We}_{\text {ref }}=\left(\frac{\rho_{\mathrm{w}} \mathrm{v}_{\mathrm{T}, \mathrm{d}_{\mathrm{m}}}^{2} \mathrm{~d}_{\mathrm{m}}}{\sigma}\right)_{\text {ref }}=\frac{1000 \times 9.075^{2} \times 0.01085}{0.072}=12408.94$.

From Equation (4.16), the splash fraction for the drops in packet 14 striking a $5 \mathrm{~mm}$ wide slat can be calculated as

$$
\begin{aligned}
\left(\overline{f_{\mathrm{s}}}\right)_{5}= & 0.01\left(6.613+8.200\left(\frac{\delta}{\mathrm{W}}\right)\right)\left(5.506 \times 10^{-1}+12.551\left(\frac{\mathrm{d}_{\mathrm{i}}}{\mathrm{W}}\right)^{-1.792}\right) \times \\
& \left(-12.550+13.700\left(\left(\frac{\mathrm{We}}{\mathrm{We}}\right)\left(\frac{\mathrm{d}_{\mathrm{i}}}{\mathrm{W}}\right)^{2}\right)^{1.628 \times 10^{-2}}\right) \\
& =0.01\left(6.613+8.200\left(\frac{0.0005}{0.005}\right)\right)\left(5.506 \times 10^{-1}+12.551\left(\frac{0.0045}{0.005}\right)^{-1.792}\right) \times \\
& =0.506
\end{aligned}
$$

Similarly, for a $10 \mathrm{~mm}$ wide slat it follows that $\left(\mathrm{f}_{\mathrm{s}}\right)_{10}=0.489$. By interpolation the mean splash fraction for a $9 \mathrm{~mm}$ slat width is $\left(\mathrm{f}_{\mathrm{s}}\right)_{9}=0.492$. The total water mass flow rate splashing from the grid, due to the drops from packet 14 striking the grid, can be expressed as

$$
\begin{aligned}
\dot{\mathrm{m}}_{\mathrm{s}} & =\left(\overline{\mathrm{f}_{\mathrm{s}}}\right) \dot{\mathrm{m}}_{\text {wi,grid }} \\
& =0.492 \times 0.05827 \\
& =0.02867 \mathrm{~kg} / \mathrm{s}
\end{aligned}
$$

Only a fraction of the water drops in packet 14 which strike the grid will form cutting drops. This fraction can be calculated from Equation (3.20) as

$$
\begin{aligned}
\overline{f_{c}} & =\left(\frac{d_{i}}{w+d_{i}}\right) \\
& =\left(\frac{0.0045}{0.009+0.0045}\right) \\
& =0.333
\end{aligned}
$$


The total mass flow rate of drops from packet 14 , leaving the grid as drops formed by cutting can be expressed as

$$
\begin{aligned}
\dot{\mathrm{m}}_{\mathrm{c}} & =\left(\overline{\mathrm{f}_{\mathrm{c}}}\right) \dot{\mathrm{m}}_{\text {wi,grid }} \\
& =0.333 \times 0.05827 \\
& =0.01942 \mathrm{~kg} / \mathrm{s}
\end{aligned}
$$

Repeating the above calculations of the splashing and cutting mass for each of the other packets of drops striking the grid it can be shown that the mass flow rate of the water dripping below the slats is (see Equation (5.24))

$$
\begin{aligned}
\dot{\mathrm{m}}_{\mathrm{d}} & =\sum_{\mathrm{j}}\left(\dot{\mathrm{M}}_{\mathrm{w}} \mathrm{L}\left(\mathrm{W}+\mathrm{d}_{\mathrm{i}, \mathrm{j}}\right)\right)-\sum_{\mathrm{j}}\left(\overline{\mathrm{f}_{\mathrm{s}, \mathrm{j}}} \dot{\mathrm{M}}_{\mathrm{w}} \mathrm{L}\left(\mathrm{W}+\mathrm{d}_{\mathrm{i}, \mathrm{j}}\right)\right)-\sum_{\mathrm{j}}\left(\overline{\mathrm{f}_{\mathrm{c}, \mathrm{j}}} \dot{\mathrm{M}}_{\mathrm{w}} \mathrm{L}\left(\mathrm{W}+\mathrm{d}_{\mathrm{i}, \mathrm{j}}\right)\right) \\
& =1.99747-0.77444-0.88166 \\
& =0.34136 \mathrm{~kg} / \mathrm{s}
\end{aligned}
$$

Assuming a mixing ratio of $\phi=0.5$ and by employing Equations (3.26) and (3.27) the mean temperature of the water film on top of the slats of the top grid can be found to be $T_{\mathrm{fi}}=42.054$ ${ }^{\circ} \mathrm{C}$.

The splash drops formed by the impact of the drops in packet 14 are distributed according to the Rosin-Rammler distribution function with the size and shape parameters defined by Equations (4.24) to (4.25). It follows that

$$
\begin{aligned}
\mathrm{n}_{\mathrm{RR}} & =\left(24.532-75.174\left(\frac{\delta}{\mathrm{d}_{\mathrm{i}}}\right)\left(\frac{\mathrm{We}_{\mathrm{i}}}{\mathrm{We}_{\mathrm{ref}}}\right)^{0.74}\right)\left(0.149+6.801 \times 10^{-4}\left(\frac{\mathrm{KE}}{\mathrm{KE}_{\mathrm{ref}}}\right)^{-0.76}\right) \\
& =\left(24.532-75.174\left(\frac{0.0005}{0.0045}\right)\left(\frac{565.69}{12408.94}\right)^{0.74}\right) \times \\
& \left(0.149+6.801 \times 10^{-4}\left(\frac{0.0045^{3} \times 2.963^{2}}{0.01085^{3} \times 9.075^{2}}\right)^{-0.76}\right) \\
& =4.189
\end{aligned}
$$

and

$$
\mathrm{d}_{50}=\mathrm{d}_{\mathrm{m}}\left(\begin{array}{l}
3.08 \times 10^{-2}+\left(-0.163+4.560 \times 10^{-2}\left(\frac{\delta}{\mathrm{d}_{\mathrm{m}}}\right)^{-0.34}\right) \times \\
\left(-0.804-0.619\left(\frac{\mathrm{We}}{\mathrm{We}_{\mathrm{ref}}}\right)^{-0.27}\right)\left(-1.738+1.980\left(\frac{\delta}{\mathrm{d}_{\mathrm{i}}}\right)^{-0.17}\right)
\end{array}\right)
$$




$$
\begin{aligned}
& =0.01085\left(\begin{array}{l}
3.08 \times 10^{-2}+\left(-0.163+4.560 \times 10^{-2}\left(\frac{0.0005}{0.01085}\right)^{-0.34}\right) \times \\
\left(-0.804-0.619\left(\frac{565.69}{12408.94}\right)^{-0.27}\right)\left(-1.738+1.980\left(\frac{0.0005}{0.0045}\right)^{-0.17}\right)
\end{array}\right) \\
& =1.248 \mathrm{~mm}
\end{aligned}
$$

and

$$
\begin{aligned}
\mathrm{d}_{\mathrm{RR}} & =\mathrm{d}_{50}\left(0.6931^{-\left(\mathrm{l} / \mathrm{n}_{\mathrm{RR}}\right)}\right) \\
& =1.248\left(0.6931^{-(1 / 4.189)}\right) \\
& =1.362 \mathrm{~mm}
\end{aligned}
$$

The water mass flow rate in a new packet, say packet $A$, formed by the splash drops of diameter $d_{s}=1.1666 \mathrm{~mm}$ can be found from the Rosin-Rammler distribution function, Equation (4.22) as

$$
\begin{aligned}
\left(\mathrm{m}_{\mathrm{s}}\right)_{\mathrm{A}} & =\dot{\mathrm{m}}_{\mathrm{s}}\left(\left(1-\exp \left(-\left(\frac{\mathrm{d}}{\mathrm{d}_{\mathrm{RR}}}\right)^{\mathrm{n}_{\mathrm{RR}}}\right)\right)_{\mathrm{i}}-\left(1-\exp \left(-\left(\frac{\mathrm{d}}{\mathrm{d}_{\mathrm{RR}}}\right)^{\mathrm{n}_{\mathrm{RR}}}\right)\right)_{\mathrm{i}-1}\right) \\
& =0.02867\left(\left(1-\exp \left(-\left(\frac{0.001333}{0.001362}\right)^{4.189}\right)\right)_{\mathrm{i}}-\left(1-\exp \left(-\left(\frac{0.001000}{0.001362}\right)^{4.189}\right)\right)_{\mathrm{i}-1}\right) \\
& =0.01032 \mathrm{~kg} / \mathrm{s}
\end{aligned}
$$

Two alternative models can be employed to describe the initial velocities and temperatures of the new packets formed by splashing after the impact of the drops from packet 14. Firstly, the drops can be assumed to start from zero velocity and with an initial temperature given by Equation (3.26), i.e.

$$
\begin{aligned}
\mathrm{T}_{\mathrm{s}} & =\phi \mathrm{T}_{\mathrm{f}}+(1-\phi) \mathrm{T}_{\mathrm{i}} \\
& =0.5 \times 42.054+(1-0.5) 42.023 \\
& =42.039{ }^{\circ} \mathrm{C}
\end{aligned}
$$

or, alternatively the upward motion of the drops above the plane of the grid can be evaluated, using the analytical approach described in Appendix D, to find the initial temperatures and velocities of the splash drops. If the analytical model is used to solve the splash drop motion during upsplash the initial upward velocity of the splash drops can be calculated from Equation (4.27). For packet A, representing splash drops with $d_{s}=1.166 \mathrm{~mm}$, it can be shown that the initial splash drop velocity is given by 


$$
\begin{aligned}
\mathrm{v}_{\mathrm{si}} & =\mathrm{v}_{\mathrm{T}}\left(\mathrm{d}_{\mathrm{m}}\right)\left(1.925 \times 10^{-2}+\left(\frac{7.372 \times 10^{-3}}{\left(\mathrm{~d}_{\mathrm{s}} / \mathrm{d}_{\mathrm{m}}\right)-0.01}\right)\right) \\
& =9.075\left(1.925 \times 10^{-2}+\left(\frac{7.372 \times 10^{-3}}{(0.001166 / 0.01085)-0.01}\right)\right) \\
& =0.861 \mathrm{~m} / \mathrm{s}
\end{aligned}
$$

Assuming an initial splash angle of $\theta=60^{\circ}$, it can be found that the splash drops in Packet A cool down from $42.039^{\circ} \mathrm{C}$ to $35.973{ }^{\circ} \mathrm{C}$ and that the initial downward velocity of the drops from the plane of the grid is $0.663 \mathrm{~m} / \mathrm{s}$. The pressure drop (average drag force divided by the air flow area, $\mathrm{A}_{\mathrm{fr}}$ ) due to the splash drop drag experienced by the drops in packet $\mathrm{A}$ can be found to be $0.00294 \mathrm{~Pa}$. If the cooling of the splash drops during upsplash is considered, there is a slight change in the air enthalpy which has to be taken into account.

The mean drop size of the drops formed by cutting from the impacts of drops from packet 14 can be found from Equation (3.28) as

$$
\begin{aligned}
\mathrm{d}_{\mathrm{c}} & =\sqrt[3]{\frac{1}{2}\left(\frac{6}{\pi}\right)\left(\frac{\pi \mathrm{d}_{\mathrm{i}}^{3}}{6}\right)} \\
& =\sqrt[3]{\frac{1}{2} \times 0.0045^{3}} \\
& =3.572 \mathrm{~mm}
\end{aligned}
$$

The initial velocity and temperature of the drops formed by cutting is assumed to be the same as that of the drops from which they were formed. The drops formed by cutting form a new packet, which is used in the further integration through the splash pack.

Before the water drips from below the grid, the water is cooled by simultaneous heat and mass transfer with the surrounding air. The Reynolds number for the air flow through the grids can be expressed as

$$
\begin{aligned}
\operatorname{Re} & =\frac{\rho_{\mathrm{am}}\left(\mathrm{v}_{\mathrm{am}} \mathrm{P} /(\mathrm{P}-\mathrm{W})\right) \mathrm{d}_{\mathrm{h}}}{\mu_{\mathrm{am}}} \\
& =\frac{1.157(1.550 \times 0.050 /(0.050-0.009))(0.062 / 2)}{1.8184 \times 10^{-5}} \\
& =3728.53
\end{aligned}
$$

The Nusselt number for heat transfer from the slats can be found from Equation (4.32): 


$$
\begin{aligned}
\mathrm{Nu} & =0.3+\sqrt{\left(0.664 \sqrt{\operatorname{Re}} \operatorname{Pr}^{1 / 3}\right)^{2}+\left(\frac{0.037 \operatorname{Re}^{0.8} \operatorname{Pr}}{1+2.443 \operatorname{Re}^{-0.1}\left(\operatorname{Pr}^{2 / 3}-1\right)}\right)^{2}} \\
& =0.3+\sqrt{\left(0.664 \sqrt{3728.53} \times 0.720^{1 / 3}\right)^{2}+\left(\frac{0.037 \times 3728.53^{0.8} \times 0.720}{1+2.443 \times 3728.53^{-0.1}\left(0.720^{2 / 3}-1\right)}\right)^{2}} \\
& =44.02
\end{aligned}
$$

The mass transfer coefficient can be found from Equation (5.6) as

$$
\begin{aligned}
K & =\frac{\mathrm{Nuk}_{\mathrm{am}}}{\operatorname{Le}_{\mathrm{f}} \mathrm{c}_{\text {pam }} \mathrm{d}_{\mathrm{h}}} \\
& =\frac{44.02 \times 0.02591}{1.0 \times 1025.871(0.062 / 2)} \\
& =0.03586 \mathrm{~kg} / \mathrm{m}^{2} \mathrm{~s}
\end{aligned}
$$

From the correlations in Appendix G, the specific heat of the water and saturation enthalpy of air at $\mathrm{T}_{\mathrm{fi}}=42.054{ }^{\circ} \mathrm{C}$ is $4176.90 \mathrm{~J} / \mathrm{kgK}$ and $186.41 \mathrm{~kJ} / \mathrm{kg}$ respectively. From Equation (5.23) it follows that

$$
\begin{aligned}
\mathrm{T}_{\mathrm{fo}} & =\mathrm{T}_{\mathrm{fi}}-\left(\frac{\mathrm{KA} \text { grid }}{\left.\dot{\mathrm{m}}_{\mathrm{d}} \mathrm{i}_{\mathrm{asw}}-\mathrm{i}_{\mathrm{a}}\right)}\right) \\
& =42.054-\left(\frac{0.03586 \times 2.79(186.41-118.023) 1000}{0.34136 \times 4176.90}\right) \\
& =37.255{ }^{\circ} \mathrm{C}
\end{aligned}
$$

Due to the heat/mass transfer occurring on the slat surface, there is a change in the enthalpy of the air across the grid equal to

$$
\begin{aligned}
\partial \mathrm{i}_{\mathrm{a}} & =\frac{\dot{\mathrm{m}}_{\mathrm{d}} \mathrm{c}_{\mathrm{pw}}\left(\mathrm{T}_{\mathrm{fi}}-\mathrm{T}_{\mathrm{fo}}\right)}{\dot{\mathrm{m}}_{\mathrm{da}}} \\
& =\frac{0.34136 \times 4176.90(42.054-37.255)}{3.948} \\
& =1.733 \mathrm{~kJ} / \mathrm{kg}
\end{aligned}
$$

The mass flow rate of drops dripping below the grid is redistributed into new packets using interpolation between the dripping drop size distribution data tables given in Appendix $\mathrm{N}$ or by employing the simple model based on the work by Yung et al. [80YU1] described in Chapters 2 and 3. From Appendix $G$ the water surface tension and density at $T_{\text {fo }}=37.255^{\circ} \mathrm{C}$ is 0.0700 $\mathrm{N} / \mathrm{m}$ and $993.34 \mathrm{~kg} \mathrm{~m}^{3}$. According to the Yung model the primary dripping drop size will be 


$$
\begin{aligned}
d_{p} & =3.0 \sqrt{\frac{\sigma}{g\left(\rho_{w}-\rho_{a}\right)}} \\
& =3.0 \sqrt{\frac{0.0700}{9.8(993.34-1.157)}} \\
& =8.051 \mathrm{~mm}
\end{aligned}
$$

According to Equation (2.38) the five small satellite drops will be linearly distributed in the range

$$
\begin{aligned}
0.24 \mathrm{~d}_{\mathrm{p}} & <\mathrm{d}_{\mathrm{s}}<0.46 \mathrm{~d}_{\mathrm{p}} \\
1.932 & <\mathrm{d}_{\mathrm{s}}<3.703 \mathrm{~mm}
\end{aligned}
$$

The drops formed by dripping start at zero velocity and an initial temperature of $37.255^{\circ} \mathrm{C}$.

\section{Predicted overall performance}

The integration process proceeds down the height of the splash pack and through the rain zone below the tower, in a similar fashion as that described above. At every grid new packets are formed by dripping, cutting and splashing. At each grid, the water mass flow rate in each of the original packets is reduced due the water mass flow rate striking the grid. The mean outlet water temperature of all the packets below the rain zone is found to be $30.832^{\circ} \mathrm{C}$. The calculated inlet air enthalpy is found to be the same as the specified inlet air enthalpy, implying that the initial choice of outlet air enthalpy was correct.

The total drag force experienced by the drops in free fall between the grids is $26.325 \mathrm{~N}$ and that due to the splash drops during upsplash is $6.3 \mathrm{~N}$. The total pressure drop due to the drag forces on the drops is

$$
\begin{aligned}
\Delta \mathrm{p}_{\text {drops }} & =\frac{\sum \mathrm{F}_{\mathrm{drag}}}{\mathrm{A}_{\mathrm{fr}}} \\
& =\frac{(26.325+6.3)}{2.25} \\
& =14.5 \mathrm{~Pa}
\end{aligned}
$$

The pressure drop across the grids is $4.5 \mathrm{~Pa}$. The predicted total pressure drop across the splash pack is $19 \mathrm{~Pa}$. This compares very well with the measured pressure drop of $17.5 \mathrm{~Pa}$.

The overall transfer characteristic can be found from the calculated outlet water temperature using the Tchebycheff integration method to be $\mathrm{KaZ} / \dot{\mathrm{M}}_{\mathrm{w}}=0.794$. This value is approximately $10 \%$ higher than the measured transfer characteristic (without the correction for inlet and outlet effects). 
APPENDIX J Measured drop acceleration data

Table J.1 Measured drop velocities.

\begin{tabular}{|c|c|c|c|c|c|c|c|c|}
\hline Test & $\mathbf{P}_{\text {atm }}$ & $\mathbf{T}_{\mathbf{H G}}$ & $\mathbf{T}_{\mathbf{a d b}}$ & $\mathbf{T}_{\mathbf{a w b}}$ & $\mathbf{T}_{\mathbf{w}}$ & $\mathbf{Z}$ & $\mathbf{d}$ & $\mathbf{v}$ \\
- & $m m H G$ & ${ }^{\circ} C$ & ${ }^{\circ} C$ & ${ }^{\circ} C$ & ${ }^{\circ} C$ & $m$ & $m \boldsymbol{m}$ & $m / s$ \\
\hline 1 & 758.15 & 20.5 & 22.3 & 15.8 & 20.5 & 1.00 & 2.538 & 4.054 \\
2 & 758.15 & 20.5 & 22.3 & 15.8 & 20.5 & 2.00 & 2.538 & 5.246 \\
3 & 758.15 & 20.5 & 22.3 & 15.8 & 20.5 & 3.00 & 2.538 & 6.010 \\
4 & 758.15 & 20.5 & 22.3 & 15.8 & 20.5 & 4.00 & 2.538 & 6.509 \\
5 & 758.15 & 20.5 & 22.3 & 15.8 & 20.5 & 5.00 & 2.538 & 6.714 \\
6 & 758.15 & 20.5 & 21.8 & 14.6 & 20.0 & 1.00 & 3.464 & 4.149 \\
7 & 758.15 & 20.5 & 21.8 & 14.6 & 20.0 & 2.00 & 3.464 & 5.523 \\
8 & 758.15 & 20.5 & 21.8 & 14.6 & 20.0 & 3.00 & 3.464 & 6.418 \\
9 & 758.15 & 20.5 & 21.8 & 14.6 & 20.0 & 4.00 & 3.464 & 7.026 \\
10 & 758.15 & 20.5 & 21.8 & 14.6 & 20.0 & 5.00 & 3.464 & 7.375 \\
11 & 758.15 & 20.5 & 21.8 & 14.6 & 20.0 & 6.00 & 3.464 & 7.743 \\
12 & 758.15 & 20.5 & 21.8 & 14.6 & 20.0 & 6.90 & 3.464 & 8.049 \\
13 & 756.90 & 20.5 & 22.3 & 15.8 & 20.5 & 1.00 & 4.174 & 4.244 \\
14 & 756.90 & 20.5 & 22.3 & 15.8 & 20.5 & 2.00 & 4.174 & 5.648 \\
15 & 756.90 & 20.5 & 22.3 & 15.8 & 20.5 & 3.00 & 4.174 & 6.681 \\
16 & 756.90 & 20.5 & 22.3 & 15.8 & 20.5 & 4.00 & 4.174 & 7.286 \\
17 & 756.90 & 20.5 & 22.3 & 15.8 & 20.5 & 5.00 & 4.174 & 7.795 \\
18 & 756.90 & 20.5 & 22.3 & 15.8 & 20.5 & 6.00 & 4.174 & 8.159 \\
19 & 756.90 & 20.5 & 22.3 & 15.8 & 20.5 & 6.95 & 4.174 & 8.542 \\
20 & 759.55 & 20.0 & 18.0 & 13.4 & 16.0 & 1.00 & 5.507 & 4.196 \\
21 & 759.55 & 20.0 & 18.0 & 13.4 & 16.0 & 2.00 & 5.561 & 5.819 \\
22 & 759.55 & 20.0 & 18.0 & 13.4 & 16.0 & 3.00 & 5.561 & 6.828 \\
23 & 759.55 & 20.0 & 18.0 & 13.4 & 16.0 & 4.00 & 5.561 & 7.537 \\
24 & 759.55 & 20.0 & 18.0 & 13.4 & 16.0 & 5.00 & 5.561 & 8.072 \\
25 & 759.55 & 20.0 & 18.0 & 13.4 & 16.0 & 6.00 & 5.561 & 8.321 \\
26 & 759.55 & 20.0 & 18.0 & 13.4 & 16.0 & 6.80 & 5.561 & 8.561 \\
27 & 759.55 & 20.0 & 18.0 & 13.8 & 17.0 & 1.00 & 6.079 & 4.253 \\
28 & 759.55 & 20.0 & 18.0 & 13.8 & 17.0 & 2.00 & 6.079 & 5.719 \\
29 & 759.55 & 20.0 & 18.0 & 13.8 & 17.0 & 3.00 & 6.079 & 6.953 \\
30 & 759.55 & 20.0 & 18.0 & 13.8 & 17.0 & 4.00 & 6.079 & 7.500 \\
31 & 759.55 & 20.0 & 18.0 & 13.8 & 17.0 & 5.00 & 6.079 & 8.133 \\
32 & 759.55 & 20.0 & 18.0 & 13.8 & 17.0 & 6.00 & 6.079 & 8.412 \\
33 & 759.55 & 20.0 & 18.0 & 13.8 & 17.0 & 6.80 & 6.079 & 8.670 \\
\hline
\end{tabular}




\section{APPENDIX K Experimentally determined water film thicknesses on the splash grids in a cooling tower}

Table K.1 Average film thicknesses measured on slats in a cooling tower.

(Measured below 10 splash grids in a cooling tower with an air flow area of $2.25 \mathrm{~m}^{2}$ )

\begin{tabular}{|c|c|c|c|c|c|c|c|}
\hline$\dot{\mathbf{m}}_{\mathbf{w}}$ & $\mathbf{v}_{\mathbf{a}}$ & $\mathbf{W}$ & $\boldsymbol{\delta}$ & $\dot{\mathbf{m}}_{\mathbf{w}}$ & $\mathbf{v}_{\mathbf{a}}$ & $\mathbf{W}$ & $\boldsymbol{\delta}$ \\
$\mathrm{kg} / \mathrm{s}$ & $\mathrm{m} / \mathrm{s}$ & $\mathrm{mm}$ & $\mathrm{mm}$ & $\mathrm{kg} / \mathrm{s}$ & $\mathrm{m} / \mathrm{s}$ & $\mathrm{mm}$ & $\mathrm{mm}$ \\
\hline 3.96 & 1.05 & 50 & 0.357 & 3.85 & 1.03 & 10 & 0.435 \\
3.98 & 2.08 & 50 & 0.456 & 3.94 & 2.05 & 10 & 0.426 \\
3.97 & 3.04 & 50 & 0.581 & 3.89 & 3.08 & 10 & 0.558 \\
6.59 & 1.07 & 50 & 0.458 & 6.69 & 1.04 & 10 & 0.534 \\
6.58 & 2.08 & 50 & 0.579 & 6.65 & 2.00 & 10 & 0.576 \\
6.61 & 3.03 & 50 & 0.636 & 6.64 & 3.06 & 10 & 0.576 \\
7.89 & 1.04 & 50 & 0.490 & 7.95 & 1.03 & 10 & 0.524 \\
7.87 & 2.12 & 50 & 0.652 & 7.87 & 2.02 & 10 & 0.536 \\
7.88 & 3.04 & 50 & 0.721 & 7.91 & 3.07 & 10 & 0.550 \\
4.02 & 1.05 & 25 & 0.563 & 3.95 & 1.02 & 5 & 0.367 \\
4.01 & 2.04 & 25 & 0.634 & 3.92 & 2.05 & 5 & 0.374 \\
3.98 & 3.00 & 25 & 0.710 & 3.97 & 3.08 & 5 & 0.400 \\
6.54 & 1.00 & 25 & 0.632 & 6.54 & 1.00 & 5 & 0.390 \\
6.56 & 2.02 & 25 & 0.633 & 6.52 & 2.08 & 5 & 0.402 \\
6.55 & 2.99 & 25 & 0.805 & 6.51 & 3.06 & 5 & 0.393 \\
8.01 & 0.97 & 25 & 0.678 & 7.95 & 0.98 & 5 & 0.374 \\
8.02 & 2.04 & 25 & 0.684 & 7.92 & 2.03 & 5 & 0.372 \\
7.99 & 3.08 & 25 & 0.678 & 7.96 & 3.04 & 5 & 0.368 \\
4.13 & 1.06 & 15 & 0.527 & 6.52 & 2.08 & 5 & 0.402 \\
4.11 & 2.00 & 15 & 0.549 & 6.51 & 3.06 & 5 & 0.393 \\
4.12 & 3.04 & 15 & 0.564 & 7.95 & 0.98 & 5 & 0.374 \\
6.56 & 1.05 & 15 & 0.491 & 7.92 & 2.03 & 5 & 0.372 \\
6.53 & 2.08 & 15 & 0.529 & 7.96 & 3.04 & 5 & 0.368 \\
6.52 & 3.03 & 15 & 0.551 & & & & \\
7.94 & 1.03 & 15 & 0.486 & & & & \\
7.96 & 2.10 & 15 & 0.514 & & & & \\
7.93 & 2.95 & 15 & 0.546 & & & & \\
\hline & & & & & & &
\end{tabular}




\section{APPENDIX L Summary of data obtained during experiments to measure} the mass of water splashing from narrow slats

Table L.1 Measured splash fraction data for $5 \mathrm{~mm}$ wide slat.

\begin{tabular}{|c|c|c|c|c|c|c|c|c|}
\hline $\begin{array}{l}\mathbf{Z} \\
m\end{array}$ & $\begin{array}{c}\mathbf{d}_{\mathbf{i}} \\
m m\end{array}$ & $\begin{array}{c}\boldsymbol{\delta} \\
m m\end{array}$ & $\begin{array}{c}\mathbf{x} \\
m m\end{array}$ & $\begin{array}{c}\mathbf{v}_{\mathbf{i}} \\
\mathrm{m} / \mathrm{s}\end{array}$ & $\begin{array}{c}\mathbf{f}_{\mathbf{s}}+\mathbf{f}_{\mathrm{c}} \\
-\end{array}$ & $\begin{array}{c}\mathbf{f}_{\mathbf{c}} \\
-\end{array}$ & $\begin{array}{c}\mathbf{f}_{\mathbf{s}} \\
-\end{array}$ & $\begin{array}{c}\mathbf{f}_{\mathbf{s}} \\
-\end{array}$ \\
\hline 1.00 & 5.57 & 0.69 & 0.00 & 4.27 & 1.197 & 0.015 & 1.182 & 0.591 \\
\hline 1.00 & 5.52 & 0.80 & 0.00 & 4.27 & 1.243 & 0.013 & 1.230 & 0.615 \\
\hline 1.00 & 5.59 & 1.43 & 0.00 & 4.27 & 1.244 & 0.016 & 1.228 & 0.614 \\
\hline 1.00 & 5.45 & 1.19 & 0.00 & 4.26 & 1.273 & 0.010 & 1.263 & 0.632 \\
\hline 1.00 & 5.55 & 0.74 & 0.00 & 4.27 & 1.197 & 0.014 & 1.183 & 0.592 \\
\hline 1.00 & 5.47 & 0.75 & 0.00 & 4.26 & 1.258 & 0.011 & 1.247 & 0.624 \\
\hline 1.75 & 5.52 & 0.75 & 0.00 & 5.49 & 1.535 & 0.013 & 1.522 & 0.761 \\
\hline 1.75 & 5.52 & 0.73 & 0.00 & 5.49 & 1.539 & 0.013 & 1.526 & 0.763 \\
\hline 2.50 & 5.54 & 0.80 & 0.00 & 6.38 & 1.594 & 0.014 & 1.580 & 0.790 \\
\hline 2.50 & 5.54 & 0.82 & 0.00 & 6.38 & 1.556 & 0.014 & 1.542 & 0.771 \\
\hline 0.50 & 5.52 & 0.75 & 0.00 & 3.07 & 1.123 & 0.013 & 1.110 & 0.555 \\
\hline 0.50 & 5.51 & 0.76 & 0.00 & 3.07 & 1.154 & 0.013 & 1.141 & 0.571 \\
\hline 0.25 & 5.57 & 0.73 & 0.00 & 2.19 & 0.814 & 0.015 & 0.799 & 0.399 \\
\hline 1.00 & 4.09 & 0.68 & 0.00 & 4.20 & 1.492 & 0.000 & 1.492 & 0.746 \\
\hline 1.00 & 4.08 & 0.68 & 0.00 & 4.20 & 1.476 & 0.000 & 1.476 & 0.738 \\
\hline 1.75 & 4.08 & 0.68 & 0.00 & 5.36 & 1.637 & 0.000 & 1.637 & 0.818 \\
\hline 1.75 & 4.08 & 0.70 & 0.00 & 5.36 & 1.643 & 0.000 & 1.643 & 0.822 \\
\hline 2.50 & 4.09 & 0.79 & 0.00 & 6.19 & 1.803 & 0.000 & 1.803 & 0.901 \\
\hline 2.50 & 4.13 & 0.72 & 0.00 & 6.19 & 1.846 & 0.000 & 1.846 & 0.923 \\
\hline 0.50 & 4.14 & 0.78 & 0.00 & 3.04 & 0.957 & 0.000 & 0.957 & 0.479 \\
\hline 0.25 & 4.16 & 0.75 & 0.00 & 2.18 & 0.701 & 0.000 & 0.701 & 0.351 \\
\hline 1.00 & 7.74 & 0.78 & 0.00 & 4.31 & 1.128 & 0.166 & 0.962 & 0.481 \\
\hline 1.00 & 7.67 & 0.75 & 0.00 & 4.31 & 1.074 & 0.161 & 0.914 & 0.457 \\
\hline 1.75 & 7.75 & 0.75 & 0.00 & 5.59 & 1.112 & 0.167 & 0.945 & 0.473 \\
\hline 1.75 & 7.67 & 0.74 & 0.00 & 5.58 & 1.134 & 0.161 & 0.973 & 0.487 \\
\hline 2.50 & 7.70 & 0.84 & 0.00 & 6.51 & 1.045 & 0.163 & 0.882 & 0.441 \\
\hline 0.50 & 7.85 & 0.79 & 0.00 & 3.09 & 0.945 & 0.174 & 0.772 & 0.386 \\
\hline 0.50 & 7.76 & 0.69 & 0.00 & 3.09 & 0.941 & 0.167 & 0.774 & 0.387 \\
\hline 0.25 & 7.68 & 0.80 & 0.00 & 2.20 & 0.779 & 0.161 & 0.618 & 0.309 \\
\hline 1.00 & 3.30 & 0.80 & 0.00 & 4.13 & 2.160 & 0.000 & 2.160 & 1.080 \\
\hline 1.00 & 3.30 & 0.84 & 0.00 & 4.13 & 2.100 & 0.000 & 2.100 & 1.050 \\
\hline 1.75 & 3.28 & 0.74 & 0.00 & 5.23 & 1.982 & 0.000 & 1.982 & 0.991 \\
\hline 1.75 & 3.28 & 0.77 & 0.00 & 5.23 & 2.214 & 0.000 & 2.214 & 1.107 \\
\hline 0.50 & 3.22 & 0.80 & 0.00 & 3.01 & 1.284 & 0.000 & 1.284 & 0.642 \\
\hline 0.50 & 3.22 & 0.80 & 0.00 & 3.01 & 1.253 & 0.000 & 1.253 & 0.626 \\
\hline 0.25 & 3.26 & 0.77 & 0.00 & 2.17 & 0.412 & 0.000 & 0.412 & 0.206 \\
\hline 0.25 & 5.57 & 0.51 & 0.00 & 2.19 & 0.789 & 0.015 & 0.774 & 0.387 \\
\hline 0.25 & 4.12 & 0.50 & 0.00 & 2.18 & 0.487 & 0.000 & 0.487 & 0.243 \\
\hline 0.25 & 7.69 & 0.44 & 0.00 & 2.20 & 0.764 & 0.162 & 0.602 & 0.301 \\
\hline 0.25 & 3.30 & 0.43 & 0.00 & 2.17 & 0.238 & 0.000 & 0.238 & 0.119 \\
\hline 0.50 & 3.30 & 0.40 & 0.00 & 3.01 & 1.092 & 0.000 & 1.092 & 0.546 \\
\hline 0.50 & 5.47 & 0.39 & 0.00 & 3.07 & 0.893 & 0.011 & 0.882 & 0.441 \\
\hline 1.00 & 5.35 & 0.39 & 0.00 & 4.26 & 1.158 & 0.006 & 1.152 & 0.576 \\
\hline 1.00 & 3.30 & 0.36 & 0.00 & 4.13 & 1.405 & 0.000 & 1.405 & 0.703 \\
\hline 1.75 & 3.30 & 0.32 & 0.00 & 5.23 & 1.695 & 0.000 & 1.695 & 0.848 \\
\hline 1.75 & 5.39 & 0.30 & 0.00 & 5.48 & 1.198 & 0.008 & 1.190 & 0.595 \\
\hline 2.50 & 5.46 & 0.37 & 0.00 & 6.37 & 1.216 & 0.010 & 1.205 & 0.603 \\
\hline 1.00 & 5.35 & 0.39 & 0.00 & 4.26 & 1.158 & 0.006 & 1.152 & 0.576 \\
\hline 1.00 & 5.38 & 1.08 & 0.00 & 4.26 & 1.325 & 0.007 & 1.318 & 0.659 \\
\hline
\end{tabular}


Table L.1 (continued) Measured splash fraction data for $5 \mathrm{~mm}$ wide slat.

\begin{tabular}{|c|c|c|c|c|c|c|c|c|}
\hline $\begin{array}{l}\mathbf{Z} \\
m\end{array}$ & $\begin{array}{c}\mathbf{d}_{\mathbf{i}} \\
m m\end{array}$ & $\begin{array}{c}\delta \\
m m\end{array}$ & $\begin{array}{c}\mathbf{x} \\
m m \\
\end{array}$ & $\begin{array}{c}\mathbf{v}_{\mathbf{i}} \\
\mathrm{m} / \mathrm{s}\end{array}$ & $\begin{array}{c}\mathbf{f}_{\mathbf{s}}+\mathbf{f}_{\mathbf{c}} \\
-\end{array}$ & $\begin{array}{c}\mathbf{f}_{\mathbf{c}} \\
- \\
\end{array}$ & $\begin{array}{c}\mathbf{f}_{\mathbf{s}} \\
- \\
\end{array}$ & $\begin{array}{l}\overline{f_{s}} \\
-\end{array}$ \\
\hline 1.00 & 5.52 & 1.05 & 0.00 & 4.27 & 1.286 & 0.013 & 1.273 & 0.637 \\
\hline 1.00 & 5.35 & 0.94 & 0.00 & 4.26 & 1.383 & 0.006 & 1.377 & 0.688 \\
\hline 1.00 & 5.46 & 0.85 & 0.00 & 4.26 & 1.356 & 0.010 & 1.345 & 0.673 \\
\hline 1.00 & 5.47 & 0.62 & 0.00 & 4.26 & 1.244 & 0.011 & 1.233 & 0.617 \\
\hline 1.00 & 5.47 & 0.74 & 0.00 & 4.26 & 1.352 & 0.011 & 1.341 & 0.671 \\
\hline 1.00 & 5.37 & 0.56 & 0.00 & 4.26 & 1.252 & 0.007 & 1.245 & 0.623 \\
\hline 1.00 & 5.51 & 0.30 & 0.00 & 4.27 & 1.156 & 0.012 & 1.143 & 0.572 \\
\hline 1.00 & 5.55 & 0.46 & 0.00 & 4.27 & 1.176 & 0.014 & 1.161 & 0.581 \\
\hline 1.00 & 5.46 & 0.37 & 0.00 & 4.26 & 1.103 & 0.011 & 1.093 & 0.546 \\
\hline 1.00 & 3.22 & 0.86 & 0.00 & 4.12 & 1.709 & 0.000 & 1.709 & 0.855 \\
\hline 1.00 & 3.27 & 0.86 & 0.00 & 4.13 & 1.604 & 0.000 & 1.604 & 0.802 \\
\hline 1.00 & 3.30 & 0.36 & 0.00 & 4.13 & 1.405 & 0.000 & 1.405 & 0.703 \\
\hline 1.00 & 3.33 & 0.89 & 0.00 & 4.14 & 1.541 & 0.000 & 1.541 & 0.770 \\
\hline 1.00 & 3.24 & 0.69 & 0.00 & 4.13 & 1.688 & 0.000 & 1.688 & 0.844 \\
\hline 1.00 & 3.24 & 0.57 & 0.00 & 4.13 & 1.583 & 0.000 & 1.583 & 0.791 \\
\hline 1.00 & 3.24 & 0.45 & 0.00 & 4.13 & 1.497 & 0.000 & 1.497 & 0.749 \\
\hline 1.00 & 3.24 & 0.23 & 0.00 & 4.13 & 1.180 & 0.000 & 1.180 & 0.590 \\
\hline 1.00 & 3.24 & 0.89 & 0.00 & 4.13 & 1.612 & 0.000 & 1.612 & 0.806 \\
\hline 1.00 & 3.24 & 0.85 & 0.00 & 4.13 & 1.682 & 0.000 & 1.682 & 0.841 \\
\hline 1.00 & 3.24 & 0.74 & 0.00 & 4.13 & 1.649 & 0.000 & 1.649 & 0.825 \\
\hline 0.25 & 3.28 & 0.91 & 0.00 & 2.21 & 0.469 & 0.000 & 0.469 & 0.235 \\
\hline 0.25 & 3.28 & 0.86 & 0.00 & 2.21 & 0.389 & 0.000 & 0.389 & 0.194 \\
\hline 0.25 & 3.28 & 0.86 & 0.00 & 2.21 & 0.425 & 0.000 & 0.425 & 0.212 \\
\hline 0.25 & 3.28 & 0.80 & 0.00 & 2.21 & 0.429 & 0.000 & 0.429 & 0.215 \\
\hline 0.25 & 3.28 & 0.56 & 0.00 & 2.21 & 0.379 & 0.000 & 0.379 & 0.190 \\
\hline 0.25 & 3.28 & 0.50 & 0.00 & 2.21 & 0.421 & 0.000 & 0.421 & 0.211 \\
\hline 0.25 & 3.28 & 0.35 & 0.00 & 2.21 & 0.415 & 0.000 & 0.415 & 0.207 \\
\hline 0.25 & 3.28 & 0.33 & 0.00 & 2.21 & 0.404 & 0.000 & 0.404 & 0.202 \\
\hline 0.25 & 3.28 & 0.28 & 0.00 & 2.21 & 0.413 & 0.000 & 0.413 & 0.207 \\
\hline 0.25 & 5.59 & 0.91 & 0.00 & 2.21 & 0.975 & 0.016 & 0.959 & 0.479 \\
\hline 0.25 & 5.65 & 0.69 & 0.00 & 2.21 & 1.033 & 0.019 & 1.014 & 0.507 \\
\hline 0.25 & 5.65 & 0.70 & 0.00 & 2.21 & 1.045 & 0.019 & 1.026 & 0.513 \\
\hline 0.25 & 5.65 & 0.58 & 0.00 & 2.21 & 1.023 & 0.019 & 1.004 & 0.502 \\
\hline 0.25 & 5.65 & 0.50 & 0.00 & 2.21 & 0.980 & 0.019 & 0.961 & 0.480 \\
\hline 0.25 & 5.65 & 0.38 & 0.00 & 2.21 & 0.947 & 0.019 & 0.928 & 0.464 \\
\hline 0.25 & 5.65 & 0.96 & 0.00 & 2.21 & 0.967 & 0.019 & 0.948 & 0.474 \\
\hline 0.25 & 5.62 & 0.64 & 0.00 & 2.21 & 0.966 & 0.018 & 0.948 & 0.474 \\
\hline 0.25 & 5.67 & 0.51 & 0.00 & 2.21 & 0.945 & 0.020 & 0.925 & 0.462 \\
\hline 0.25 & 5.66 & 0.28 & 0.00 & 2.21 & 0.857 & 0.020 & 0.837 & 0.419 \\
\hline 0.25 & 7.95 & 0.33 & 0.00 & 2.21 & 0.807 & 0.181 & 0.626 & 0.313 \\
\hline 0.25 & 7.78 & 0.51 & 0.00 & 2.21 & 0.837 & 0.169 & 0.668 & 0.334 \\
\hline 0.25 & 7.84 & 0.79 & 0.00 & 2.21 & 0.914 & 0.173 & 0.741 & 0.370 \\
\hline 0.35 & 2.30 & 0.40 & 0.00 & 2.62 & 0.000 & 0.000 & 0.000 & 0.000 \\
\hline 0.15 & 3.30 & 0.40 & 0.00 & 1.71 & 0.000 & 0.000 & 0.000 & 0.000 \\
\hline 0.09 & 4.20 & 0.40 & 0.00 & 1.29 & 0.000 & 0.000 & 0.000 & 0.000 \\
\hline 1.00 & 6.98 & 0.63 & 0.00 & 4.30 & 1.114 & 0.109 & 1.005 & 0.502 \\
\hline 1.00 & 6.92 & 0.63 & 0.00 & 4.30 & 0.986 , & 0.105 & 0.881 & 0.441 \\
\hline 1.00 & 7.62 & 0.91 & 0.00 & 4.31 & 1.074 & 0.157 & 0.917 & 0.458 \\
\hline 1.00 & 2.58 & 0.86 & 0.00 & 4.03 & 1.313 & 0.000 & 1.313 & 0.656 \\
\hline 1.00 & 2.58 & 0.85 & 0.00 & 4.03 & 1.276 & 0.000 & 1.276 & 0.638 \\
\hline 1.00 & 2.58 & 0.57 & 0.00 & 4.03 & 1.588 & 0.000 & 1.588 & 0.794 \\
\hline 1.00 & 2.58 & 0.34 & 0.00 & 4.03 & 1.425 & 0.000 & 1.425 & 0.713 \\
\hline 1.00 & 2.58 & 0.34 & 0.00 & 4.03 & 1.355 & 0.000 & 1.355 & 0.678 \\
\hline 1.00 & 2.58 & 0.65 & 0.00 & 4.03 & 1.530 & 0.000 & 1.530 & 0.765 \\
\hline
\end{tabular}


Table L.2 Measured splash fraction data for $10 \mathrm{~mm}$ wide slat.

\begin{tabular}{|c|c|c|c|c|c|c|c|}
\hline $\begin{array}{l}\mathbf{Z} \\
m\end{array}$ & $\begin{array}{c}\mathbf{d}_{\mathbf{i}} \\
m m\end{array}$ & $\begin{array}{c}\delta \\
m m\end{array}$ & $\begin{array}{c}\mathbf{x} \\
m m \\
\end{array}$ & $\begin{array}{c}\mathbf{v}_{\mathbf{i}} \\
m / s\end{array}$ & $\begin{array}{c}f_{s}+f_{c} \\
-\end{array}$ & $\begin{array}{l}\mathbf{f}_{\mathbf{c}} \\
- \\
\end{array}$ & $\begin{array}{l}\mathbf{f}_{\mathbf{s}} \\
- \\
\end{array}$ \\
\hline 0.25 & 3.28 & 0.29 & 0.17 & 2.17 & 0.406 & 0.000 & 0.406 \\
\hline 0.25 & 3.28 & 0.29 & 0.13 & 2.17 & 0.313 & 0.000 & 0.313 \\
\hline 0.25 & 3.28 & 0.52 & 0.22 & 2.17 & 0.099 & 0.000 & 0.099 \\
\hline 0.25 & 3.28 & 0.34 & 4.27 & 2.17 & 0.215 & 0.188 & 0.027 \\
\hline 0.25 & 3.28 & 0.34 & 4.27 & 2.17 & 0.191 & 0.188 & 0.003 \\
\hline 0.25 & 3.28 & 0.49 & 4.27 & 2.17 & 0.139 & 0.188 & 0.000 \\
\hline 0.25 & 3.28 & 0.83 & 2.53 & 2.17 & 0.000 & 0.000 & 0.000 \\
\hline 0.25 & 4.13 & 0.26 & 0.23 & 2.18 & 0.579 & 0.089 & 0.490 \\
\hline 0.25 & 4.13 & 0.52 & 0.13 & 2.18 & 0.407 & 0.000 & 0.407 \\
\hline 0.25 & 4.13 & 0.75 & 0.22 & 2.18 & 0.407 & 0.000 & 0.407 \\
\hline 0.25 & 4.13 & 0.75 & 4.88 & 2.18 & 0.225 & 0.458 & 0.000 \\
\hline 0.25 & 4.13 & 0.52 & 4.98 & 2.18 & 0.468 & 0.494 & 0.000 \\
\hline 0.25 & 4.13 & 0.30 & 4.98 & 2.18 & 0.562 & 0.494 & 0.067 \\
\hline 0.25 & 5.60 & 0.30 & 0.62 & 2.19 & 0.630 & 0.000 & 0.630 \\
\hline 0.25 & 5.60 & 0.55 & 0.35 & 2.19 & 0.552 & 0.000 & 0.552 \\
\hline 0.25 & 5.60 & 0.79 & 0.25 & 2.19 & 0.519 & 0.000 & 0.519 \\
\hline 0.25 & 5.60 & 0.79 & 4.12 & 2.19 & 0.827 & 0.271 & 0.556 \\
\hline 0.25 & 5.60 & 0.51 & 4.07 & 2.19 & 0.854 & 0.259 & 0.594 \\
\hline 0.25 & 5.60 & 0.31 & 4.15 & 2.19 & 0.662 & 0.279 & 0.383 \\
\hline 0.25 & 5.60 & 0.41 & 4.10 & 2.19 & 0.776 & 0.267 & 0.509 \\
\hline 0.25 & 7.57 & 0.50 & 0.15 & 2.20 & 0.528 & 0.000 & 0.528 \\
\hline 0.25 & 7.57 & 0.34 & 0.18 & 2.20 & 0.525 & 0.000 & 0.525 \\
\hline 0.25 & 7.57 & 0.79 & 0.13 & 2.20 & 0.554 & 0.000 & 0.554 \\
\hline 0.25 & 7.57 & 0.79 & 4.43 & 2.20 & 0.760 & 0.388 & 0.371 \\
\hline 0.25 & 7.57 & 0.52 & 4.57 & 2.20 & 0.426 & 0.415 & 0.011 \\
\hline 0.25 & 7.57 & 0.34 & 4.55 & 2.20 & 0.361 & 0.411 & 0.000 \\
\hline 1.00 & 7.60 & 0.75 & 0.12 & 4.31 & 1.094 & 0.000 & 1.094 \\
\hline 1.00 & 7.60 & 0.47 & 0.13 & 4.31 & 1.012 & 0.000 & 1.012 \\
\hline 1.00 & 7.60 & 0.38 & 1.45 & 4.31 & 0.953 & 0.003 & 0.950 \\
\hline 1.00 & 7.60 & 0.28 & 3.63 & 4.31 & 0.899 & 0.242 & 0.657 \\
\hline 1.00 & 7.60 & 0.53 & 4.10 & 4.31 & 0.962 & 0.326 & 0.636 \\
\hline 1.00 & 7.60 & 0.80 & 4.78 & 4.31 & 0.806 & 0.457 & 0.349 \\
\hline 1.00 & 7.60 & 0.71 & 4.72 & 4.31 & 0.754 & 0.444 & 0.310 \\
\hline 1.00 & 5.49 & 0.34 & 0.78 & 4.27 & 1.025 & 0.000 & 1.025 \\
\hline 1.00 & 5.49 & 0.52 & 0.92 & 4.27 & 1.181 & 0.000 & 1.181 \\
\hline 1.00 & 5.49 & 0.86 & 0.73 & 4.27 & 1.321 & 0.000 & 1.321 \\
\hline 1.00 & 5.49 & 0.84 & 5.00 & 4.27 & 0.795 & 0.500 & 0.295 \\
\hline 1.00 & 5.49 & 0.48 & 4.75 & 4.27 & 0.871 & 0.432 & 0.439 \\
\hline 1.00 & 5.49 & 0.36 & 4.53 & 4.27 & 0.849 & 0.374 & 0.475 \\
\hline 1.00 & 4.12 & 0.37 & 0.95 & 4.20 & 1.229 & 0.000 & 1.229 \\
\hline 1.00 & 4.12 & 0.52 & 0.92 & 4.20 & 1.421 & 0.000 & 1.421 \\
\hline 1.00 & 4.12 & 0.75 & 0.72 & 4.20 & 1.591 & 0.000 & 1.591 \\
\hline 1.00 & 4.12 & 0.48 & 4.43 & 4.20 & 1.406 & 0.299 & 1.107 \\
\hline 1.00 & 4.12 & 0.35 & 4.57 & 4.20 & 1.218 & 0.345 & 0.874 \\
\hline 1.00 & 4.12 & 0.79 & 4.50 & 4.20 & 1.624 & 0.322 & 1.303 \\
\hline 1.00 & 3.13 & 0.34 & 0.72 & 4.11 & 1.421 & 0.000 & 1.421 \\
\hline 1.00 & 3.13 & 0.51 & 0.05 & 4.11 & 1.647 & 0.000 & 1.647 \\
\hline 1.00 & 3.13 & 0.84 & 0.03 & 4.11 & 1.430 & 0.000 & 1.430 \\
\hline 1.00 & 3.13 & 0.64 & 0.65 & 4.11 & 1.474 & 0.000 & 1.474 \\
\hline 1.00 & 3.13 & 0.79 & 4.95 & 4.11 & 0.317 & 0.476 & 0.000 \\
\hline 1.00 & 3.13 & 0.79 & 4.42 & 4.11 & 0.835 & 0.234 & 0.601 \\
\hline 1.00 & 3.13 & 0.48 & 4.45 & 4.11 & 1.106 & 0.247 & 0.858 \\
\hline 1.00 & 3.13 & 0.29 & 4.48 & 4.11 & 1.499 & 0.261 & 1.237 \\
\hline
\end{tabular}


Table L.2 (continued) Measured splash fraction data for $10 \mathrm{~mm}$ wide slat.

\begin{tabular}{|c|c|c|c|c|c|c|c|}
\hline $\mathbf{Z}$ & $\mathbf{d}_{\mathbf{i}}$ & $\delta$ & $\mathbf{x}$ & $\begin{array}{c}\mathbf{v}_{\mathbf{i}} \\
m\end{array}$ & $\begin{array}{c}\mathbf{f}_{\mathbf{s}}+\mathbf{f}_{\mathbf{c}} \\
m m\end{array}$ & $\mathbf{f}_{\mathbf{c}}$ & \begin{tabular}{c}
$\mathbf{f}_{\mathbf{s}}$ \\
\hline$m$
\end{tabular} \\
\hline 1.00 & 2.41 & 0.34 & 0.10 & 3.99 & 1.120 & 0.000 & 1.120 \\
1.00 & 2.41 & 0.52 & 0.48 & 3.99 & 1.283 & 0.000 & 1.283 \\
1.00 & 2.41 & 0.80 & 0.50 & 3.99 & 1.213 & 0.000 & 1.213 \\
2.40 & 7.61 & 0.37 & 0.65 & 6.40 & 1.030 & 0.000 & 1.030 \\
2.40 & 7.61 & 0.86 & 0.18 & 6.40 & 1.261 & 0.000 & 1.261 \\
2.40 & 7.61 & 0.49 & 0.22 & 6.40 & 1.123 & 0.000 & 1.123 \\
2.40 & 7.61 & 0.48 & 5.35 & 6.40 & 0.853 & 0.569 & 0.284 \\
2.40 & 7.61 & 0.75 & 5.00 & 6.40 & 1.068 & 0.500 & 0.568 \\
2.50 & 5.61 & 0.75 & 0.10 & 6.38 & 1.389 & 0.000 & 1.389 \\
2.50 & 5.61 & 0.75 & 4.68 & 6.38 & 1.377 & 0.416 & 0.962 \\
2.50 & 5.61 & 0.51 & 4.75 & 6.38 & 1.300 & 0.433 & 0.867 \\
2.50 & 5.61 & 0.52 & 0.30 & 6.38 & 1.415 & 0.000 & 1.415 \\
2.50 & 5.61 & 0.30 & 0.13 & 6.38 & 1.153 & 0.000 & 1.153 \\
2.50 & 5.61 & 0.27 & 4.72 & 6.38 & 1.071 & 0.425 & 0.646 \\
2.50 & 3.31 & 0.28 & 0.88 & 6.00 & 1.930 & 0.000 & 1.930 \\
2.50 & 3.31 & 0.29 & 4.08 & 6.00 & 2.221 & 0.127 & 2.094 \\
2.50 & 3.31 & 0.28 & 0.15 & 6.00 & 1.783 & 0.000 & 1.783 \\
2.50 & 3.31 & 0.53 & 1.55 & 6.00 & 2.054 & 0.000 & 2.054 \\
2.50 & 4.14 & 0.46 & 0.50 & 6.20 & 1.843 & 0.000 & 1.843 \\
2.50 & 4.14 & 0.48 & 0.50 & 6.20 & 1.750 & 0.000 & 1.750 \\
2.50 & 4.14 & 0.48 & 4.17 & 6.20 & 1.620 & 0.214 & 1.406 \\
2.50 & 4.14 & 0.33 & 4.32 & 6.20 & 1.596 & 0.261 & 1.335 \\
2.50 & 4.14 & 0.34 & 0.08 & 6.20 & 1.537 & 0.000 & 1.537 \\
2.50 & 4.14 & 0.75 & 0.78 & 6.20 & 1.899 & 0.000 & 1.899 \\
\hline
\end{tabular}


Table L.3 Measured splash fraction data for $25 \mathrm{~mm}$ wide slat.

\begin{tabular}{|c|c|c|c|c|c|c|c|}
\hline $\begin{array}{l}\mathbf{Z} \\
m\end{array}$ & $\begin{array}{c}\mathbf{d}_{\mathbf{i}} \\
m m\end{array}$ & $\begin{array}{c}\delta \\
m m\end{array}$ & $\begin{array}{c}\mathbf{x} \\
m m \\
\end{array}$ & $\begin{array}{c}\mathbf{v}_{\mathbf{i}} \\
m / s\end{array}$ & $\begin{array}{c}f_{s}+f_{c} \\
-\end{array}$ & $\begin{array}{c}\mathbf{f}_{\mathbf{c}} \\
- \\
\end{array}$ & $\begin{array}{c}\mathbf{f}_{\mathbf{s}} \\
- \\
\end{array}$ \\
\hline 2.50 & 5.58 & 0.95 & 9.28 & 6.38 & 2.466 & 0.000 & 2.466 \\
\hline 2.50 & 5.58 & 0.92 & 9.33 & 6.38 & 1.991 & 0.000 & 1.991 \\
\hline 2.50 & 5.58 & 0.91 & 0.00 & 6.38 & 2.031 & 0.000 & 2.031 \\
\hline 2.50 & 5.58 & 0.90 & 6.25 & 6.38 & 1.855 & 0.000 & 1.855 \\
\hline 2.50 & 5.58 & 0.90 & 12.50 & 6.38 & 1.355 & 0.500 & 0.855 \\
\hline 2.50 & 5.58 & 0.76 & 6.25 & 6.38 & 1.821 & 0.000 & 1.821 \\
\hline 2.50 & 5.58 & 0.74 & 12.42 & 6.38 & 1.282 & 0.478 & 0.804 \\
\hline 2.50 & 5.58 & 0.71 & 0.00 & 6.38 & 1.584 & 0.000 & 1.584 \\
\hline 2.50 & 5.58 & 0.70 & 9.20 & 6.38 & 1.534 & 0.000 & 1.534 \\
\hline 2.50 & 5.58 & 0.57 & 6.25 & 6.38 & 1.519 & 0.000 & 1.519 \\
\hline 2.50 & 5.58 & 0.51 & 0.00 & 6.38 & 1.478 & 0.000 & 1.478 \\
\hline 2.50 & 5.58 & 0.51 & 6.25 & 6.38 & 1.511 & 0.000 & 1.511 \\
\hline 2.50 & 5.58 & 0.51 & 12.64 & 6.38 & 1.217 & 0.538 & 0.679 \\
\hline 2.50 & 5.58 & 0.50 & 9.67 & 6.38 & 1.378 & 0.000 & 1.378 \\
\hline 2.50 & 5.58 & 0.45 & 0.00 & 6.38 & 1.333 & 0.000 & 1.333 \\
\hline 2.50 & 5.58 & 0.31 & 6.25 & 6.38 & 1.270 & 0.000 & 1.270 \\
\hline 2.50 & 5.58 & 0.30 & 9.37 & 6.38 & 1.287 & 0.000 & 1.287 \\
\hline 2.50 & 5.58 & 0.30 & 12.55 & 6.38 & 1.084 & 0.513 & 0.571 \\
\hline 2.50 & 5.58 & 0.28 & 0.00 & 6.38 & 1.255 & 0.000 & 1.255 \\
\hline 2.50 & 7.79 & 0.95 & 12.26 & 6.51 & 1.663 & 0.453 & 1.210 \\
\hline 2.50 & 7.79 & 0.94 & 0.16 & 6.51 & 2.204 & 0.000 & 2.204 \\
\hline 2.50 & 7.79 & 0.92 & 0.93 & 6.51 & 1.941 & 0.000 & 1.941 \\
\hline 2.50 & 7.79 & 0.89 & 5.09 & 6.51 & 1.794 & 0.000 & 1.794 \\
\hline 2.50 & 7.79 & 0.88 & 14.66 & 6.51 & 0.937 & 0.873 & 0.064 \\
\hline 2.50 & 7.79 & 0.88 & 10.02 & 6.51 & 1.677 & 0.087 & 1.590 \\
\hline 2.50 & 7.79 & 0.88 & 7.49 & 6.51 & 1.792 & 0.000 & 1.792 \\
\hline 2.50 & 7.79 & 0.74 & 0.33 & 6.51 & 1.623 & 0.000 & 1.623 \\
\hline 2.50 & 7.79 & 0.63 & 14.95 & 6.51 & 0.869 & 0.909 & 0.000 \\
\hline 2.50 & 7.79 & 0.61 & 12.80 & 6.51 & 1.250 & 0.558 & 0.692 \\
\hline 2.50 & 7.79 & 0.61 & 6.36 & 6.51 & 1.339 & 0.000 & 1.339 \\
\hline 2.50 & 7.79 & 0.59 & 9.61 & 6.51 & 1.365 & 0.045 & 1.320 \\
\hline 2.50 & 7.79 & 0.48 & 0.13 & 6.51 & 1.229 & 0.000 & 1.229 \\
\hline 2.50 & 7.79 & 0.39 & 15.71 & 6.51 & 0.762 & 0.978 & 0.000 \\
\hline 2.50 & 7.79 & 0.29 & 9.50 & 6.51 & 1.060 & 0.037 & 1.023 \\
\hline 2.50 & 7.79 & 0.27 & 4.36 & 6.51 & 0.995 & 0.000 & 0.995 \\
\hline 2.50 & 7.79 & 0.27 & 1.54 & 6.51 & 0.962 & 0.000 & 0.962 \\
\hline 2.50 & 7.79 & 0.26 & 11.99 & 6.51 & 0.943 & 0.401 & 0.542 \\
\hline 2.50 & 4.17 & 0.91 & 0.95 & 6.20 & 1.678 & 0.000 & 1.678 \\
\hline 2.50 & 4.17 & 0.89 & 0.06 & 6.20 & 1.625 & 0.000 & 1.625 \\
\hline 2.50 & 4.17 & 0.86 & 6.36 & 6.20 & 1.679 & 0.000 & 1.679 \\
\hline 2.50 & 4.17 & 0.86 & 10.11 & 6.20 & 1.156 & 0.000 & 1.156 \\
\hline 2.50 & 4.17 & 0.85 & 10.02 & 6.20 & 1.202 & 0.000 & 1.202 \\
\hline 2.50 & 4.17 & 0.83 & 12.53 & 6.20 & 0.882 & 0.511 & 0.371 \\
\hline 2.50 & 4.17 & 0.63 & 0.45 & 6.20 & 1.487 & 0.000 & 1.487 \\
\hline 2.50 & 4.17 & 0.58 & 1.58 & 6.20 & 1.457 & 0.000 & 1.457 \\
\hline 2.50 & 4.17 & 0.57 & 9.88 & 6.20 & 1.127 & 0.000 & 1.127 \\
\hline 2.50 & 4.17 & 0.56 & 7.17 & 6.20 & 1.358 & 0.000 & 1.358 \\
\hline 2.50 & 4.17 & 0.56 & 12.42 & 6.20 & 0.895 & 0.471 & 0.424 \\
\hline 2.50 & 4.17 & 0.55 & 6.52 & 6.20 & 1.445 & 0.000 & 1.445 \\
\hline 2.50 & 4.17 & 0.40 & 9.88 & 6.20 & 0.971 & 0.000 & 0.971 \\
\hline 2.50 & 4.17 & 0.36 & 7.41 & 6.20 & 0.964 & 0.000 & 0.964 \\
\hline 2.50 & 4.17 & 0.35 & 0.12 & 6.20 & 1.185 & 0.000 & 1.185 \\
\hline 2.50 & 4.17 & 0.35 & 12.17 & 6.20 & 0.905 & 0.382 & 0.523 \\
\hline 2.50 & 4.17 & 0.35 & 12.17 & 6.20 & 0.848 & 0.382 & 0.466 \\
\hline
\end{tabular}


Table L.3 (continued) Measured splash fraction data for $25 \mathrm{~mm}$ wide slat.

\begin{tabular}{|c|c|c|c|c|c|c|c|}
\hline $\begin{array}{l}\mathbf{Z} \\
m\end{array}$ & $\underset{m m}{\mathbf{d}_{\mathbf{i}}}$ & $\underset{m m}{\boldsymbol{\delta}}$ & $\underset{m m}{\mathbf{x}}$ & $\begin{array}{c}\mathbf{v}_{\mathbf{i}} \\
\mathrm{m} / \mathrm{s}\end{array}$ & $\begin{array}{c}f_{s}+f_{c} \\
-\end{array}$ & $\begin{array}{l}\mathbf{f}_{\mathbf{c}} \\
-\end{array}$ & $\begin{array}{c}f_{s} \\
-\end{array}$ \\
\hline 2.50 & 4.17 & 0.34 & 6.12 & 6.20 & 0.997 & 0.000 & 0.997 \\
\hline 2.50 & 4.17 & 0.27 & 0.20 & 6.20 & 1.028 & 0.000 & 1.028 \\
\hline 2.50 & 3.30 & 0.84 & 0.44 & 5.99 & 1.120 & 0.000 & 1.120 \\
\hline 2.50 & 3.30 & 0.84 & 0.11 & 5.99 & 1.113 & 0.000 & 1.113 \\
\hline 2.50 & 3.30 & 0.83 & 0.10 & 5.99 & 1.160 & 0.000 & 1.160 \\
\hline 2.50 & 3.30 & 0.81 & 6.28 & 5.99 & 1.281 & 0.000 & 1.281 \\
\hline 2.50 & 3.30 & 0.81 & 6.36 & 5.99 & 1.235 & 0.000 & 1.235 \\
\hline 2.50 & 3.30 & 0.65 & 9.61 & 5.99 & 0.958 & 0.000 & 0.958 \\
\hline 2.50 & 3.30 & 0.63 & 12.39 & 5.99 & 0.640 & 0.450 & 0.190 \\
\hline 2.50 & 3.30 & 0.59 & 0.55 & 5.99 & 1.387 & 0.000 & 1.387 \\
\hline 2.50 & 3.30 & 0.58 & 6.47 & 5.99 & 1.327 & 0.000 & 1.327 \\
\hline 2.50 & 3.30 & 0.57 & 12.31 & 5.99 & 0.495 & 0.415 & 0.080 \\
\hline 2.50 & 3.30 & 0.57 & 12.28 & 5.99 & 0.550 & 0.401 & 0.149 \\
\hline 2.50 & 3.30 & 0.53 & 9.49 & 5.99 & 1.049 & 0.000 & 1.049 \\
\hline 2.50 & 3.30 & 0.51 & 6.89 & 5.99 & 1.238 & 0.000 & 1.238 \\
\hline 2.50 & 3.30 & 0.39 & 12.44 & 5.99 & 0.934 & 0.472 & 0.462 \\
\hline 2.50 & 3.30 & 0.38 & 6.67 & 5.99 & 1.140 & 0.000 & 1.140 \\
\hline 2.50 & 3.30 & 0.38 & 0.23 & 5.99 & 1.273 & 0.000 & 1.273 \\
\hline 2.50 & 3.30 & 0.37 & 12.42 & 5.99 & 0.964 & 0.465 & 0.499 \\
\hline 2.50 & 3.30 & 0.35 & 0.38 & 5.99 & 1.338 & 0.000 & 1.338 \\
\hline 2.50 & 3.30 & 0.34 & 6.77 & 5.99 & 1.070 & 0.000 & 1.070 \\
\hline 2.50 & 3.30 & 0.33 & 12.36 & 5.99 & 0.952 & 0.436 & 0.516 \\
\hline 2.50 & 3.30 & 0.33 & 9.56 & 5.99 & 1.010 & 0.000 & 1.010 \\
\hline 1.00 & 2.56 & 0.79 & 7.44 & 4.02 & 0.003 & 0.000 & 0.003 \\
\hline 1.00 & 2.56 & 0.79 & 1.29 & 4.02 & 0.100 & 0.000 & 0.100 \\
\hline 1.00 & 2.56 & 0.75 & 9.28 & 4.02 & 0.000 & 0.000 & 0.000 \\
\hline 1.00 & 2.56 & 0.74 & 13.07 & 4.02 & 0.000 & 0.812 & 0.000 \\
\hline 1.00 & 2.56 & 0.62 & 1.10 & 4.02 & 0.779 & 0.000 & 0.779 \\
\hline 1.00 & 2.56 & 0.59 & 7.46 & 4.02 & 0.812 & 0.000 & 0.812 \\
\hline 1.00 & 2.56 & 0.59 & 9.49 & 4.02 & 0.514 & 0.000 & 0.514 \\
\hline 1.00 & 2.56 & 0.59 & 7.40 & 4.02 & 0.000 & 0.000 & 0.000 \\
\hline 1.00 & 2.56 & 0.53 & 12.50 & 4.02 & 0.943 & 0.500 & 0.443 \\
\hline 1.00 & 2.56 & 0.37 & 12.43 & 4.02 & 1.251 & 0.457 & 0.794 \\
\hline 1.00 & 2.56 & 0.36 & 7.47 & 4.02 & 1.089 & 0.000 & 1.089 \\
\hline 1.00 & 2.56 & 0.35 & 9.71 & 4.02 & 1.181 & 0.000 & 1.181 \\
\hline 1.00 & 2.56 & 0.35 & 0.95 & 4.02 & 1.035 & 0.000 & 1.035 \\
\hline 1.00 & 3.31 & 0.82 & 0.83 & 4.13 & 0.625 & 0.000 & 0.625 \\
\hline 1.00 & 3.31 & 0.82 & 7.13 & 4.13 & 0.452 & 0.000 & 0.452 \\
\hline 1.00 & 3.31 & 0.76 & 12.25 & 4.13 & 0.705 & 0.388 & 0.317 \\
\hline 1.00 & 3.31 & 0.62 & 0.89 & 4.13 & 0.701 & 0.000 & 0.701 \\
\hline 1.00 & 3. & 0.61 & 7.00 & 4.13 & 0.941 & 0.000 & 0.941 \\
\hline 1.00 & 3.31 & 0.59 & 12.37 & 4.13 & 1.026 & 0.440 & 0.586 \\
\hline 1.00 & 3.31 & 0.59 & 12.25 & 4.13 & 1.105 & 0.388 & 0.717 \\
\hline 1.00 & 3.31 & 0.38 & 0.71 & 4.13 & 1.000 & 0.000 & 1.000 \\
\hline 1.00 & 3.31 & 0.37 & 6.93 & 4.13 & 0.962 & 0.000 & 0.962 \\
\hline 1.00 & 3.31 & 0.36 & 12.67 & 4.13 & 0.960 & 0.577 & 0.383 \\
\hline 1.00 & 4.13 & 0.82 & 9.34 & 4.20 & 1.139 & 0.000 & 1.139 \\
\hline 1.00 & 4.13 & 0.82 & 9.54 & 4.20 & 1.159 & 0.000 & 1.159 \\
\hline 1.00 & 4.13 & 0.81 & 12.99 & 4.20 & 0.735 & 0.675 & 0.060 \\
\hline 1.00 & 4.13 & 0.76 & 0.15 & 4.20 & 0.955 & 0.000 & 0.955 \\
\hline 1.00 & 4.13 & 0.60 & 0.10 & 4.20 & 1.083 & 0.000 & 1.083 \\
\hline 1.00 & 4.13 & 0.57 & 13.00 & 4.20 & 0.731 & 0.678 & 0.053 \\
\hline 1.00 & 4.13 & 0.57 & 9.54 & 4.20 & 1.194 & 0.000 & 1.194 \\
\hline
\end{tabular}


Table L.3 (continued) Measured splash fraction data for $25 \mathrm{~mm}$ wide slat.

\begin{tabular}{|c|c|c|c|c|c|c|c|}
\hline $\begin{array}{l}\mathbf{Z} \\
m\end{array}$ & $\begin{array}{c}\mathbf{d}_{\mathbf{i}} \\
m m\end{array}$ & $\begin{array}{c}\delta \\
m m\end{array}$ & $\begin{array}{c}\mathbf{x} \\
m m\end{array}$ & $\begin{array}{c}\mathbf{v}_{\mathbf{i}} \\
\mathrm{m} / \mathrm{s}\end{array}$ & $\begin{array}{c}f_{s}+f_{c} \\
-\end{array}$ & $\begin{array}{c}\mathbf{f}_{c} \\
-\end{array}$ & $\begin{array}{l}f_{s} \\
- \\
\end{array}$ \\
\hline 1.00 & 4.13 & 0.38 & 13.13 & 4.20 & 0.720 & 0.722 & 0.000 \\
\hline 1.00 & 4.13 & 0.38 & 13.15 & 4.20 & 0.602 & 0.728 & 0.000 \\
\hline 1.00 & 4.13 & 0.35 & 9.43 & 4.20 & 0.872 & 0.000 & 0.872 \\
\hline 1.00 & 4.13 & 0.35 & 9.03 & 4.20 & 0.859 & 0.000 & 0.859 \\
\hline 1.00 & 4.13 & 0.33 & 0.03 & 4.20 & 1.104 & 0.000 & 1.104 \\
\hline 1.00 & 5.62 & 0.89 & 0.42 & 4.27 & 1.345 & 0.000 & 1.345 \\
\hline 1.00 & 5.62 & 0.88 & 12.24 & 4.27 & 1.251 & 0.430 & 0.821 \\
\hline 1.00 & 5.62 & 0.87 & 14.14 & 4.27 & 0.682 & 0.888 & 0.000 \\
\hline 1.00 & 5.62 & 0.63 & 14.32 & 4.27 & 0.663 & 0.918 & 0.000 \\
\hline 1.00 & 5.62 & 0.61 & 0.61 & 4.27 & 1.105 & 0.000 & 1.105 \\
\hline 1.00 & 5.62 & 0.54 & 12.43 & 4.27 & 1.013 & 0.480 & 0.533 \\
\hline 1.00 & 5.62 & 0.40 & 14.19 & 4.27 & 0.669 & 0.897 & 0.000 \\
\hline 1.00 & 5.62 & 0.39 & 12.18 & 4.27 & 0.928 & 0.414 & 0.514 \\
\hline 1.00 & 5.62 & 0.37 & 0.19 & 4.27 & 0.958 & 0.000 & 0.958 \\
\hline 1.00 & 7.63 & 0.83 & 0.35 & 4.31 & 1.453 & 0.000 & 1.453 \\
\hline 1.00 & 7.63 & 0.79 & 12.77 & 4.31 & 1.104 & 0.553 & 0.551 \\
\hline 1.00 & 7.63 & 0.64 & 0.80 & 4.31 & 1.107 & 0.000 & 1.107 \\
\hline 1.00 & 7.63 & 0.58 & 12.98 & 4.31 & 0.928 & 0.594 & 0.334 \\
\hline 1.00 & 7.63 & 0.42 & 0.64 & 4.31 & 0.901 & 0.000 & 0.901 \\
\hline 1.00 & 7.63 & 0.30 & 11.78 & 4.31 & 0.873 & 0.360 & 0.513 \\
\hline 0.25 & 7.60 & 0.87 & 0.90 & 4.31 & 0.519 & 0.000 . & 0.519 \\
\hline 0.25 & 7.60 & 0.83 & 9.73 & 4.31 & 0.726 & 0.050 & 0.676 \\
\hline 0.25 & 7.60 & 0.81 & 12.39 & 4.31 & 0.540 & 0.478 & 0.062 \\
\hline 0.25 & 7.60 & 0.63 & 0.80 & 4.31 & 0.509 & 0.000 & 0.509 \\
\hline 0.25 & 7.60 & 0.56 & 11.88 & 4.31 & 0.700 & 0.379 & 0.321 \\
\hline 0.25 & 7.60 & 0.56 & 9.71 & 4.31 & 0.771 & 0.048 & 0.723 \\
\hline 0.25 & 7.60 & 0.45 & 0.70 & 4.31 & 0.460 & 0.000 & 0.460 \\
\hline 0.25 & 7.60 & 0.40 & 9.77 & 4.31 & 0.667 & 0.054 & 0.613 \\
\hline 0.25 & 7.60 & 0.38 & 11.91 & 4.31 & 0.568 & 0.384 & 0.184 \\
\hline 0.25 & 5.70 & 0.90 & 0.70 & 2.19 & 0.366 & 0.000 & 0.366 \\
\hline 0.25 & 5.70 & 0.73 & 0.60 & 2.19 & 0.391 & 0.000 & 0.391 \\
\hline 0.25 & 5.70 & 0.61 & 9.20 & 2.19 & 0.413 & 0.000 & 0.413 \\
\hline 0.25 & 5.70 & 0.57 & 12.23 & 2.19 & 0.314 & 0.429 & 0.000 \\
\hline 0.25 & 5.70 & 0.57 & 14.60 & 2.19 & 0.678 & 0.953 & 0.000 \\
\hline 0.25 & 5.70 & 0.54 & 0.70 & 2.19 & 0.587 & 0.000 & 0.587 \\
\hline 0.25 & 5.70 & 0.43 & 9.19 & 2.19 & 0.511 & 0.000 & 0.511 \\
\hline 0.25 & 5.70 & 0.39 & 12.46 & 2.19 & 0.358 & 0.489 & 0.000 \\
\hline 0.25 & 5.70 & 0.37 & 14.00 & 2.19 & 0.626 & 0.858 & 0.000 \\
\hline 0.25 & 5.70 & 0.33 & 0.70 & 2.19 & 0.490 & 0.000 & 0.490 \\
\hline 0.25 & 3.29 & 0.77 & 13.20 & 2.17 & 0.589 & 0.800 & 0.000 \\
\hline 0.25 & 3.29 & 0.77 & 12.35 & 2.17 & 0.402 & 0.432 & 0.000 \\
\hline 0.25 & 3.29 & 0.75 & 10.42 & 2.17 & 0.000 & 0.000 & 0.000 \\
\hline 0.25 & 3.29 & 0.71 & 6.25 & 2.17 & 0.000 & 0.000 & 0.000 \\
\hline 0.25 & 3.29 & 0.41 & 11.85 & 2.17 & 0.467 & 0.219 & 0.248 \\
\hline 0.25 & 3.29 & 0.41 & 9.13 & 2.17 & 0.314 & 0.000 & 0.314 \\
\hline 0.25 & 3.29 & 0.39 & 6.37 & 2.17 & 0.152 & 0.000 & 0.152 \\
\hline 0.25 & 3.29 & 0.39 & 13.20 & 2.17 & 0.821 & 0.800 & 0.021 \\
\hline 0.25 & 4.17 & 0.83 & 0.70 & 2.18 & 0.132 & 0.000 & 0.132 \\
\hline 0.25 & 4.17 & 0.60 & 0.70 & 2.18 & 0.180 & 0.000 & 0.180 \\
\hline 0.25 & 4.17 & 0.43 & 0.70 & 2.18 & 0.241 & 0.000 & 0.241 \\
\hline
\end{tabular}




\section{APPENDIX M Summary of drop size distribution data obtained from drop splashing and cutting experiments}

Table M.1 Measured splash drop size distribution.

\begin{tabular}{|c|c|c|c|c|c|c|c|c|}
\hline $\mathbf{d}_{\mathbf{i}}, m m$ & .32 & 3.29 & 3.32 & 3.29 & 5.56 & 5.56 & 5.61 & 5.56 \\
\hline$\delta, \mathrm{mm}$ & 0.37 & 0.38 & 0.83 & 0.84 & 0.37 & 0.40 & 0.46 & 0.78 \\
\hline $\mathbf{Z}, m$ & 1.00 & 2.50 & 1.00 & 2.50 & 0.25 & 1.00 & 2.50 & 0.25 \\
\hline $\mathbf{v}_{\mathbf{i}}, \mathrm{m} / \mathrm{s}$ & 4.14 & 5.99 & 4.14 & 5.99 & 2.19 & 4.27 & 6.38 & 2.19 \\
\hline $\mathbf{d}_{\mathbf{s}}, m m$ & $\mathbf{R},-$ & R, - & R, - & $\mathbf{R}$, - & R, - & $\mathbf{R},-$ & $\mathbf{R},-$ & R, - \\
\hline 0.05 & 000 & 000 & 000 & .000 & 0.000 & 0.000 & 0.000 & 0.000 \\
\hline 0.15 & 00 & 00 & 000 & 000 & 000 & .000 & .000 & .000 \\
\hline 0.25 & 05 & 006 & 002 & 001 & .002 & .004 & .003 & 0.000 \\
\hline 0.35 & 15 & 18 & 03 & 002 & .004 & .011 & 0.015 & 0.000 \\
\hline 0.45 & 46 & 55 & 07 & 005 & .013 & .025 & 0.049 & 0.001 \\
\hline 0.55 & 25 & & 15 & 14 & 039 & .054 & 111 & 002 \\
\hline 0.65 & 257 & 52 & c & 34 & 070 & 128 & 242 & 005 \\
\hline 0.75 & 04 & & & 1 & 13 & 226 & 96 & 09 \\
\hline 0.85 & 5 & & & & 08 & 91 & .595 & 0.019 \\
\hline 0.95 & 3 & & & 0 & 288 & .519 & 0.770 & 0.022 \\
\hline 1.05 & 9 & & $c$ & 239 & 320 & 0.650 & 0.894 & 0.028 \\
\hline 1.15 & 0 & 62 & c & 54 & 550 & 0.783 & 0.963 & 0.060 \\
\hline 1.25 & 00 & 962 & c & 470 & 643 & 0.857 & 1.000 & 0.079 \\
\hline 1.35 & 00 & 00 & 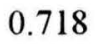 & 580 & 3 & 896 & 1.000 & 0.133 \\
\hline 1.45 & 00 & & & 780 & 848 & .000 & 1.000 & 0.252 \\
\hline 1.55 & 00 & & & & 18 & 000 & .000 & 0.379 \\
\hline 1.65 & & & & & 00 & 00 & 00 & 0.518 \\
\hline 1.75 & & & & & 00 & 00 & 000 & 0.616 \\
\hline 1.85 & & & & & 00 & 000 & 000 & 0.711 \\
\hline 1.95 & & & & & 000 & 000 & .000 & 0.754 \\
\hline 2.05 & & & & 00 & 000 & .000 & 1.000 & 0.863 \\
\hline 2.15 & & & & 00 & 00 & 1.000 & 1.000 & 0.863 \\
\hline & & & & 00 & 00 & 1.000 & 1.000 & 0.863 \\
\hline 2.35 & & & & 00 & 00 & 1.000 & 1.000 & 0.902 \\
\hline 2. & 00 & & & 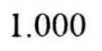 & 00 & 1.000 & 1.000 & 0.945 \\
\hline 2.55 & 00 & & & 00 & 00 & 1.000 & 1.000 & 0.945 \\
\hline 2.65 & 00 & & & & 00 & 1.000 & 1.000 & 1.000 \\
\hline 2.75 & 00 & & & & & 1.000 & 1.000 & 1.000 \\
\hline 2.85 & & & & & & 1.000 & .000 & 1.000 \\
\hline 2.95 & 1.000 & 1.000 & 1.000 & 1.000 & 1.000 & 1.000 & 1.000 & 1.000 \\
\hline 3.05 & 1.000 & 1.000 & 1.000 & 1.000 & 1.000 & 0 & 1.000 & 1.000 \\
\hline
\end{tabular}


Table M.1 (continued) Measured splash drop size distribution.

\begin{tabular}{|c|c|c|c|c|c|c|c|c|}
\hline $\mathbf{d}_{\mathbf{i}}, m m$ & 5.56 & 5.61 & 7.48 & 7.7 & 7.48 & 7.53 & 7.70 & 7.48 \\
\hline$\delta, m m$ & 0.85 & 0.80 & 0.41 & 0.38 & 0.46 & 0.83 & 0.83 & 0.75 \\
\hline $\mathbf{Z}, m$ & 1.00 & 2.50 & 0.25 & 1.00 & 2.50 & 0.25 & 1.00 & 2.50 \\
\hline $\mathbf{v}_{\mathbf{i}}, \mathrm{m} / \mathrm{s}$ & 4.27 & 6.38 & 2.20 & 4.31 & 6.50 & 2.20 & 4.31 & 6.50 \\
\hline $\mathbf{d}_{\mathbf{s}}, \mathrm{mm}$ & $\mathbf{R},-$ & $\mathbf{R},-$ & $\mathbf{R},-$ & $\mathbf{R},-$ & $\mathbf{R},-$ & $\mathbf{R},-$ & $\mathbf{R},-$ & $\mathbf{R},-$ \\
\hline 0.05 & 0.000 & 0.000 & 0.000 & 0.000 & 0.000 & 0.000 & 0.000 & 0.000 \\
\hline 0.15 & 0.000 & 0.000 & 0.000 & 0.000 & 0.000 & 0.000 & 0.000 & 0.000 \\
\hline 0.25 & 0.001 & 0.001 & 0.000 & 0.002 & 0.003 & 0.000 & 0.000 & 0.001 \\
\hline 0.35 & 0.002 & 0.005 & 0.003 & 0.005 & 0.010 & 0.001 & 0.001 & 0.004 \\
\hline 0.45 & 0.006 & 0.013 & 0.008 & 0.014 & 0.032 & 0.003 & 0.004 & 0.011 \\
\hline 0.55 & 0.010 & 0.025 & 0.027 & 0.037 & 0.066 & 0.006 & 0.010 & 0.026 \\
\hline 0.65 & 0.017 & 0.042 & 0.065 & 0.071 & 0.122 & 0.014 & 0.019 & 0.052 \\
\hline 0.75 & 0.036 & 0.082 & 0.145 & 0.117 & 0.200 & 0.022 & 0.032 & 0.080 \\
\hline 0.85 & 0.055 & 0.137 & 0.226 & 0.183 & 0.302 & 0.037 & 0.056 & 0.116 \\
\hline 0.95 & 0.114 & 0.207 & 0.301 & 0.270 & 0.405 & 0.041 & 0.070 & 0.158 \\
\hline 1.05 & 0.201 & 0.296 & 0.402 & 0.347 & 0.523 & 0.073 & 0.100 & 0.204 \\
\hline 1.15 & 0.269 & 0.398 & 0.558 & 0.456 & 0.643 & 0.121 & 0.137 & 0.277 \\
\hline 1.25 & 0.362 & 0.546 & 0.661 & 0.558 & 0.770 & 0.163 & 0.178 & 0.350 \\
\hline 1.35 & 0.477 & 0.641 & 0.757 & 0.680 & 0.861 & 0.197 & 0.230 & 0.395 \\
\hline 1.45 & 0.589 & 0.735 & 0.792 & 0.749 & 0.936 & 0.274 & 0.277 & 0.483 \\
\hline 1.55 & 0.702 & 0.838 & 0.861 & 0.818 & 0.957 & 0.360 & 0.319 & 0.558 \\
\hline 1.65 & 0.820 & 0.901 & 0.881 & 0.891 & 0.969 & 0.418 & 0.417 & 0.628 \\
\hline 1.75 & 0.860 & 0.958 & 0.919 & 0.920 & 0.969 & 0.440 & 0.464 & 0.690 \\
\hline 1.85 & 0.932 & 0.974 & 0.919 & 0.952 & 0.978 & 0.469 & 0.545 & 0.750 \\
\hline 1.95 & 0.957 & 0.974 & 0.937 & 0.964 & 1.000 & 0.541 & 0.592 & 0.796 \\
\hline 2.05 & 0.957 & 0.974 & 1.000 & 0.964 & 1.000 & 0.668 & 0.635 & 0.837 \\
\hline 2.15 & 0.957 & 0.974 & 1.000 & 1.000 & 1.000 & 0.676 & 0.671 & 0.847 \\
\hline 2.25 & 1.000 & 1.000 & 1.000 & 1.000 & 1.000 & 0.783 & 0.752 & 0.869 \\
\hline 2.35 & 1.000 & 1.000 & 1.000 & 1.000 & 1.000 & 0.783 & 0.752 & 0.895 \\
\hline 2.45 & 1.000 & 1.000 & 1.000 & 1.000 & 1.000 & 0.783 & 0.824 & 0.895 \\
\hline 2.55 & 1.000 & 1.000 & 1.000 & 1.000 & 1.000 & 0.783 & 0.864 & 0.911 \\
\hline 2.65 & 1.000 & 1.000 & 1.000 & 1.000 & 1.000 & 0.907 & 0.888 & 0.930 \\
\hline 2.75 & 1.000 & 1.000 & 1.000 & 1.000 & 1.000 & 0.907 & 0.938 & 0.972 \\
\hline 2.85 & 1.000 & 1.000 & 1.000 & 1.000 & 1.000 & 0.907 & 0.965 & 0.972 \\
\hline 2.95 & 1.000 & 1.000 & 1.000 & 1.000 & 1.000 & 0.907 & 0.965 & 0.972 \\
\hline 3.05 & 1.000 & 1. & 1.000 & 1.000 & 1 & 1 & 0 & 1 \\
\hline
\end{tabular}


Table M.2 Measured size distribution of drops formed by cutting (W=0.3 $\mathrm{mm}$ ).

\begin{tabular}{|c|c|c|c|c|c|c|c|c|}
\hline $\begin{array}{l}\mathbf{d}_{\mathbf{i}}, m m \\
\mathbf{W} \mathbf{e}_{\mathbf{i}},- \\
\mathbf{x}, m m\end{array}$ & $\begin{array}{l}3.3 \\
183 \\
0.0 \\
\end{array}$ & $\begin{array}{c}3.3 \\
784 \\
0.0 \\
\end{array}$ & $\begin{array}{c}3.3 \\
1645 \\
0.0 \\
\end{array}$ & $\begin{array}{l}5.6 \\
373 \\
0.0 \\
\end{array}$ & $\begin{array}{c}5.6 \\
1416 \\
0.0 \\
\end{array}$ & $\begin{array}{c}5.6 \\
3170 \\
0.0 \\
\end{array}$ & $\begin{array}{c}5.6 \\
1416 \\
1.5 \\
\end{array}$ & $\begin{array}{c}5.6 \\
3170 \\
1.5 \\
\end{array}$ \\
\hline $\mathbf{d}_{\mathbf{c}}, m m$ & $\mathbf{R},-$ & $\mathbf{R},-$ & $\mathbf{R},-$ & $\mathbf{R},-$ & $\mathbf{R},-$ & R, - & R, - & R, - \\
\hline 0.17 & 0.000 & 0.000 & 0.000 & 0.000 & 0.000 & 0.000 & 0.000 & 0.000 \\
\hline 0.28 & 0.000 & 0.000 & 0.001 & 0.000 & 0.000 & 0.000 & 0.000 & 0.000 \\
\hline 0.39 & 0.000 & 0.001 & 0.002 & 0.000 & 0.001 & 0.001 & 0.000 & 0.001 \\
\hline 0.50 & 0.001 & 0.003 & 0.006 & 0.000 & 0.001 & 0.003 & 0.001 & 0.003 \\
\hline 0.61 & 0.003 & 0.007 & 0.013 & 0.001 & 0.003 & 0.006 & 0.001 & 0.006 \\
\hline 0.72 & 0.004 & 0.013 & 0.016 & 0.002 & 0.004 & 0.009 & 0.003 & 0.009 \\
\hline 0.83 & 0.009 & 0.019 & 0.023 & 0.003 & 0.006 & 0.014 & 0.006 & 0.013 \\
\hline 0.94 & 0.012 & 0.028 & 0.028 & 0.005 & 0.007 & 0.019 & 0.010 & 0.018 \\
\hline 1.06 & 0.018 & 0.028 & 0.030 & 0.007 & 0.009 & 0.027 & 0.016 & 0.027 \\
\hline 1.17 & 0.022 & 0.034 & 0.030 & 0.008 & 0.013 & 0.035 & 0.021 & 0.034 \\
\hline 1.28 & 0.025 & 0.034 & 0.030 & 0.011 & 0.020 & 0.047 & 0.024 & 0.046 \\
\hline 1.39 & 0.029 & 0.041 & 0.030 & 0.011 & 0.023 & 0.054 & 0.029 & 0.054 \\
\hline 1.50 & 0.029 & 0.055 & 0.030 & 0.014 & 0.024 & 0.062 & 0.029 & 0.061 \\
\hline 1.61 & 0.029 & 0.055 & 0.035 & 0.015 & 0.027 & 0.066 & 0.029 & 0.071 \\
\hline 1.72 & 0.029 & 0.061 & 0.035 & 0.020 & 0.028 & 0.072 & 0.033 & 0.077 \\
\hline 1.83 & 0.033 & 0.069 & 0.035 & 0.020 & 0.028 & 0.078 & 0.038 & 0.092 \\
\hline 1.94 & 0.033 & 0.078 & 0.063 & 0.020 & 0.028 & 0.081 & 0.038 & 0.096 \\
\hline 2.06 & 0.033 & 0.089 & 0.063 & 0.020 & 0.028 & 0.094 & 0.038 & 0.107 \\
\hline 2.17 & 0.033 & 0.140 & 0.077 & 0.020 & 0.028 & 0.098 & 0.038 & 0.117 \\
\hline 2.28 & 0.033 & 0.186 & 0.092 & 0.020 & 0.031 & 0.114 & 0.038 & 0.138 \\
\hline 2.39 & 0.033 & 0.186 & 0.197 & 0.020 & 0.034 & 0.126 & 0.038 & 0.169 \\
\hline 2.50 & 0.033 & 0.264 & 0.238 & 0.020 & 0.034 & 0.133 & 0.038 & 0.189 \\
\hline 2.61 & 0.033 & 0.286 & 0.300 & 0.020 & 0.034 & 0.142 & 0.038 & 0.189 \\
\hline 2.72 & 0.033 & 0.439 & 0.444 & 0.025 & 0.043 & 0.142 & 0.038 & 0.200 \\
\hline 2.83 & 0.033 & 0.587 & 0.567 & 0.025 & 0.048 & 0.142 & 0.038 & 0.216 \\
\hline 2.94 & 0.033 & 0.715 & 0.697 & 0.025 & 0.048 & 0.142 & 0.056 & 0.233 \\
\hline 3.06 & 0.033 & 0.865 & 0.773 & 0.025 & 0.048 & 0.142 & 0.056 & 0.262 \\
\hline 3.17 & 0.055 & 0.907 & 0.816 & 0.025 & 0.055 & 0.156 & 0.056 & 0.288 \\
\hline 3.28 & 0.312 & 1.000 & 0.949 & 0.025 & 0.064 & 0.156 & 0.056 & 0.305 \\
\hline 3.39 & 0.940 & 1.000 & 1.000 & 0.025 & 0.064 & 0.156 & 0.056 & 0.322 \\
\hline 3.50 & 1.000 & 1.000 & 1.000 & 0.025 & 0.073 & 0.174 & 0.056 & 0.342 \\
\hline 3.61 & & & & 0.025 & 0.106 & 0.174 & 0.056 & 0.355 \\
\hline 3.72 & & & & 0.025 & 0.154 & 0.174 & 0.093 & 0.355 \\
\hline 3.83 & & & & 0.025 & 0.206 & 0.224 & 0.093 & 0.366 \\
\hline 3.94 & & & & 0.059 & 0.220 & 0.280 & 0.186 & 0.366 \\
\hline 4.06 & & & & 0.113 & 0.236 & 0.342 & 0.234 & 0.385 \\
\hline 4.17 & & & & 0.133 & 0.236 & 0.440 & 0.234 & 0.385 \\
\hline 4.28 & & & & 0.154 & 0.253 & 0.655 & 0.292 & 0.385 \\
\hline 4.39 & & & & 0.178 & 0.292 & 0.733 & 0.292 & 0.409 \\
\hline 4.50 & & & & 0.178 & 0.334 & 0.817 & 0.292 & 0.409 \\
\hline 4.61 & & & & 0.178 & 0.357 & 0.904 & 0.368 & 0.483 \\
\hline 4.72 & & & & 0.206 & 0.382 & 1.000 & 0.680 & 0.483 \\
\hline 4.83 & & & & 0.298 & 0.458 & 1.000 & 0.767 & 0.483 \\
\hline 4.94 & & & & 0.330 & 0.571 & 1.000 & 0.767 & 0.516 \\
\hline 5.06 & & & & 0.365 & 0.719 & 1.000 & 0.767 & 0.586 \\
\hline 5.17 & & & & 0.365 & 0.782 & 1.000 & 0.767 & 0.586 \\
\hline 5.28 & & & & 0.365 & 0.849 & 1.000 & 0.767 & 0.739 \\
\hline 5.39 & & & & 0.365 & 0.959 & 1.000 & 1.000 & 0.920 \\
\hline 5.50 & & & & 0.365 & 0.959 & 1.000 & 1.000 & 1.000 \\
\hline 5.61 & & & & 0.849 & 1.000 & 1.000 & & \\
\hline 5.72 & & & & 1.000 & 1.000 & 1.000 & & \\
\hline
\end{tabular}




\section{5}

Table M.3 Measured drop size distribution of drops formed by cutting (W=2 $\mathrm{mm}$ ).

\begin{tabular}{|c|c|c|c|c|c|}
\hline $\mathbf{d}_{\mathbf{i}}, m m$ & 6.2 & 6.2 & 6.2 & 6.2 & 6.2 \\
$\mathbf{W e}_{\mathbf{i}},-$ & 802 & 1552 & 2255 & 2902 & 3492 \\
$\mathbf{x}, m m$ & 0.0 & 0.0 & 0.0 & 0.0 & 0.0 \\
\hline $\mathbf{d}_{\mathbf{c}}, m m$ & $\mathbf{R},-$ & $\mathbf{R},-$ & $\mathbf{R},-$ & $\mathbf{R},-$ & $\mathbf{R},-$ \\
\hline 0.1 & 0.000 & 0.000 & 0.000 & 0.000 & 0.000 \\
0.3 & 0.000 & 0.001 & 0.003 & 0.007 & 0.007 \\
0.5 & 0.004 & 0.019 & 0.026 & 0.052 & 0.055 \\
0.7 & 0.016 & 0.077 & 0.132 & 0.146 & 0.193 \\
0.9 & 0.033 & 0.168 & 0.258 & 0.274 & 0.375 \\
1.1 & 0.061 & 0.244 & 0.366 & 0.384 & 0.468 \\
1.3 & 0.073 & 0.281 & 0.433 & 0.507 & 0.576 \\
1.5 & 0.088 & 0.332 & 0.467 & 0.589 & 0.711 \\
1.7 & 0.088 & 0.407 & 0.538 & 0.617 & 0.789 \\
1.9 & 0.129 & 0.432 & 0.607 & 0.617 & 0.819 \\
2.1 & 0.129 & 0.501 & 0.637 & 0.617 & 0.900 \\
2.3 & 0.129 & 0.541 & 0.637 & 0.617 & 0.900 \\
2.5 & 0.159 & 0.541 & 0.637 & 0.617 & 0.900 \\
2.7 & 0.159 & 0.541 & 0.637 & 0.617 & 1.000 \\
2.9 & 0.159 & 0.541 & 0.637 & 0.617 & 1.000 \\
3.1 & 0.159 & 0.541 & 0.637 & 0.617 & 1.000 \\
3.3 & 0.159 & 0.541 & 1.000 & 0.789 & 1.000 \\
3.5 & 0.159 & 0.541 & 1.000 & 1.000 & 1.000 \\
3.7 & 0.159 & 0.541 & 1.000 & 1.000 & 1.000 \\
3.9 & 0.159 & 1.000 & 1.000 & 1.000 & 1.000 \\
4.1 & 0.297 & 1.000 & 1.000 & 1.000 & 1.000 \\
4.3 & 0.607 & 1.000 & 1.000 & 1.000 & 1.000 \\
4.5 & 0.779 & 1.000 & 1.000 & 1.000 & 1.000 \\
4.7 & 0.779 & 1.000 & 1.000 & 1.000 & 1.000 \\
4.9 & 1.000 & 1.000 & 1.000 & 1.000 & 1.000 \\
\hline & & & & &
\end{tabular}




\section{APPENDIX N Summary of drop size distribution data for water dripping below different slats}

Table N.1 Measured dripping drop size distribution for slat A.

\begin{tabular}{|c|c|c|c|c|c|c|c|c|c|c|c|c|}
\hline & \multicolumn{6}{|c|}{ FREQUENCY } & \multicolumn{6}{|c|}{ CUMULATIVE MASS FRACTION } \\
\hline $\begin{array}{c}\Gamma, g / m s \\
\text { d, } m m\end{array}$ & $\begin{array}{l}7.9 \\
\mathbf{N} \\
\end{array}$ & $\begin{array}{l}16 \\
\mathrm{~N} \\
\end{array}$ & $\begin{array}{l}30 \\
\mathbf{N} \\
\end{array}$ & $\begin{array}{l}77 \\
\mathbf{N} \\
\end{array}$ & $\begin{array}{l}64 \\
\mathbf{N} \\
\end{array}$ & $\begin{array}{c}113 \\
\mathbf{N}\end{array}$ & $\begin{array}{l}7.9 \\
\mathbf{R},- \\
\end{array}$ & $\begin{array}{l}16 \\
\mathbf{R},- \\
\end{array}$ & $\begin{array}{c}30 \\
\mathbf{R},- \\
\end{array}$ & $\begin{array}{c}47 \\
\text { R, - } \\
\end{array}$ & $\begin{array}{c}64 \\
R,- \\
\end{array}$ & $\begin{array}{l}113 \\
\mathbf{R},-\end{array}$ \\
\hline 0.25 & 0 & 21 & 7 & 24 & 5 & 11 & 0.000 & 0.000 & 0.000 & 0.000 & 0.000 & 0.000 \\
\hline 0.75 & 4 & 6 & 9 & 15 & 17 & 21 & 0.000 & 0.000 & 0.000 & 0.000 & 0.000 & 0.000 \\
\hline 1.25 & 14 & 50 & 26 & 28 & 93 & 38 & 0.000 & 0.000 & 0.000 & 0.000 & 0.000 & 0.000 \\
\hline 1.75 & 48 & 178 & 77 & 52 & 96 & 34 & 0.003 & 0.004 & 0.004 & 0.004 & 005 & 0.002 \\
\hline 2.25 & 42 & 146 & 88 & 55 & 56 & 27 & 0.026 & 0.038 & 0.030 & 19 & 0.016 & 0.005 \\
\hline 2.75 & 24 & 33 & 59 & 47 & 61 & 40 & 0.066 & 0.091 & 0.090 & 0.054 & 0.029 & 0.011 \\
\hline 3.25 & 14 & 14 & 58 & 52 & 63 & 26 & 0.107 & 0.113 & 0.161 & 0.108 & 0.056 & 0.027 \\
\hline 3.75 & 2 & 28 & 26 & 54 & 66 & 28 & 0.149 & 0.129 & 0.283 & 0.202 & 0.102 & 0.045 \\
\hline 4.25 & 3 & 6 & 19 & 30 & 69 & 29 & 0.165 & 0.178 & 0.365 & 0.353 & 0.178 & 0.075 \\
\hline 4.75 & 1 & 0 & 10 & 30 & 66 & 30 & 0.184 & 0.193 & 0.449 & 0.474 & 0.294 & 0.118 \\
\hline 5.25 & 0 & 0 & 1 & 12 & 39 & 25 & 0.192 & 0.193 & 0.514 & 0.648 & 0.443 & 0.182 \\
\hline 5.75 & 0 & 0 & 2 & 0 & 22 & 25 & 0.192 & 0.193 & 0.524 & 0.741 & 0.564 & 0.251 \\
\hline 6.25 & 0 & 0 & 0 & 5 & 14 & 24 & 0.192 & 0.193 & 0.546 & 0.831 & 0.655 & 0.346 \\
\hline 6.75 & 3 & 0 & 3 & $?$ & 9 & 15 & 0.192 & 0.193 & 0.546 & 0.898 & 0.728 & 0.459 \\
\hline 7.25 & 14 & 18 & 0 & 0 & 3 & 12 & 0.278 & 0.193 & 0.604 & 0.950 & 0.787 & 0.548 \\
\hline 7.75 & 4 & 31 & 9 & 2 & 5 & 4 & 0.750 & 0.438 & 0.604 & 950 & 812 & 0.633 \\
\hline 8.25 & 2 & 5 & 3 & 0 & 4 & 4 & 0.903 & 0.911 & 0.867 & 1.000 & 863 & 0.667 \\
\hline 8.75 & 0 & 0 & 1 & 0 & 5 & 5 & 1.000 & 00 & 0.963 & .000 & 0.912 & 0.711 \\
\hline 9.25 & 0 & 0 & 0 & 0 & 1 & 0 & 1.000 & 00 & 1.000 & 1.000 & 0.984 & 0.776 \\
\hline 9.7 & 0 & 0 & 0 & 0 & 0 & 4 & 1.000 & 0 & 1.000 & 1.000 & 1.000 & 0.776 \\
\hline 10.25 & 0 & 0 & 0 & 0 & 0 & 0 & 1.000 & 1.000 & 1.000 & 1.000 & 1.000 & 0.849 \\
\hline 10.75 & 0 & 0 & 0 & 0 & 0 & 1 & 1.000 & 1.000 & 1.000 & 1.000 & 1.000 & 0.849 \\
\hline 11.25 & 0 & 0 & 0 & 0 & 0 & 1 & 1.000 & 1.000 & 1.000 & 1.000 & 1.000 & 0.874 \\
\hline 11.75 & 0 & 0 & 0 & 0 & 0 & 1 & 1.000 & 1.000 & 1.000 & 1.000 & 1.000 & 0.901 \\
\hline 12.25 & 0 & 0 & 0 & 0 & 0 & 2 & 1.000 & 1.000 & 1.000 & 1.000 & 1.000 & 0.932 \\
\hline 12.75 & 0 & 0 & 0 & 0 & 0 & 0 & 1.000 & 1.000 & 1.000 & 1.000 & 1.000 & 1.000 \\
\hline 13.25 & 0 & 0 & 0 & 0 & 0 & 0 & 1.000 & 1.000 & 1.000 & 1.000 & 1.000 & 1.000 \\
\hline Total & 176 & 536 & 398 & 418 & 694 & 407 & & & & & & \\
\hline
\end{tabular}


Table N.2 Measured dripping drop size distribution for slat B.

\begin{tabular}{|c|c|c|c|c|c|c|c|c|c|c|}
\hline & \multicolumn{5}{|c|}{ FREQUENCY } & \multicolumn{5}{|c|}{ CUMULATIVE MASS FRACTION } \\
\hline $\begin{array}{c}\Gamma, g / m s \\
\text { d, } m m\end{array}$ & $\begin{array}{l}19 \\
\mathbf{N}\end{array}$ & $\begin{array}{l}36 \\
\mathbf{N}\end{array}$ & $\begin{array}{l}57 \\
\mathbf{N}\end{array}$ & $\begin{array}{l}77 \\
\mathbf{N}\end{array}$ & $\begin{array}{c}136 \\
\mathbf{N}\end{array}$ & $\begin{array}{c}19 \\
\mathbf{R},-\end{array}$ & $\begin{array}{c}36 \\
\mathbf{R},-\end{array}$ & $\begin{array}{c}57 \\
\mathbf{R},-\end{array}$ & $\begin{array}{c}77 \\
\text { R. - }\end{array}$ & $\begin{array}{l}136 \\
\mathbf{R},-\end{array}$ \\
\hline 0.25 & 4 & 0 & 0 & 2 & 13 & 0.000 & 0.000 & 0.000 & 0.000 & 0.000 \\
\hline 0.75 & 5 & 13 & 8 & 6 & 25 & 0.000 & 0.000 & 0.000 & 0.000 & 0.000 \\
\hline 1.25 & 58 & 43 & 32 & 55 & 34 & 0.000 & 0 . & 00 & 0.000 & 0.000 \\
\hline 1.75 & 87 & 40 & 31 & 50 & 34 & 06 & & 0.005 & 0.004 & 0.002 \\
\hline 2.25 & 43 & 34 & 19 & 44 & 18 & 27 & & 0.016 & 0.013 & 0.006 \\
\hline 2.75 & 24 & 32 & 27 & 45 & 34 & 0.048 & 46 & 0.031 & 0.029 & 0.011 \\
\hline 3.25 & 24 & 22 & 29 & 41 & 23 & 0.071 & 0.087 & 0.070 & 0.060 & 0.028 \\
\hline 3.75 & 11 & 19 & 36 & 44 & 27 & 0.108 & 0.133 & 0.136 & 0.106 & 0.047 \\
\hline 4.25 & 5 & 15 & 37 & 51 & 30 & 0.133 & 0.199 & 0.269 & 0.181 & 0.079 \\
\hline 4.75 & 1 & 3 & 20 & 38 & 32 & 0.151 & 0.271 & 0.461 & 0.307 & 0.134 \\
\hline 5.25 & . & 4 & 14 & 24 & 15 & 0.156 & 0. & 0.608 & 0.438 & 0.212 \\
\hline 5.75 & 0 & 0 & 3 & 18 & 18 & 0.156 & 0. & 0.744 & 0.551 & 0.264 \\
\hline 6.25 & 0 & 1 & 6 & 10 & 20 & 0.156 & 0.327 & 0.782 & 0.660 & 0.343 \\
\hline 6.75 & 6 & 0 & 1 & 10 & 14 & 0.156 & 0.344 & 0.883 & 0.738 & 0.459 \\
\hline 7.25 & 38 & 17 & 1 & 4 & 14 & 0.246 & 0. & 0.904 & 0.837 & 0.563 \\
\hline 7.75 & 4 & 6 & 2 & 4 & 9 & 0.893 & & 0.932 & 0.883 & 0.690 \\
\hline 8.25 & 0 & 2 & 0 & 2 & 3 & 0.972 & 35 & 1.000 & 0.941 & 0.789 \\
\hline 8.75 & 1 & . & 0 & 1 & 3 & 0.972 & 00 & 1.000 & 0.977 & 0.827 \\
\hline 9.2 & 0 & 0 & 0 & 0 & 3 & 1.000 & 1 & 1.000 & 1.000 & 0.873 \\
\hline 9.7 & 0 & 0 & 0 & 0 & 3 & 1.000 & 1.000 & 1.000 & 1.000 & 0.933 \\
\hline 10.2 & 0 & 0 & 0 & 0 & 0 & 1.000 & 1.000 & 1.000 & 1.000 & 1.000 \\
\hline 10.75 & 0 & 0 & 0 & 0 & 0 & 1.000 & 1.000 & 1.000 & 1.000 & 1.000 \\
\hline 11.25 & 0 & 0 & 0 & 0 & 0 & 1.000 & 1.000 & 1.000 & 1.000 & 1.000 \\
\hline 11.75 & 0 & 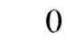 & 0 & 0 & 0 & 1.000 & 1.000 & 1.000 & 1.000 & 1.000 \\
\hline 12.25 & 0 & 8 & 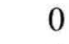 & 0 & 0 & 1.000 & 1.000 & 1.000 & 1.000 & 1.000 \\
\hline 12.7 & 0 & 0 & 0 & 0 & 0 & 1.000 & 1.000 & 1.000 & 1.000 & 1.000 \\
\hline 13.25 & 0 & 0 & $\underline{0}$ & 0 & 0 & 1.000 & 1.000 & 1.000 & 1.000 & 1.000 \\
\hline Total & 311 & 251 & 266 & 449 & 372 & & & & & \\
\hline
\end{tabular}


Table N.3 Measured dripping drop size distribution for slat C.

\begin{tabular}{|c|c|c|c|c|c|c|c|c|c|c|}
\hline & \multicolumn{5}{|c|}{ FREQUENCY } & \multicolumn{5}{|c|}{ CUMULATIVE MASS FRACTION } \\
\hline $\begin{array}{c}\Gamma, g / m s \\
\text { d, } m m\end{array}$ & $\begin{array}{l}18 \\
\mathrm{~N}\end{array}$ & $\begin{array}{l}35 \\
\mathbf{N}\end{array}$ & $\begin{array}{l}55 \\
\mathrm{~N}\end{array}$ & $\begin{array}{l}75 \\
N\end{array}$ & $\begin{array}{c}133 \\
\mathbf{N}\end{array}$ & $\begin{array}{l}18 \\
\mathrm{R},-\end{array}$ & $\begin{array}{c}35 \\
\mathbf{R},-\end{array}$ & $\begin{array}{c}55 \\
\mathbf{R},-\end{array}$ & $\begin{array}{c}75 \\
\mathbf{R},-\end{array}$ & $\begin{array}{l}133 \\
\mathbf{R},-\end{array}$ \\
\hline 0.25 & 0 & 2 & 0 & 6 & 30 & 0.000 & 0.000 & 0.000 & 0.000 & 0.000 \\
\hline 0.75 & 21 & 13 & 2 & 2 & 18 & 0.000 & 0.000 & 0.000 & 0.000 & 0.000 \\
\hline 1.25 & 75 & 60 & 24 & 32 & 30 & 0.001 & 0.000 & 0.000 & 0.000 & 0.000 \\
\hline 1.75 & 55 & 58 & 20 & 42 & 28 & 0.007 & 0.006 & 0.003 & 0.002 & 0.002 \\
\hline 2.25 & 46 & 35 & 27 & 33 & 17 & 19 & 0.018 & 0.010 & 0.010 & 0.006 \\
\hline 2.75 & 28 & 32 & 24 & 29 & 36 & 13 & 35 & 0.025 & 0.021 & 0.012 \\
\hline 3.25 & 4 & 39 & 30 & 38 & 23 & 0.065 & 0.063 & 0.053 & 0.041 & 0.033 \\
\hline 3.75 & 2 & 11 & 31 & 44 & 36 & 0.070 & 0.119 & 0.110 & 0.083 & 0.056 \\
\hline 4.25 & 0 & 10 & 27 & 50 & 26 & 0.075 & 0.142 & 0.204 & 0.158 & 0.109 \\
\hline 4.75 & 0 & 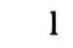 & 22 & 33 & 36 & 0.075 & 0.175 & 0.316 & 0.279 & 0.165 \\
\hline 5.25 & 0 & 0 & 14 & 35 & 20 & 0.075 & 0.179 & 0.444 & 0.392 & 0.274 \\
\hline 5.75 & 0 & 1 & 11 & 16 & 17 & 0.075 & 79 & 0.550 & 0.548 & 0.354 \\
\hline 6.25 & 31 & 3 & 6 & 15 & 20 & 0.075 & 0.187 & 0.666 & 0.645 & 0.443 \\
\hline 6.75 & 40 & 40 & 4 & 10 & 12 & 0.421 & 0.216 & 0.747 & 0.759 & 0.580 \\
\hline 7.25 & 4 & 14 & 8 & 2 & 0 & 0.935 & & 0.816 & 0.855 & 0.684 \\
\hline 7.75 & 0 & 2 & 1 & 4 & 5 & 1. & & 0.977 & 0.880 & 0.778 \\
\hline 8.25 & 0 & 0 & 0 & 1 & 1 & & 0 & 1.000 & 0.940 & 0.841 \\
\hline 8 . & 0 & 0 & 0 & 2 & 1 & 1. & 0 & 1.000 & 0.958 & 0.856 \\
\hline 9.2 & 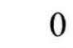 & . & 0 & 0 & the & 1.000 & 1.000 & 1.000 & 1.000 & 0.874 \\
\hline 9.7 & 0 & 0 & 0 & 0 & 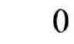 & 1.000 & 1.000 & 1.000 & 1.000 & 0.895 \\
\hline 10.25 & 0 & 0 & 0 & $\sigma$ & 1 & 1.000 & 1.000 & 1.000 & 1.000 & 0.895 \\
\hline 10.75 & 0 & 0 & 0 & 0 & 1 & 1.000 & 1.000 & 1.000 & 1.000 & 0.926 \\
\hline 11.25 & 8 & 0 & 0 & 0 & 1 & 1.000 & 1.000 & 1.000 & 1.000 & 0.959 \\
\hline 11.75 & 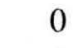 & . & 0 & 0 & v & 1.000 & 1.000 & 1.000 & 1.000 & 1.000 \\
\hline 12.25 & 0 & 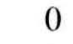 & 0 & 0 & 0 & 1.000 & 1.000 & 1.000 & 1.000 & 1.000 \\
\hline 12.75 & 0 & 0 & 0 & 0 & 0 & 1.000 & 1.000 & 1.000 & 1.000 & 1.000 \\
\hline 13.25 & 0 & 0 & 0 & 0 & $\underline{0}$ & 1.000 & 1.000 & 1.000 & 1.000 & 1.000 \\
\hline Total & 306 & 321 & 251 & 394 & 369 & & & & & \\
\hline
\end{tabular}


Table N.4 Measured dripping drop size distribution for slat D.

\begin{tabular}{|c|c|c|c|c|c|c|c|c|c|c|}
\hline & \multicolumn{5}{|c|}{ FREQUENCY } & \multicolumn{5}{|c|}{ CUMULATIVE MASS FRACTION } \\
\hline $\begin{array}{c}\Gamma, g / m s \\
\mathbf{d}, m m\end{array}$ & $\begin{array}{l}19 \\
\mathbf{N}\end{array}$ & $\begin{array}{l}36 \\
\mathbf{N}\end{array}$ & $\begin{array}{l}56 \\
\mathbf{N}\end{array}$ & $\begin{array}{l}77 \\
\mathbf{N}\end{array}$ & $\begin{array}{c}135 \\
\mathbf{N}\end{array}$ & $\begin{array}{l}19 \\
\mathbf{R},-\end{array}$ & $\begin{array}{c}36 \\
\mathbf{R},-\end{array}$ & $\begin{array}{c}56 \\
\mathbf{R},-\end{array}$ & $\begin{array}{c}77 \\
\mathbf{R},-\end{array}$ & $\begin{array}{l}135 \\
\mathbf{R},-\end{array}$ \\
\hline 0.25 & 11 & 8 & 7 & 9 & 10 & 0.000 & 0.000 & 0.000 & 0.000 & 0.000 \\
\hline 0.75 & 9 & 39 & 31 & 43 & 9 & 0.000 & 0.000 & 0.000 & 0.000 & 0.000 \\
\hline 1.25 & 32 & 57 & 72 & 73 & 26 & 0.000 & 0.001 & 0.001 & 0.001 & 0.000 \\
\hline 1.75 & 66 & 62 & 56 & 50 & 38 & 0.004 & 0.005 & 0.007 & 0.006 & 0.002 \\
\hline 2.25 & 50 & 63 & 40 & 35 & 35 & 0.025 & 0.017 & 0.022 & 0.016 & 0.007 \\
\hline 2.75 & 29 & 54 & 37 & 27 & 24 & 0.057 & 0.042 & 0.042 & 0.030 & 0.017 \\
\hline 3.25 & 24 & 37 & 30 & 22 & 27 & 0.089 & 0.081 & 0.079 & 0.051 & 0.030 \\
\hline 3.75 & 8 & 25 & 28 & 42 & 17 & 0.135 & 0.124 & 0.128 & 0.078 & 0.053 \\
\hline 4.25 & 3 & 12 & 40 & 45 & 24 & 0.158 & 0.170 & 0.195 & 0.160 & 0.076 \\
\hline 4.75 & 1 & 6 & 29 & 32 & 39 & 0.170 & 0.200 & 0.339 & 0.288 & 0.121 \\
\hline 5.25 & 0 & 3 & 13 & 34 & 26 & 0.175 & 0.224 & 0.484 & 0.417 & 0.225 \\
\hline 5.75 & 0 & 1 & 5 & 15 & 33 & 0.175 & 0.239 & 0.573 & 0.597 & 0.320 \\
\hline 6.25 & 0 & 10 & 2 & 10 & 20 & 0.175 & 0.245 & 0.619 & 0.700 & 0.481 \\
\hline 6.75 & 13 & 16 & o & 4 & 18 & 0.175 & 0.331 & 0.642 & 0.786 & 0.602 \\
\hline 7.25 & 16 & 21 & o & 8 & 6 & 0.399 & 0.508 & 0.774 & 0.831 & 0.741 \\
\hline 7.75 & 8 & 10 & 2 & 2 & 3 & 0.736 & 0.777 & 0.933 & 0.944 & 0.798 \\
\hline 8.25 & 2 & 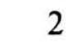 & 1 & 1 & 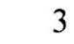 & 0.941 & 0.936 & 0.975 & 0.978 & 0.835 \\
\hline 8.75 & 0 & 0 & a & 0 & 2 & 1.000 & 0.974 & 1.000 & 1.000 & 0.875 \\
\hline 9.25 & 0 & 1 & 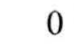 & 0 & 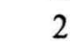 & 1.000 & 0.974 & 1.000 & 1.000 & 0.911 \\
\hline 9.75 & 0 & 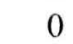 & 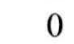 & 0 & 2 & 1.000 & 1.000 & 1.000 & 1.000 & 0.950 \\
\hline 10.25 & 0 & 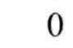 & 0 & 0 & 0 & 1.000 & 1.000 & 1.000 & 1.000 & 1.000 \\
\hline 10.75 & 0 & 0 & 0 & 0 & 0 & 1.000 & 1.000 & 1.000 & 1.000 & 1.000 \\
\hline 11.25 & 0 & 0 & 0 & 0 & 0 & 1.000 & 1.000 & 1.000 & 1.000 & 1.000 \\
\hline 11.75 & 0 & 0 & 0 & 0 & 0 & 1.000 & 1.000 & 1.000 & 1.000 & 1.000 \\
\hline 12.25 & 0 & 0 & 0 & 0 & 0 & 1.000 & 1.000 & 1.000 & 1.000 & 1.000 \\
\hline 12.75 & 0 & 0 & 0 & 0 & 0 & 1.000 & 1.000 & 1.000 & 1.000 & 1.000 \\
\hline 13.25 & 0 & 0 & 0 & 0 & 0 & 1.000 & 1.000 & 1.000 & 1.000 & 1.000 \\
\hline Total & 272 & 427 & 411 & 452 & 364 & & & & & \\
\hline
\end{tabular}


Table N.5 Measured dripping drop size distribution for slat E.

\begin{tabular}{|c|c|c|c|c|c|c|c|c|c|c|}
\hline & \multicolumn{5}{|c|}{ FREQUENCY } & \multicolumn{5}{|c|}{ CUMULATIVE MASS FRACTION } \\
\hline $\begin{array}{c}\Gamma, g / m s \\
\text { d, } m m\end{array}$ & $\begin{array}{l}18 \\
\mathbf{N}\end{array}$ & $\begin{array}{l}35 \\
\mathbf{N}\end{array}$ & $\begin{array}{l}54 \\
\mathbf{N}\end{array}$ & $\begin{array}{l}74 \\
\text { N }\end{array}$ & $\begin{array}{c}131 \\
\mathbf{N}\end{array}$ & $\begin{array}{c}18 \\
\mathbf{R},-\end{array}$ & $\begin{array}{c}35 \\
\mathbf{R},-\end{array}$ & $\begin{array}{c}54 \\
\mathbf{R},-\end{array}$ & $\begin{array}{l}74 \\
\text { R. - }\end{array}$ & $\begin{array}{l}131 \\
\mathbf{R},-\end{array}$ \\
\hline 0.25 & 8 & 4 & $\overline{6}$ & 5 & 12 & 0.000 & 0.000 & 0.000 & 0.000 & 0.000 \\
\hline 0.75 & 34 & 20 & 44 & 52 & 22 & 0.000 & 0.000 & 0.000 & 0.000 & 0.000 \\
\hline 1.25 & 131 & 47 & 29 & 36 & 11 & 0.001 & 0.000 & 0.001 & 0.001 & 0.000 \\
\hline 1.75 & 83 & 59 & 35 & 33 & 22 & 0.009 & 0.004 & 0.003 & 0.003 & 0.001 \\
\hline 2.25 & 83 & 43 & 33 & 42 & 13 & 0.023 & 0.014 & 0.010 & 0.010 & 0.004 \\
\hline 2.75 & 34 & 40 & 44 & 51 & 11 & 0.053 & 0.031 & 0.025 & 0.028 & 0.008 \\
\hline 3.25 & 5 & 28 & 62 & 51 & 28 & 0.073 & 0.060 & 0.060 & 0.068 & 0.015 \\
\hline 3.75 & 2 & 33 & 68 & 40 & 24 & 0.079 & 0.091 & 0.142 & 0.133 & 0.040 \\
\hline 4.25 & 1 & 25 & 58 & 41 & 30 & 0.082 & 0.147 & 0.277 & 0.209 & 0.074 \\
\hline 4.75 & 2 & 19 & 25 & 33 & 30 & 0.084 & 0.209 & 0.440 & 0.324 & 0.138 \\
\hline 5.25 & 5 & 14 & 19 & 28 & 16 & 0.092 & 0.275 & 0.538 & 0.451 & 0.226 \\
\hline 5.75 & 3 & 13 & 15 & 16 & 12 & 0.116 & 0.344 & 0.639 & 0.596 & 0.290 \\
\hline 6.25 & 48 & 32 & 11 & 18 & 12 & 0.134 & 0.424 & 0.746 & 0.705 & 0.353 \\
\hline 6.75 & 48 & 28 & 11 & 8 & 11 & 0.526 & 0.685 & 0.846 & 0.861 & 0.434 \\
\hline 7.25 & 2 & 3 & 1 & 2 & 9 & 0.976 & 0.966 & 0.969 & 0.951 & 0.526 \\
\hline 7.75 & 0 & 0 & 1 & 0 & 3 & 1.000 & 1.000 & 0.982 & 0.976 & 0.619 \\
\hline 8.25 & 0 & 0 & 0 & 0 & 3 & 1.000 & 1.000 & 1.000 & 0.976 & 0.655 \\
\hline 8.75 & 0 & 0 & 0 & 1 & 2 & 1.000 & 1.000 & 1.000 & 0.976 & 0.702 \\
\hline 9.25 & 0 & 0 & 0 & 0 & 2 & 1.000 & 1.000 & 1.000 & 1.000 & 0.739 \\
\hline 9.75 & 0 & 0 & 0 & 0 & 2 & 1.000 & 1.000 & 1.000 & 1.000 & 0.781 \\
\hline 10.25 & 0 & 0 & 0 & 0 & 1 & 1.000 & 1.000 & 1.000 & 1.000 & 0.832 \\
\hline 10.75 & 0 & 0 & 0 & 0 & 1 & 1.000 & 1.000 & 1.000 & 1.000 & 0.862 \\
\hline 11.25 & 0 & 0 & 0 & 0 & 0 & 1.000 & 1.000 & 1.000 & 1.000 & 0.894 \\
\hline 11.75 & 0 & 0 & 0 & 0 & 1 & 1.000 & 1.000 & 1.000 & 1.000 & 0.894 \\
\hline 12.25 & 0 & 0 & 0 & 0 & 0 & 1.000 & 1.000 & 1.000 & 1.000 & 0.941 \\
\hline 12.75 & 0 & 0 & 0 & 0 & 1 & 1.000 & 1.000 & 1.000 & 1.000 & 0.941 \\
\hline 13.25 & 0 & 0 & 0 & 0 & 0 & 1.000 & 1.000 & 1.000 & 1.000 & 1.000 \\
\hline Total & 489 & 408 & 462 & 457 & 279 & & & & & \\
\hline
\end{tabular}




\section{APPENDIX $O$ Summary of data obtained from experiments to determine the heat transfer coefficients on the outside of tubes with different profiles}

Table 0.1 Experimental data for heat transfer from tubes of different profiles.

\begin{tabular}{|c|c|c|c|c|c|c|c|c|}
\hline $\begin{array}{c}\text { Tube } \\
-\end{array}$ & $\begin{array}{c}\mathbf{T}_{\text {steam }} \\
{ }^{\circ} \mathrm{C}\end{array}$ & $\begin{array}{c}\mathbf{v}_{\mathbf{a}} \\
\mathrm{m} / \mathrm{s}\end{array}$ & $\begin{array}{l}\mathbf{T}_{\mathbf{a}} \\
{ }^{\circ} \mathrm{C}\end{array}$ & $\begin{array}{c}\mathbf{m}_{\text {cond }} \\
g\end{array}$ & $\begin{array}{l}\mathrm{t} \\
s\end{array}$ & $\begin{array}{c}\mathbf{Q}_{\text {total }} \\
W\end{array}$ & $\begin{array}{c}\mathbf{R e}_{\mathbf{b}} \\
- \\
\end{array}$ & $\begin{array}{c}\mathbf{N} \mathbf{u}_{\mathbf{b}} \\
- \\
\end{array}$ \\
\hline $\mathrm{A}$ & 99.7 & 0.62 & 25.5 & 55.7 & 600 & 209.5 & 795.5 & 15.94 \\
\hline A & 99.7 & 1.02 & 26.5 & 65.9 & 600 & 248.0 & 1305.4 & 20.60 \\
\hline A & 99.7 & 1.50 & 26.5 & 45.4 & 360 & 284.8 & 1919.7 & 24.77 \\
\hline A & 99.7 & 1.50 & 26.3 & 77.1 & 600 & 290.3 & 1921.0 & 25.30 \\
\hline A & 99.7 & 2.00 & 25.0 & 85.4 & 600 & 321.5 & 2569.6 & 28.31 \\
\hline A & 99.7 & 2.00 & 25.0 & 86.9 & 600 & 327.0 & 2569.6 & 28.92 \\
\hline A & 99.7 & 2.50 & 24.0 & 95.9 & 600 & 361.0 & 3220.4 & 32.25 \\
\hline A & 99.7 & 2.85 & 23.5 & 82.3 & 480 & 387.0 & 3676.0 & 34.86 \\
\hline B & 99.7 & 0.62 & 22.5 & 56.1 & 600 & 211.3 & 808.2 & 13.87 \\
\hline B & 99.7 & 1.00 & 22.0 & 64.4 & 600 & 242.5 & 1305.3 & 16.93 \\
\hline B & 99.7 & 1.50 & 22.0 & 78.1 & 600 & 294.0 & 1958.0 & 22.12 \\
\hline B & 99.7 & 2.00 & 21.0 & 88.9 & 600 & 334.4 & 2617.4 & 25.83 \\
\hline B & 99.7 & 2.50 & 21.0 & 99.6 & 600 & 374.8 & 3271.8 & 29.86 \\
\hline B & 99.7 & 2.90 & 21.0 & 86.9 & 480 & 408.8 & 3795.3 & 33.24 \\
\hline $\mathrm{C}$ & 99.8 & 0.62 & 19.0 & 59.4 & 605 & 221.5 & 1157.4 & 19.90 \\
\hline $\mathrm{C}$ & 99.8 & 1.00 & 19.0 & 68.4 & 600 & 257.2 & 1866.8 & 24.84 \\
\hline $\mathrm{C}$ & 99.8 & 1.50 & 19.5 & 81.0 & 600 & 305.0 & 2796.6 & 31.64 \\
\hline $\mathrm{C}$ & 99.8 & 2.00 & 20.5 & 91.3 & 600 & 343.5 & 3719.1 & 37.49 \\
\hline $\mathrm{C}$ & 99.8 & 2.50 & 20.5 & 101.6 & 600 & 382.1 & 4648.8 & 42.89 \\
\hline $\mathrm{C}$ & 99.8 & 2.85 & 20.8 & 87.9 & 480 & 413.3 & 5296.2 & 47.41 \\
\hline D & 99.8 & 0.63 & 25.0 & 53.7 & 600 & 202.1 & 623.1 & 10.50 \\
\hline $\mathrm{D}$ & 99.8 & 1.04 & 24.9 & 64.0 & 600 & 240.6 & 1028.8 & 13.60 \\
\hline $\mathrm{D}$ & 99.8 & 1.52 & 25.0 & 75.2 & 600 & 282.9 & 1503.3 & 17.03 \\
\hline $\mathrm{D}$ & 99.8 & 2.01 & 24.8 & 84.0 & 600 & 316.0 & 1988.9 & 19.64 \\
\hline $\mathrm{D}$ & 99.8 & 2.49 & 24.8 & 92.8 & 600 & 349.0 & 2463.8 & 22.30 \\
\hline D & 99.8 & 2.90 & 24.5 & 99.4 & 600 & 374.0 & 2871.8 & 24.20 \\
\hline$E$ & 99.8 & 0.65 & 23.5 & 57.1 & 600 & 214.9 & 1086.9 & 18.96 \\
\hline$E$ & 99.8 & 1.02 & 22.5 & 65.9 & 600 & 248.0 & 1710.0 & 23.03 \\
\hline$E$ & 99.8 & 1.50 & 22.0 & 77.1 & 600 & 290.2 & 2518.0 & 28.40 \\
\hline$E$ & 99.8 & 2.00 & 22.0 & 73.8 & 510 & 326.8 & 3357.4 & 33.18 \\
\hline$E$ & 99.8 & 2.50 & 21.5 & 77.6 & 490 & 357.6 & 4202.2 & 36.97 \\
\hline$E$ & 99.8 & 2.90 & 21.5 & 82.8 & 480 & 389.2 & 4874.6 & 41.07 \\
\hline $\mathrm{F}$ & 99.8 & 0.64 & 21.5 & 88.9 & 780 & 257.2 & 1074.0 & 14.31 \\
\hline $\mathrm{F}$ & 99.8 & 1.04 & 22.0 & 80.1 & 600 & 301.3 & 1743.0 & 18.42 \\
\hline $\mathrm{F}$ & 99.8 & 1.51 & 22.2 & 93.7 & 600 & 352.7 & 2529.4 & 23.12 \\
\hline $\mathrm{F}$ & 99.8 & 2.00 & 23.0 & 106.4 & 600 & 400.5 & 3343.3 & 27.77 \\
\hline $\mathrm{F}$ & 99.8 & 2.50 & 24.5 & 118.2 & 600 & 444.6 & 4162.8 & 32.50 \\
\hline $\mathrm{F}$ & 99.8 & 2.90 & 25.4 & 103.5 & 480 & 486.8 & 4817.6 & 36.92 \\
\hline
\end{tabular}


APPENDIX P Summary of drop size distribution data obtained for the water distribution system

Table P.1 Measured drop size distribution for the water distribution system of the cooling tower packing material test facility.

\begin{tabular}{|c|c|c|c|c|c|c|}
\hline$\dot{\mathbf{m}}_{\mathbf{w}}, \mathrm{kg} / \mathrm{s}$ & 3.1 & 4.4 & 6.2 & 7.3 & 8.9 & 10.6 \\
\hline $\mathbf{d}, \mathrm{mm}$ & $\mathbf{R},-$ & $\mathbf{R},-$ & $\mathbf{R},-$ & $\mathbf{R},-$ & $\mathbf{R},-$ & $\mathbf{R},-$ \\
\hline 0.25 & 0.0000 & 0.0001 & 0.0001 & 0.0001 & 0.0002 & 0.0005 \\
0.75 & 0.0013 & 0.0020 & 0.0042 & 0.0029 & 0.0070 & 0.0203 \\
1.25 & 0.0049 & 0.0066 & 0.0116 & 0.0142 & 0.0247 & 0.0477 \\
1.75 & 0.0105 & 0.0127 & 0.0181 & 0.0289 & 0.0484 & 0.0802 \\
2.25 & 0.0151 & 0.0199 & 0.0272 & 0.0488 & 0.0759 & 0.1123 \\
2.75 & 0.0252 & 0.0325 & 0.0372 & 0.0756 & 0.1086 & 0.1416 \\
3.25 & 0.0539 & 0.0579 & 0.0531 & 0.1080 & 0.1534 & 0.1779 \\
3.75 & 0.1107 & 0.0931 & 0.0757 & 0.1534 & 0.2041 & 0.2205 \\
4.25 & 0.2262 & 0.1690 & 0.1121 & 0.2006 & 0.2583 & 0.2826 \\
4.75 & 0.4254 & 0.2852 & 0.1647 & 0.2762 & 0.3224 & 0.3493 \\
5.25 & 0.6727 & 0.4197 & 0.2513 & 0.3650 & 0.3963 & 0.4273 \\
5.75 & 0.8457 & 0.5670 & 0.3480 & 0.4242 & 0.4970 & 0.5298 \\
6.25 & 0.9093 & 0.6513 & 0.4069 & 0.4868 & 0.5712 & 0.5854 \\
6.75 & 0.9492 & 0.7430 & 0.4645 & 0.5544 & 0.6527 & 0.6364 \\
7.25 & 0.9705 & 0.8202 & 0.5360 & 0.6522 & 0.7088 & 0.6838 \\
7.75 & 0.9791 & 0.8923 & 0.5858 & 0.7119 & 0.7727 & 0.7707 \\
8.25 & 1.0000 & 0.9458 & 0.6661 & 0.7582 & 0.8388 & 0.7823 \\
8.75 & 1.0000 & 0.9777 & 0.7319 & 0.8073 & 0.8914 & 0.8518 \\
9.25 & 1.0000 & 0.9872 & 0.7813 & 0.8726 & 0.9147 & 0.8846 \\
9.75 & 1.0000 & 0.9872 & 0.8392 & 0.9065 & 0.9511 & 0.9038 \\
10.25 & 1.0000 & 1.0000 & 0.8969 & 0.9361 & 0.9617 & 0.9485 \\
10.75 & 1.0000 & 1.0000 & 0.9746 & 0.9703 & 0.9860 & 1.0000 \\
11.25 & 1.0000 & 1.0000 & 1.0000 & 0.9703 & 1.0000 & 1.0000 \\
11.75 & 1.0000 & 1.0000 & 1.0000 & 1.0000 & 1.0000 & 1.0000 \\
\hline
\end{tabular}




\section{APPENDIX Q Drop size distribution data from measurements in a cooling tower fitted with splash pack}

Table Q.1 Drop size distribution in a cooling tower fitted with splash pack.

\begin{tabular}{|c|c|c|c|c|}
\hline $\mathbf{W}, m m$ & 9 & 9 & 25 & 25 \\
$\mathbf{M}_{\mathbf{w}}, \mathrm{kg} / \mathrm{m}^{2} s$ & 1.8 & 3.0 & 1.8 & 3.0 \\
\hline $\mathbf{d}, m m$ & $\mathbf{R},-$ & $\mathbf{R},-$ & $\mathbf{R},-$ & $\mathbf{R}-$ \\
\hline 0.1 & 0.0000 & 0.0000 & 0.0000 & 0.0000 \\
0.3 & 0.0005 & 0.0003 & 0.0004 & 0.0002 \\
0.5 & 0.0039 & 0.0030 & 0.0031 & 0.0020 \\
0.7 & 0.0140 & 0.0102 & 0.0104 & 0.0078 \\
0.9 & 0.0301 & 0.0233 & 0.0247 & 0.0177 \\
1.1 & 0.0496 & 0.0380 & 0.0441 & 0.0320 \\
1.3 & 0.0724 & 0.0535 & 0.0627 & 0.0472 \\
1.5 & 0.0926 & 0.0710 & 0.0835 & 0.0616 \\
1.7 & 0.1179 & 0.0908 & 0.1091 & 0.0795 \\
1.9 & 0.1419 & 0.1123 & 0.1378 & 0.0975 \\
2.1 & 0.1671 & 0.1299 & 0.1669 & 0.1187 \\
2.3 & 0.1931 & 0.1504 & 0.1905 & 0.1415 \\
2.5 & 0.2141 & 0.1640 & 0.2188 & 0.1665 \\
2.7 & 0.2451 & 0.1854 & 0.2513 & 0.1924 \\
2.9 & 0.2885 & 0.2090 & 0.2958 & 0.2223 \\
3.1 & 0.3208 & 0.2381 & 0.3284 & 0.2502 \\
3.3 & 0.3541 & 0.2564 & 0.3656 & 0.2725 \\
3.5 & 0.3886 & 0.2864 & 0.4030 & 0.2974 \\
3.7 & 0.4106 & 0.3217 & 0.4364 & 0.3363 \\
3.9 & 0.4452 & 0.3447 & 0.4873 & 0.3604 \\
4.1 & 0.4977 & 0.3685 & 0.5124 & 0.3790 \\
4.3 & 0.5273 & 0.3998 & 0.5706 & 0.4146 \\
4.5 & 0.5569 & 0.4093 & 0.5991 & 0.4434 \\
4.7 & 0.5899 & 0.4307 & 0.6623 & 0.4855 \\
4.9 & 0.6343 & 0.4350 & 0.6766 & 0.5289 \\
5.1 & 0.6697 & 0.4534 & 0.7010 & 0.5716 \\
5.3 & 0.7106 & 0.4848 & 0.7284 & 0.5849 \\
5.5 & 0.7546 & 0.5311 & 0.7585 & 0.6314 \\
5.7 & 0.8239 & 0.5444 & 0.7809 & 0.6643 \\
5.9 & 0.8455 & 0.5659 & 0.8307 & 0.6828 \\
6.1 & 0.8817 & 0.5739 & 0.8865 & 0.7037 \\
6.3 & 0.9232 & 0.6182 & 0.9316 & 0.7499 \\
6.5 & 0.9525 & 0.6674 & 0.9316 & 0.7993 \\
6.7 & 0.9525 & 0.7102 & 0.9692 & 0.7993 \\
6.9 & 0.9525 & 0.7219 & 0.9692 & 0.8139 \\
7.1 & 0.9525 & 0.7347 & 0.9692 & 0.8475 \\
7.3 & 0.9525 & 0.7488 & 0.9692 & 0.8475 \\
7.5 & 0.9755 & 0.7635 & 0.9692 & 0.8475 \\
7.7 & 1.0000 & 0.8115 & 0.9692 & 0.8679 \\
7.9 & 1.0000 & 0.8285 & 1.0000 & 0.9118 \\
8.1 & 1.0000 & 0.9217 & 1.0000 & 0.9352 \\
8.3 & 1.0000 & 0.9421 & 1.0000 & 0.9352 \\
8.5 & 1.0000 & 0.9421 & 1.0000 & 0.9352 \\
8.7 & 1.0000 & 0.9421 & 1.0000 & 0.9644 \\
8.9 & 1.0000 & 0.9421 & 1.0000 & 0.9644 \\
9.1 & 1.0000 & 0.9421 & 1.0000 & 0.9644 \\
9.3 & 1.0000 & 1.0000 & 1.0000 & 1.0000 \\
\hline & & & & \\
\hline
\end{tabular}


APPENDIX R Summary of measured pressure drop across experimental splash grids during dry operation

Table R.1 Measured single phase pressure drop across the experimental splash grids.

\begin{tabular}{|c|c|c|c|c|c|c|}
\hline Layout & $\begin{array}{l}\mathbf{N} \\
-\end{array}$ & $\begin{array}{l}\beta \\
-\end{array}$ & $\begin{array}{c}\mathbf{v}_{\mathbf{a}} \\
\mathrm{m} / \mathrm{s}\end{array}$ & $\begin{array}{c}\mathbf{R e} \\
- \\
\end{array}$ & $\begin{array}{l}\Delta \mathbf{p} \\
P a\end{array}$ & $\begin{array}{c}K_{\Delta p, \text { grid }} \\
-\end{array}$ \\
\hline A & 10 & 0.820 & 1.50 & 864 & 4.0 & 0.304 \\
\hline A & 10 & 0.820 & 2.00 & 1152 & 8.3 & 0.354 \\
\hline A & 10 & 0.820 & 2.53 & 1458 & 13.0 & 0.347 \\
\hline A & 10 & 0.820 & 3.09 & 1781 & 19.5 & 0.349 \\
\hline A & 10 & 0.820 & 3.50 & 2017 & 25.4 & 0.354 \\
\hline A & 10 & 0.820 & 4.09 & 2357 & 36.2 & 0.370 \\
\hline B & 10 & 0.820 & 1.49 & 869 & 4.0 & 0.306 \\
\hline B & 10 & 0.820 & 2.03 & 1184 & 6.5 & 0.268 \\
\hline B & 10 & 0.820 & 2.53 & 1475 & 9.4 & 0.249 \\
\hline B & 10 & 0.820 & 3.11 & 1813 & 15.2 & 0.267 \\
\hline B & 10 & 0.820 & 3.53 & 2058 & 18.1 & 0.246 \\
\hline B & 10 & 0.820 & 4.10 & 2390 & 28.5 & 0.288 \\
\hline C & 7 & 0.820 & 1.51 & 888 & 3.0 & 0.317 \\
\hline C & 7 & 0.820 & 2.04 & 1200 & 5.7 & 0.330 \\
\hline C & 7 & 0.820 & 2.53 & 1488 & 8.0 & 0.301 \\
\hline C & 7 & 0.820 & 3.08 & 1812 & 13.0 & 0.330 \\
\hline C & 7 & 0.820 & 3.53 & 2077 & 17.4 & 0.337 \\
\hline C & 7 & 0.820 & 4.08 & 2400 & 24.3 & 0.352 \\
\hline D & 10 & 0.892 & 1.50 & 899 & 2.0 & 0.148 \\
\hline D & 10 & 0.892 & 2.03 & 1217 & 3.6 & 0.146 \\
\hline D & 10 & 0.892 & 2.57 & 1541 & 5.2 & 0.131 \\
\hline D & 10 & 0.892 & 3.11 & 1865 & 8.4 & 0.145 \\
\hline D & 10 & 0.892 & 3.55 & 2129 & 10.5 & 0.139 \\
\hline D & 10 & 0.892 & 4.08 & 2447 & 14.3 & 0.143 \\
\hline E & 10 & 0.892 & 1.48 & 888 & 1.3 & 0.099 \\
\hline E & 10 & 0.892 & 2.03 & 1218 & 2.5 & 0.102 \\
\hline E & 10 & 0.892 & 2.54 & 1524 & 4.2 & 0.109 \\
\hline E & 10 & 0.892 & 3.08 & 1847 & 5.1 & 0.090 \\
\hline E & 10 & 0.892 & 3.53 & 2117 & 7.0 & 0.094 \\
\hline E & 10 & 0.892 & 4.12 & 2471 & 9.8 & 0.097 \\
\hline E & 10 & 0.892 & 4.11 & 2465 & 12.1 & 0.120 \\
\hline $\mathrm{F}$ & 7 & 0.892 & 1.50 & 880 & 1.5 & 0.161 \\
\hline $\mathrm{F}$ & 7 & 0.892 & 2.06 & 1209 & 2.3 & 0.131 \\
\hline $\mathrm{F}$ & 7 & 0.892 & 2.55 & 1496 & 3.5 & 0.130 \\
\hline $\mathrm{F}$ & 7 & 0.892 & 3.06 & 1795 & 5.0 & 0.129 \\
\hline $\mathrm{F}$ & 7 & 0.892 & 3.51 & 2059 & 6.5 & 0.128 \\
\hline $\mathrm{F}$ & 7 & 0.892 & 4.10 & 2406 & 9.0 & 0.130 \\
\hline G & 10 & 0.667 & 1.49 & 2431 & 11.8 & 0.899 \\
\hline G & 10 & 0.667 & 2.01 & 3279 & 23.0 & 0.962 \\
\hline G & 10 & 0.667 & 2.54 & 4143 & 36.2 & 0.949 \\
\hline G & 10 & 0.667 & 3.07 & 5008 & 53.5 & 0.960 \\
\hline G & 10 & 0.667 & 3.51 & 5726 & 71.0 & 0.974 \\
\hline G & 10 & 0.667 & 4.03 & 6574 & 97.8 & 1.018 \\
\hline $\mathrm{H}$ & 10 & 0.667 & 1.49 & 2376 & 10.8 & 0.833 \\
\hline $\mathrm{H}$ & 10 & 0.667 & 2.01 & 3205 & 21.0 & 0.890 \\
\hline $\mathrm{H}$ & 10 & 0.667 & 2.51 & 4003 & 33.0 & 0.897 \\
\hline $\mathrm{H}$ & 10 & 0.667 & 3.06 & 4880 & 49.3 & 0.902 \\
\hline $\mathrm{H}$ & 10 & 0.667 & 3.50 & 5582 & 64.5 & 0.902 \\
\hline $\mathrm{H}$ & 10 & 0.667 & 4.05 & 6459 & 88.5 & 0.924 \\
\hline
\end{tabular}


Table R.1 (continued) Measured single phase pressure drop across the experimental splash grids.

\begin{tabular}{|c|c|c|c|c|c|c|}
\hline Layout & $\mathbf{N}$ & $\boldsymbol{\beta}$ & $\mathbf{v}_{\mathbf{a}}$ & $\mathbf{R e}$ & \multicolumn{1}{|c|}{$\boldsymbol{\Delta} \mathbf{p}$} & $\mathbf{K}_{\Delta \mathbf{p}, \mathbf{g r i d}}$ \\
& - & - & $m / s$ & - & $P a$ & - \\
\hline $\mathrm{I}$ & 7 & 0.667 & 1.49 & 2480 & 8.2 & 0.883 \\
$\mathrm{I}$ & 7 & 0.667 & 2.01 & 3345 & 15.0 & 0.888 \\
$\mathrm{I}$ & 7 & 0.667 & 2.54 & 4227 & 25.0 & 0.926 \\
$\mathrm{I}$ & 7 & 0.667 & 3.06 & 5093 & 36.7 & 0.937 \\
$\mathrm{I}$ & 7 & 0.667 & 3.50 & 5825 & 48.5 & 0.947 \\
$\mathrm{I}$ & 7 & 0.667 & 4.05 & 6741 & 67.0 & 0.977 \\
$\mathrm{~J}$ & 10 & 0.800 & 1.49 & 2428 & 5.5 & 0.420 \\
$\mathrm{~J}$ & 10 & 0.800 & 2.03 & 3309 & 10.2 & 0.420 \\
$\mathrm{~J}$ & 10 & 0.800 & 2.54 & 4140 & 16.0 & 0.420 \\
$\mathrm{~J}$ & 10 & 0.800 & 3.11 & 5069 & 24.3 & 0.426 \\
$\mathrm{~J}$ & 10 & 0.800 & 3.5 & 5737 & 31.7 & 0.434 \\
$\mathrm{~J}$ & 10 & 0.800 & 4.12 & 6715 & 44.0 & 0.439 \\
$\mathrm{~K}$ & 10 & 0.800 & 1.48 & 2395 & 5.5 & 0.427 \\
$\mathrm{~K}$ & 10 & 0.800 & 2.04 & 3301 & 10.2 & 0.417 \\
$\mathrm{~K}$ & 10 & 0.800 & 2.54 & 4110 & 16.3 & 0.430 \\
$\mathrm{~K}$ & 10 & 0.800 & 3.08 & 4984 & 23.0 & 0.412 \\
$\mathrm{~K}$ & 10 & 0.800 & 3.53 & 5712 & 30.5 & 0.416 \\
$\mathrm{~K}$ & 10 & 0.800 & 4.12 & 6666 & 41.7 & 0.418 \\
$\mathrm{~L}$ & 7 & 0.860 & 1.50 & 2452 & 3.8 & 0.407 \\
$\mathrm{~L}$ & 7 & 0.800 & 1.99 & 3253 & 7.0 & 0.426 \\
$\mathrm{~L}$ & 7 & 0.800 & 2.54 & 4152 & 11.3 & 0.422 \\
$\mathrm{~L}$ & 7 & 0.800 & 3.08 & 5034 & 17.0 & 0.432 \\
$\mathrm{~L}$ & 7 & 0.800 & 3.51 & 5737 & 22.2 & 0.434 \\
$\mathrm{~L}$ & 7 & 0.800 & 4.08 & 6669 & 30.7 & 0.444 \\
\hline
\end{tabular}




\section{APPENDIX S Program for the simulation of the thermal performance of counterflow cooling tower splash pack}

The source code for the simulation program is available from:

Prof. P.J. Erens

Department of Mechanical Engineering

University of Stellenbosch

Stellenbosch

7600

South Africa 